\title{
Colloid-Facilitated Transport of Low- Solubility Radionuclides: A Field, Experimental, and Modeling Investigation
}

Editors: A. B. Kersting, P. W. Reimus Contributors: A. Abdel-Fattah, P. G. Allen, I. Anghel, F. C. Benedict, B. K. Esser, N. Lu, K. S. Kung, J. Nelson, M. P. Neu, S. D. Reilly, D. K. Smith, E. R. Sylwester, L. Wang, S. D. Ware, R. G. Warren, R. W. Williams, Z. Zavarin, $P$. Zhao

U.S. Department of Energy

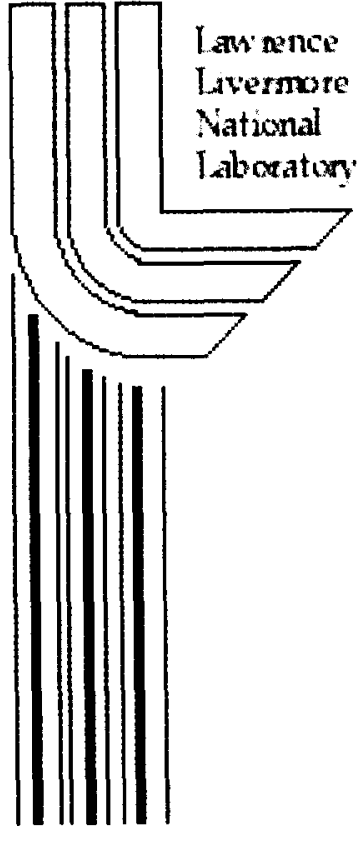

February 2003 


\section{DISCLAIMER}

This document was prepared as an account of work sponsored by an agency of the United States Government. Neither the United States Government nor the University of California nor any of their employees, makes any warranty, express or implied, or assumes any legal liability or responsibility for the accuracy, completeness, or usefulness of any information, apparatus, product, or process disclosed, or represents that its use would not infringe privately owned rights. Reference herein to any specific commercial product, process, or service by trade name, trademark, manufacturer, or otherwise, does not necessarily constitute or imply its endorsement, recommendation, or favoring by the United States Government or the University of California. The views and opinions of authors expressed herein do not necessarily state or reflect those of the United States Government or the University of California, and shall not be used for advertising or product endorsement purposes.

This report has been reproduced directly from the best available copy.

Available electronically at http://www.doc.gov/bridge

Available for a processing fee to U.S. Department of Energy

And its contractors in paper from

U.S. Department of Energy

Office of Scientific and Technical Information

P.O. Box 62

Oak Ridge, TN 37831-0062

Telephone: (865) 576-8401

Facsimile: (865) 576-5728

E-mail: reports@adonis.osti.gov

Available for the sale to the public from

U.S. Department of Commerce

National Technical Information Service

5285 Port Royal Road

Springfield, VA 22161

Telephone: (800) 553-6847

Facsimile: (703) 605-6900

E-mail: orders@ntis.fedworld.gov

Online ordering: http://www.ntis.gov/ordering.htm

\section{OR}

Lawrence Livermore National Laboratory

Technical Information Department's Digital Library

http://www.llnl.gov/tid/Library.html 


\title{
Colloid-Facilitated Transport of Low-Solubility Radionuclides: A Field, Experimental, and Modeling Investigation
}

\author{
Editors
}

Annie B. Kersting

Paul W. Reimus

Contributors:
A. Abdel-Fattah ${ }^{2}$
P. G. Allen ${ }^{1}$
I. Anghel ${ }^{2}$
F. C. Benedict ${ }^{4}$
B. K. Esser $^{1}$
N. $\mathrm{Lu}^{2}$
K.S. Kung ${ }^{2}$
J. Nelson ${ }^{1}$
M. P. $\mathrm{Neu}^{2}$
S. D. Reilly ${ }^{2}$
D. K. Smith ${ }^{1}$
E. R. Sylwester ${ }^{1}$
L. Wang ${ }^{3}$
S. D. Ware
R. G. Warren ${ }^{2}$
R. W. Williams ${ }^{1}$
M. Zavarin ${ }^{1}$
P. Zhao ${ }^{1}$

February, 2003
(1)LLNL
(2) LANL

(3) University of Michigan

(4) Shaw E\&I Corporation

This work was performed under the auspices of the U.S. Department of Energy by the University of California, Lawrence Livermore National Laboratory under Contract No. W-7405-Eng-48. 


\section{Table of Contents}

\section{Chapter}

$\underline{\text { Title }}$

$\underline{\text { Page }}$

Executive

Summary

i

Introduction $\quad$ vi

Chapter 1 Background Literature Review on Colloids in Groundwater

1

Chapter 2 Characterization of Colloids in Groundwater Collected at the

Nevada Test Site

Chapter 3 Characterization of Colloids in Water From a Vadose Zone:

Rainier Mesa, Nevada Test Site

Chapter 4

Sorption of $\mathrm{Pu}(\mathrm{IV})$ on Mineral Colloids

Chapter 5

Sorption of $\mathrm{Pu}(\mathrm{V})$ on Mineral Colloids

67

Chapter 6

Summary of Radionuclide-Colloid Batch Sorption and

Desorption Measurements for the Yucca Mountain Project

Chapter 7

Colloid-Facilitated Plutonium Fracture Transport

93

Experiments

Chapter 8

Quantitative Interpretation of Fracture Transport Experiments and Associated Batch Sorption/Desorption Experiments

Chapter 9

Characterization of Fracture Cores post Colloid Flow-

156 Through Experiments

Chapter 10 Colloid Transport Parameters Derived From Field-Scale

183 Tracer Tests and Laboratory Studies 
Chapter 11 Implications of Experimental Results and Interpretations and Previous Studies for CAU-Scale Colloid-Facilitated Radionuclide Transport

Chapter 12 Recommendations

Appendix A Optical Petrography and Scanning Electron Microscope

Descriptions of Representative Fracture Surfaces from Cores Used in Transport Experiments

Appendix B

Appendix C

Appendix D

Appendix E
Appendix F

Stability Studies of Ca-Montmorillonite Colloids

Plots of Normalized Concentrations and Recoveries of Tritiated Water, Colloids, and Plutonium in Each of the Fracture Transport Experiments

Simultaneous RELAP Fits to Tritiated Water Data in Each Pair of Fracture Experiments for a Given Colloid

RELAP Fits to Colloid Data in Each Pair of Fracture Experiments for a Given Colloid

Model Simulations of Colloid and Pu Responses in all Fracture Transport Experiments Using the Numerical Model Described in Chapter 8

Appendix G Thermodynamic Data Used to fit Pu Sorption Data 255

249 


\section{Executive Summary}

Until recently, radionuclides that have a low solubility in groundwater (e.g. $\mathrm{Pu}, \mathrm{Am}, \mathrm{Eu}$ ) were considered immobile due to their ability to strongly sorb to rock and sediment. Yet, there is an increasing body of evidence to suggest that the existence of naturally occurring inorganic and organic colloids (1-1000 nm sized particles) may enhance contaminant transport through sorption (Buddemeier and Hunt, 1998; Degueldre et al., 1989; Kim, 1991; McDowell-Boyer, 1992; McCarthy et al., 1998; Kersting et al., 1999). Due to their small size, colloids have the ability to be transported with groundwater. In addition, colloids have the capacity to sorb significant quantities of contaminants due to their large surface area per unit mass.

Kersting et al. (1999) recently showed that low levels of Pu and other radionuclides were transported over $1 \mathrm{~km}$ in a saturated, fracture-flow volcanic system at the Nevada Test Site (NTS). The low-solubility radionuclides detected were associated with the colloidal fraction of the groundwater. The observation that $\mathrm{Pu}$ can and has migrated in the subsurface has forced scientists to rethink the basic assumptions regarding the hydrologic and geochemical transport pathways of low-solubility radionuclides. If colloid-facilitated transport is the primary mechanism for transporting low-solubility radionuclides in the subsurface then current transport models based solely on solubility arguments and retardation estimates may underestimate the flux and rate of Pu migration.

Currently, the role of colloids in facilitating the transport of low-solubility radionuclides is not understood well enough to effectively model contaminant transport. A fundamental understanding of the role that colloids play in the transport of contaminants is necessary in order to predict contaminant transport, design remediation strategies and provide risk assessments. Ryan and Elimelech (1996) have argued that in order to evaluate the potential for colloids to transport radionuclides, several criteria must be met: 1) colloids must exist and be stable, 2) radionuclides must have a high sorption affinity for the colloids, and 3) colloids must be transported. In order to evaluate the potential for colloids to transport $\mathrm{Pu}$ and other low-solubility radionuclides, the Underground Test Area (UGTA) program has pursued a field, laboratory, and modeling approach to gain a better understanding of colloids and their ability to transport contaminants.

In this report we integrate the results from a series of field, laboratory and modeling studies funded by the UGTA program to evaluate the potential for colloids to transport low-solubility radionuclides at the NTS. Only then can we understand the conditions under which colloids facilitate transport of contaminants.

The major findings from the field studies are:

- Colloids exist in all groundwater sampled at the NTS and appear physically and chemically stable.

- Low-solubility radionuclides, such as $\mathrm{Pu}$ and $\mathrm{Am}$, are associated with the colloidal fraction of the groundwater collected in both saturated and vadose zone environments. 
- Inorganic colloids consist of common secondary minerals found in volcanic rock; clays, zeolites, silica, and calcite. The mineralogy of the colloids reflects the secondary mineralogy of the host rock aquifer as well as fracture lining minerals.

- The colloidal size distribution tends to be mono-modal with the majority of colloids less than $200 \mathrm{~nm}$ in diameter, and

- The mass of colloids measured in NTS groundwaters should be viewed as a maximum as the high pump rates typically used for groundwater sampling tend to mobilize colloids near the wells, introducing a high-concentration bias to the samples.

- A finite fraction of colloidal tracers have traveled without retardation in every forced-gradient tracer test that has been conducted in fractured volcanic rocks at the NTS.

Results from UGTA field studies provided the framework for UGTA experimental studies investigating the geochemical mechanisms controlling the sorption of $\mathrm{Pu}$ on colloids observed in NTS groundwater. The major findings from our $\mathrm{Pu}$ sorption experiments are:

- $\mathrm{Pu}(\mathrm{IV})$ and $\mathrm{Pu}(\mathrm{V})$ are the dominant oxidation states of $\mathrm{Pu}$ under equilibrium conditions for groundwater compositions measured at the NTS.

- Pu sorbed to all minerals investigated, although the extent, rates and mechanisms for sorption are dependent on the mineral colloid.

- The sorption rate of $\mathrm{Pu}(\mathrm{IV})$ on the various colloids investigated was rapid, occurring within the first few hours.

- The final oxidation state of the sorbed $\mathrm{Pu}$ on the mineral surfaces was $\mathrm{Pu}(\mathrm{IV})$.

- The percent sorption on clinoptilolite was consistently high, but varies as a function of $\mathrm{pH}$ from approximately $70 \%$ to over $90 \%$. The lowest sorption rate occurred at pHs similar to those found at NTS.

- The sorption rate of $\mathrm{Pu}(\mathrm{V})$ to various colloids investigated varied as a function of mineral. $\mathrm{Pu}(\mathrm{V})$ sorbed rapidly to Fe- and Mn-oxides, but much more slowly to silicates.

- The soluble $\mathrm{Pu}(\mathrm{V})$ was ultimately reduced to $\mathrm{Pu}(\mathrm{IV})$ on all the minerals investigated. Even the slowest reduction of $\mathrm{Pu}(\mathrm{V})$ to $\mathrm{Pu}(\mathrm{IV})$ occurs within three weeks.

- The sorption of $\mathrm{Pu}(\mathrm{V})$ on clinoptilolite and possibly other silicates will be controlled by the disproportionation rate in solution of $\mathrm{Pu}(\mathrm{V})$ to $\mathrm{Pu}(\mathrm{IV})$ and $\mathrm{Pu}(\mathrm{VI})$ and then rapid sorption of soluble $\mathrm{Pu}(\mathrm{IV})$ to the mineral surface.

- In contrast, sorption of $\mathrm{Pu}(\mathrm{V})$ on Fe- and Mn-oxide minerals will sorb quickly as $\mathrm{Pu}(\mathrm{V})$ to the mineral surface and will subsequently reduce to $\mathrm{Pu}(\mathrm{IV})$ on the mineral surface.

- Regardless of the different mechanisms for sorption of soluble $\mathrm{Pu}(\mathrm{V})$, $\mathrm{Pu}(\mathrm{IV})$ will be the final sorbed species on the mineral surfaces. 
- Desorption of $\mathrm{Pu}(\mathrm{IV})$ on clinoptilolite is low (2-8\% in 7 months) and this suggests the majority of $\mathrm{Pu}(\mathrm{IV})$ can remain on the mineral colloids with the potential to be transported. However, some Pu(IV) does desorb from clinoptilolite colloids.

The experiments undertaken by UGTA have shown that if $\mathrm{Pu}$ is present in groundwater in either the $\mathrm{Pu}(\mathrm{IV})$ or $\mathrm{Pu}(\mathrm{V})$ oxidation state it will sorb to the common mineral colloids observed at the NTS. The batch desorption experiments on clinoptilolite, silica and montmorillonite show little desorption, suggesting that the majority of $\mathrm{Pu}(\mathrm{IV})$ remain attached to colloids and available for transport over significant time and distance scales

The major findings from the UGTA fracture transport experiments are:

- Pu was eluted in all the fracture experiments, but it was significantly attenuated relative to the colloids in all cases except when the $\mathrm{Pu}$ was associated with clinoptilolite colloids. Injection of Pu-free colloids into the fractures after the initial pulse of $\mathrm{Pu}$ had eluted resulted in negligible remobilization of the sorbed $\mathrm{Pu}$ from the fracture cores.

- The colloids arrive at the same time or slightly faster than the tritiated water.

- Each colloid was significantly more attenuated at the lower flow rate in each fracture.

- The clinoptilolite colloids facilitated the transport of Pu more efficiently than either the montmorillonite or silica colloid. The montmorillonite colloids facilitated the transport of Pu more efficiently than the silica.

- Except in the case of the clinoptilolite colloids, Pu desorbed much faster in the fractures than predicted from batch experiments. This suggests that fracture lining minerals (Mn-oxides) can effectively compete with colloids for $\mathrm{Pu}$.

- Microscopic colloid attachment and detachments experiments were conducted in parallel-plate flow channels to study colloid attachment and detachment processes on smooth surfaces. The major findings from these experiments are:

- Many colloids appeared to be irreversibility attached to the surface.

- Gravitational effects play a dominant role in the attachment and detachment kinetics of silica colloids.

- Polystyrene and silica colloids exhibit attachment residence time distributions at flow channel surfaces that imply a non-linear detachment rate expression.

- Attachment and detachments rates increase linearly with flow rate. Attachment rates increase as ionic strength increases.

- Colloids and colloid attachment sites have distributions of properties that may result in non-linear macroscopic transport behavior that is not readily described by first-order rate expressions.

Sensitivity studies using models developed to interpret the batch sorption and fracture transport experiments lead to the following conclusions for colloid-facilitated radionuclide transport over large time and distance scales: 
- It is not unreasonable to expect a small fraction of radionuclide mass from a discrete source to move essentially at the rate of water flow through fractures over significant distances while sorbed onto colloids. The key requirements for such transport are:

○ the radionuclide desorption rate from the colloids must be slow enough that a fraction of the mass sorbed to the colloids at the source remains sorbed to the colloids during the time it takes to move the distance in question, and

- the irreversible filtration rate of the colloids must be slow enough that a fraction of the colloids at the source are not irreversibly filtered during the time it takes groundwater to move the distance in question.

- The colloid concentrations in the system, the rates of reversible attachment and detachment of colloids onto fracture surfaces, and the rates of radionuclide sorption and desorption onto fracture surfaces all affect the fraction of radionuclide mass from the source that will effectively move at the rate of water flow through the flow system while sorbed to colloids. However, the two conditions listed above must be met before any radionuclide mass will move unretarded through the system.

- The only time colloids can facilitate the transport of radionuclides under conditions of fast radionuclide sorption and desorption rates (e.g., equilibrium conditions) is if there are very high colloid concentrations or very large radionuclide-colloid partition coefficients $\left(\mathrm{K}_{\mathrm{d}}\right.$ values for radionuclides onto colloids) relative to partition coefficients onto fracture surfaces.

In summary, the colloid studies funded by UGTA shows that, 1) colloids exist and are stable in groundwater at the NTS, 2) Pu and Am are overwhelmingly associated with the colloidal fraction of groundwater in both the unsaturated and saturated fracture flow environments at the NTS, 3) Pu has a high sorption affinity and concomitant low desorption extent for inorganic mineral colloids found in groundwater at the NTS, such as zeolites, clays, Mn-oxides, Fe-oxides, and silica, 4) Pu-doped mineral colloids were transported through saturated fractured rock cores from Pahute Mesa, and 5) modeling results suggest that it is not unreasonable to expect a small fraction of radionuclide mass from a discrete source to move essentially at the rate of water flow through fractures over significant distances while sorbed onto colloids. The results from colloid studies in this report demonstrate that colloids can facilitate the transport of low levels low-solubility radionuclides and this is the dominant mechanism for the rapid transport of low-solubility radionuclides in groundwater. 


\section{References}

Buddemeier R. W. and Hunt J. R. (1988) Transport of Colloidal Contaminants in Groundwater: Radionuclide Migration at the Nevada Test Site. Applied Geochemistry 3, 535-548.

Degueldre, C., B. Baeyans, W. Goerlich, et al. (1989). Colloids in Water from a Subsurface Fracture in Granitic Rock, Grimsel Test Site, Switzerland. Geochimica Cosmochimica Acta 53, 603-610.

Kersting A. B., Efurd D. W., Finnegan D. L., Rokop D. J., Smith D. K., and Thompson J. L. (1999) Migration of Plutonium in Groundwater at the Nevada Test Site. Nature 397, 56-59.

Kim J. I. (1991) Actinide Colloid Generation in Groundwater. Radiochim. Acta 52/52, 71-81.

McCarthy, J. F., K. R. Czerwinski, W. E. Sanford, et al. (1998). Mobilization of Actinides from Disposal Trenches by Natural Organic Matter. Journal of Contaminant Hydrology 30, 49.

McDowell-Boyer L. M. (1992) Chemical Mobilization of Micron Sized Particles in Saturated Porous Media under Steady Flow Conditions. Environmental Science and Technology 26, 586-593.

Ryan J. N. and Elimelech M. (1996) Colloid Mobilzation and Transport in Groundwater. Colloids and Surfaces A: Physicochem. and Engin. Aspects 107, 1-56. 


\section{Introduction}

For the last several years, the Underground Test Area (UGTA) program has funded a series of studies carried out by scientists to investigate the role of colloids in facilitating the transport of low-solubility radionuclides in groundwater, specifically plutonium $(\mathrm{Pu})$. Although the studies were carried out independently, the overarching goals of these studies has been to determine if colloids in groundwater at the NTS can and will transport low-solubility radionuclides such as $\mathrm{Pu}$, define the geochemical mechanisms under which this may or may not occur, determine the hydrologic parameters that may or may not enhance transport through fractures and provide recommendations for incorporating this information into future modeling efforts.

The initial motivation for this work came from the observation in 1997 and 1998 by scientists from Lawrence Livermore National Laboratory (LLNL) and Los Alamos National Laboratory (LANL) that low levels of $\mathrm{Pu}$ originally from the Benham underground nuclear test were detected in groundwater from two different aquifers collected from wells $1.3 \mathrm{~km}$ downgradient (Kersting et al., 1999). Greater than $90 \%$ of the $\mathrm{Pu}$ and other radionuclides were associated with the naturally occurring colloidal fraction ( $<1$ micron particles) in the groundwater. The colloids consisted mainly of zeolite (mordenite, clinoptilolite/heulandite), clays (illite, smectite) and cristobalite $\left(\mathrm{SiO}_{2}\right)$. These minerals were also identified as alteration mineral components in the host rock aquifer, a rhyolitic tuff. The observation that $\mathrm{Pu}$ can and has migrated in the subsurface at the NTS has forced a rethinking of our basic assumptions regarding the mechanical and geochemical transport pathways of low-solubility radionuclides. If colloid-facilitated transport is the primary mechanism for transporting low-solubility radionuclides in the subsurface, then current transport models based solely on solubility arguments and retardation estimates may underestimate the flux and rate of $\mathrm{Pu}$ transport.

Currently, the role of colloids in facilitating the transport of low-solubility radionuclides is not understood well enough to effectively model contaminant transport. A fundamental understanding of the role that colloids may or may not play in the transport of low-solubility radionuclides is needed in order to predict contaminant transport, design remediation strategies and provide risk assessments. Ryan and Elimelech (1996) have argued that in order to evaluate the potential for colloids to transport radionuclides, several criteria must be met: 1) colloids must exist and be stable, 2) radionuclides must have a high sorption affinity for the colloids, and 3) colloids must be transported. Only then can we understand the conditions where colloids can and will facilitate transport of radionuclides.

In this report we compile the results from a series of field, laboratory and modeling studies funded by the UGTA program in order to evaluate the potential for colloids to transport low-solubility radionuclides at the NTS. The studies presented in this report fall under three general areas of investigation:

- Characterization of natural colloids in groundwater at NTS, 
- $\mathrm{Pu}$ sorption/desorption experiments on colloid minerals identified in NTS groundwater, and

- Transport of Pu-doped colloids through fractured rock core.

Chapter 1 is a background review of our current understanding of colloids and their role in facilitating contaminant transport.

Chapters 2, and 3 are field studies that focused on characterizing natural colloids at different hydrologic environments at the NTS and address Ryan and Elimelech's (1996) first criteria regarding the existence and stability of colloids.

Chapters 4, 5 and 6 are laboratory experimental studies that investigate the sorption/desorption behavior of $\mathrm{Pu}$ and other low-solubility radionuclides on colloid minerals observed in NTS groundwater. These studies evaluate Ryan and Elimelech's (1996) second criteria that the affinity of Pu for colloids must be high.

Chapters 7, 8, 9, and 10 are laboratory studies that focus on whether colloids can be transported through fractures. These transport studies address Ryan and Elimelech's (1996) third criteria that colloids must be transported.

Chapter 11 discusses the implications of the fracture flow experimental results.

Chapter 12 provides recommendations for future work that would help reduce uncertainties associated with the prediction of colloid-facilitated radionuclide transport at the NTS. 


\section{References}

Kersting A. B., Efurd D. W., Finnegan D. L., Rokop D. J., Smith D. K., and Thompson J. L. (1999) Migration of Plutonium in Groundwater at the Nevada Test Site. Nature 397, 56-59.

Ryan J. N. and Elimelech M. (1996) Colloid Mobilzation and Transport in Groundwater. Colloids and Surfaces A: Physicochem. and Engin. Aspects 107, 1-56. 


\title{
-Chapter 1- \\ Background Literature Review on Colloids in Groundwater
}

\author{
Annie B. Kersting and Paul W. Reimus
}

\subsection{Introduction}

It has long been thought that radionuclides that do not readily dissolve in groundwater (low-solubility) and have a high sorption (attachment) capacity for rocks would not move far in the subsurface. Most models used to predict transport were based on laboratory solubility studies that determined to what extent different radionuclides dissolve and can migrate in groundwater. Low solubility radionuclides, such as $\mathrm{Pu}$ and $\mathrm{Am}$, were not expected to move far from their original source because of their high $\mathrm{K}_{\mathrm{d}}$ values (affinity for solid phase over the liquid phase). Yet, there is a growing body of evidence from both field and laboratory studies that suggests that low-solubility radionuclides can and in fact do migrate in groundwater. Low solubility radionuclides can either "hitch a ride" by sorbing to an organic or inorganic colloid, sometimes referred to as a pseudocolloid. In the case of $\mathrm{Pu}$, it can either form a pseudocolloid or can form its own colloid sometimes referred to as an intrinsic colloid. Somehow our models are falling short in predicting the fundamental processes that control the dominant transport mechanisms operating in a complex, natural field setting. Recent studies suggest that the existence of naturally occurring inorganic and organic colloids (1-1000 nm sized particles) may enhance contaminant transport through sorption (Buddemeier and Hunt, 1998; Degueldre et al., 1989; Kim , 1991; McDowell-Boyer, 1992; and McCarthy et al., 1998; Kersting et al., 1999). In this chapter, the current understanding of colloids and their ability to facilitate transport is summarized.

\subsection{Definition and Origin of Colloids}

Colloids are ubiquitous, naturally occurring, sub-micron particles found in groundwater (Figure 1.1). As a result of their small size, colloids have the ability to remain suspended and be transported in groundwater. Colloids have high surface area per unit mass and a high capacity for sorption of low-solubility contaminants. Colloids are generally defined as being between 1 and $1000 \mathrm{~nm}$ in diameter (Stumm, 1992). This definition is based on physical properties. Due to their small size, they have the ability to remain suspended by Brownian motion and ultimately transported in groundwater. Particles greater than a few microns (2000 $-3000 \mathrm{~nm}$ ) will generally settle out of a fluid due to gravitational forces and those particles less than $1 \mathrm{~nm}$ are thought to behave as dissolved species.

Colloids found in groundwater are naturally occurring particles that are composed of inorganic minerals or organic (humic and fulvic) species. Although the properties of inorganic and organic colloids may require different thermodynamic treatments, there is recent recognition organic and inorganic colloids co-exist where organic surfaces sometimes cover inorganic mineral colloids in waters where organic material is present (Degueldre et al., 2000). Humic colloids originate from soil zones or organic deposits in sediments. Inorganic colloids originate from either mechanical abrasion of aquifer minerals or chemical precipitation of super-saturated mineral phases. Most groundwater 
colloids originate from mechanical abrasion of existing mineral phases or organic materials (McCarthy and Degueldre, 1993).
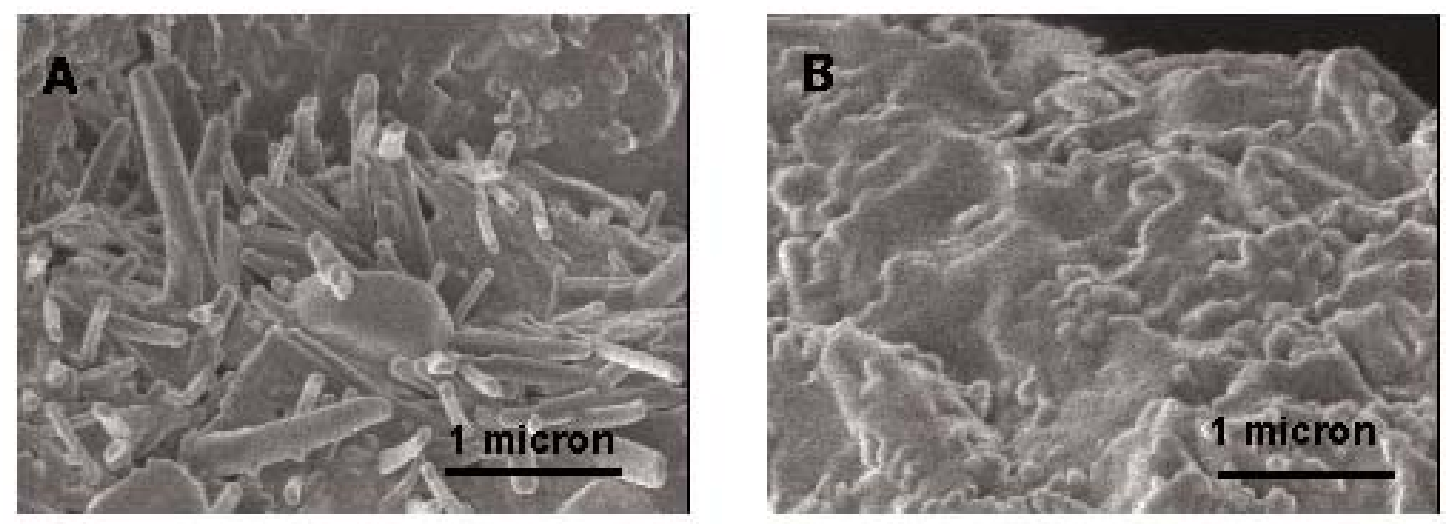

Figure 1.1. Scanning electron images of natural colloids filtered from groundwater collected at the NTS. Colloids consist predominantly of zeolites (A) and clays (B). Scale bar located at bottom right.

Both laboratory experiments and field studies have shown that when organic substances are present the behavior of the actinide ions is predominantly controlled by the humic acids (Kim et al., 1992). When organic substances are present with inorganic colloids they act to enhance the ability of colloids to transport radionuclides (Kim, 1991). Organic colloids are common in surface and shallow waters. In contrast, the organic content of deep waters in fractured rock is generally very low and these systems are dominated by inorganic colloids (Smith and Degueldre, 1993). For example, the total organic content of a deep groundwater, J-13, a water used as a standard for the potential Yucca Mountain Repository (YMR), was low and measured between 0.15 to $0.50 \mathrm{mg} / \mathrm{L}$ (Moulin et al., 1999).

\subsection{Nature of Groundwater Colloids}

In order to develop a conceptual model of colloidal transport of radionuclides, the following information about colloids is needed: 1) the concentration in groundwater, 2) the composition, 3) the size distribution, 4) the generation and the physical and chemical stability in groundwater. Sampling groundwater colloids without introducing artifacts has been difficult and significant effort has been invested in developing techniques designed to minimize contamination (Buddemeier, 1986; McCarthy and Degueldre, 1993) (See chapter 2). Many things can inadvertently alter the sample and lead to erroneous results including: over-pumping of the aquifer, contamination of well construction materials, surface contamination in the collected sample, and poor handling of the sample after collection that alters the water chemistry (McCarthy and Degueldre, 1993; Degueldre et al., 1996). For example, most pump rates used to collect groundwater are high and result in over-pumping or overestimating the concentration of colloids (Puls et al., 1992) and possibly biasing samples toward larger particles (McCarthy and Degueldre, 1993). Therefore, even though care has been taken to minimize biases in 
many studies, estimates of the concentration and size of colloids in groundwater should be taken as upper limits, unless demonstrated otherwise.

\subsection{Concentration and Composition of Groundwater Colloids}

McCarthy and Degueldre (1993) compiled literature data on the concentration and composition of natural colloids, with their respective groundwater chemistry from wells at potential high-level waste sites around the world. Host rock types ranged from granite to sandstone to high-silica volcanic rock. When all the studies were compared, the following generalizations were made:

- colloids were found in every sample, although there is a large variation in concentration,

- the concentrations of colloids in fracture flow systems ranged between 0.0002 $200 \mathrm{mg} / \mathrm{L}$,

- the most common minerals identified were clays, zeolites, Fe-oxides, Mn-oxides, and silica,

- colloid concentrations are lowest in the deepest fracture flow environments, and

- in general, the mineral composition of the colloids is reflective of the host rock mineralogy or the fracture lining minerals; those minerals in contact with the groundwater.

Ccolloids are present in all groundwater and their composition is dominated by the presence of secondary minerals such as clays, zeolites, silica and iron-oxides. Determining the natural concentration of colloids in groundwater is difficult, but careful studies show that concentrations vary significantly with deeper water having fewer colloids than shallower water. Deeper groundwater is dominated by inorganic rather than organic colloids. Although previous studies demonstrate that colloids are present and available for transport, they must be stable in order to be mobile over significant distances.

\subsection{Generation and Mobilization of Natural Groundwater Colloids}

Inorganic colloids are primarily generated by mechanical fragmentation of the host rock in contact with the groundwater caused by either chemical or physical perturbations. This change can result from either a chemical process ( $\mathrm{pH}$, ionic strength) and/or a change in the flow rate of a groundwater system. Chemical changes in ionic strength, $\mathrm{pH}$ and fluid composition have been found to greatly increase colloid generation. Changes in ionic strength can occur when groundwater is diluted by infiltration or precipitation.

The most important physical perturbations in groundwater systems result from an increase or decrease in the flow rate of the water. Rapid infiltration of water through soils can induce shear that may generate colloids. Colloid generation can also be induced by changes in groundwater flow rate in fractured rock. Degueldre et al. (1989) showed that colloids were continuously produced from water flowing through fractured granite at the Grimsel Test Site, Switzerland. Ryan and Gschwend (1994) investigated colloid release rates in the laboratory with a hematite-quartz system and showed that release rates were correlated with increases in flow rates and decreases in ionic strength of solutions. 
Extensive theoretical treatment of the physics of colloid generation, filtration and DLVO theory can be found in the literature. A review of this literature is beyond the scope for this report, however, the reader is referred to the following references for additional information: Ryan and Elimelech, 1996; Roy and Dzombak, 1996; and Elimelech et al., 1995.

\subsection{Modeling of Colloid Transport}

Field observations of colloid-facilitated transport of contaminants have prompted modeling efforts to describe this phenomenon. Most of the work on colloid transport in the past three decades has focused on determining the dependencies of the attachment and detachment rate constants on factors such as colloid size and density, colloid and media surface properties (including air bubbles as a "media" under unsaturated conditions), solution ionic strength, solution $\mathrm{pH}$, flow rates, and media geometry. Ryan and Elimelech (1996) provide an excellent review of how most of these variables affect colloid filtration and detachment. Recent efforts have focused on incorporating the effects of media heterogeneity on colloid transport (Sun et al., 2001; Chen et al., 2001).

Early models considered equilibrium partitioning of contaminants between the solution phase, colloid surfaces, and media surfaces (Hwang et al., 1989; Smith and Degueldre, 1993; Grindrod, 1993). These efforts focused on describing transport through fractured media. The equilibrium assumption simplified considerably the system of coupled equations to be solved and even allowed analytical or semi-analytical solutions to be implemented (Hwang et al., 1989; Grindrod, 1993). Corapcioglu and Jiang (1993) introduced a numerical model with first-order reversible rate expressions to describe contaminant sorption onto both colloid and media surfaces as well as first-order expressions describing colloid attachment and detachment to media surfaces. Their model (with some adjustments) was later used to describe ${ }^{137} \mathrm{Cs}$ transport facilitated by silica colloids through glass bead columns (Noell et al., 1998). Ibaraki and Sudicky (1995a, 1995b) were the first to implement kinetic expressions in a model to explicitly describe colloid-facilitated contaminant transport in discrete fractures and fracture networks, although their approach was essentially mathematically identical to that of Corapcioglu and Jiang (1993). Oswald and Ibaraki (2001) later extended the model to account for matrix diffusion of colloids, a phenomenon that was observed in laboratory experiments conducted in a fractured saprolite of high matrix porosity.

\subsection{Stability of Natural Groundwater Colloids}

The stability of colloids in a given aquifer setting is a function of $\mathrm{pH}$, redox potential, water chemistry and status of the hydrogeochemical system (Degueldre et al., 2000). For steady state conditions, the chemistry of the water plays a major role in determining the stability of colloids. Degueldre et al., (2000) found that alkaline element $\left(\mathrm{Na}^{+}, \mathrm{K}^{+}\right)$ concentration below $10^{-2} \mathrm{M}$ and alkaline earth $\left(\mathrm{Mg}^{2+}, \mathrm{Ca}^{2+}\right)$ concentrations below $10^{-4} \mathrm{M}$ increase colloid stability. Recent work by Reimus and Kung (personal communication) document a similar dependence of colloid concentration on cation concentration in groundwater at the potential YMR. The presence of organic material has a strong stabilizing effect on the inorganic colloids (e.g. Kim, 1991; Ryan et al., 1999). Most field 
and laboratory experimental studies have demonstrated that lowering the ionic strength of groundwater results in the generation and stability of existing colloids, whereas, increasing the ionic strength tends to decrease colloid concentration by promoting coagulation (Roy and Dzombak, 1996).

\subsection{Field Studies of Actinide Movement in Groundwater}

Although there have been a growing number of field studies documenting movement of actinides in the subsurface, a consensus as to the mechanisms responsible for the apparent observations has been slow to emerge. This is due, in part, to the natural geologic complexity of field environments making definitive conclusions somewhat difficult. This is juxtaposed by laboratory experiments demonstrating strong sorption of $\mathrm{Pu}$ and $\mathrm{Am}$ onto rock minerals and two-phase transport models that predict immobility. In addition, colloid-filtration theory predicts limited transport on the order of meters to tens of meters (McDowell-Boyer, 1992; Ryan and Elimelech, 1996).

Pioneering field studies examining the association of colloids and transport of radionuclides were conducted at the Nevada Test Site (NTS), down gradient from an underground nuclear test (Buddemeier and Hunt, 1988). Buddemeier and Hunt (1988) collected and filtered groundwater sampled from a well that was screened in fractured volcanic tuff $300 \mathrm{~m}$ down gradient of an underground nuclear test. Approximately $98 \%$ of the low-solubility radionuclides, $\mathrm{Co}, \mathrm{Ce}, \mathrm{Eu}$, detected in the groundwater were associated with the colloidal fraction. The colloids were not identified in this study. These findings provide strong support that colloids play a role in transport of lowsolubility radionuclides in a saturated, fracture flow aquifer.

In a recent study, low levels of $\mathrm{Pu}, \mathrm{Cs}, \mathrm{Co}$, and $\mathrm{Eu}$, and high levels of tritium were detected in groundwater collected from two different aquifers down gradient of a nuclear test at the NTS (Kersting et al., 1999). Am was detected in groundwater from one of the aquifers (Thompson, 1999). Groundwater samples collected from the two wells were filtered and greater than $98 \%$ of the $\mathrm{Pu}$ was associated with the colloidal fraction. The colloids consisted of clays, zeolites and silica and had a mean size range between 100$150 \mathrm{~nm}$. The $\mathrm{Pu}$ isotopic ratio $\left({ }^{240} \mathrm{Pu} /{ }^{239} \mathrm{Pu}\right)$ of the groundwater samples was measured and matched one test exactly, located $1.3 \mathrm{~km}$ upgradient from the sampling wells. This work clearly establishes that the low-levels of Pu were associated with the colloids and not the dissolved fraction of the groundwater and that $\mathrm{Pu}$ is not immobile in the subsurface, but can be transported significant distances.

In another field study conducted at the Rocky Flats Environmental Technology Site (RFETS), groundwater from a well was collected, filtered and analyzed for radionuclides (Harnish et al., 1984). Low levels of Pu were detected and the majority of the Pu was associated with the particulate and colloidal fractions of the groundwater. Colloid concentrations were very low, estimated at less than $1 \mathrm{mg} / \mathrm{L}$. Clay minerals were detected in the colloidal fraction. Organic material was suspected, but not analyzed. Although this study does not address transport of radionuclides, it does document that the $\mathrm{Pu}$ is in the colloidal, rather than dissolved fraction of the groundwater. 
In a separate study at RFETS, $\mathrm{Pu}$ and $\mathrm{Am}$ transported in surface water was overwhelmingly associated with the particulate and colloidal fraction of the groundwater (Santschi et al. 2002). Less than one percent of the Pu was in the dissolved fraction. The composition of the colloidal fraction was predominantly organic colloids. This study documents the existence of colloids, the association of organic colloids with the radionuclides and the transport of low-solubility radionuclides with surface water.

The release and migration of trace amounts of actinides ( $\mathrm{Pu}$ and/or $\mathrm{Am}$ ) was observed at the Idaho National Engineering and Environmental Laboratory (INEEL) (Rawson et al., 1991). Like the Rocky Flats Plant, INEEL was part of the DOE's nuclear weapons complex, established in 1949 as a chemical separations facility and was the site of the largest concentration of nuclear reactors in the world. Trace Pu and/or Am was detected in two different sandy interbeds located 34 and 73 meters beneath the ground surface of an area where transuranic and low-level radioactive mixed waste was stored. The waste was stored for approximately 30 years on 1 meter of sediment, which was underlain by fractured basalt flows and sedimentary interbeds. Rawson et al. (1991) argues that due to the low solubility of $\mathrm{Pu}$, the concentrations of $\mathrm{Pu}$ detected are too high to be the result of transport as a dissolved species. Colloids were not analyzed in the study, but Rawson et al. (1991) suggests that the $\mathrm{Pu}$ may have migrated in the groundwater associated with colloids.

Ccollectively these field studies provide a compelling case for colloids to facilitate the transport of low-solubility radionuclides in the subsurface. In addition, these studies suggest that colloids are the dominant mechanism for the transport of low-solubility radionuclides in groundwater.

\subsection{Summary}

Colloids are present in all groundwater and their composition is dominated by the presence of secondary minerals such as clays, zeolites, silica and iron-oxides. Determining the natural concentration of colloids in groundwater is difficult, but careful studies show that concentrations vary significantly with deeper water having fewer colloids than shallower water. Deeper groundwater is dominated by inorganic rather than organic colloids. Although studies demonstrate that colloids are present and available for transport, they must also be stable in order to be mobile over significant distances. The stability of colloids in a given aquifer setting is a function of $\mathrm{pH}$, redox potential, water chemistry and status of the steady state hydrogeochemical system. The effect on the stability of colloids as a result of changes in geochemical conditions is poorly understood.

Collectively, the field studies discussed show that colloids can and do facilitate transport of low-solubility radionuclides significant distances in fracture flow systems. The field studies to date suggest that colloids are the dominant mechanism for the transport of lowsolubility radionuclides in groundwater.

Currently, the role of colloids in facilitating the transport of low-solubility radionuclides is not understood well enough to effectively model contaminant transport. For example, the nature of $\mathrm{Pu}$ in the subsurface is not known. Low concentrations of $\mathrm{Pu}$ in the 
environment have prevented efforts to apply even the latest spectroscopic techniques to determining the intrinsic or pseudocolloid nature of the Pu. This lack of understanding about the nature of $\mathrm{Pu}$ in the subsurface greatly complicates modeling efforts to predict transport. In addition, the current lack of detailed knowledge regarding the stability and concentration of colloids in the natural environment inhibits our ability to develop reliable predictive models for transport of low solubility radionuclides. Without the capability to predict when colloids are stable and at what quantities, we will not be able to make rational risk assessments regarding the transport of $\mathrm{Pu}$ and other low-solubility radionuclides. 


\subsection{References}

Buddemeier R. W. (1986) Sampling the Subsurface Environment for Colloidal Materials: Issues Problems and Techniques. Transport of contaminants in the subsurface: the role of organic and inorganic colloidal particles.

Buddemeier R. W. and Hunt J. R. (1988) Transport of Colloidal Ctaminants in Groundwater: Radionuclide Migration at the Nevada Test Site. Applied Geochemistry 3, 535-548.

Chen, J. Y., C. H. Ko, S. Bhattacharjee, and M. Elimelech (2001). Role of Spatial

Distribution of Porous Medium Surface Charge Heterogeneity in Colloid Transport, Colloids Surf. A, 191(1-2/SISI), 3-15.

Corapcioglu, M. Y. and Jiang, S. (1993). Colloid-Facilitated Groundwater Contaminant Transport, Water Resour. Res., 29(7), 2215-2226.

Degueldre, C., B. Baeyans, and W. Goerlich (1989). Colloids in Water from a Subsurface

Fracture in Granitic Rock, Grimsel Test Site, Switzerland. Geochimica Cosmochimica Acta 53, 603-610.

Degueldre c., Pfeiffer H. R., Alexander W., Wernli B., and Bruetsch R. (1996) Colloid Properties in Granitic Groundwater Systems. I: Sampling and Characterization. Applied Geochem. 11, 677-695.

Degueldre C., Triay I., Kim J. I., Vilks P., Laaksoharju M., and Miekeley N. (2000) Groundwater Colloid Properties: a Global Approach. Applied Geochemistry 15, 1043-1051.

Elimelech M., Gregory J., Jia X., and and Williams R. (1995) Particle deposition and aggregation. Butterworth-Heinmann.

Grindrod, P. (1993). The Impact of Colloids on the Migration and Dispersal of Radionuclides within Fractured rock, J. Contam. Hydrol., 13, 167-181.

Harnish, R. A., D. M. McKnight and J. F. Ranville (1984) Particulate, colloidal, and dissolved-phase associations of plutonium and americium in a water sample from well 1587 at the Rocky Flats Plant, Colorado. U.S.: Geological Survey WaterResources Investigations. Report 93-4175.

Hwang, Y., T. H. Pigford, W. W.-L. Lee, and P. L. Chambre (1989). Analytic Solution of Pseudocolloid Migration in Fractured Rock, Proc. Am. Nucl. Soc., Winter Meeting, San Francisco, CA, Nov. 26-30, 1989.

Ibaraki, M. and E. A. Sudicky (1995a). Colloid-Facilitated Contaminant Transport in

Discretely Fractured Porous Media 1. Numerical Formulation and Sensitivity

Analysis, Water Resour. Res., 31(12), 2945-2960.

Ibaraki, M. and E. A. Sudicky (1995b). Colloid-Facilitated Contaminant Transport in Discretely Fractured Porous Media 2. Fracture Network Examples, Water Resour. Res., 31(12), 2961-2969.

Kersting A. B., Efurd D. W., Finnegan D. L., Rokop D. J., Smith D. K., and Thompson J. L. (1999) Migration of Plutonium in Groundwater at the Nevada Test Site. Nature 397, 56-59.

Kim J. I. (1991) Actinide Colloid Generation in Groundwater. Radiochim. Acta 52/52, 71-81.

Kim J. I., Zeh P., and Delakowitz B. (1992) Chemical Interaction of Actinide Ions with Groundwater Colloids in Gorleben Aquifer Systems. Radiochim. Acta 58/59, 14754. 
McCarthy J. F. and Degueldre C. (1993) Environmental Particles, Vol. 2, Ch 6 (ed. J. Buffle and H. P. van Leeuwen), pp. 426. Lewis Publisher.

McCarthy, J. F., K. R. Czerwinski, W. E. Sanford, et al. (1998). Mobilization of Actinides from Disposal Trenches by Natural Organic Matter. Journal of Contaminant Hydrology 30, 49.

McDowell-Boyer L. M. (1992) Chemical Mobilization of Micron Sized Particles in Saturated Porous Media under Steady Flow Conditions. Environmental Science and Technology 26, 586-593.

Moulin, V., P. Reiller, G. Plancque, I. Laszak and C. Moulin (1999) Complexation of $\mathrm{Eu}(\mathrm{III}), \mathrm{Th}(\mathrm{IV})$ and U(VI) by Humic Substances. In Effects of humic substances on the migration of radionuclides: complexation and transport of actinides. Second technical progress report (ed.) Karlsruhe: Forschungszentrum Karlsruhe GmbH., pp.

Noell, A. L., J. L. Thompson, M. Y. Corapcioglu, and I. R. Triay (1998). The Role of Silica Colloids on Facilitated Cesium Transport Through Glass Bead Columns and Modeling, J. Contam. Hydrol., 31, 23-56.

Oswald, J. G. and M. Ibaraki (2001). Migration of Colloids in Discretely Fractured Porous Media: Effect of Colloidal Matrix Diffusion, J. Contam. Hydrol., 52, 213244.

Puls R. W., Clark D. A., Bledsoe B., and Powell R. M. (1992) Metals in Ground Water Sampling Artifacts and Reproducibility. Hazardous waste \& Hazardous Materials 9, 149-162.

Rawson S. A., Walton J. C., and R.G. B. (1991) Migration of Actinides from a Transuranic Waste Disposal Site in the Vadose Zone. Radiochimica Acta 52/53, 477-486.

Roy S. B. and Dzombak D. A. (1996) Colloid Release and Transport Processes in Natural and Model Porous Media. Colloids and Surfaces A: Physicochem. Engin. Aspects 107, 245-262.

Ryan J. N. and Elimelech M. (1996) Colloid Mobilzation and Transport in Groundwater. Colloids and Surfaces A: Physicochem. and Engin. Aspects 107, 1-56.

Ryan J. N. and Gschwend P. M. (1994) Effects of Ionic Strength and Flow Rate on Colloid Release: Relating Kinetics to Intersurface Potential Energy. Journal of Colloid and Interfe Science 164, 21-34.

Ryan, J. N., M. Elimelech M., R. A. Ard, R. W. Harvey, and P. R. Johnson (1999).

Bacteriophage PRD1 and Silica Colloid Transport and Recovery in an Iron OxideCoated Sand Aquifer, Environ. Sci. Tech., 33(1), 63-73.

Santschi, P.H., Roberts, K.A., and Guo, L. (2002) The Organic Nature of Colloidal Actinides Transported in Surface Water Enviroments. Environmental Science \& Technology. 36(17), 3711-3719.

Smith P. A. and Degueldre C. (1993) Colloid-Facilitated Transport of Radionuclides Through Fractured Media. J. Contam. Hydrol. 13(1-49), 143-66.

Stumm W. (1992) Chemistry of the solid-water interface: processes at the mineral-water and particle-water interface in natural systems. Wiley Press, Chicago .

Sun, N., M. Elimelech, N.-Z. Sun, and J. N. Ryan (2001). A Novel Two-Dimensional Model for Colloid Transport in Physically and Geochemically Heterogeneous Porous Media, J. Contam. Hydrol., , 173-199. 
Thompson J. L. (1999) Laboratory and Field Studies Related to Radionuclide Migration at the Nevada Test Site: Oct. 1 1997-Sept. 30, 1998. pp. 1-28. Los Alamos National Laboratory. 


\title{
-Chapter 2- \\ Characterization of Colloids in Groundwater Collected at the Nevada Test Site
}

\author{
Axel Brachmann and Annie B. Kersting
}

\subsection{Introduction}

In 1997 and 1998, low levels of $\mathrm{Pu}$ were detected in groundwater collected from wells ER-20-5-1 and ER-20-3. The Pu originated $1.3 \mathrm{~km}$ from the BENHAM underground nuclear test, $1.3 \mathrm{~km}$ upgradient (Kersting et al. 1999). The Pu and other low-solubility radionuclides were associated with the colloidal fraction of the groundwater. This observation raised many questions concerning the ability of colloids to transport radionuclides, specifically radionuclides that have a low solubility in groundwater. Efforts were undertaken by the UGTA program to better understand, the mineralogy of the colloids, the range of colloid concentrations in the groundwater, and the size distribution of the colloids.

In this chapter, we report results from analysis of water samples from two locations at the NTS that were collected from wells screened in volcanic tuff. Samples were collected from well cluster ER-20-5 (wells \#1 and \#3), and drill hole U20n PS1 DDH, a post-shot hole for the CHESHIRE underground nuclear test. Both the ER-20-5 and CHESHIRE drill holes are located on Pahute Mesa in Area 20 at the NTS (Fig. 2.1). The composition, size distribution, and concentration of colloidal material in groundwater were characterized. Efforts were made to determine if sample collection methods compromised results. A second colloid characterization study (Kung and Fresquez (2000)), funded by UGTA and previously published, will be compared at the end of this chapter. In addition, this study is compared with other colloid studies in order to better understand the nature of colloids worldwide.

\subsection{Characterization of Colloids Collected From NTS wells; ER20-5 \#1, ER20-5 \#3, and CHESHIRE.}

Groundwater samples from ER-20-5 \#3 were collected on April 30, 1998 and samples from well ER-20-5 \#1 were collected on July 9, 1998. Groundwater samples from the CHESHIRE region were sampled from two distinct production levels from well U20n PS1 DDH. The first sample (CHESHIRE upper) was taken on July 28, 1998 from the upper perforation interval between 2665 - $2995 \mathrm{ft}(812-913 \mathrm{~m})$ above a bridge plug. After drilling through the bridge plug, the second sample (CHESHIRE lower) was collected on September 21, 1998 from the lower perforation level between $4100-4110 \mathrm{ft}$ $(1250-1253 \mathrm{~m})$, within the test cavity.

In this study, special care was taken to evaluate the potential effect of sampling and experimental protocols on the detection of colloids. We wanted to make sure that our 
results regarding the composition, size, and concentration of colloids in groundwater at the NTS were not impacted by our sampling methods.

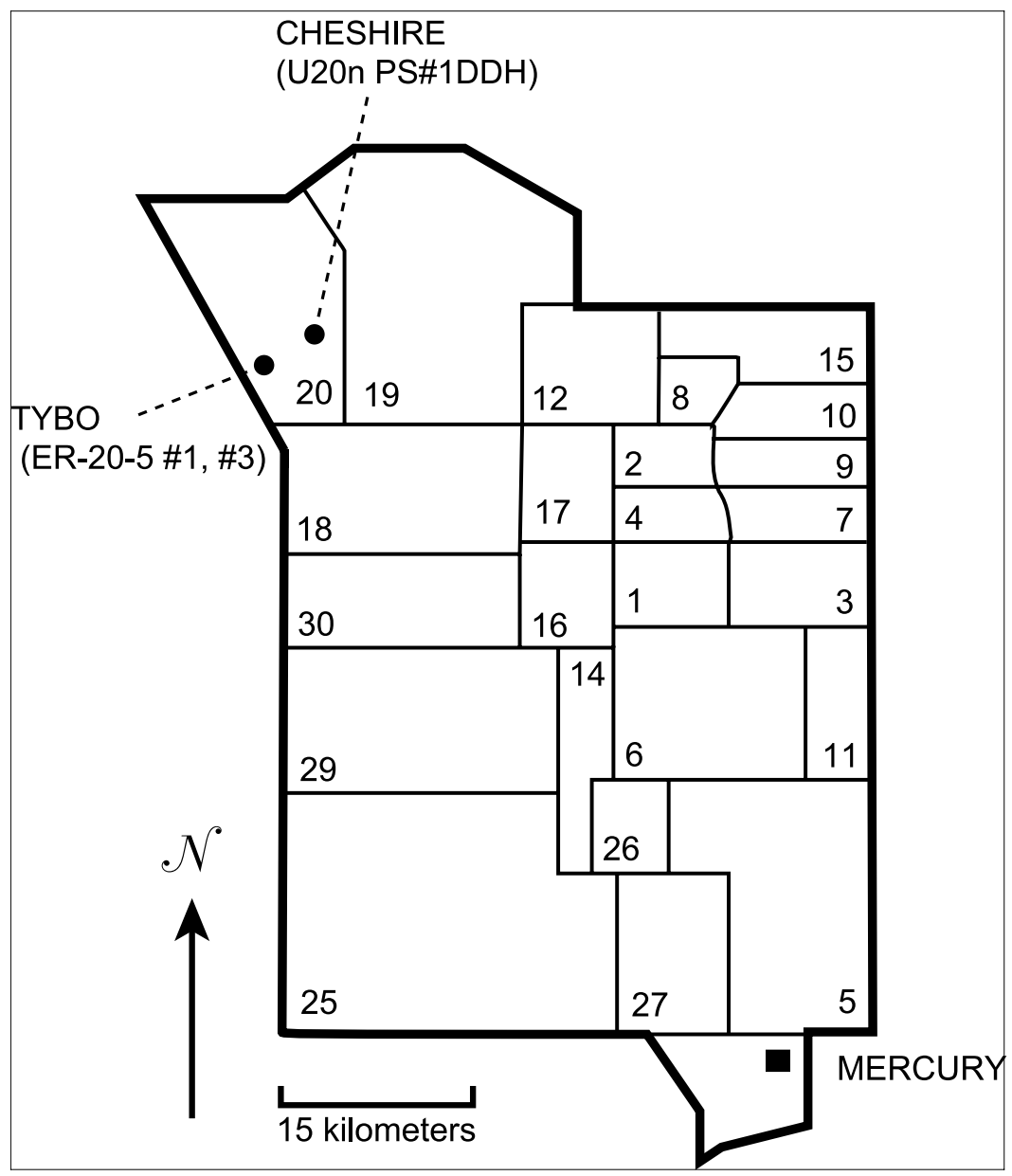

Figure 2.1. Map of the Nevada Test Site showing the location of the ER-20-5 and CHESHIRE wells on Pahute Mesa.

\section{Experimental Techniques}

Groundwater was filtered using a high-performance ultra-filtration system combined with a Pellicon cassette system. The groundwater samples were filtered using a 100,000 NMW $(\sim 7 \mathrm{~nm})$ Millipore filter $(1 \mathrm{~mm}$ equals $1000 \mathrm{~nm})$. The tangential flow system separates the ultra-filtrate (fluid $<7 \mathrm{~nm}$ ) and retentate (colloids $>7 \mathrm{~nm}+$ fluid). What passed 
through the filter (ultra-filtrate) was considered the dissolved fraction. The concentrated retentate was then freeze-dried and prepared for x-ray diffraction (XRD) and scanning electron microscopy (SEM) analysis. The groundwater samples collected at ER-20-5 are dilute bicarbonate waters.

This procedure is slightly different from the serial filtration techniques used on the groundwater samples collected in 1997 (Kersting et al. 1999). In previous filtration exercises, 200 liters of groundwater were filtered and the filterable material deposited on the various filters used. A small portion of this material was then scraped off the filters and analyzed. Additional details can be found in 1998 LANL and LLNL reports (Thompson et al. 1998). In this study, less than 5 liters of the groundwater sampled in 1998 were filtered for analysis, and only one size filter was used $(\sim 7 \mathrm{~nm}, 100,000$ NMW). Instead of collecting the colloids on the filters, the colloids were concentrated in the retentate and freeze-dried. This eliminated any potential chemical interaction between the colloids and the filters.

Freeze-drying compared with air drying the colloid sample has the advantage of preventing minerals from precipitating as the solution becomes more concentrated (e.g., NTS waters are close to calcite saturation). This technique is also better for preserving the morphology of the clay minerals for resolution on the SEM.

\section{Composition of Colloids from ER-20-5 well Cluster}

The colloids collected from ER-20-5 \#1 and ER-20-5 \#3 groundwater were analyzed by XRD. The results of the analyses show that ER-20-5 \#1 consists of zeolites (mordenite, clinoptilolite/heulandite), clays (illite, smectite), and cristobalite $\left(\mathrm{SiO}_{2}\right)$. The colloids collected from ER-20-5 \#3 groundwater consist of clays (illite, smectite), and minor zeolites.

The XRD patterns of the two samples were strikingly similar, primarily consisting of clays and zeolites. The major difference between the two samples was the higher concentration of clay minerals relative to zeolites in groundwater colloids from ER-20-5 \#3. In contrast, the dominant colloid in ER-20-5 \#1 groundwater was the zeolite, mordenite. It should be noted that even though clinoptilolite and heulandite are closely related, they are still distinct zeolites. However, these minerals are not easily distinguished on the basis of XRD or optical properties, therefore the term heulandite/clinoptilolite is used. The colloidal minerals identified in the ER-20-5 \#1 sample were the same as previously identified in groundwater collected in 1997. The two different filtration methods produced identical results, and we conclude that the filters did not chemically react with the colloids.

The physical appearance between the groundwater samples from ER-20-5 \#1 and \#3, as observed by SEM analysis, was dramatic. The SEM used in this study was a highresolution Hitachi S4500 capable of imaging very small particles. Routine analysis using this SEM can resolve images on the order of tens of nanometers $(\mathrm{nm})$ in size. Figures 2.2 and 2.3 are SEM images taken of the groundwater colloids from ER-20-5 \#1 and \#3, 
respectively. The colloids in ER-20-5 \#1 were predominantly rod shaped minerals, the rod shape being a common habit of mordenite. This is consistent with the quantitative results from the XRD analysis. In contrast, the dominant morphology in the ER-20-5 \#3 sample was the rounded, platy minerals characteristic of clay minerals. This is again consistent with the quantitative results from the XRD analysis. The minerals were too small for quantitative identification by SEM.

Side-wall cores and rock cuttings taken from well ER-20-5\#1 and \#3 were also analyzed by XRD to determine if the minerals identified in the colloidal fraction of the groundwater were also present in the rock aquifers. Rock cuttings from well ER-20-5 \#1 collected from $2400 \mathrm{ft}, 2500 \mathrm{ft}$, and $2600 \mathrm{ft}$ depths were analyzed. These samples bracket the rock aquifer from ER-20-5 \#1. No core was recovered from well ER-20-5 \#1. Core samples from ER-20-5 \#3, collected at depths of $3500 \mathrm{ft}, 3750 \mathrm{ft}$, and $3890 \mathrm{ft}$, were analyzed. These samples bracket the rock aquifer in ER-20-5 \#3.

The mineralogy of rock units from $2400 \mathrm{ft}$ to $2600 \mathrm{ft}$ in well ER-20-5 \#1 is fairly uniform, consisting of common silicic volcanic minerals (quartz, sanidine and microcline) and minor secondary alteration minerals (clays and zeolites). The clays consist of illite/smectite and zeolites heulandite/clinoptilolite and mordenite. Although zeolites and clays are the minor phases in the rock aquifer, they dominate the groundwater colloids. The mineralogy of rock units from $3500 \mathrm{ft}$ to $3890 \mathrm{ft}$ in well ER20-5 \#3 is also fairly uniform. These rock units are strongly altered and are predominately clays and zeolites. Specifically, the zeolites are mordenite, and heulandite/clinoptilolite and the clay is illite/smectite. Both host aquifer rocks contain clays and zeolites. Therefore, it is not surprising that the colloidal minerals identified in the groundwater from ER-20-5 well clusters are minerals also found in the host rock aquifer.

Composition of Colloids from the CHESHIRE Drill-back well (Well U20n PS1 DDH)

The colloids collected in groundwater from both the upper and lower aquifers at CHESHIRE, were analyzed by XRD and predominantly consist of clays (illite, smectite), and zeolites (mordenite).

SEM photos of the different mineral morphologies are shown in Figure 2.4. Both samples had minor amounts of the rod shaped minerals indicative of zeolites, while the minerals with the more platy appearance, had the common habit of clay minerals. Again, the individual minerals were much too small for quantitative analysis using the SEM. Energy dispersive analysis (EDS), however, gave an average chemical analysis of a single area of 

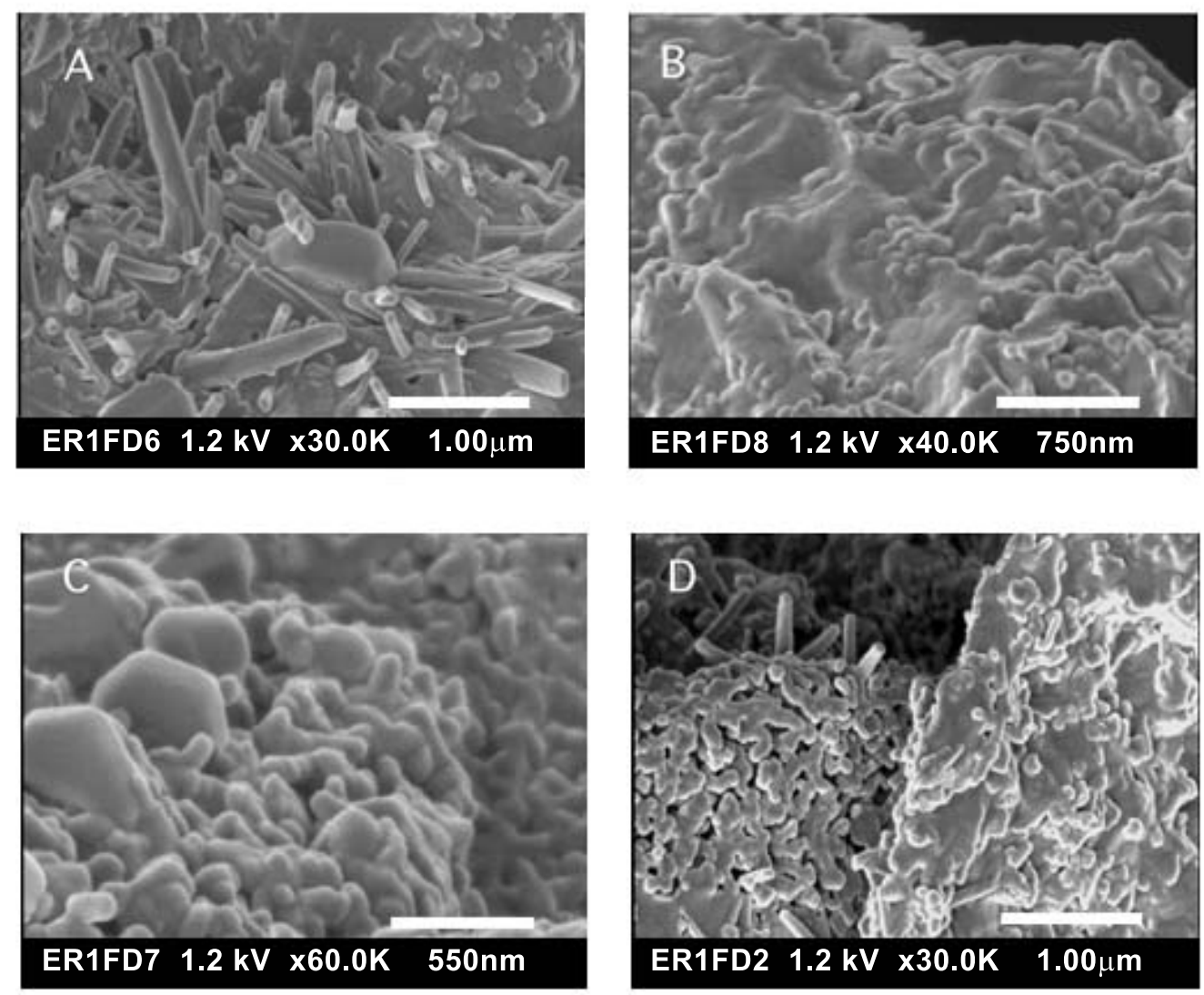

Figure 2.2. SEM photos of colloids in ER-20-5 \#1 groundwater. The rod-shaped minerals shown in (A) are mordenite. The flat, platy appearing minerals in (B) are clays. The blocky minerals shown in (A) and (C) are probably heulandite. All minerals were identified by XRD and only tentatively identified here by their characteristic morphology. Scale bars are shown as the white lines displayed in the bottom right. 

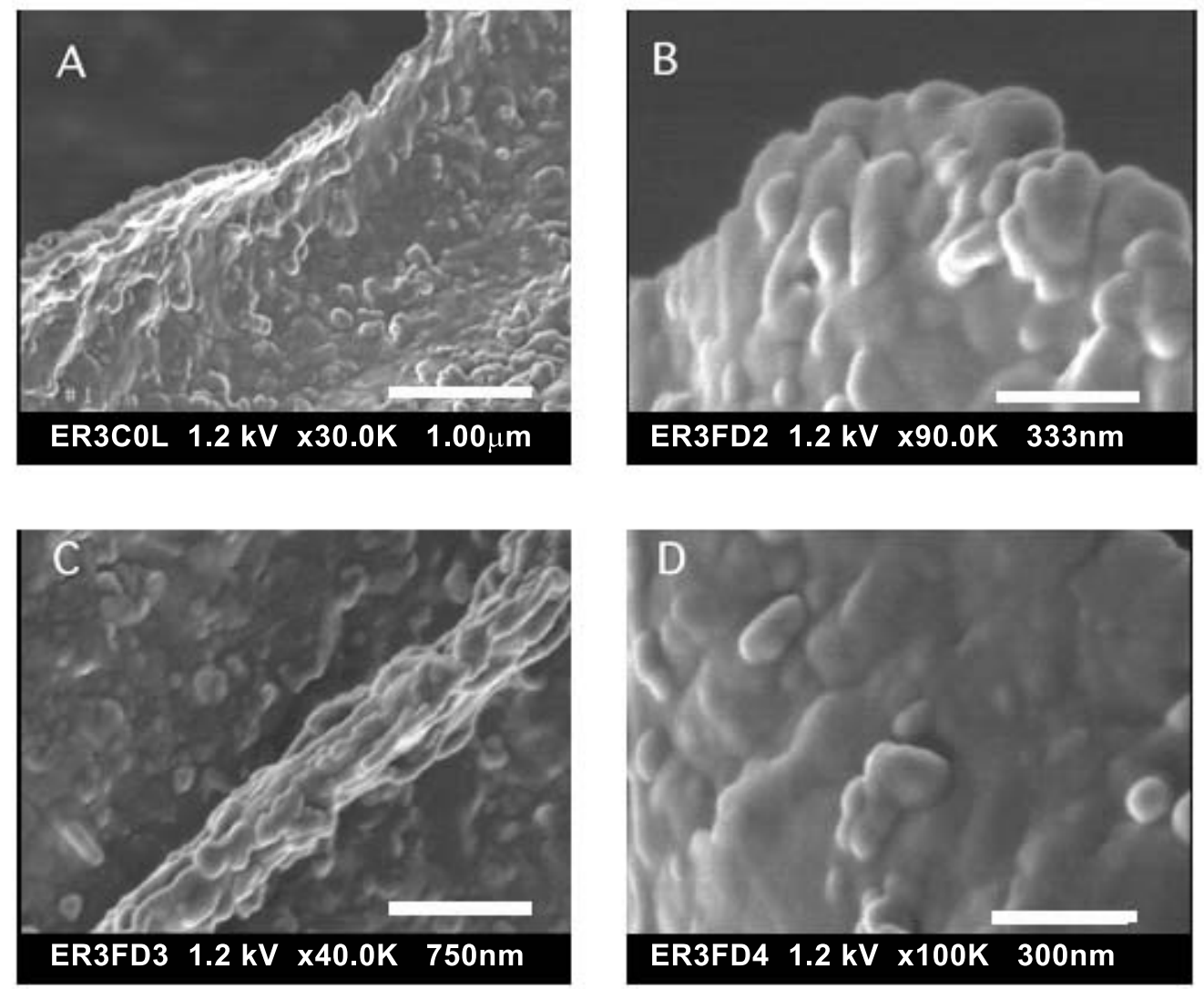

Figure 2.3. SEM photos of colloids in ER-20-5 \#3 groundwater. The minerals shown in (A) are predominantly clays. Mordenite appears infrequently as small rod-shaped laths. All minerals were identified by XRD and only tentatively identified here by their characteristic morphology. Scale bars are shown the white lines displayed at the bottom right. 

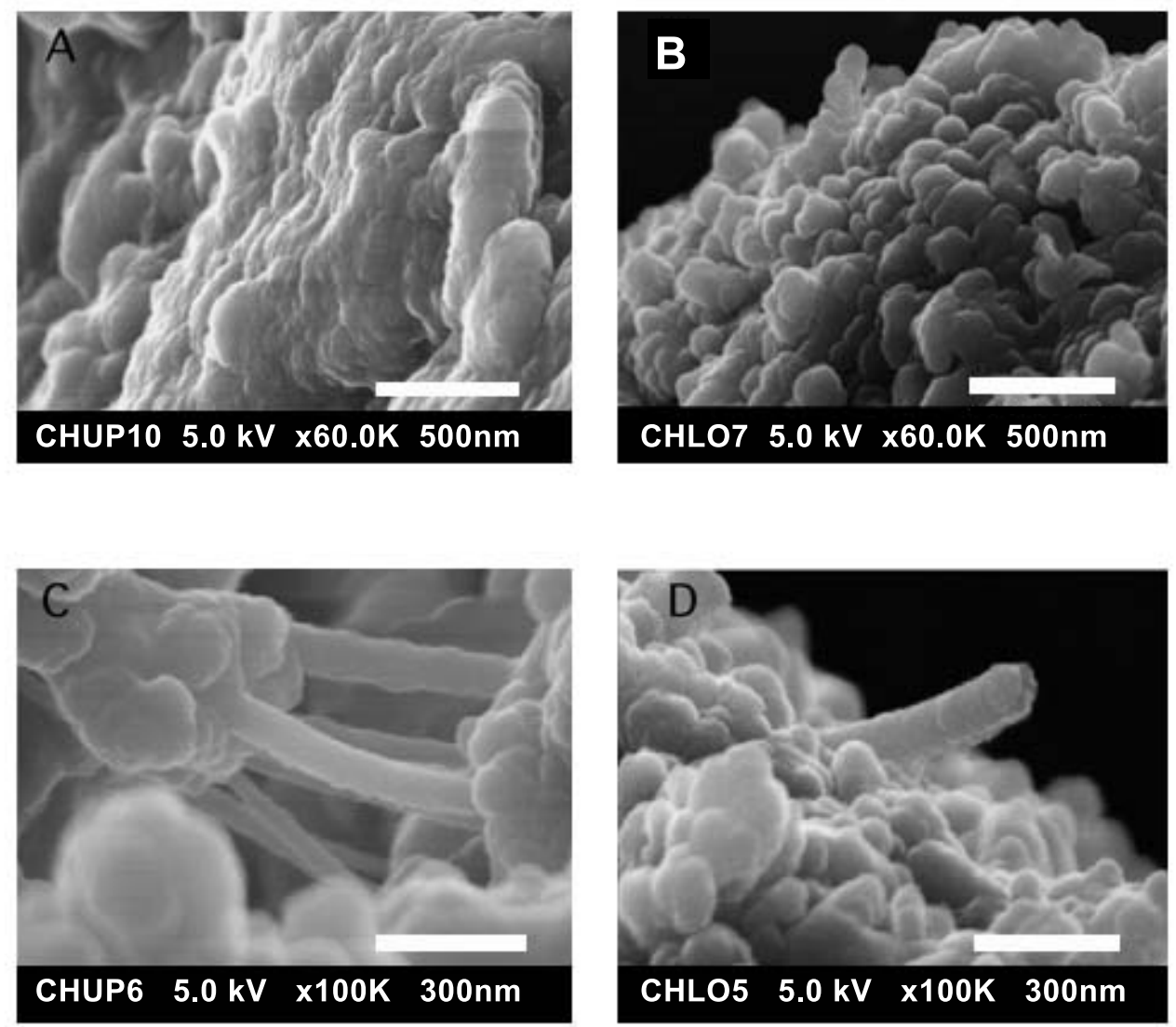

Figure 2.4. SEM photos of colloids in groundwater from U20n PS1 DDH. The rodshaped minerals shown in (C) and (D) are thought to be the zeolites identified in the XRD patterns. The flat, platy appearing minerals that dominate the morphology are thought to be clays. All minerals were identified by XRD and only tentatively identified here by their characteristic morphology. Scale bars are shown as the white lines displayed in the bottom right.

colloids that was consistent with, but not diagnostic of silicates, zeolites, and clays. The EDS analysis consisted of $\mathrm{Si}>\mathrm{Al}>\mathrm{Na}>\mathrm{Fe}+\mathrm{Ca}$. This is similar to the chemical spectra obtained for the colloids in ER-20-5 samples.

Unfortunately, no rock cuttings were available for mineral analysis from either aquifer unit at CHESHIRE. However, the host rocks are classified as fractured silicic rhyolites and thus it would not be unexpected to observe both clays and zeolites in the host units at the CHESHIRE site. 


\section{Size Distribution and Concentration of Colloids}

Our objectives for this study were to determine: 1) the concentration and particle size of the colloids, 2) whether the type of sampling container compromised the analyses, 3) if micro-bacterial growth compromised the analyses, and 4) if the length of the storage time compromised the analyses.

In order to answer these questions, samples were collected in plastic, glass, and Teflon ${ }^{\mathrm{TM}}$ containers. All sample containers were acid-leached and rinsed prior to sampling. One set of containers was spiked with preservative $\left(\mathrm{NaNO}_{3}\right)$ to kill any potential microbacterial growth. In addition, samples were analyzed repeatedly over a period of several months.

Photon correlation spectroscopy (PCS) was used to measure the size distribution and concentration of colloids in the ER-20-5 groundwater. PCS is a well established particle sizing technique used to determine particle size distribution for a wide variety of scientific and industrial fields (e.g., Allen, 1981; Chu, 1974). PCS is commonly used in the fields of physics and chemistry to characterize colloidal and polymer dispersions, gels, nucleation and aggregation processes, chemical reaction kinetics, and phase transitions.

One of the characteristics of particles in the nanometer to micrometer range that are suspended in a fluid is that they perform a constant, random thermal, or Brownian motion. This causes the intensity of light scattered by the particles to vary with time. Since larger particles move more slowly than smaller particles, the rate of fluctuations of the scattered light will also be slower. The PCS method uses the fluctuation rate of the scattered light to determine the size distribution of the particles suspended in the fluid.

For particle concentration analysis, the static intensity of the scattered light is used. Since standard particle solutions provide the calibration over the concentration range of interest, a set of standards allows the calculation of the concentration calibration functions for each desired particle size. To calibrate the light scattering intensity as a function of particle size and concentration, polystyrene particle standards are used $(54 \mathrm{~nm}, 105 \mathrm{~nm}$, $198 \mathrm{~nm}, 496 \mathrm{~nm}$ and $895 \mathrm{~nm}$ ). The calibration curves are then fitted to linear equations, the $y$-intercept being a function of the particle concentration. As a result, the particle concentration can be derived from the light scattering intensity.

The PCS system at LLNL was designed using a INNOVA 70 Ar-ion laser and MALVERN K7032-8 correlator, manufactured by MALVERN Instruments Ltd., Malvern, Worcs., UK (Fig. 2.5). For each analysis, $4 \mathrm{ml}$ of sample was placed in the sample chamber and analyzed. No dilutions were necessary, although filtrations were performed to determine if there were multiple population sizes in each individual sample. 


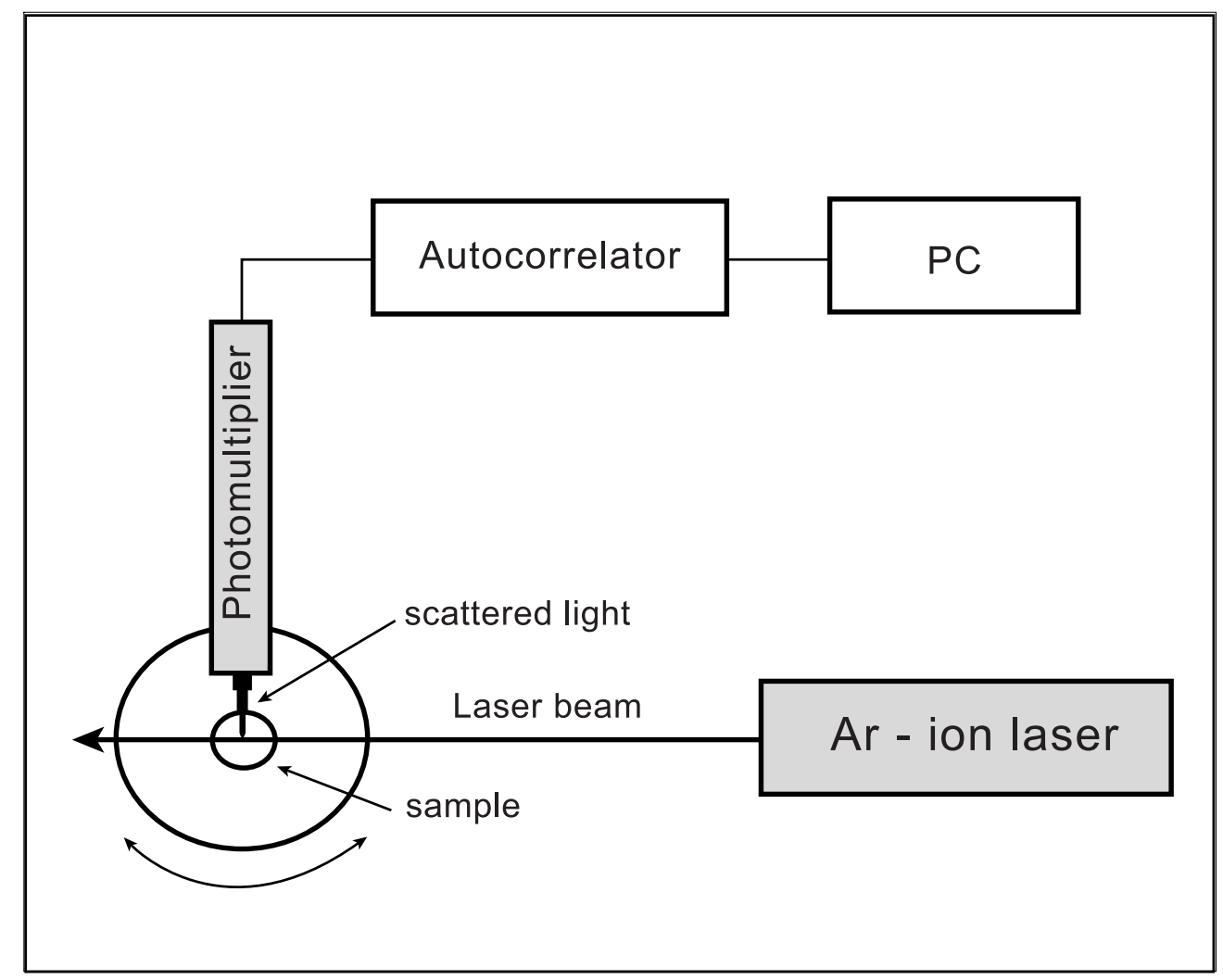

Figure 2.5. Schematic diagram of the PCS experimental set-up designed to measure particle size in liquid.

\section{ER-20-5 well Cluster}

The size distribution of colloids measured from ER-20-5 \#1 is shown in Figure 2.6 and 2.7, and Table 2.1. Figure 2.6 shows the variation in colloid size for a representative sample. The samples were filtered to determine if there were multiple size populations. The samples were all mono-modal, with all measured colloids falling within a narrow range from 80 to $100 \mathrm{~nm}$. The average colloid size was $90.9 \mathrm{~nm}$ (Table 2.1). The SEM photos qualitatively confirm this result, since the average size of the colloids in the photos is around $100 \mathrm{~nm}$.

Table 2.1. Colloid size for ER-20-5 \#1.

\begin{tabular}{|l|c|c|c|}
\hline $\begin{array}{l}\text { Container } \\
\text { Type }\end{array}$ & $\begin{array}{c}\text { Time in container } \\
\text { Following sampling } \\
\text { (days) }\end{array}$ & $\begin{array}{c}\text { Average Colloid } \\
\text { Size } \\
\text { (nm) }\end{array}$ & $\begin{array}{c}\text { Standard } \\
\text { Error }\end{array}$ \\
\hline & & & \\
\hline LDPE* & 6 & 86.47 & 5.85 \\
\hline & 20 & 95.62 & 4.19 \\
\hline & & & \\
\hline LDPE & 7 & 93.00 & 3.23 \\
\hline & 20 & 93.73 & 6.47 \\
\hline & 72 & 90.02 & 3.25 \\
\hline
\end{tabular}




\begin{tabular}{|c|c|c|c|}
\hline Glass & 7 & 87.74 & 5.14 \\
\hline & 20 & 87.70 & 6.94 \\
\hline Glass & 7 & 9630 & 484 \\
\hline (with preservative) & 20 & 91.82 & 6.38 \\
\hline & 72 & 85.37 & 3.41 \\
\hline Teflon ${ }^{\mathrm{TM}}$ & 6 & 96.34 & 3.68 \\
\hline & 20 & 86.82 & 6.56 \\
\hline Teflon ${ }^{\mathrm{TM}}$ & 7 & 89.26 & 6.44 \\
\hline (with preservative) & 20 & 93.15 & 0.59 \\
\hline & 72 & 90.12 & 2.14 \\
\hline & Summed Average: & 90.90 & \\
\hline
\end{tabular}

* low density polyethylene plastic

Figure 2.7 is a plot of the particle size versus time for the samples stored in different containers. The plot shows that the size distribution the colloids in this sample is independent of the type of sampling container used (polyethylene, glass, or Teflon ${ }^{\mathrm{TM}}$ ), addition of preservative, or time between sampling and analysis.

The size distribution of the colloids measured from ER-20-5 \#3 is shown in Figure 2.6, 2.8 and Table 2.2.. Samples were not collected in Teflon ${ }^{\mathrm{TM}}$ containers. All measured colloids were mono-modal and fell within a narrow range of 70 to $85 \mathrm{~nm}$. The average colloid size was $80.8 \mathrm{~nm}$ (Table 2.2). As observed with the colloids from ER-20-5 \#1, these data were independent of sample container, addition of preservative, or time between sampling and analysis. The average size of colloids from the ER-20-5 \#3 sample was slightly smaller than colloids from ER-20-5 \#1.

The colloid concentrations in groundwater from both ER-20-5 \#1 and \#3 are shown in Figure 2.9 and Table 2.3, and Figure 2.10 and Table 2.4, respectively. The average number of colloids in groundwater from ER-20-5 \#1 was $3.02 \times 10^{10}$ colloid particles $/ \mathrm{ml}$. In contrast, the average number for ER-20-5 \#3 was $7.85 \times 10^{10}$ colloid particles $/ \mathrm{ml}$, indicating that groundwater from ER-20-5 \#3 contains approximately 2.6 times more colloids than groundwater from ER-20-5 \#1. These differences represent qualitative observations during the filtration process. It was noted that more groundwater had to be filtered from ER-20-5 \#1 than from ER-20-5 \#3 for approximately the same volume of colloidal material. Groundwater was pumped from both wells at the same rate $(0.03$ $\left.\mathrm{m}^{3} / \mathrm{min}\right)$. 


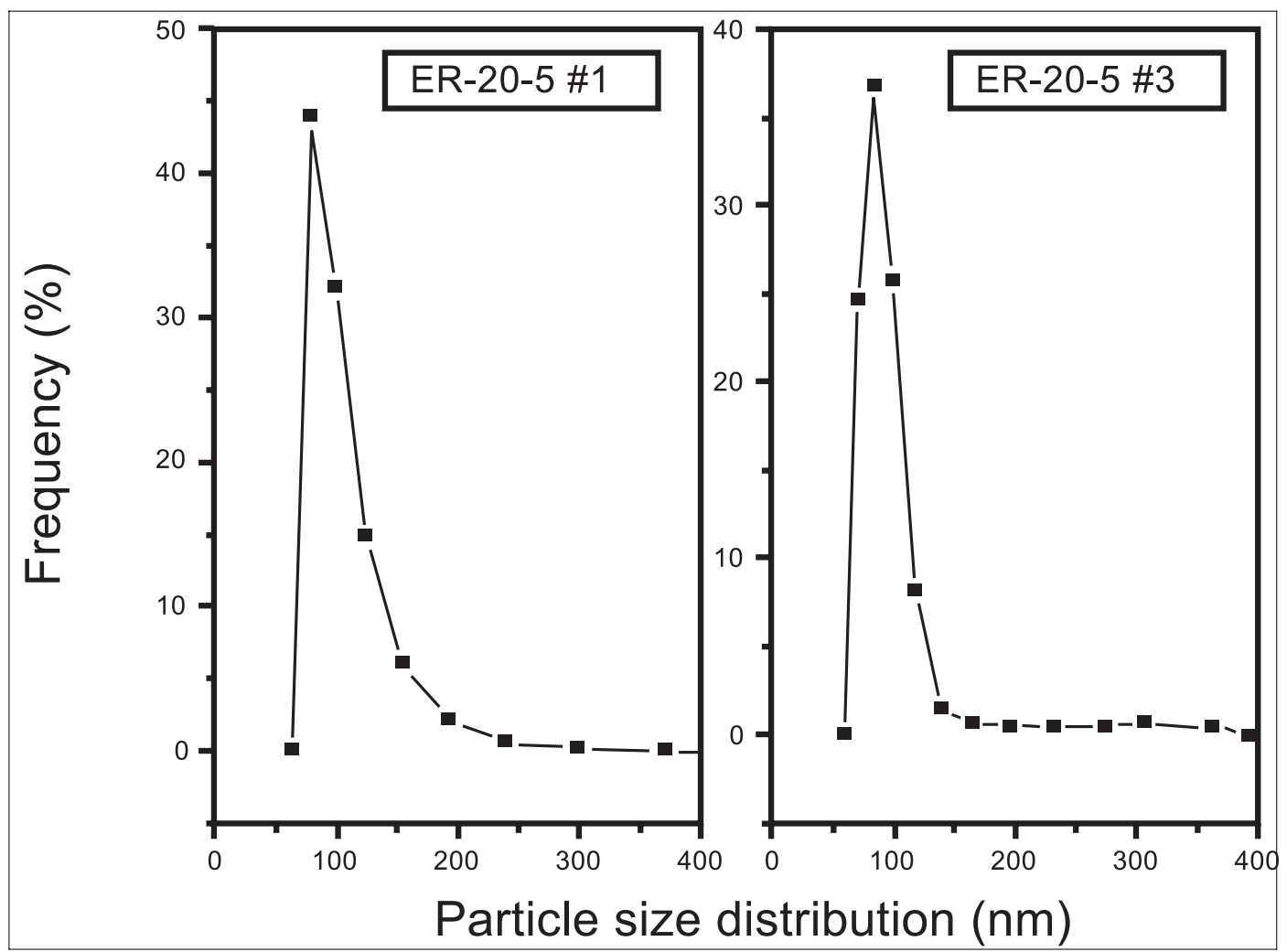

Figure 2.6. Representative particle size distribution for groundwater from ER-20-5 \#1 and ER-20-5 \#3. 

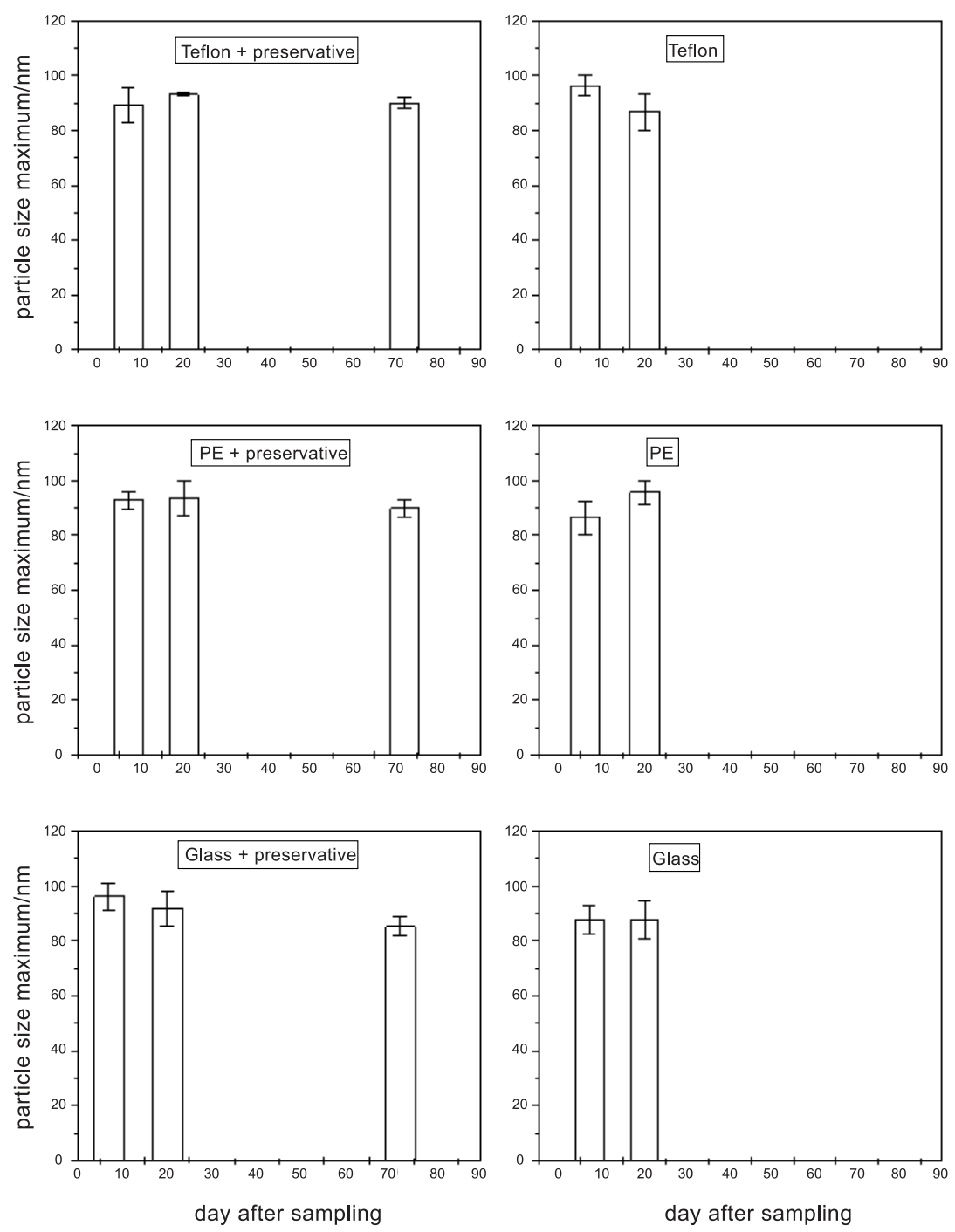

Figure 2.7. Colloid Size Distribution for groundwater samples from ER-20-5 \#1 as a function of time, preservative, and container type. Containers used were glass, Teflon ${ }^{\mathrm{TM}}$, and polyethylene plastic (PE). 
Table 2.2. Colloid size for ER-20-5 \#3.

\begin{tabular}{|c|c|c|c|}
\hline $\begin{array}{l}\text { Container } \\
\text { Type }\end{array}$ & $\begin{array}{c}\text { Time in container } \\
\text { Following sampling } \\
\text { (days) }\end{array}$ & $\begin{array}{c}\text { Average Colloid } \\
\text { Size } \\
(\mathbf{n m})\end{array}$ & $\begin{array}{c}\text { Standard } \\
\text { Error }\end{array}$ \\
\hline \multirow[t]{6}{*}{ LDPE } & 21 & 81.47 & 4.01 \\
\hline & 30 & 79.34 & 4.61 \\
\hline & 35 & 77.35 & 4.93 \\
\hline & 41 & 80.74 & 2.39 \\
\hline & 48 & 77.36 & 3.64 \\
\hline & 133 & 79.59 & 2.70 \\
\hline LDPE & 22 & 81.85 & 4.24 \\
\hline \multirow[t]{6}{*}{ (with preservative) } & 29 & 76.64 & 2.87 \\
\hline & 30 & 84.78 & 7.8 \\
\hline & 35 & 79.89 & 2.70 \\
\hline & 41 & 84.94 & 5.95 \\
\hline & 48 & 79.90 & 5.47 \\
\hline & 133 & 82.78 & 3.65 \\
\hline \multirow[t]{7}{*}{ Glass } & 21 & 81.67 & 4.23 \\
\hline & 29 & 82.60 & 4.84 \\
\hline & 35 & 85.30 & 4.11 \\
\hline & 41 & 80.96 & 3.87 \\
\hline & 48 & 81.64 & 3.48 \\
\hline & 133 & 76.40 & 5.79 \\
\hline & Summed Average: & 80.80 & \\
\hline
\end{tabular}


Table 2.3. Colloid Concentration for ER-20-5 \#1.

\begin{tabular}{|c|c|c|c|}
\hline $\begin{array}{l}\text { Container } \\
\text { Type }\end{array}$ & $\begin{array}{l}\text { Time in container } \\
\text { Following sampling } \\
\text { (days) }\end{array}$ & $\begin{array}{l}\text { Number of } \\
\text { Colloids } \\
\text { (particles/ml) }\end{array}$ & $\begin{array}{l}\text { Standard } \\
\text { Error }\end{array}$ \\
\hline \multirow[t]{3}{*}{$\overline{\mathrm{LDPE}}$} & 6 & $3.60 \times 10^{10}$ & $5.57 \times 10^{8}$ \\
\hline & 20 & $2.32 \times 10^{10}$ & $3.59 \times 10^{8}$ \\
\hline & 63 & $2.95 \times 10^{10}$ & $8.80 \times 10^{8}$ \\
\hline LDPE & 7 & $2.72 \times 10^{10}$ & $4.22 \times 10^{8}$ \\
\hline \multirow[t]{3}{*}{ (with preservative) } & 20 & $2.57 \times 10^{10}$ & $3.98 \times 10^{8}$ \\
\hline & 63 & $2.72 \times 10^{10}$ & $4.34 \times 10^{8}$ \\
\hline & 72 & $2.94 \times 10^{10}$ & $1.97 \times 10^{8}$ \\
\hline \multirow[t]{3}{*}{ Glass } & 7 & $3.42 \times 10^{10}$ & $5.29 \times 10^{8}$ \\
\hline & 20 & $3.60 \times 10^{10}$ & $5.57 \times 10^{8}$ \\
\hline & 63 & $3.59 \times 10^{10}$ & $8.4 \times 10^{8}$ \\
\hline Glass & 7 & $2.31 \times 10^{10}$ & $3.58 \times 10^{8}$ \\
\hline \multirow[t]{2}{*}{ (with preservative) } & 63 & $2.82 \times 10^{10}$ & $2.64 \times 10^{8}$ \\
\hline & 72 & $3.83 \times 10^{10}$ & $2.23 \times 10^{8}$ \\
\hline \multirow[t]{3}{*}{ Teflon ${ }^{\mathrm{TM}}$} & 6 & $2.04 \times 10^{10}$ & $3.16 \times 10^{8}$ \\
\hline & 20 & $3.76 \times 10^{10}$ & $5.83 \times 10^{8}$ \\
\hline & 63 & $2.86 \times 10^{10}$ & $7.55 \times 10^{8}$ \\
\hline Teflon ${ }^{\mathrm{TM}}$ & 7 & $3.28 \times 10^{10}$ & $5.07 \times 10^{8}$ \\
\hline \multirow[t]{4}{*}{ (with preservative) } & 20 & $1.97 \times 10^{10}$ & $3.05 \times 10^{8}$ \\
\hline & 63 & $2.90 \times 10^{10}$ & $2.14 \times 10^{8}$ \\
\hline & 72 & $2.87 \times 10^{10}$ & $4.14 \times 10^{8}$ \\
\hline & Summed Average: & $3.02 \times 10^{10}$ & \\
\hline
\end{tabular}


Table 2.4. Colloid Concentration for ER-20-5 \#3.

\begin{tabular}{|c|c|c|c|}
\hline $\begin{array}{l}\text { Container } \\
\text { Type }\end{array}$ & $\begin{array}{c}\text { Time in container } \\
\text { Following sampling } \\
\text { (days) }\end{array}$ & $\begin{array}{c}\text { Number of } \\
\text { Colloids } \\
\text { (particles/ml) }\end{array}$ & $\begin{array}{c}\text { Standard } \\
\text { Error }\end{array}$ \\
\hline \multirow[t]{6}{*}{ LDPE } & 21 & $7.48 \times 10^{10}$ & $1.66 \times 10^{9}$ \\
\hline & 30 & $9.02 \times 10^{10}$ & $1.58 \times 10^{9}$ \\
\hline & 35 & $9.60 \times 10^{10}$ & $1.76 \times 10^{9}$ \\
\hline & 41 & $7.59 \times 10^{10}$ & $9.14 \times 10^{8}$ \\
\hline & 48 & $9.47 \times 10^{10}$ & $7.34 \times 10^{8}$ \\
\hline & 133 & $7.95 \times 10^{10}$ & $1.25 \times 10^{8}$ \\
\hline LDPE & 22 & $7.03 \times 10^{10}$ & $5.76 \times 10^{8}$ \\
\hline \multirow[t]{6}{*}{ (with preservative) } & 29 & $1.04 \times 10^{11}$ & $7.00 \times 10^{8}$ \\
\hline & 30 & $6.61 \times 10^{10}$ & $1.03 \times 10^{9}$ \\
\hline & 35 & $8.43 \times 10^{10}$ & $1.04 \times 10^{9}$ \\
\hline & 41 & $5.75 \times 10^{10}$ & $3.68 \times 10^{8}$ \\
\hline & 48 & $7.98 \times 10^{10}$ & $9.27 \times 10^{8}$ \\
\hline & 133 & $6.50 \times 10^{10}$ & $6.55 \times 10^{8}$ \\
\hline \multirow[t]{7}{*}{ Glass } & 21 & $6.77 \times 10^{10}$ & $3.24 \times 10^{9}$ \\
\hline & 29 & $7.24 \times 10^{10}$ & $7.50 \times 10^{8}$ \\
\hline & 35 & $5.80 \times 10^{10}$ & $3.05 \times 10^{8}$ \\
\hline & 41 & $7.65 \times 10^{10}$ & $6.85 \times 10^{8}$ \\
\hline & 48 & $7.68 \times 10^{10}$ & $1.29 \times 10^{9}$ \\
\hline & 133 & $1.03 \times 10^{11}$ & $2.56 \times 10^{9}$ \\
\hline & Summed Average: & $7.86 \times 10^{10}$ & \\
\hline
\end{tabular}




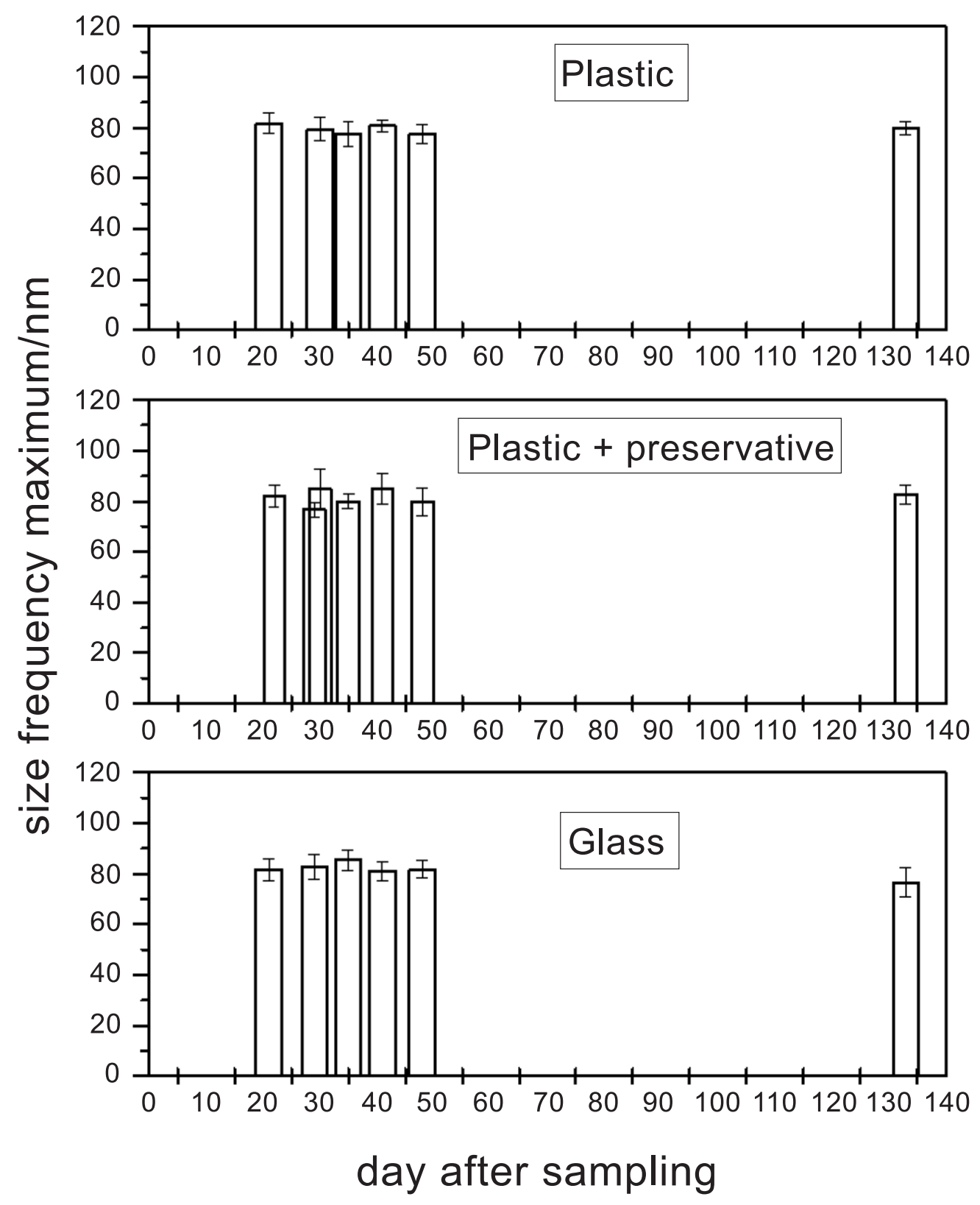

Figure 2.8. Colloid size distribution for groundwater samples from ER-20-5 \#3. 

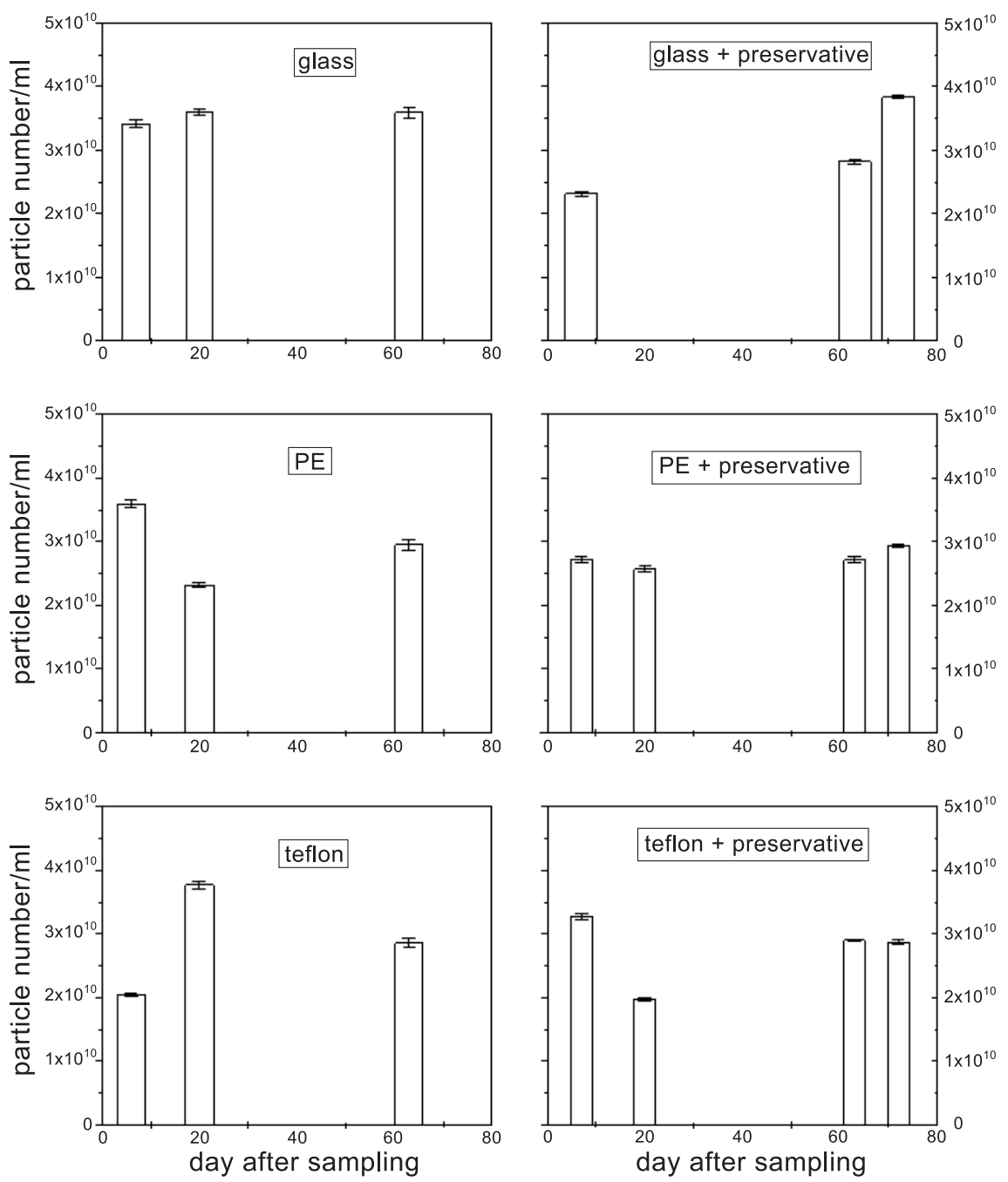

Figure 2.9. Concentration of colloids in groundwater from ER-20-5 \#1 as a function of time. 

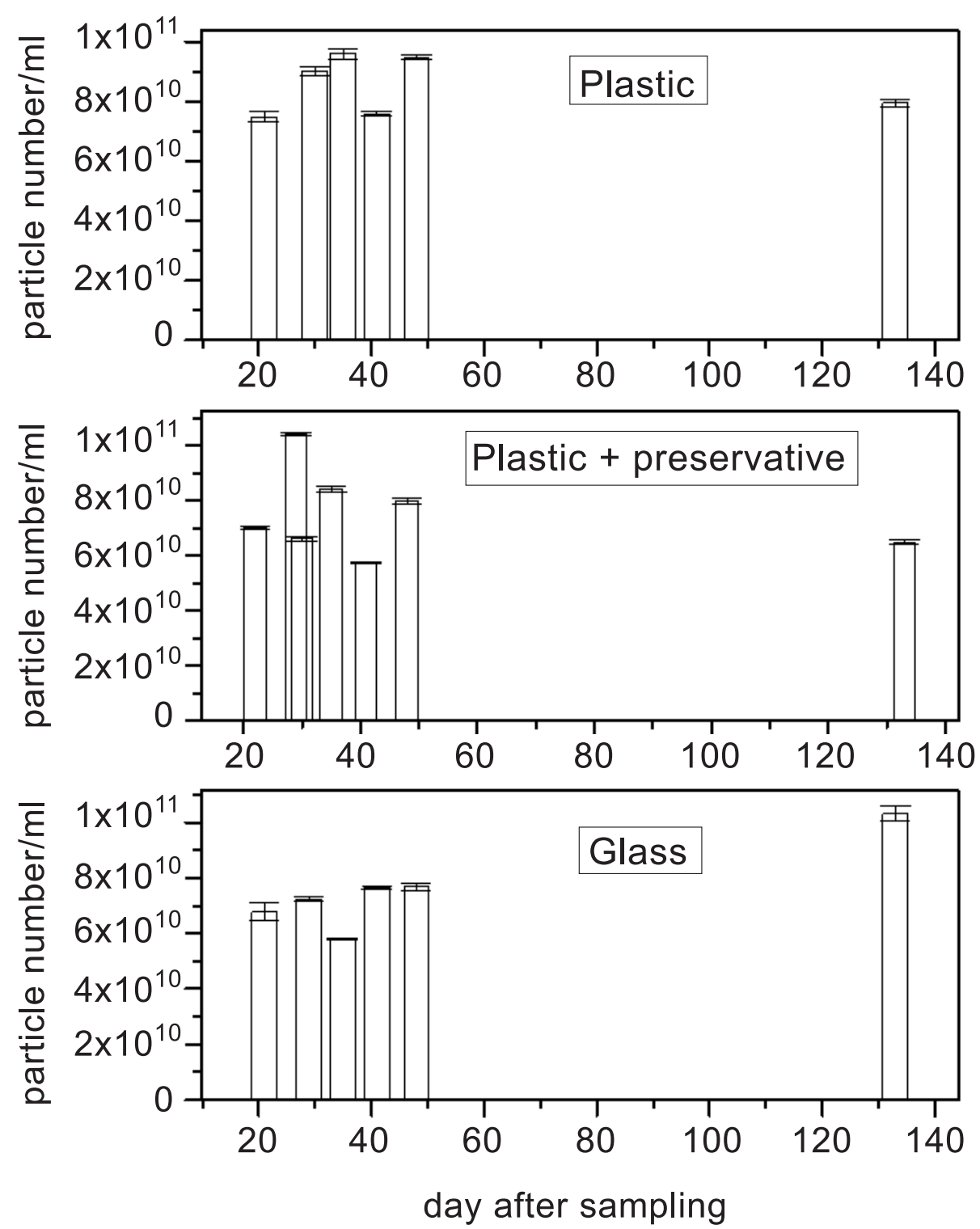

Figure 2.10. Concentration of colloids in groundwater from ER-20-5\#3 as a function of time. 


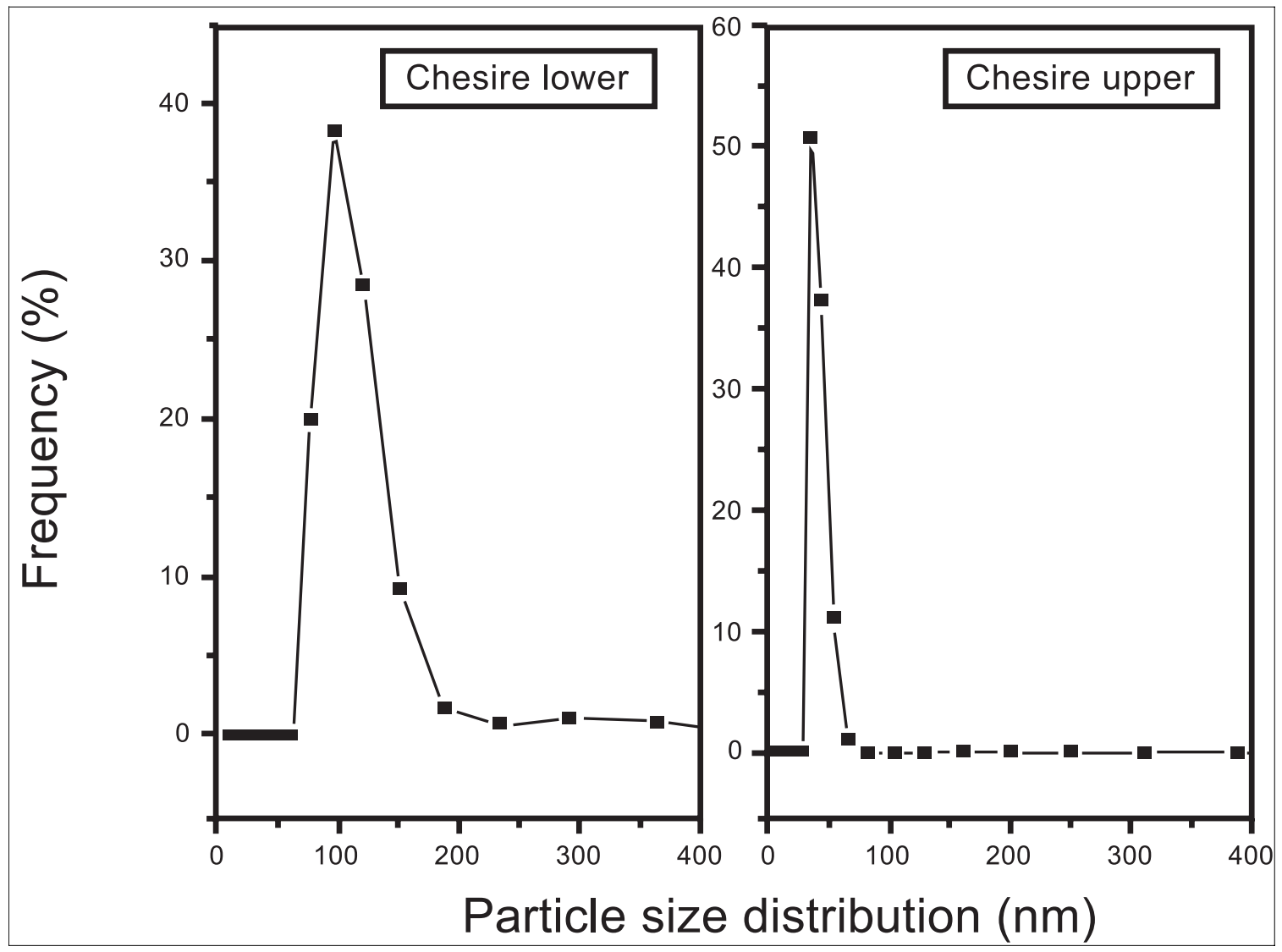

Figure 2.11. Representative particle size distribution for groundwater from the upper and lower aquifer at CHESHIRE.

\section{CHESHIRE drill-back (Well U20n PS1 DDH)}

Having determined that the results from colloid analyses are independent of the sample container used, we collected all of the CHESHIRE samples in plastic 1-liter bottles. All sample bottles were acid-leached prior to sampling. Samples were kept very cool (on ice) until they could be analyzed at LLNL.

Figure 2.11 shows the variation in colloid size for a representative sample from both the upper and lower aquifer units at CHESHIRE. The samples were filtered to determine if there were multiple size populations. The samples were all mono-modal, with the majority of colloids falling within a narrow range from 50 to $150 \mathrm{~nm}$. The average size of colloids from the lower CHESHIRE sample was approximately $90 \mathrm{~nm}$. The average size of colloids from the upper CHESHIRE sample was smaller, averaging approximately 60-70 $\mathrm{nm}$. The SEM photos qualitatively confirm this result, as the average size colloids in the photos are small $(<<500 \mathrm{~nm})$.

All four colloid samples had mono-modal colloid size distributions. Although the average colloid size was different, ranging from 50 to $150 \mathrm{~nm}$, the range was still quite 
small over all. It is likely, that under the hydrologic conditions on Pahute Mesa the $<200$ $\mathrm{nm}$ size colloid is dominant.

\subsection{Comparison of Results with Previous Colloid Characterization Studies at and Near the NTS}

McCarthy and Degueldre (1993) compiled literature data on the concentration and composition of natural colloids, with their respective groundwater chemistry from wells at potential high-level waste sites around the world. When all the studies were compared, the following generalizations were made:

- Colloids were found in every sample, although there was a large variation in concentration $(0.0002-200 \mathrm{mg} / \mathrm{L})$.

- The most common minerals identified were clays, zeolites, Fe-oxides, Mn-oxides, and silica, and in general the colloid composition is reflective of the fracture rock or host rock mineralogy.

- Colloid concentrations are lowest in the deepest fracture flow environments.

Our findings that colloids are ubiquitious, and that the mineralogy of the colloids are consistent with the host rock or fracture mineralogy are consistent with the overall results by McCarthy and Degueldre (1993). In addition, two separate studies have analyzed the concentration and composition of groundwater colloids from wells at the NTS and one well near the Yucca Mountain Repository, J-13 (Viani and Martin, 1996; Kung and Fresquez, 2000). Viani and Martin (1996) found that the colloid masses from water from the 4 wells at the NTS and J-13 ranged between $3.12 \mathrm{mg} / \mathrm{L}$ to $0.03 \mathrm{mg} / \mathrm{L}$.

Kung and Fresquez (2000) analyzed colloids from the ER-20-5 cluster, the CHESHIRE drill-back as well as colloids filtered from J-13. Special effort was given to understanding the effect of sample collection and preparation to colloid concentration and size distribution. As with our studies, Kung and Fresquez (2000) observed that a prolonged waiting period after sampling was found to affect both the colloid size distribution as well as colloid concentration resulting from changes in water chemistry. They found that colloids collected from the ER20-5-1 and ER-20-5-3 have similar particle size distributions. Most of these colloidal particles are less than $100 \mathrm{~nm}$ in size. More than $98 \%$ of the colloids were smaller than $100 \mathrm{~nm}$ in size. For the particle size range between 50- and 200-nm, about ten trillion (1E10) colloidal particles per liter are present in these water samples. Furthermore, it was found that the smaller the sizes of colloid, the higher the colloid concentration present in the water. Although Kung and Fresquez (2000) used different techniques at a different laboratory to measure the size, concentration and composition of colloids most observations agree with our findings.

\subsection{Summary}

The combination of XRD, SEM, and PCS analysis was successfully used to characterize the colloids in groundwater collected from four different aquifers on Pahute Mesa at the NTS. To date, from these results we can conclude: 
- The colloids in the NTS groundwater samples are mineralogically similar, although the actual abundance of each mineral may vary from aquifer to aquifer.

- The colloidal material is composed primarily of clays and zeolites. This suggests that the potentially important Pu-sorbing minerals are clays and zeolites for groundwater hosted in silicic volcanic units. This does not rule out the importance of minor colloidal minerals, those present at less than $10 \%$.

- The same clays and zeolites identified in the groundwater samples are also present in the host rock aquifers at ER-20-5. The colloid minerals mimic the minor, host rock minerals from which they originated, and most likely represent the fracture lining minerals.

- The size distribution of the colloids from all four groundwater samples is monomodal, consisting of small particles $<200 \mathrm{~nm}$. We suggest that the relatively small colloid size is a reflection of the maximum size that the aquifer has the ability to transport. The ability of aquifers to transport colloids is a function of the host rock mineralogy, water chemistry, width of the fractures, and groundwater flow rate.

- The concentration of colloids in ER-20-5 \#1 is approximately $3 \times 10^{10}$ colloids/ml; ER-20-5 \#3 is $8 \times 10^{10}$ colloids/ml. Groundwater from ER-20-5 \#3 has $~ 2.6$ times more colloids/ml than ER-20-5 \#1 (groundwater was pumped from both wells at the same rate of $0.03 \mathrm{~m}^{3} / \mathrm{min}$ ). The actual concentration of the colloids must be interpreted with extreme caution however, since the pumping rates during sampling were very high. These high pumping rates most likely produced an artificially high value for the measured colloid load. Therefore, the concentrations measured in this study most likely represent maximum concentrations of colloidal material.

- The results of the PCS measurements are independent of sample container used, addition of preservative, or time between sampling and analysis. This result gives us confidence that the size, composition, and concentration of the colloids that we measure reflect what is actually collected in the field. 


\subsection{References}

Allen, T. (1981) Particle Size Measurement. 3rd Ed. Chapman \& Hall, New York, p. 678.

Chu, B. (1974) Laser Light Scattering. Academic Press, New York, p. 317.

Kersting, A. B., D. W. Efurd, et al. (1999). Migration of Plutonium in Groundwater at the Nevada Test Site." Nature 397: 56-59.

Kung, S.K. and P.R. Fresquez. (2000) Colloid Characterization and Quantification in Groundwater Samples. Los Alamos National Laboratory. LA-13727-MS. P.41. http:/lib-www.lanl.gov/cgi-gin/getfile?00538241.pdf.

McCarthy, J.F. and Degueldre, C. (1993) Environmental particles. Eds. Buffle, J. and van Leeuwen, H.P. Boca Raton, Florida: Lewis Publisher. 2, p. 426.

Thompson, J. L., Ed. (1998). Laboratory and Field Studies Related to Radionuclide Migration at the Nevada Test Site October 1, 1996 - September 30, 1997. Los Alamos, Los Alamos National Laboratory: p. 31.

Viani, B. E. and S. I. Martin (1996) Groundwater Colloid Characterization: Lawrence Livermore National Laboratory. UCRL-ID-132087. p. 30. 


\title{
-Chapter 3- \\ Characterization of Colloids in Water From a Vadose Zone: Rainier Mesa, Nevada Test Site
}

\author{
Annie B. Kersting, Dave K. Smith, and Lumin Wang
}

\subsection{Introduction}

Kersting et al. (1999) showed that the Pu transported $1.3 \mathrm{~km}$ in two different aquifers from an underground nuclear test at the Nevada Test Site (NTS) was associated with the colloidal and not the dissolved fraction of the groundwater. In a previous study at the NTS, the low-solubility radionuclides, $\mathrm{Co}, \mathrm{Ce}, \mathrm{Eu}$, were detected in groundwater collected $300 \mathrm{~m}$ downgradient from the CHESHIRE underground nuclear test (Buddeimeier and Hunt, 1988). The majority of the radionuclides were associated with the colloidal fraction of the groundwater. Both these studies investigated water collected below the water table, in saturated fracture flow systems. These two studies suggest that colloids in saturated fracture flow systems can and do facilitate the transport of lowsolubility radionuclides. Yet, many questions remain as to the importance of colloids as a transport mechanisms for contaminants. Are there other geologic settings where colloidfacilitated transport of low-solubility radionuclides is important? At the NTS, two-thirds of all the tests were detonated in the vadose zone, or unsaturated subsurface located above the water table. Can colloids facilitate transport of low-solubility radionuclides in vadose zone environments?

In the following study, groundwater containing radionuclides was collected from an unsaturated fracture flow environment at the NTS in order to evaluate whether or not colloids are associated with radionuclides in a vadose zone. Groundwater was collected dripping from fracture surfaces exposed in tunnel bores near the location of past underground nuclear tests. The tunnels are located in Rainier Mesa, in fractured volcanic tuff, in a unit called the Tunnel Formation (14 Ma). The Tunnel Formation lies stratigraphically below the Calico Hills Formation (12.9 Ma) (Laczniak et al., 1996).

The purpose of this project was to, 1) collect water dripping from fractures surfaces inside the tunnels in Rainier Mesa, 2), analyze the water samples for radionuclides, 3) characterize the colloids in the groundwater, and 4) compare findings to earlier work on colloid characteization in saturated groundwater systems at the NTS.

\section{2. $\quad$ Fieldwork in the Rainier Mesa Tunnels, NTS}

In August of 1998, scientists from LLNL in conjunction with Defense Threat Reduction Agency (DTRA), spent two days collecting water samples from the tunnels on Rainier Mesa, NTS (Figure 3.1). Underground nuclear tests were fired in a series of tunnel emplacement holes drilled into the side of a hill in fractured volcanic tuff. The tunnel system provides unprecedented access to waters contaminated with radionuclides as a result of previous underground nuclear testing, without the complication of pumping groundwater from wells. 


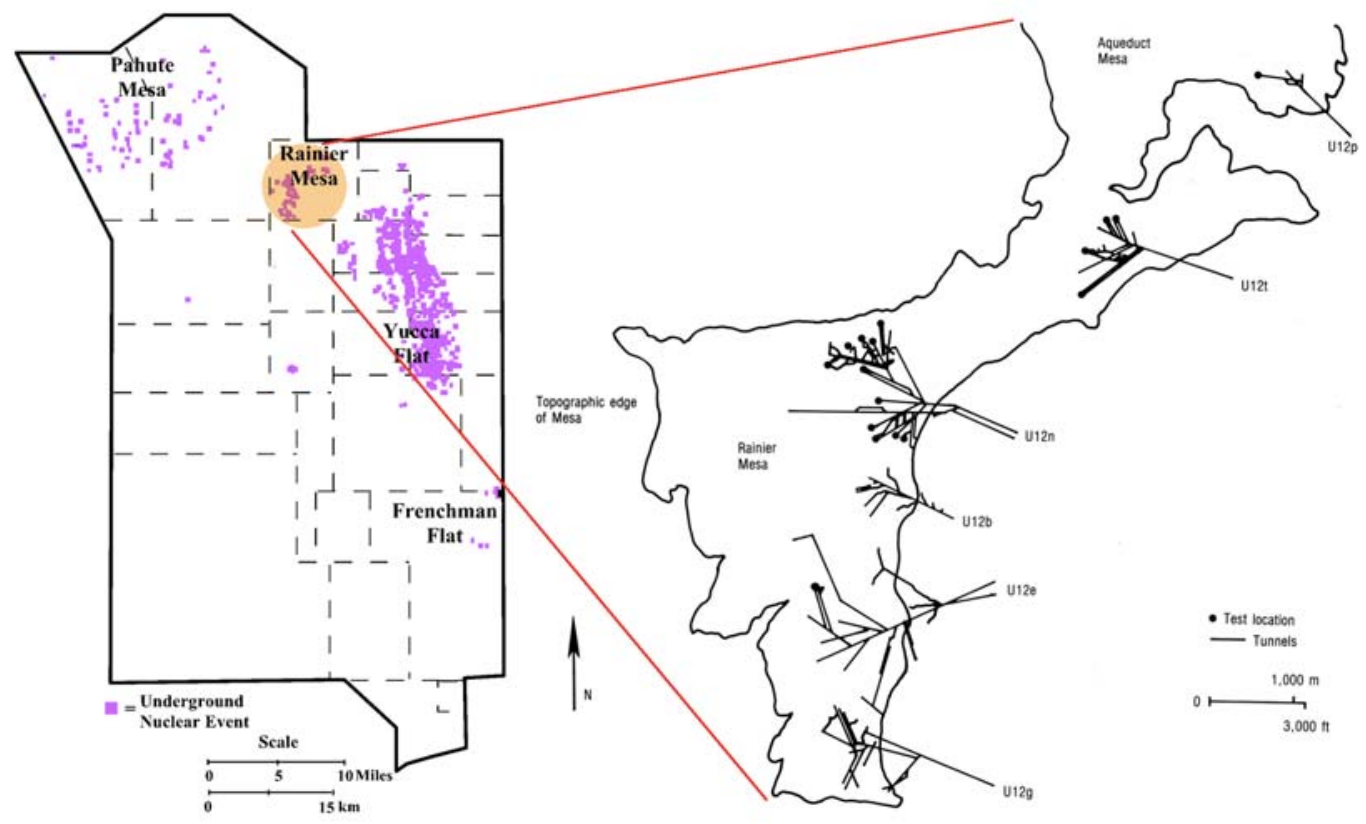

Figure 3.1. Map view of the Nevada Test Site showing an expanded view of the field area, Rainier Mesa. Purple and magenta dots represent the location of each underground nuclear test. Expanded area of Rainier Mesa shows the location of underground tunnel system.

Samples were collected from three tunnels; U12E, U12T and U12N. The majority of the water samples were collected from pipes sampling vadose zone water collected behind cement portals (Figure 3.2A). In tunnel U12T, we were able to collect water that was dripping off the tunnel walls unencumbered by the engineered cement barriers (Figure 3.2B). This water is the most representative natural vadose zone water, not in contact with engineered barriers. Therefore, all colloid characterization work was carried out using these samples (U12T-A, U12T-B, U12T-C, U12T-D, and U12T-E). Samples from $\mathrm{U} 12 \mathrm{~T}$ were collected at different locations along the tunnel bore. 

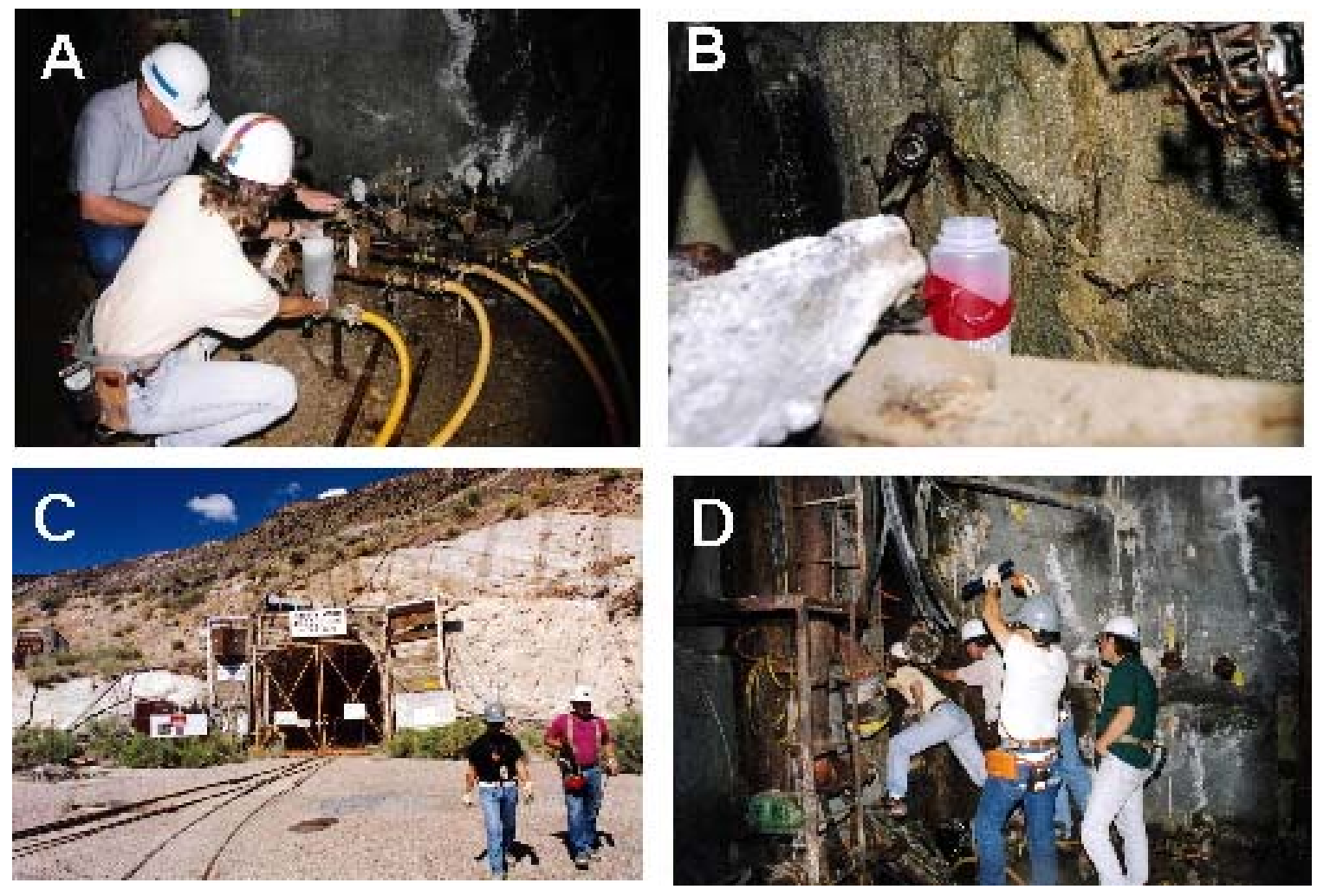

Figure 3.2. A) water collected from pipes in tunnel U12E. B) water collected from the rock wall of tunnel U12T, C) entrance to T-tunnel, D) water collection location in TTunnel

\subsection{Groundwater Composition}

Water samples were selected analyzed for groundwater composition, colloid content, gamma-emitting radionuclides, $\mathrm{Pu}, \mathrm{Am}$ and ${ }^{90} \mathrm{Sr}$. Figure 3.3 is a comparison of the cation composition of water collected in U12T-A with J-13, WW-20, and ER20-5. WW-20 and ER20-5 are wells located in the saturated zone hosted volcanic rocks in Pahute Mesa, NTS. J-13 is representative groundwater from the proposed Yucca Mountain Repository, located adjacent to the NTS. The water in the tunnels is similar to water on Pahute Mesa, although the $\mathrm{Na}$ concentration is much higher. 


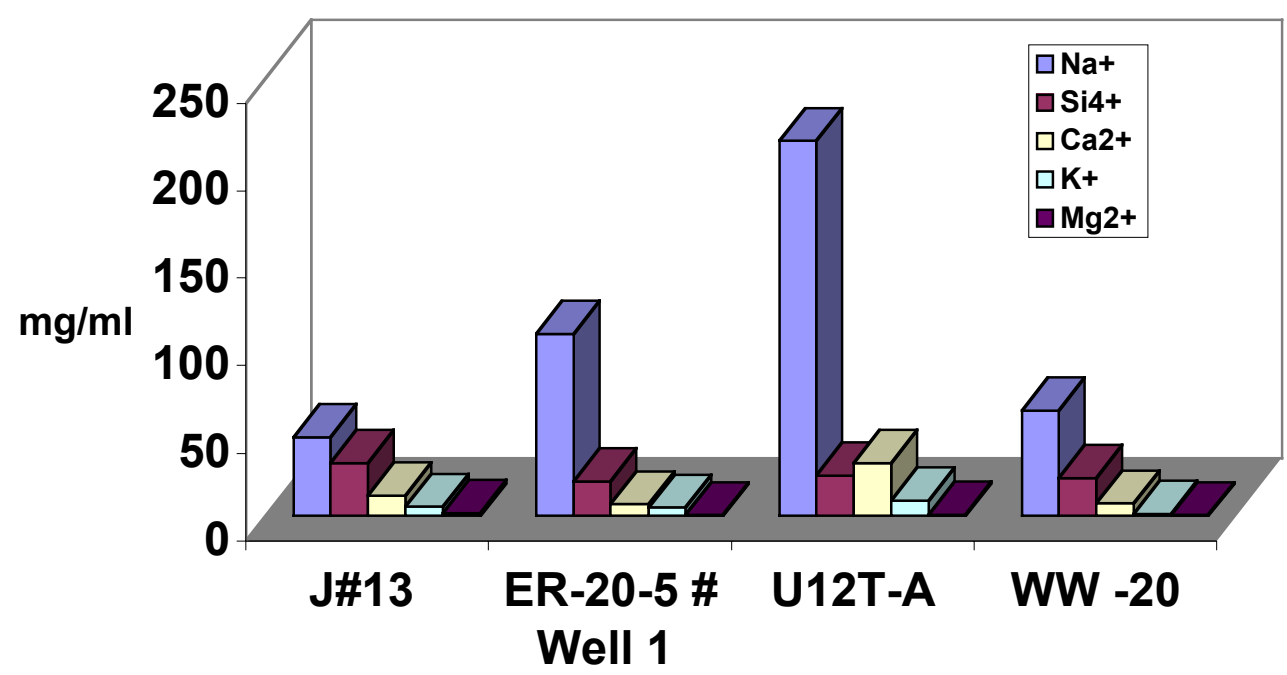

Figure 3.3. Comparison of the cation composition of groundwater from U12T and other NTS water samples.

\subsection{Radiochemical Analysis}

All the water samples collected in the tunnels contained tritium $\left({ }^{3} \mathrm{H}\right)$. Figure 3.4 is a plot of the tritium $\left({ }^{3} \mathrm{H}\right)$ concentrations in the groundwater samples from Rainier Mesa. All the samples collected have tritium levels above the EPA drinking water limit (black line). Sample U12T-2100 is the closest to the nuclear detonations (2100 feet from the tunnel entrance) and has the highest level of tritium. Samples T-A, T-B, T-D and T-E are all samples collected farthest from the nuclear detonations, on the rockwalls of the tunnel. 


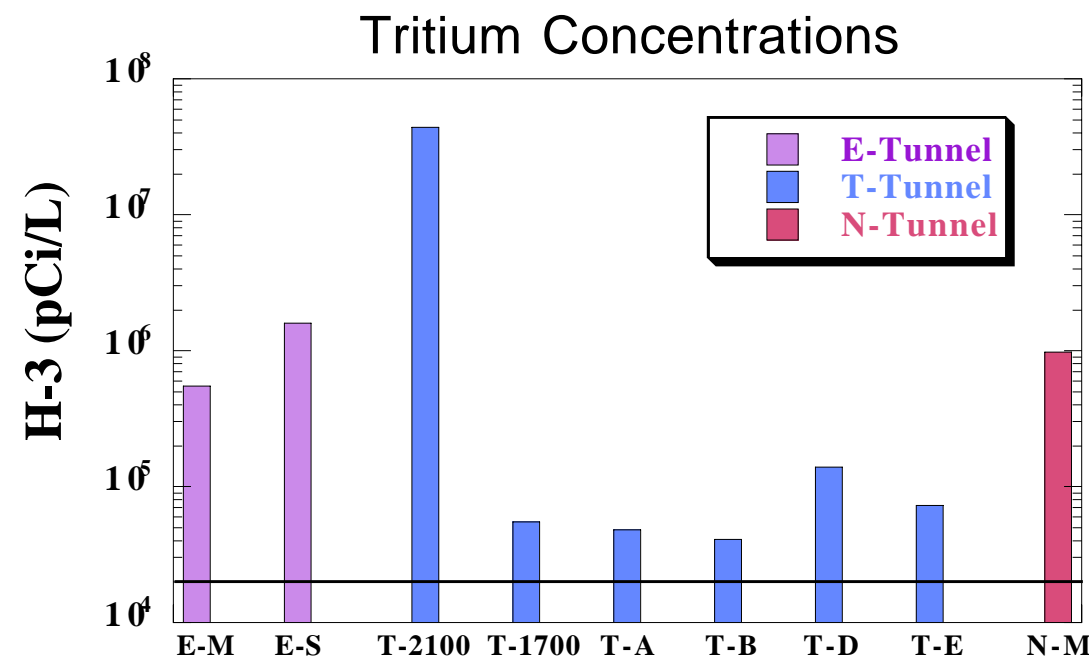

Sample Locations

Figure 3.4. Tritium concentrations of Rainier Mesa water samples (concentrations corrected to 9/99). Black line represents the EPA maximum drinking water standard.

Representative water samples were selected for radiochemical analysis. Low levels of ${ }^{137} \mathrm{Cs},{ }^{! 25} \mathrm{Sb}$ and ${ }^{106} \mathrm{Ru}$ were the only gamma-emitting radionuclides detected in the water samples. Low levels of $\mathrm{Pu}, \mathrm{Am}$ and ${ }^{90} \mathrm{Sr}$ were detected in the samples. Table 3.1 shows the samples that had detectable concentration of ${ }^{137} \mathrm{Cs},{ }^{125} \mathrm{Sb},{ }^{106} \mathrm{Ru}, \mathrm{Pu}, \mathrm{Am}$, and ${ }^{90} \mathrm{Sr}$.

Table 3.1. Radionuclides detected in water samples from Rainier Mesa Tunnels. $X=$ detected, ND $=$ non detect, NA $=$ not analyzed, $*=$ near reporting limit

\begin{tabular}{|l|c|c|c|c|c|c|}
\hline Sample ID & ${ }^{137} \mathrm{Cs}$ & ${ }^{125} \mathrm{Sb}$ & ${ }^{106} \mathrm{Ru}$ & $\mathrm{Pu}$ & $\mathrm{Am}$ & ${ }^{90} \mathrm{Sr}$ \\
\hline U12E-M & $\mathrm{X}$ & $\mathrm{ND}$ & $\mathrm{ND}$ & $\mathrm{X}$ & $\mathrm{X}$ & $\mathrm{X}$ \\
\hline U12E-S & $\mathrm{X}$ & $\mathrm{ND}$ & $\mathrm{ND}$ & $\mathrm{NA}$ & $\mathrm{NA}$ & $\mathrm{NA}$ \\
\hline U12T-2100 & $\mathrm{X}$ & $\mathrm{X}$ & $\mathrm{X}$ & $\mathrm{X}$ & $\mathrm{X}^{*}$ & $\mathrm{X}$ \\
\hline U12T-1700 & $\mathrm{ND}$ & $\mathrm{ND}$ & $\mathrm{ND}$ & $\mathrm{X}$ & $\mathrm{ND}^{*}$ & $\mathrm{NA}$ \\
\hline U12T-B & $\mathrm{ND}$ & $\mathrm{ND}$ & $\mathrm{ND}$ & $\mathrm{X}$ & $\mathrm{X}$ & $\mathrm{ND}$ \\
\hline U12T-E & $\mathrm{ND}$ & $\mathrm{ND}$ & $\mathrm{ND}$ & $\mathrm{NA}$ & $\mathrm{NA}$ & $\mathrm{NA}$ \\
\hline U12N-M & $\mathrm{X}$ & $\mathrm{ND}$ & $\mathrm{ND}$ & $\mathrm{X}$ & $\mathrm{X}$ & $\mathrm{X}$ \\
\hline
\end{tabular}

\section{Mass Spectrometry Results}

Four of the tunnel water samples (U12T-A, U12T-B, U12T-2100, and U12E-M) were analyzed for plutonium concentration and isotopic composition by thermal ionization mass spectrometry (TIMS). This is a much more sensitive technique than standard alpha counting, provides a lower background and an independent confirmation for the alpha 
spectrometry results. Separate analyses of the dissolved and the filterable solids fractions for three of these samples (U12T-B, U12T-2100, and U12E-M) were made by standard radiochemical methods.

$\mathrm{Pu}$ was detected in all four water samples. No $\mathrm{Pu}$ was detected in the reagent and processing blank. The minimum detectable levels as determined from the 2-sigma uncertainties on the isotope/spike ratio measurement in the blank are ${ }^{239} \mathrm{Pu}<0.010 \mathrm{pg}$, ${ }^{240} \mathrm{Pu}<0.022 \mathrm{pg},{ }^{241} \mathrm{Pu}<0.010 \mathrm{pg}$ and ${ }^{242} \mathrm{Pu}<0.037 \mathrm{pg}$. Figure 3.5 shows excellent agreement between the two methods used.

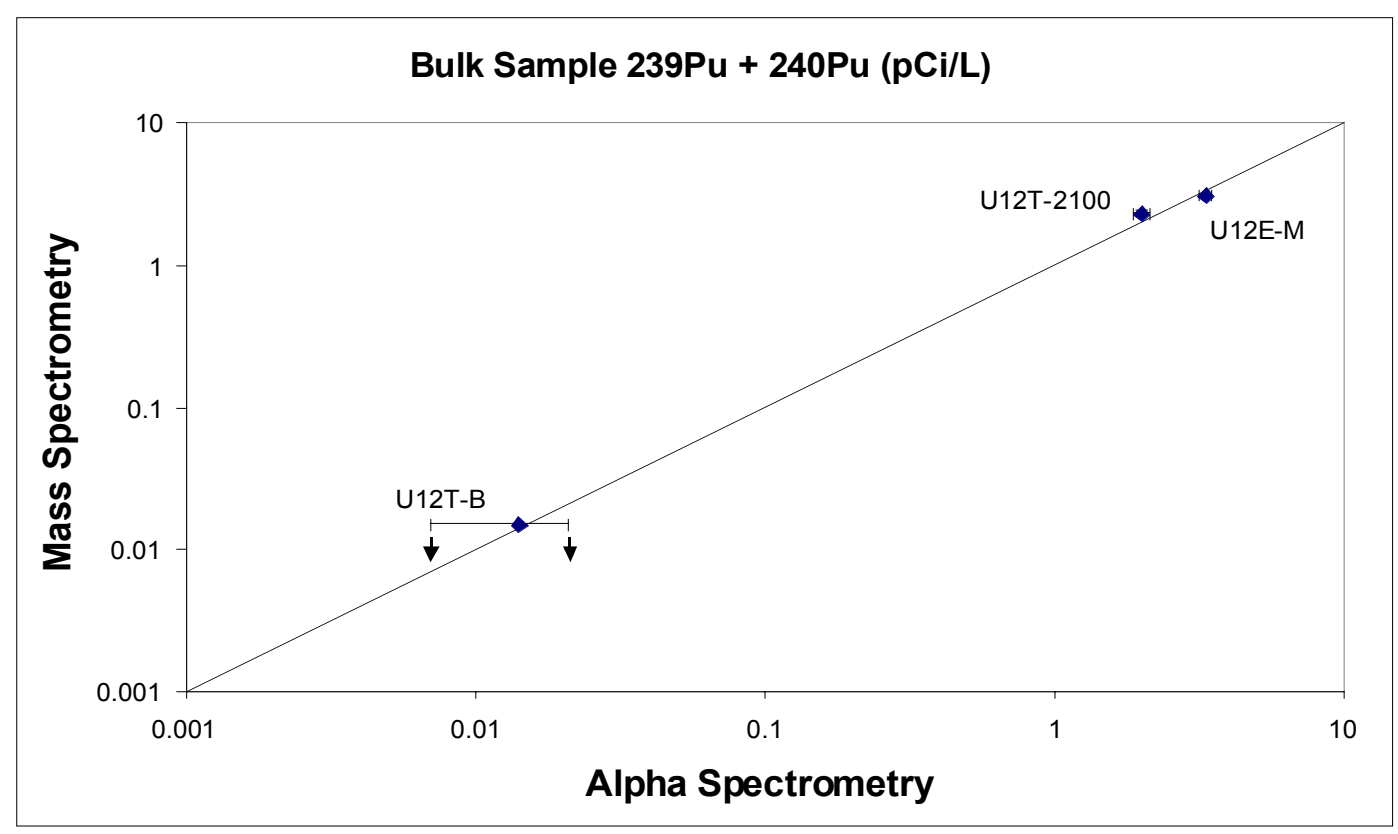

Figure 3.5. Comparison of concentrations of $239 \mathrm{Pu}+240 \mathrm{Pu}$ using two different analytical methods. Agreement is excellent.

The overwhelming majority of the $\mathrm{Pu}, \mathrm{Am}$, and ${ }^{90} \mathrm{Sr}$ was detected on the filterable, or colloidal fraction. For example, greater than $95 \%$ of the $\mathrm{Pu}$ was found in the colloidal, not the dissolved fraction.

\subsection{Characterization of Mineral Colloids}

Photon correlation spectroscopy (PCS) was used to determine the size distribution of the colloids in the water samples. The majority of the colloids were between 80-200 nm. Figure 3.6 shows the colloid size distribution for a selected set of samples collected. 


\section{Colloid size distribution}

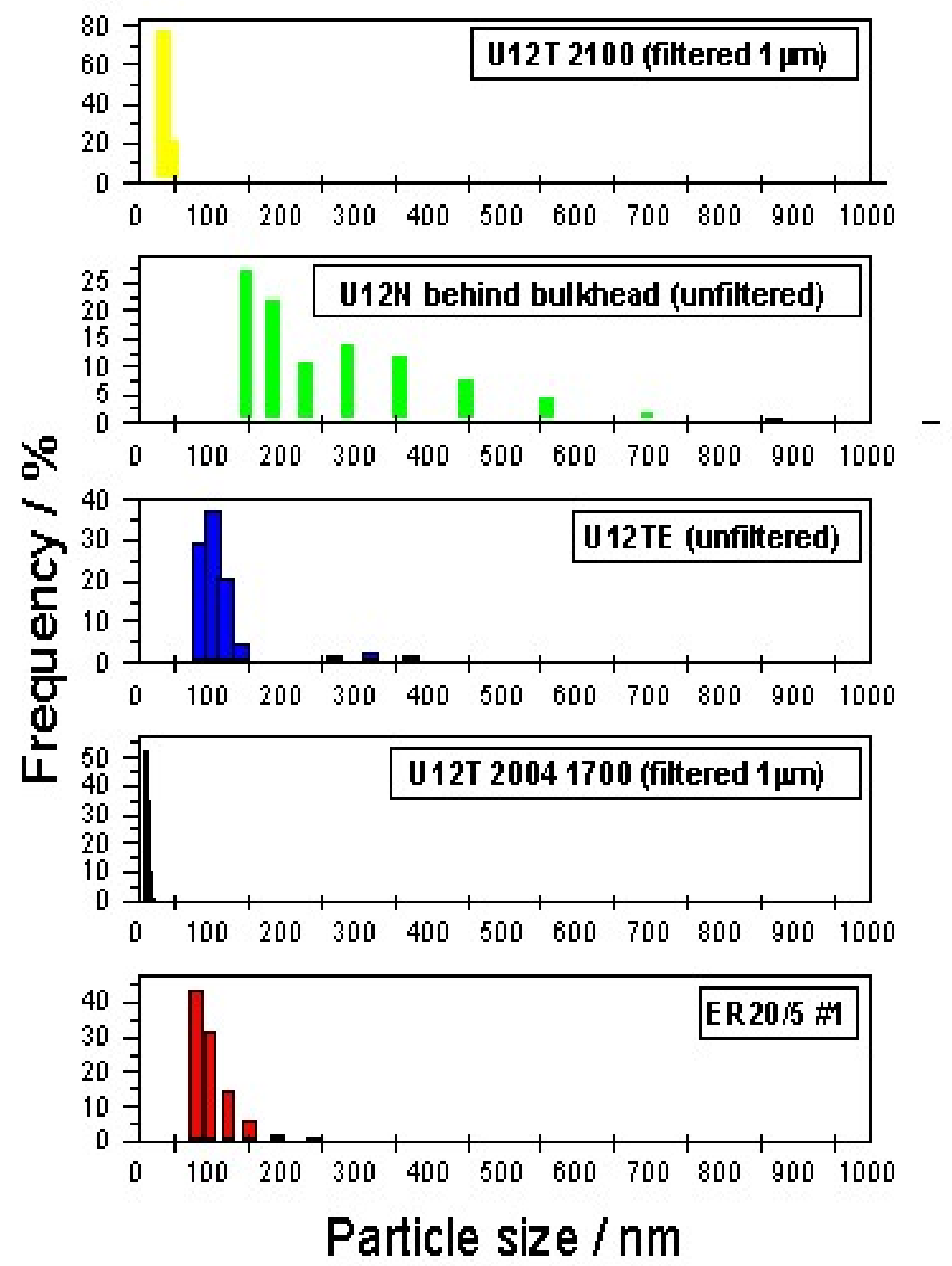

Figure 3.6. Photon correlation spectroscopy data showing the particle size distribution of the colloids in Rainier Mesa water samples. U12N was added for comparison. U12N water was in contact with the concrete portal and was not analyzed for colloids.

Water sample U12T-A was filtered and the colloidal fraction identified by Scanning Electron Microscope (SEM), X-ray diffraction (XRD), and Transmission Electron Microscopy (TEM). Each of these micro analytical techniques offer either quantitative or qualitative chemical or morphological information, that when used together helps to positively identify the mineral colloids. The physical appearance of the colloidal minerals can be seen from SEM images shown in Figure 3.7. Calcite and possibly the 
clay, illite were identified by XRD analysis. TEM analysis confirmed the presence of the clay as illite, calcite and also identified the zeolite, clinoptilolite (Figure 3.8).
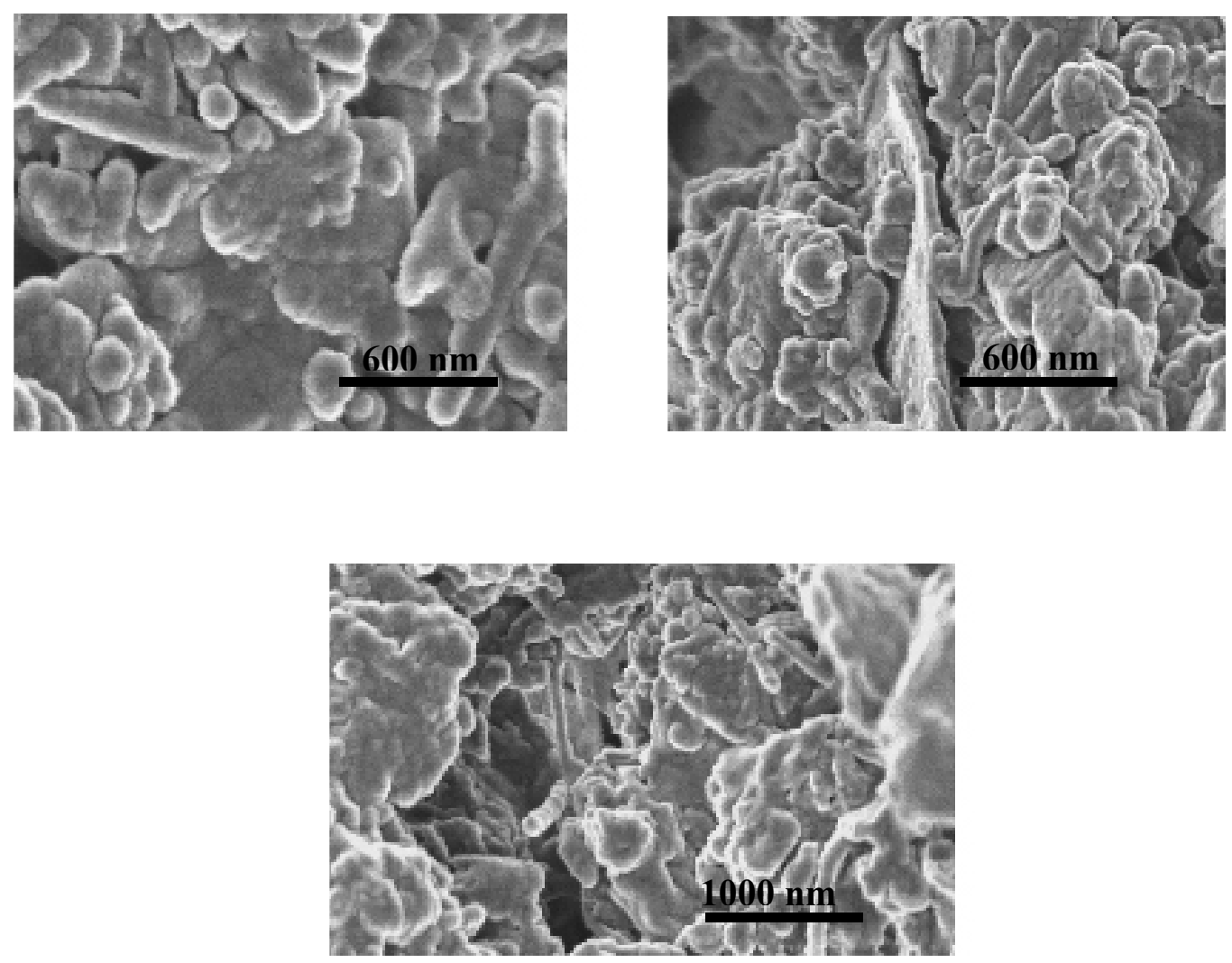

Figure 3.7. SEM images of colloids found in U12T-A groundwater. The flat platy minerals are most likely clays and the rod shaped minerals are probably zeolite. 

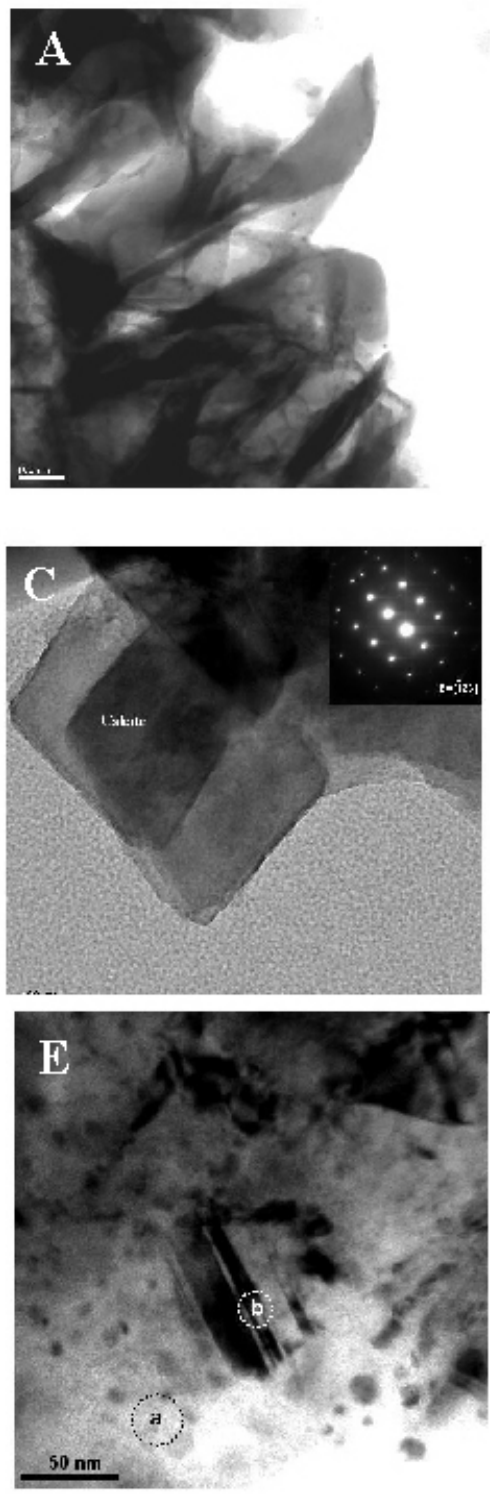
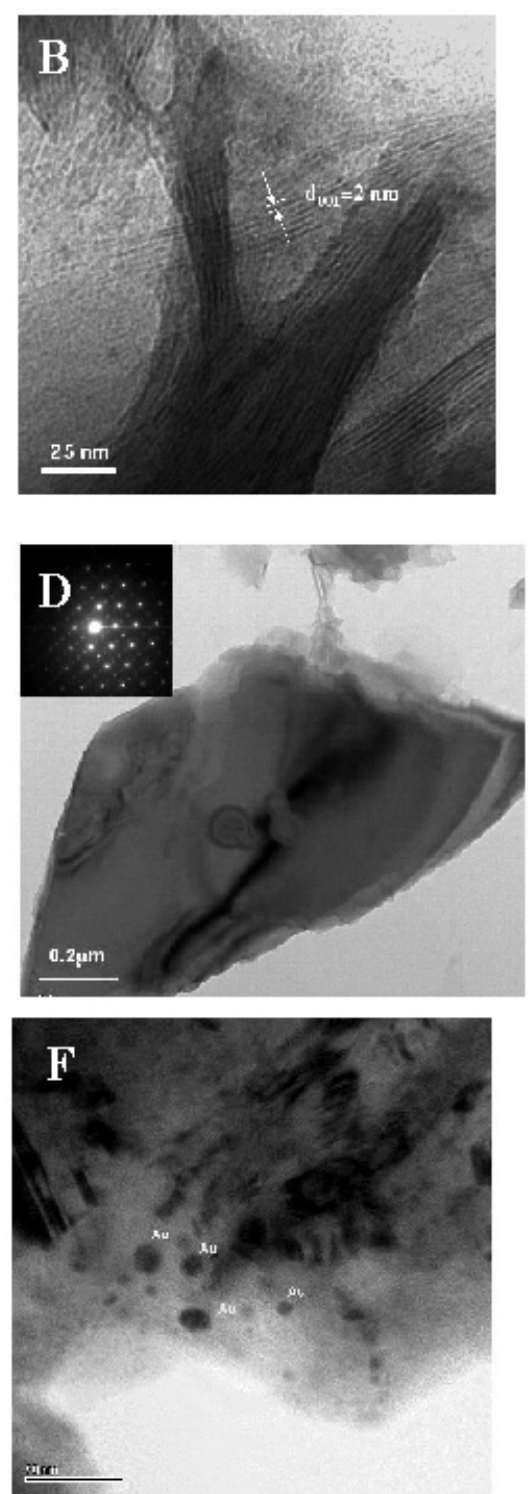

Figure 3.8. TEM images of A) Bright Field TEM image of illite grains, B) High Resolution TEM image of illite grains, C) Bright Field TEM image and diffraction pattern of calcite, D) Bright Field TEM image and SAD pattern from a zeolite grain, E) Bright Field TEM image of a Ru-rich colloid, F) Bright Field TEM image showing Au nano-particles

\subsection{Summary}

The most important finding of this study is that low-solubility radionuclides detected in the vadose zone water samples are overwhelmingly associated with the colloidal fraction of the groundwater and not the dissolved fraction. In both this study of a vadose zone fracture flow system and previous work in the saturated fracture flow system at Pahute Mesa, NTS (Buddemeier and Hunt, 1988; Kersting et al., 1999) the Pu detected in the groundwater is associated with the colloidal fraction of the groundwater. With the exception of calcite, the colloid minerals identified in the T-Tunnel, Rainier Mesa are the 
same minerals identified in the Cheshire and ER20-5 groundwater. The size distribution of the colloids is also similar to the size distribution $(<200 \mathrm{~nm})$ for colloids measured in the saturated groundwater samples previously analyzed from the NTS.

We caution that this study was not a transport study, but argue instead that if conditions permit transport of colloids, low-solubility radionuclides have the potential to be transported with colloids in both vadose zone and saturated fracture flow environments.

\section{Acknowledgements}

Special thanks to Wayne Griffin from Defense Threat Reduction Agency (DTRA) for providing permission to collect water samples, and logistical and technical support in the field. Additional assistance in the field by T. Rose, J. Kenneally was greatly appreciated. Efforts by J. Kenneally for working with DTRA and NNSA/NV to obtain access to the tunnels at the NTS are appreciated. Thanks to Ross Williams for the ICP-MS analyses. This work was partially funded by a Tech. Development grant from LLNL to A.B. Kersting. 


\subsection{References}

Buddemeier R. W. and Hunt J. R. (1988) Transport of Colloidal Contaminants in Groundwater: Radionuclide Migration at the Nevada Test Site. Applied Geochemistry 3, 535-548.

Kersting A. B., Efurd D. W., Finnegan D. L., Rokop D. J., Smith D. K., and Thompson J. L. (1999) Migration of Plutonium in Groundwater at the Nevada Test Site. Nature 397, 56-59.

Laczniak R. J., Cole J. C., Sawyer D. A., and Trudeau D. A. (1996) Summary of Hydrologic Controls on Ground-water Flow at the Nevada Test Site, Nye county, Nevada, pp. 59. U.S. Geological Survey. 


\title{
-Chapter 4- \\ Sorption of Pu(IV) on Mineral Colloids
}

\author{
Annie B. Kersting, Pihong Zhao, Mavrik Zavarin, Eric R. Sylwester, Patrick G. Allen, \\ Lumin Wang, Erik J. Nelson, and Ross W. Williams
}

\subsection{Introduction}

Results from the UGTA field studies discussed in Chapters 2 and 3 provide the framework for designing an experimental program to determine the geochemical controls on colloid-facilitated transport of low-solubility radionuclides. Field studies at the NTS have demonstrated that inorganic colloids exist in all the groundwater sampled, and that when low-solubility radionuclides such as $\mathrm{Pu}$ and $\mathrm{Am}$ are present in the groundwater they are associated with the colloidal fraction, not the dissolved fraction, as is tritium. Although the mineral percentages vary from groundwater sample to groundwater sample, the majority of colloids are clays and zeolites with minor tridymite, and calcite. These minerals, in addition to Fe- and Mn-oxide, are common secondary minerals found in altered volcanic rocks and fracture lining minerals. In addition, the mechanism of colloid-facilitated transport can operate in both a saturated and unsaturated fracture flow hydrologic setting. The majority of colloids sampled in NTS water were small and mono-modal, ranging between 50-200 $\mathrm{nm}$.

Our experimental focus was to investigate the low solubility radionuclide, $\mathrm{Pu}$. Plutonium is part of the residual radionuclide inventory deposited in the subsurface as a result of underground nuclear testing at the NTS. It is highly toxic to human health and has a very long half-life (e.g. ${ }^{239} \mathrm{Pu}$ half-life $=24,000 \mathrm{yrs}$ ). Therefore, the behavior of $\mathrm{Pu}$ in the subsurface is of major concern to the UGTA program. Plutonium has been described as one of the most complicated elements known. In solution, Pu can exist in five different oxidation states (Pu- III, IV, V, VI, VII), and four of them have similar reduction potentials. The oxidation state of $\mathrm{Pu}$ affects its chemical behavior in solution (Choppin, 1991). Each oxidation state of $\mathrm{Pu}$ has a unique chemical behavior, and therefore modeling the geochemical behavior of $\mathrm{Pu}$ must include knowledge of its oxidation state. For example, $\mathrm{Pu}$ (III) and $\mathrm{Pu}(\mathrm{IV})$ are relatively insoluble compared to $\mathrm{Pu}(\mathrm{V})$ and $\mathrm{Pu}(\mathrm{VI})$ (Clark, 2000). $\mathrm{Pu}(\mathrm{IV})$ and $\mathrm{Pu}(\mathrm{V})$ are the dominant oxidation states of $\mathrm{Pu}$ under equilibrium conditions for groundwater compositions measured at the NTS (see Figure 4.1) (Nitsche et al., 1992). Therefore, our experiments focus on understanding the sorption behavior of the two dominant oxidation states, $\mathrm{Pu}(\mathrm{IV})$ and $\mathrm{Pu}(\mathrm{V})$.

Although Pu sorption experiments have previously been carried on some minerals, not all mineral have been investigated (e.g. Triay et al., 1996). In addition, laboratory experiments have not identified the mechanisms by which $\mathrm{Pu}$ may sorb to colloids and travel relatively unimpeded in the subsurface, as this requires understanding how $\mathrm{Pu}$ desorbs from the colloid. Some data suggest that $\mathrm{Pu}$ sorption to colloids is a very fast process while desorption is very slow or simply does not occur (Lu et al., 1998) This could allow $\mathrm{Pu}$ sorbed to a colloid to travel much farther than if sorption were an equilibrium process. However, Pu sorption (and particularly desorption) data in the 
literature are scant and sometimes contradictory. In some cases, $\mathrm{Pu}$ desorption is rather fast, with rates dependent on colloid mineralogy (Runde et al., 2000; Lu et al., 1998; Lu et al., 2000). Moreover, the effect of sorption and desorption kinetics (as well as other mechanisms) on colloid-facilitated transport at the field scale has not been thoroughly evaluated. This is, in part, due to limitations in colloid transport models as well as sorption/desorption models.

In an effort to better understand the dominant mechanisms that control colloid-facilitated $\mathrm{Pu}$ transport, we have performed a series of sorption/desorption experiments using mineral colloids and $\mathrm{Pu}(\mathrm{IV})$ and $\mathrm{Pu}(\mathrm{V})$. We focused on natural colloidal minerals present in water samples collected from both saturated and vadose zone waters at the Nevada Test Site.

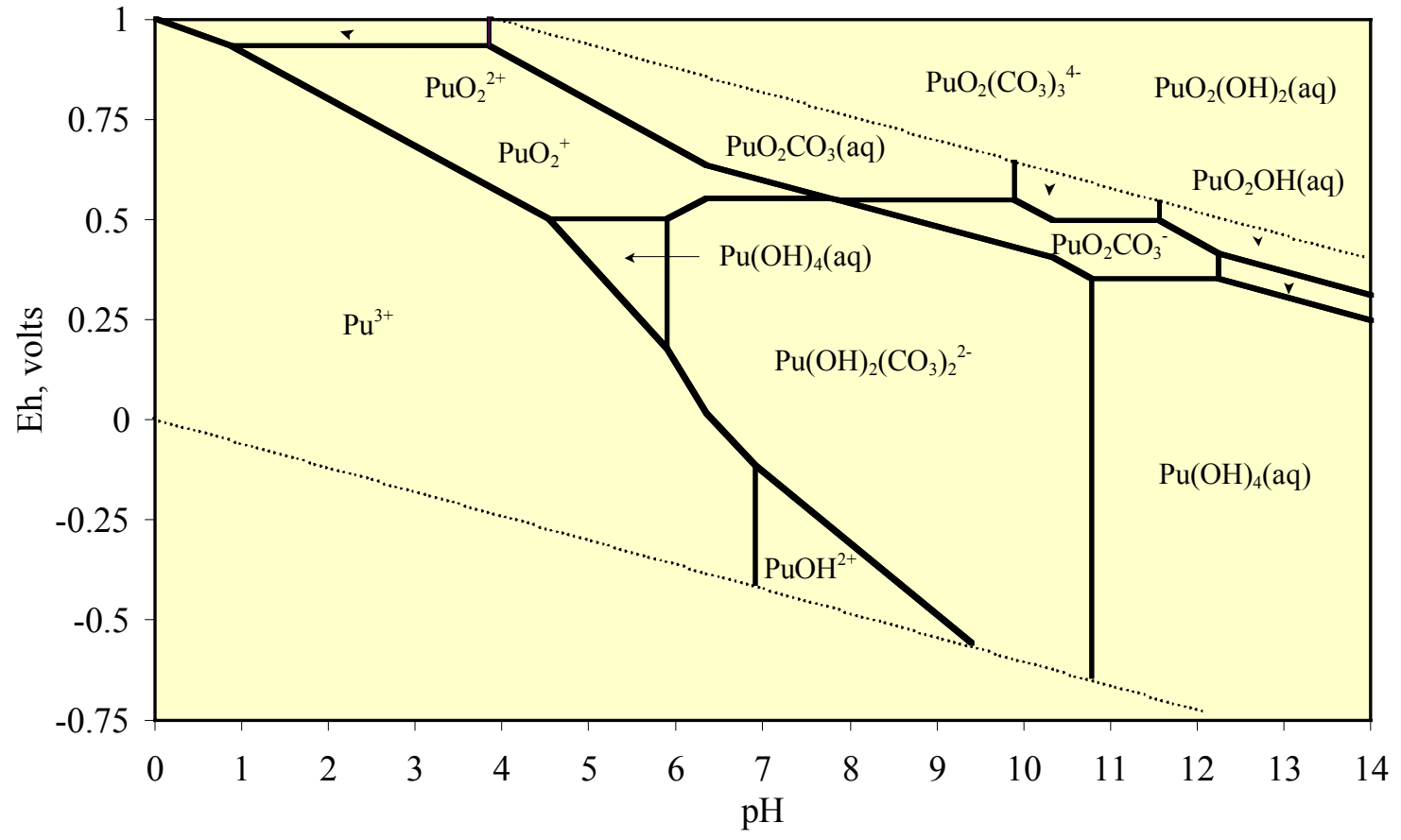

Figure 4.1. $\mathrm{Eh}-\mathrm{pH}$ diagram for the speciation of $\mathrm{Pu}$ in equilibrium in $\mathrm{J}-13$ type water. $\mathrm{Pu}(\mathrm{IV})$ and $\mathrm{Pu}(\mathrm{V})$ are the dominant species in groundwater at near neutral $\mathrm{pH}(8)$ found in low-bicarbonate, NTS water.

A series of laboratory experiments were undertaken to determine how Pu sorbs to one of the most common colloid minerals detected in the NTS groundwater, the zeolite mineral, clinoptilolite. Specifically, we wanted to understand: the forward (sorption) and reverse rate (desorption) of sorption, the redox controls on sorption, and the sorption rate of clinoptilolite relative to other colloid minerals detected in NTS groundwater. In order to answer the above questions we carried out the following experiments:

- Sorption of Pu(IV) on clinoptilolite colloids,

- Determination of oxidation state of sorbed $\mathrm{Pu}$ on clinoptilolite by X-ray absorption spectroscopy (XAS),

○ XAS of $\mathrm{Pu}$ sorbed to birnessite and montmorillonite,

- Modeling Pu(IV) sorption on clinoptilolite colloids, 
- Desorption of Pu(IV) sorbed on clinoptilolite,

- Modeling Pu(IV) desorption on clinoptilolite,

- Sorption of $\mathrm{Pu}(\mathrm{V})$ on clinoptilolite colloids,

- Sorption of $\mathrm{Pu}(\mathrm{V})$ on other mineral surfaces (e.g. Mn-oxides, Fe-oxide, clays, calcite and silica),

- XAS of $\mathrm{Pu}(\mathrm{V})$ sorbed onto all minerals investigated, and

- Factors influencing the speciation and sorption behavior of $\mathrm{Pu}(\mathrm{V})$

$\circ$ Disproportionation.

In Chapter 4, results from $\mathrm{Pu}(\mathrm{IV})$ sorption and desorption experiments on clinoptilolite will be presented. In Chapter 5, results from $\mathrm{Pu}(\mathrm{V})$ sorption experiments on clinoptilolite as well as Mn-oxide, Fe-oxide, calcite and silica minerals will be presented.

\subsection{Sorption of $\mathrm{Pu}(\mathrm{IV})$ on Clinoptilolite (Zeolite) Colloids}

Laboratory batch sorption experiments were carried out at room temperature to evaluate the effects of time and solution $\mathrm{pH}$ on the sorption of $\mathrm{Pu}(\mathrm{IV})$ on clinoptilolite colloids under conditions observed at the NTS. Although the $\mathrm{pH}$ of most NTS water is near $\mathrm{pH} 8$, the effect of Pu sorption at $\mathrm{pHs}$ between 4 and 10 was investigated to more accurately model our results.

\section{Experimental Techniques}

The groundwater solution was prepared using synthetic groundwater composition similar to the Yucca Mountain standard, J-13 (Figure 4.2). Our synthetic groundwater composition, J-13S2, is a low bicarbonate water similar in composition to groundwater at the NTS. A synthetic water composition was used in order to better control the effect of trace constituents and to more accurately model our results. The J-13 surrogate sample $(\mathrm{J}-13 \mathrm{~S} 2)$ is lower in $\mathrm{Si}$ and $\mathrm{Ca}$ than natural $\mathrm{J}-13$ groundwater. This was done to eliminate the possibility of supersaturation with respect to silica and calcite over the range of $\mathrm{pH}$ investigated.

$\mathrm{Pu}$ solution: The $\mathrm{Pu}(\mathrm{IV})$ concentration used was $4.4 \times 10^{-9} \mathrm{M}$ (this concentration is below the solubility limit for $\mathrm{Pu}(\mathrm{IV})$ under the conditions investigated). The plutonium solution was purified using an anion exchange column. The Pu isotopic compositions (mass \%) are ${ }^{238} \mathrm{Pu}(1.94 \%),{ }^{239} \mathrm{Pu}(57.47 \%),{ }^{240} \mathrm{Pu}(26.57 \%),{ }^{241} \mathrm{Pu}(7.28 \%),{ }^{242} \mathrm{Pu}(6.74 \%)$. The oxidation state of $\mathrm{Pu}(\mathrm{IV})$ in the stock solution was confirmed using UV/VIS spectrometry. The $\mathrm{Pu}(\mathrm{IV})$ solution was filtered through a $20 \mathrm{~nm}$ pore size filter prior to use in the sorption experiments. A liquid scintillation analyzer was used for Pu $\alpha$-activity measurements. The $\mathrm{Pu}$ solution was $96 \% \mathrm{Pu}(\mathrm{IV})$ at the start of the sorption experiments.

Colloidal solution: Pure clinoptilolite (zeolite) crystals were hand picked from high purity minerals that were crushed to achieve greater than $99 \%$ mineral purity. The mineral was ground to a fine powder, then the particles in the size range 100-200 nm were separated in J-132 water using ultra-centrifugation. The particle sizes of the colloids were measured using photon correlation spectroscopy (PCS). The results indicate that $>90 \%$ 
of the colloids have particle sizes between 100 to $200 \mathrm{~nm}$ (Figure 4.3). The concentration of colloidal solution used was $0.14 \mathrm{mg} / \mathrm{mL}$.

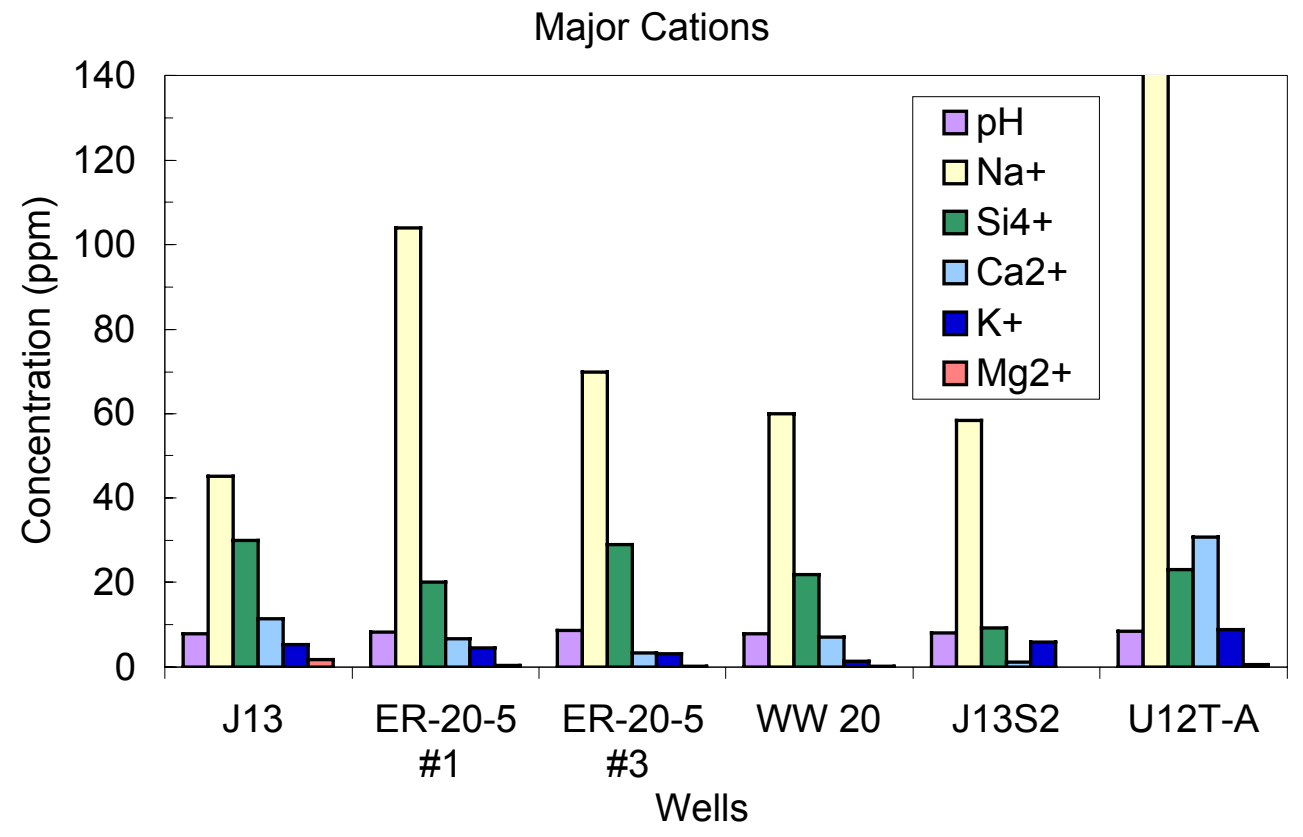

Figure 4.2. Comparison of the cation composition and $\mathrm{pH}$ of groundwater samples from the NTS, J-13 and the synthetic J13S(2) water used in our experiments. J-13 is the Yucca Mountain standard, ER20-5 \#1 \& 3 and WW20 are wells located on Pahute Mesa, and U12T-A is a water sample from T-Tunnel, Rainier Mesa, NTS (see Chapter 4).

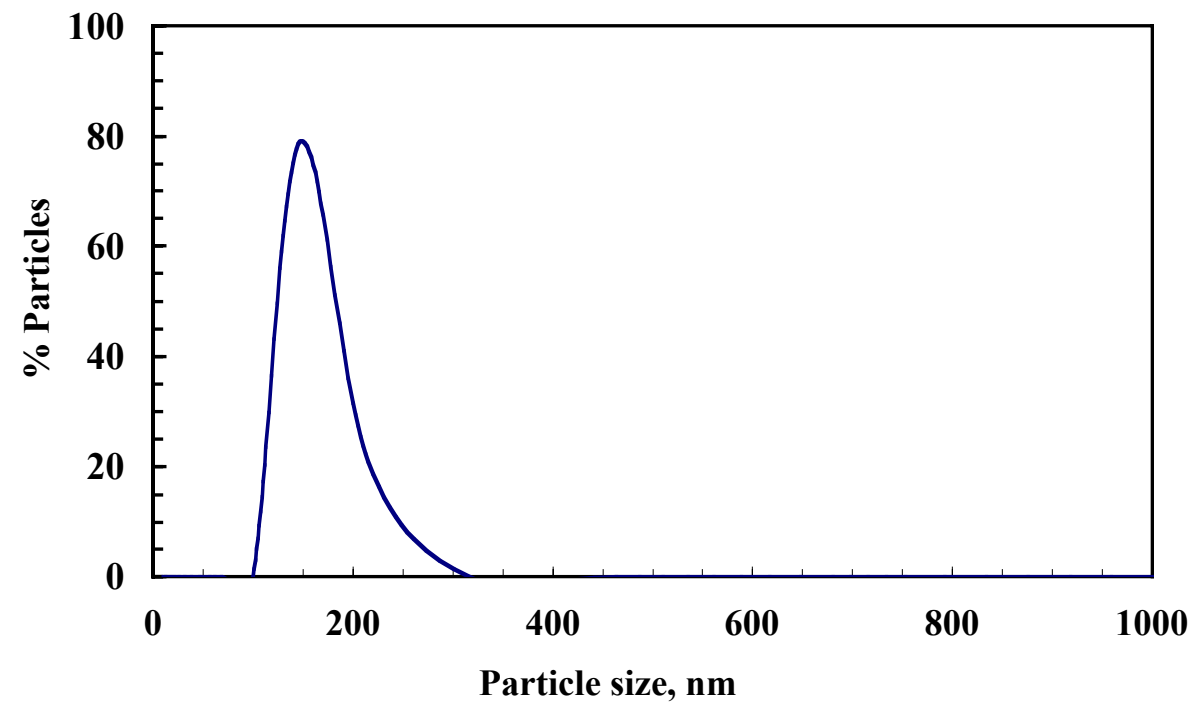

Figure 4.3. PCS measurement of clinoptilolite colloidal size distribution used in our experiments. 
For each set of sorption experiments, a second series of "control" solutions were prepared under the exact same experimental conditions but in the absence of the colloids. The controls were used to monitor any precipitation or sorption of $\mathrm{Pu}$ on the container during the experiments. Samples and controls were kept in closed polyethylene containers and were gently shaken with a slow and stable shaking speed of $300 \mathrm{rpm}$, until equilibrium was reached. Sorption experiments were carried out from $\mathrm{pH} 4$ through 10. Prior to the beginning of the sorption experiment, the solutions and controls were monitored for stability at the different $\mathrm{pHs}$. After 10 days, the $\mathrm{pH}$ solutions were stable and the experiments began. The $\mathrm{pH}$ was not adjusted during the course of the experiment. The first samples were taken after 1.5 hours. Partition coefficients $(\mathrm{mL} / \mathrm{g})$ were calculated according to the following equation (1):

$$
K_{d}=\frac{\left(\frac{\left(A_{i}-A\right)}{m}\right)}{\left(\frac{A}{V}\right)}
$$

Where $\mathrm{A}_{\mathrm{i}}$ and $\mathrm{A}$ are the activity $(\mathrm{cpm} / \mathrm{mL})$ in the initial and final solution, respectively; $\mathrm{m}$ is the mass $(\mathrm{g})$ of colloid used; and $\mathrm{V}$ is the volume $(\mathrm{mL})$ of solution.

\section{Results and Discussion}

The sorption of $\mathrm{Pu}(\mathrm{IV})$ on clinoptilolite colloids as a function of time between $\mathrm{pH} 4$ and 10 is plotted in Figure 4.4A. The sorption of $\mathrm{Pu}(\mathrm{IV})$ was rapid occurring within the first few hours. Equilibrium was reached by day two. The experiments were continued for 63 days with no change in sorption behavior after day two. The percent sorption is consistently high, but varies as a function of $\mathrm{pH}$ from approximately $70 \%$ to over $90 \%$ as shown in Figure 4.4B. The lowest sorption occurs between $\mathrm{pH} 7$ and 9.5.

\subsection{Determination of Oxidation State of Sorbed Pu on Clinoptilolite by XAS}

Another set of sorption experiments were carried out to determine the oxidation state and structure of the Pu sorbed to clinoptilolite. X-Ray absorption spectroscopy (XAS) was used to determine the oxidation state and structure of $\mathrm{Pu}$ on the clinoptilolite surface. XAS provides information on sorption mechanisms that increase our fundamental understanding of the chemical processes involved at the molecular level. XAS can provide a direct picture of the average actinide molecular structure and oxidation state insitu, non-destructively, at ppm concentrations, in amorphous, crystalline, and mixed solid/liquid samples. Identifying specific sorption mechanisms allows us to design experiments targeting the most important chemical processes in sorption, identify environmental systems where these processes occur, and develop a better predictive capability through modeling. In our experiments, XAS was used to determine both the oxidation state of the sorbed $\mathrm{Pu}$ and the short-range structure. 

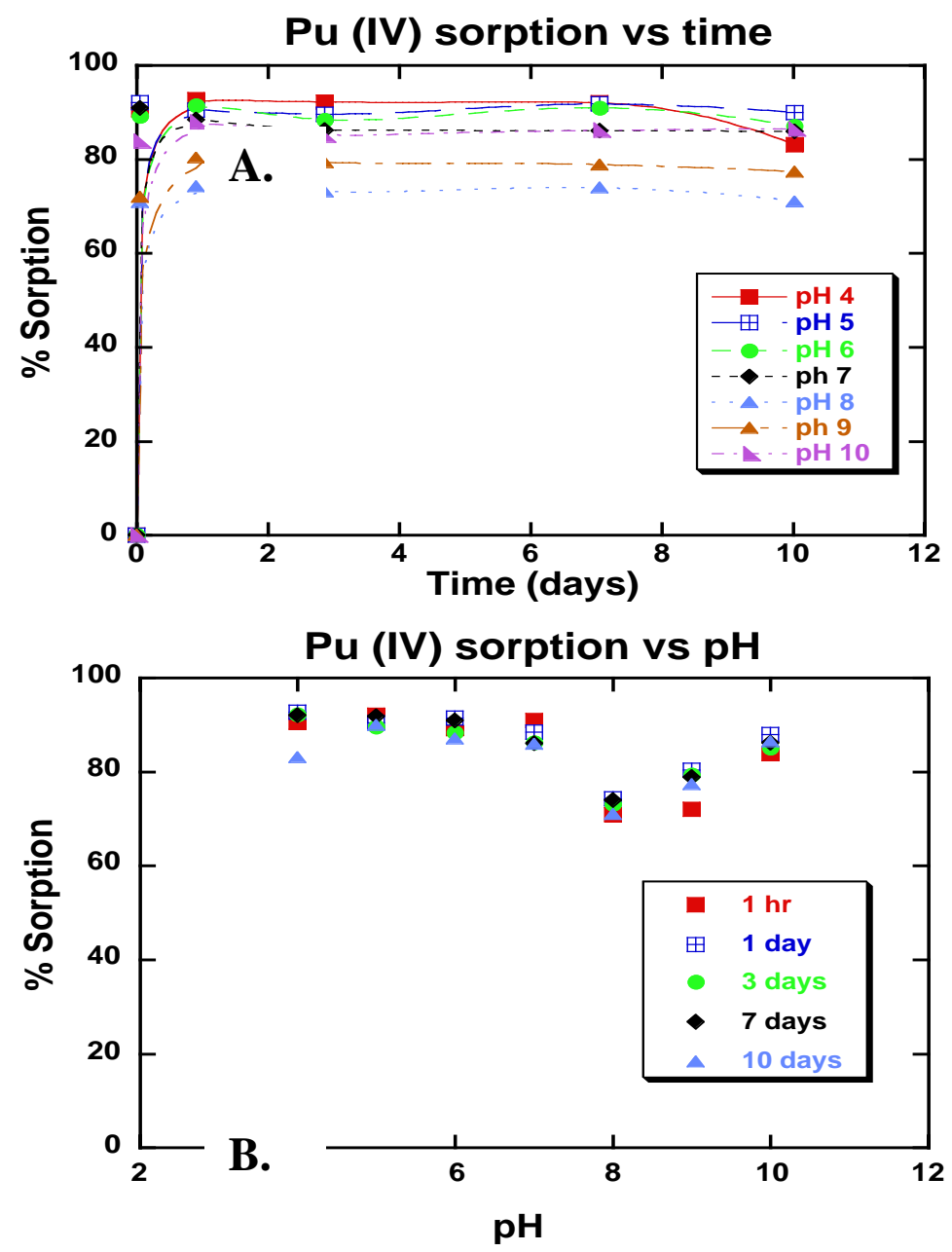

Figure 4.4. $\mathrm{Pu}(\mathrm{IV})$ sorption as a function of time and $\mathrm{pH}$. The experiment was carried out for 63 days, although no change in sorption behavior was observed after day two.

\section{Experimental Techniques}

Aqueous $\mathrm{Pu}(\mathrm{IV})$ stock solution was mixed with $\mathrm{J}-132 \mathrm{~S}$ to a concentration of $\sim 5 \times 10^{-6} \mathrm{M}$ at $\mathrm{pH}$ 8.4. This solution was then mixed with $20 \mathrm{mg}$ of clinoptilolite colloid. After seven days, the sample was centrifuged and the solution drawn off to leave a wet paste consisting of $\mathrm{Pu}$ sorbed to the colloidal material as the final sample form. The wet paste sample was studied directly using XAS.

Two additional $\mathrm{Pu}(\mathrm{IV})$ sorption experiments were run to compare the clinoptilolite sorption results with other mineral surfaces. The minerals chosen were a Mn-oxide, birnessite and a clay, Na-montmorillonite.

All XAS analysis was performed on BL 4-1 at the Stanford Synchrontron Radiation Laboratory (SSRL). The spectra were energy calibrated by simultaneously measuring the 
absorption spectrum for the reference standard $\mathrm{PuO}_{2}$, and setting the energy of the first inflection point, $\mathrm{E}_{\mathrm{r}}$, at $22258.0 \mathrm{eV}$. Plutonium $\mathrm{L}_{\mathrm{II}}$-edge XAS spectra were measured on wiggler beamline 4-1 under normal ring operating conditions (3.0 GeV, 50-100 MA). Energy scans were obtained using a Si (220) double-crystal monochromator and a vertical slit width of $0.5 \mathrm{~mm}$. All samples were measured in fluorescence mode using a Ge solid state detector. XAS data reduction and normalization was done using the EXAFSPAK suite of programs developed by G. George of SSRL.

\section{Results and Discussion}

In all three samples the X-ray absorption near edge structure (XANES) region of the energy spectra shows that $\mathrm{Pu}(\mathrm{IV})$ remains in the $\mathrm{Pu}^{4+}$ oxidation state (orange line Fig. 4.5). There are two major features that identify the Pu oxidation state as (IV) in the sorbed samples. First, the position of the absorption peak in the samples is that of the $\mathrm{Pu}(\mathrm{IV})$ reference. Second, the structure of the XANES spectra contain a high energy shoulder which is the direct result of X-ray scattering from the short, linear, $\mathrm{O}=\mathrm{Pu}=\mathrm{O}$ structure in the $\mathrm{Pu}(\mathrm{IV})$ ion; the high energy shoulder is absent in the $\mathrm{Pu}(\mathrm{V})$ reference. 


\section{$\mathrm{Pu}^{4+}$ Sorption on Minerals $(\mathrm{pH} 8)$}

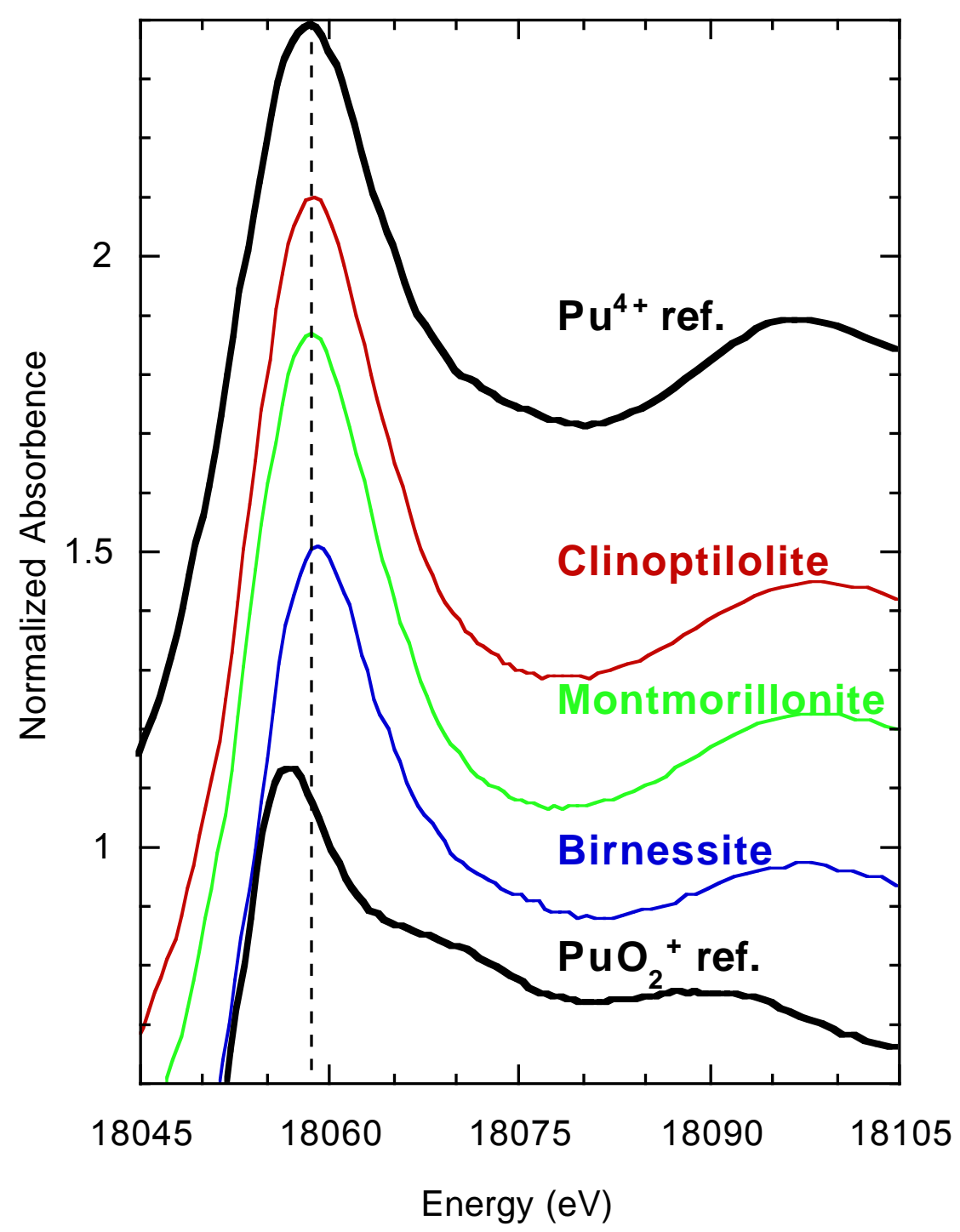

Figure 4.5. The normalized absorbance vs. energy of the XANES region is shown. The $\mathrm{Pu}$ sorbed on clinoptilolite at $\mathrm{pH} 8.4$ is compared to reference standards, $\mathrm{Pu}^{4+}$ and $\mathrm{PuO}_{2}{ }^{+}$ spectra. All the $\mathrm{Pu}$ sorbed samples match the $\mathrm{Pu}^{4+}$ reference spectrum.

Analysis of the extended $\mathrm{x}$-ray absorption fine structure (EXAFS) region of the spectra was performed on one sample for $\mathrm{k}=2-9.5 \AA^{-1}$ to determine the structural bonding of the $\mathrm{Pu}$ to the surface of the clinoptilolite. The fourier transformed EXAFS spectrum for this sample is shown in Figure 4.6. The spectrum represents a pseudo-radial average distribution of atoms neighboring the $\mathrm{Pu}$ atoms. Shells of similar atoms usually appear as 
peaks in the spectrum, with the size of the peak dependent on the number and $\mathrm{Z}$ of the neighboring atom and the position of the peak dependent on the distance between the neighbor and the $\mathrm{Pu}$ center. In the fourier transformed low- $\mathrm{Z}$ neighbors are shifted $\sim 0.2$ $0.5 \AA$ closer to the central atom than their true radial distance due to their EXAFS phase shift. Resolution limits in the technique may result in two or more shells appearing as a single peak, if their phase-shifted radial distances are similar.

In this sample we observe three peaks, a large peak at $1.5 \AA$ and two smaller, more distant peaks at 2.6 and $3.2 \AA$. A non-linear least squares fitting procedure was used to determine the identity, coordination number $(\mathrm{N})$, radial distance (i.e. bond length) $(\mathrm{R}, \AA)$, and Debye-Waller disorder $\left(\sigma^{2}, \AA^{2}\right)$ for each shell of atoms contributing to these peaks. Table 4.1 gives the results of this fitting, while the overall fit is shown in Figure 4.6.

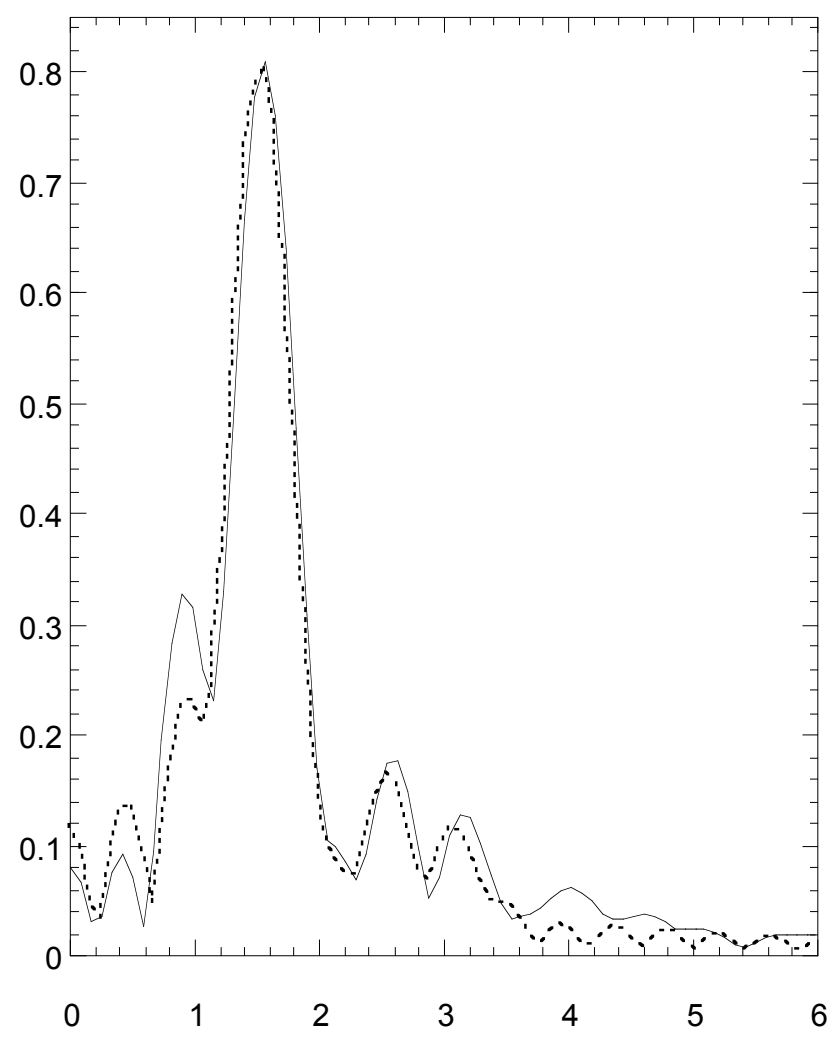

Figure 4.6. Fourier transformed EXAFS spectrum of the data (solid line) and fit (dashed line) for the $\mathrm{Pu}^{4+}(\mathrm{aq})$ in $\mathrm{J}-13 \mathrm{~S} 2$.

Results of fitting the first peak show that it is composed of two distinct shells of oxygen atoms with bond lengths of 2.24 and $2.50 \AA$. This split of the oxygen nearest neighbors into two shells is indicative of two different types of oxygen bonds in the structure. This effect has previously been observed in sorption complexes that contain both surface- 
binding and non-surface-binding (i.e., hydration) oxygen near-neighbors, which show different bond lengths, as well as crystals with complex structures.

The second peak has been identified as a Pu-Si shell occurring at $3.09 \AA$. This bond distance is characteristic of bidentate actinide-silica bonds; for example, coffinite $\left(\mathrm{U}^{\mathrm{IV}} \mathrm{SiO}_{4}\right)$ contains bidentate U-Si bonds at $3.13 \AA$. Observation of Pu-Si interactions in our sample provides evidence for inner-sphere complexation between the $\mathrm{Pu}(\mathrm{IV})$ and the clinoptilolite colloid via bidentate complexation to the silica tetrahedra.

These interactions would also be observed in the case where silica, dissolved from the colloid, complexes with plutonium in solution to form a co-precipitate that then precipitates on the colloid surface. If a co-precipitate is forming, we would also expect to observe Pu-Pu interactions based on the oligomeric structure of such co-precipitates.

Table 4.1. Fit parameters for sample $\mathrm{Pu}^{4+}(\mathrm{aq})$ in $\mathrm{J} 13 \mathrm{~S} 2$, $\mathrm{pH}$ 8.4.

\begin{tabular}{llccc}
\hline $\begin{array}{l}\text { Proposed } \\
\text { Structure }\end{array}$ & Shell & $\mathrm{N}$ & $\mathrm{R}(\AA)$ & $\sigma^{2}\left(\AA^{2}\right)$ \\
\hline Silicate & $\mathrm{Pu}-\mathrm{O}$ & 7.8 & 2.24 & 0.00984 \\
& $\mathrm{Pu}-\mathrm{O}$ & 4.0 & 2.50 & 0.00984 \\
& $\mathrm{Pu}-\mathrm{Si}$ & 2.0 & 3.09 & 0.01176 \\
& $\mathrm{Pu}-\mathrm{Si}{ }^{*}$ & 1.5 & 3.83 & 0.01176 \\
\hline \multicolumn{3}{c}{ * preliminary assignment } & &
\end{tabular}

Results of the least-squares fitting analysis show the presence of two oxygen shells, which is a strong indicator for inner-sphere surface complexation to the clinoptilolite surface. The analysis also shows a silicon shell at $\sim 3.09 \AA$, a bond length commensurate with bidentate complexation to silica. Analysis of the third peak yields tentative evidence for the presence of a second type of complexation with the surface, monodentate complexation. More importantly, analysis of the third peak is not consistent with the presence of $\mathrm{Pu}-\mathrm{Pu}$ interactions that would indicate co-precipitate formation, and therefore provides evidence for direct sorption of the plutonium to the clinoptilolite.

The XANES and EXAFS spectra from the X-ray absorption spectroscopy shows that the aqueous $\mathrm{Pu}(\mathrm{IV})$ remained as $\mathrm{Pu}(\mathrm{IV})$ on the clinoptilolite, montmorillonite, and birnessite surfaces at $\mathrm{pH} 8$ (EXAFS spetra not shown for montmorillonite and birnessite). The $\mathrm{Pu}(\mathrm{IV})$ sorbed directly to the clinoptilolite surface as an inner-sphere surface complex and not as a co-precipitate.

\subsection{Modeling Pu(IV) Sorption on Clinoptilolite Colloids}

The interaction of $\mathrm{Pu}(\mathrm{IV})$ with clinoptilolite colloids was modeled using both the nonelectrostatic and the diffuse layer surface complexation models. In particular, efforts to understand the decrease in sorption of $\mathrm{Pu}(\mathrm{IV})$ at $\mathrm{pH}$ values between 7 and 9 were undertaken (see Figure 4.4b). A description of the models used is presented below and justification for their use is discussed. 
Surface complexation and ion exchange reactions that occur between the fracture lining minerals and $\mathrm{Pu}$ in solution will act to reduce the mobility of $\mathrm{Pu}$ by sorption. ${ }^{1}$ In contrast these same processes occur on mobile mineral colloids and act to transport $\mathrm{Pu}$. So it is important to understand the extent to which these processes occur, both in the context of reducing mobility and also enhancing mobility of low-solubility radionuclides. Surface complexation and ion exchange reactions provide a mechanistic approach to modeling sorption and can account for the effect of changing environmental conditions on sorption.

In modeling radionuclide migration in the environment, partitioning $K_{d}$ constants are often used to model sorption. Although the $K_{d}$ approach can adequately describe the sorptive behavior of a particular sediment at a particular $\mathrm{pH}$ and solution composition, the many factors that affect radionuclide sorption cannot be accounted for in dynamic geologic environments. Therefore an overall understanding of the dominant geochemical processes occurring over a range of $\mathrm{pH}$ values becomes important.

Surface complexation reactions involve mineral surface functional groups and aqueous species. Factors that influence surface complexation on a particular mineral include:

- surface area,

- $\mathrm{pH}$,

- aqueous complexation,

- ionic strength, and

- surface charge.

There are many models that have been used to describe surface complexation reactions (non-electrostatic, constant capacitance, diffuse layer, triple layer, and others) Here, we fit Pu surface complexation using two of the simpler models: the non-electrostatic model (NEM) and the diffuse layer model (DLM). Unlike all other surface complexation models, the NEM (Kurbatov et al., 1951) assumes that surface electrical charge does not affect equilibrium surface complexation reactions. Although the NEM approach oversimplifies the factors affecting surface complexation, several investigators have used this model approach to describe sorption reactions (Bradbury and Baeyens, 1997; Davis et al., 1998; Zachara et al., 1994). Davis et al. (1998) argued that the NEM approach may be the most appropriate for complex environmental applications since the surface charging behavior of non-ideal natural mineral phases is not well known. The electrostatic models typically contain one or more parameters that account for surface charge effects. The DLM is one of the simplest electrostatic models and relies on a modified Gouy-Chapman theory to describe the effect of electrostatics on sorption.

\section{Data Fitting Routine and Aqueous Speciation Data for both Pu(IV) and Pu(V)}

Sorption data were fit using a modified version of the fitting program FITEQL (Herbelin and Westall, 1994). The code was modified to allow for:

\footnotetext{
${ }^{1}$ Ion exchange reactions are not discussed in this report because surface complexation is considered to be the primary mechanism by which $\mathrm{Pu}$ sorbs to clinoptilolite. Ion exchange is not expected to be significant due to steric hindrance.
} 
- automated activity correction using the extended Debye Huckel model,

- automated retrieval of aqueous speciation data from a database,

- an ion exchange capability, and

- code stability in cases of large species matrices.

Aqueous speciation of major cations and anions was based on the GEMBOCHS thermodynamic data base version data.com.V8.R6 (Johnson and Lundeen, 1997). Pu speciation information was gathered from a variety of sources, as will be briefly summarized below. A more detailed explanation of the thermodynamic data used to fit our experimental data can be found in Appendix G.

The majority of the data was taken from the recent Nuclear Energy Agency (NEA) volume on the chemical thermodynamics of plutonium (Lemire et al., 2001). However, some additional aqueous species were used based on fits to amorphous $\mathrm{PuO}_{2}$ solubility data reported in Rai et al. (1999). These data were not included in the NEA volume but the aqueous plutonium hydroxycarbonate species evaluated based on these data are critical to our evaluation of $\mathrm{Pu}(\mathrm{IV}$ ) sorption. Rai et al. (1999) examined the solubility of amorphous $\mathrm{PuO}_{2}$ across a range of $\mathrm{pH}$ and carbonate alkalinity conditions. The solubility data of Rai et al. (1999) (as well as Yamaguchi et al. (1994)) showed that carbonate complexation will increase the solubility of amorphous $\mathrm{PuO}_{2}$ at carbonate concentrations as low as $10^{-3} \mathrm{~mol} / \mathrm{L}$. Since the carbonate alkalinity of J-13 type waters is $\sim 10^{-2.7} \mathrm{~mol} / \mathrm{L}$, the results suggest that $\mathrm{Pu}(\mathrm{IV})$-hydroxycarbonate complexation will be significant at the NTS. Two Pu(IV)-hydroxycarbonate species were used to fit the solubility data of Rai et al. (1999) and Yamaguchi et al. (1994): $\mathrm{Pu}(\mathrm{OH})_{2}\left(\mathrm{CO}_{3}\right)_{2}{ }^{2-}$ and $\mathrm{Pu}(\mathrm{OH})_{4}\left(\mathrm{CO}_{3}\right)_{2}{ }^{4-}$. Details regarding these species are found in Appendix G.

The speciation of $\mathrm{Pu}(\mathrm{IV})$ for the water composition used in our experiments was modeled using the thermodynamic data presented in Appendix G. The carbonate concentration in groundwater is sensitive to changes in $\mathrm{pH}$ and is important for modeling the $\mathrm{Pu}$ speciation. The measured carbonate concentration as a function of $\mathrm{pH}$ in the J-13 surrogate water is shown in Figure 4.7a. Figure 4.7b shows the dominant species present in the water used in our experiments. The positively charged hydroxide species dominate speciation at low $\mathrm{pH}$, negatively charged carbonate (or hydroxycarbonate) species dominate at neutral to high $\mathrm{pH}$, and the neutral $\mathrm{Pu}(\mathrm{OH})_{4}(\mathrm{aq})$ species dominates at intermediate to low $\mathrm{pH}$ as well as very high $\mathrm{pH}(\sim 11)$. Given that sorption often decreases under conditions where negatively charged aqueous species dominate, the speciation data suggests that sorption at intermediate to high pHs (7-10) will be suppressed by the formation of Pu-carbonate species in solution. This would result in a decrease in $\mathrm{Pu}$ sorption at these $\mathrm{pHs}$ and a greater amount of $\mathrm{Pu}$ in solution as a carbonate complex. 

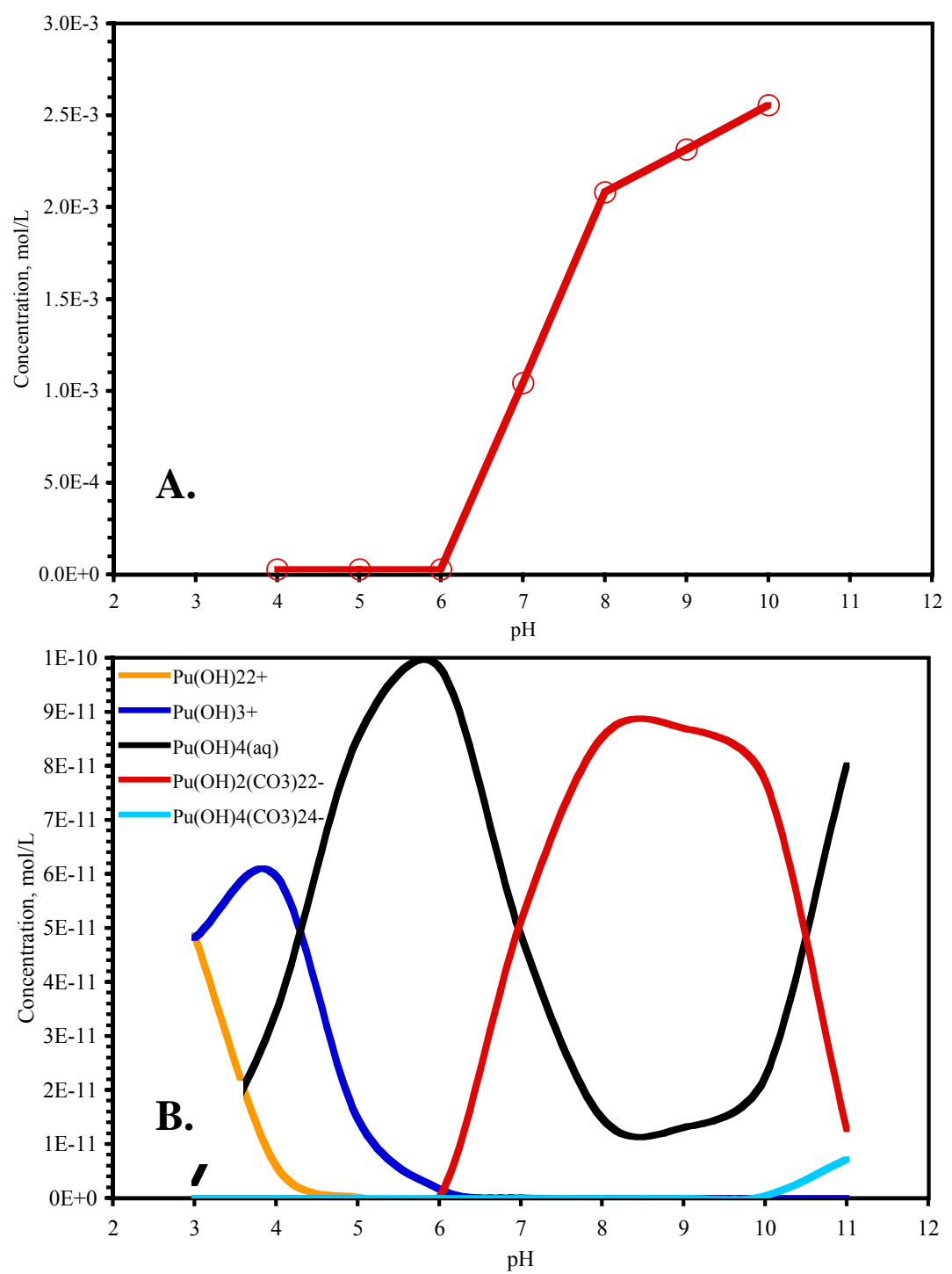

Figure 4.7. A) Measured carbonate concentration J13S2 as a function of pH. B) Speciation of $\mathrm{Pu}(\mathrm{IV})$ under the experimental conditions used. Note that at the $\mathrm{pH}$ of most NTS water $(\sim 8)$, the $\mathrm{Pu}(\mathrm{IV})$ hydroxy-carbonate is the dominant species in solution (red line).

\section{Modeling of Pu(IV) sorption data}

In the following section, we describe our modeling of $\mathrm{Pu}(\mathrm{IV})$ sorption to clinoptilolite colloids in J-13S2 as a function of $\mathrm{pH}$. Aqueous speciation of $\mathrm{Pu}(\mathrm{IV})$ in batch sorption solutions is shown in Figure 4.7b. As suggested in the previous section, Pu(IV)hydroxycarbonate complexes may play a significant role in $\mathrm{Pu}(\mathrm{IV})$ speciation in these solutions. In particular, between $\mathrm{pH} 7$ and $10, \mathrm{Pu}(\mathrm{OH})_{2}\left(\mathrm{CO}_{3}\right)_{2}{ }^{2-}$ aqueous complex is predicted to be the dominant $\mathrm{Pu}(\mathrm{IV})$ species in solution. Since the $\mathrm{Pu}(\mathrm{IV})$ hydroxycarbonate complex is negatively charged, it is also likely that $\mathrm{Pu}(\mathrm{IV})$ sorption will be reduced under those conditions. 
The sorption data used in surface complexation modeling is presented in Figure 4.8 along with several model fits. Sorption of $\mathrm{Pu}(\mathrm{IV})$ occurred very quickly and did not change as a function of time. Thus, in this plot, we do not distinguish sorption data points with regards to time. The kinetics of $\mathrm{Pu}(\mathrm{IV})$ sorption was presented in Figure 4.4. It is also presented in Figure 4.9, where the aqueous $\mathrm{Pu}(\mathrm{IV})$ concentration (instead of \% sorbed) is plotted as a function of $\mathrm{pH}$ and time. The data in Figure 4.9 clearly illustrate that the aqueous $\mathrm{Pu}(\mathrm{IV})$ concentration decreases dramatically and reaches equilibrium within 1-2 days. The higher equilibrium $\mathrm{Pu}$ concentration in the $\mathrm{pH} 7$ to 9 region is consistent with the predominance of aqueous $\mathrm{Pu}(\mathrm{IV})$-hydroxycarbonate species in solution and is simply the inverse of the sorption minimum shown in Figure 4.8. Because the sorption kinetics are very fast, we assume in our model that $\mathrm{Pu}$ sorption can be regarded as an equilibrium problem. Therefore, we ignore sorption kinetics and regard all $\mathrm{Pu}(\mathrm{IV})$ sorption data as equilibrium data points. A discussion of $\mathrm{Pu}(\mathrm{IV})$ sorption modeling follows.

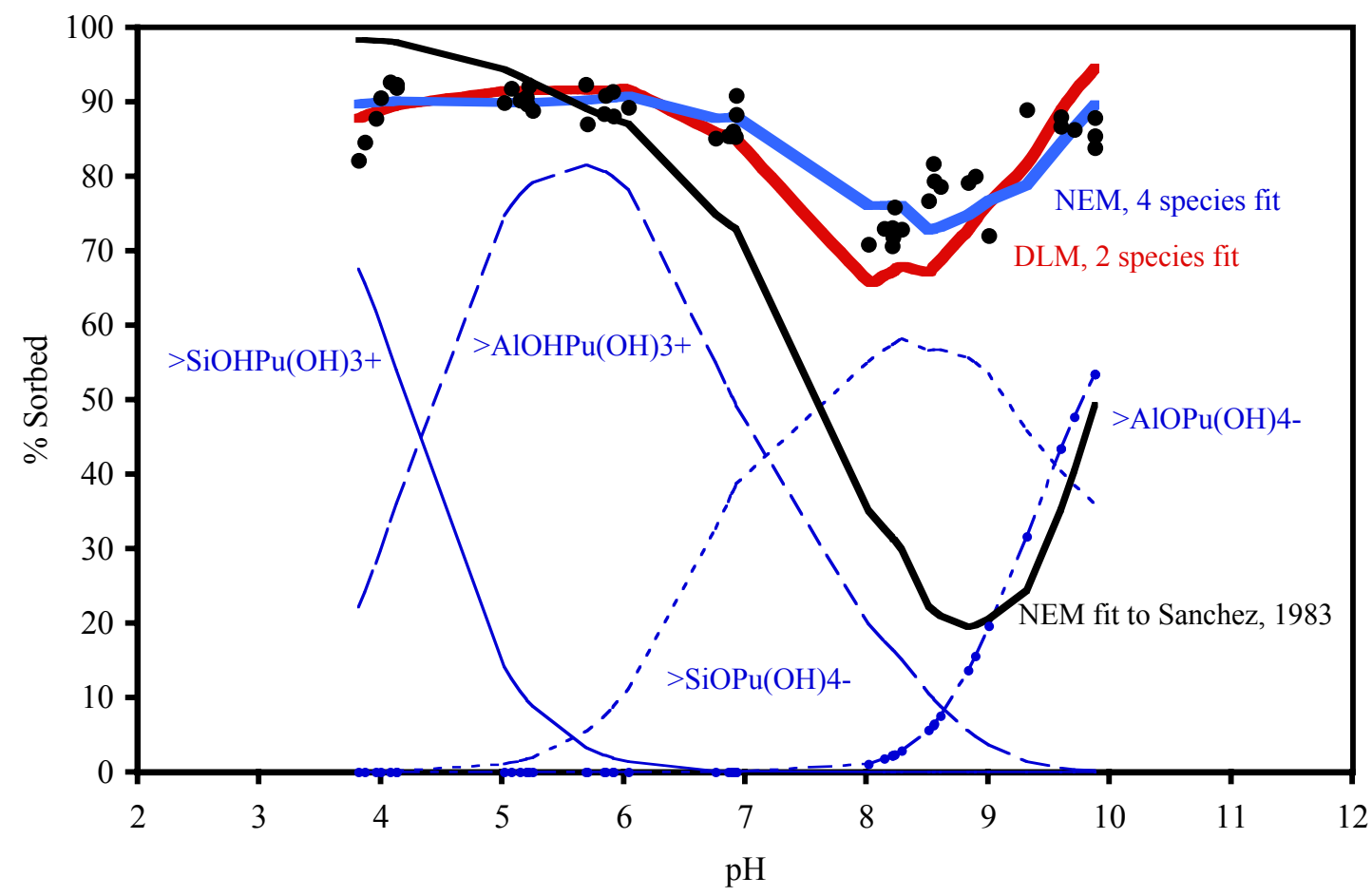

Figure 4.8. $\mathrm{Pu}(\mathrm{IV})$ sorption and clinoptilolite colloid data (black dots) as a function of percent sorption vs. $\mathrm{pH}$. $\mathrm{Pu}(\mathrm{IV})$ sorption to clinoptlilolite colloids is compared to several model fits. Non-electrostatic 4 surface species model fit (blue), diffuse layer model 2 species fit (red), and predicted sorption based on non-electrostatic 3 species model fit to data of Sanchez et al., 1983 (black). 


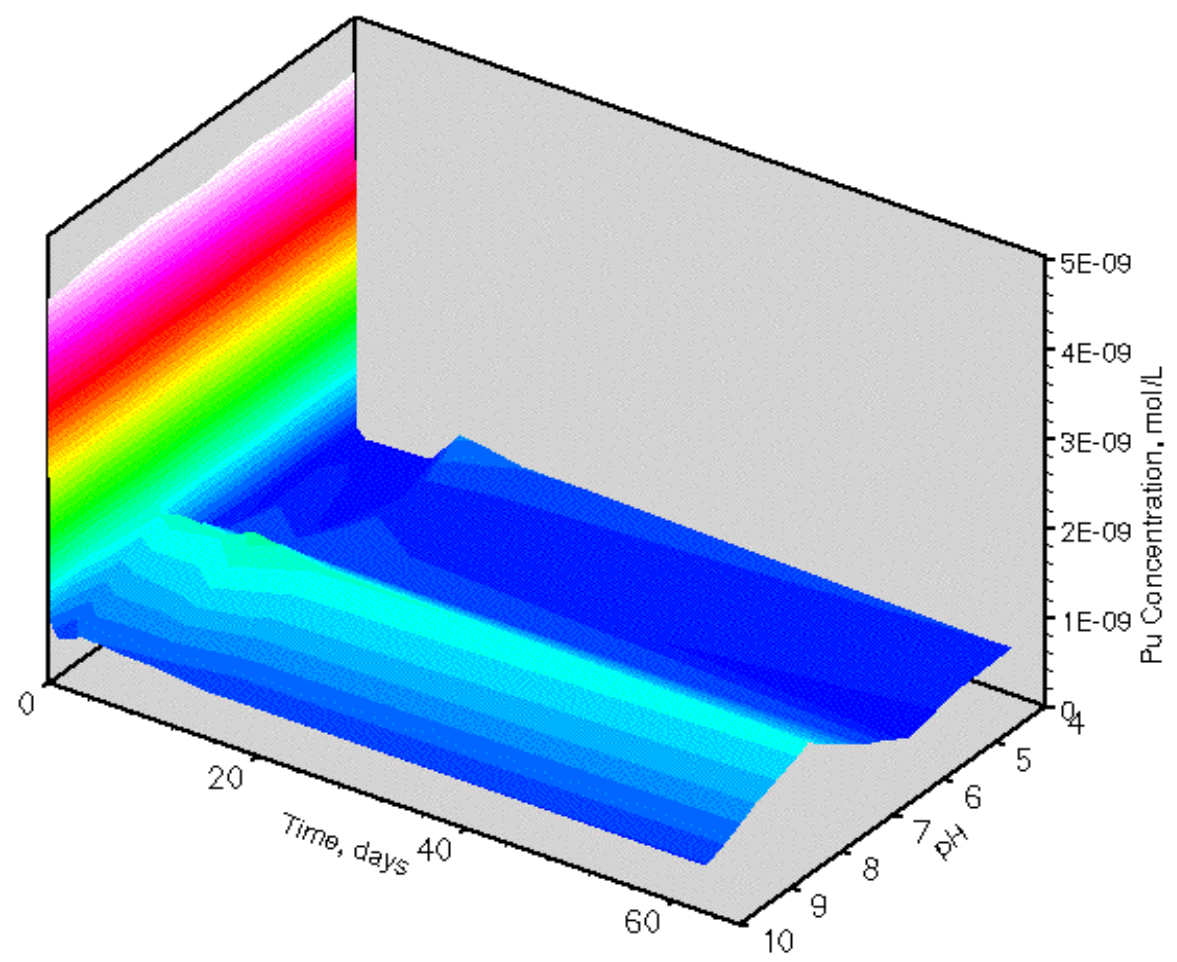

Figure 4.9. Aqueous $\mathrm{Pu}(\mathrm{IV})$ concentration as a function of time and $\mathrm{pH}$ in $\mathrm{Pu}(\mathrm{IV})$ sorption experiments.

The $\mathrm{Pu}(\mathrm{IV})$ sorption to clinoptilolite colloids batch sorption experiments were conducted using $0.14 \mathrm{mg} / \mathrm{mL}$ zeolite colloids and $4.4 \mathrm{E}-9 \mathrm{~mol} / \mathrm{L} \mathrm{Pu}(\mathrm{IV})$. The colloid particle size was measured by PCS to be $171 \pm 25 \mathrm{~nm}$ in diameter. Based on a two standard of deviation range of particle size, the surface area of the colloids ranges from 12.7 to 23.3 $\mathrm{m}^{2} / \mathrm{g}$ (assuming cubic particles). In our sorption modeling, we used a surface area of 23.3 $\mathrm{m}^{2} / \mathrm{g}$ since it is likely that particle surface areas are underestimated when assuming ideal cubic particle morphology. ${ }^{2}$ Based on a reactive site density of 2.31 sites $/ \mathrm{nm}^{2}$ (previously used in Zavarin and Bruton (2001)), a colloid reactive site concentration of $1.25 \times 10^{-5} \mathrm{~mol} / \mathrm{L}$ was estimated. Based on a 0.15:0.85 ratio of Al:Si in clinoptilolite, it was estimated that $15 \%$ of the surface sites were $>\mathrm{AlOH}$ and $85 \%$ were $>\mathrm{SiOH}$. The water composition varied to some degree as a function of $\mathrm{pH}$. The water composition was taken into account during modeling; the solution composition as a function of $\mathrm{pH}$ is reported in Table 4.2 .

Based on the solution composition in Table 4.2 and estimates of the reactive site concentrations discussed above, both non-electrostatic and diffuse layer models were

\footnotetext{
${ }^{2}$ For example, we previously used colloids that were $250 \pm 25 \mathrm{~nm}$ in size. Based on the same arguments, we predict a surface area of 9.4 to $14.1 \mathrm{~m}^{2} / \mathrm{g}$. BET measurements of surface area yielded $15.13 \mathrm{~m}^{2} / \mathrm{g}$, higher than the upper range of surface area based on cubic particle morphology.
} 
used to examine the $\mathrm{Pu}(\mathrm{IV})$ sorption data. The best fit reaction constants are reported in Table 4.3 for several modeling scenarios. Fits were accomplished by a least squares fit of predicted to measured aqueous $\mathrm{Pu}(\mathrm{IV})$ concentrations in solution. Not surprisingly, the decrease in $\mathrm{Pu}(\mathrm{IV})$ sorption in the $\mathrm{pH} 8$ to 9 range could be simulated by both the nonelectrostatic and electrostatic models. Also, the goodness of fit increased with the number of surface species used in the model. Given our limited ability to constrain the surface species and the limited $\mathrm{Pu}(\mathrm{IV})$ sorption data, the significance of the modeling results should not be over-estimated. It interesting to note, however, that the 3 species NEM fit to these sorption data is in approximate agreement with the Pu-smectite sorption data fits to the data of Sanchez et al. (1983) (see Zavarin and Bruton, 2001 and Table 4. .3). The reaction constants based on the data of Sanchez et al. (1983) somewhat overestimate sorption at low $\mathrm{pH}$ and underestimate sorption at high $\mathrm{pH}$ (Figure 4.8). While there is not enough sorption data to develop a robust surface complexation model for $\mathrm{Pu}(\mathrm{IV})$ sorption to clinoptilolite, the sorption modeling reaction constants are useful for developing hypotheses regarding the effects of $\mathrm{pH}$ and carbonate alkalinity on $\mathrm{Pu}(\mathrm{IV})$ reactive transport. The extrapolation of these reaction constants beyond the solution conditions examined here should be regarded with significant uncertainty. Notwithstanding the limitations of our modeling, it appears that the 4 species NEM and the 3 species DLM best fit our sorption data.

Table 4.2. Composition of batch sorption solutions for selected cations and $\mathrm{HCO}_{3}{ }^{-}$.

\begin{tabular}{rcccccc}
\hline $\mathrm{pH}$ & $\mathrm{Ca}$ & $\mathrm{Mg}$ & $\mathrm{Na}$ & $\mathrm{Si}$ & $\mathrm{K}$ & $\mathrm{HCO}_{3}{ }^{-}$ \\
& & \multicolumn{5}{c}{$\mathrm{mol} / \mathrm{L}$} \\
4 & $3.53 \mathrm{E}-05$ & $8.04 \mathrm{E}-05$ & $2.58 \mathrm{E}-03$ & $3.31 \mathrm{E}-04$ & $6.86 \mathrm{E}-04$ & $2.89 \mathrm{E}-05$ \\
5 & $3.51 \mathrm{E}-05$ & $7.93 \mathrm{E}-05$ & $2.98 \mathrm{E}-03$ & $3.24 \mathrm{E}-04$ & $1.76 \mathrm{E}-03$ & $2.89 \mathrm{E}-05$ \\
6 & $3.53 \mathrm{E}-05$ & $8.08 \mathrm{E}-05$ & $2.71 \mathrm{E}-03$ & $3.38 \mathrm{E}-04$ & $8.21 \mathrm{E}-04$ & $2.95 \mathrm{E}-05$ \\
7 & $3.39 \mathrm{E}-05$ & $7.83 \mathrm{E}-05$ & $2.51 \mathrm{E}-03$ & $3.25 \mathrm{E}-04$ & $8.32 \mathrm{E}-04$ & $1.04 \mathrm{E}-03$ \\
8 & $2.82 \mathrm{E}-05$ & $7.33 \mathrm{E}-05$ & $2.54 \mathrm{E}-03$ & $3.34 \mathrm{E}-04$ & $1.49 \mathrm{E}-04$ & $2.08 \mathrm{E}-03$ \\
9 & $3.59 \mathrm{E}-05$ & $8.23 \mathrm{E}-05$ & $2.96 \mathrm{E}-03$ & $3.45 \mathrm{E}-04$ & $5.86 \mathrm{E}-04$ & $2.31 \mathrm{E}-03$ \\
10 & $2.90 \mathrm{E}-05$ & $7.88 \mathrm{E}-05$ & $3.85 \mathrm{E}-03$ & $3.33 \mathrm{E}-04$ & $4.54 \mathrm{E}-04$ & $2.55 \mathrm{E}-03$ \\
\hline
\end{tabular}

Table 4.3. Reaction constants that describe the sorption of $\mathrm{Pu}(\mathrm{IV})$ to clinoptilolite colloids. $\dagger$

\begin{tabular}{|c|c|c|c|}
\hline Model Type & Surface Species & $\log K$ & $\begin{array}{c}\text { Goodness of } \\
\text { Fit }^{\S}\end{array}$ \\
\hline \multirow{3}{*}{$\begin{array}{l}3 \text { species NEM (fit to data of } \\
\text { Sanchez, 1983) }\end{array}$} & $>\mathrm{SiOPu}(\mathrm{OH})_{2}^{+}$ & -2.32 & \\
\hline & $>\mathrm{AlOPu}(\mathrm{OH})_{2}^{+}$ & -5.95 & \\
\hline & $>\mathrm{AlOPu}(\mathrm{OH})_{4}^{-}$ & 11.93 & \\
\hline \multirow[t]{3}{*}{3 species NEM (fit to batch data) } & $>\mathrm{SiOPu}(\mathrm{OH})_{2}^{+}$ & -1.24 & 23 \\
\hline & $>\mathrm{AlOPu}(\mathrm{OH})_{2}^{+}$ & -6.28 & \\
\hline & $>\mathrm{AlOPu}(\mathrm{OH})_{4}^{-}$ & 10.66 & \\
\hline \multirow{2}{*}{4 species NEM (fit to batch data) } & $>\mathrm{SiOPu}(\mathrm{OH})_{2}^{+}$ & -1.39 & 8.2 \\
\hline & $>\mathrm{SiOPu}(\mathrm{OH})_{4}^{-}$ & 9.82 & \\
\hline
\end{tabular}




$$
\begin{aligned}
& >\mathrm{AlOPu}(\mathrm{OH})_{2}{ }^{+} \quad-6.14 \\
& >\mathrm{AlOPu}(\mathrm{OH})_{4}{ }^{-} \quad 11.20 \\
& 2 \text { species DLM (fit to batch data) } \quad>\operatorname{SiOPu}(\mathrm{OH})_{2}{ }^{+} \quad-3.53 \\
& >\mathrm{AlOPu}(\mathrm{OH})_{4}^{-} \quad 7.15 \\
& >\mathrm{SiOPu}(\mathrm{OH})_{4}^{-} \quad 9.74 \\
& >\mathrm{AlOPu}(\mathrm{OH})_{4}^{-} \quad 7.37
\end{aligned}
$$$$
3 \text { species DLM (fit to batch data) } \quad>\mathrm{SiOPu}(\mathrm{OH})_{2}{ }^{+} \quad-3.47
$$

$\dagger$ For all surface complexation model fits, pKa's of the surface were based on DLM reaction constants reported in Turner (1995) for silica and $\mathrm{a}-\mathrm{Al}_{2} \mathrm{O}_{3}$. The $\log \mathrm{K}$ constants are the following: $>\mathrm{SiO}^{-}=7.20,>\mathrm{AlO}^{-}=9.73,>\mathrm{AlOH}_{2}{ }^{+}=-8.33$.

$\S$ Goodness-of-fit calculated as the weighted sum of squares divided by the degrees of freedom. A goodness-of-fit of $<20$ is considered a reasonably good fit. See Herbelin and Westall (1994) for further details.

Efforts to model the $\mathrm{Pu}(\mathrm{IV})$ sorption to clinoptilolite were able to match our sorption data as a function of $\mathrm{pH}$. Model data show that $\mathrm{Pu}(\mathrm{IV})$ sorption at intermediate to high $\mathrm{pHs}$ (7-10) will be slightly suppressed by the formation of Pu-carbonate species in solution. In particular, between $\mathrm{pH} 7$ and $10, \mathrm{Pu}(\mathrm{OH})_{2}\left(\mathrm{CO}_{3}\right)_{2}{ }^{2-}$ aqueous complex is predicted to be the dominant $\mathrm{Pu}(\mathrm{IV})$ species in solution. The decrease in $\mathrm{Pu}(\mathrm{IV})$ sorption in the $\mathrm{pH} 8$ to 9 range could be simulated by both the non-electrostatic and electrostatic models. This model predicts a decrease in $\mathrm{Pu}$ sorption at $\mathrm{pHs}$ between $\mathrm{pH} 7$ and 10 and an increase in the amount of $\mathrm{Pu}$ in solution as a carbonate complex. At moderate $\mathrm{pH}$ values, like those observed at the NTS, the overwhelming majority of $\mathrm{Pu}(\mathrm{IV})$ in solution would be expected to sorb to colloids, while a small percentage could remain in solution. This is consistent with Kersting et al.'s (1999) data where greater than $95 \%$ of the $\mathrm{Pu}$ was associated with the colloids and a small percentage was detected in the dissolved fraction.

\subsection{Desorption of $\mathrm{Pu}(\mathrm{IV})$ sorbed on Clinoptilolite}

Long-term batch desorption experiments were carried out to determine how much $\mathrm{Pu}(\mathrm{IV})$ would desorb after initially sorbing on the clinoptilolite surface. A critical question that needs to be addressed before assessing the importance of colloidal-facilitated transport of $\mathrm{Pu}$, involves determining if $\mathrm{Pu}$, once sorbed, will remain on the colloids. If $\mathrm{Pu}(\mathrm{IV})$ is not easily and/or quickly desorbed from clinoptilolite, its transport is much more likely. Desorption experiments were begun with the final solutions remaining from the $\mathrm{Pu}(\mathrm{IV})$ sorption experiments discussed in Section 4.2. Sorption experiments of $\mathrm{Pu}(\mathrm{IV})$ on clinoptilolite were run for 63 days before the desorption experiments began.

\section{Experimental Techniques}

The colloids containing $\mathrm{Pu}(\mathrm{IV})$ from the initial sorption experiments were centrifuged from the supernatant and the supernatant removed by pipette. The J-13 surrogate water was pre-adjusted with respect to $\mathrm{pH}(\mathrm{pH} 4-10)$ and added to each tube with colloids. Because the sorption of $\mathrm{Pu}(\mathrm{IV})$ on the clinoptilolite was consistently high across the $\mathrm{pH}$ range investigated, the initial concentration of $\mathrm{Pu}(\mathrm{IV})$ on the colloids ranged from 3 to 4 $\mathrm{x} 10^{-9} \mathrm{M}$. Samples were continuously shaken at a slow rate of $300 \mathrm{rpm}$. The first samples were taken after 1 hour and the last sample were taken 218 days later. 


\section{Results and Discussion}

The desorption of $\mathrm{Pu}(\mathrm{IV})$ on clinoptilolite was slow and the percentage desorbed remained low throughout the experiment. Figure 4.10 shows the percentage of $\mathrm{Pu}(\mathrm{IV})$ remaining on the colloid surface as a function of $\mathrm{pH}$ and time. There was no noticeable increase in desorption with time up to 218 days. After 7 months, only $2-8 \%$ of the $\mathrm{Pu}$ was removed from the clinoptilolite surface. The overwhelming majority of the $\mathrm{Pu}(\mathrm{IV})$ remained on the colloids at all $\mathrm{pHs}$ investigated under the experimental conditions investigated. This suggests that $\mathrm{Pu}(\mathrm{IV})$ sorbed to colloids has the potential to be transported. However, $\mathrm{Pu}(\mathrm{IV})$ does desorb to some degree (2-8\% in 7 months) and reach a steady state solution condition, suggesting that $\mathrm{Pu}(\mathrm{IV})$ can desorb (albeit very slowly) from clinoptilolite colloids.
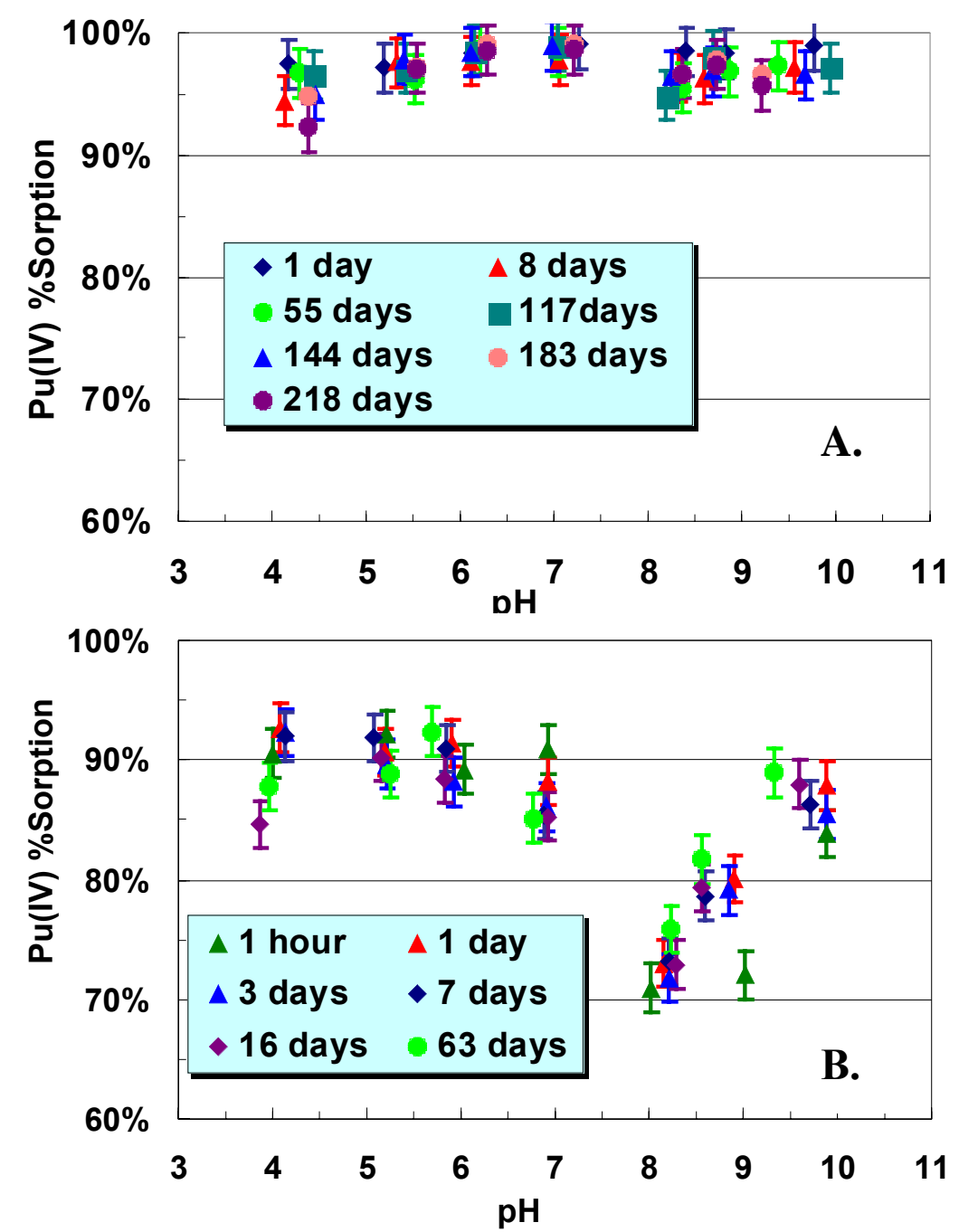

Figure 4.10. A. The desorption of $\mathrm{Pu}(\mathrm{IV})$ on clinoptilolite is shown as a function of percent $\mathrm{Pu}(\mathrm{IV})$ still remaining on the colloids. B. Sorption of $\mathrm{Pu}(\mathrm{IV})$ in original sorption experiment, shown for comparison. 
Figure 4.10B is shown for comparison and is a replotting of the sorption of $\mathrm{Pu}(\mathrm{IV})$ on clinoptilolite. The desorption experiments do not show a commensurate increase in desorption percentage between $\mathrm{pH}$ 7-9. We suggest that aqueous carbonate complexation cannot successfully compete for the $\mathrm{Pu}(\mathrm{IV})$ ion once the ion has sorbed on the clinoptilolite surface.

\subsection{Modeling Pu(IV) Desorption on Clinoptilolite}

$\mathrm{Pu}(\mathrm{IV})$ desorption was examined for the same set of batch sorption samples discussed above. However, it was found that the concentration of desorbed $\mathrm{Pu}(\mathrm{IV})$ never reached the expected value based on sorption data (desorption for 200 days) (Compare Figures $4.10 \mathrm{a}$ and $4.10 \mathrm{~b}$ ). This result can be interpreted in more than one manner. For example, the result can be interpreted as very slow desorption kinetics. The result can also be interpreted as permanently bound $\mathrm{Pu}$ that will never desorb. Finally, the result can be interpreted as sorption hysteresis.

Figure 4.11 is a plot of aqueous $\mathrm{Pu}(\mathrm{IV})$ as a function of time and $\mathrm{pH}$ based on our desorption experiments. The effect of $\mathrm{pH}$ is qualitatively similar to sorption experiment results, with a sorption maximum at $\mathrm{pH} 5$ to 7 and reduced sorption at the lower and higher pHs. It appears that the $\mathrm{Pu}$ solutions reach a steady state in a relatively short period of time. ${ }^{3}$ Steady state conditions were reached in both the sorption and desorption experiments. However, the steady state concentration in the desorption experiments is significantly lower than that in the sorption experiments $\left(\sim 5 \times 10^{-11}\right.$ to $2 \times 10^{-10}$ versus $4 \times 10^{-10}$ to $1 \times 10^{-9} \mathrm{~mol} / \mathrm{L}$, respectively). This is consistent with both hysteresis or permanently bound $\mathrm{Pu}$, but not with very slow desorption kinetics since, under those conditions, $\mathrm{Pu}(\mathrm{IV})$ should continue to desorb as a function of time.

To distinguish between permanently bound $\mathrm{Pu}$ and sorption hysteresis, one needs to evaluate whether $\mathrm{Pu}$ would desorb from the colloids if a stronger sorbing agent were present. After the 200 day desorption time period, ethylenediametetraacetic acid (EDTA) was added to the sorption solutions. EDTA is a strong organic complexing agent that was used extensively in processing plutonium. EDTA can alter the solubility of $\mathrm{Pu}$ and as a result increase the mobility of $\mathrm{Pu}$ by forming stable complex $\mathrm{Pu}(\mathrm{IV}$ )-EDTA (Hakem et al., 2001). With the addition of EDTA almost all the Pu was removed from the colloid surface (within the accuracy of the techniques used) suggesting that $\mathrm{Pu}$ will desorb from colloid when a higher $\mathrm{Pu}$ affinity compound is added. However, the EDTA result is qualitative because colloid surface conditions may have been altered by the EDTA. This experiment serves to demonstrate that care that should be taken when discussing the 'irreversibility' of a given reaction, such as $\mathrm{Pu}(\mathrm{IV})$ sorbed on clinoptilolite. Clearly, under some conditions, such as in the presence of EDTA, sorption of $\mathrm{Pu}(\mathrm{IV})$ on clinoptilolite can be reversed. Further study of the factors affecting $\mathrm{Pu}$ desorption is necessary to understand the conditions under which $\mathrm{Pu}$ can be desorb from mineral surfaces.

\footnotetext{
${ }^{3}$ That is not to say that sorption and desorption rates are equivalent but only that desorption and sorption rates are on the scale of days or less and certainly not months.
} 


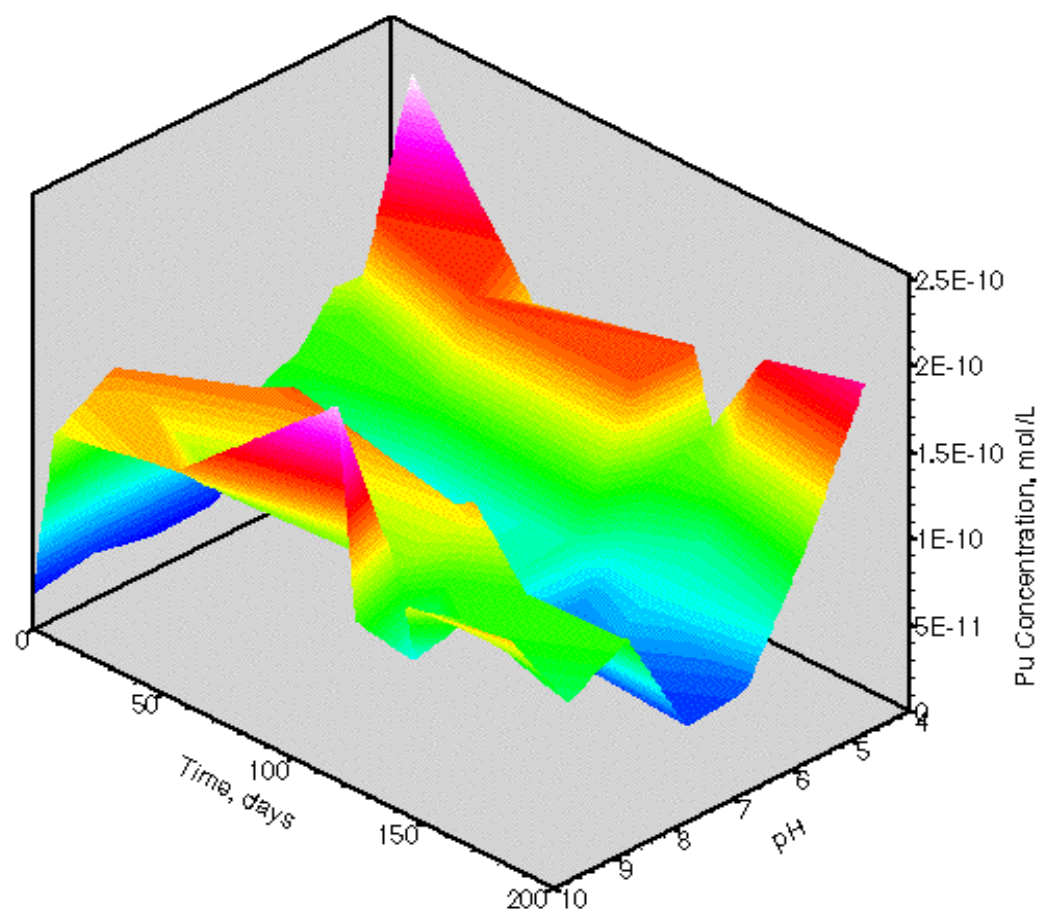

Figure 4.11. Aqueous $\mathrm{Pu}(\mathrm{IV})$ concentration as a function of time and $\mathrm{pH}$ in batch desorption experiments.

With the hypothesis that the difference between sorption and desorption is largely a hysteresis effect, we can apply surface complexation modeling to the steady-state desorption data. For modeling purposes, all batch desorption data collected at $>2$ days was assumed to have reached equilibium and used in sorption modeling. The Day 1 data was not included in the desorption modeling because the desorption process had not reached steady state (see Figure 4.11). The desorption data and NEM 4 species fit are shown in Figure 4.12. Best fit reaction constants are reported in Table 4.4. Due to the significant variability in the aqueous concentrations (Figure 4.11), the fitting routine used to model the sorption data could not simultaneously fit all sorption constants. Instead only a small number were varied; the reduced number of fitting parameters did not significant affect our ability to fit these data. For the 4 species NEM fit, The $>\mathrm{SiOPu}(\mathrm{OH})_{4}{ }_{4}$ and $>\mathrm{AlOPu}(\mathrm{OH})_{2}{ }^{+}$species reaction constants were adjusted from 9.82 and $-6.14(\mathrm{Pu}(\mathrm{IV})$ sorption data fit) to 8.64 and -6.84 while the other two species were not adjusted. Thus, sorption to two of the four surface species had to be increased by nearly an order of magnitude to account for the difference between the steady-state sorption and desorption results. For the 3 species DLM fit, the $>\mathrm{SiOPu}(\mathrm{OH})_{4}{ }^{-}$species reaction constant was adjusted from $9.74(\mathrm{Pu}(\mathrm{IV})$ sorption data fit) to 8.34 while the other two species were not adjusted. In this case, sorption to one of three species had to be increased by nearly 1.5 orders of magnitude to account for the difference between the steady-state sorption and desorption results.

Regardless of the surface complexation model, we find that the reaction constants fit to the sorption data are not adequate for describing the desorption data. This implies that $\mathrm{Pu}$ 
affinity for colloid surfaces based on sorption data alone will likely underestimate the potential for colloid mediated transport of plutonium.

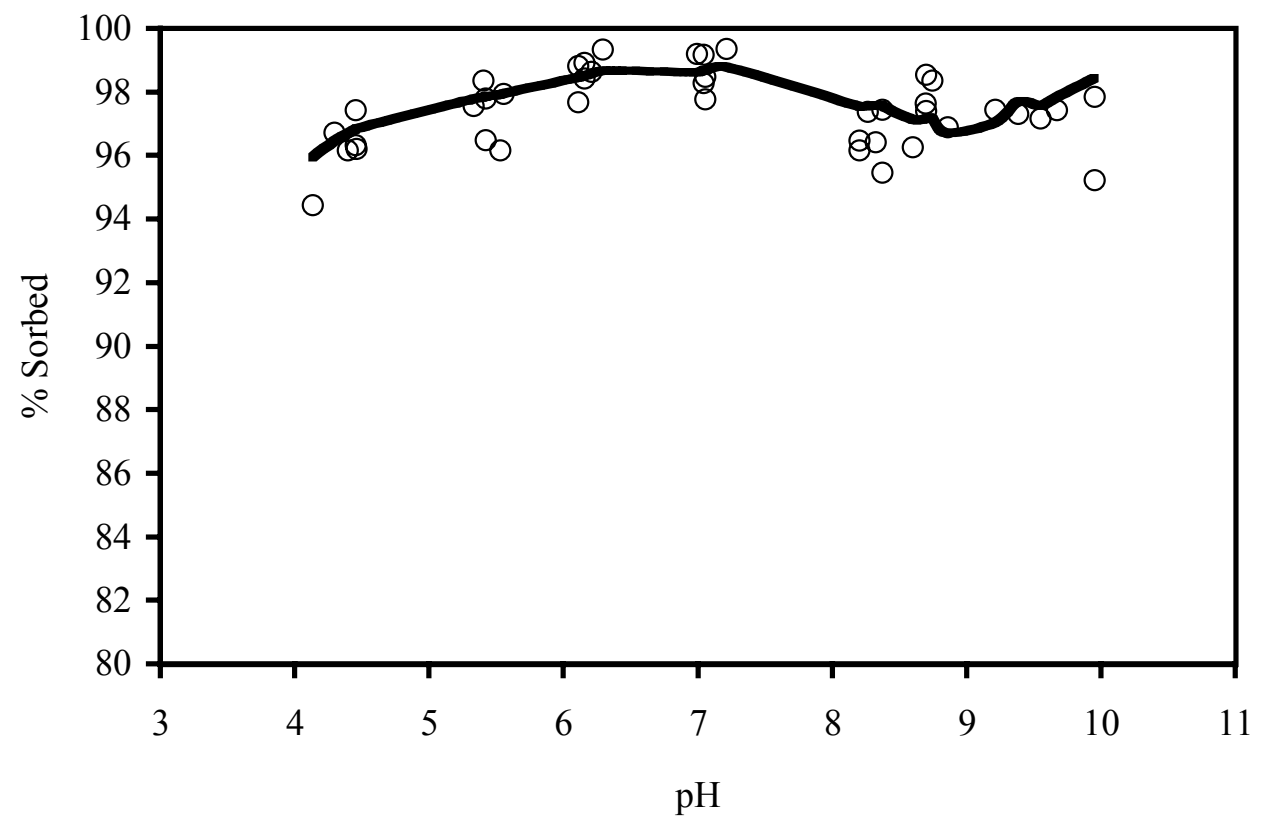

Figure 4.12. $\mathrm{Pu}(\mathrm{IV})$ desorption as a function of $\mathrm{pH}$ and 4 species $\mathrm{NEM}$ model fit to data.

Table 4.4. Reaction constants that describe the desorption of $\mathrm{Pu}(\mathrm{IV})$ to clinoptilolite colloids. $\dagger$

\begin{tabular}{|c|c|c|c|}
\hline Model Type & Surface Species & $\log K$ & $\begin{array}{l}\text { Goodness of } \\
\text { fit }\end{array}$ \\
\hline \multirow{3}{*}{$\begin{array}{l}3 \text { species NEM (fit to data of } \\
\text { Sanchez, 1983) }\end{array}$} & $>\mathrm{SiOPu}(\mathrm{OH})_{2}^{+}$ & -1.24 & \\
\hline & $>\mathrm{AlOPu}(\mathrm{OH})_{2}^{+}$ & -6.28 & \\
\hline & $>\mathrm{AlOPu}(\mathrm{OH})_{4}^{-}$ & 10.66 & \\
\hline \multirow[t]{4}{*}{4 species NEM (fit to batch data) } & $>\mathrm{SiOPu}(\mathrm{OH})_{2}^{+}$ & -1.39 & \multirow[t]{4}{*}{18} \\
\hline & $>\mathrm{SiOPu}(\mathrm{OH})_{4}^{-}$ & $8.64^{\pi}$ & \\
\hline & $>\mathrm{AlOPu}(\mathrm{OH})_{2}^{+}$ & -6.84 & \\
\hline & $>\mathrm{AlOPu}(\mathrm{OH})_{4}^{-}$ & 11.20 & \\
\hline \multirow[t]{3}{*}{3 species DLM (fit to batch data) } & $>\mathrm{SiOPu}(\mathrm{OH})_{2}^{+}$ & -3.47 & \multirow[t]{3}{*}{33} \\
\hline & $>\mathrm{SiOPu}(\mathrm{OH})_{4}^{-}$ & 8.34 & \\
\hline & $>\mathrm{AlOPu}(\mathrm{OH})_{4}^{-}$ & 7.37 & \\
\hline
\end{tabular}

$\dagger$ For all surface complexation model fits, $\mathrm{pKa}$ 's of the surface were based on DLM reaction constants reported in Turner (1995) for silica and $\alpha-\mathrm{Al}_{2} \mathrm{O}_{3}$. The $\log \mathrm{K}$ constants are the following: $>\mathrm{SiO}^{-}=7.20,>\mathrm{AlO}^{-}=9.73,>\mathrm{AlOH}_{2}^{+}=-8.33$.

If Only those reaction constants in italics were fitted; all other reaction constants remained the same as $\mathrm{Pu}(\mathrm{IV})$ sorption fits. 


\subsection{Summary}

The sorption of $\mathrm{Pu}(\mathrm{IV})$ on the zeolite, clinoptilolite, colloids as a function of time between $\mathrm{pH} 4$ and 10 was rapid, occurring within the first few hours. Equilibrium was reached within a few days. The percent sorption is consistently high, but varies as a function of $\mathrm{pH}$ from approximately $70 \%$ to over $90 \%$. The lowest sorption percentage occurs between $\mathrm{pH} 7$ and 9.5.

The EXAFS for clinoptilolite and the XANES spectra shows that the aqueous Pu(IV) sorbed as $\mathrm{Pu}(\mathrm{IV})$ on the clinoptilolite, montmorillonite, and birnessite surfaces at $\mathrm{pH} 8$. No change in oxidation state was observed. The $\mathrm{Pu}(\mathrm{IV})$ sorbed directly to the clinoptilolite surface as an inner-sphere surface complex and not as a co-precipitate.

The data for $\mathrm{Pu}(\mathrm{IV})$ sorption to clinoptilolite were successfully modeled as a function of $\mathrm{pH}$. Model data show that $\mathrm{Pu}(\mathrm{IV})$ sorption is consistently high across the $\mathrm{pH}$ range investigated, but at intermediate to high pHs (7-10) sorption will be slightly suppressed by the formation of $\mathrm{Pu}$-carbonate species in solution. In particular, between $\mathrm{pH} 7$ and 10, $\mathrm{Pu}(\mathrm{OH})_{2}\left(\mathrm{CO}_{3}\right)_{2}{ }^{2-}$ aqueous complex is predicted to be the dominant $\mathrm{Pu}(\mathrm{IV})$ species in solution.

At moderate $\mathrm{pH}$ values, like those observed at the NTS, the overwhelming majority of $\mathrm{Pu}(\mathrm{IV})$ in solution would be expected to sorb to colloids, while a small percentage could remain in solution as a Pu-carbonate species. This is consistent with Kersting et al.'s (1999) data where greater than $95 \%$ of the $\mathrm{Pu}$ was associated with the colloids and a small percentage was detected in the dissolved fraction.

In our desorption experiments, the majority of the $\mathrm{Pu}(\mathrm{IV})$ remained on the colloids at all $\mathrm{pHs}$ investigated under the experimental conditions investigated. This suggests that $\mathrm{Pu}(\mathrm{IV})$, once sorbed to the colloid surface has the potential to be transported. However, $\mathrm{Pu}(\mathrm{IV})$ does desorb to some degree (2-8\% in 7 months) and reaches a steady state solution condition, suggesting that $\mathrm{Pu}(\mathrm{IV})$ can desorb (albeit very slowly) from clinoptilolite colloids. The desorption rates of $\mathrm{Pu}(\mathrm{IV})$ are the important rate parameters needed to model the potential for $\mathrm{Pu}$ transport.

\section{Acknowledgments}

We thank Ken Moody for graciously supplying all the Pu stock solutions used in these experiments. We thank Nadia L. Hakem for some of the preliminary experimental work. Additional funding to A.B. Kersting in support of the Pu sorption experiments came from Laboratory Directed Research and Development grant, LLNL. 


\subsection{References}

Bradbury M. H. and Baeyens B. (1997) A Mechanistic Description of Ni and Zn Sorption on Na- Montmorillonite .2. Modelling. Journal of Contaminant Hydrology 27(34), 223-248.

Choppin G. R. (1991) Redox Speciation of Plutonium in Natural Waters. J. Radio analytical and Nuclear Chemistry, Articles 147(1), 109-116.

Clark D. L. (2000) The Chemical Complexities of Plutonium. In Challenges in Plutonium Science, Vol. 2 (ed. N. G. Cooper), pp. 493. Los Alamos National Laboratory, LA-UR-00 4100.

Davis J. A., Coston J. A., Kent D. B., and Fuller C. C. (1998) Application of the Surface Complexation Concept to Complex Mineral Assemblages. Environmental Science \& Technology 32(19), 2820-2828.

Hakem N. L., Allen P. G., and Sylwester E. R. (2001) Effect of EDTA on Plutonium Migration. J. Radioanalytical and Nuclear Chemistry 250(1), 47-53.

Herbelin A. L. and Westall J. C. (1994) FITEQL, A Computer Program for Determination of Chemical Equilibrium Constants from Experimental Data. Department of Chemistry, Oregon State University.

Johnson J. W. and Lundeen S. R. (1997) GEMBOCHS Thermodynamic Datafiles for use with the EQ3/6 Modeling Package. Lawrence Livermore National Laboratory.

Kurbatov M. H., Wood G. B., and Kurbatov J. D. (1951) Isothermal Adsorption of Cobalt from Dilute Solutions. Journal of Physical Chemistry 55, 1170-1182.

Lemire R. J., Fuger J., Spahiu K., Nitsche H., Ullman W. J., Potter P., Vitorge P., Rand M. H., Wanner H., and Rydberg J. (2001) Chemical Thermodynamics of Neptunium and Plutonium. Elsevier.

Lemire R. J. and Tremaine P. R. (1980) Uranium and Plutonium Equilibria in Aqueous Solutions to 200C. J. Chemical Eng. Data 25, 361-370.

Lu, Ningping, I. R. Triay, C. R. Cotter, H. D. Kitten, and J. Bentley (1998a). Reversibility of sorption of plutonium-239 onto colloids of hematite, goethite, smectite, and silica. LA-UR-98-3057, Los Alamos National Laboratory Unrestricted Report, Los Alamos, NM.

Lu, Ningping, C. R. Cotter, H. D. Kitten, and J. Bentley (1998b). Reversibility of sorption of plutonium-239 onto colloids of hematite and goethite. Radiochim. Acta 83, 167-173.

Nitsche H., Muller A., Standifer E. M., Deinhammer R. S., Becraft K., Prussin T., and Gatti R. C. (1992) Dependence of Actinide Solubility and Speciation on Carbonate concentration and Ionic Strength in Groundwater. Radiochimica Acta 58-59(P1)(9), 27-32.

Rai D., Hess N. J., Felmy A. R., Moore D. A., Yui M., and Vitorge P. (1999) A Thermodynamic Model for the Solubility of $\mathrm{PuO}_{2}(\mathrm{am})$ in the Aqueous $\mathrm{K}^{+}-\mathrm{HCO}_{3}$-$\mathrm{CO}_{3}$ 2--OH-- $\mathrm{H}_{2} \mathrm{O}$ system. Radiochimica Acta 86(3-4), 89-99.

Runde W, Conradson SD, Efurd DW, Lu NP, VanPelt CE, Tait CD, 2002. Solubility and sorption of redox-sensitive radionuclides $(\mathrm{Np}, \mathrm{Pu})$ in $\mathrm{J}-13$ water from the Yucca Mountain site: comparison between experiment and theory. Applied Geochemistry 17: 837-853.

Sanchez A. L. (1983) Chemical Speciation and Adsorption Behavior of Plutonium in Natural Waters, University of Washington. 
Triay, I.R., Cotter, C.R., Draus, S.M., Huddleston, M.H., Chipera, S.J., and Bish, D.L. (1996) Radionuclide Sorption in Yucca Mountain Tuffs with J-13 well water: Neptunium, Uranium, and Plutonium. Los Alamos National Laboratory. LA12779-MS.

Turner D. R. (1995) A Uniform Approach to Surface Complexation Modeling of Radionuclide Sorption, pp. 103. Center for Nuclear Waste Regulatory Analyses.

Yamaguchi T., Sakamoto Y., and Ohnuki T. (1994) Effect of the Complexation of Solubility of $\mathrm{Pu}(\mathrm{IV})$ in Aqueous Carbonate System. Radiochimica Acta 66/67, 914.

Zachara J. M., Resch C. T., and Smith S. C. (1994) Influence of Humic Substances on Co2+ Sorption by a Subsurface Mineral Separate and its Mineralogic Components. Geochimica et Cosmochimica Acta 58(2), 553-566.

Zavarin M. and Bruton C. J. (2000) A Non-Electrostatic Surface Complexation Approach to Modeling Radionuclide Migration at the Nevada Test Site: Aluminosilicates. Lawrence Livermore National Laboratory. UCRL-JC-133474 


\title{
-Chapter 5- Sorption of $\mathrm{Pu}(\mathrm{V})$ on Mineral Colloids
}

\author{
Annie B. Kersting, Pihong Zhao, Mavrik Zavarin, Eric R. Sylwester, \\ Patrick G. Allen, and Ross W. Williams
}

\subsection{Introduction}

In natural waters, the behavior of $\mathrm{Pu}$ depends on its oxidation state. As mentioned in Chapter 4, Pu can exist in five different oxidation states (Pu-III, IV, V, VI, VII). Pu(IV) and $\mathrm{Pu}(\mathrm{V})$ are the dominant oxidation states of $\mathrm{Pu}$ under equilibrium conditions for groundwater compositions measured at the NTS (Figure 4.1) (Nitsche et al., 1992). $\mathrm{Pu}(\mathrm{V})$ is the most soluble form of $\mathrm{Pu}$ in NTS type groundwater. As shown in Chapter 4, $\mathrm{Pu}(\mathrm{IV})$ sorbs quite quickly and strongly to zeolites, clays and Mn-oxides. This is consistent with results of (Penrose et al., 1987) who concluded that $\mathrm{Pu}$ (III) and $\mathrm{Pu}(\mathrm{IV}$ ) sorb strongly to most natural mineral surfaces, and that $\mathrm{Pu}(\mathrm{V})$ and $\mathrm{Pu}(\mathrm{VI})$ bind more weakly. In this chapter, the sorption of $\mathrm{Pu}(\mathrm{V})$ to mineral surfaces was investigated in the following experiments:

- Sorption of $\mathrm{Pu}(\mathrm{V})$ on clinoptilolite colloids

○ Sorption of $\mathrm{Pu}(\mathrm{V})$ on other colloids minerals: Fe-oxides, Mn-oxides, calcite, clay and silica

- X-ray absorption spectroscopy (XAS) of Pu sorbed on all minerals investigated, and

- Factors influencing the speciation and sorption behavior of $\mathrm{Pu}(\mathrm{V})$

○ Disproportionation

\subsection{Sorption of $\mathrm{Pu}(\mathrm{V})$ on Clinoptilolite Colloids}

Batch sorption experiments of $\mathrm{Pu}(\mathrm{V})$ on clinoptilolite colloids were carried out under the same experimental protocol as those of $\mathrm{Pu}(\mathrm{IV})$ sorption. Earlier sorption experiments of $\mathrm{Pu}(\mathrm{V})$ on hematite and goethite by $\mathrm{Lu}$ et al. (1998) suggest that $\mathrm{Pu}(\mathrm{V})$ sorbs slowly in contrast to sorption of $\mathrm{Pu}(\mathrm{IV})$. However, there are no internally consistent data available on sorption rates of both $\mathrm{Pu}(\mathrm{IV})$ and $\mathrm{Pu}(\mathrm{V})$ to mineral colloids that also includes oxidation state information of the sorbed species. Sorption data combined with speciation information would provide a framework for understanding the mechanisms that may control different sorption behavior between $\mathrm{Pu}(\mathrm{IV})$ and $\mathrm{Pu}(\mathrm{V})$, and allows accurate interpretation of desorption experiments.

\section{Experimental Techniques}

$\mathrm{Pu}(\mathrm{V})$ sorption experiments were undertaken under the same experimental protocol as those of $\mathrm{Pu}(\mathrm{IV})$ (Chapter 5). The $\mathrm{Pu}$ solution was $98 \% \mathrm{Pu}(\mathrm{V})$ at the start of the sorption experiments. The oxidation state of $\mathrm{Pu}(\mathrm{V})$ in the stock solution was confirmed using UV/VIS spectrometry. The starting concentration of $\mathrm{Pu}(\mathrm{V})$ solution was $2.1 \times 10^{-9} \mathrm{M}$. The $\mathrm{Pu}(\mathrm{V})$ solution was filtered through a $20 \mathrm{~nm}$ pore size filter prior to use in the sorption experiments. The $\mathrm{pH}$ range investigated ranged from 4 to 10 . The colloids ranged between 100-200 $\mathrm{nm}$ in size and the concentration used in the experiments was $0.14 \mathrm{mg} / \mathrm{mL}$. Sorption experiments were carried out for 77 days. 
For each set of sorption experiments, a second series of "control" solutions were prepared under the exact same experimental conditions but in the absence of the colloids. The controls were used to monitor any precipitation or sorption of $\mathrm{Pu}$ on the container during the experiments. Samples and controls were kept in closed polyethylene containers and were gently shaken with a slow and stable shaking speed of $300 \mathrm{rpm}$, until equilibrium was reached. Prior to the beginning of the sorption experiment, the solutions and controls were monitored for stability with respect to their $\mathrm{pH}$. After the $\mathrm{pH}$ solutions were stable, the experiments began. The $\mathrm{pH}$ was not adjusted during the course of the experiments.

A second set of $\mathrm{Pu}(\mathrm{V})$ sorption experiments were undertaken to compare the sorption of clinoptilolite with other common colloid minerals found in NTS water. These experiments were designed to use XAS to analyze the oxidation state and average local structure of the $\mathrm{Pu}$ sorbed to the colloids. Minerals investigated were: Mn-oxide, birnessite and pyrolusite; calcite; zeolite, clinoptilolite; Fe-oxide, goethite; clay, montmorillonite; and silica. Experiments were carried out at $\mathrm{pH} 8$ only, using a colloid size between $270-330 \mathrm{~nm}$. The concentration of $\mathrm{Pu}$ was $3.110^{-6} \mathrm{M}$, and the solution was $98 \% \mathrm{Pu}(\mathrm{V})$ at the start of the experiment. After 15 days the sorption experiments were stopped and the samples were prepared for XAS analysis.

\section{Results and Discussion}

The sorption of $\mathrm{Pu}(\mathrm{V})$ on clinoptilolite colloids as a function of time between $\mathrm{pH} 4$ and 10 is shown in Figure 5.1. The sorption rate of $\mathrm{Pu}(\mathrm{V})$ is slow, but continues to increase even after 77 days at $\mathrm{pHs}$ greater than 4. Although the sorption rate of $\mathrm{Pu}(\mathrm{V})$ on clinoptilolite varies as a function of $\mathrm{pH}$, the rate is low at the $\mathrm{pH}$ range observed in NTS water (7-9). After 2.5 months, only $20 \%$ of the Pu had sorbed on the colloids at $\mathrm{pH} 8$; the maximum sorption of $80 \%$ occurred between $\mathrm{pH} 5-6$. 
A.

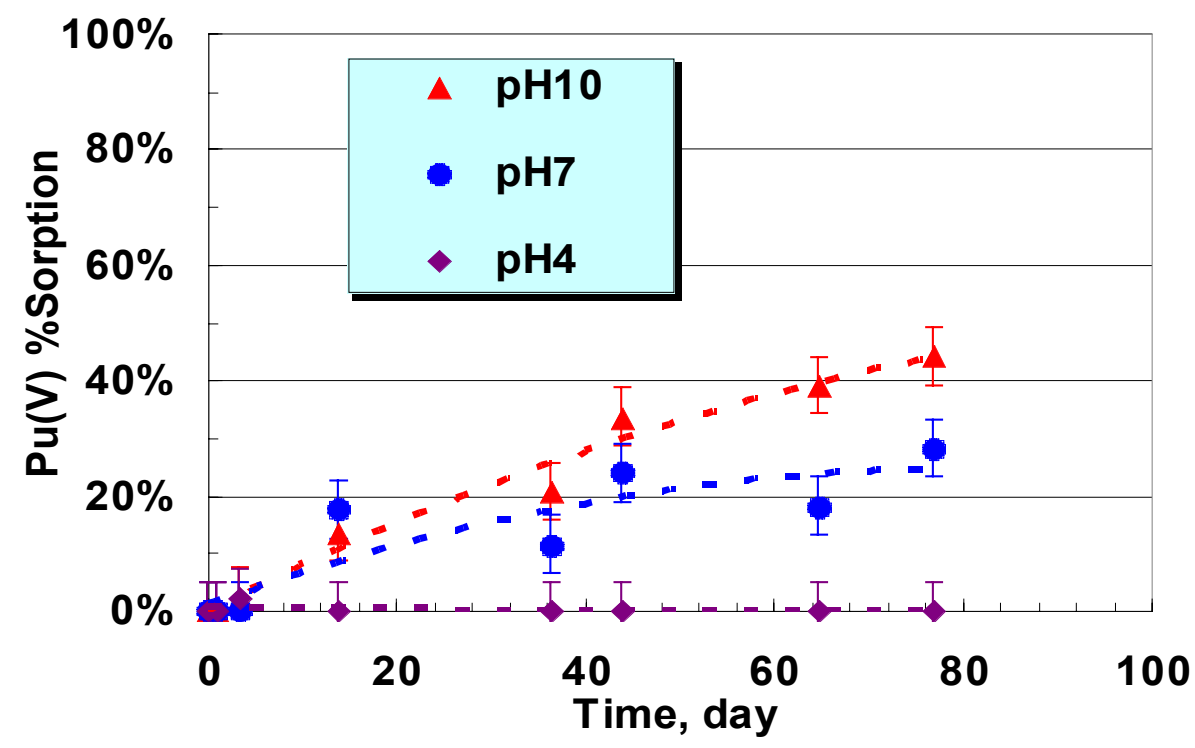

B.

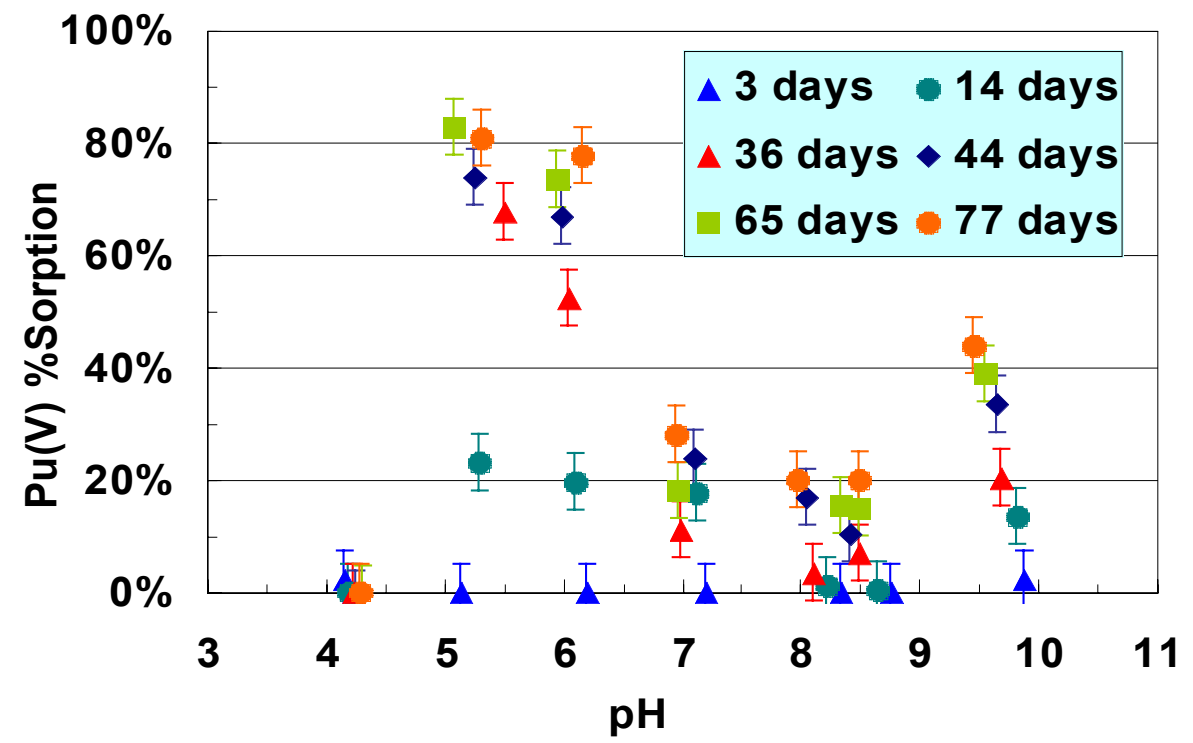

Figure 5.1. $\mathrm{Pu}(\mathrm{V})$ sorption on clinoptilolite as a function of time and $\mathrm{pH}$.

The sorption of $\mathrm{Pu}(\mathrm{V})$ on clinoptilolite is strongly dependent on $\mathrm{pH}$ (Figure 5.1b). Even after 77 days, the sorption continues to slowly increase at $\mathrm{pH}$ greater than 5 . As with the sorption data for $\mathrm{Pu}(\mathrm{IV})$, there is a decease in sorption at the moderate $\mathrm{pH}$ range (7-9), from $80 \%$ sorption at $\mathrm{pH} 5$ to $20 \%$ at $\mathrm{pH} 8$ and $45 \%$ at $\mathrm{pH} 10$.

In an effort to compare the sorption rate of $\mathrm{Pu}(\mathrm{V})$ on clinoptilolite to other minerals, another series of $\mathrm{Pu}(\mathrm{V})$ sorption experiments were conducted using the following minerals: Mn-oxide, birnessite and pyrolusite; calcite; zeolite, clinoptilolite; Fe-oxide, goethite; clay, montmorillonite; and silica. In this set of experiments, the experimental conditions were slightly different (see Experimental Techniques) so the Pu sorbed to the colloids could be analyzed by XAS. 
The distribution coefficient $\left(\mathrm{K}_{\mathrm{d}}\right)$ for $\mathrm{Pu}$ on the various minerals was calculated for comparison purposes. $\mathrm{K}_{\mathrm{d}} \mathrm{s}$ are typically reported as a ratio of total sorbed concentration $(\mathrm{mol} / \mathrm{g})$ to total aqueous concentration $(\mathrm{mol} / \mathrm{mL})$. A $\mathrm{K}_{d}$ for $\mathrm{Pu}$ of greater than one represents an affinity for the solid phase over the dissolved. The distribution coefficients calculated for the sorption of $\mathrm{Pu}(\mathrm{V})$ on the minerals investigated and are compared in Figure 5.2.

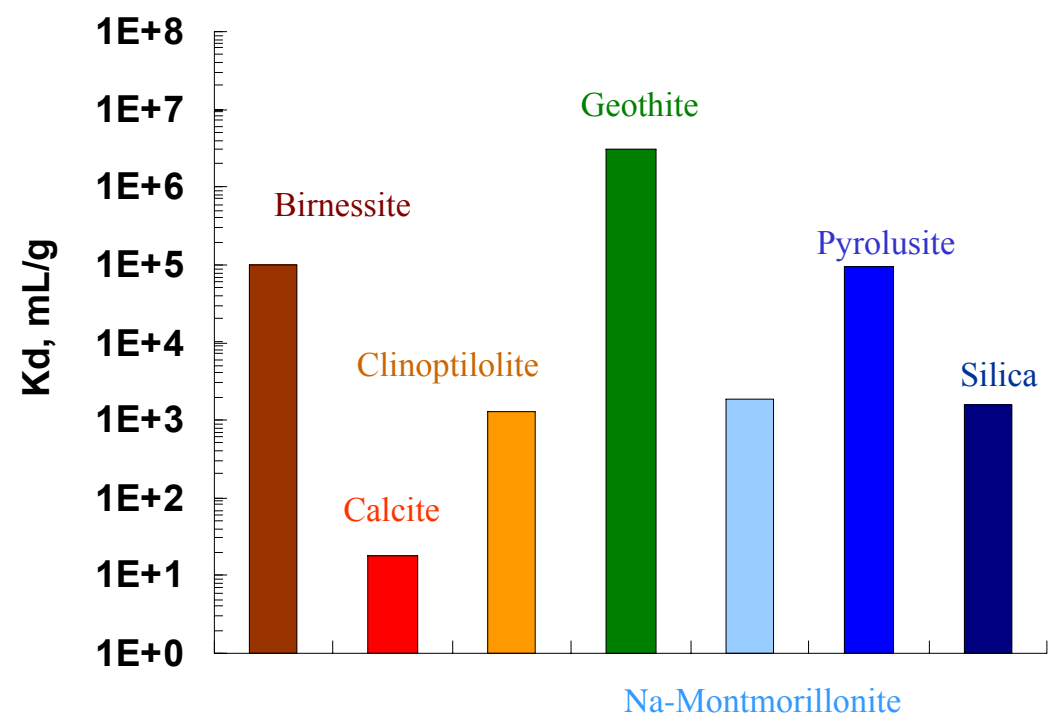

Figure 5.2. The distribution coefficient $\left(\mathrm{K}_{\mathrm{d}}\right)$ calculated for $\mathrm{Pu}(\mathrm{V})$ sorbed on the following minerals: Mn-oxide, birnessite and pyrolusite; calcite; zeolite, clinoptilolite; Fe-oxide, goethite; clay, montmorillonite; and silica.

Plutonium (V) sorbed to all seven minerals investigated, although the extent of sorption and the rates vary as a function of mineralogy. Greater than $90 \%$ of the $\mathrm{Pu}$ sorbed to birnessite and goethite in the first hour. In contrast, only $54 \%$ of the Pu had sorbed to the clinoptilolite after eight days, and only $8 \%$ of the $\mathrm{Pu}$ had sorbed to the silica after eight days. The $\mathrm{Pu}(\mathrm{V})$ sorbs the strongest to the $\mathrm{Mn}$ - and Fe- oxides and the weakest to calcite and silica. The different sorption rates of $\mathrm{Pu}(\mathrm{V})$ on different minerals suggests that the mechanism for $\mathrm{Pu}(\mathrm{V})$ sorption is different for different minerals; a hypothesis that will be further evaluated in section 5.4.

\subsection{X-ray Absorption Spectroscopy of $P u(V)$ Sorbed on all Minerals Investigated} As previously discussed in section 5.3, X-Ray absorption spectroscopy (XAS) is a method for studying the speciation of elements in a sample. The information gained from XAS includes the oxidation state of the element targeted as well as average local structure around that element. Structural details include the identity, coordination number and radial distances (i.e. bond lengths) for atoms within $\sim 4-5 \AA$ of the absorbing atom. In addition, XAS provides information on sorption mechanisms involved at the 
molecular level. Identifying specific sorption mechanisms will allow us to design experiments targeting the most important chemical processes in sorption, identify environmental systems where these processes occur, and develop better predictive capability through modeling.

In this study, we investigated the sorption of soluble $\mathrm{Pu}(\mathrm{V})$ on clinoptilolite colloids as a function of $\mathrm{pH}$, as well as sorption of $\mathrm{Pu}(\mathrm{V})$ on Fe-oxides, Mn-oxides, calcite, clays, and silica at $\mathrm{pH}$ 8. XAS was used to determine the oxidation state of the Pu sorbed on the mineral surfaces, as well as determine the average local structure of the $\mathrm{Pu}$ on the clinoptilolite.

\section{Experimental Techniques}

After 16 days, the $\mathrm{Pu}(\mathrm{V})$ sorption experiments were stopped and the sample was centrifuged. The solution was drawn off to leave a wet paste consisting of Pu sorbed to the colloidal material as the final sample form. The wet paste sample was studied directly using XAS.

All spectroscopic analysis was performed on BL 4-1 at the Stanford Synchrontron Radiation Laboratory (SSRL). The spectra were energy calibrated by simultaneously measuring the absorption spectrum for the reference standard $\mathrm{PuO}_{2}$, and setting the energy of the first inflection point, $\mathrm{E}_{\mathrm{r}}$, at $18053.1 \mathrm{eV}$. Plutonium $\mathrm{L}_{\mathrm{III}}$-edge XAS spectra were measured on wiggler beamline 4-1 under normal ring operating conditions (3.0 $\mathrm{GeV}, 50-100 \mathrm{~mA}$ ). Energy scans were obtained using a Si (220) double-crystal monochromator and a vertical slit width of $0.5 \mathrm{~mm}$. All samples were measured in fluorescence mode using a Ge solid state detector. Extended X-ray absorption fine structure (EXAFS) data reduction and normalization was done using the EXAFSPAK, suite of programs developed by G.George of SSRL. A hypothetical model compound, $\mathrm{Pu}^{4+} \mathrm{M}(\mathrm{O}, \mathrm{OH})_{6} .8 \mathrm{H}_{2} \mathrm{O}$ (where $\mathrm{M}=\mathrm{MN}, \mathrm{Fe}$ ) was derived from the crystal structures of Birnessite and Goethite, in order to model $\mathrm{Pu}-\mathrm{Mn}$ and $\mathrm{Pu}-\mathrm{Fe}$ EXAFS scattering, respectively.

\section{Results and Discussion}

The $\mathrm{Pu} \mathrm{L}_{\mathrm{III}} \mathrm{X}$-ray absorption near edge structure (XANES) results for the sorption of $\mathrm{Pu}(\mathrm{V})$ on clinoptilolite as a function of $\mathrm{pH}$ is shown in Figure 5.3. The $\mathrm{PuO}_{2}(\mathrm{Pu}-\mathrm{IV})$ and $\mathrm{PuO}_{2}{ }^{+}(\mathrm{Pu}-\mathrm{V})$ reference standards are shown for comparison. The soluble $\mathrm{Pu}(\mathrm{V})$ appears to have been reduced to $\mathrm{Pu}(\mathrm{IV})$ at all the $\mathrm{pHs}$ investigated. The absorption peaks of the sorbed samples now have the position of the $\mathrm{Pu}(\mathrm{IV})$ reference and the spectra display a $\sim 3 \mathrm{eV}$ shift in the main peak energy to the right of the $\mathrm{Pu}(\mathrm{V})$ reference peak (see vertical black line). Also, the samples have lost the high energy shoulder that identifies the plutonyl, $\mathrm{Pu}(\mathrm{V})$ structure. The shoulder is caused by multiple-scattering along the $\mathrm{O}=\mathrm{Pu}=\mathrm{O}$ axis and it's loss corresponds to a loss of the 'plutonyl' structure. These results show that reduction of soluble $\mathrm{Pu}(\mathrm{V})$ to $\mathrm{Pu}(\mathrm{IV})$ occurs at all $\mathrm{pHs}$ investigated. 


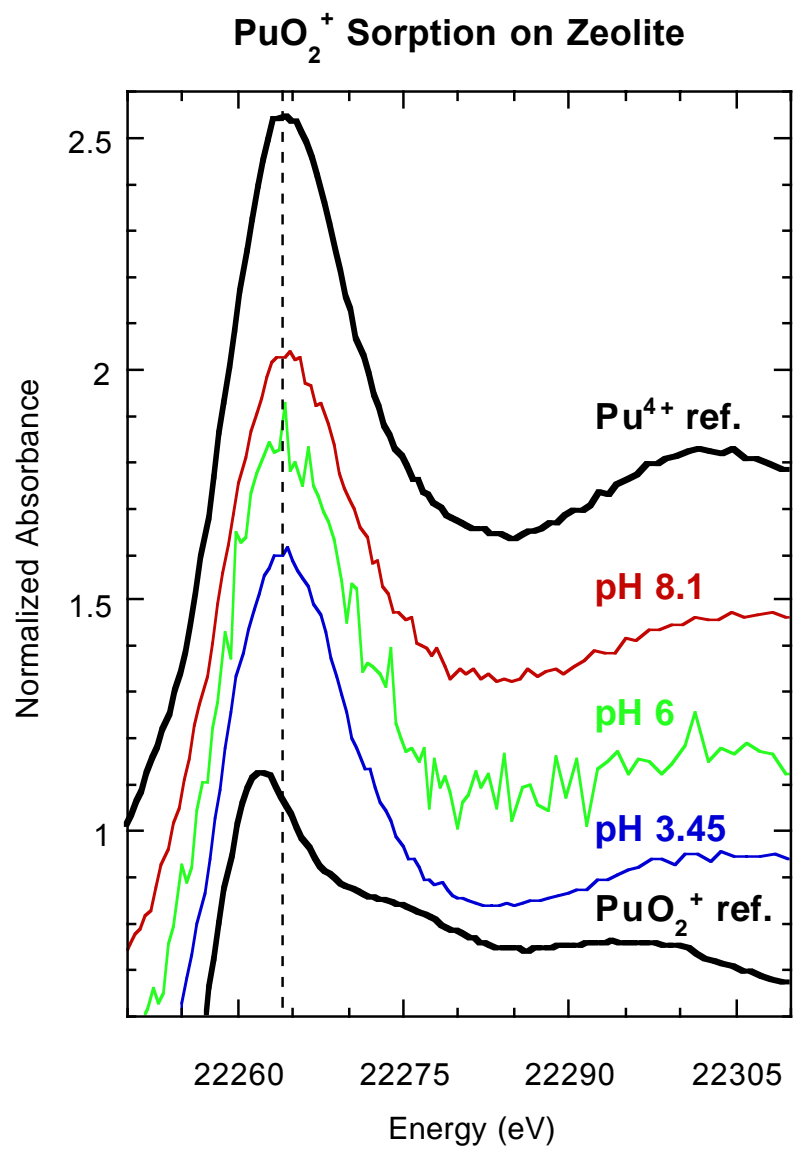

Figure 5.3. The normalized absorbence vs. energy of the XANES region is shown. The $\mathrm{Pu}$ sorbed on the clinoptilolite is in the +4 oxidation state at all $\mathrm{pHs}$ investigated.

The XANES results for $\mathrm{Pu}(\mathrm{V})$ sorption to various minerals at $\mathrm{pH} 8$ are shown in Figure 5.4. The $\mathrm{PuO}_{2}(\mathrm{Pu}-\mathrm{IV})$ and $\mathrm{PuO}_{2}^{+}(\mathrm{Pu}-\mathrm{V})$ reference standards are shown for comparison. As seen with the clinoptilolite as a function of $\mathrm{pH}$, all of the soluble $\mathrm{Pu}(\mathrm{V})$ appears to have been reduced to $\mathrm{Pu}(\mathrm{IV})$ on all seven minerals. The absorption peaks of the sorbed samples now have the position of the $\mathrm{Pu}(\mathrm{IV})$ reference and the spectra display a $\sim 3 \mathrm{eV}$ shift in the main peak energy to the right of the $\mathrm{Pu}(\mathrm{V})$ reference peak (see vertical black line). Also, the samples have lost the high energy shoulder that identifies the plutonyl, $\mathrm{Pu}(\mathrm{V})$ structure. The shoulder is caused by multiple-scattering along the $\mathrm{O}=\mathrm{Pu}=\mathrm{O}$ axis and it's loss corresponds to a loss of the 'plutonyl' structure. These results show that the reduction of soluble $\mathrm{Pu}(\mathrm{V})$ to $\mathrm{Pu}(\mathrm{IV})$ occurs on all mineral surfaces investigated. 


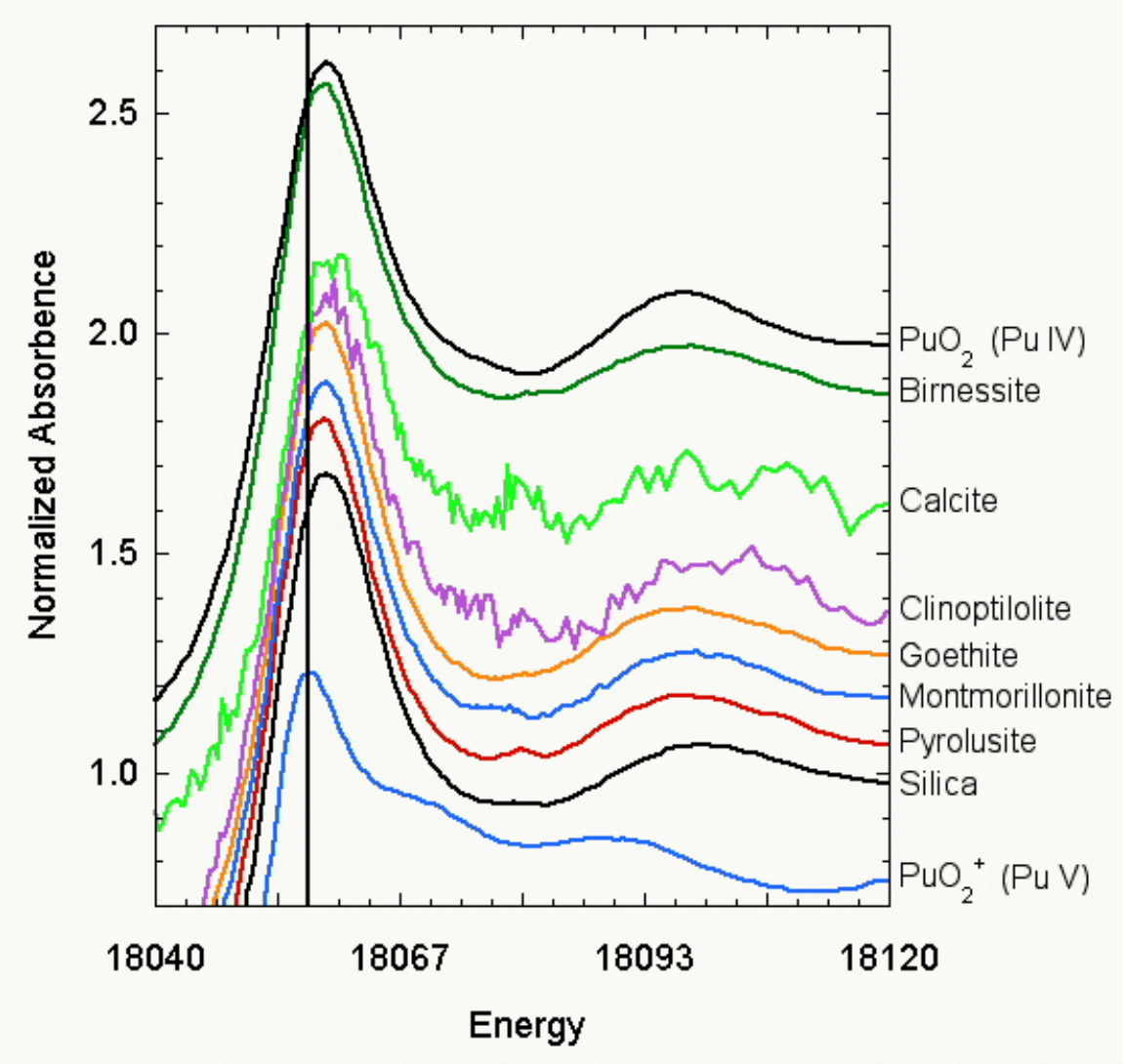

Figure 5.4. The normalized abundance vs. energy of the XANES region is shown. The $\mathrm{Pu}$ sorbed on colloid minerals at $\mathrm{pH} 8.4$ is compared to reference standards, $\mathrm{Pu}^{4+}$ and $\mathrm{PuO}_{2}^{+}$spectra.

Extended X-ray absorption fine structure (EXAFS) results were modeled and confirm an inner-sphere complexation mechanism, with some oligomerization occurring in the silica containing minerals. Figure 5.5 shows the raw, $\mathrm{k}^{3}$-weighted extended (EXAFS; left) spectrum and its fourier transform (right) for all $\mathrm{Pu}$ sorbed samples, including the two reference spectra at the bottom. The fourier transform spectra shows a pseudo-radial distribution of peaks corresponding to individual shells of atoms around the Pu center, at shorter distance than the true bond distance due to the EXAFS phase shift. 


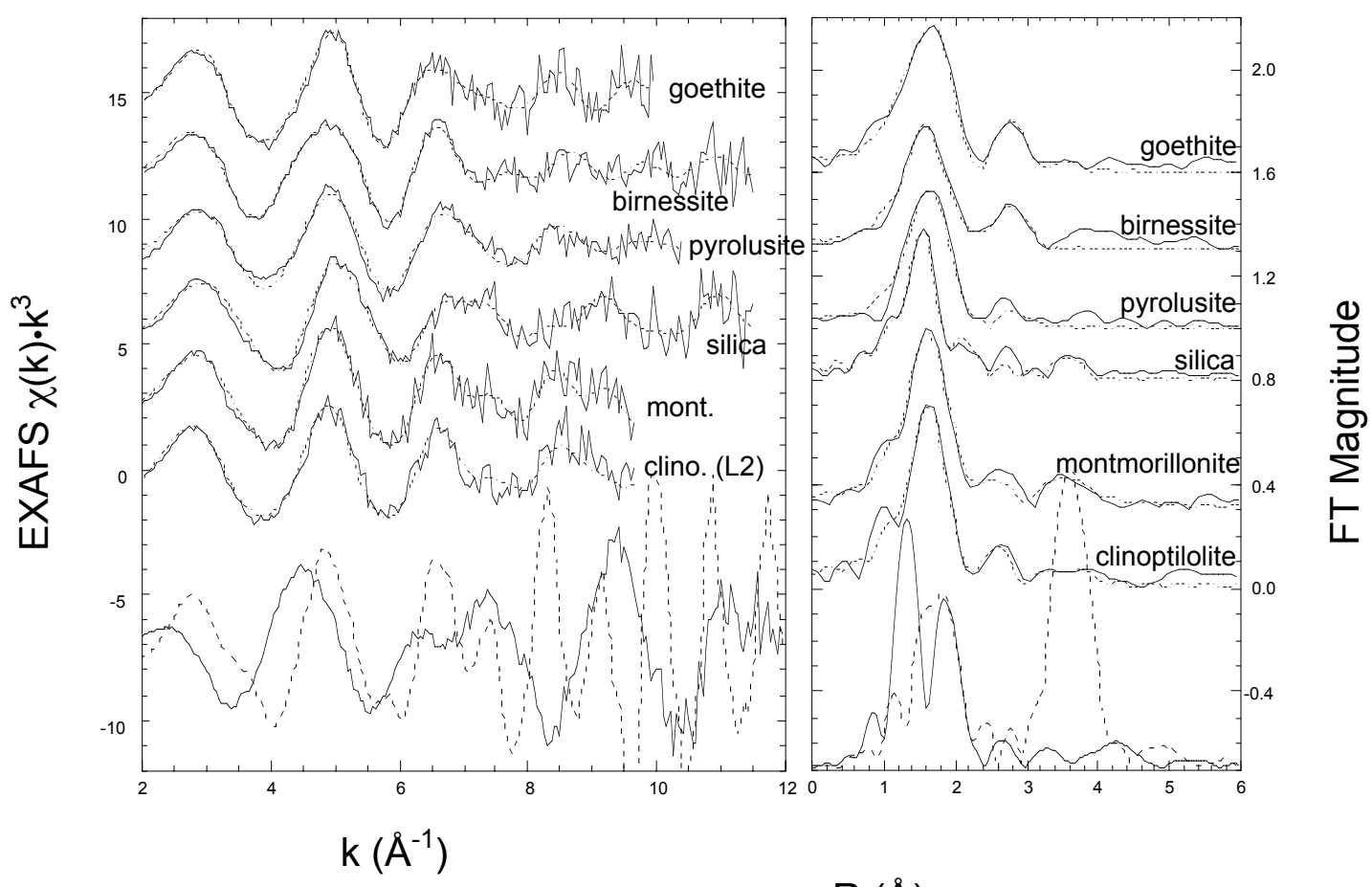

$\mathrm{R}(\AA)$

Figure 5.5. Raw, $\mathrm{k}^{3}$-weighted EXAFS (left) and fourier transformed EXAFS (right) spectra for Pu sorbed to goethite, birnessite, pyrolusite, silica, clinoptilolite, and montmorillonite. Samples are shown with a solid line for the data and a dashed line for the least-squares fit to the data. Shown at the bottom of the plots are spectra for $\mathrm{PuO}_{2}^{+}$(aq) (solid line) and $\mathrm{PuO}_{2}$ (dashed line).

In the raw EXAFS the spectral pattern of the samples at $\mathrm{k} \sim 2.75 \AA^{-1}, 5 \AA^{-1}$, and $6.5 \AA^{-1}$ corresponds directly to the scattering pattern in $\mathrm{PuO}_{2}$ and differs significantly from the EXAFS of $\mathrm{PuO}_{2}{ }^{+}$(aq) in the same region. This provides further evidence of reduction to the $\mathrm{Pu}^{4+}$ form. In the fourier transform spectra we observe a first peak at $1.6 \AA$, the same position as the first peak in $\mathrm{PuO}_{2}$ and $0.3 \AA$ longer than the peak in corresponding to the axial $\mathrm{Pu}-\mathrm{O}$ bond in $\mathrm{PuO}_{2}^{+}(\mathrm{aq})$. The primary contribution to the EXAFS scattering and the first peak in the fourier transform spectra comes from the first oxygen shell associated with $\mathrm{Pu}(\mathrm{IV})-\mathrm{O}$ bonds in the sample. We also observe additional peaks between 2.4-3 $\AA$ in the fourier transform spectra, which do not correspond to any significant interactions in $\mathrm{PuO}_{2}$, and which can therefore be attributed to direct $\mathrm{Pu}$-surface interactions. In montmorillonite and silica we observe a peak at $\sim 3.6 \AA$, approximately the same position as the large $\mathrm{Pu}-\mathrm{Pu}$ interaction in $\mathrm{PuO}_{2}$, and which may therefore indicate the formation of a polymer or surface precipitate in these samples.

These qualitative results are confirmed by the non-linear least-squares fits, which are given in Table 5.1. The first fourier transform peak is fit with two separate $\mathrm{Pu}-\mathrm{O}$ shells at ca. $2.2 \AA$ and $2.5 \AA$ (phase corrected) distance, and having a total coordination of 6-10. 
Both coordination $(\mathrm{N})$ and bond length $(\mathrm{R})$ are consistent with $\mathrm{Pu}(\mathrm{IV})-\mathrm{O}$ characteristic structures, and the split into two shells is evidence for heterogeneous Pu-surface and $\mathrm{Pu}-$ hydration interactions. The second fourier transform peak is well modeled in all samples by a $\mathrm{Pu}-\mathrm{M}$ interaction where $\mathrm{M}=\mathrm{Fe}, \mathrm{Mn}$, or $\mathrm{Si}$, and is direct evidence for inner-sphere $\mathrm{Pu}$-colloid bonding. We also observe Pu-carbonate interactions in the goethite sample, and $\mathrm{Pu}-\mathrm{Pu}$ interactions in the three silica-containing samples. The latter indicates the formation of a surface precipitate or oligomer.

Table 5.1. Results of non-linear least-squares fitting for Pu-bearing samples.

\begin{tabular}{|c|c|c|c|c|c|c|c|c|c|}
\hline Sample & Shell & $\mathrm{N}$ & $\mathrm{R}(\AA)^{\mathrm{a}}$ & $\sigma^{2}\left(\AA^{2}\right)^{b}$ & Sample & Shell & $\mathrm{N}^{\mathrm{a}}$ & $\mathrm{R}(\AA)^{\mathrm{a}}$ & $\sigma^{2}\left(\AA^{2}\right)$ \\
\hline \multirow[t]{8}{*}{ Goethite } & $\mathrm{Pu}-\mathrm{O}$ & 2.8 & 2.19 & 0.00769 & \multirow{5}{*}{$\begin{array}{l}\text { Clinoptilolite } \\
\text { (L } \mathrm{L}_{\text {II }} \text { edge) }\end{array}$} & $\mathrm{Pu}-\mathrm{O}$ & 7.4 & 2.31 & 0.01133 \\
\hline & $\mathrm{Pu}-\mathrm{O}$ & 5.0 & 2.35 & 0.00769 & & $\mathrm{Pu}-\mathrm{O}$ & 3.0 & 2.52 & 0.01133 \\
\hline & $\mathrm{Pu}-\mathrm{C}$ & 1.7 & 2.97 & 0.01036 & & $\mathrm{Pu}-\mathrm{Si}$ & 2.7 & 3.14 & 0.01542 \\
\hline & $\mathrm{Pu}-\mathrm{O}-\mathrm{C}$ & 6.7 & 3.88 & 0.02078 & & $\mathrm{Pu}-\mathrm{Pu}$ & 1.9 & 3.78 & 0.01219 \\
\hline & $\mathrm{Pu}-\mathrm{C}-\mathrm{O}(3)$ & 3.3 & 4.28 & 0.02078 & & & & & \\
\hline & $\mathrm{Pu}-\mathrm{C}-\mathrm{O}(4)$ & 1.7 & 4.28 & 0.02078 & \multirow[t]{3}{*}{ Montmorillonite } & $\mathrm{Pu}-\mathrm{O}$ & 8.1 & 2.29 & 0.01152 \\
\hline & $\mathrm{Pu}-\mathrm{Fe}$ & 0.9 & 3.43 & 0.00682 & & $\mathrm{Pu}-\mathrm{Si}$ & 1.9 & 3.14 & 0.00444 \\
\hline & & & & & & $\mathrm{Pu}-\mathrm{Pu}$ & 1 & 3.78 & 0.00104 \\
\hline \multirow[t]{4}{*}{ Birnessite } & $\mathrm{Pu}-\mathrm{O}$ & 3.5 & 2.23 & 0.00646 & & & & & \\
\hline & $\mathrm{Pu}-\mathrm{O}$ & 3.9 & 2.39 & 0.00646 & \multirow[t]{6}{*}{ Silica gel } & $\mathrm{Pu}-\mathrm{O}$ & 4.3 & 2.20 & 0.00586 \\
\hline & $\mathrm{Pu}-\mathrm{Mn}$ & 2.1 & 3.33 & 0.01208 & & $\mathrm{Pu}-\mathrm{O}$ & 3.3 & 2.38 & 0.00586 \\
\hline & & & & & & $\mathrm{Pu}-\mathrm{Si}$ & 3.1 & 3.11 & 0.02764 \\
\hline \multirow[t]{3}{*}{ Pyrolusite } & $\mathrm{Pu}-\mathrm{O}$ & 2.5 & 2.22 & 0.00373 & & $\mathrm{Pu}-\mathrm{Pu}$ & 1.4 & 3.81 & 0.01009 \\
\hline & $\mathrm{Pu}-\mathrm{O}$ & 3.2 & 2.37 & 0.00373 & & & & & \\
\hline & $\mathrm{Pu}-\mathrm{Mn}$ & 1 & 3.42 & 0.01663 & & & & & \\
\hline
\end{tabular}

We modeled Pu-surface interaction geometries based on a simple representation of $\mathrm{Fe}$ and $\mathrm{Mn}$ as a $\mathrm{M}(\mathrm{O}, \mathrm{OH})_{6}$ octahedron and $\mathrm{Si}$ as a $\mathrm{Si}(\mathrm{O}, \mathrm{OH})_{4}$ tetrahedron. Depending on whether the $\mathrm{Pu}$ hydration sphere shares one (point-sharing), two (edge-sharing), or three (face-sharing) oxygen with the polyhedral structure of the mineral, different $\mathrm{Pu}-\mathrm{M}$ distances are predicted. Table 5.2 gives the model-specific predictions for each structure along with observed bond distances.

Table 5.2. Comparison of observed and model-specific predicted $\mathrm{Pu}-\mathrm{M}$ interactions

\begin{tabular}{|c|c|c|c|c|}
\hline \multirow[t]{2}{*}{ Sample } & \multirow[t]{2}{*}{$\mathrm{Pu}-\mathrm{M}(\AA)$} & \multicolumn{3}{|c|}{$\mathrm{R}_{\text {Pred. }}(\AA)$} \\
\hline & & Face-sharing & Edge-Sharing & Point-Sharing \\
\hline Goethite $(\mathrm{Fe})$ & 3.43 & 3.05 & 3.65 & 4.35 \\
\hline Birnessite (Mn) & 3.33 & 2.77 & 3.56 & 4.22 \\
\hline Pyrolusite (Mn) & 3.42 & 2.77 & 3.56 & 4.22 \\
\hline Clinoptilolite (Si) & 3.14 & & 3.14 & 3.83 \\
\hline Montmorillonite (Si) & 3.14 & & 3.14 & 3.83 \\
\hline Silica (Si) & 3.11 & & 3.14 & 3.83 \\
\hline
\end{tabular}

Based on these calculations, the interactions we observe in all our samples are most consistent with edge-sharing between the plutonium hydration sphere and Fe- or Mnoctahedra, or Si tetrahedra. In the case of the silicate minerals, the calculated and observed $\mathrm{Pu}-\mathrm{Si}$ distances are the same within the error margins given for EXAFS 
distance ( $\mathrm{R}, \AA$ ) determinations. The exact correlation between expected and observed $\mathrm{Pu}$ Si distances in montmorillonite and clinoptilolite provides evidence for silica tetrahedra bonding rather than alumina octahedra bonding, even though aluminum and silicon are themselves indistinguishable via the EXAFS technique.

The combined XANES and EXAFS spectra from XAS shows that the aqueous $\mathrm{Pu}(\mathrm{V})$ sorbed as $\mathrm{Pu}(\mathrm{IV})$ on the surfaces of clinoptilolite, birnessite, calcite, goethite, montmorillonite, pyrolusite and silica. The reduction of $\mathrm{Pu}(\mathrm{V})$ to $\mathrm{Pu}(\mathrm{IV})$ on clinoptilolite was also observed between pHs 3 to 8 .

\subsection{Factors Influencing the Speciation and Sorption Behavior of $\mathrm{Pu}(\mathrm{V})$}

Our XAS results showed that at the end of the sorption experiment (3 weeks), all the soluble $\mathrm{Pu}(\mathrm{V})$ is reduced to $\mathrm{Pu}(\mathrm{IV})$ on all the mineral colloids investigated. In addition, our $\mathrm{Pu}(\mathrm{V})$ sorption experiments showed that the rate of sorption on the various minerals investigated varied by over four orders of magnitude. These two findings are in contrast to those found in our $\mathrm{Pu}(\mathrm{IV})$ sorption experiments where sorption of $\mathrm{Pu}(\mathrm{IV})$ was consistently fast and high ( $>90 \%$ within a day) and no change in oxidation state was observed on the minerals. We undertook an additional set of experiments to try and explain both the redox change and sorption behavior of $\mathrm{Pu}(\mathrm{V})$.

There are two possible pathways (mechanisms) for the soluble $\mathrm{Pu}(\mathrm{V})$ to reduce to $\mathrm{Pu}(\mathrm{IV})$ on mineral surfaces. Figure 5.6 shows a cartoon of the different reaction pathways by which the $\mathrm{Pu}$ can sorb to the mineral surface. Pathway 1 describes $\mathrm{Pu}(\mathrm{V})$ sorbing directly to the colloid surface as $\mathrm{Pu}(\mathrm{V})$ and then reducing to $\mathrm{Pu}(\mathrm{IV})$ on the surface (blue route). Pathway 2 involves the initial disproportionation of $\mathrm{Pu}(\mathrm{V})$ to $\mathrm{Pu}(\mathrm{IV})$ in the aqueous phase and $\mathrm{Pu}(\mathrm{IV})$ subsequent sorption to the mineral surface (red route).

\section{Disproportionation}

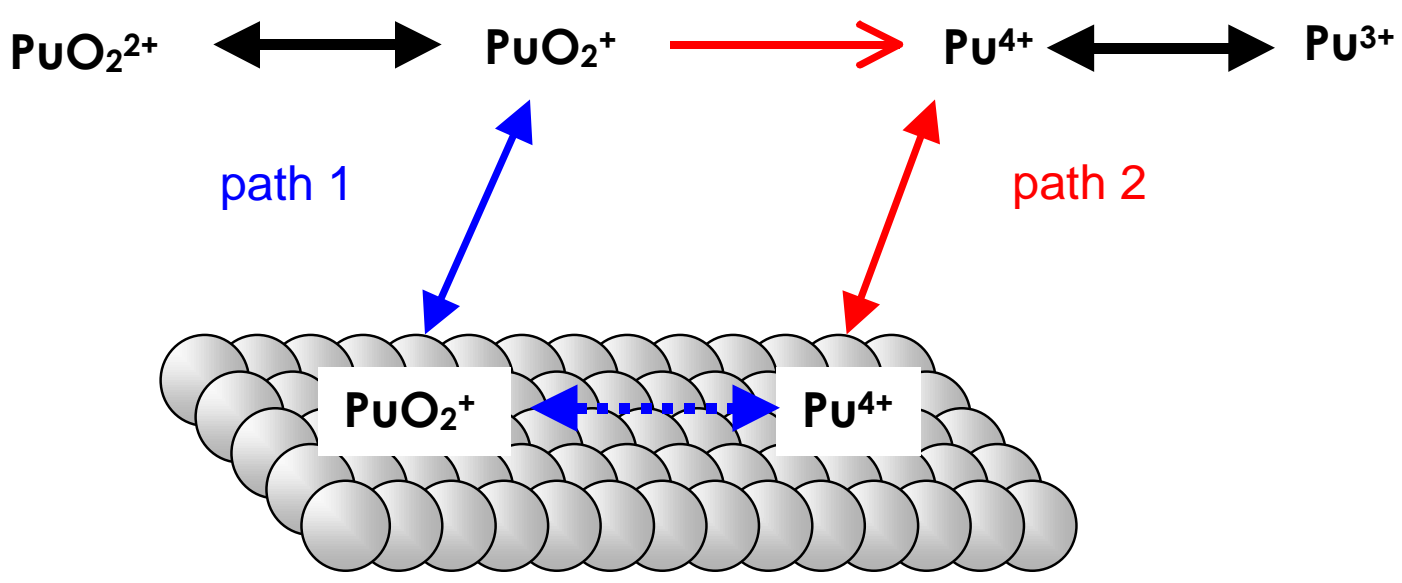

Figure 5.6. Cartoon showing the possible reaction pathways for sorption of $\mathrm{Pu}$ on mineral surfaces. Path 1 (blue): $\mathrm{Pu}(\mathrm{V})$ is sorbed directly on the mineral, then is reduced to $\mathrm{Pu}(\mathrm{IV})$ on the surface. Path 2 (red): $\mathrm{Pu}(\mathrm{V})$ is reduced to $\mathrm{Pu}(\mathrm{IV})$ through its disproportionation in the solution, then $\mathrm{Pu}(\mathrm{IV})$ aqueous species is sorbed on the mineral 
surface.

Disproportionation is a reaction whereby a species reacts with itself and causes autooxidation and auto-reduction. For example, $\mathrm{Pu}(\mathrm{V})$ disproportionates to $\mathrm{Pu}(\mathrm{IV})$ and $\mathrm{Pu}(\mathrm{VI})$, thereby increasing the concentration of $\mathrm{Pu}(\mathrm{IV})$ and $\mathrm{Pu}(\mathrm{VI})$ and decreasing that of $\mathrm{Pu}(\mathrm{V})$ present in solution. The disproportionation of $\mathrm{Pu}(\mathrm{V})$ is represented by the reaction below (Silver, 2000):

$2 \mathrm{PuO}_{2}^{+}+4 \mathrm{H}^{+}=\mathrm{Pu}^{4+}+\mathrm{PuO}_{2}^{2+}+2 \mathrm{H}_{2} \mathrm{O}$

Disproportionation reactions are unique in that they do not rely on the oxidation/reduction of water or the oxidation/reduction of any other element in solution. Instead, the rate of disproportionation is a strong function of concentration and $\mathrm{pH}$, with higher concentrations having an increased number of interactions resulting in a faster rate of disproportionation than lower Pu concentrations (Silver, 1983).

In the case of soluble $\mathrm{Pu}(\mathrm{V})$, the rate of path 2 (Figure 5.6) would be controlled by both the rate of disproportionation of $\mathrm{Pu}(\mathrm{V})$, and the subsequent rate of $\mathrm{Pu}(\mathrm{IV})$ sorption to the specific mineral surface. However, based on our experimental data, we have demonstrated that $\mathrm{Pu}(\mathrm{IV})$ sorbs rapidly onto mineral surfaces. Thus, $\mathrm{Pu}(\mathrm{V})$ disproportionation in solution is likely to be rate limiting. Another interesting aspect of path 2 is that, since $\mathrm{Pu}(\mathrm{IV})$ sorbs very strongly to mineral surfaces, the sorption of $\mathrm{Pu}(\mathrm{IV})$ provides a constant sink for $\mathrm{Pu}(\mathrm{V})$ which is controlled by disproportionation. In contrast, in path 1 , the initial sorption rate would be controlled by the rate of $\mathrm{Pu}(\mathrm{V})$ sorption to the mineral surface. Depending on the relative sorption affinity of $\mathrm{Pu}(\mathrm{V})$ versus $\mathrm{Pu}(\mathrm{IV})$ on a particular mineral surface, the long-term rate may or may not be controlled by the reduction of $\mathrm{Pu}(\mathrm{V})$ to $\mathrm{Pu}(\mathrm{IV})$ on the mineral surface.

In order to test if the sorption rate of $\mathrm{Pu}(\mathrm{V})$ on the different minerals investigated is controlled by path 1 or 2 (Figure 5.6), two different sets of experiments were designed. First a series of $\mathrm{Pu}(\mathrm{V})$ disproportionation experiments were carried out in the absence of colloids (first part of path 2). Second, a series of fast sorption/desorption experiments were carried out on three different mineral colloids in an attempt to elucidate the initial oxidation state of the sorbed $\mathrm{Pu}$ (first part of path 1).

\section{Experimental Techniques}

Three aqueous $\mathrm{Pu}(\mathrm{V})$ stability experiments were carried out to measure the rate of $\mathrm{Pu}(\mathrm{V})$ disproportionation in $\mathrm{J}-13$ surrogate water. The concentrations of $\mathrm{Pu}$ and dominant isotope (by alpha activity) in the different stock solutions used in the three experiments were, 3.0x $10^{-6} \mathrm{~mol} / \mathrm{L}{ }^{238} \mathrm{Pu} ; 3.9 \times 10^{-9} \mathrm{~mol} / \mathrm{L}{ }^{238} \mathrm{Pu}$; and $4.2 \times 10^{-9} \mathrm{~mol} / \mathrm{L}{ }^{242} \mathrm{Pu}$. Disproportionation experiments with two different $\mathrm{Pu}(\mathrm{V})$ concentrations were used to evaluate possible effects of concentration on the rate of disproportionation. In addition, two different $\mathrm{Pu}$ isotope compositions were used to evaluate possible radiolysis effects. All experiments were performed in $\mathrm{pH} \sim 8 \mathrm{~J}-13$ surrogate water. 
A second set of experiments were undertaken in an effort to try and determine the oxidation state of $\mathrm{Pu}$ that initially sorbs to the colloid surface. We know from our XAS results that the final state of $\mathrm{Pu}$ on the mineral surface is $\mathrm{Pu}(\mathrm{IV})$. XAS experiments involving Pu at LLNL have the limitation of taking one to two weeks to analyze after the end of the sorption experiment, due to sample preparation, transport to SLAC and analysis. Therefore, XAS analysis cannot determine the oxidation state of the Pu under initial sorption conditions. Short-term $\mathrm{Pu}(\mathrm{V})$ sorption experiments, followed by desorption and evaluation of oxidation state were carried out. The minerals investigated were, clinoptilolite, goethite and birnessite. Desorption was accomplished by using standard solvent extraction techniques and oxidation states were determined by standard UV/VIS spectrometry.

\section{Results and Discussion of the Pu(V) Disproportionation Experiments}

Results from the disproportionation experiments are shown on Figure 5.7. While $\mathrm{Pu}(\mathrm{V})$ disproportionation produced some $\mathrm{Pu}(\mathrm{III})$, (IV), and (VI), $\mathrm{Pu}(\mathrm{IV})$ was the dominant (or at least a major) product of $\mathrm{Pu}(\mathrm{V})$ disproportionation. $\mathrm{The} \mathrm{Pu}(\mathrm{V})$ disproportionation rates in all three experiments are very similar and appear independent of the isotopic composition of the $\mathrm{Pu}(\mathrm{V})$ solution, or the range in $\mathrm{Pu}$ concentration investigated. Although the third disproportionation experiment $\left(4.2 \times 10^{-9} \mathrm{M}^{242} \mathrm{Pu}\right)$ showed the same general rate of disproportionation as the other two, the data measuring the oxidation state had large errors and the percentages plotted for $\mathrm{Pu}(\mathrm{IV}), \mathrm{Pu}(\mathrm{VI})$ and $\mathrm{Pu}(\mathrm{III})$ and colloidal $\mathrm{Pu}$ are not reliable. Nevertheless, even at the highest concentrations $\left(\mathrm{Pu}=10^{-6} \mathrm{~mol} / \mathrm{L}\right)$ the reaction rate is fairly slow (Figure 5.7b). It is not until after approximately 10 days that there is an appreciable amount of $\mathrm{Pu}(\mathrm{IV})(10 \%)$ in solution.

Based on his experiments using sea water, Choppin (1991) suggested that a empirical kinetic equation could be used to describe the decrease in $\mathrm{Pu}(\mathrm{V})$ in solution (in the absence of light) as a function of time:

$\mathrm{R}=1.06 \times 10^{-4} \mathrm{~mol} / \mathrm{L} / \mathrm{hr} . \times[\mathrm{Pu}(\mathrm{V})]^{0.86}$,

which is close to a first order reaction with respect to $\mathrm{Pu}(\mathrm{V}) .{ }^{1}$ Based on first order reaction kinetics:

$$
\frac{d P u(V)}{d t}=k \times P u(V)
$$

(that can be integrated to $\ln (P u(V))=-k t+\ln \left(P u(V)_{0}\right)$ where $\mathrm{Pu}(\mathrm{V})_{0}$ is the initial $\mathrm{Pu}(\mathrm{V})$ concentration in solution), fits to two data sets of Choppin (1991), resulted in reaction constants of $4.3 \times 10^{-4}$ and $1.0 \times 10^{-3} \mathrm{~mol} / \mathrm{L} / \mathrm{hr}$. We fit our three $\mathrm{Pu}(\mathrm{V})$ disproportionation data sets using first order kinetics, which yielded reaction constants of $5.36 \times 10^{-4}$, $6.14 \times 10^{-4}$, and $3.66 \times 10^{-4} \mathrm{~mol} / \mathrm{L} / \mathrm{hr}$. for the $3.0 \times 10^{-6} \mathrm{~mol} / \mathrm{L}{ }^{242} \mathrm{Pu}(\mathrm{V}), 3.9 \times 10^{-9} \mathrm{~mol} / \mathrm{L}$ ${ }^{238} \mathrm{Pu}(\mathrm{V})$, and $4.2 \times 10^{-9} \mathrm{~mol} / \mathrm{L}^{242} \mathrm{Pu}(\mathrm{V}) /$ disproportionation experiments, respectively (fits

${ }^{1}$ Choppin (1991) noted that the reaction rate was significantly faster in the presence of light, possibly due to the interaction of humics in solution with sunlight. 
shown in Figure 5.8). These results are in good agreement with fits to the data of Choppin (1991). The results also suggest that the disproportionation is not isotopedependent. The resulting reaction constants are equivalent to a $\mathrm{Pu}(\mathrm{V})$ disproportionation half-life of 0.16 years. However, we have not attempted to develop any back-reactions (such as $\mathrm{Pu}(\mathrm{IV})$ disproportionation back to $\mathrm{Pu}(\mathrm{V})$ ); sufficient data were not available to predict other disproportionation reactions or their rates. 

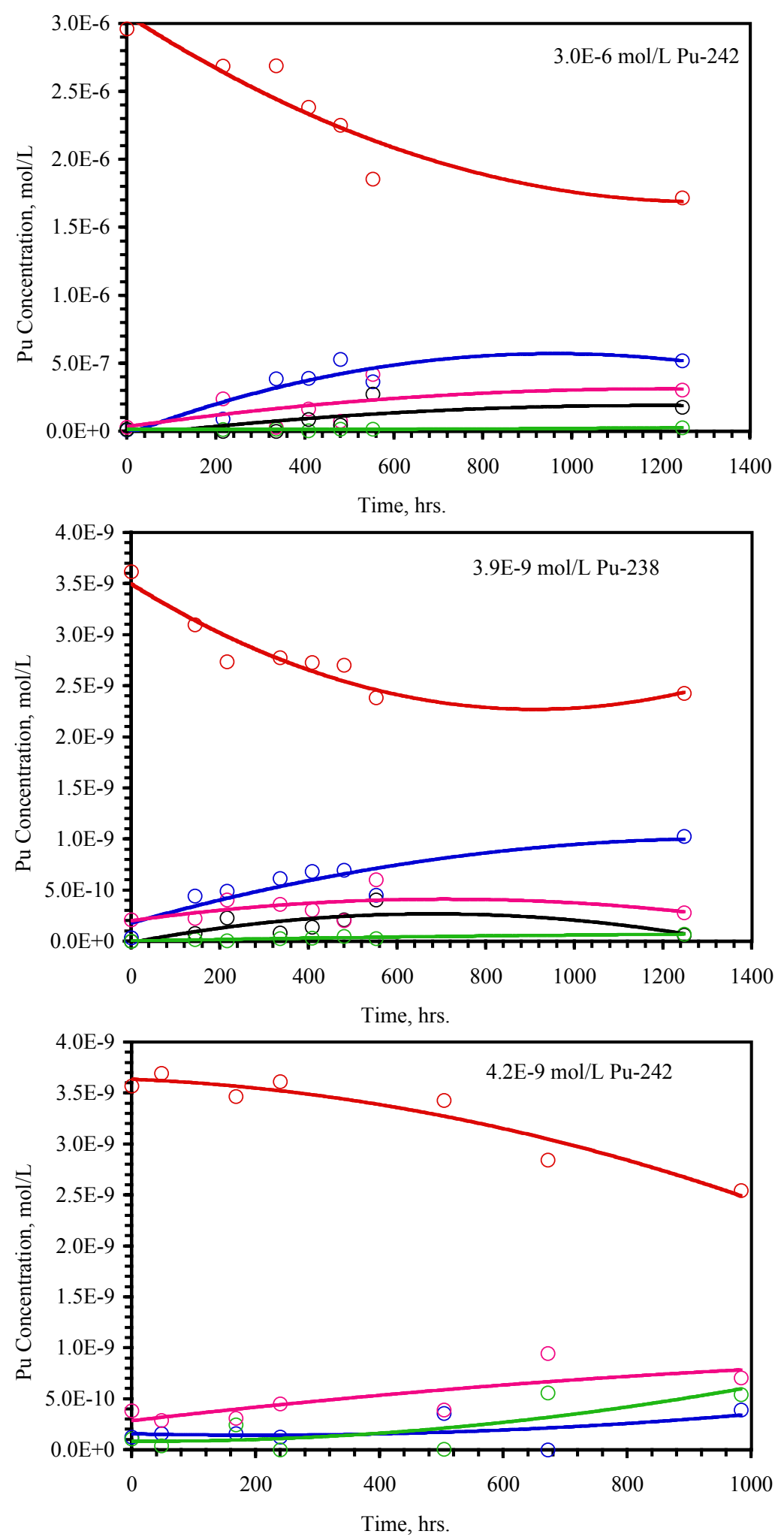

Figure 5.7. Results from three $\mathrm{Pu}(\mathrm{V})$ disproportionation experiments in $\mathrm{J}-13$ surrogate water in the absence of colloids. Data are plotted as a function of Pu concentration vs. time. $\mathrm{Pu}(\mathrm{V})=$ red, $\mathrm{Pu}(\mathrm{IV})=$ blue, $\mathrm{Pu}(\mathrm{VI})=$ pink, $\mathrm{Pu}(\mathrm{III})=$ black, and $\mathrm{Pu}$ colloid=green. 


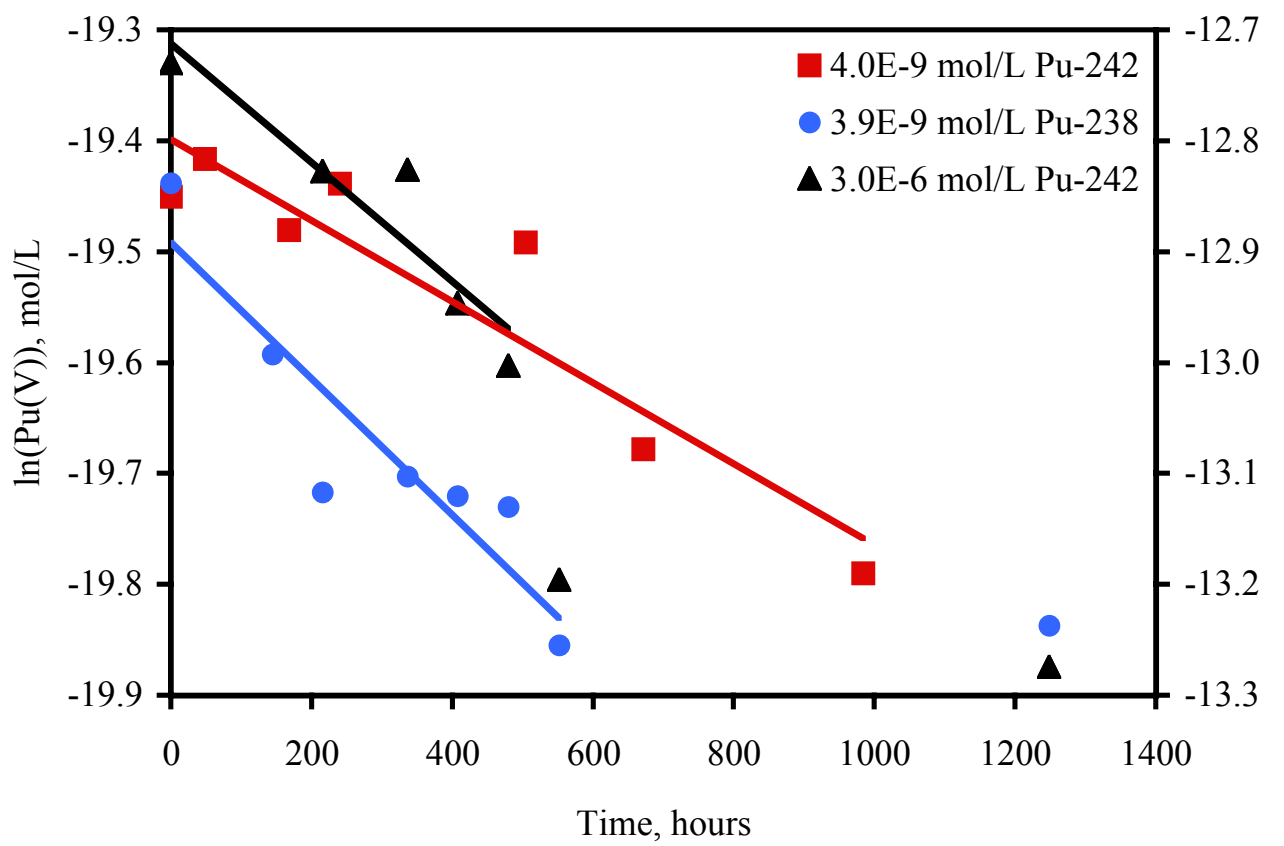

Figure 5.8. First order disproportionation reactions fit to data of Choppin (1991).

\section{Results and Discussion of the Pu(V)Fast Sorption/Desorption Experiments}

One of our limitations of using the XAS technique at LLNL and then doing the analysis at SLAC is that is that we are unable to stop our $\mathrm{Pu}(\mathrm{V})$ sorption experiment and immediately analyze our sample. Therefore, our XAS results provide information on the final oxidation state on the colloid surface. Fast sorption experiments followed by desorption and oxidation state analysis were carried out on clinoptilolite, goethite and birnessite.

Table 5.3 summarizes the results of our sorption/desorption experiments. In less than 1 hour, $88 \%$ and $85 \%$ of the $\mathrm{Pu}(\mathrm{V})$ sorbed to the goethite and birnessite, respectively. The sorption to clinoptilolite was much slower. After 35 days, only $16 \%$ of the Pu had sorbed to the colloid surface. The initial oxidation state of the goethite was $\mathrm{Pu}(\mathrm{V})$ and on the clinoptilolite was $\mathrm{Pu}(\mathrm{IV})$. The initial oxidation state on the birnessite surface was surprisingly, $\mathrm{Pu}(\mathrm{VI})$.

Table 5.3. Determination of oxidation state and percent sorption for $\mathrm{Pu}(\mathrm{V})$

\begin{tabular}{|l|l|l|l|}
\hline Minerals & Sorption time & $\%$ Pu Sorption & $\begin{array}{c}\text { Major Pu species on } \\
\text { colloids }\end{array}$ \\
\hline Geothite & 15 min. & $88 \%$ & $87 \% \pm 5 \quad \mathrm{Pu}(\mathrm{V})$ \\
\hline Clinoptilolite & 35 days & $16 \%$ & $79 \% \pm 10 \quad \mathrm{Pu}(\mathrm{IV})$ \\
\hline Birnessite & 30 min. & $85 \%$ & $98 \% \pm 2 \quad \mathrm{Pu}(\mathrm{VI})^{*}$ \\
\hline
\end{tabular}


Clearly, the geothite $\mathrm{Pu}(\mathrm{V})$ sorption rates are too fast to result from disproportionation, followed by aqueous $\mathrm{Pu}(\mathrm{IV})$ sorption (path 2 in Figure 5.6). Sorption occurs within minutes and disproportionation is on the order of days to weeks. In addition, the dominant initial species of $\mathrm{Pu}$ is $\mathrm{Pu}(\mathrm{V})$. The data are best explained by $\mathrm{Pu}(\mathrm{V})$ sorbing to geothite as $\mathrm{Pu}(\mathrm{V})$ and then reducing to $\mathrm{Pu}(\mathrm{IV})$ on the mineral surface (path 1).

The sorption rate of $\mathrm{Pu}(\mathrm{V})$ on birnessite is also too fast to result from disproportionation, followed by aqueous $\mathrm{Pu}(\mathrm{IV})$ sorption to the mineral surface (path 2). In this experiment, the dominant initial oxidation state measured was $\mathrm{Pu}(\mathrm{VI})$. We think this is due to an oxidation reaction occurring between birnessite and strong acid $(\mathrm{pH} 1)$ used to desorb the $\mathrm{Pu}$ from the surface for analysis. Therefore, our method of using solvent extraction to desorb $\mathrm{Pu}$ from the mineral surface probably resulted in altering the initial oxidation state of $\mathrm{Pu}$ sorbed on the surface. XAS analysis was later used to confirm the presence of $\mathrm{Pu}(\mathrm{VI})$ on the surface of birnessite when in the presence of a strong acid, further suggesting that the acid caused the $\mathrm{Pu}$ to oxidize. We suggest, that as with Fe-oxides, the sorption mechanism that controls Mn-oxides is the intitial sorption of $\mathrm{Pu}(\mathrm{V})$ to the mineral surface, followed by $\mathrm{Pu}(\mathrm{V})$ reduction to $\mathrm{Pu}(\mathrm{IV})$ on the mineral surface (path 1 ).

In contrast to the $\mathrm{Fe}$ - and $\mathrm{Mn}$-oxides, the $\mathrm{Pu}(\mathrm{V})$ sorption rate on clinoptilolite is much slower. $\mathrm{Pu}(\mathrm{V})$ sorption as a function of time and $\mathrm{pH}$ is plotted in Figure 5.9. The little to no sorption of $\mathrm{Pu}(\mathrm{V})$ at early time suggests that $\mathrm{Pu}(\mathrm{V})$ sorbs to clinoptilolite colloid very weakly if at all. This is particularly true when compared to the sorption behavior of $\mathrm{Pu}(\mathrm{IV})$. After 80 days, the sorption profile as a function of $\mathrm{pH}$ begin to appear similar to that of $\mathrm{Pu}(\mathrm{IV})$ sorption experiments (Figure 5.4b), with a sorption minimum at $\mathrm{pH} 7$ to 9 .

The sorption rate of $\mathrm{Pu}$ onto clinoptilolite is slow enough that the sorption mechanism could be ascribed to either path 1 or path 2 . Yet, the determination that $\mathrm{Pu}(\mathrm{IV})$ is the dominant initial surface species on the clinoptilolite (Table 5.3) suggests that pathway 1, where the initial $\mathrm{Pu}(\mathrm{V})$ sorbs to the mineral surface as $\mathrm{Pu}(\mathrm{V})$ and then reduces to $\mathrm{Pu}(\mathrm{IV})$ is not likely. Experimental data of the initial oxidation state of $\mathrm{Pu}$ on the clinoptilolite surface $(\mathrm{Pu}(\mathrm{IV}))$ in conjunction with a moderate disproportionation rate is most consistent with the sorption rate of $\mathrm{Pu}(\mathrm{V})$ on clinoptilolite being controlled by first the disproportionation rate of $\mathrm{Pu}(\mathrm{V})$, then followed by the rapid sorption of aqueous $\mathrm{Pu}(\mathrm{IV})$ to the colloid surface (path 2).

The behavior of $\mathrm{Pu}(\mathrm{V})$ sorption onto clinoptilolite can be qualitatively modeled using the $\mathrm{Pu}(\mathrm{V})$ disproportionation rate constant and $\mathrm{Pu}(\mathrm{IV})$ surface complexation modeling data.

In Figure 5.9b, we predict the behavior of $\mathrm{Pu}(\mathrm{V})$ sorption experiments based on an average $\mathrm{Pu}(\mathrm{V})$ disproportionation constant $\left(\mathrm{k}=5.05 \times 10^{-4} \mathrm{~mol} / \mathrm{L} / \mathrm{hr}\right)$, assuming disproportionation is $\mathrm{pH}$ independent, assuming that $\mathrm{Pu}(\mathrm{IV})$ is the dominant product of $\mathrm{Pu}(\mathrm{V})$ disproportionation, and applying the four species non-electrostatic surface complexation reaction constants fitted to the $\mathrm{Pu}(\mathrm{IV})$ sorption data (Table 5.3). 

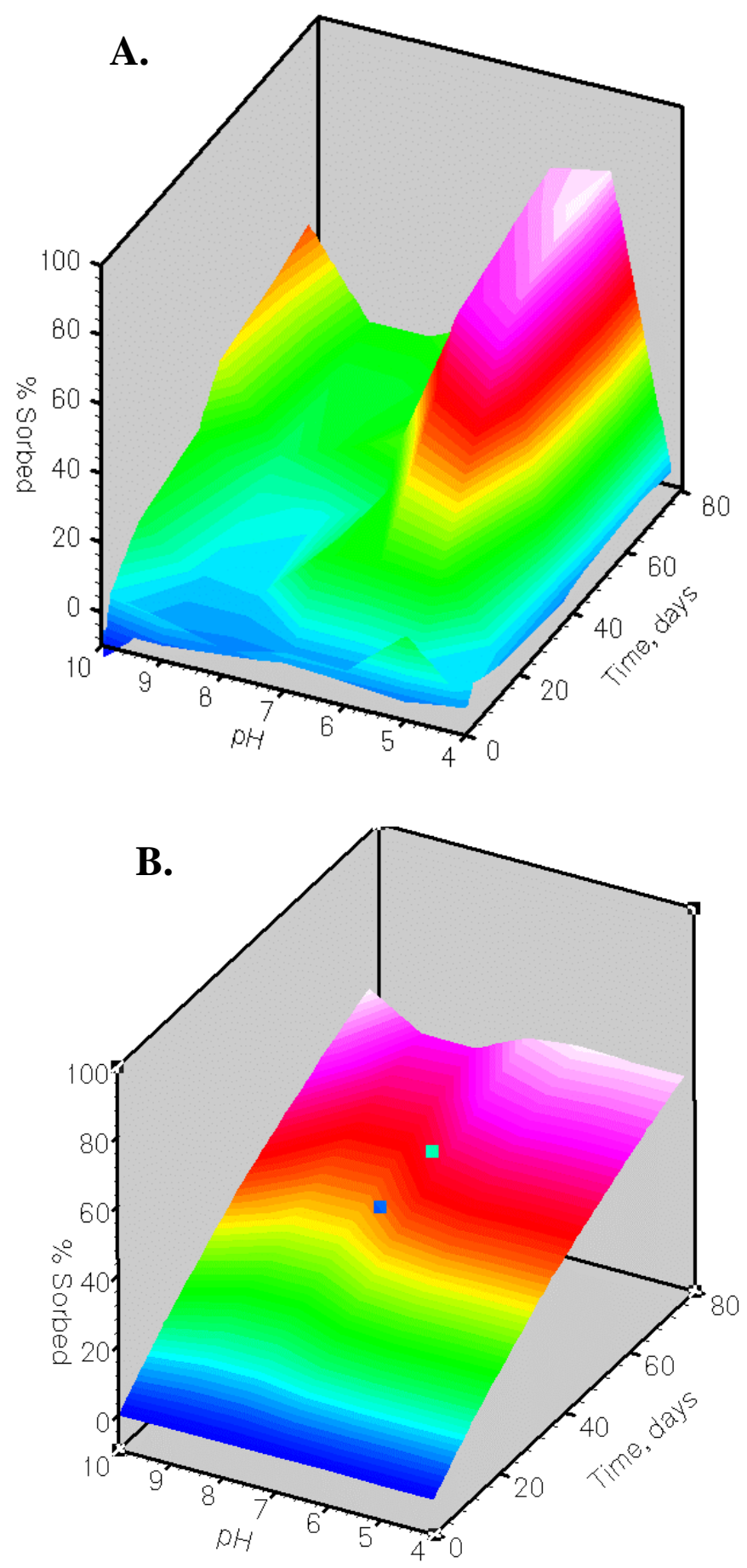

Figure 5.9. A) Sorption of $\mathrm{Pu}(\mathrm{V})$ on clinoptilolite as a function of $\mathrm{pH}$ and time. B) Predicted sorption behavior of a $\mathrm{Pu}(\mathrm{V})$ solution using the disproportionation rate of $\mathrm{Pu}(\mathrm{V})$ to $\mathrm{Pu}(\mathrm{IV})$ and subsequent sorption rate of aqueous $\mathrm{Pu}(\mathrm{IV})$ to clinoptilolite. 
Qualitatively, the modeling results are in agreement with experimental data, suggesting that the model adequately accounts for the dominant mechanism controlling $\mathrm{Pu}(\mathrm{V})$ sorption to clinoptilolite. For example, the sorption minimum at $\mathrm{pH} 7$ to 9 is predicted by our model. Also, the magnitude of $\mathrm{Pu}$ sorption at $\mathrm{pH} 5$ to 7 is accurately predicted. Furthermore, the increase in $\mathrm{Pu}$ sorption at $\mathrm{pH} 10$ relative to $\mathrm{pH} 9$ is matched.

While our model qualitatively predicts the behavior of $\mathrm{Pu}(\mathrm{V})$ sorption to clinoptilolite surfaces, some differences between experimental and modeling results are worth discussing. Our model over predicts sorption rates in the $\mathrm{pH} 7$ to 9 range even though our disproportionation model is based on experiments performed in this $\mathrm{pH}$ range. However, the results are in agreement if we account for the uncertainty in the disproportionation constant. For example, the average and standard deviations for $\mathrm{Pu}(\mathrm{V})$ disproportionation rate is $5.05 \times 10^{-4} \pm 1.3 \times 10^{-4} \mathrm{~mol} / \mathrm{L} / \mathrm{hr}$. Based on a two standard deviation range, the half-life of $\mathrm{Pu}(\mathrm{V})$ will range from 0.10 to 0.31 years. Over 80 days, we predict that between 23 and 62 percent of $\mathrm{Pu}(\mathrm{V})$ will remain in solution. Based on this level of uncertainty in the disproportionation constant, our modeling results are consistent with the sorption data. Furthermore, we assumed in our model that the product of $\mathrm{Pu}(\mathrm{V})$ disproportionation is $\mathrm{Pu}(\mathrm{IV})$. Data suggest that some $\mathrm{Pu}(\mathrm{III})$ and $\mathrm{Pu}(\mathrm{VI})$ will be produced as well, which will also affect sorption data. Thus, considering the complexity of $\mathrm{Pu}$ disproportionation and redox reactions and the simplifications included in our model, our ability to predict the behavior of $\mathrm{Pu}(\mathrm{V})$ in solution with clinoptilolite colloids as a function of $\mathrm{pH}$ and time is quite good.

Another difference worth noting is that our model does not accurately predict the low sorption of $\mathrm{Pu}(\mathrm{V})$ at $\mathrm{pH} 4$ over the time period of the experiment. Clearly, several disproportionation reactions exist and these reactions may all be affected by $\mathrm{pH}$, aqueous speciation, or other factors. Furthermore, equilibrium modeling under mildly reducing conditions (Eh $\sim 1$ volt) suggest that $\mathrm{Pu}(\mathrm{V})$ should be more stable than $\mathrm{Pu}(\mathrm{IV})$ at low $\mathrm{pH}$ (see Figure 5.1). Further study of the effect of $\mathrm{pH}$, aqueous speciation, and $\mathrm{Pu}$ concentration on disproportionation would be required to adequately address the factors affecting $\mathrm{Pu}(\mathrm{V})$ disproportionation.

We have shown that disproportionation is the most likely rate controlling step for the slow $\mathrm{Pu}(\mathrm{V})$ sorption to clinoptilolite surfaces. We further suggest that like clinoptilolite, the sorption mechanism of $\mathrm{Pu}(\mathrm{V})$ on other silicatesis first controlled by the rate of disproportionation of $\mathrm{Pu}(\mathrm{V})$ in solution, followed by rapid sorption of soluble $\mathrm{Pu}(\mathrm{IV})$ to the mineral surface. In contrast, the mechanism for sorption of $\mathrm{Pu}(\mathrm{V})$ on minerals such as Fe- and Mn-oxides is not controlled by disproportionation of $\mathrm{Pu}(\mathrm{V})$. The $\mathrm{Pu}(\mathrm{V})$ sorbs rapidly, on the mineral surface as $\mathrm{Pu}(\mathrm{V})$ and reduces to $\mathrm{Pu}(\mathrm{IV})$ on the mineral surface. We speculate that the rapid sorption of $\mathrm{Pu}(\mathrm{V})$ on the Fe- and Mn-oxides, followed by reduction of $\mathrm{Pu}$ on the mineral surface is controlled by the Fe and $\mathrm{Mn}$ redox couples of the minerals, respectively. 


\subsection{Summary}

The sorption of $\mathrm{Pu}(\mathrm{V})$ on clinoptilolite colloids as a function of time between $\mathrm{pH} 4$ and 10 is slow. Although the sorption rate of $\mathrm{Pu}(\mathrm{V})$ on clinoptilolite varies as a function of $\mathrm{pH}$, the sorption extent is low at the $\mathrm{pH}$ range observed in NTS water ( $\mathrm{pH} 7-9$ ). After 2.5 months, only $20 \%$ of the $\mathrm{Pu}$ sorbed on the colloids at $\mathrm{pH} 8$; the maximum sorption of $80 \%$ occurred between $\mathrm{pH} 5-6$.

Plutonium (V) sorbed to all seven minerals investigated; zeolite, clinoptilolite; Fe-oxide, goethite; Mn-oxides, birnessite and pyrolusite; calcite; clay, montmorillonite; and silica, although sorption $\mathrm{K}_{\mathrm{d}} \mathrm{s}$ vary as a function of mineralogy. $\mathrm{The} \mathrm{Pu}(\mathrm{V})$ sorbs the strongest to the $\mathrm{Mn}$ - and $\mathrm{Fe}$ - oxides and the weakest to calcite and silica.

The XAS data show that the initial soluble $\mathrm{Pu}(\mathrm{V})$ has ultimately been reduced to the $\mathrm{Pu}(\mathrm{IV})$ state on all the minerals: clinoptilolite, birnessite, calcite, goethite, montmorillonite, pyrolusite and silica. The reduction of $\mathrm{Pu}(\mathrm{V})$ to $\mathrm{Pu}(\mathrm{IV})$ on clinoptilolite was also observed at different $\mathrm{pHs}$.

We have shown that there are two possible mechanisms for the soluble $\mathrm{Pu}(\mathrm{V})$ to reduce to $\mathrm{Pu}(\mathrm{IV})$ on mineral surfaces. The first pathway is where $\mathrm{Pu}(\mathrm{V})$ is sorbed directly on the mineral, and then is reduced to $\mathrm{Pu}(\mathrm{IV})$ as a result of interaction with the mineral surface. The second pathway is where $\mathrm{Pu}(\mathrm{V})$ is reduced to aqueous $\mathrm{Pu}(\mathrm{IV})$ through its disproportionation in solution, and then $\mathrm{Pu}(\mathrm{IV})$ aqueous species is rapidly sorbed on the mineral surface.

Our data suggest that disproportionation is the most likely rate-controlling step for the slow $\mathrm{Pu}(\mathrm{V})$ sorption to clinoptilolite surfaces. The disproportionation of $\mathrm{Pu}(\mathrm{V})$ is then followed by rapid sorption of aqueous $\mathrm{Pu}(\mathrm{IV})$ to the clinoptilolite surface. We further suggest that like clinoptilolite, the sorption mechanism of $\mathrm{Pu}(\mathrm{V})$ on other silicates must be first controlled by the rate of disproportionation of $\mathrm{Pu}(\mathrm{V})$, followed by rapid sorption of soluble $\mathrm{Pu}(\mathrm{IV})$ to the mineral surface.

In contrast, the mechanism for sorption of $\mathrm{Pu}(\mathrm{V})$ on minerals such as Fe- and Mn-oxides is not controlled by disproportionation of $\mathrm{Pu}(\mathrm{V})$. The $\mathrm{Pu}(\mathrm{V})$ sorbs rapidly, directly on the mineral surface as $\mathrm{Pu}(\mathrm{V})$ and reduces to $\mathrm{Pu}(\mathrm{IV})$ as a result of interaction with the mineral surface. We speculate that the rapid sorption of $\mathrm{Pu}(\mathrm{V})$ on the Fe- and Mn-oxides, followed by reduction of $\mathrm{Pu}$ on the mineral surface is controlled by the Fe and Mn redox couples of the minerals, respectively.

We have shown that if $\mathrm{Pu}$ is present in water in either the $\mathrm{Pu}(\mathrm{IV})$ or $\mathrm{Pu}(\mathrm{V})$ oxidation state it will sorb to the common mineral colloids observed at the NTS. Pu(IV) sorbs quite rapidly. For $\mathrm{Pu}(\mathrm{V})$, the sorption rate and mechanism is a function of the mineral colloid, but ultimately all the $\mathrm{Pu}(\mathrm{V})$ is reduced to $\mathrm{Pu}(\mathrm{IV})$ relatively quickly, on the order of weeks. 


\section{Acknowledgments}

We thank Ken Moody for graciously supplying all the Pu stock solutions used in these experiments. We thank Nadia L. Hakem for some of the preliminary experimental work. Additional funding to A.B. Kersting in support of the Pu sorption experiments came from Laboratory Directed Research and Development grant, LLNL. 


\subsection{References}

Choppin G. R. (1991) Redox speciation of plutonium in natural waters. Journal of Radioanalytical and Nuclear Chemistry 147(1), 109-116.

Lu, N., Cotter, C.R., Kitten, H.D., Bentley, J. and Triay, I.R. (1998) Reversibility of sorption of plutonium-239 onto hematite and goethite colloids. Radiochimica Acta 83, 167-173.

Nitsche H., Muller A., Standifer E. M., Deinhammer R. S., Becraft K., Prussin T., and Gatti R. C. (1992) Dependence of actinide solubility and speciation on carbonate concentration and ionic strength in groundwater. Radiochimica Acta 5859(P1)(9), 27-32.

Penrose W. R., Metta D. N., Hylko J. M., and Rinckel L. A. (1987) The reduction of plutonium (V) by aquatic sediments. J. Environmental Radioactivity 5, 169-184.

Silver G. L. (1988) On the representation of soluble plutonium by four oxidation states. Inorganica Chimica Acta 154, 123-127.

Silver G. L. (2000) Proportional equations in plutonium chemistry. Journal of Radioanalytical and Nuclear Chemistry 245(2), 229-232. 


\title{
-Chapter 6- \\ Summary of Radionuclide-Colloid Batch Sorption and Desorption \\ Measurements for the Yucca Mountain Project
}

\author{
Ningping Lu and Paul W. Reimus
}

\subsection{Introduction}

The Yucca Mountain Site Characterization Project (YMP) has long recognized the potential for colloid-facilitated transport of radionuclides, particularly strongly sorbing ones, in both the unsaturated zone and the saturated zone near Yucca Mountain, NV, potential site of the nation's first high-level nuclear waste repository. In 1993, a workshop was held to discuss the importance of colloid-facilitated radionuclide transport to the YMP. At this workshop, colloid stability and the strength and reversibility of radionuclide sorption onto colloids were identified as key processes that would have the greatest potential influence on colloid-facilitated radionuclide transport (Triay et al., 1995). Subsequently, the YMP conducted a small number of laboratory colloid stability studies and embarked on a long-term program to measure colloid concentrations and size distributions in ground waters near Yucca Mountain. The YMP also initiated a laboratory experimental program to study radionuclide sorption and desorption onto colloids. The stability studies and early groundwater colloid concentration measurements are summarized in Triay et al. (1996). More recent measurements of natural colloid concentrations in ground waters are discussed in Chapter 2 and Kung (2000). In this chapter, the results of several years of YMP sorption and desorption studies of radionuclides onto colloids are summarized.

\subsection{Radionuclide Sorption onto Colloids of Hematite, Goethite, Montmorillonite, and Silica}

Between 1997 and 2000, Lu et al. (1998a, 1998b, 2000) conducted several batch sorption and desorption experiments to measure the sorption of $\mathrm{Pu}(\mathrm{IV}), \mathrm{Pu}(\mathrm{V}), \mathrm{Am}(\mathrm{III}), \mathrm{Np}(\mathrm{V})$, and U(VI) onto hematite, goethite, montmorillonite, and silica colloids using filtered waters from well J-13 in Area 25 at the NTS (near Yucca Mountain) and synthetic J-13 water. The Pu(IV) was always introduced as a polymeric colloid $(\leq 10 \mathrm{~nm}$ diameter). The hematite and goethite were selected to represent colloids that could form as a result of the corrosion of iron-containing waste package materials in the potential Yucca Mountain repository. Experiments were conducted at different colloid concentrations, temperatures, and ionic strengths. The reader is referred to the $\mathrm{Lu}$ references above for details of the experiments.

Figure 6.1 graphically depicts the ranges of radionuclide partition coefficients $\left(\mathrm{K}_{\mathrm{d}}\right.$ values, $\left.\mathrm{ml} / \mathrm{g}\right)$ measured for the various radionuclide-colloid combinations at colloid concentrations of 200 $\mathrm{mg} / \mathrm{L}$ at $20^{\circ} \mathrm{C}$ in natural J-13 water. Some of the key observations in the experiments, involving $\mathrm{Pu}(\mathrm{IV})$ and $\mathrm{Pu}(\mathrm{V})$, including the effects of changing the temperature, ionic strength, and colloid concentrations are listed in brief summary fashion for each radionuclide and each colloid below. 


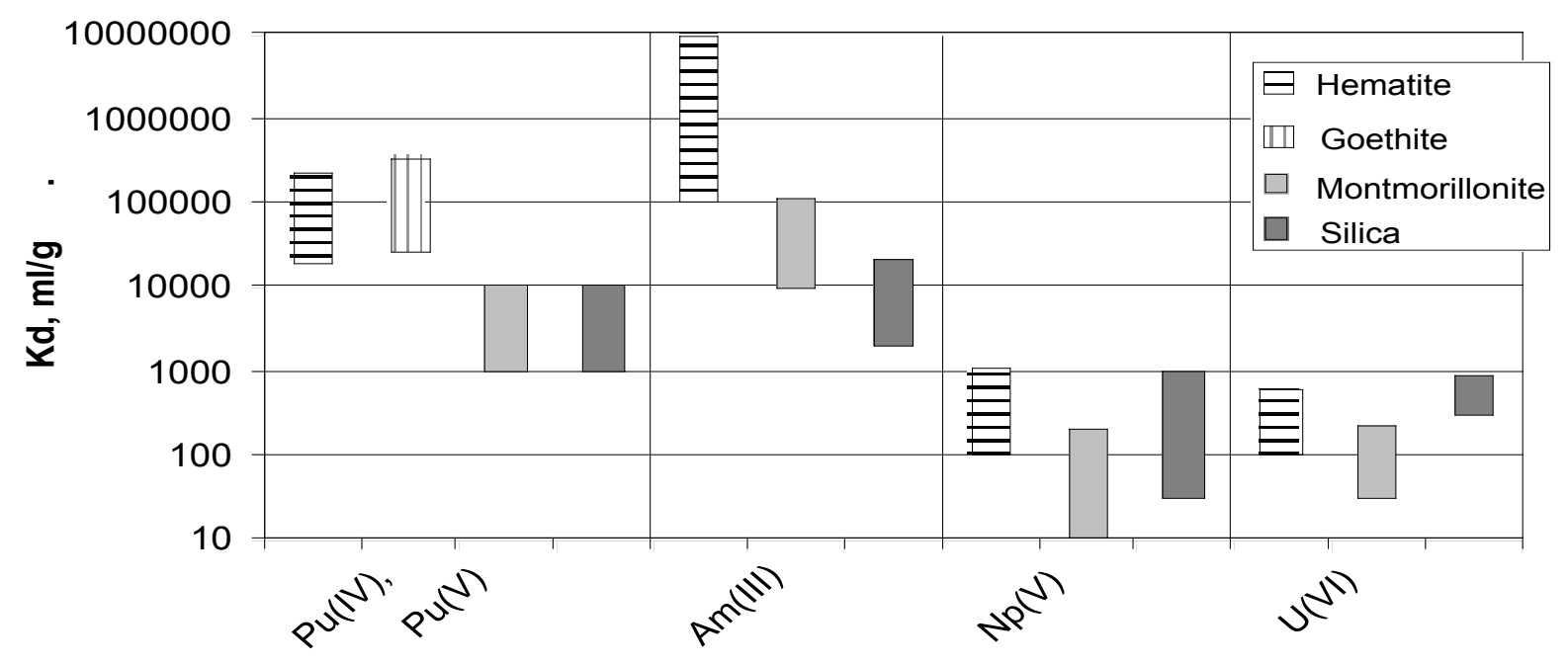

Figure 6.1. Ranges of $K_{d}$ values measured for actinide sorption onto colloids at $200 \mathrm{mg} / \mathrm{L}$ colloid concentrations in well J-13 water at $20^{\circ} \mathrm{C}$ (Lu et al., 1998a, 1998b, 2000).

$\mathrm{Pu}(\mathrm{IV})$ and $\mathrm{Pu}(\mathrm{V})$ sorption to hematite colloids:

$\mathrm{Pu}(\mathrm{IV})$ sorption is much faster than $\mathrm{Pu}(\mathrm{V})$ sorption, although final $\mathrm{K}_{d}$ s were quite similar.

$\mathrm{K}_{\mathrm{d}}$ range $2 \times 10^{4}-2 \times 10^{5} \mathrm{ml} / \mathrm{g}$ at $20 \mathrm{C}$, $\mathrm{J} 13$ water, $200 \mathrm{mg} / \mathrm{L}$ colloid concentration

$\mathrm{K}_{\mathrm{d}}$ increase to $5 \times 10^{6}$ as temperature increases to $80^{\circ} \mathrm{C}$

$\mathrm{K}_{\mathrm{d}}$ increase to $1 \times 10^{7}$ as ionic strength increases to $0.07 \underline{\mathrm{M}}$ or greater

Slight $\mathrm{K}_{\mathrm{d}}$ increase (about a factor of 2 ) at low colloid concentrations (down to $10 \mathrm{mg} / \mathrm{L}$ )

1 -step desorption tests showed some desorption, suggesting additional desorption experiments were warranted

$\mathrm{Pu}(\mathrm{IV})$ and $\mathrm{Pu}(\mathrm{V})$ sorption to goethite colloids:

$\mathrm{Pu}(\mathrm{IV})$ sorption is much faster than $\mathrm{Pu}(\mathrm{V})$ sorption, although final $\mathrm{K}_{\mathrm{d}}$ s were quite similar.

$\mathrm{K}_{\mathrm{d}}$ range $2.5 \times 10^{4}-2 \times 10^{5} \mathrm{ml} / \mathrm{g} @ 20 \mathrm{C}$, J 13 water, $200 \mathrm{mg} / \mathrm{L}$ colloid concentration

No measurements as a function of temperature, ionic strength, or colloid concentration

1 -step desorption tests showed some desorption, suggesting additional desorption experiments were warranted

$\mathrm{Pu}(\mathrm{IV})$ and $\mathrm{Pu}(\mathrm{V})$ sorption to montmorillonite colloids:

$\mathrm{Pu}(\mathrm{IV})$ sorption is much faster than $\mathrm{Pu}(\mathrm{V})$ sorption, although final $\mathrm{K}_{\mathrm{d}} \mathrm{s}$ were quite similar.

$\mathrm{K}_{\mathrm{d}}$ range $10^{3}-10^{4} \mathrm{ml} / \mathrm{g} @ 20 \mathrm{C}, \mathrm{J} 13$ water, $200 \mathrm{mg} / \mathrm{L}$ colloid concentration

$\mathrm{K}_{\mathrm{d}}$ increases to $2 \times 10^{5}$ as temperature increases to $80 \mathrm{C}$

No significant effect of ionic strength on $\mathrm{K}_{\mathrm{d}}$ values

$\mathrm{K}_{\mathrm{d}}$ increases to $3 \times 10^{4} \mathrm{ml} / \mathrm{g}$ at low colloid concentrations

1-step desorption tests showed some desorption, suggesting additional desorption experiments were warranted 
$\mathrm{Pu}(\mathrm{IV})$ and $\mathrm{Pu}(\mathrm{V})$ sorption to silica colloids:

$\mathrm{Pu}(\mathrm{IV})$ sorption is much faster than $\mathrm{Pu}(\mathrm{V})$ sorption, although final $\mathrm{K}_{\mathrm{d}} \mathrm{s}$ were quite similar.

$\mathrm{K}_{\mathrm{d}}$ range $10^{3}-10^{4} \mathrm{ml} / \mathrm{g} @ 20 \mathrm{C}$, J 13 water, $200 \mathrm{mg} / \mathrm{L}$ colloid concentration

$\mathrm{K}_{\mathrm{d}}$ upper limit of $10^{4} \mathrm{ml} / \mathrm{g}$ as temperature increases to $80 \mathrm{C}$

$\mathrm{K}_{\mathrm{d}}$ lower limit of $10^{3} \mathrm{ml} / \mathrm{g}$ as ionic strength increases to $0.1 \underline{\mathrm{M}}$

$\mathrm{K}_{\mathrm{d}}$ increases to $3 \times 10^{4} \mathrm{ml} / \mathrm{g}$ at low colloid concentrations

1-step desorption tests showed some desorption, suggesting additional desorption experiments were warranted

\subsection{Pu Desorption From Montmorillonite and Silica Colloids}

A series of laboratory experiments conducted for the Yucca Mountain Project in which $\mathrm{Pu}$ desorption from smectite (montmorillonite) and silica colloids were measured are of particular relevance to the UGTA program (unpublished results, but data are available in YMP online database). These experiments differed from most desorption experiments conducted for the YMP in that they involved multiple batch desorption steps; i.e., Pu-free water was introduced on nine different occasions into test tubes containing Pu-bearing colloids, instead of just once. The $\mathrm{Pu}$-bearing colloids came from a batch sorption experiment. Each desorption step involved centrifuging to separate the colloids from solution (after the colloids and solution had been shaking together for a specified time), decanting off most of the solution, and then introducing fresh water and restarting shaking. Additional details of the batch sorption and desorption experiments are provided in Chapter 7.

Figure 6.2 shows the results of the YMP desorption experiments (expressed as the percentage of $\mathrm{Pu}$ remaining sorbed to the colloids vs. time since desorption started) as well as model fits to the data. The model used to fit the data is described in detail in Chapter 8. The desorption experiments were preceded by a batch $\mathrm{Pu}(\mathrm{V})$ sorption experiment of $144 \mathrm{hrs}$ (6 days).

The key point associated with the experimental results shown in Figure 6.2 is that the desorption of $\mathrm{Pu}$ from both types of colloids is extremely slow after the first 2-3 desorption steps. In the case of the silica colloids, this result is especially surprising because of the relatively small amount of $\mathrm{Pu}$ adsorbed to the colloids after 6 days $(\sim 65 \%)$, which, in the absence of the desorption data, might be interpreted as an indication of a relatively rapid desorption rate. The model fits suggest a desorption rate constant of $0.00004 \mathrm{hr}^{-1}\left(0.35 \mathrm{yr}^{-1}\right)$ for the smectite colloids, and a rate constant of $0.000014 \mathrm{hr}^{-1}\left(0.12 \mathrm{yr}^{-1}\right)$ for at least a portion of the $\mathrm{Pu}$ mass sorbed to the silica colloids. These desorption rate constants are both small enough that a significant mass of $\mathrm{Pu}$ would be expected to remain sorbed to the colloids after several years. Thus, these rate constants are consistent with the possibility of colloid-facilitated $\mathrm{Pu}$ transport over significant distances in ground water provided that $\mathrm{Pu}$-bearing colloids are not rapidly and irreversibly filtered. 


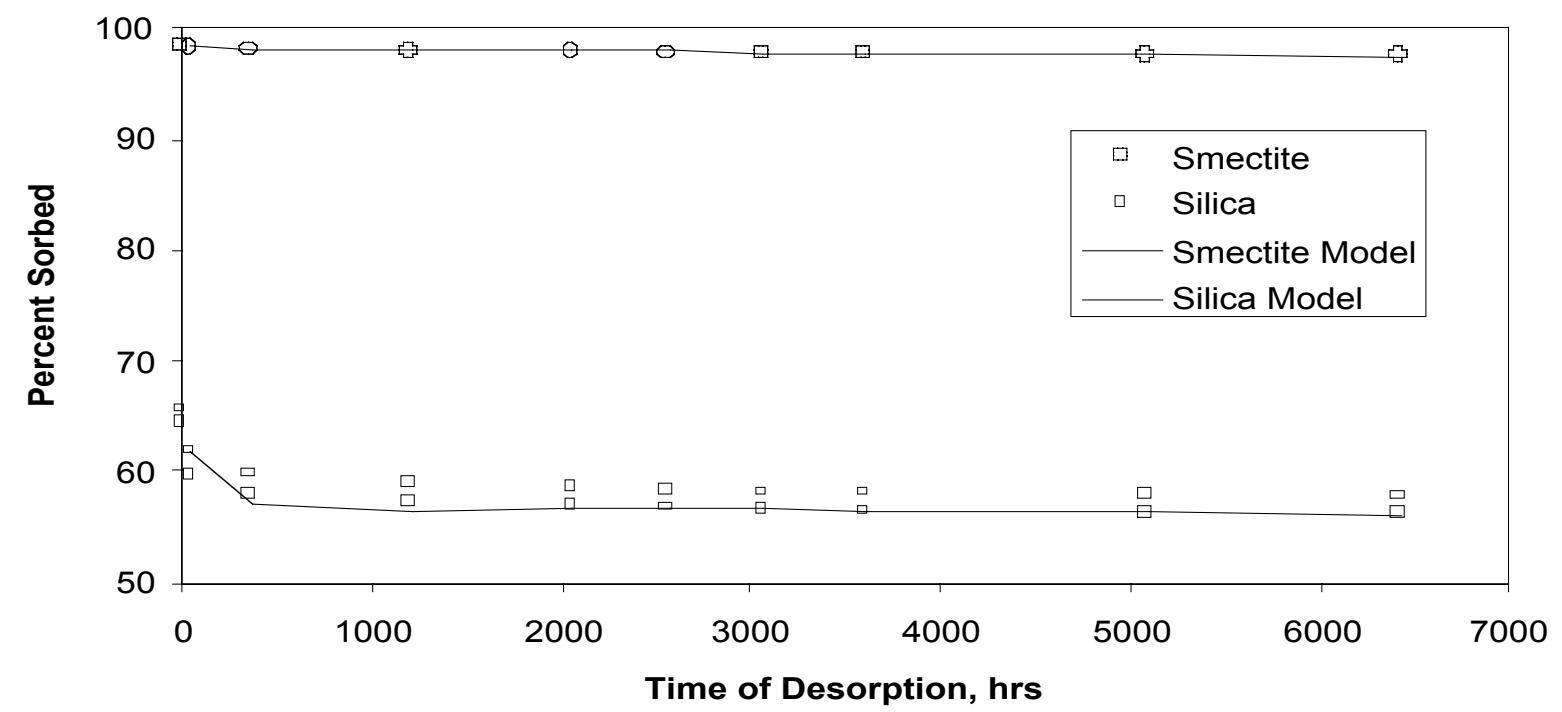

Figure 6.2. Results of Yucca Mountain Pu desorption experiments in which $\mathrm{Pu}(\mathrm{V})$ was sorbed onto montmorillonite and silica colloids for 6 days and then the $\mathrm{Pu}$ (either IV or V) was desorbed in multiple batch steps in which Pu-free water was placed in contact with the colloids. Experiments were conducted at room temperature in J-13 well water. (Source: Yucca Mountain Project database.) 


\subsection{References}

Kung, S.K. and P.R. Fresquez. (2000) Colloid Characterization and Quantification in Groundwater Samples. Los Alamos National Laboratory. LA-13727-MS. P.41. http:/libwww.lanl.gov/cgi-gin/getfile?00538241.pdf.

Lu, Ningping, I. R. Triay, C. R. Cotter, H. D. Kitten, and J. Bentley (1998a). Reversibility of sorption of plutonium-239 onto colloids of hematite, goethite, smectite, and silica. LAUR-98-3057, Los Alamos National Laboratory Unrestricted Report, Los Alamos, NM.

Lu, Ningping, C. R. Cotter, H. D. Kitten, and J. Bentley (1998b). Reversibility of sorption of plutonium-239 onto colloids of hematite and goethite. Radiochim. Acta 83, 167-173.

Lu, Ningping, J. Conca, G. R. Parker, P. A. Leonard, B. Moore, E. A. Strietelmeier, and I. R. Triay (2000). Adsorption of Actinides onto Colloids as a Function of Time, Temperature, Ionic Strength and Colloid Concentration. LA-UR-00-5121, Los Alamos National Laboratory Unrestricted Report, Los Alamos, NM.

Triay, I. R., A. Simmons, S. Levy, S. Nelson, H. Nuttall, B. Robinson, W. Steinkampf, and B. Vianni (1995). Colloid-Facilitated Radionuclide Transport at Yucca Mountain.

LA-12779-MS, Los Alamos National Laboratory Manuscript Series Report, Los Alamos, NM.

Triay, I. R., W. W. Lemons, C. R. Cotter, A. O. Wistrom, and C. Degueldre (1996). Progress report on colloid-facilitated transport at Yucca Mountain, Yucca Mountain Site Characterization Program Milestone 3383, LA-12959-MS, Los Alamos National Laboratory Manuscript Series Report, Los Alamos, NM. 


\title{
-Chapter 7- \\ Colloid-Facilitated Plutonium Fracture \\ Transport Experiments
}

\author{
Paul W. Reimus, S. D. Ware, Ningping Lu, K. S. Kung, Amr Abdel-Fattah, \\ Ioana Anghel, Mary P. Neu, and Sean D. Reilly
}

\subsection{Introduction}

In FY 2000 and 2001, the UGTA program sponsored several laboratory experiments in which $\mathrm{Pu}(\mathrm{V})$ was sorbed onto either montmorillonite or silica colloids, and then the resulting solutions were injected into saturated, fractured cores from Pahute Mesa (along with a conservative tracer, tritiated water). Experiments were also conducted in which $\mathrm{Pu}(\mathrm{IV})$ was sorbed onto clinoptilolite colloids (at LLNL) and then injected into fractured cores. The primary objective of these experiments was to determine whether inorganic colloids known to be present in ground waters at NTS could facilitate the transport of plutonium sufficiently to account for the observance of $\mathrm{Pu}$ from the BENHAM cavity in borehole ER-20-5 \#1, located $\sim 1.3 \mathrm{~km}$ from the BENHAM cavity, $\sim 30$ years after the BENHAM shot.

In general, the fracture transport experiments proceeded with the following steps:

- Preparation of ${ }^{239} \mathrm{Pu}(\mathrm{V})$ solutions, and spectroscopic verification of the Pu oxidation state.

- Preparation of dilute ( 200 mg/L) colloid suspensions.

- Batch sorption experiments in which $\mathrm{Pu}(\mathrm{V})$ and colloids were brought into contact for specified periods of time to determine rates and magnitudes of $\mathrm{Pu}$ sorption onto the colloids, and to prepare solutions for injection into the fractured cores, or

- Measurement of partitioning of $\mathrm{Pu}(\mathrm{IV})$ between solution and colloids in the clinoptilolite colloid suspension provided by LLNL (prior to injecting into fractured cores).

- Batch desorption experiments to determine the rates and magnitude of $\mathrm{Pu}(\mathrm{IV})$ and/or $\mathrm{Pu}(\mathrm{V})$ desorption from the colloids over the duration of the fracture transport experiments as well as over longer time periods.

- Fractured core transport experiments in which the Pu-colloid solutions were injected (along with tritiated water) into the naturally-fractured cores at a specified flow rate.

- Analysis of the column effluents for tritium and ${ }^{239} \mathrm{Pu}$ by liquid scintillation counting and for colloids using a high-sensitivity liquid in-situ particle spectrometer.

- Dismantling of the fractured cores and qualitative analysis of the location of Pu on the fracture surfaces using autoradiography and gross alpha counting with a hand monitor.

- Interpretations of the batch sorption/desorption data using a rate-limited Pu sorption/ desorption model.

- Interpretations of the fracture transport experiments using transport models featuring ratelimited colloid filtration (attachment) and detachment as well as rate-limited sorption and desorption of $\mathrm{Pu}$ onto/from colloids.

All but the last three activities will be described in detail in this chapter. The autoradiography work is briefly discussed in this chapter in the context of the fracture experiment results; however, more details on the autoradiography investigations and other post-test destructive 
analysis methods are discussed in Chapter 9. The interpretations of the batch sorption/ desorption and column transport experiments are discussed in detail in Chapter 8.

\subsection{Materials}

\section{U-20WW Water}

A natural water from Well U-20WW on Pahute Mesa at the Nevada Test Site (NTS), which will be referred to as WW20 water, was used in most of the batch sorption/desorption and fracture transport experiments. The water was collected by IT Corp. in a 55-gal polyethylene drum on November 5, 1997, and it was immediately shipped to Los Alamos where it was analyzed for cations, anions, and $\mathrm{pH}$, and then used in various laboratory experiments, including several not discussed in this report. The water chemistry measured at Los Alamos is summarized in Table 7.1. The water was filtered with a $0.05-\mu \mathrm{m}$ filter prior to use in any of the experiments involving colloids.

\section{Synthetic Groundwater}

A synthetic groundwater based on the natural WW20 water chemistry was used in some of the earlier batch sorption/desorption and fracture transport experiments. This water was called synthetic (syn-) WW20 water, and its recipe is given in Table 7.2. The ionic strength of the water is $\sim 0.003 \underline{\mathrm{M}}$ (natural WW20 water is about $0.0035 \underline{\mathrm{M}}$ ). There are two important differences between the synthetic WW20 water and natural WW20 water. First, sodium was used in place of calcium in the synthetic water to minimize colloid aggregation induced by multivalent cations. Second, silica was not included in the recipe (natural WW20 water is approximately saturated in silica) because it is difficult to dissolve, and we did not want it to interfere with the $\mathrm{Pu}$ sorption measurements onto silica colloids. The synthetic water was prepared by dissolving the ingredients of Table 7.2 in nanopure deionized water and then adjusting the $\mathrm{pH}$ to approximately 8.5 using a small amount of sodium hydroxide. The $\mathrm{pH}$ was adjusted to this value so that $\mathrm{CO}_{2}$ gas would neither be absorbed nor significantly evolved during the experiments (at the elevation of Los Alamos). We wanted to avoid significant changes in carbonate/bicarbonate concentrations over time because carbonate can complex with $\mathrm{Pu}$ and thus potentially affect the sorption and transport measurements. The $\mathrm{pH}$ of 8.5 is also reasonably consistent with measured

pHs of waters from Pahute Mesa. Natural WW20 water was used in the later experiments (including all column experiments involving silica colloids) because of concerns that the undersaturation of the syn-WW20 water with respect to silica could cause the silica colloids to partially dissolve.

\section{Montmorillonite Colloid Solutions}

Cheto Ca-montmorillonite and Otay Ca-montmorillonite mineral powders (particle size $<1.0$ $\mu \mathrm{m})$ were obtained from University of Missouri, Columbia, Source Clay Mineral Repository. The Cheto montmorillonite mineral consisted of $52.0 \%$ of $\mathrm{SiO}_{2}, 16.2 \%$ of $\mathrm{Al}_{2} \mathrm{O}_{3}, 1.34 \%$ of $\mathrm{Fe}_{2} \mathrm{O}_{3}, 5.16 \%$ of $\mathrm{MgO}, 1.88 \%$ of $\mathrm{CaO}, 0.98 \%$ of $\mathrm{Na}_{2} \mathrm{O}, 0.12 \%$ of $\mathrm{K}_{2} \mathrm{O}$ and $8.17 \%$ of water as well as $\mathrm{CO}_{2}$ and volatile organic compounds. X-ray diffraction showed that the mineral was a Ca-montmorillonite. The cation exchange capacity (CEC) of the Cheto montmorillonite was 
Table 7.1. Major Ion Chemistry of Natural WW20 Water Used in Experiments.

\begin{tabular}{ll}
\hline Ion & Conc., $\mathbf{~ m g} / \mathbf{L}$ \\
\hline $\mathrm{pH}$ & 8.0 \\
\hline $\mathrm{Na}^{+}$ & 60 \\
\hline $\mathrm{Ca}^{2+}$ & 7.2 \\
\hline $\mathrm{K}^{+}$ & 1.3 \\
\hline $\mathrm{Mg}^{2+}$ & 0.3 \\
\hline $\mathrm{SiO}_{2}$ & 46.9 \\
\hline $\mathrm{HCO}_{3}{ }^{-}$ & 110 \\
\hline $\mathrm{SO}_{4}{ }^{2-}$ & 32 \\
\hline $\mathrm{Cl}^{-}$ & 11.6 \\
\hline $\mathrm{F}^{-}$ & 2.6 \\
\hline $\mathrm{NO}_{3}{ }^{-}$ & 1.5 \\
\hline
\end{tabular}

Table 7.2. Synthetic WW20 Water Recipe used in Experiments.

\begin{tabular}{|c|c|}
\hline Chemical & Conc., mg/L \\
\hline $\mathrm{NaF}$ & 5.715 \\
\hline $\mathrm{H}_{4} \mathrm{SiO}_{4}$ & 75.18 \\
\hline $\mathrm{NaHCO}_{3}$ & 151.53 \\
\hline $\mathrm{NaCl}$ & 17.825 \\
\hline $\mathrm{KCl}$ & 2.51 \\
\hline $\mathrm{NaNO}_{3}$ & 2.06 \\
\hline
\end{tabular}

$152.37 \mathrm{cmol} \mathrm{kg}^{-1}$ (meq kg${ }^{-1}$ ), which was determined using a method described by Sumner and Miller (1996). The chemical composition, CEC, and quantitative mineralogy of the Otay montmorillonite were not determined. Otay montmorillonite solutions were prepared from two different batches of Otay montmorillonite, which will be referred to as batch 1 and batch 2 .

Colloidal solutions of the Cheto and Otay montmorillonite were prepared by dispersing some of the montmorillonite powder in nanopure deionized water in an ultrasonic bath for 3 minutes. The dispersed suspension was transferred to a one-liter cylinder and allowed to stand for 5 hours at room temperature to allow larger particles to settle. Thereafter, the supernatant solutions were carefully collected as stock colloidal solutions. The $\mathrm{pH}$ of both the Cheto and Otay montmorillonite colloidal solutions was 6.9 , respectively. The mass of the colloids in the solutions was determined by the difference in the weights before and after air-drying and ovendrying $\left(105^{\circ} \mathrm{C}\right)$ a given amount of each solution (both with and without colloids so that corrections could be made for total dissolved solids). Working colloidal solutions with colloid concentrations of $200 \mathrm{mg} / \mathrm{L}$ were prepared by diluting a measured volume of stock colloidal solution into 1 liter of synthetic WW20 water (Cheto) or natural WW20 water (Otay batches 1 and 2). The $\mathrm{pH}$ of the working solutions was 8.5 for the Cheto and 8.1 for the Otay 
montmorillonite. The surface charge (expressed as zeta potential) of the montmorillonite colloids was measured using a Coulter Delsa 440 SX mobility measurement system at the University of New Mexico. The zeta potential on the surface of Cheto montmorillonite colloidal particles in synthetic WW20 water was $(-34.3 \pm 3.8) \mathrm{mV}$. The surface zeta potential of the batch 1 Otay montmorillonite colloids in natural WW20 water was $(-21.0 \pm 5.7) \mathrm{mV}$ at the time of the first set of experiments conducted with these colloids, and it was measured as $(-22.5 \pm 8.6) \mathrm{mV}$ when the colloids were used again over a year later. The zeta potential on the surface of the batch 2 Otay montmorillonite colloids in natural WW20 water was $(-25.7 \pm 6.5) \mathrm{mV}$. Measurements of particle size distributions using a high-sensitivity liquid in-situ particle spectrometer, HSLIS-S50, manufactured by Particle Measuring System, Inc. (Boulder, Colorado), indicated that all types of montmorillonite colloids had a broad size distribution with a mode (highest number frequency) occurring between 50 and $100 \mathrm{~nm}$ diameter. High-resolution transmission electron microscopy (TEM) images of the Cheto and Otay montmorillonite colloids are shown in Figures 7.1 and 7.2, respectively.

\section{Silica Colloid Solutions}

Synthetic colloidal silica, designated as PST-1 and MP1040 by the manufacturer, were purchased from Nissan Chemical Industries Ltd. The solutions were manufactured by dispersing negatively charged amorphous silica particles in water. The particles were spherical, and the stock solutions were $20 \% \mathrm{SiO}_{2}$ in PST- 1 and $40 \% \mathrm{SiO}_{2}$ in MP1040 by weight. The following dialysis procedure was used to remove all surfactants from the solutions. Approximately $50 \mathrm{ml}$ of original colloidal solution was diluted to $200 \mathrm{ml}$ with nanopure water and transferred into a 12,000-14,000 molecular weight cut-off membrane, referred to as dialysis tubing. The tubing was sealed and placed in a container with nanopure water. Measurements of $\mathrm{Na}, \mathrm{Ca}, \mathrm{Mg}$ and $\mathrm{Si}$ concentration or conductivity before and after dialysis verified the removal of surfactant. Almost all of the $\mathrm{Na}_{2} \mathrm{O}$ in the PST-1 solution was removed by a 50-day dialysis procedure. The conductivity of original MP1040 solution was $9.27 \mathrm{mS}$. After 50 days of dialysis, the conductivity of the cleaned MP1040 colloidal solution was $4.9 \mu \mathrm{S}$. At the end of dialysis procedure, the $\mathrm{pH}$ of the cleaned silica colloidal solution was adjusted to 8.0 using a saturated $\mathrm{Na}_{2} \mathrm{CO}_{3}$ solution. The mass of colloids was determined by applying the same method used for the montmorillonite colloidal solutions. Two diluted working solutions were prepared by diluting a certain volume of cleaned colloidal solution with synthetic WW20 (PST-1) or natural WW20 groundwater (MP1040). Both solutions had a concentration of $200 \mathrm{mg}$ colloids/L. The $\mathrm{pH}$ of the working solutions was 8.5 for PST-1 and 8.2 for MP1040. The particle size and zeta potential of colloidal particles in the solution were determined in the same way as the montmorillonite colloids. The zeta potential of the PST-1 silica in syn-WW20 water was $(-51.8 \pm 36.7) \mathrm{mV}$, while the zeta potential of the MP1040 silica in natural WW20 water was $(-61.7 \pm 13.4) \mathrm{mV}$. The average particle size of both silica colloids, determined by TEM and high-sensitivity liquid in-situ particle spectrometry, was 90-100 nm. The cation exchange capacity for the silica was not measured but was presumed to be much lower than for the montmorillonite. TEM images of the PST-1 and MP1040 colloids are shown in Figures 7.3 and 7.4, respectively. 


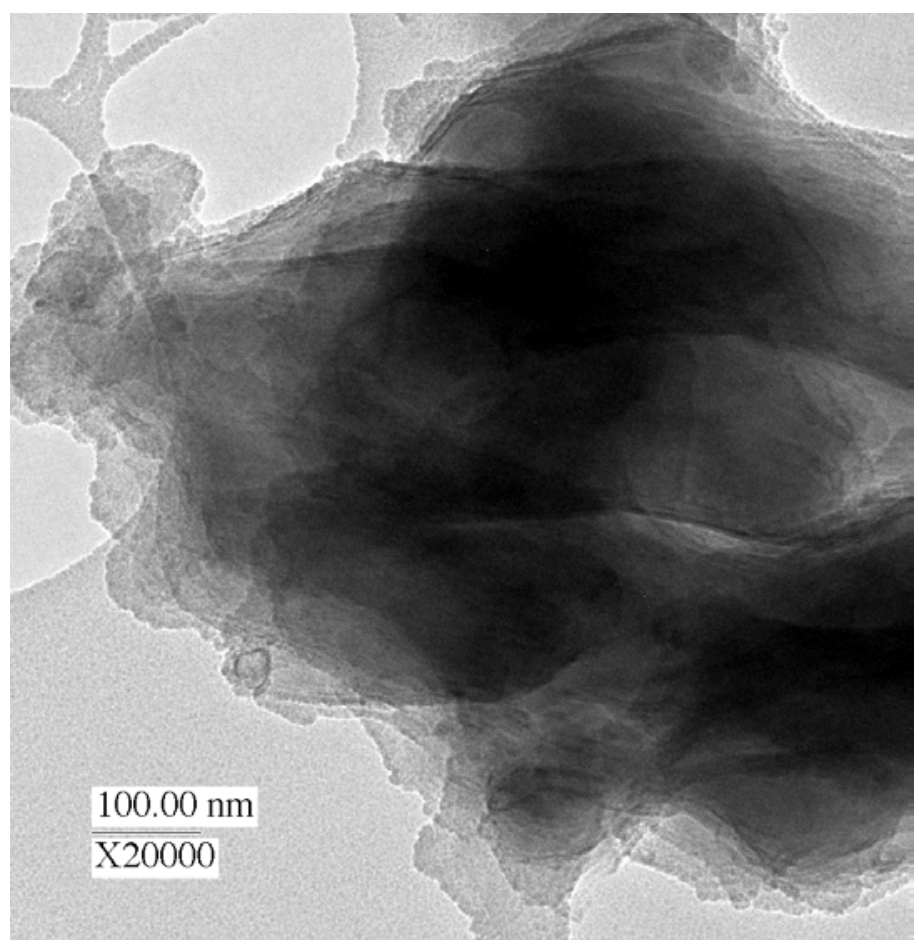

Figure 7.1. High-resolution TEM image of a Cheto montmorillonite colloid.

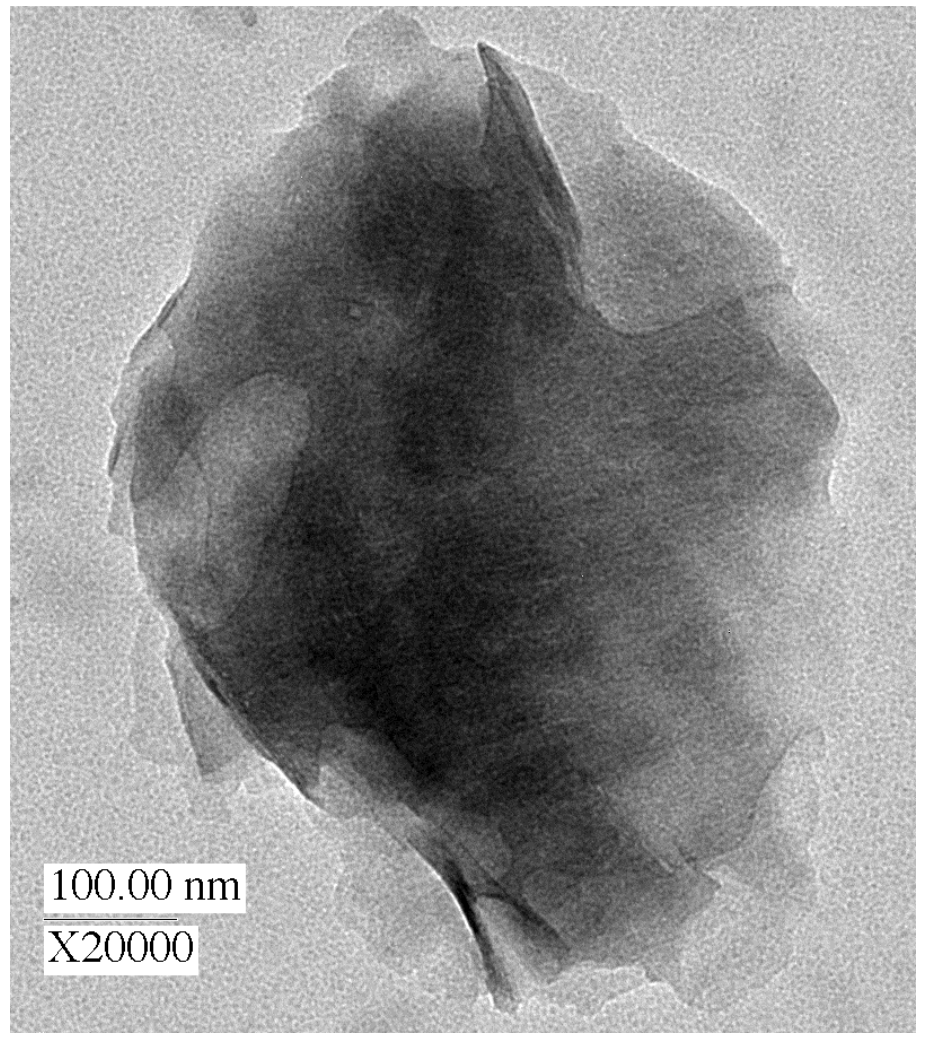

Figure 7.2. High-resolution TEM image of an Otay montmorillonite colloid. 


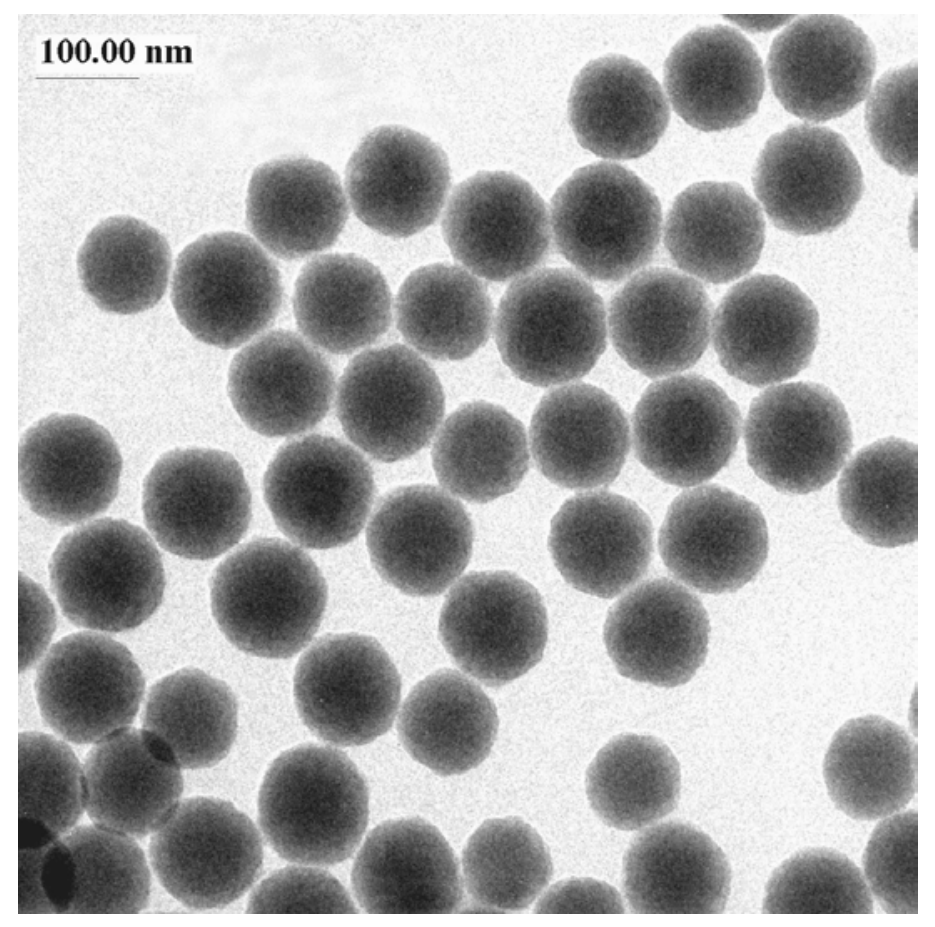

Figure 7.3. Low-resolution TEM image of PST-1 silica colloids.

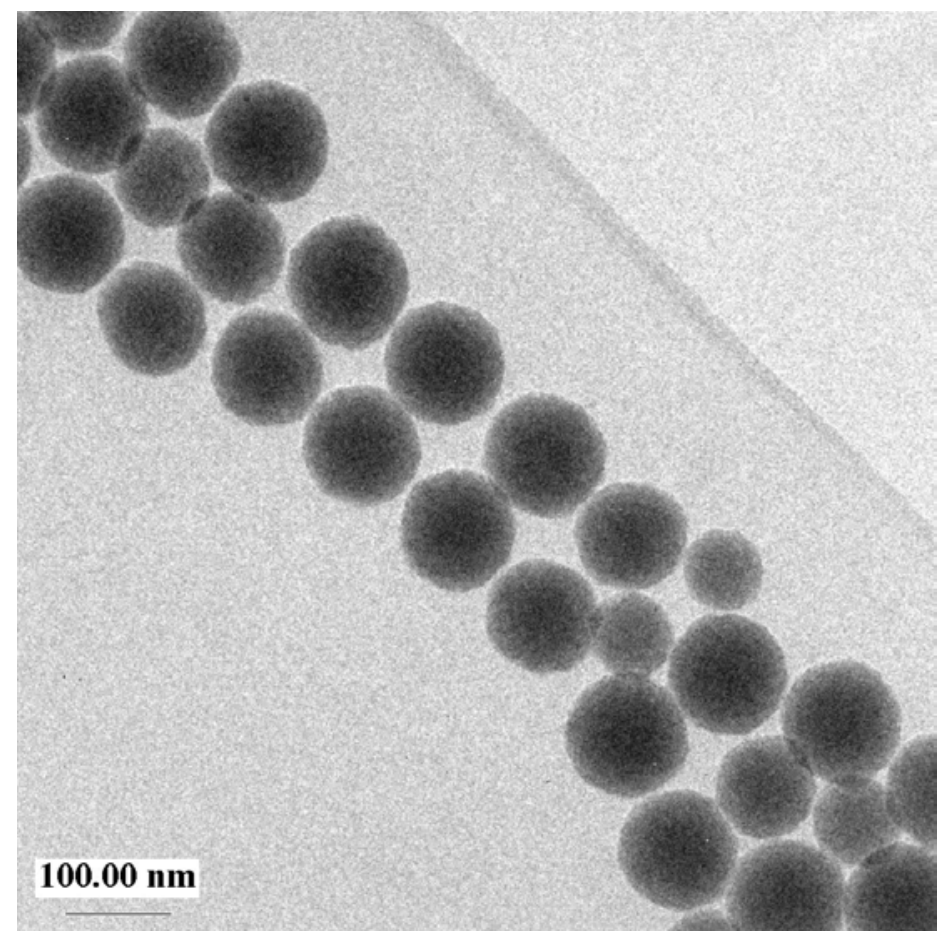

Figure 7.4. Low-resolution TEM image of MP1040 silica colloids. 


\section{Clinoptilolite Colloid Solutions}

The Pu(IV)-clinoptilolite colloid solutions were prepared by LLNL and shipped to Los Alamos for injection into the fractured cores. The quantity received was insufficient to conduct colloid characterizations similar to that conducted for the other colloids. LLNL reported that the colloid concentration was approximately $200 \mathrm{mg} / \mathrm{L}$. The size distribution of the colloids measured in association with the fracture transport experiments was similar to that of the montmorillonite colloids.

\section{Plutonium Tracer Solutions}

Three different $\mathrm{Pu}(\mathrm{V})$ stock solutions (referred to as $\mathrm{A}, \mathrm{B}$, and $\mathrm{C}$ ) were prepared for the experiments, one for the experiments in synthetic WW20 water and the UE20c fractured cores (Cheto montmorillonite and PST-1 silica), one for the experiments in natural WW20 water in the UE20c fractured cores (batch 1 Otay montmorillonite and MP1040 silica), and the third for the experiments in natural WW20 water in the PM-1 and PM-2 fractured cores (batch 2 Otay montmorillonite and one final experiment with batch 1 Otay montmorillonite). The first solution contained $\sim 7.65 \times 10^{-4} \mathrm{M} \mathrm{Pu}$ with $99.7 \%$ of $\mathrm{Pu}(\mathrm{V}), 0.3 \%$ of $\mathrm{Pu}(\mathrm{VI})$, and consisted of $93.9 \%$

${ }^{239} \mathrm{Pu}$. The second and third solutions also consisted of $>99 \% \mathrm{Pu}(\mathrm{V})$ at a lower concentration of $\sim 1 \times 10^{-5} \mathrm{M} \mathrm{Pu}$, but the isotopic composition was different from the first two solutions and is still being verified. The ratio of Pu oxidation states was determined by UV-VIS spectroscopy. In all cases, diluted $\mathrm{Pu}(\mathrm{V})$ working solutions in deionized water were prepared containing 1.0 to $2.4 \mathrm{x}$ $10^{-7} \mathrm{M}$ of $\mathrm{Pu}(\mathrm{V})$. The solutions were diluted further to between $5 \times 10^{-9}$ and $1 \times 10^{-8} \mathrm{M} \mathrm{Pu}$ for use in the batch sorption and column transport experiments.

\section{Fractured Cores}

A total of four fractured cores were used in the fracture transport experiments; two from borehole UE20c at depths of 2851 and $2858 \mathrm{ft}$ below surface, one from $4177 \mathrm{ft}$ below surface in borehole PM-2, and one from $4823 \mathrm{ft}$ below surface in borehole PM-1.

The two cores from UE20c were obtained from a heavily fractured interval of the Topopah Spring Tuff Unit. The material is described as a devitrified moderately welded tuff from the Pahute Mesa lobe of the Topopah Spring Tuff of the Paintbrush Group. It comprises a welded tuff aquifer within the Topopah Spring Aquifer (Drellack and Prothro, 1997). These cores were chosen for fracture transport experiments because

- this borehole is within $2 \mathrm{~km}$ of the ER-20-5 wells where colloid-facilitated Pu transport was observed, and it is very close to the nuclear test cavity (BENHAM) from which the $\mathrm{Pu}$ found in the ER-20-5 wells originated,

- the core was taken from the same unit of moderately-welded Topopah Spring Tuff that ER-20-5 \#1 is completed in (ER-20-5 \#1 had the highest concentrations of Pu from the BENHAM cavity), and

- the cores offered a rare opportunity to test for reproducibility and consistency of transport in fractures from the same unit at the same location.

The core from PM-2, $4177 \mathrm{ft}$ below surface is described as a devitrified lava from the dacite of Mt. Helen of the Volcanics of Quartz Mountain. This material comprises a lava flow aquifer within the Pre-Belted Range Composite Unit (Drellack and Prothro, 1997). The core from PM$1,4823 \mathrm{ft}$ below surface is described as a quartzofeldspathic altered non-welded tuff from the 
mafic-poor Bullfrog Tuff of the Crater Flat Group. This material comprises a confining unit within the Bullfrog Confining Unit (Drellack and Prothro, 1997). Descriptions of optical petrography and scanning electron microscope (SEM) investigations of the fracture surfaces and matrices of these cores are provided in Appendix A.

All four cores were bisected by a single natural axial fracture that was at least partially coated with alteration minerals (see Appendix A). The UE20c cores both had significant manganese oxide coatings on their fracture surfaces.

\subsection{Methods}

The experimental matrices for both the batch sorption/desorption experiments and the fracture transport experiments are summarized in Table 7.3. The procedures followed for these experiments are discussed in the following sections.

\section{Pu-Colloid Sorption Procedure}

Laboratory batch sorption experiments were conducted to evaluate: 1) the sorption kinetics and equilibrium partitioning of $\mathrm{Pu}(\mathrm{IV})$ and $\mathrm{Pu}(\mathrm{V})$ onto the different colloids and 2) the desorption of ${ }^{239} \mathrm{Pu}$ from the ${ }^{239} \mathrm{Pu}$-loaded colloids as a function of time. The clinoptilolite colloid suspension was supplied (by LLNL) already pre-loaded with $\mathrm{Pu}(\mathrm{IV})$, so only the partitioning of $\mathrm{Pu}(\mathrm{IV})$ between colloids and solution at the time of injection into the fractures and the desorption kinetics of the $\mathrm{Pu}$ from the colloids were measured.

For all $\mathrm{Pu}(\mathrm{V})$-colloid mixtures, sorption kinetics experiments were performed in duplicate at room temperature $\left(\sim 25^{\circ} \mathrm{C}\right)$. A $20 \mathrm{ml}$ aliquot of montmorillonite or silica colloidal solution and 1 $\mathrm{ml}$ of Pu working solution were placed into an Teflon centrifuge tube $(50 \mathrm{ml})$, and then agitated on an orbital shaker at $150 \mathrm{rpm}$. Contact periods for Cheto montmorillonite and PST-1 silica in synthetic WW20 water were 1, 4, 24, 48, 96, 240, 480, 720, and 960 hours. The contact periods for batch 1 Otay montmorillonite and MP-1040 silica in natural WW20 water were 1, 4, 24, 48, $96,240,480$, and 720 hours. The contact periods for batch 2 Otay montmorillonite with natural WW20 water were 576, 1224, 1920, and 2400 hours, and a final batch 1 Otay montmorillonite experiment was conducted with a contact period of $816 \mathrm{hrs}$ in natural WW20 water (to coincide with a final fracture injection). Batch sorption measurements were always conducted at the same time as injections into fractured cores so that the fraction of $\mathrm{Pu}(\mathrm{IV})$ or $\mathrm{Pu}(\mathrm{V})$ sorbed onto the colloids at the time of the injections was known.

For each duplicate set of test tubes and each contact period, two control samples containing 20$\mathrm{ml}$ aliquots of colloid-free synthetic or natural WW20 water and $1 \mathrm{ml}$ of Pu solution were shaken with the colloid-containing samples. At the end of each contact period, the samples and controls were centrifuged twice at $12000 \mathrm{rpm}$ for 2 hours each. This treatment should have caused all particles with size $\geq 28 \mathrm{~nm}$ to settle to the bottom of the tubes. After the first centrifugation, $\sim 15$ $\mathrm{ml}$ of supernatant was transferred to a second Teflon centrifuge tube and the centrifugation was repeated. $\sim 10 \mathrm{ml}$ of supernatant from the second tube was then collected for $\alpha$ activity and $\mathrm{pH}$ measurements. The adsorbed values of $\mathrm{Pu}(\mathrm{V})$ onto colloids of montmorillonite and silica were corrected for sorption of $\mathrm{Pu}$ to the test tube walls using the results from the control samples. No 
Table 7.3. Experimental matrices for the batch Pu sorption/desorption and column transport experiments.

Batch Sorption/Desorption Experiments:

\begin{tabular}{|c|c|c|c|}
\hline Experiment & Pu stock solution $^{(1)}$ & Colloid & Water \\
\hline 1 & $\mathrm{~A}-\mathrm{Pu}(\mathrm{V})$ & PST-1 Silica & Syn. WW-20 \\
\hline 2 & $\mathrm{~A}-\mathrm{Pu}(\mathrm{V})$ & Cheto-montmorillonite & Syn. WW-20 \\
\hline 3 & $\mathrm{~B}-\mathrm{Pu}(\mathrm{V})$ & MP1040 Silica & WW-20 \\
\hline 4 & $\mathrm{~B}-\mathrm{Pu}(\mathrm{V})$ & Otay-montmorillonite & WW-20 \\
\hline $5 *$ & $\mathrm{Pu}(\mathrm{IV})$ & Clinoptilolite & WW-20 \\
\hline 6 & $\mathrm{C}-\mathrm{Pu}(\mathrm{V})$ & Otay -montmorillonite ${ }^{(2)}$ & WW-20 \\
\hline 7 & $\mathrm{C}-\mathrm{Pu}(\mathrm{V})$ & Otay -montmorillonite & WW-20 \\
\hline
\end{tabular}

(1) Different letters denote different Pu batches.

(2) A 2nd batch of Otay-montmorillonite.

*Clinoptilolite colloids tagged with $\mathrm{Pu}(\mathrm{IV})$ were provided by LLNL for desorption kinetics and column transport experiments.

Column Transport Experiments:

\begin{tabular}{|c|c|c|c|c|}
\hline Experiment $^{(1)}$ & Borehole-Core $^{(2)}$ & Colloids & Water $^{(3)}$ & $\begin{array}{l}\text { Flow Rate, } \\
\mathrm{ml} / \mathrm{h}\end{array}$ \\
\hline $1 \mathrm{a}$ & UE20c-2851 & Cheto-montmorillonite & Syn. WW-20 (2) & 1.55 \\
\hline $1 \mathrm{~b}$ & UE20c-2858 & Cheto-montmorillonite & Syn. WW-20 (2) & 1.53 \\
\hline $2 \mathrm{a}$ & UE20c-2851 & Cheto-montmorillonite & Syn. WW-20 (2) & 0.575 \\
\hline $2 b$ & UE20c-2858 & Cheto-montmorillonite & Syn. WW-20 (2) & 0.587 \\
\hline $3 \mathrm{a}$ & UE20c-2851 & MP1040 Silica & WW-20 (3) & 1.43 \\
\hline $3 b$ & UE20c-2858 & MP1040 Silica & WW-20 (3) & 1.43 \\
\hline $4 a$ & UE20c-2851 & MP1040 Silica & WW-20 (3) & 5.23 \\
\hline $4 \mathrm{~b}$ & UE20c-2858 & MP1040 Silica & WW-20 (3) & 4.75 \\
\hline $5 a$ & UE20c-2851 & Otay-montmorillonite & WW-20 (4) & 1.42 \\
\hline $5 b$ & UE20c-2858 & Otay-montmorillonite & WW-20 (4) & 1.43 \\
\hline $6 a$ & UE20c-2851 & Otay-montmorillonite & WW-20 (4) & 4.84 \\
\hline $6 b$ & UE20c-2858 & Otay-montmorillonite & WW-20 (4) & 4.79 \\
\hline $7 \mathrm{a}$ & PM2-4177 & Clinoptilolite & WW-20(5) & 1.41 \\
\hline $7 b$ & PM1-4823 & Clinoptilolite & WW-20(5) & 1.41 \\
\hline $8 a$ & PM2-4177 & Clinoptilolite & WW-20(5) & 0.53 \\
\hline $8 b$ & PM1-4823 & Clinoptilolite & WW-20(5) & 0.54 \\
\hline $9 \mathrm{a}$ & PM2-4177 & Otay-montmorillonite ${ }^{*}$ & WW-20(6) & 1.40 \\
\hline $9 \mathrm{~b}$ & PM1-4823 & Otay-montmorillonite & WW-20(6) & 1.63 \\
\hline $10 \mathrm{a}$ & PM2-4177 & Otay-montmorillonite ${ }^{*}$ & WW-20(6) & 0.42 \\
\hline $10 \mathrm{~b}$ & PM1-4823 & Otay-montmorillonite ${ }^{*}$ & WW-20(6) & 0.45 \\
\hline $11 \mathrm{a}$ & PM2-4177 & Otay-montmorillonite & WW-20(7) & 1.47 \\
\hline
\end{tabular}

(1) $a$ and $b$ experiments were run at the same time (side by side).

${ }^{(2)}$ Numbers indicate depth (below surface) from which cores were taken from borehole UE-20c.

${ }^{(3)}$ Number in parentheses indicates the batch sorption experiment that supplied Pu-colloids.

*2nd batch of Otay-Montmorillonite 
controls were run with the $\mathrm{Pu}(\mathrm{IV})$-clinoptilolite samples because of a lack of $\mathrm{Pu}(\mathrm{IV})$ solution at Los Alamos.

The sorption distribution coefficient $\left(\mathrm{K}_{\mathrm{d}}\right.$ value) of $\mathrm{Pu}$ onto colloids was calculated using the following equation (Lu et al., 1998):

$$
\mathrm{K}_{\mathrm{d}} \text { value }(\mathrm{ml} / \mathrm{g})=\left[\left(\mathrm{A}_{\mathrm{o}} \mathrm{V}_{\mathrm{o}}-\mathrm{A}_{\mathrm{f}} \mathrm{V}_{\mathrm{f}}\right) / \mathrm{M}\right] / \mathrm{A}_{\mathrm{f}}
$$

where $A_{o}$ is the initial $\alpha$ activity of $\mathrm{Pu}(\mathrm{V})$ per ml solution; $\mathrm{V}_{\mathrm{o}}$ is the initial volume $(\mathrm{ml})$ of $\mathrm{Pu}$ working solution in contact with colloids; $\mathrm{A}_{\mathrm{f}}$ is the final $\alpha$ activity of $\mathrm{Pu}(\mathrm{V})$ per ml solution after sorption (minus the activity of $\mathrm{Pu}(\mathrm{V})$ determined from the controls to be sorbing to test tube walls), $V_{\mathrm{f}}$ is the final volume ( $\left.\mathrm{ml}\right)$ of the solution after sorption, and $\mathrm{M}(\mathrm{g})$ is the mass of colloids used in the sorption process. Alpha activities were determined by liquid scintillation counting.

\section{Pu-Colloid Desorption Procedure}

Desorption experiments, including the desorption of $\mathrm{Pu}(\mathrm{IV})$ from the clintoptilolite colloids, were conducted in duplicate under the same conditions as the sorption experiments. In the experiments with Cheto montmorillonite and PST-1 silica in synthetic WW20 water, two ${ }^{239} \mathrm{Pu}-$ loaded colloid samples from each of the 96-, 240-, and 720- hr sorption experiments were used for desorption experiments. A 4-hr desorption experiment was conducted using the colloids from the 96-hr sorption test, and 24-hr and 320-day desorption experiments were conducted using colloids from the 240- and 720-hr sorption tests, respectively. In the experiments with batch 1 Otay montmorillonite and silica MP-1040, six ${ }^{239} \mathrm{Pu}$-loaded colloid samples from the 240-hr sorption experiments were used for duplicate desorption experiments of 24-hr, 48-hr, and 225-day durations. Desorption of $\mathrm{Pu}(\mathrm{IV})$ from clinoptilolite colloids was measured after $24 \mathrm{hrs}$, $48 \mathrm{hrs}, 61$ days, and 126 days of desorption time. The sorption contact period prior to desorption was $456 \mathrm{hrs}$ in all cases except for the 126-day desorption experiment, which had a sorption contact period of $984 \mathrm{hrs}$ (41 days). Desorption experiments with the batch 2 Otay montmorillonite in natural WW20 water were conducted for $24 \mathrm{hrs}, 48 \mathrm{hrs}, 41$ days, and 100 days, with the sorption contact periods being $576 \mathrm{hrs}, 984 \mathrm{hrs}, 80$ days, and 100 days, respectively. Finally, additional desorption experiments with the batch 1 Otay montmorillonite were conducted for 240, 480, and $960 \mathrm{hrs}$ after an 816-hr sorption contact period in natural WW20 water.

At the end of the sorption process, the colloids were concentrated at the bottom of the test tubes by centrifuging at 12,000 rpm for $2 \mathrm{hr}$. All of the supernatant was carefully removed, and the wet weights of the colloids were measured. $20 \mathrm{ml}$ of Pu-free synthetic or natural WW20 water was then added to the test tube to start the desorption experiment. The desorption experiments were conducted in the same way as the sorption experiments. The $\alpha$ activity of ${ }^{239} \mathrm{Pu}$ in the solutions was measured using liquid scintillation counting. The residual $\mathrm{Pu}$ remaining in solution after the sorption experiment was accounted for when calculating the amount of $\mathrm{Pu}$ that remained sorbed to the colloids after the desorption experiment. 


\section{Fracture Transport Experimental Procedure}

The fractured cores were cut perpendicular to their axes such that the fracture bisected the length of the resulting core section. The core was then encapsulated in epoxy (while carefully keeping epoxy out of the fractures) and fitted with end caps that allowed water to be introduced/collected uniformly across the fracture trace on either end of the core (Figure 7.5). The final dimensions of the cut cores are listed in Tables 7.4 through 7.14. After assembly, the cores were saturated by (1) evacuating them under vacuum, (2) introducing $\mathrm{CO}_{2}$ gas to displace oxygen and nitrogen, (3) evacuating the $\mathrm{CO}_{2}$ using vacuum, (4) slowly introducing degassed water while still maintaining vacuum, and finally (5) keeping the cores under water and under vacuum until they no longer evolved gas bubbles. The $\mathrm{CO}_{2}$ was introduced because it dissolves in water more readily than oxygen or nitrogen. This method has proven to significantly reduce times required for saturation.

Once the cores were saturated, steady flow of degassed synthetic WW20 water was established through the fractures using syringe pumps ( $\mathrm{K}_{\mathrm{D}}$ Scientific, Inc.). A pulse of tracer solution was introduced by simply switching syringes from one charged with WW20 water to one charged with $\mathrm{Pu}$-colloid product solution from the batch sorption experiments. In all but one set of experiments, the syringes were switched back so that tracer-free WW-20 water was injected for the remainder of the test after the tracer solution was injected. The switchover was done with negligible flow interruption. In one set of montmorillonite experiments in the PM1 and PM2 fractures, the WW-20 water injected into the fractures immediately after the Pu-colloid injection contained montmorillonite colloids at the same concentration as in the Pu-colloid pulse (but with no $\mathrm{Pu}$ in the solution). These experiments were intended to determine if the $\mathrm{Pu}$ recovery could be increased by continuously injecting colloids into the fractures, thus providing mobile surface area for the $\mathrm{Pu}$ to sorb onto. The percentage of $\mathrm{Pu}$ sorbed to the colloids at the time of tracer injection into the cores was determined from batch sorption measurements that were conducted within one day of the column injections. Prior to injection, the tracer solution was spiked with tritiated water so that tritium could be used as a conservative (non-sorbing) tracer in the experiments. The exact concentration of tritium was not important because all effluent concentrations were normalized to the injection concentrations.

Effluent from the fractured cores was collected using an automatic fraction collector. The experimental setup is illustrated in Figure 7.6. Fractions averaged about $1 \mathrm{ml}$ each until well after the peak tracer concentrations were established, at which time the fraction volume was increased to about $6 \mathrm{ml}$ to offer better analytical sensitivity. Teflon tubing was used to connect the syringes to the columns and the columns to the fraction collector. The fractions were split into two roughly equal-volume aliquots with one aliquot for colloid analyses and the other for tritium and plutonium analyses using liquid scintillation counting. The colloid aliquot was immediately diluted with ultrafiltered deionized water to stabilize the colloids before analysis. The dilution factor used was about 10, although the samples had to be diluted considerably more prior to analysis. Separate colloid stability experiments (Appendix B) indicated that measured 


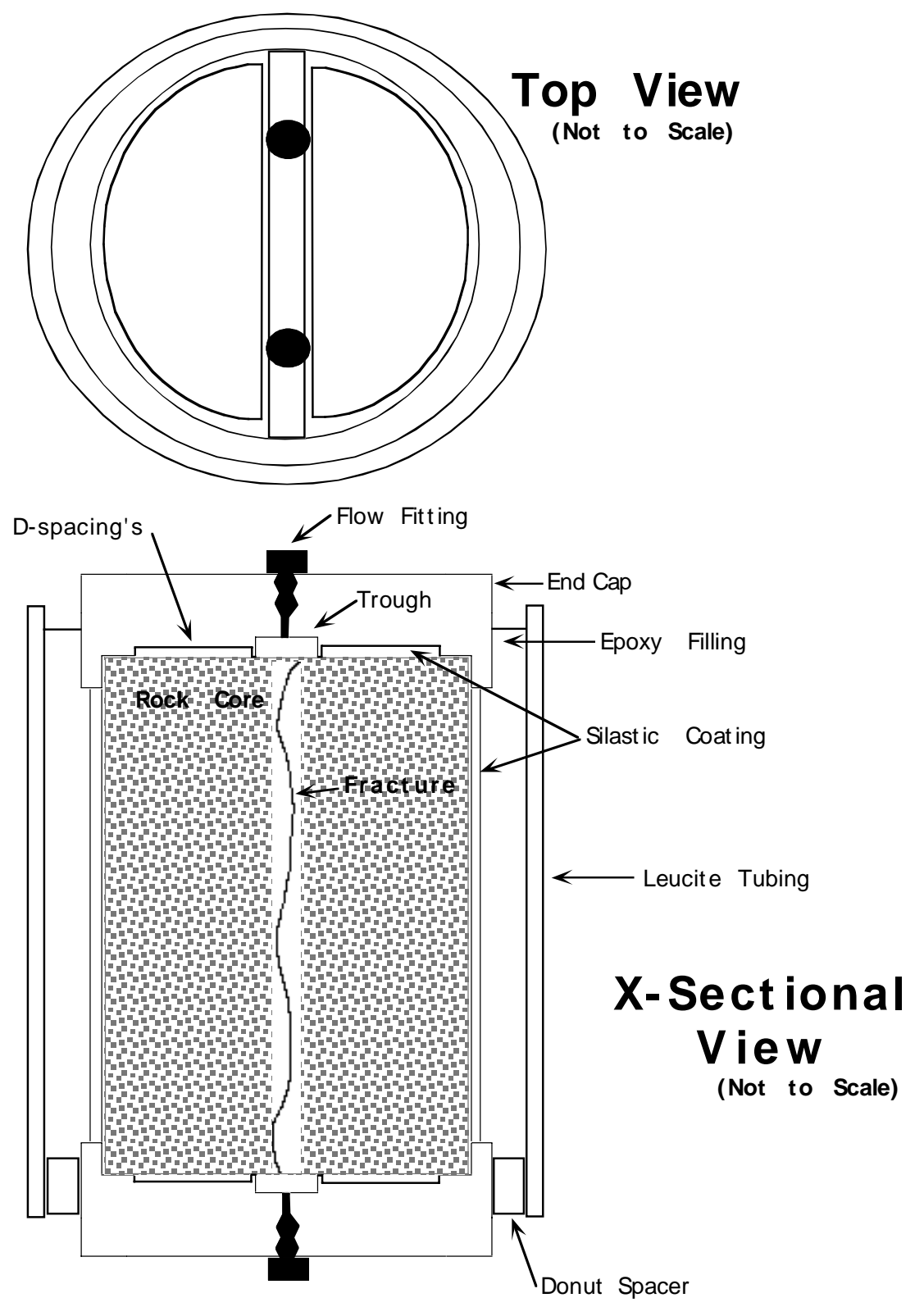

Figure 7.5 Fracture column assembly used in $\mathrm{Pu}$-colloid experiments.

colloid concentrations changed significantly over a 3-week period in syn. WW20 water, but the samples remained stable for several weeks after dilution in deionized water.

The colloids were analyzed using a 16-channel high sensitivity liquid in-situ particle spectrometer, HSLIS-S50, manufactured by Particle Measuring System, Inc. (Boulder, Colorado). This instrument is configured to measure total colloid concentrations within the diameter range of 50 to $1000 \mathrm{~nm}$. Size distributions were measured for all colloid injection solutions and for all effluent samples, but they are not reported here. Although some of the montmorillonite colloids were smaller than the lower end of this range, it was assumed that the response within this range was reasonably representative of the overall colloid response. 


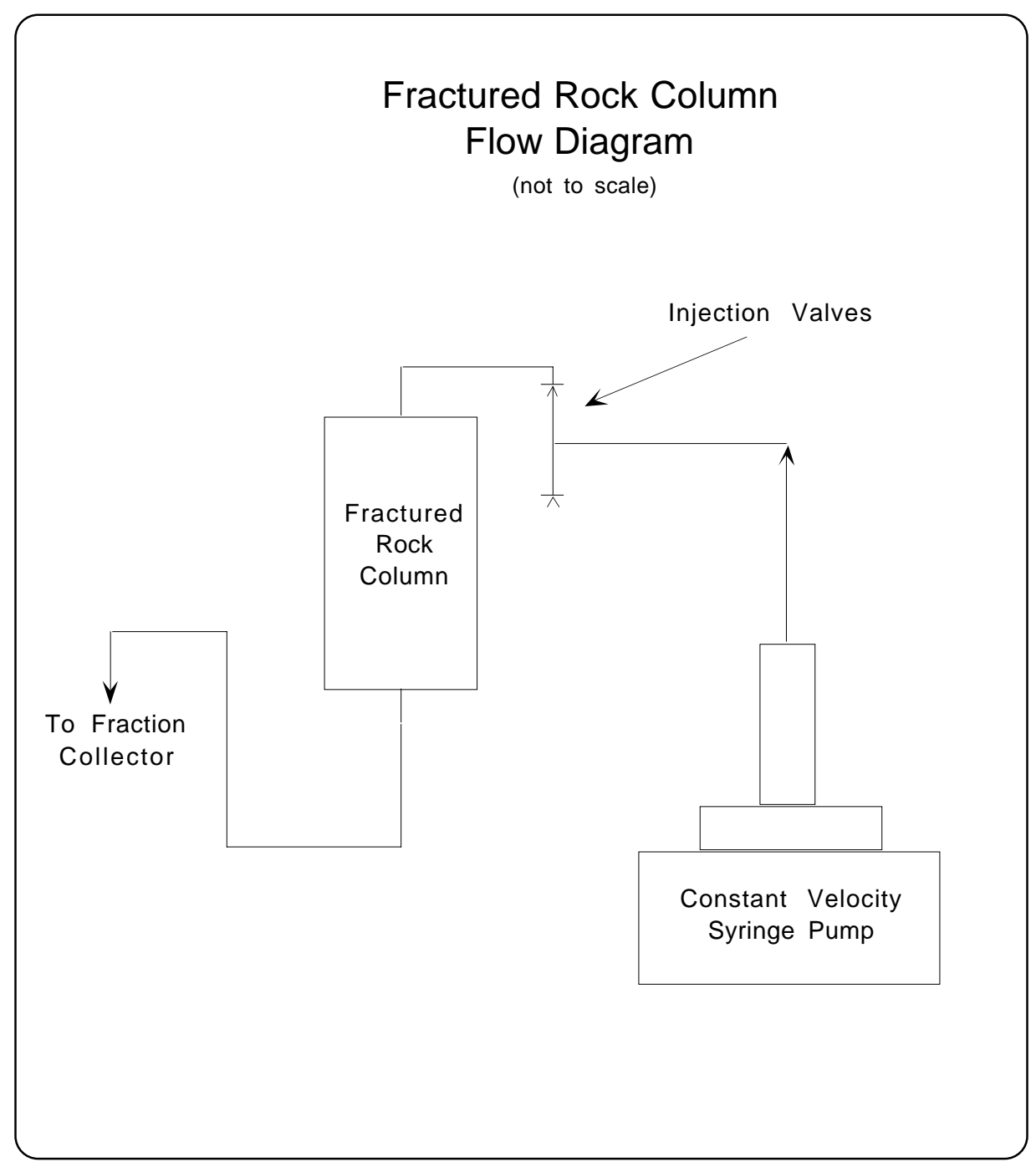

Figure 7.6. Experimental setup for fractured column experiments.

In the case of the silica colloids, the size distributions were very narrow, and all the colloids fell within the size range of the instrument. The colloid size distributions also did not change appreciably over time in the column effluents, provided that the effluents were immediately diluted in deionized water. Tritium and plutonium were analyzed simultaneously using a liquid scintillation counter equipped with alpha/beta discriminating capabilities (Packard $2550 \mathrm{TR} / \mathrm{AB}$ ).

The experimental conditions in all of fracture experiments are summarized in Tables 7.4 through 7.14. For each of the different Pu-colloid combinations, two experiments were run in each of two fractured cores. The first experiment was always conducted at approximately $1.5 \mathrm{ml} / \mathrm{hr}$, and the second was conducted at either $\sim 0.5 \mathrm{ml} / \mathrm{hr}$ or $\sim 5 \mathrm{ml} / \mathrm{hr}$, depending on the $\mathrm{Pu}$ and colloid responses in the first experiment. The flow rate was lowered in the second experiment if there were relatively high recoveries of both $\mathrm{Pu}$ and colloids in the first experiment (as in the 
Table 7.4. Experimental parameters in the two Cheto montmorillonite experiments conducted in the fracture from UE20c $2851 \mathrm{ft}$.

\begin{tabular}{|l|c|c|}
\hline \multicolumn{2}{|l|}{ Length: $16.7 \mathrm{~cm}$} & Diameter: $8.7 \mathrm{~cm}$ \\
\hline $\begin{array}{l}\text { Experiment } \\
\text { Number }\end{array}$ & $1 \mathrm{a}$ & $2 \mathrm{a}$ \\
\hline $\begin{array}{l}\text { Flow Rate } \\
\text { (mL/hr) }\end{array}$ & 1.55 & 0.575 \\
\hline $\begin{array}{l}\text { Injection } \\
\text { Duration (hr) }\end{array}$ & 7.73 & 24.4 \\
\hline $\begin{array}{l}\text { Injection } \\
\text { Concentrations }\end{array}$ & $\begin{array}{l}{ }^{3} \mathrm{H}-1069 \mathrm{CPM} / \mathrm{g} \\
\text { Colloids }-3.95 \times 10^{9} / \mathrm{ml}\end{array}$ & $\begin{array}{l}{ }^{3} \mathrm{H}-1072 \mathrm{CPM} / \mathrm{g} \\
\text { Colloids }-1.99 \times 10^{9} / \mathrm{ml}\end{array}$ \\
\hline
\end{tabular}

Note: The matrix porosity of this rock was measured to be $\sim 0.21$.

Table 7.5. Experimental parameters in the two Cheto montmorillonite experiments conducted in the fracture from UE20c $2858 \mathrm{ft}$.

\begin{tabular}{|l|c|c|}
\hline \hline \multicolumn{2}{|l|}{ Length: $21.9 \mathrm{~cm}$} & Diameter: $8.7 \mathrm{~cm}$ \\
\hline \hline $\begin{array}{l}\text { Experiment } \\
\text { Number }\end{array}$ & $1 \mathrm{~b}$ & $2 \mathrm{~b}$ \\
\hline $\begin{array}{l}\text { Flow Rate } \\
\text { (mL/hr) }\end{array}$ & 1.53 & 0.587 \\
\hline $\begin{array}{l}\text { Injection } \\
\text { Duration (hr) }\end{array}$ & 7.52 & 23.4 \\
\hline $\begin{array}{l}\text { Injection } \\
\text { Concentrations }\end{array}$ & $\begin{array}{l}{ }^{3} \mathrm{H}-1069 \mathrm{CPM} / \mathrm{g} \\
{ }^{239} \mathrm{Pu}-296 \mathrm{CPM} / \mathrm{g} \\
\text { Colloids }-3.95 \times 10^{9} / \mathrm{ml}\end{array}$ & $\begin{array}{l}{ }^{3} \mathrm{H}-651 \mathrm{CPM} / \mathrm{g} \\
{ }^{239} \mathrm{Pu}-393 \mathrm{CPM} / \mathrm{g} \\
\text { Colloids }-2.25 \times 10^{9} / \mathrm{ml}\end{array}$ \\
\hline
\end{tabular}

Note: The matrix porosity of this rock was measured to be $\sim 0.21$.

Table 7.6. Experimental parameters in the two silica experiments conducted in the fracture from UE20c $2851 \mathrm{ft}$.

\begin{tabular}{|l|c|c|}
\hline \hline \multicolumn{2}{|l|}{ Length: $16.7 \mathrm{~cm}$} & Diameter: $8.7 \mathrm{~cm}$ \\
\hline \hline $\begin{array}{l}\text { Experiment } \\
\text { Number }\end{array}$ & $3 \mathrm{a}$ & $4 \mathrm{a}$ \\
\hline $\begin{array}{l}\text { Flow Rate } \\
\text { (mL/hr) }\end{array}$ & 1.43 & 4.70 \\
\hline $\begin{array}{l}\text { Injection } \\
\text { Duration (hr) }\end{array}$ & 16.4 & 5.23 \\
\hline $\begin{array}{l}\text { Injection } \\
\text { Concentrations }\end{array}$ & $\begin{array}{l}{ }^{3} \mathrm{H}-669 \mathrm{CPM} / \mathrm{g} \\
\mathrm{Cu}-396 \mathrm{CPM} / \mathrm{g} \\
\text { Colloids }-4.8 \times 10^{10} / \mathrm{ml}\end{array}$ & $\begin{array}{l}{ }^{3} \mathrm{H}-3884 \mathrm{CPM} / \mathrm{g} \\
\text { Colloids }-4.8 \times 10^{10} / \mathrm{ml}\end{array}$ \\
\hline
\end{tabular}

Note: The matrix porosity of this rock was measured to be $\sim 0.21$. 
Table 7.7. Experimental parameters in the two silica experiments conducted in the fracture from UE20c $2858 \mathrm{ft}$.

\begin{tabular}{|l|c|c|}
\hline \hline \multicolumn{2}{|l|}{ Length: $21.9 \mathrm{~cm}$} & Diameter: $8.7 \mathrm{~cm}$ \\
\hline \hline $\begin{array}{l}\text { Experiment } \\
\text { Number }\end{array}$ & $3 \mathrm{~b}$ & $4 \mathrm{~b}$ \\
\hline $\begin{array}{l}\text { Flow Rate } \\
\text { (mL/hr) }\end{array}$ & 1.43 & 4.75 \\
\hline $\begin{array}{l}\text { Injection } \\
\text { Duration (hr) }\end{array}$ & 16.3 & 5.18 \\
\hline $\begin{array}{l}\text { Injection } \\
\text { Concentrations }\end{array}$ & $\begin{array}{l}{ }^{3} \mathrm{H}-665 \mathrm{CPM} / \mathrm{g} \\
\mathrm{23u}-399 \mathrm{CPM} / \mathrm{g}\end{array}$ & $\begin{array}{l}{ }^{3} \mathrm{H}-3884 \mathrm{CPM} / \mathrm{g} \\
\mathrm{23} \mathrm{Pu}-389 \mathrm{CPM} / \mathrm{g} \\
\text { Colloids }-4.8 \times 10^{10} / \mathrm{ml}-4.8 \times 10^{10} / \mathrm{ml}\end{array}$ \\
\hline
\end{tabular}

Note: The matrix porosity of this rock was measured to be $\sim 0.21$.

Table 7.8. Experimental parameters in the two batch 1 Otay montmorillonite experiments conducted in the fracture from UE20c $2851 \mathrm{ft}$.

\begin{tabular}{|l|c|c|}
\hline \hline \multicolumn{2}{|l|}{ Length: $16.7 \mathrm{~cm}$} & Diameter: $8.7 \mathrm{~cm}$ \\
\hline \hline $\begin{array}{l}\text { Experiment } \\
\text { Number }\end{array}$ & $5 \mathrm{a}$ & $6 \mathrm{a}$ \\
\hline $\begin{array}{l}\text { Flow Rate } \\
\text { (mL/hr) }\end{array}$ & 1.42 & 4.84 \\
\hline $\begin{array}{l}\text { Injection } \\
\text { Duration (hr) }\end{array}$ & 10.07 & 4.60 \\
\hline $\begin{array}{l}\text { Injection } \\
\text { Concentrations }\end{array}$ & $\begin{array}{l}{ }^{3} \mathrm{H}-2650 \mathrm{CPM} / \mathrm{g} \\
\mathrm{2u}-678 \mathrm{CPM} / \mathrm{g} \\
\text { Colloids }-6.47 \times 10^{9} / \mathrm{ml}\end{array}$ & $\begin{array}{l}{ }^{3} \mathrm{H}-2220 \mathrm{CPM} / \mathrm{g} \\
\mathrm{2} 9 \mathrm{Pu}-506 \mathrm{CPM} / \mathrm{g} \\
\text { Colloids }-5.65 \times 10^{9} / \mathrm{ml}\end{array}$ \\
\hline
\end{tabular}

Note: The matrix porosity of this rock was measured to be $\sim 0.21$.

Table 7.9. Experimental parameters in the two batch 1 Otay montmorillonite experiments conducted in the fracture from UE20c $2858 \mathrm{ft}$.

\begin{tabular}{|c|c|c|}
\hline \multicolumn{2}{|c|}{ Length: $21.9 \mathrm{~cm}$} & Diameter: $8.7 \mathrm{~cm}$ \\
\hline $\begin{array}{l}\text { Experiment } \\
\text { Number }\end{array}$ & $5 b$ & $6 \mathrm{~b}$ \\
\hline $\begin{array}{l}\text { Flow Rate } \\
(\mathrm{mL} / \mathrm{hr})\end{array}$ & 1.43 & 4.79 \\
\hline $\begin{array}{l}\text { Injection } \\
\text { Duration (hr) } \\
\end{array}$ & 9.78 & 4.42 \\
\hline $\begin{array}{l}\text { Injection } \\
\text { Concentrations }\end{array}$ & $\begin{array}{l}{ }^{3} \mathrm{H}-2650 \mathrm{CPM} / \mathrm{g} \\
{ }^{239} \mathrm{Pu}-678 \mathrm{CPM} / \mathrm{g} \\
\text { Colloids }-6.47 \times 10^{9} / \mathrm{ml}\end{array}$ & $\begin{array}{l}{ }^{3} \mathrm{H}-2220 \mathrm{CPM} / \mathrm{g} \\
{ }^{239} \mathrm{Pu}-506 \mathrm{CPM} / \mathrm{g} \\
\text { Colloids }-5.65 \times 10^{9} / \mathrm{ml}\end{array}$ \\
\hline
\end{tabular}

Note: The matrix porosity of this rock was measured to be $\sim 0.21$. 
Table 7.10. Experimental parameters in the two clinoptilolite experiments conducted in the fracture from PM2-4177 ft.

\begin{tabular}{|l|c|c|}
\hline \hline \multicolumn{2}{|l|}{ Length: $12.6 \mathrm{~cm}$} & Diameter: $8.7 \mathrm{~cm}$ \\
\hline \hline $\begin{array}{l}\text { Experiment } \\
\text { Number }\end{array}$ & $7 \mathrm{a}$ & $8 \mathrm{a}$ \\
\hline $\begin{array}{l}\text { Flow Rate } \\
\text { (mL/hr) }\end{array}$ & 1.41 & 0.53 \\
\hline $\begin{array}{l}\text { Injection } \\
\text { Duration }(\mathrm{hr})\end{array}$ & 16.61 & 51.6 \\
\hline $\begin{array}{l}\text { Injection } \\
\text { Concentrations }\end{array}$ & $\begin{array}{l}{ }^{3} \mathrm{H}-5537.13 \mathrm{CPM} / \mathrm{g} \\
\mathrm{Pu}-232.4 \mathrm{CPM} / \mathrm{g}\end{array}$ & $\begin{array}{l}{ }^{3} \mathrm{H}-5615.71 \mathrm{CPM} / \mathrm{g} \\
\text { Colloids }-5.215 \times 10^{10} / \mathrm{ml}-236.38 \mathrm{CPM} / \mathrm{g} \\
\text { Colloids }-5.215 \times 10^{10} / \mathrm{ml}\end{array}$ \\
\hline
\end{tabular}

Note: The matrix porosity of this rock was measured to be $\sim 0.17$.

Table 7.11. Experimental parameters in the two Clinoptilolite experiments conducted in the fracture from PM1-4823 ft.

\begin{tabular}{|l|c|c|}
\hline \hline \multicolumn{2}{|l|}{ Length: $16.3 \mathrm{~cm}$} & Diameter: $8.7 \mathrm{~cm}$ \\
\hline \hline $\begin{array}{l}\text { Experiment } \\
\text { Number }\end{array}$ & $7 \mathrm{~b}$ & $8 \mathrm{~b}$ \\
\hline $\begin{array}{l}\text { Flow Rate } \\
\text { (mL/hr) }\end{array}$ & 1.41 & 0.54 \\
\hline $\begin{array}{l}\text { Injection } \\
\text { Duration (hr) }\end{array}$ & 16.61 & 51.6 \\
\hline $\begin{array}{l}\text { Injection } \\
\text { Concentrations }\end{array}$ & $\begin{array}{l}{ }^{3} \mathrm{H}-5537.13 \mathrm{CPM} / \mathrm{g} \\
{ }^{239} \mathrm{Pu}-232.4 \mathrm{CPM} / \mathrm{g} \\
\text { Colloids }-5.215 \times 10^{10} / \mathrm{ml}\end{array}$ & $\begin{array}{l}{ }^{3} \mathrm{H}-5586.67 \mathrm{CPM} / \mathrm{g} \\
\mathrm{239} \mathrm{Pu}-236.19 \mathrm{CPM} / \mathrm{g} \\
\text { Colloids }-5.215 \times 10^{10} / \mathrm{ml}\end{array}$ \\
\hline
\end{tabular}

Note: The matrix porosity of this rock was measured to be $\sim 0.24$.

Table 7.12. Experimental parameters in the two batch 2 Otay montmorillonite experiments conducted in the fracture from PM2-4177 ft.

\begin{tabular}{|c|c|c|}
\hline \multicolumn{2}{|c|}{ Length: $12.6 \mathrm{~cm}$} & Diameter: $8.7 \mathrm{~cm}$ \\
\hline $\begin{array}{l}\text { Experiment } \\
\text { Number }\end{array}$ & $9 a$ & $10 \mathrm{a}$ \\
\hline $\begin{array}{l}\text { Flow Rate } \\
(\mathrm{mL} / \mathrm{hr})\end{array}$ & 1.40 & 0.42 \\
\hline $\begin{array}{l}\text { Injection } \\
\text { Duration (hr) }\end{array}$ & 13.42 & 35.55 \\
\hline $\begin{array}{l}\text { Injection } \\
\text { Concentrations }\end{array}$ & $\begin{array}{l}{ }^{3} \mathrm{H}-4828.16 \mathrm{CPM} / \mathrm{g} \\
{ }^{239} \mathrm{Pu}-1592.01 \mathrm{CPM} / \mathrm{g} \\
\text { Colloids }-8.33 \times 10^{9} / \mathrm{ml}\end{array}$ & $\begin{array}{l}{ }^{3} \mathrm{H}-4940.88 \mathrm{CPM} / \mathrm{g} \\
{ }^{239} \mathrm{Pu}-4172.69 \mathrm{CPM} / \mathrm{g} \\
\text { Colloids }-1.21 \times 10^{10} / \mathrm{ml}\end{array}$ \\
\hline
\end{tabular}

Note: The matrix porosity of this rock was measured to be $\sim 0.17$. 
Table 7.13. Experimental parameters in the two batch 2 Otay montmorillonite experiments conducted in the fracture from PM1-4823 ft.

\begin{tabular}{|l|c|c|}
\hline \hline \multicolumn{2}{|l|}{ Length: $16.3 \mathrm{~cm}$} & Diameter: $8.7 \mathrm{~cm}$ \\
\hline \hline $\begin{array}{l}\text { Experiment } \\
\text { Number }\end{array}$ & $9 \mathrm{~b}$ & $10 \mathrm{~b}$ \\
\hline $\begin{array}{l}\text { Flow Rate } \\
\text { (mL/hr) }\end{array}$ & 1.63 & 0.45 \\
\hline $\begin{array}{l}\text { Injection } \\
\text { Duration (hr) }\end{array}$ & 13.42 & 35.55 \\
\hline $\begin{array}{l}\text { Injection } \\
\text { Concentrations }\end{array}$ & $\begin{array}{l}{ }^{3} \mathrm{H}-4839.75 \mathrm{CPM} / \mathrm{g} \\
\mathrm{2u}-1558.07 \mathrm{CPM} / \mathrm{g}\end{array}$ & $\begin{array}{l}{ }^{3} \mathrm{H}-4963.48 \mathrm{CPM} / \mathrm{g} \\
\text { Colloids }-8.33 \times 10^{9} / \mathrm{ml}-4120.47 \mathrm{CPM} / \mathrm{g} \\
\text { Colloids }-1.21 \times 10^{10} / \mathrm{ml}\end{array}$ \\
\hline
\end{tabular}

Note: The matrix porosity of this rock was measured to be $\sim 0.24$.

Table 7.14. Experimental parameters in the batch 1 Otay-montmorillonite experiment conducted in the fracture from PM2-4177 ft (experiment 11).

\begin{tabular}{|l|c|}
\hline \hline \multicolumn{2}{|l|}{ Length: $12.6 \mathrm{~cm}$; Diameter: $8.7 \mathrm{~cm}$} \\
\hline $\begin{array}{l}\text { Flow Rate } \\
\text { (mL/hr) }\end{array}$ & 1.47 \\
\hline $\begin{array}{l}\text { Injection } \\
\text { Duration (hr) }\end{array}$ & 24.92 \\
\hline $\begin{array}{l}\text { Injection } \\
\text { Concentrations }\end{array}$ & $\begin{array}{l}{ }^{3} \mathrm{H}-5296.68 \mathrm{CPM} / \mathrm{g} \\
\text { Colloids }-1.46 \times 10^{10} / \mathrm{ml}\end{array}$ \\
\hline
\end{tabular}

Note: The matrix porosity of this rock was measured to be $\sim 0.17$.

case of the Cheto-montmorillonite), and the flow rate was raised in the second experiment if the recoveries of either the $\mathrm{Pu}$ or colloids were very low in the first. The rationale was that it was desirable to try to obtain a measurable response of all injected species, but it was also desirable to try to keep flow rates as low as possible to approximate field conditions more closely. 0.5 $\mathrm{ml} / \mathrm{hr}$ is about the lowest flow rate the syringe pumps could deliver at a reasonably steady rate. The flow rate in the two sets of experiments was varied by approximately a factor of 3 so that matrix diffusion in the cores could be quantified by comparing the tritium responses at the two rates. The response at the lower flow rate was expected to be slightly retarded and lower in normalized concentration than the response at the higher flow rate because the increased residence time allows more opportunity for tritium diffusion into the matrix.

In the lower flow rate experiments involving $\mathrm{Pu}(\mathrm{V})$ and the batch 2 Otay montmorillonite colloids (PM-1 and PM-2 fractures), Pu-free colloids were continuously injected into the fractures (at a constant concentration) after the tritium and $\mathrm{Pu}$ injections had ceased. The purpose of these experiments was to determine if any of the immobilized $\mathrm{Pu}$ in the fractures would sorb onto the mobile colloids and be transported out of the fractures. 
At the conclusion of the fracture transport experiments, the flow systems were cut open to expose the fracture surfaces for post-test analyses. These analyses included:

(1) assessing the microbial activity in the fractures at the conclusion of the tests (UE20c fractures only),

(2) determining in a very qualitative way where the alpha activity was concentrated on the fracture surfaces using a hand-and-foot monitor,

(3) conducting macro- and micro-autoradiographic determinations of where Pu activity was concentrated on the surfaces and what mineral phases it might be associated with, and

(4) conducting laser-ablation secondary ion mass spectrometry (SIMS) measurements to try to identify elements and mineral phases that sorbed $\mathrm{Pu}$ is associated with on the fracture surfaces.

The autoradiography and SIMS work, which was conducted at LLNL (with the exception of a single set of macro-autoradiographs of one of the UE20c fracture surfaces), is summarized in Chapter 9. The single set macro-autoradiographs at Los Alamos was obtained using BioMax MS films (8"x10") in combination with a low energy intensifying screen (BioMax Transcreen LE), both from Kodak Scientific Imaging. The procedure was as follows:

1- A double-stick tape, $\sim 4 \mathrm{~mm}$ thick, was placed around the edges of the fracture to prevent direct contact between the intensifying screen and the fracture surface.

2- In a dark room, one film sheet was placed in the intensifying screen folder, and then the folder was placed on top of the fracture surface.

3- A 3-mm-thick piece of cardboard was placed on top of the screen/folder, and the whole assembly was put in a darkroom cloth bag.

4- The bag, with the sample inside, was left in a freezer (to decrease the activation energy of the screen coating material as much as possible) for about 7 days. A uniform weight was placed on top of the bag to minimize any movements of the intensifying screen relative to the fracture (e.g., from vibrations).

In a dark room, the film was developed according to the following procedures:

1 - The film was submerged in a Kodak GBX developer and replenisher solution for $\sim 5$ minutes at $\sim 68^{\circ} \mathrm{F}$ with no agitation applied,

2- The film was rinsed for about 30 seconds with fresh water,

3- The film was submerged in a Kodak GBX fixer and replenisher solution for $\sim 10$ minutes at $\sim 68^{\circ} \mathrm{F}$, with moderate agitation applied,

4- The film was then rinsed carefully with fresh water for $\sim 10$ minutes and air-dried.

\subsection{Results of Batch Sorption/Desorption Experiments}

\section{Sorption of Pu onto Colloids}

The results from the batch sorption experiments are summarized in Tables 7.15 through 7.21; a separate table is provided for each set of experiments with a different combination of $\mathrm{Pu}$ and colloids. These tables list the percentages of $\mathrm{Pu}$ sorbed to the colloids and the $\mathrm{K}_{\mathrm{d}}$ values associated with each of the measurement times. A composite plot of the percentages sorbed as 
Table 7.15. Percentages of $\mathrm{Pu}$ adsorbed and calculated $\mathrm{K}_{\mathrm{d}}$-values $(\mathrm{ml} / \mathrm{g})$ for $\mathrm{Pu}(\mathrm{V})$ sorption onto Cheto montmorillonite colloids as a function of time in synthetic WW20 groundwater. Percentages adsorbed and $\mathrm{K}_{\mathrm{d}}$-values associated with desorption experiments are also listed.

Adsorption Experiments

\begin{tabular}{|c|c|c|c|}
\hline \multicolumn{2}{|l|}{ Time, $\mathrm{hr}$} & $\%$ Adsorbed (avg. of 2 ) & $\mathrm{K}_{\mathrm{d}}$ value, $\mathrm{ml} / \mathrm{g}$ (avg. of 2 ) \\
\hline \multicolumn{2}{|l|}{1} & 4.8 & 267 \\
\hline \multicolumn{2}{|l|}{4} & 24.8 & 1738 \\
\hline \multicolumn{2}{|l|}{24} & 29.9 & 2234 \\
\hline \multicolumn{2}{|l|}{48} & 54.8 & 6983 \\
\hline \multicolumn{2}{|l|}{96} & 53.5 & 6132 \\
\hline \multicolumn{2}{|l|}{240} & 63.4 & 9089 \\
\hline \multicolumn{2}{|l|}{480} & 76.6 & 17666 \\
\hline \multicolumn{2}{|l|}{720} & 77.5 & 18247 \\
\hline \multicolumn{2}{|l|}{960} & 60.3 & 7971 \\
\hline \multicolumn{4}{|c|}{ Desorption Experiments } \\
\hline Start, hr & End, hr & $\%$ Adsorbed (avg. of 2) & $\mathrm{K}_{\mathrm{d}}$ value, $\mathrm{ml} / \mathrm{g}$ (avg. of 2 ) \\
\hline 96 & 100 & 44.9 & 5894 \\
\hline 240 & 264 & 61.4 & 9266 \\
\hline 720 & 8400 & 55.7 & 4490 \\
\hline
\end{tabular}

Table 7.16. Percentages of $\mathrm{Pu}$ adsorbed and calculated $\mathrm{K}_{\mathrm{d}}$-values $(\mathrm{ml} / \mathrm{g})$ for $\mathrm{Pu}(\mathrm{V})$ sorption onto PST-1 silica colloids as a function of time in synthetic WW20 groundwater. Percentages adsorbed and $\mathrm{K}_{\mathrm{d}}$-values associated with desorption experiments are also listed.

Adsorption Experiments

\begin{tabular}{|c|c|c|c|}
\hline \multicolumn{2}{|l|}{ Time, $\mathrm{hr}$} & $\%$ Adsorbed (avg. of 2 ) & $\mathrm{K}_{\mathrm{d}}$ value, $\mathrm{ml} / \mathrm{g}$ (avg. of 2 ) \\
\hline \multicolumn{2}{|l|}{1} & 34.1 & 2734 \\
\hline \multicolumn{2}{|l|}{4} & 33.5 & 2968 \\
\hline \multicolumn{2}{|l|}{24} & 57.6 & 7113 \\
\hline \multicolumn{2}{|l|}{48} & 61.5 & 8412 \\
\hline \multicolumn{2}{|l|}{96} & 63.4 & 9067 \\
\hline \multicolumn{2}{|l|}{240} & 70.9 & 13535 \\
\hline \multicolumn{2}{|l|}{480} & 71.9 & 13501 \\
\hline \multicolumn{2}{|l|}{720} & 82.3 & 34169 \\
\hline \multicolumn{2}{|l|}{960} & 54.7 & 6336 \\
\hline \multicolumn{4}{|c|}{ Desorption Experiments } \\
\hline Start, hr & End, hr & $\%$ Adsorbed (avg of 2 ) & $\mathrm{K}_{\mathrm{d}}$ value, $\mathrm{ml} / \mathrm{g}$ (avg. of 2 ) \\
\hline 96 & 100 & 64.2 & 35787 \\
\hline 240 & 264 & 78.6 & 71137 \\
\hline 720 & 8400 & 61.0 & 5199 \\
\hline
\end{tabular}


Table 7.17. Percentages of $\mathrm{Pu}$ adsorbed and calculated $\mathrm{K}_{\mathrm{d}}$-values $(\mathrm{ml} / \mathrm{g})$ for $\mathrm{Pu}(\mathrm{V})$ sorption onto batch 1 Otay montmorillonite colloids as a function of time in natural WW20 groundwater. Percentages adsorbed and $\mathrm{K}_{\mathrm{d}}$-values associated with desorption experiments are also listed.

Adsorption Experiments

\begin{tabular}{|c|c|c|c|}
\hline \multicolumn{2}{|l|}{ Time, hr } & $\%$ Adsorbed (avg. of 2) & $\mathrm{K}_{\mathrm{d}}$ value, $\mathrm{ml} / \mathrm{g}$ (avg. of 2) \\
\hline \multicolumn{2}{|l|}{1} & 7.0 & 438 \\
\hline \multicolumn{2}{|l|}{4} & 19.8 & 1450 \\
\hline \multicolumn{2}{|l|}{24} & 33.2 & 2905 \\
\hline \multicolumn{2}{|l|}{48} & 53.4 & 8005 \\
\hline \multicolumn{2}{|l|}{96} & 55.8 & 7384 \\
\hline \multicolumn{2}{|l|}{240} & $86.0^{*}$ & $45435^{*}$ \\
\hline \multicolumn{2}{|l|}{480} & 93.6 & 85732 \\
\hline \multicolumn{2}{|l|}{720} & 88.1 & 43508 \\
\hline \multicolumn{4}{|c|}{ Desorption Experiments } \\
\hline Start, hr & End, hr & $\%$ Adsorbed (avg. of 2) & $K_{d}$ value, $\mathrm{ml} / \mathrm{g}$ (avg. of 2) \\
\hline 240 & 264 & 79.7 & 291645 \\
\hline 240 & 288 & 86.8 & 85864 \\
\hline 240 & 5640 & 63.9 & 16898 \\
\hline
\end{tabular}

* The 240-hr values are an average of 6 measurements.

Table 7.18. Percentages of $\mathrm{Pu}$ adsorbed and calculated $\mathrm{K}_{\mathrm{d}}$-values $(\mathrm{ml} / \mathrm{g})$ for $\mathrm{Pu}(\mathrm{V})$ sorption onto MP-1040 silica colloids as a function of time in natural WW20 groundwater. Percentages adsorbed and $\mathrm{K}_{\mathrm{d}}$-values associated with desorption experiments are also listed.

Adsorption Experiments

\begin{tabular}{|c|c|c|c|}
\hline \multicolumn{2}{|l|}{ Time, hr } & $\%$ Adsorbed (avg. of 2 ) & $\mathrm{K}_{\mathrm{d}}$ value, $\mathrm{ml} / \mathrm{g}$ (avg. of 2 ) \\
\hline \multicolumn{2}{|l|}{1} & 1.7 & 98 \\
\hline \multicolumn{2}{|l|}{4} & 42.2 & 4013 \\
\hline \multicolumn{2}{|l|}{24} & 66.5 & 10927 \\
\hline \multicolumn{2}{|l|}{48} & 59.6 & 8122 \\
\hline \multicolumn{2}{|l|}{96} & 60.8 & 8564 \\
\hline \multicolumn{2}{|l|}{240} & $64.8^{*}$ & 10188* \\
\hline \multicolumn{2}{|l|}{480} & 63.2 & 9475 \\
\hline \multicolumn{2}{|l|}{720} & 69.1 & 12317 \\
\hline \multicolumn{4}{|c|}{ Desorption Experiments } \\
\hline Start, hr & End, hr & $\%$ Adsorbed (avg of 2) & $\mathrm{K}_{\mathrm{d}}$ value, $\mathrm{ml} / \mathrm{g}$ (avg. of 2 ) \\
\hline 240 & 264 & 59.2 & 33562 \\
\hline 240 & 288 & 58.4 & 27013 \\
\hline 240 & 5640 & 46.6 & 8049 \\
\hline
\end{tabular}

* The 240-hr values are an average of 6 measurements. 
Table 7.19. Percentages of $\mathrm{Pu}$ adsorbed and calculated $\mathrm{K}_{\mathrm{d}}$-values $(\mathrm{ml} / \mathrm{g})$ for $\mathrm{Pu}(\mathrm{IV})$ sorption onto clinoptilolite colloids as a function of time in natural WW20 groundwater. Percentages adsorbed and $\mathrm{K}_{\mathrm{d}}$-values associated with desorption experiments are also listed.

Adsorption Experiments

\begin{tabular}{|c|c|c|c|}
\hline \multicolumn{2}{|l|}{ Time, $\mathrm{hr}$} & $\%$ Adsorbed (avg. of 2) & $\mathrm{K}_{\mathrm{d}}$ value, $\mathrm{ml} / \mathrm{g}$ (avg. of 2 ) \\
\hline \multicolumn{2}{|l|}{456} & $91.3 *$ & $52645^{*}$ \\
\hline \multicolumn{2}{|l|}{984} & 91.2 & 51994 \\
\hline \multicolumn{4}{|c|}{ Desorption Experiments } \\
\hline Start, hr & End, hr & $\%$ Adsorbed (avg. of 2) & $\mathrm{K}_{\mathrm{d}}$ value, $\mathrm{ml} / \mathrm{g}$ (avg. of 2 ) \\
\hline 456 & 480 & 91.6 & 238509 \\
\hline 456 & 504 & 91.4 & 175570 \\
\hline 456 & 1920 & 91.8 & 146834 \\
\hline 984 & 4008 & 87.8 & 102254 \\
\hline
\end{tabular}

* The 456-hr values are an average of 6 measurements.

Table 7.20. Percentages of $\mathrm{Pu}$ adsorbed and calculated $\mathrm{K}_{\mathrm{d}}$-values $(\mathrm{ml} / \mathrm{g})$ for $\mathrm{Pu}(\mathrm{V})$ sorption onto batch 2 Otay montmorillonite colloids as a function of time in natural WW20 groundwater. Percentages adsorbed and $\mathrm{K}_{\mathrm{d}}$-values associated with desorption experiments are also listed.

Adsorption Experiments

\begin{tabular}{|c|c|c|c|}
\hline \multicolumn{2}{|l|}{ Time, $\mathrm{hr}$} & $\%$ Adsorbed (avg. of 2 ) & $\mathrm{K}_{\mathrm{d}}$ value, $\mathrm{ml} / \mathrm{g}$ (avg. of 2 ) \\
\hline \multicolumn{2}{|l|}{576} & 69.2 & 11878 \\
\hline \multicolumn{2}{|l|}{1224} & 81.8 & 25748 \\
\hline \multicolumn{2}{|l|}{1920} & 83.8 & 29711 \\
\hline \multicolumn{2}{|l|}{2400} & 84.1 & 32365 \\
\hline \multicolumn{4}{|c|}{ Desorption Experiments } \\
\hline Start, hr & End, hr & $\%$ Adsorbed (avg. of 2) & $K_{d}$ value, $\mathrm{ml} / \mathrm{g}$ (avg. of 2 ) \\
\hline 576 & 600 & 69.3 & 184966 \\
\hline 1224 & 1272 & 82.2 & 108479 \\
\hline 1920 & 2904 & 79.5 & 57708 \\
\hline 2400 & 4800 & 81.4 & 71571 \\
\hline
\end{tabular}

Table 7.21. Percentages of $\mathrm{Pu}$ adsorbed and calculated $\mathrm{K}_{\mathrm{d}}$-values $(\mathrm{ml} / \mathrm{g})$ for $\mathrm{Pu}(\mathrm{V})$ sorption onto batch 1 Otay montmorillonite colloids as a function of time in natural WW20 groundwater. Percentages adsorbed and $\mathrm{K}_{\mathrm{d}}$-values associated with desorption experiments are also listed.

Adsorption Experiments

\begin{tabular}{|c|c|c|c|}
\hline \multicolumn{2}{|l|}{ Time, hr } & $\%$ Adsorbed (avg. of 6 ) & $\mathrm{K}_{\mathrm{d}}$ value, $\mathrm{ml} / \mathrm{g}$ (avg. of 6 ) \\
\hline 816 & & 85.7 & 29751 \\
\hline \multicolumn{4}{|c|}{ Desorption Experiments } \\
\hline Start, hr & End, hr & $\%$ Adsorbed (avg. of 2 ) & $K_{d}$ value, $\mathrm{ml} / \mathrm{g}$ (avg. of 2) \\
\hline 816 & 1056 & 87.3 & 232024 \\
\hline 816 & 1296 & 85.7 & 145643 \\
\hline 816 & 1776 & 86.0 & 145660 \\
\hline
\end{tabular}


a function of time for each of the Pu-colloid combinations is provided in Figure 7.7. It is apparent that the sorption of $\mathrm{Pu}(\mathrm{IV})$ onto the clinoptilolite colloids was a much faster process than the sorption of $\mathrm{Pu}(\mathrm{V})$ to the other colloids (note that the first three $\mathrm{Pu}(\mathrm{IV})$-clinoptilolite data points were obtained from separate experiments conducted at LLNL). It is also apparent that $\mathrm{Pu}(\mathrm{V})$ sorbed more rapidly to the silica colloids than the montmorillonite colloids.

Sorption measurements were made more frequently in the earlier batch experiments and less frequently in the later experiments due to time and budget constraints. In the experiment with the $\mathrm{Pu}(\mathrm{IV})$ and clinoptilolite colloids from LLNL and in the final experiment with $\mathrm{Pu}(\mathrm{V})$ and the batch 1 Otay montmorillonite colloids, sorption measurements were conducted only at the times of injection into the fractured cores. There also were no early sorption measurements conducted for $\mathrm{Pu}(\mathrm{V})$ sorption onto the batch 2 Otay montmorillonite.

Interpretations of the batch sorption and desorption experiments are discussed in Chapter 8. The apparent decrease in the amount of $\mathrm{Pu}$ sorbed as a function of time in some of the experiments (note particularly the decrease between $720 \mathrm{hrs}$ and $960 \mathrm{hrs}$ for the Cheto montmorillonite and PST-1 silica) could indicate net desorption of $\mathrm{Pu}$ from the colloids over time. However, it is also possible that there was some variability in the separation of the colloids from solution during centrifuging. The occurrences of lower sorption percentages at later times may have been the result of poor colloid-solution separations due to partial resuspension of colloids during handling of the centrifuge tubes. This hypothesis is supported by the fact that the 960-hr Cheto montmorillonite and PST-1 silica colloids were centrifuged at the same time. Replicate measurements were generally in very good agreement (even if the overall trend from previous measurements was not followed), suggesting that variability among test tubes centrifuged and processed at the same time was very small.

\section{Desorption of Pu from Colloids}

The results from the batch desorption experiments are summarized in Tables 7.15 through 7.21 along with the sorption results (at the bottom of each table). The tables list the percentages of $\mathrm{Pu}$ that remained sorbed to the colloids after desorption (these can be compared with the percentages sorbed after sorption in the upper part of each table) and the $\mathrm{K}_{\mathrm{d}}$ values asssociated with these percentages at each of the measurement times. In most cases, very little Pu desorbed from the colloids in the first 1-2 days of desorption, which corresponds to the approximate residence times of the $\mathrm{Pu}$ and colloids in the fractured core transport experiments. After much longer times (days to weeks), more significant amounts of $\mathrm{Pu}$ desorbed from some of the colloids (e.g., the Cheto montmorillonite, the batch 1 Otay montmorillonite, and the silica colloids). However, desorption was very minimal from the clinoptilolite colloids and the batch 2 Otay montmorillonite colloids. Plots showing the percentage of $\mathrm{Pu}$ remaining sorbed to the colloids after desorption are provided in Chapter 8 where experiment interpretations are discussed.

\section{Discussion of Possible Sorption and Desorption Mechanisms}

In this section, we discuss potential sorption and desorption mechanisms for $\mathrm{Pu}(\mathrm{V})$ on the montmorillonite and silica colloids (the $\mathrm{Pu}(\mathrm{IV})$ and clinoptilolite colloids are discussed in Chapter 4). Chapter 5 presents a more detailed discussion of $\mathrm{Pu}(\mathrm{V})$ sorption mechanisms onto 


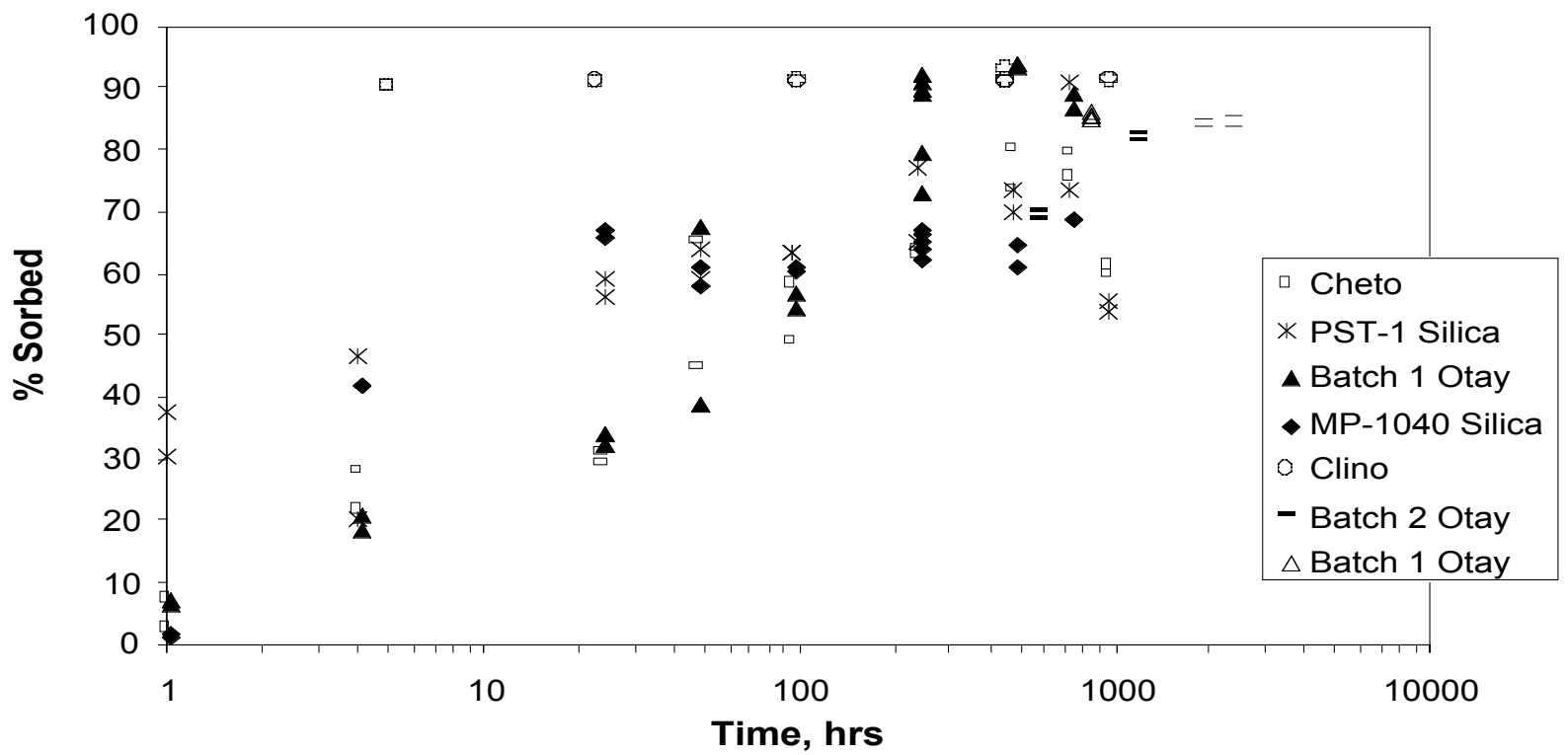

Figure 7.7. Percentages of $\mathrm{Pu}$ sorbed in each of the adsorption experiments conducted in association with the fracture transport experiments. All replicate measurements are shown on this plot (Tables 7.15 through 7.21 list averages).

various inorganic colloids, which is supported by detailed mechanistic experiments. The discussion in this section is intended to supplement the Chapter 5 discussion based on observations made during the sorption/desorption experiments conducted at LANL.

The experiments presented in Chapter 5 provide clear evidence that when $\mathrm{Pu}(\mathrm{V})$ is sorbed onto silica and montmorillonite colloids, the predominant oxidation state on the colloid surfaces is $\mathrm{Pu}(\mathrm{IV})$ (Figure 5.4). The experiments of Chapter 5 were not designed to determine whether the reduction of $\mathrm{Pu}(\mathrm{V})$ to $\mathrm{Pu}(\mathrm{IV})$ occurred before or after $\mathrm{Pu}$ sorption onto the colloids (i.e., Paths 1 or 2 of Figure 5.6). However, it was pointed out that the slow rate of $\mathrm{Pu}(\mathrm{V})$ sorption onto clinoptilolite colloids was consistent with Path $2(\mathrm{Pu}(\mathrm{V})$ reduction in solution followed by $\mathrm{Pu}(\mathrm{IV})$ sorption) because the observed sorption rate was qualitatively similar to the rate of $\mathrm{Pu}(\mathrm{V})$ disproportionation in separate experiments. Sorption rates for $\mathrm{Pu}(\mathrm{V})$ onto montmorillonite colloids were not discussed in Chapter 5, but Figure 7.7 shows that $\mathrm{Pu}(\mathrm{V})$ sorption rates onto montmorillonite colloids tended to be slower than onto silica colloids in the sorption experiments conducted at LANL, so Path 2 would also be implicated for montmorillonite.

The LANL sorption experiments did not involve direct measurements of Pu oxidation states on colloid surfaces. However, indirect evidence associated with the experiments seem to be consistent with a Path 1 sorption mechanism onto silica and montmorillonite colloids (sorption of $\mathrm{Pu}(\mathrm{V})$ followed by reduction to $\mathrm{Pu}(\mathrm{IV})$ on the surfaces) rather than a Path 2 mechanism. The solutions used in the sorption experiments were prepared by diluting relatively high concentration $\mathrm{Pu}(\mathrm{V})$ stock solutions $\left(10^{-4}\right.$ to $\left.10^{-5} \mathrm{M}\right)$ of $\mathrm{pH} \sim 7$ that were checked periodically by 
UV-VIS spectroscopy during the course of the experiments to verify $\mathrm{Pu}$ oxidation state. The higher $\mathrm{Pu}$ concentration and lower $\mathrm{pH}$ of the stock solutions relative to the water used in the batch experiments should have resulted in more disproportionation of $\mathrm{Pu}(\mathrm{V})$ to $\mathrm{Pu}(\mathrm{IV})$ and $\mathrm{Pu}(\mathrm{VI})$ in the stock solutions than in the experiments. Thus, as long as the $\mathrm{Pu}$ in the stock solutions remained $\mathrm{Pu}(\mathrm{V})$, significant disproportionation should not have occurred in the batch experiments. In all cases, the $\mathrm{Pu}$ in the stock solutions remained $\mathrm{Pu}(\mathrm{V})$ throughout the sorption phase of the experiments (the oxidation state was not monitored for the entire desorption time, which in some cases, approached a year). Of course, the possibility that $\mathrm{Pu}(\mathrm{V})$ could have been reduced in solution by a mechanism other than disproportionation cannot be ruled out (e.g., oxygen depletion, the presence of small amounts of reducing agents in the waters). However, the stock solutions were stored as closely as possible under the same ambient conditions that the batch experiments were conducted at, so any differences in the behavior of $\mathrm{Pu}$ in the different solutions cannot be attributed to environmental factors.

The second line of evidence supporting a Path 1 sorption mechanism onto the silica and montmorillonite colloids in the LANL experiments is that the sorption rates shown in Figure 7.7 are all far greater than the $\mathrm{Pu}(\mathrm{V})$ disproportionation rates discussed in Chapter 5. Thus, although the qualitative similarity of sorption and disproportionation rates in Chapter 5 apparently supports a Path 2 sorption mechanism, the same comparison of rates supports a Path 1 mechanism in the LANL experiments. Again, however, it must be pointed out that the observations in the LANL experiments do not rule out the possibility of $\mathrm{Pu}(\mathrm{V})$ reduction in solution (prior to sorption) by a process other than disproportionation.

It is difficult to explain why sorption rates of $\mathrm{Pu}(\mathrm{V})$ onto silica colloids were so different in the LLNL and LANL experiments. One possibility is that the $\mathrm{Pu}$ concentrations in the LLNL experiments were over two orders of magnitude higher than in the LANL experiments, which could have resulted in partial "saturation" of surface sorption sites and a corresponding suppression of sorption rates that would not have been observed at the lower concentrations in the LANL experiments. Normalizing the sorption rates to colloid surface area to allow a more valid comparison of rates was not possible because surface areas were not measured by either LLNL or LANL. Other differences between the LANL and LLNL experiments that could have affected sorption rates include the use of different waters (real or synthetic U-20WW water vs. synthetic J-13 water), , and different ambient pressures (7200 ft above sea level vs. close to sea level), which affects ambient oxygen concentrations as well as carbonate/bicarbonate equilibria. It must also be emphasized the the two sets of experiments had two very different objectives: the LLNL experiments were designed to conduct XAS analyses to determine surface oxidation states

of $\mathrm{Pu}$, and the LANL experiments were designed to measure sorption parameters and generate solutions for injections into fractures. Neither set of experiments was designed specifically to determine sorption mechanisms.

\subsection{Results of Fracture Transport Experiments Tritium, Colloid and Pu Responses}

The tritium, colloid, and Pu responses in the 21 fractured core transport experiments (normalized concentrations and fractional or percent recoveries vs. volume eluted in $\mathrm{ml}$ ) are shown in Figures $\mathrm{C}-1$ through $\mathrm{C}-21$ in Appendix $\mathrm{C}$ in the order that the experiments were conducted. Examples 
are provided in Figures 7.8 and 7.9 , which show $\mathrm{Pu}(\mathrm{V})$ facilitated by Cheto montmorillonite colloids at $\sim 1.5 \mathrm{ml} / \mathrm{hr}$ in the UE20c 2851 fracture and $\mathrm{Pu}(\mathrm{IV})$ facilitated by clinoptilolite colloids at $\sim 1.5 \mathrm{ml} / \mathrm{hr}$ in the PM-2 fracture. Even without detailed quantitative interpretations of these experiments, the following observations/conclusions can be drawn from the plots in Appendix C:

- The tritium responses at the two different flow rates in the same fracture for each pair of colloid experiments (with the same colloid) are consistent with matrix diffusion behavior in the cores; i.e., peak concentrations are lower and tails are longer in the lower flow rate tests. However, the tritium elution behavior over time was not completely reproducible in experiments that were conducted at the same flow rate with different colloids. Figures 7.10 and 7.11 show the tritium recoveries as a function of volume eluted in all of the tests in the UE20c cores and in the PM-1 and PM-2 cores, respectively. Different symbols are used for each different colloid and flow rate. It is apparent that while the recovery at any particular volume tends to be greater at higher flow rates in all cores (as expected), the responses vary considerably, especially in core 2851 , where the 2 nd experiment at $\sim 1.5$ $\mathrm{ml} / \mathrm{hr}$ (Silica 1.5) has the lowest recovery curve even though another test was conducted at a significantly lower flow rate (Cheto 0.5 ). Likewise, the lowest flow rate test in the PM-1 core (Old Otay 0.4 ) had the highest overall tritium recovery curve in that core. Some, but not all, of this behavior can be attributed to different tracer injection volumes in the different experiments. However, in general, these results suggest that subtle differences in experimental conditions and possibly changes in the cores over time, such as buildup of biofilms on the surfaces or changes in the fracture aperture due to expansion or contraction of the flow systems, may significantly influence the tracer responses. These results illustrate the importance of simultaneously injecting species instead of conducting separate experiments for the different species that are to be compared. Comparing the responses of simultaneously injected tracers will always involve less uncertainty than comparing the responses of species injected in different tests, even if the tests are run under what are intended to be identical conditions.

- Except in the case of the batch 1 Otay montmorillonite colloids in the PM-2 core, the montmorillonite colloid responses were always attenuated relative to tritiated water (lower overall recoveries) in each experiment. The silica and clinoptilolite colloids both had recoveries that were comparable to tritium in each experiment, suggesting that these colloids experienced significantly less filtration than montmorillonite in the cores. No direct comparison could be made between the silica and clinoptilolite colloids because these colloids were not eluted through the same fractures.

- All colloids tended to arrive at the same time or slightly earlier than the tritium, and they reached peak concentrations sooner than the tritium. This early first and peak arrival time behavior is consistent with numerous field observations of colloid transport in forced-gradient tests, including several at the Nevada Test Site (Reimus et al. 1999, Reimus and Haga, 1999), as well as laboratory experiments in fractured media (Reimus 1995, Becker et al. 1999). 


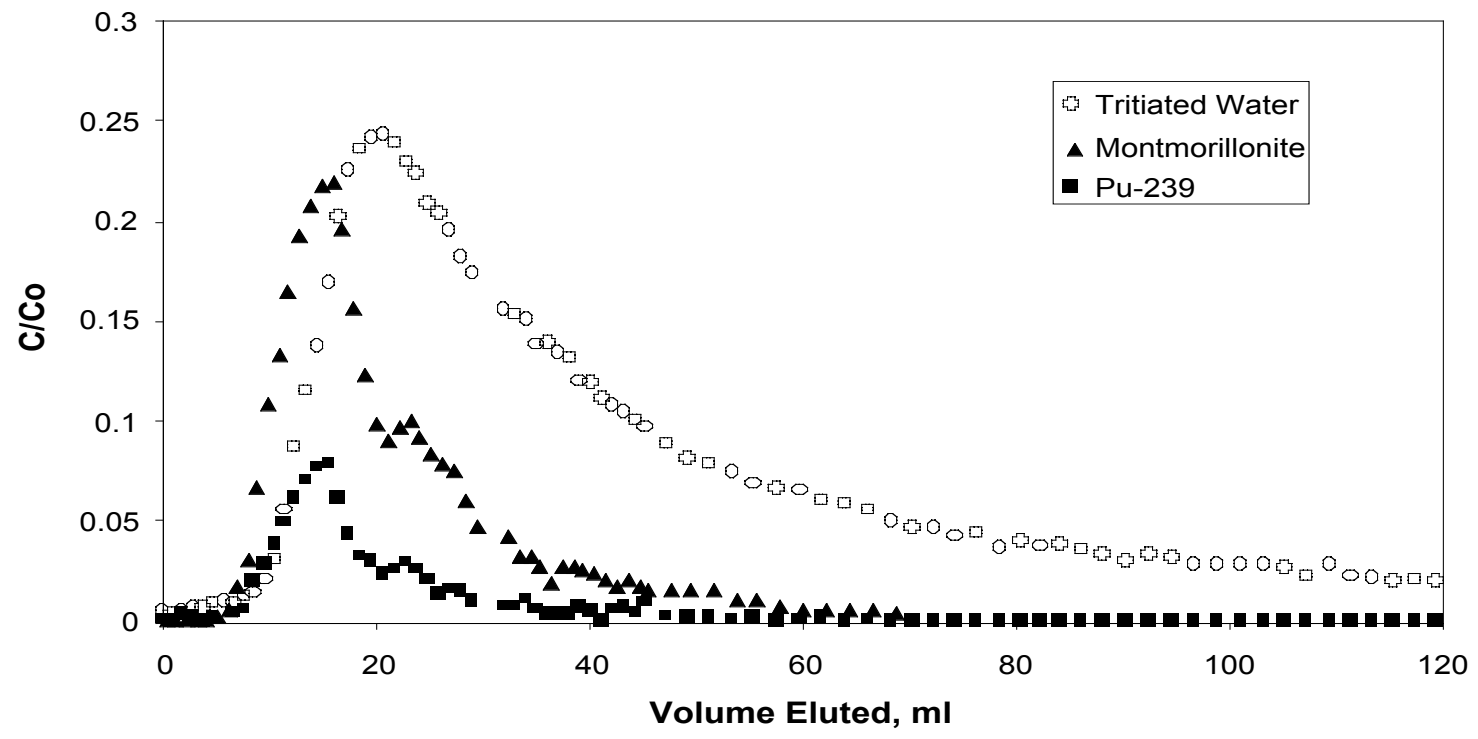

Figure 7.8. Tritiated water, Cheto montmorillonite, and ${ }^{239} \mathrm{Pu}$ responses in fractured core UE20c, $2858 \mathrm{ft}$ at $\sim 1.5 \mathrm{ml} / \mathrm{hr}$ (Experiment 1b).

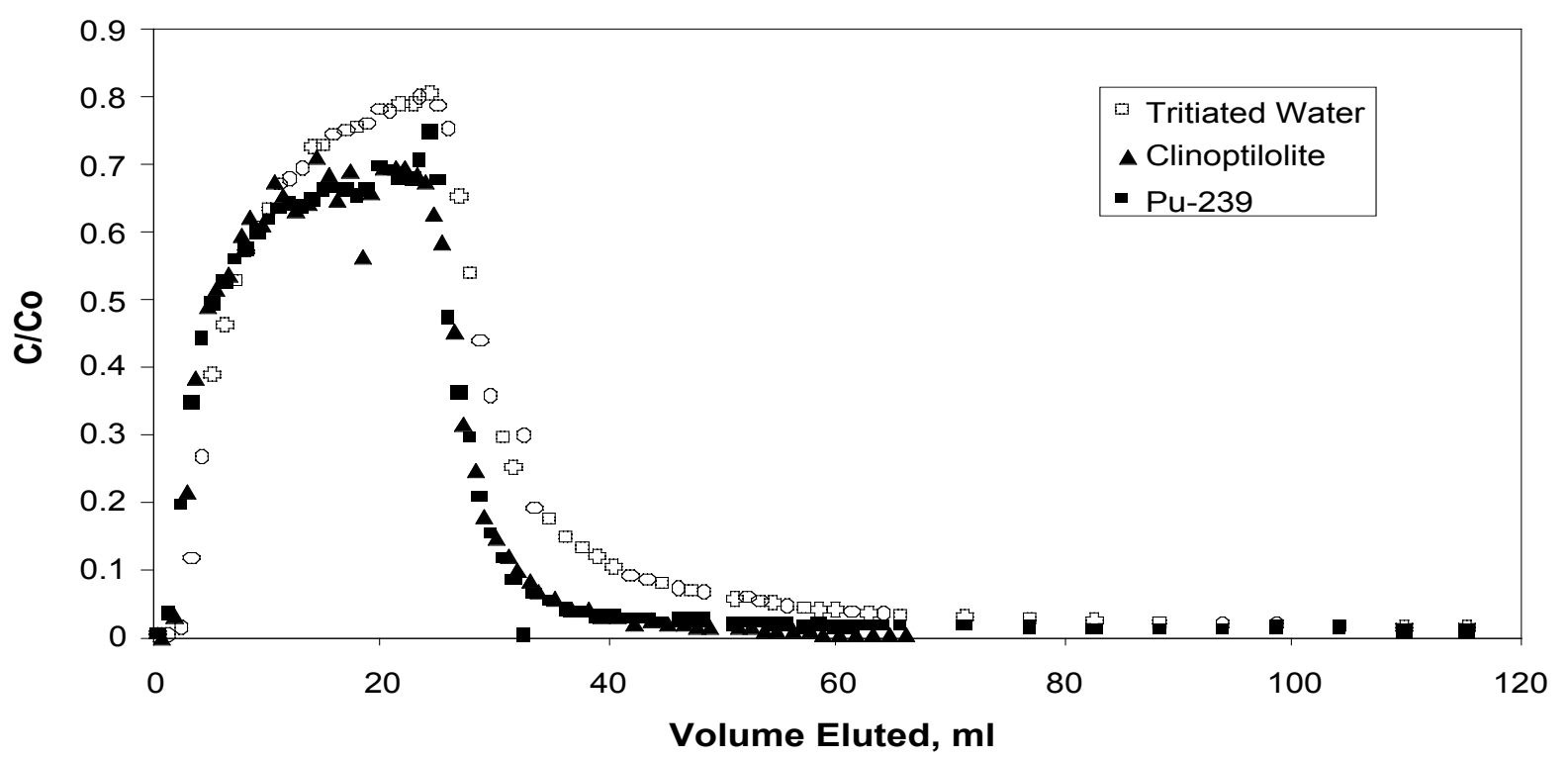

Figure 7.9. Tritiated water, clinoptilolite, and ${ }^{239} \mathrm{Pu}$ responses in fractured core $\mathrm{PM}-2,4177 \mathrm{ft}$ at $\sim 1.5 \mathrm{ml} / \mathrm{hr}$ (Experiment 7a). 
2851

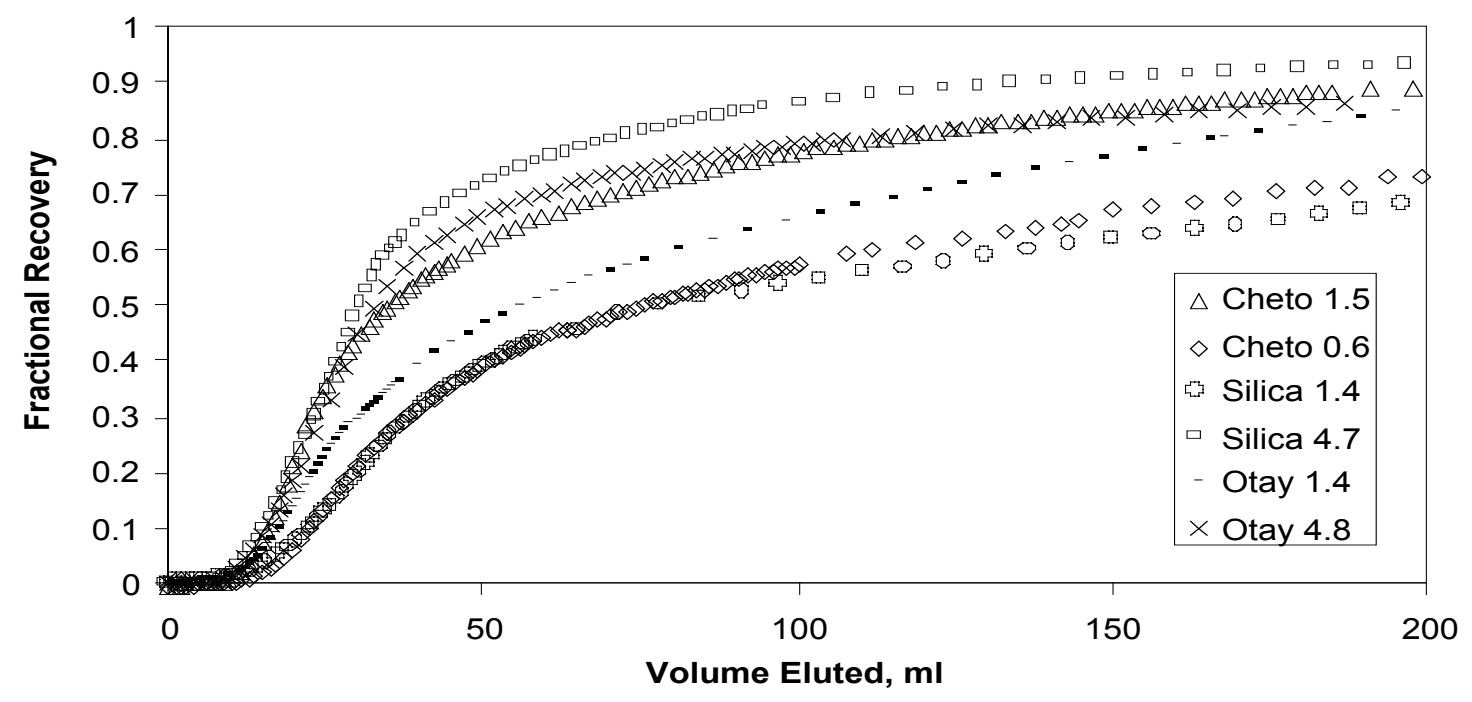

2858

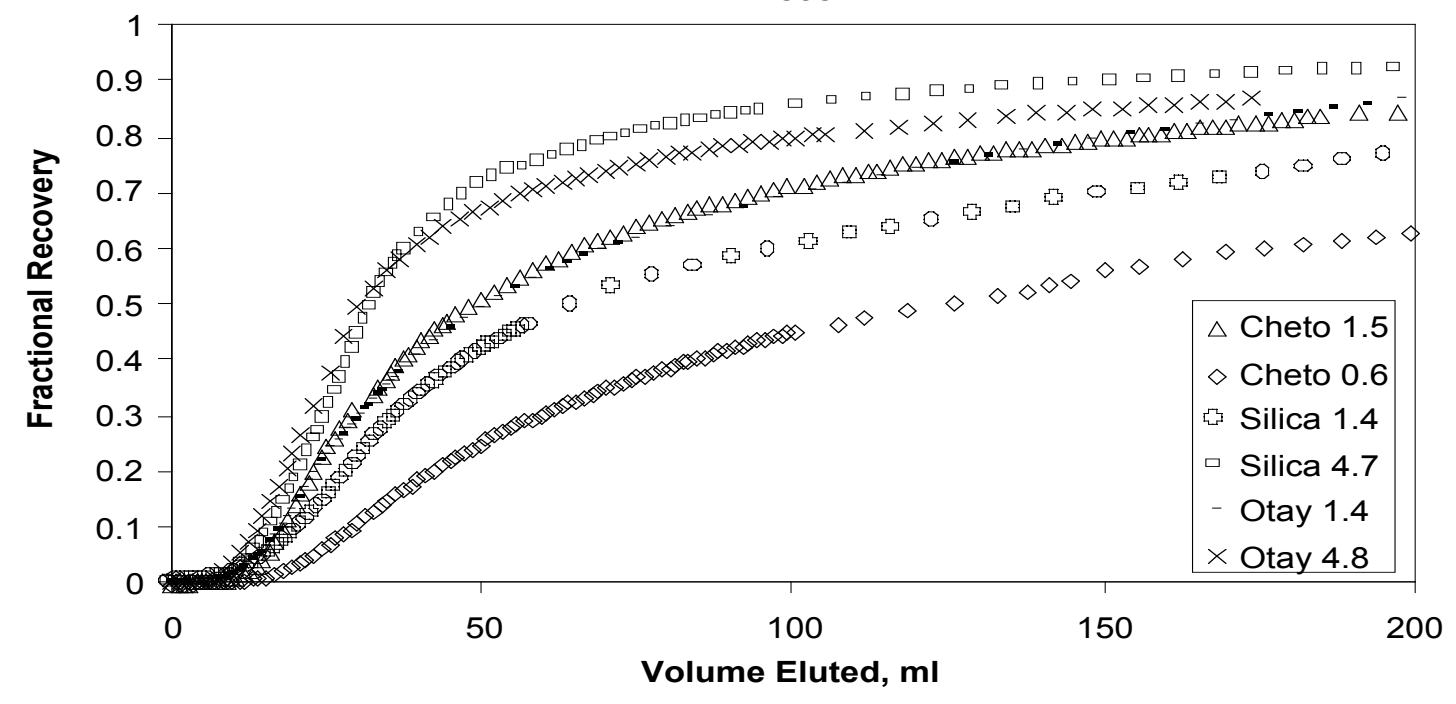

Figure 7.10. Tritiated water recoveries in each of the experiments conducted in the UE20c fractured cores (2851 top, 2858 bottom). Legend indicates the colloid used in each experiment, and the number is the flow rate in $\mathrm{ml} / \mathrm{hr}$. 
PM-2

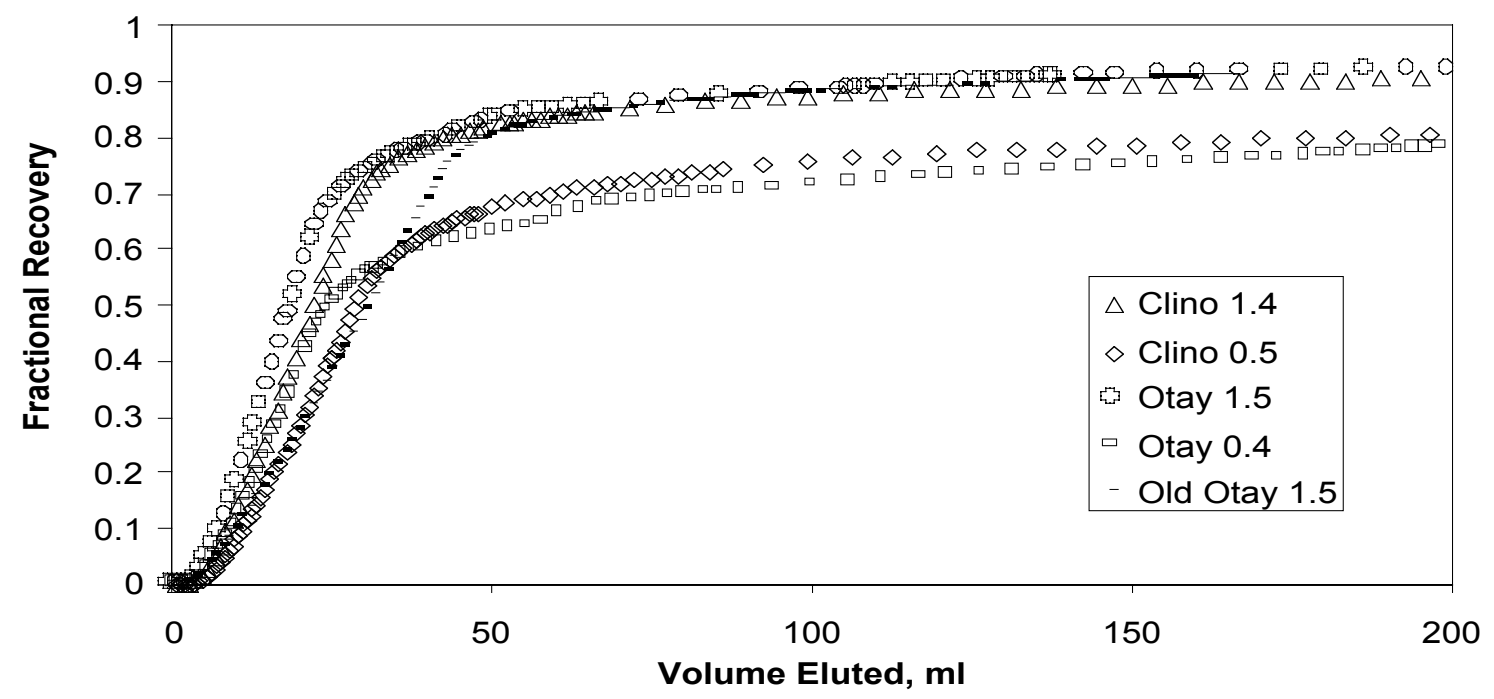

PM-1

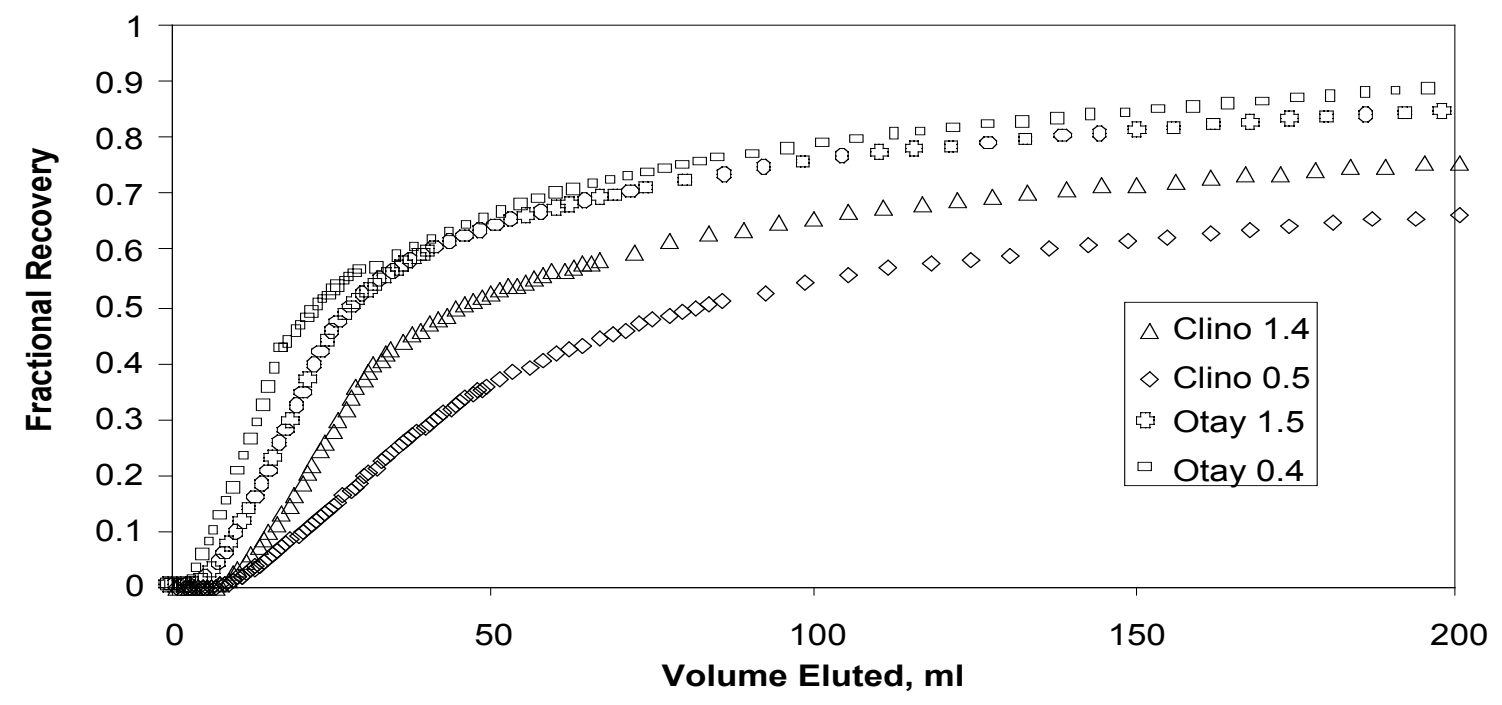

Figure 7.11. Tritiated water recoveries in each of the experiments conducted in the PM-2 and PM-1 fractured cores (PM-2 top, PM-1 bottom). Legend indicates the colloid used in each experiment, and the number is the flow rate in $\mathrm{ml} / \mathrm{hr}$. 
- Each colloid was more attenuated at the lower flow rate in each fracture, indicating that more filtration occurred at the lower flow rate. This result was expected because at lower flow rates, greater residence times allow more opportunity for colloid contact with the fracture surfaces.

- In the montmorillonite and silica colloid experiments, the Pu responses were nearly identical in shape to that of the colloids but lower in normalized concentration. Even the small second peak in the colloid response at $\sim 23 \mathrm{ml}$ into the $1.5 \mathrm{ml} / \mathrm{hr}$ Cheto montmorillonite test in the core from $2858 \mathrm{ft}$ was mirrored in the Pu response (see Figure 7.8). This behavior suggests that the $\mathrm{Pu}$ migrated through the fractures exclusively with the colloids (while sorbed to the colloids), although a significant amount of the $\mathrm{Pu}$ was desorbed from the colloids during the time in the cores. Filtration of selected fracture effluent samples confirmed that all of the $\mathrm{Pu}$ exiting the columns was sorbed to colloids (no measurable $\mathrm{Pu}$ activity in the filtrates).

- The $\mathrm{Pu}$ and clinoptilolite colloid responses were nearly identical (within experimental error), indicating that desorption of Pu(IV) from the clinoptilolite in the PM1 and PM2 fractures was negligible. The clinoptilolite colloids clearly facilitated the transport of $\mathrm{Pu}$ more efficiently than either the montmorillonite or the silica colloids in the fractured cores.

- Although the batch 1 Otay-montmorillonite colloids were more attenuated in the UE20c fractures than the silica colloids at comparable flow rates, the $\mathrm{Pu}(\mathrm{V})$ responses were greater with the Otay-montmorillonite colloids. This result suggests that the Otaymontmorillonite colloids facilitated the transport of the Pu more efficiently than the silica colloids, probably because of a slower desorption rate of the $\mathrm{Pu}$ from the montmorillonite colloids in the WW20 water.

- The continuous injection of the batch 2 Otay montmorillonite colloids into the PM-1 and PM-2 fractures after the tritium and $\mathrm{Pu}$ injection pulses ceased resulted in no apparent remobilization of previously-immobilized $\mathrm{Pu}$ in these cores (see Figures $\mathrm{C}-19$ and C-20). This result suggests that the sorption of $\mathrm{Pu}$ (or of filtered colloids) onto the fracture surfaces was strong enough that the $\mathrm{Pu}$ did not desorb from the fracture surfaces onto mobile Pu-free colloids.

- The batch Otay-montmorillonite colloids were more attenuated than the Chetomontmorillonite colloids at the same flow rate $(\sim 1.5 \mathrm{ml} / \mathrm{hr})$ in the UE20c fractures. This result may be due to the fact that the Cheto montmorillonite colloids were eluted in synthetic WW20 water and the batch Otay montmorillonite colloids were eluted in natural WW20 water. The natural water has a significant concentration of divalent cations (mainly $\mathrm{Ca}^{2+}$ ), which would tend to collapse the stabilizing electrical doublelayers (and hence promote attachment to fracture surfaces) more than the synthetic water, which had no divalent cations. This hypothesis is supported by the larger negative zeta potential of the Cheto montmorillonite colloids in the synthetic WW20 water $(-34.3 \pm$ 3.8) $\mathrm{mV}$ compared to the zeta potential of the Otay montmorillonite colloids in natural WW20 water $(-21.0 \pm 5.7) \mathrm{mV}$.

A semi-quantitative measure of the facilitation of $\mathrm{Pu}$ transport by the colloids was obtained for the fracture transport experiments in the following way: 
1) The "predicted" recovery of $\mathrm{Pu}$ in each experiment was calculated based on the batch sorption and desorption data as follows:

$$
R_{P u}=\left(R_{\text {col }}\right)\left(f_{\text {ads }}\right)\left(1-f_{\text {des }}\right)_{\tau}
$$

where, $\mathrm{R}_{\mathrm{Pu}}=\mathrm{Pu}$ recovery (predicted)

$\mathrm{R}_{\mathrm{col}}=$ colloid recovery (actual)

$\mathrm{f}_{\mathrm{ads}}=$ fraction of Pu sorbed to colloids in injection solution (from batch tests)

$\left(1-f_{\text {des }}\right)_{\tau}=$ fraction of $\mathrm{Pu}$ remaining adsorbed to colloids during residence time in column $(\tau)$ (again, from batch tests).

This calculation assumes that any $\mathrm{Pu}$ that was initially in the solution phase and any $\mathrm{Pu}$ that desorbed from the colloids during the residence time in the column became adsorbed to the fracture walls in the column and was not recovered. It also assumes that any $\mathrm{Pu}$ that was initially sorbed to colloids that were filtered in the column was retained in the column with the colloids.

2) The actual Pu recovery was then compared to the "predicted" Pu recovery. If the actual recovery was less than the predicted recovery, it was taken as an indication that the fracture surfaces were able to effectively compete with the colloid surfaces for Pu. The ratio of actual to predicted recoveries was taken as a measure of the facilitation of $\mathrm{Pu}$ transport provided by the colloids.

Table 7.22 lists the colloid recoveries, "predicted" Pu recoveries, and actual Pu recoveries for each of the column experiments, and Figure 7.12 graphically depicts the data. Figure 7.13 shows the ratios of actual to predicted $\mathrm{Pu}$ recoveries in each of the experiments. The ratios of greater than one for the clinoptilolite colloids are taken as an indication that the batch experiments slightly underestimated the fraction of Pu sorbed to the colloids. The ratios in Figure 7.13 are, from greatest to least (i.e., the order of greatest to least facilitation of Pu transport):

1) $\mathrm{Pu}(\mathrm{IV})$ on Clinoptilolite in WW20 water

2) $\mathrm{Pu}(\mathrm{V})$ on Cheto-montmorillonite in syn. WW20 water

3) $\mathrm{Pu}(\mathrm{V})$ on Otay-montmorillonite in WW20 water

4) $\mathrm{Pu}(\mathrm{V})$ in Silica in $\mathrm{WW} 20$ water

We suspect that the greater facilitation associated with the Cheto montmorillonite relative to the Otay-montmorillonite may have been due to the fact that the Cheto montmorillonite experiments were conducted in the divalent-cation-free synthetic WW20 water. As mentioned in Section 7.3, the divalent cations in the natural WW20 may have competed more effectively with the Pu for colloid sorption sites than the sodium in the synthetic water, thus resulting in less Pu desorption from the colloids in the synthetic water. With the exception of the clinoptilolite experiments, the ratio of actual to predicted $\mathrm{Pu}$ recoveries (Figure 7.13) was lower at the lower flow rates and longer residence times in each set of experiments in the same fractured core. The fact that the ratio was lower at the lower flow rates suggests that some of the Pu may have transferred from 
Table 7.22. Colloid recoveries, "predicted" Pu recoveries, and actual Pu recoveries for all of the fractured core experiments. See text for discussion of predicted and actual $\mathrm{Pu}$ recoveries.

\begin{tabular}{|c|c|c|c|}
\hline Experiment & Colloid Recovery & Predicted & Pu Rec. Actual Pu Rec. \\
\hline $2851,1.5 \mathrm{ml} / \mathrm{hr}$ Cheto-mont. & 0.319 & 0.17 & 0.1 \\
\hline $2851,0.6 \mathrm{ml} / \mathrm{hr}$ Cheto-mont. & 0.133 & 0.062 & 0.012 \\
\hline $2858,1.5 \mathrm{ml} / \mathrm{hr}$ Cheto-mont. & 0.275 & 0.15 & 0.079 \\
\hline $2858,0.6 \mathrm{ml} / \mathrm{hr}$ Cheto-mont. & 0.075 & 0.035 & 0.017 \\
\hline $2851,5 \mathrm{ml} / \mathrm{hr} \mathrm{Si}$ & 0.897 & 0.54 & 0.076 \\
\hline $2851,1.5 \mathrm{ml} / \mathrm{hr} \mathrm{Si}$. & 0.344 & 0.21 & 0.0003 \\
\hline $2858,5 \mathrm{ml} / \mathrm{hr} \mathrm{Si}$. & 0.923 & 0.55 & 0.15 \\
\hline $2858,1.5 \mathrm{ml} / \mathrm{hr} \mathrm{Si}$ & 0.628 & 0.38 & 0.028 \\
\hline 2851, $5 \mathrm{ml} / \mathrm{hr}$ Otay-M. & 0.553 & 0.46 & 0.24 \\
\hline $2851,1.5 \mathrm{ml} / \mathrm{hr}$ Otay-M. & 0.108 & 0.090 & 0.003 \\
\hline 2858, 5 ml/hr Otay-M. & 0.485 & 0.40 & 0.12 \\
\hline $2858,1.5 \mathrm{ml} / \mathrm{hr}$ Otay-M. & 0.158 & 0.13 & 0.013 \\
\hline PM-2, 1.4 ml/hr, Clinop. & 0.692 & 0.63 & 0.72 \\
\hline PM-2, $0.5 \mathrm{ml} / \mathrm{hr}$, Clinop. & 0.479 & 0.44 & 0.485 \\
\hline PM-1, 1.4 ml/hr, Clinop. & 0.696 & 0.63 & 0.685 \\
\hline PM-1, 0.5 ml/hr, Clinop. & 0.493 & 0.45 & 0.57 \\
\hline PM-2, $1.4 \mathrm{ml} / \mathrm{hr}$, Batch 2 Otay & 0.485 & 0.33 & 0.079 \\
\hline PM-2, $0.4 \mathrm{ml} / \mathrm{hr}$, Batch 2 Otay* & 0.3 & 0.24 & 0.015 \\
\hline PM-1, $1.6 \mathrm{ml} / \mathrm{hr}$, Batch 2 Otay. & 0.569 & 0.39 & 0.083 \\
\hline PM-1, $0.4 \mathrm{ml} / \mathrm{hr}$, Batch 2 Otay* & 0.25 & 0.20 & 0.006 \\
\hline PM-2, $1.5 \mathrm{ml} / \mathrm{hr}$, Batch 1 Otay & 0.81 & 0.67 & 0.402 \\
\hline
\end{tabular}

*These experiments involved the continuous injection of colloids after the tritiated water and $\mathrm{Pu}$ pulse had ceased.

from the colloids to the fracture surfaces when the colloids collided with the fracture surfaces. More collisions would be expected over longer residence times. It is also possible that the $\mathrm{Pu}$ had greater affinity for the manganese oxide fracture coatings known to be present on the UE20c fracture surfaces than for the colloids if the two surfaces came into contact and there was opportunity for exchange.

\section{Post-Test Fracture Characterizations (at Los Alamos)}

After the two UE-20c fractured cores were split open for post-test examinations, it was immediately apparent that there was a considerable amount of microbial growth in the fractures, which was discernable as a white-colored substance on the surfaces, although it tended to be concentrated in certain areas, not widely distributed. Twelve samples were collected from the fracture surfaces (six from each core) by scraping selected areas (including some areas with no 

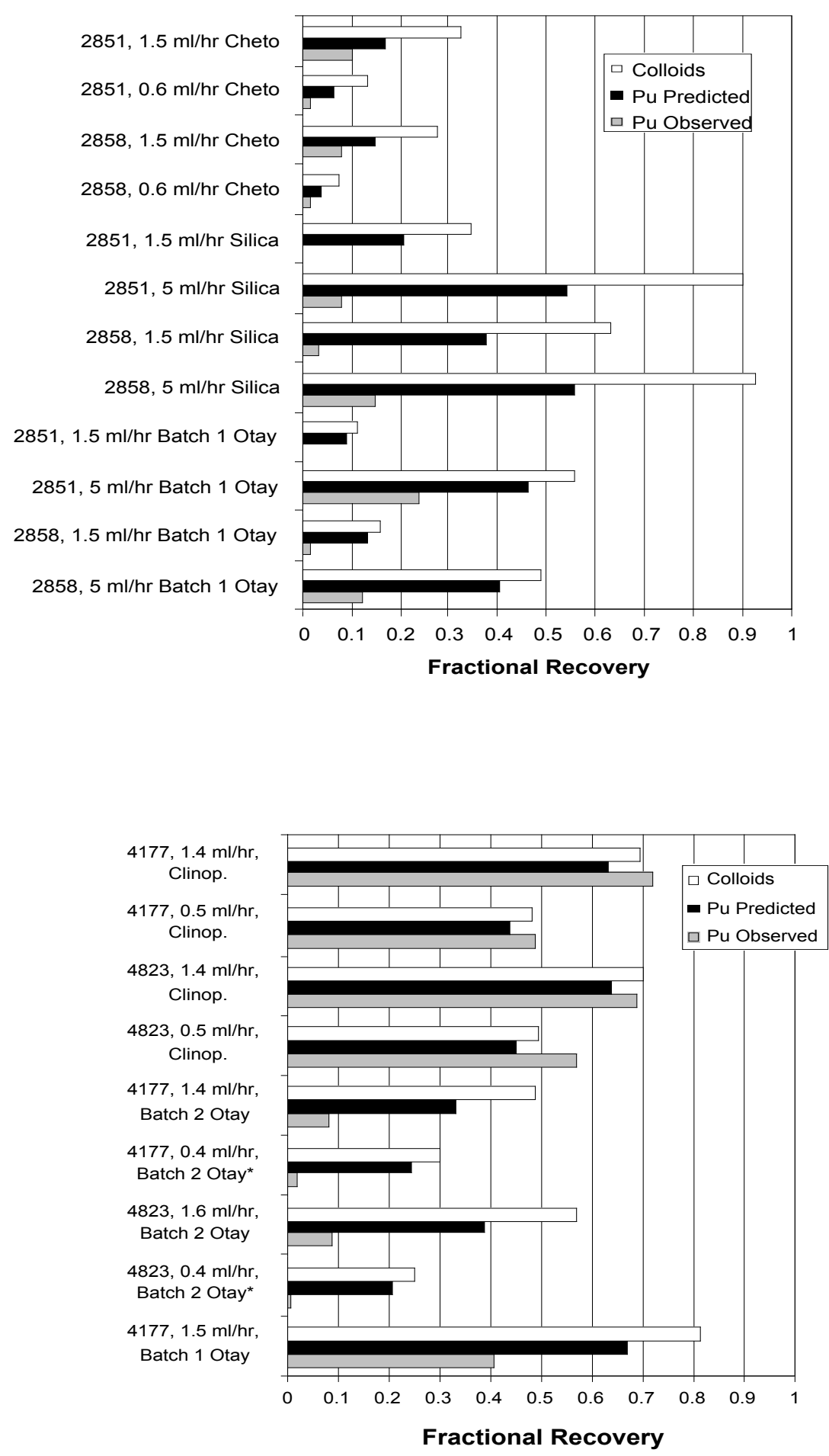

Figure 7.12. Colloid recoveries, "predicted" Pu recoveries, and actual Pu recoveries for all of the fractured core experiments. See Table 7.22 for actual data. 


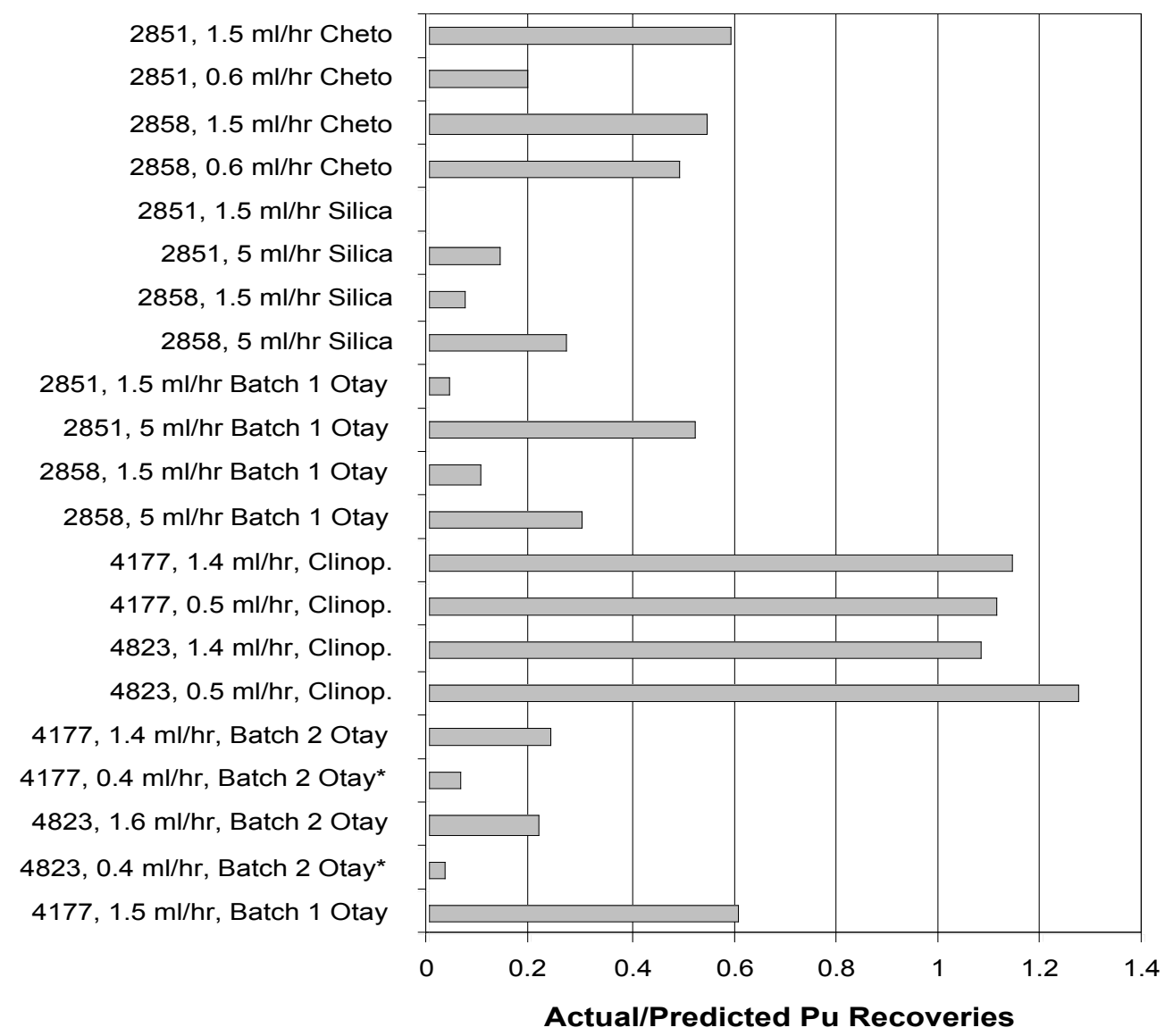

Figure 7.13. Ratio of actual to predicted $\mathrm{Pu}$ recoveries in each of the fractured core experiments.

visible white substance) with sterile $10 \mu$ plastic loops. Each loop was placed in a 10-ml sterile plastic test tube that contained $1 \mathrm{ml}$ sterile water. These tubes containing the loops were vortexed in order to detach the sample particles from the plastic and give an even suspension for inoculating MPN (most probable number) broth tubes. All samples were inoculated in duplicate: $0.1 \mathrm{ml}$ in $5 \mathrm{ml}$ broth and $0.01 \mathrm{ml}$ in $5 \mathrm{ml}$ broth. The culture tubes were incubated at room temperature. Growth occurred in all of the $0.1 \mathrm{ml}$ inoculations and in all but one of the $0.01 \mathrm{ml}$ inoculations. These results confirmed the presence of microbes in the fractures even in areas where there was no visible evidence on the fracture surfaces. Some of the cultures were subsequently DAPI-stained and examined under an epifluorescent microscope, which revealed that the microbes predominantly had a rod-shaped morphology.

Gross alpha scanning of all cores using a hand and foot monitor immediately after splitting them open indicated that the majority of the alpha activity was concentrated near the entrance end of 
the fractures. Two of the cores (UE20c, $2858 \mathrm{ft}$ below surface and PM-1 $4823 \mathrm{ft}$ below surface) were sent to LLNL for autoradiography work and for cutting the surfaces more finely for analysis by other techniques (see Chapter 9).

The UE20c, 2858 fracture surface was macro-autoradiographed at Los Alamos prior to sending it to LLNL. Figure 7.15 shows both a standard photograph and an autoradiograph of one of the two fracture surfaces. As this figure indicates, most of the activity is concentrated in the inlet region of the fracture, consistent with the observations of Eaton and Kersting (Appendix B of FY 00 UGTA Progress Report on Colloid-Facilitated Transport Work). This result is also consistent with the gross alpha scanning of the core using a hand and foot monitor. Figure 7.15 also suggests that the disposition of $\mathrm{Pu}$ almost matches the distribution of a dark-brown mineral on the surface. This mineral is a manganese oxide. 


\section{䓀}

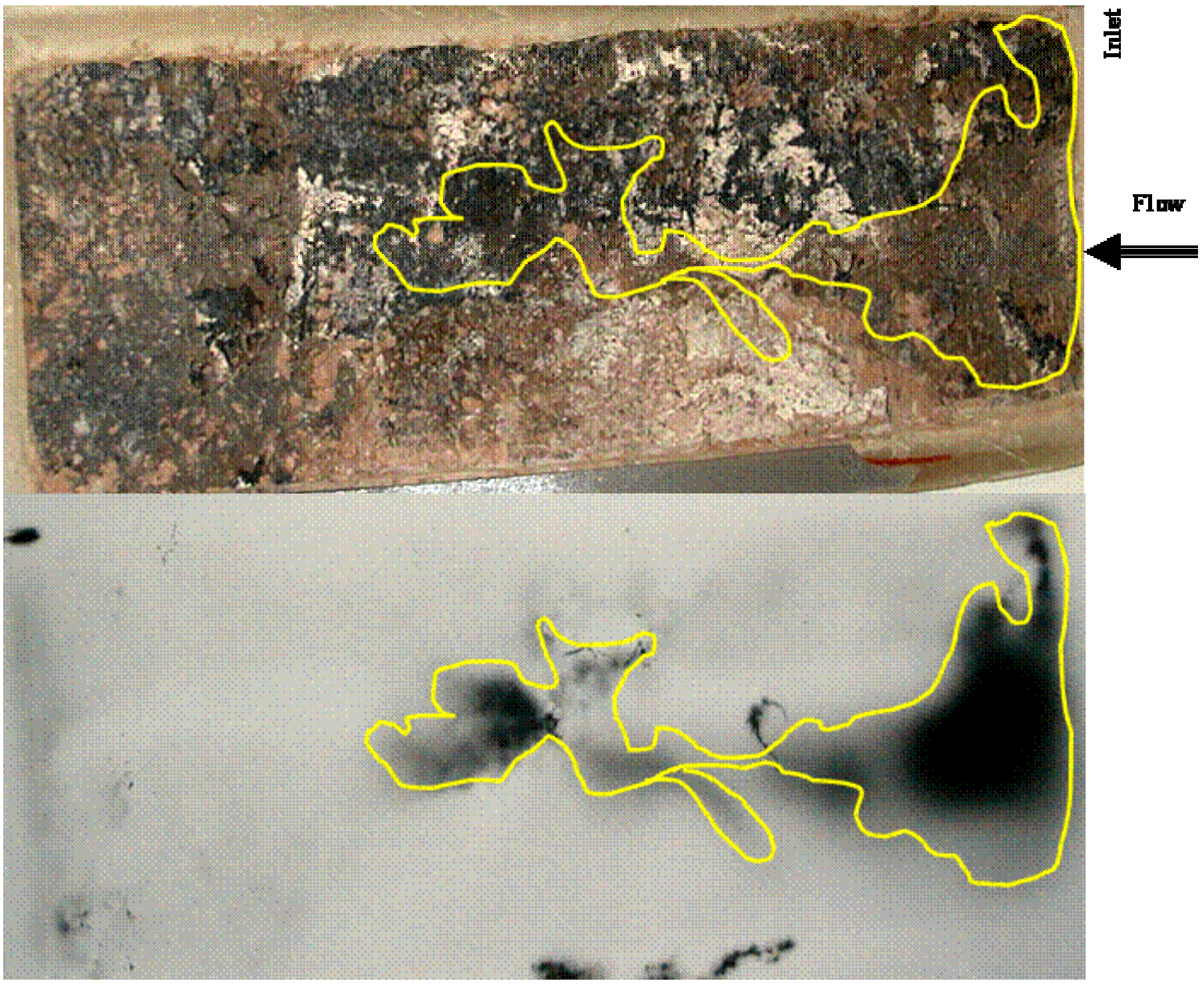



Figure 8.15. Autoradiograph (bottom) showing the disposition of Pu on one of the UE20c-2858 $\mathrm{ft}$ fracture surfaces (top). 


\subsection{References}

Becker, M. W., Reimus, P. W. and P. Vilks (1999). Transport and Attenuation of CarboxylateModified Latex Microspheres in Fractured Rock Laboratory and Field Tracer Tests, Ground Water, 37(3), 387-395.

Drellack, S. L. Jr., and L. B. Prothro (1997). Descriptive Narrative for the Hydrogeologic Model of Western and Central Pahute Mesa Corrective Action Units. Bechtel Nevada Draft Report.

Lu, N., C. R. Cotter, H. D. Kitten, J. Bentley, and I. R. Triay (1998). Reversibility of Sorption of Plutonium Onto Hematite and Goethite colloids, Radiochim. Acta, 83, 167-173.

Reimus, P. W. (1995). The Use of Synthetic Colloids in Tracer Transport Experiments in Saturated Rock Fractures, LA-13004-T, Los Alamos National Laboratory Thesis Report, Los Alamos, NM.

Reimus, P. W., A. Adams, M. J. Haga, A. R. Humphrey, T. J. Callahan, I. Anghel, and D. A. Counce (1999). Results and Interpretation of Hydraulic and Tracer Testing in the Prow Pass Tuff at the C-Holes, Yucca Mountain Site Characterization Project Milestone Report SP32E7M4, Los Alamos National Laboratory, Los Alamos, NM.

Reimus, P. W. and M. J. Haga (1999). Analysis of Tracer Responses in the BULLION ForcedGradient Experiment, $L A-13615-M S$, Los Alamos National Laboratory Manuscript Series Report, Los Alamos, NM.

Sumner, M. E., and W. P. Miller (1996) Cation Exchange Capacity and Exchange Coefficients. In: Method of Soil Analysis, Part 3. D. L. Sparks ed. SSSA book series No. 5, pp. 1215-1218, ASA and SSSA, Madison, WI. 


\title{
-Chapter 8- \\ Quantitative Interpretation of Fracture Transport Experiments and Associated Batch Sorption/Desorption Experiments
}

\author{
Paul W. Reimus
}

\subsection{Introduction}

In this chapter, quantitative interpretations of the fracture transport experiments and the associated batch sorption/desorption experiments conducted for UGTA (see Chapter 7) are presented. $\mathrm{Pu}$ sorption and desorption parameters were estimated from both the batch experiments and the fracture transport experiments, and these parameters were then compared to see how well the batch results could be used as a predictor of colloid-facilitated $\mathrm{Pu}$ transport under fracture flow conditions. Additionally, estimates of colloid filtration and detachment parameters were obtained for the colloids used in the fracture transport experiments.

\subsection{Interpretation of Batch Sorption and Desorption Experiments}

Two different conceptual models of sorption onto the colloids were used to "fit" the sorption and desorption data from each set of batch experiments. In the first model, two different types of sorption sites were assumed to exist on the colloids, with each site having potentially different sorption and desorption rate constants. The model equations for this conceptual model, called "the two-site model" are:

$$
\begin{aligned}
\left(\frac{\mathrm{V}}{\mathrm{M}_{\mathrm{col}}}\right) \frac{\mathrm{dC}}{\mathrm{dt}} & =-\mathrm{k}_{1 \mathrm{f}} \mathrm{C}\left(1-\frac{\mathrm{S}_{1}}{\mathrm{~S}_{1}^{0}}\right)-\mathrm{k}_{2 \mathrm{f}} \mathrm{C}\left(1-\frac{\mathrm{S}_{2}}{\mathrm{~S}_{2}^{0}}\right)+\mathrm{k}_{1 \mathrm{~b}} \mathrm{~S}_{1}+\mathrm{k}_{2 \mathrm{~b}} \mathrm{~S}_{2} \\
\frac{\mathrm{dS} \mathrm{S}_{1}}{\mathrm{dt}} & =\mathrm{k}_{1 \mathrm{f}} \mathrm{C}\left(1-\frac{\mathrm{S}_{1}}{\mathrm{~S}_{1}^{0}}\right)-\mathrm{k}_{1 \mathrm{~b}} \mathrm{~S}_{1} \\
\frac{\mathrm{dS} \mathrm{S}_{2}}{\mathrm{dt}} & =\mathrm{k}_{2 \mathrm{f}} \mathrm{C}\left(1-\frac{\mathrm{S}_{2}}{\mathrm{~S}_{2}^{0}}\right)-\mathrm{k}_{2 \mathrm{~b}} \mathrm{~S}_{2}
\end{aligned}
$$

where, $\mathrm{C}=$ radionuclide concentration in solution, $\mathrm{g} / \mathrm{g} \mathrm{H}_{2} \mathrm{O}$

$\mathrm{S}_{1}=$ radionuclide concentration sorbed to site type 1 on colloids, $\mathrm{g} / \mathrm{g}$ colloid

$\mathrm{S}_{2}=$ radionuclide concentration sorbed to site type 2 on colloids, $\mathrm{g} / \mathrm{g}$ colloid

$\mathrm{V}=$ mass of solution, $\mathrm{g}$

$\mathrm{M}_{\mathrm{col}}=$ mass of colloids in solution, $\mathrm{g}$ colloid

$\mathrm{k}_{1 \mathrm{f}}=$ rate constant for sorption to site type $1, \mathrm{~g} \mathrm{H}_{2} \mathrm{O} / \mathrm{g}$ colloid-hr

$\mathrm{k}_{2 \mathrm{f}}=$ rate constant for sorption to site type $2, \mathrm{~g} \mathrm{H}_{2} \mathrm{O} / \mathrm{g}$ colloid-hr

$\mathrm{k}_{1 \mathrm{~b}}=$ rate constant for desorption from site type $1,1 / \mathrm{hr}$

$\mathrm{k}_{2 \mathrm{~b}}=$ rate constant for desorption from site type $2,1 / \mathrm{hr}$

$\mathrm{S}_{1}^{0}=$ maximum radionuclide concentration sorbed to site type 1 on colloids, $\mathrm{g} / \mathrm{g}$ colloid

$\mathrm{S}_{2}^{0}=$ maximum radionuclide concentration sorbed site to type 2 on colloids, g/g colloid. 
This model does not have a firm mechanistic basis; it simply allows for two independent sorption and desorption rates on the colloid surfaces. However, one might consider a faster sorption site to be associated with $\mathrm{Pu}(\mathrm{V})$ sorption onto the surfaces without immediate reduction to $\mathrm{Pu}(\mathrm{IV})$, and a slower, less reversible, sorption site to be associated with $\mathrm{Pu}(\mathrm{V})$ sorption that involves immediate reduction to $\mathrm{Pu}(\mathrm{IV})$ on the surface. The electron transfer step associated with the reduction of $\mathrm{Pu}(\mathrm{V})$ to $\mathrm{Pu}(\mathrm{IV})$ is likely to be slow compared to the sorption of $\mathrm{Pu}(\mathrm{V})$ without immediate reduction, but once the $\mathrm{Pu}$ is reduced to $\mathrm{Pu}(\mathrm{IV})$ it is also likely to be much more strongly and less reversibly sorbed than $\mathrm{Pu}(\mathrm{V})$. Alternatively, all $\mathrm{Pu}$ sorbed onto the colloids could be considered to be $\mathrm{Pu}$ (IV) (consistent with the LLNL XAS experiments), and the two different sites could represent a distribution of sites with different affinities for binding $\mathrm{Pu}(\mathrm{IV})$ once it has been reduced. The two-site model can also mathematically accomodate the possibility of reduction of $\mathrm{Pu}(\mathrm{V})$ to $\mathrm{Pu}(\mathrm{IV})$ in solution prior to sorption (Path 2 in Figure 5.6) or even a catalytic reduction of $\mathrm{Pu}(\mathrm{V})$ at the colloid surfaces without sorption of $\mathrm{Pu}(\mathrm{V})$ followed by rapid sorption of $\mathrm{Pu}(\mathrm{IV})$ from the solution phase. In these cases, the $\mathrm{Pu}(\mathrm{V})$ to $\mathrm{Pu}(\mathrm{IV})$ reduction is considered the rate-limiting step, and the net result is a slow sorption reaction. Thus, although the two-site model is not strictly mechanistic, it is flexible in that it can mathematically accommodate many different possible mechanisms.

The second conceptual sorption model has a more mechanistic basis that is consistent with Path 1 of Figure 5.6. As discussed in Chapter 7, the Path 1 mechanism is believed to be more consistent with the LANL batch sorption/desorption experiments than the Path 2 mechanism. The model equations for this conceptual model, called the "Path 1 model" are:

$$
\begin{aligned}
& \left(\frac{V}{M_{c o l}}\right) \frac{d C}{d t}=-k_{1 f} C\left(1-\frac{S_{1}}{S_{1}^{0}+S_{2}^{0}}\right)+k_{1 b} S_{1} \\
& \frac{d S_{1}}{d t}=k_{1 f} C\left(1-\frac{S_{1}}{S_{1}^{0}+S_{2}^{0}}\right)+k_{2 b} S_{2}-k_{2 f} S_{1}\left(1-\frac{S_{2}}{S_{2}^{0}}\right)-k_{1 b} S_{1} \\
& \frac{d S_{2}}{d t}=k_{2 f} S_{1}\left(1-\frac{S_{2}}{S_{2}^{0}}\right)-k_{2 b} S_{2}
\end{aligned}
$$

where, $\mathrm{S}_{1}=\mathrm{Pu}(\mathrm{V})\left(\right.$ or $\left.\mathrm{PuO}_{2}^{+}\right)$concentration on colloids, $\mathrm{g} / \mathrm{g} \mathrm{H}_{2} \mathrm{O}$

$\mathrm{S}_{2}=\mathrm{Pu}(\mathrm{IV})$ concentration on colloids, $\mathrm{g} / \mathrm{g} \mathrm{H}_{2} \mathrm{O}$

$\mathrm{k}_{1 \mathrm{f}}=$ rate constant for sorption of $\mathrm{Pu}(\mathrm{V})\left(\right.$ or $\left.\mathrm{PuO}_{2}{ }^{+}\right)$to colloids, $\mathrm{g} \mathrm{H}_{2} \mathrm{O} / \mathrm{g}$ colloid-hr

$\mathrm{k}_{2 \mathrm{f}}=$ rate constant for reduction of $\mathrm{Pu}(\mathrm{V})$ to $\mathrm{Pu}(\mathrm{IV})$ on surfaces, $1 / \mathrm{hr}$

$\mathrm{k}_{1 \mathrm{~b}}=$ rate constant for desorption of $\mathrm{Pu}(\mathrm{V})\left(\right.$ or $\left.\mathrm{PuO}_{2}{ }^{+}\right)$from colloids, $1 / \mathrm{hr}$

$\mathrm{k}_{2 \mathrm{~b}}=$ rate constant for oxidation of $\mathrm{Pu}(\mathrm{IV})$ to $\mathrm{Pu}(\mathrm{V})$ on surfaces, $1 / \mathrm{hr}$

$\mathrm{S}_{1}^{0}=$ maximum $\mathrm{Pu}(\mathrm{V})\left(\right.$ or $\left.\mathrm{PuO}_{2}^{+}\right)$concentration sorbed on colloids, $\mathrm{g} / \mathrm{g}$ colloid

$\mathrm{S}_{2}^{0}=$ maximum $\mathrm{Pu}(\mathrm{IV})$ concentration sorbed on colloids, g/g colloid.

This model considers only a single reversible sorption reaction for $\mathrm{Pu}(\mathrm{V})$ onto the colloid surfaces. However, once the $\mathrm{Pu}(\mathrm{V})$ is sorbed onto the surfaces, it can be reduced to $\mathrm{Pu}(\mathrm{IV})$ by means of an electron-transfer reaction. This electron-transfer reaction can be specified as fast or 
slow, reversible or irreversible. Parameters in the model can be specified such that $\mathrm{Pu}(\mathrm{V})$ reduces immediately to $\mathrm{Pu}(\mathrm{IV})$ when it sorbs to the colloid surface (as was the case in the LLNL $\mathrm{Fe}$ - and $\mathrm{Mn}$-oxide experiments) or such that it reduces to $\mathrm{Pu}(\mathrm{IV})$ more slowly at the surface. In the latter case, there will be greater sorption reversibility at early times, consistent with LANL experiments involving silica and montmorillonite colloids, but the $\mathrm{Pu}$ can still end up predominantly as $\mathrm{Pu}(\mathrm{IV})$ on the surfaces over time (consistent with the LLNL EXAFS measurements). In this conceptual model, the only way that $\mathrm{Pu}(\mathrm{IV})$ can desorb from colloid surfaces is to first be oxidized back to $\mathrm{Pu}(\mathrm{V})$ and then to desorb as $\mathrm{Pu}(\mathrm{V})$. Although clearly a simplification with no direct evidence to support it, this assumption implicitly acknowledges the much higher solubility of $\mathrm{Pu}(\mathrm{V})$ relative to $\mathrm{Pu}(\mathrm{IV})$ under oxidizing (ambient) conditions.

The assumed reversible sorption of $\mathrm{Pu}(\mathrm{V})$ onto colloid surfaces without immediate reduction to $\mathrm{Pu}(\mathrm{IV})$ in both of the conceptual models above is not supported by direct experimental evidence, but it is certainly plausible given the structural and chemical similarity between $\mathrm{Pu}(\mathrm{V}) \mathrm{O}_{2}{ }^{+}$and $\mathrm{Np}(\mathrm{V}) \mathrm{O}_{2}{ }^{+}$, and the fact that $\mathrm{Np}(\mathrm{V}) \mathrm{O}_{2}{ }^{+}$has been observed to sorb significantly and reversibly to inorganic colloids ( $\mathrm{Lu}$ et al., The primary reason that $\mathrm{Pu}(\mathrm{V})$ consistently sorbs more strongly to inorganic surfaces than $\mathrm{Np}(\mathrm{V}) \mathrm{O}_{2}{ }^{+}$may be that $\mathrm{Pu}(\mathrm{V}) \mathrm{O}_{2}{ }^{+}$is more easily reduced than $\mathrm{Np}(\mathrm{V}) \mathrm{O}_{2}{ }^{+}$ at the surface (as predicted from thermodynamics - Langmuir, 1997), thus forming a more stable and strongly bound tetravalent species on the surface.

Equations (8.1) through (8.3) and (8.4) through (8.6) were encoded into separate FORTRAN models and solved simultaneously using a fourth-order Runge-Kutta numerical differential equation solver. In both cases, a model with only a single reversible sorption reaction was first used (by setting the rate constants for the second reaction equal to zero) to try to fit the experimental data before resorting to the more complex models. However, with the exception of $\mathrm{Pu}(\mathrm{IV})$ sorption onto the clinoptilolite colloids, it was quickly discovered that a single-site model could not accurately fit both the early and late portions of the sorption data, and the fits were especially poor when the desorption data were also considered. The adequate fit of a single-site model to the $\mathrm{Pu}(\mathrm{IV})$-clinoptilolite sorption data is presumably due to the fact that the starting $\mathrm{Pu}$ oxidation state was $\mathrm{Pu}(\mathrm{IV})$, so there was effectively only a single species with a high affinity for the colloid surfaces present throughout the experiments. For the other colloids, the fits to the data were improved considerably when the more complex models were employed. These models also ultimately resulted in better matches to the fracture transport data in all cases except for the $\mathrm{Pu}(\mathrm{IV})$ sorbed to the clinoptilolite colloids.

Model fits to the Pu-colloid sorption and desorption data are shown in Figures 8.1 through 8.7. Each plot has a log time axis to allow the early time data to be seen more clearly. The model parameters associated with the fits of the two-site model are provided in Table 8.1, and the parameters associated with the fits of the Path 1 model are provided in Table 8.2. Note that although the capability existed to treat both sorption reactions as reversible in the two-site model, one of the reactions was always considered irreversible $\left(k_{2 b}=0\right)$ (except in the case of the clinoptilolite colloids, where only a single reversible reaction was used). In Figures 8.1 through 8.7 , only fits in which the faster sorption reaction was considered reversible in the two-site model are shown. However, Table 8.1 also provides fitted model parameters obtained assuming that the slower reaction was reversible, with an irreversible fast reaction. These two variations of the two-site model offered fits of similar quality, although the desorption data were fitted 
slightly better when a slow irreversible reaction was assumed. It should be noted that, when specifying an irreversible reaction, it was also necessary to limit the number of irreversible sorption sites to avoid most or all of the Pu ending up sorbed to irreversible sites by the end of the experiments (contrary to the desorption data). Fits to the data assuming two reversible reactions with an unlimited number of sites were not quite as good as the fits with an irreversible reaction, but this is not surprising given that they involved one less adjustable fitting parameter. Also, the fits with a limited number of sites resulted in slightly better matches to the fracture transport data.

For the fits involving the Path 1 model shown in Figures 8.1 through 8.7, an unlimited number of sorption sites were specified $\left(\mathrm{S}_{1}^{0}\right.$ and $\mathrm{S}_{2}^{0}$ were assigned very large values). Given the $\mathrm{Pu}$ concentrations in the LANL batch experiments and reasonable estimates of colloid surface areas in the experiments, there were several hundred to over a thousand $\mathrm{nm}^{2}$ of surface area per $\mathrm{Pu}$ atom sorbed to the colloids, which intuitively seems far less than maximum surface coverage. However, it is also possible that there are only a limited number of preferred sites for sorption on the colloid surfaces, thus making the effective maximum coverage far less than the theoretical coverage. An additional fitting constraint used with the Path 1 model (besides simply fitting the sorption and desorption data) was that at least three-fourths of the Pu on the colloid surfaces after 30 days of sorption had to be associated with $\mathrm{Pu}(\mathrm{IV})$ sites. This constraint ensured consistency with the LLNL XAS experiments, in which little or no $\mathrm{Pu}(\mathrm{V})$ was observed on any of the inorganic colloid surfaces after approximately 30 days of sorption. Such a constraint was not used with the two-site model above because that model does not explicitly associate Pu oxidation states with sorption sites.

In Figures 8.1 through 8.7, the sorption and desorption data include points obtained assuming that there was no $\mathrm{Pu}$ sorption to the test tube walls and points assuming that the fraction of $\mathrm{Pu}$ sorbed to the walls in control samples ( $\mathrm{Pu}$ with no colloids) was the same as in the colloid samples. It is apparent that the correction for wall effects has very little influence on the majority of the data.

In Figures 8.1 through 8.4 and Figure 8.6, model fits assuming a single reversible sorption reaction (single site model), the two-site model, and the Path 1 model are shown. Also shown are model predictions of desorption for the two-site and Path 1 models, which offered better matches to the desorption data and fracture transport data in all cases. For the silica colloids, a single reversible reaction fitted the sorption data quite well (Figures 8.2 and 8.4), but it did not offer a very good match to the desorption data.

Figure 8.5 shows only a single model fit, corresponding to a single reversible reaction, for $\mathrm{Pu}(\mathrm{IV})$ sorption onto the clinoptilolite colloids. The inclusion of a second reaction in this case provided an insignificant improvement to the model fit. As discussed above, the good fit of a single-site model to the $\mathrm{Pu}(\mathrm{IV})$-clinoptilolite sorption data is probably due to the fact that the starting $\mathrm{Pu}$ oxidation state was $\mathrm{Pu}(\mathrm{IV})$, so there was effectively only a single species with a high affinity for the colloid surfaces present throughout the experiments. Figure 8.7 shows only the fits of the two-site and Path 1 models to the batch 1 Otay montmorillonite data. The "fits" in this case have the same model parameters as the fits of these models to the batch 1 Otay 
montmorillonite data in Figure 8.3. Thus, a single set of model parameters was capable of fitting both batch sorption/desorption data sets in which the same montmorillonite colloids were used.

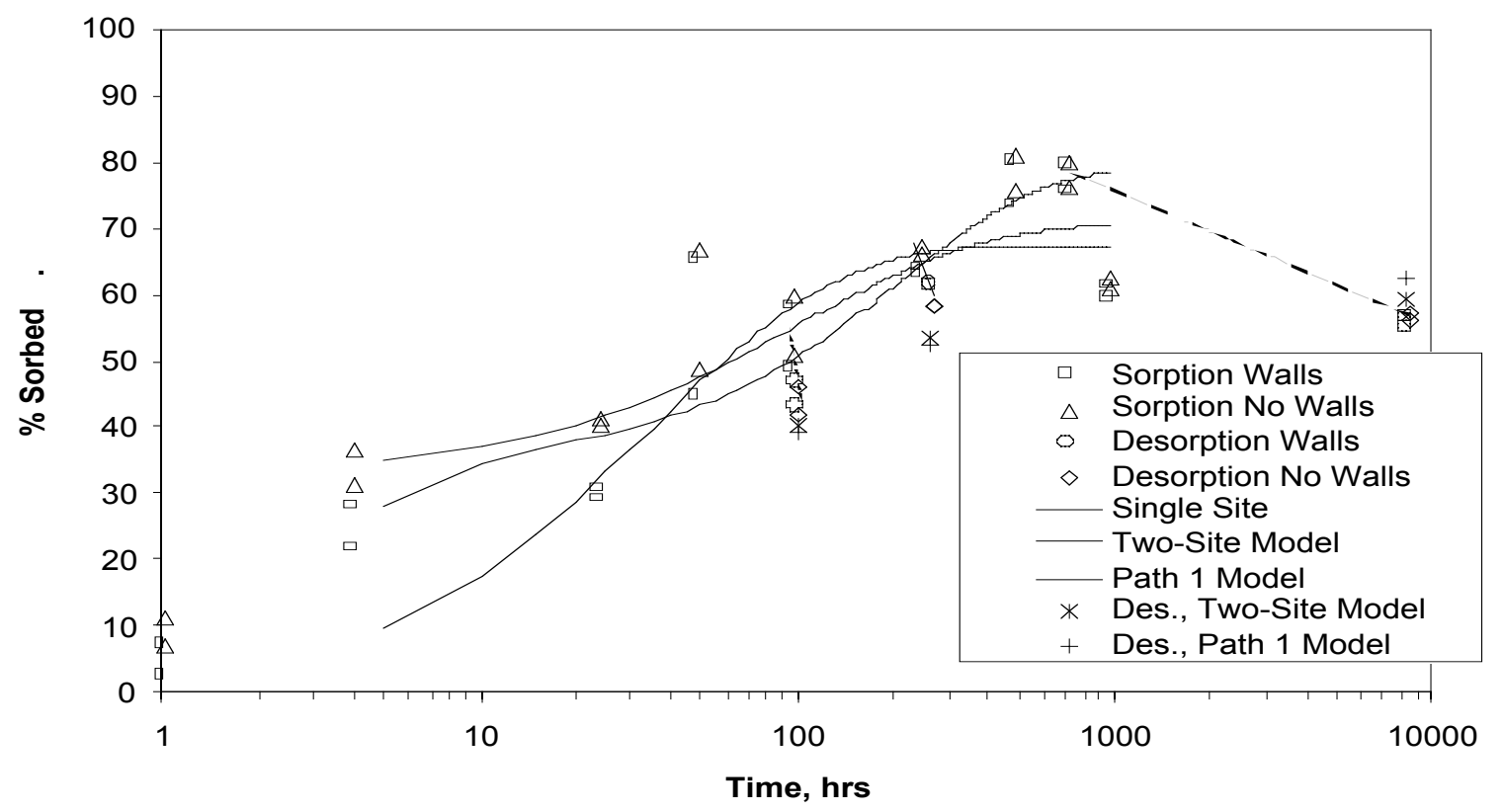

Figure 8.1. Model fits to sorption and desorption data for $\mathrm{Pu}(\mathrm{V})$ sorption onto Cheto montmorillonite colloids. Dashed lines are drawn from start time to end time of desorption. Asterisks indicate model results for desorption. Model fits (solid lines) are discussed in text.

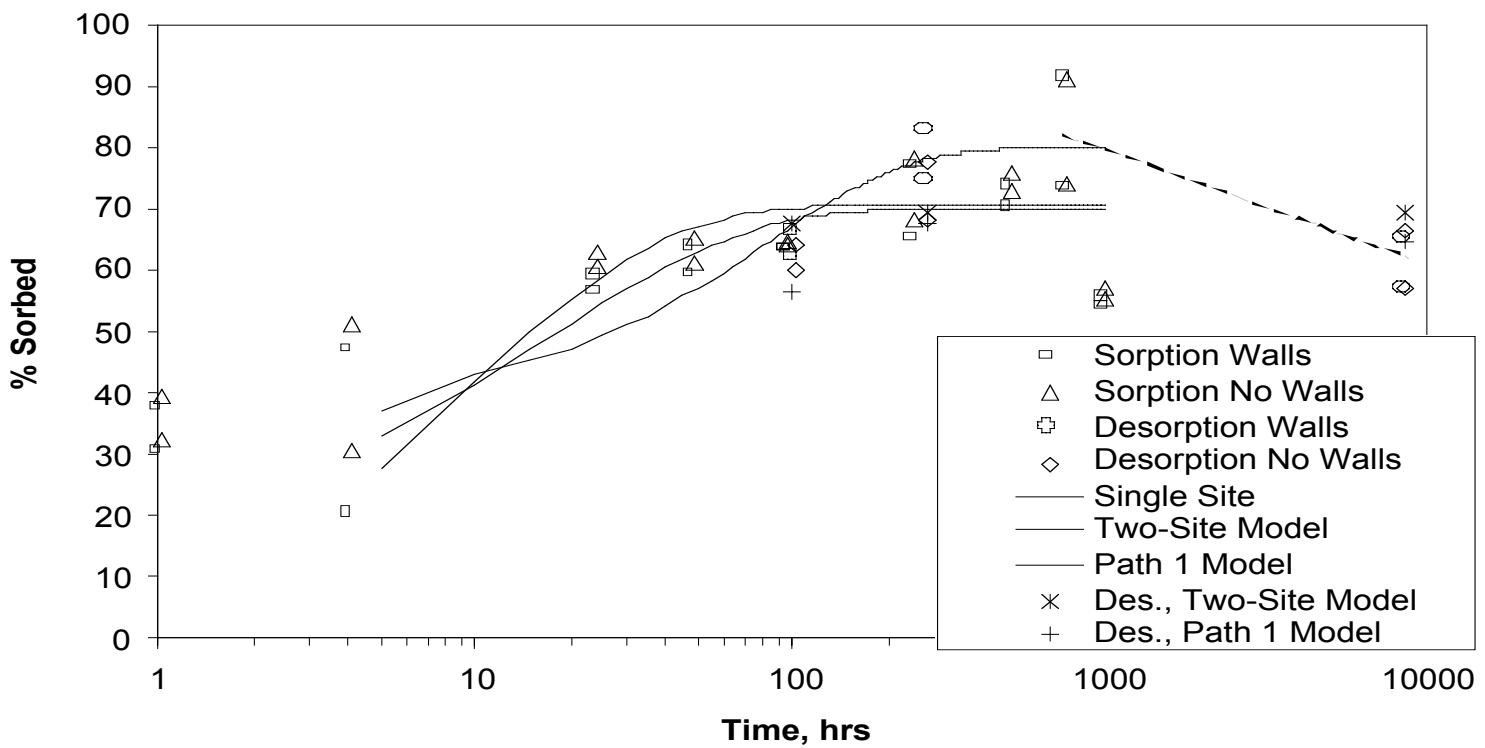

Figure 8.2. Model fits to sorption and desorption data for $\mathrm{Pu}(\mathrm{V})$ sorption onto PST-1 silica colloids. Dashed lines are drawn from start time to end time of desorption. Asterisks indicate model results for desorption. Model fits (solid lines) are discussed in text. 


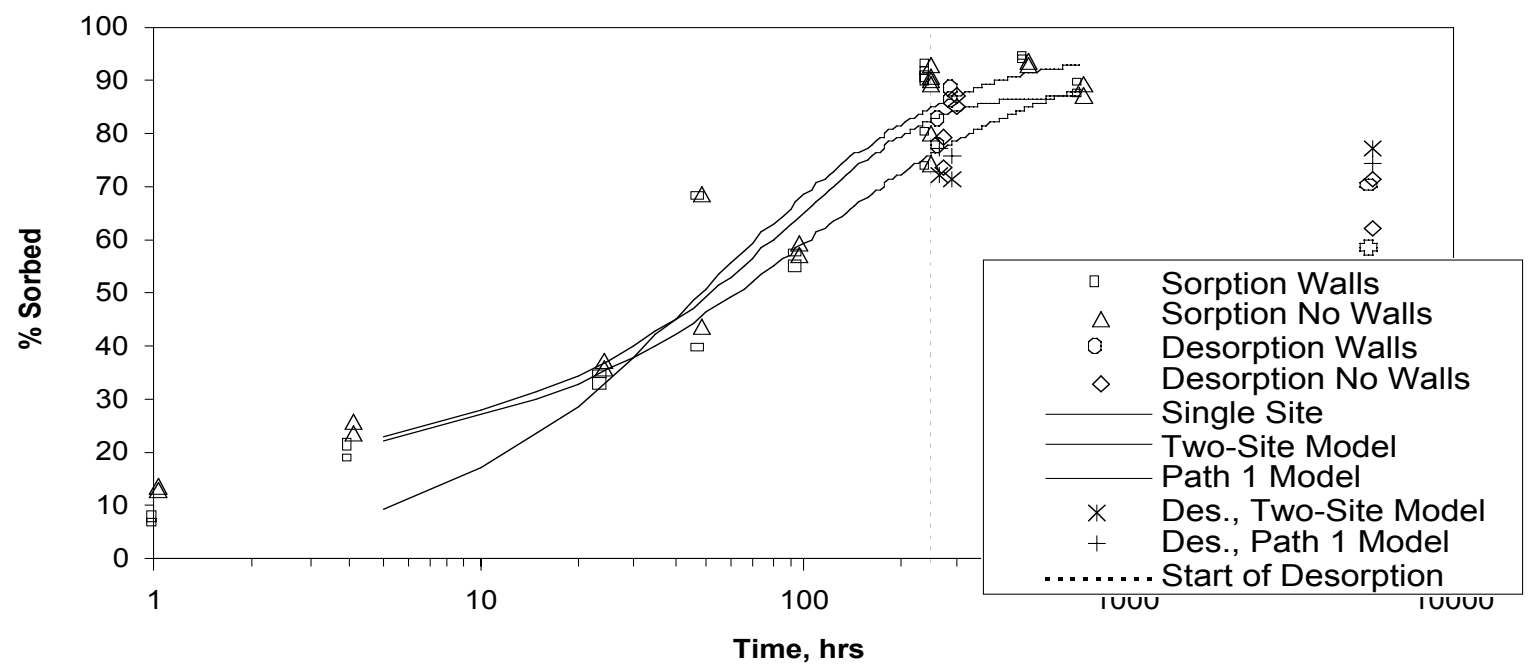

Figure 8.3. Model fits to sorption and desorption data for $\mathrm{Pu}(\mathrm{V})$ sorption onto batch 1 Otay montmorillonite colloids. Dashed line shows when desorption experiments started. Asterisks indicate model results for desorption. Model fits (solid lines) are discussed in text.

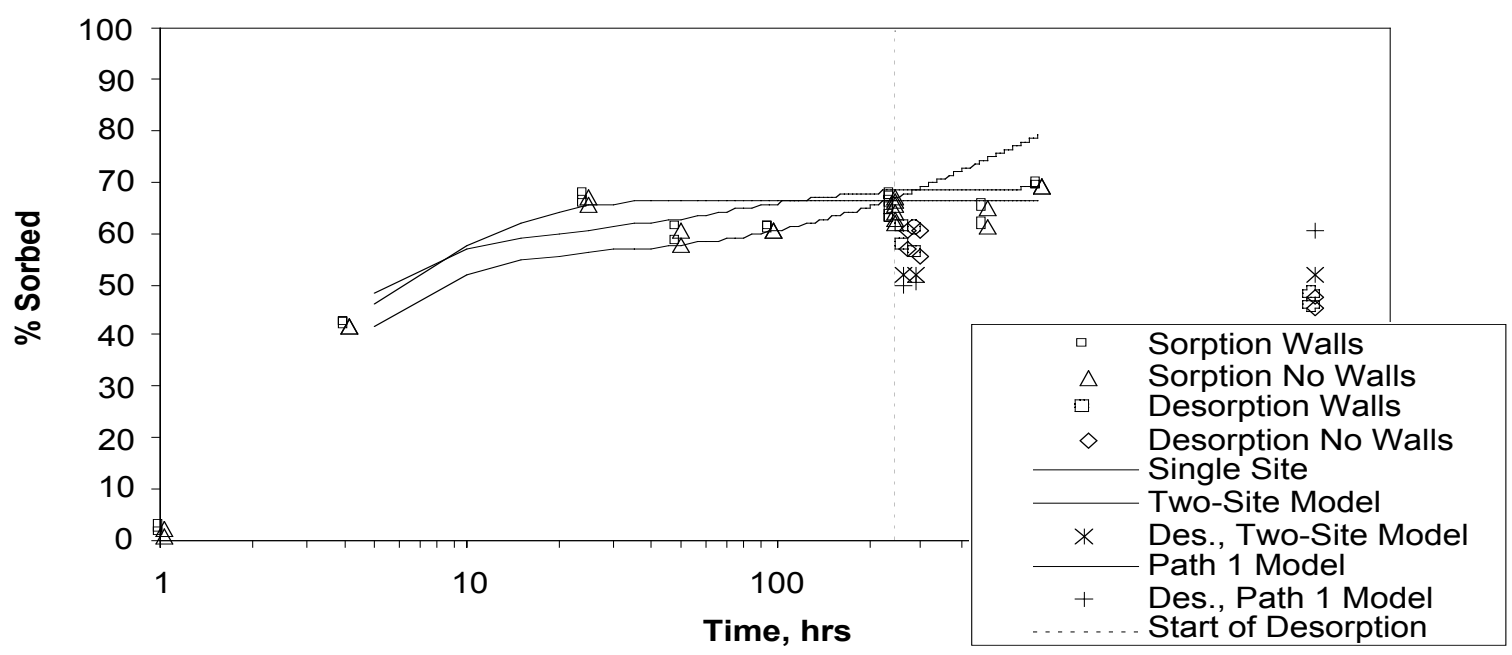

Figure 8.4. Model fits to sorption and desorption data for $\mathrm{Pu}(\mathrm{V})$ sorption onto MP-1040 silica colloids. Dashed line shows when desorption experiments started. Asterisks indicate model results for desorption. Model fits (solid lines) are discussed in text. 


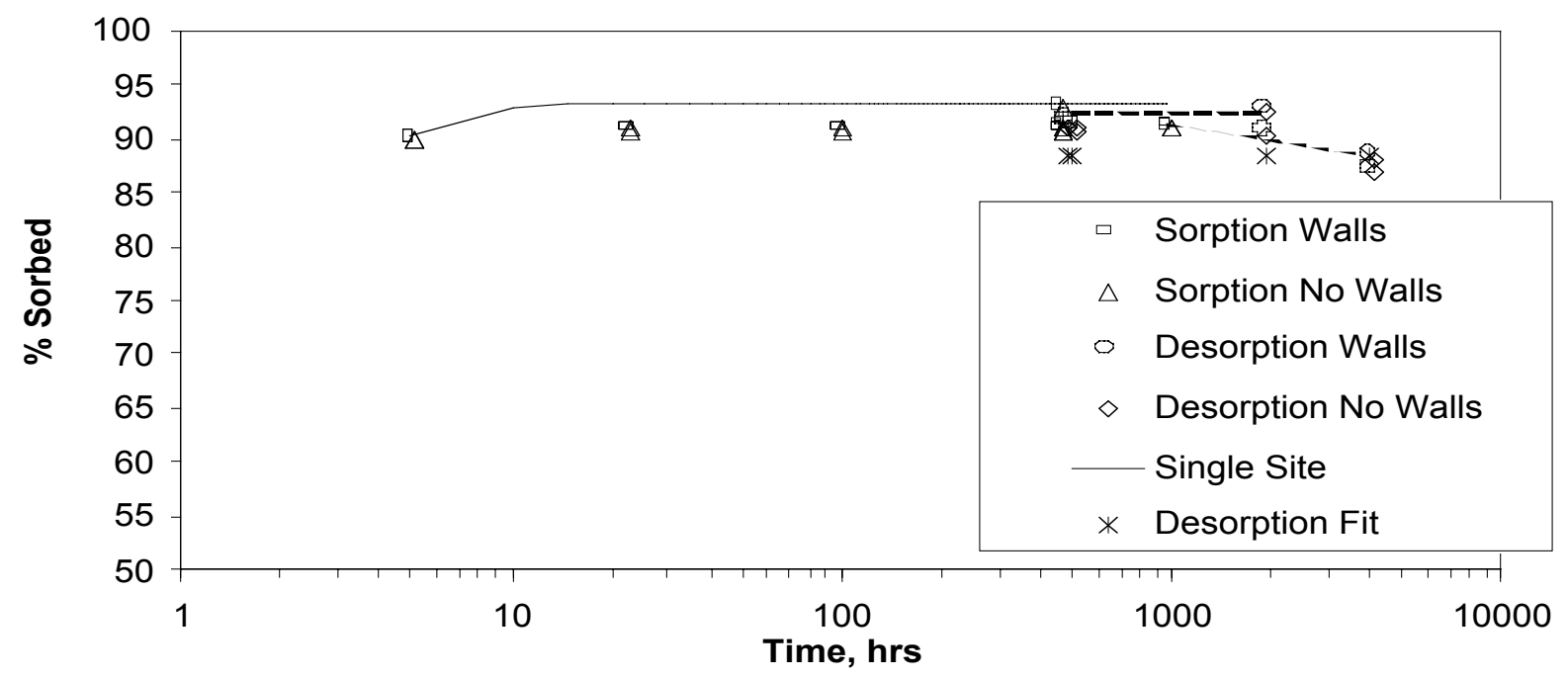

Figure 8.5. Single-site model fit to sorption and desorption data for $\mathrm{Pu}(\mathrm{V})$ sorption onto clinoptilolite colloids (first 3 sorption data points are from LLNL experiments in J-13 well water at $\mathrm{pH}$ 8). Dashed lines are drawn from start time to end time of desorption. Asterisks indicate model results for desorption.

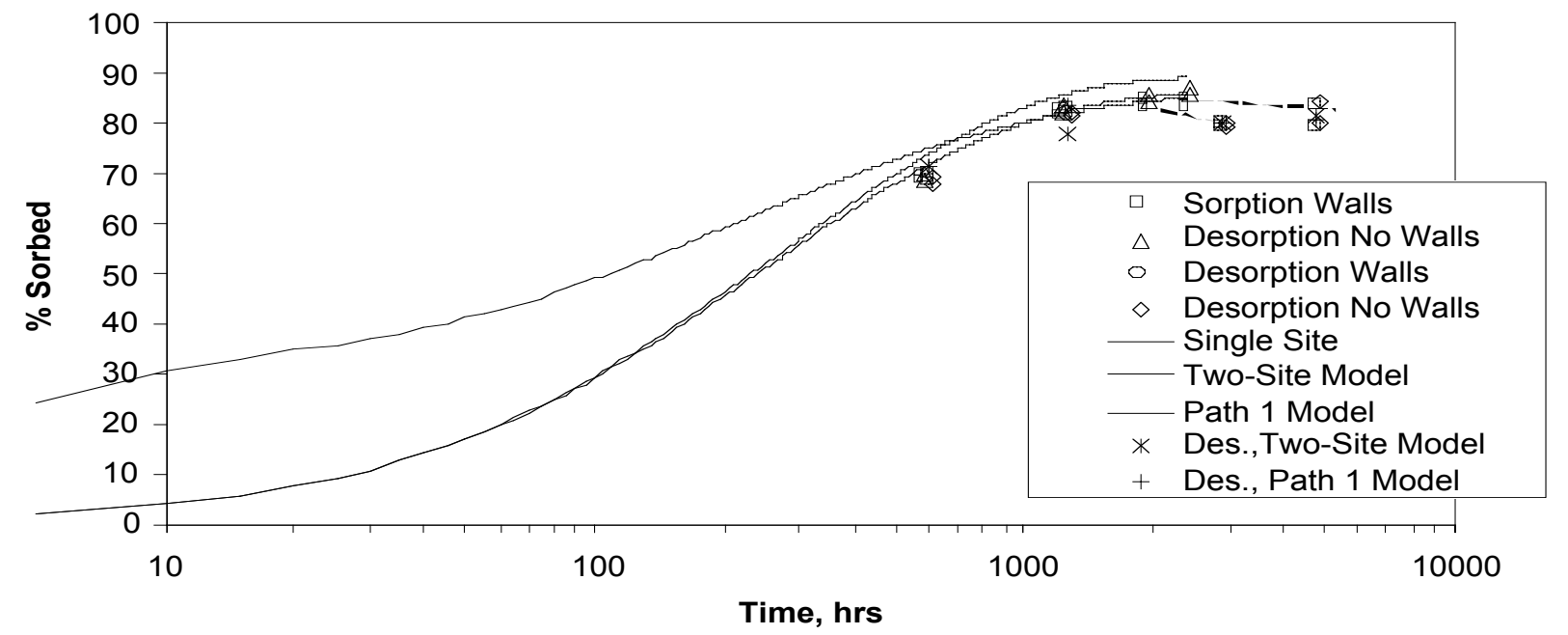

Figure 8.6. Model fits to sorption and desorption data for $\mathrm{Pu}(\mathrm{V})$ sorption onto batch 2 Otay montmorillonite colloids. Dashed lines are drawn from start time to end time of desorption. Asterisks indicate model results for desorption. Model fits (solid lines) are discussed in text. 


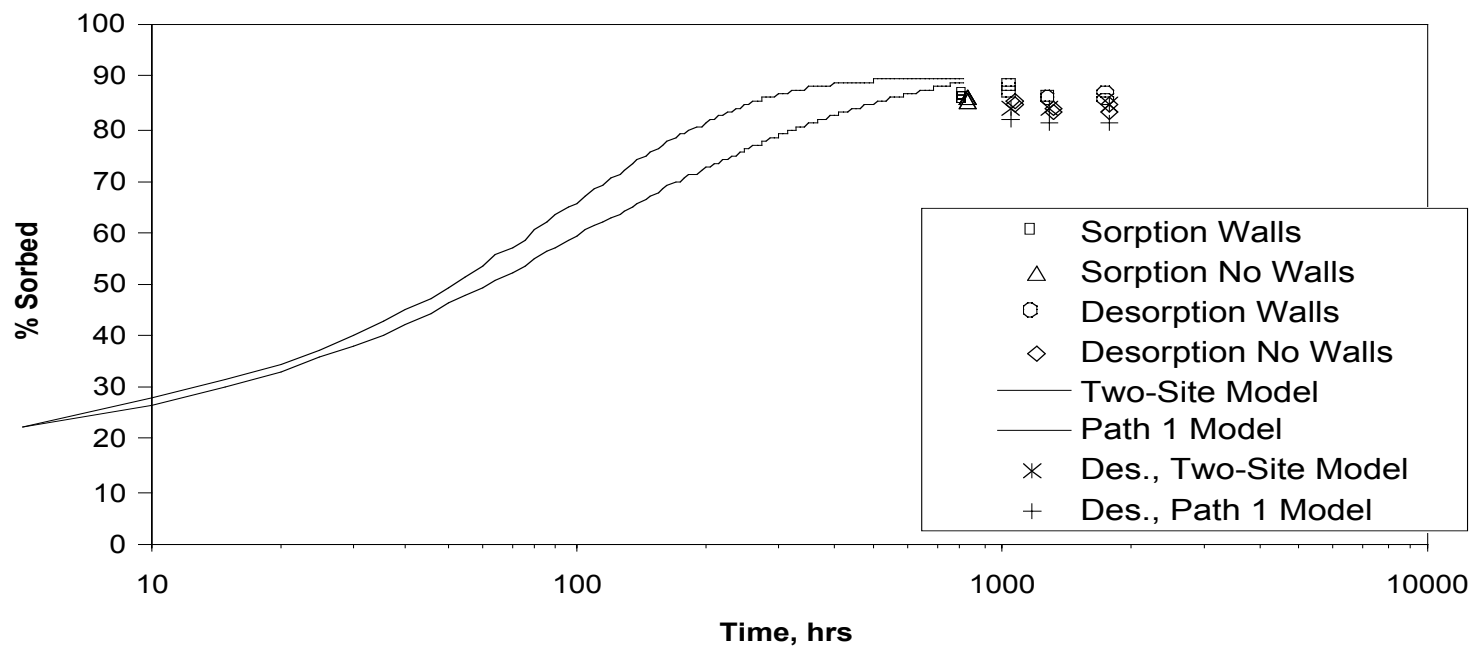

Figure 8.7. Fast, reversible 2-site model fit to sorption and desorption data for $\mathrm{Pu}(\mathrm{V})$ sorption onto batch 1 Otay montmorillonite colloids. Asterisks indicate model results for desorption (all started at $816 \mathrm{hrs}$ ). Model fit is same as for batch 1 Otay montmorillonite in Figure 8.3.

Table 8.1. Sorption/desorption model parameters associated with the single-site and two-site model fits shown in Figures 8.1 through 8.7. Also provided are the best-fitting parameters associated with the two-site model when the irreversible sorption reaction is faster than the reversible sorption reaction.

$\begin{array}{lllll}\text { Cheto } & \text { PST-1 } & \text { Otay (1) } & \text { MP-1040 } & \text { Clino. Otay (2) }\end{array}$

\section{Single-Site}

\begin{tabular}{|c|c|c|c|c|c|c|}
\hline $\mathrm{k}_{1 \mathrm{f}}, \mathrm{g} / \mathrm{g}-\mathrm{hr}$ & 110 & 350 & 100 & 1000 & 5000 & 20 \\
\hline $\mathrm{k}_{1 \mathrm{~b}}, 1 / \mathrm{hr}$ & 0.0011 & 0.025 & 0.002 & 0.0175 & 0.024 & 0.000175 \\
\hline $\mathrm{S}_{1}^{0}, \mathrm{~g} / \mathrm{g}$ & 0.0045 & 0.01 & 0.0066 & 0.0048 & 0.0072 & 0.0072 \\
\hline
\end{tabular}

\section{2-Site, Fast Rev.}

\begin{tabular}{|c|c|c|c|c|c|c|}
\hline $\mathrm{k}_{1 \mathrm{f}}, \mathrm{g} / \mathrm{g}-\mathrm{hr}$ & 2000 & 2250 & 650 & 950 & --- & 500 \\
\hline $\mathrm{k}_{1 \mathrm{~b}}, 1 / \mathrm{hr}$ & 0.15 & 0.002 & 0.01 & 0.15 & --- & 0.006 \\
\hline $\mathrm{k}_{2 \mathrm{f}}, \mathrm{g} / \mathrm{g}-\mathrm{hr}$ & 35 & 200 & 75 & 55 & --- & 20 \\
\hline $\mathrm{k}_{2 \mathrm{~b}}, 1 / \mathrm{hr}$ & 0 & 0 & 0 & 0 & --- & 0 \\
\hline $\mathrm{S}_{1}^{0}, \mathrm{~g} / \mathrm{g}$ & 0.0024 & 0.0012 & 0.0012 & 0.01 & --- & 0.0018 \\
\hline $\mathrm{S}_{2}^{0}, \mathrm{~g} / \mathrm{g}$ & 0.0026 & 0.003 & 0.0048 & 0.0018 & --- & 0.00136 \\
\hline
\end{tabular}

2-Site, Fast Irrev.

\begin{tabular}{|c|c|c|c|c|c|c|}
\hline $\mathrm{k}_{1 \mathrm{f}}, \mathrm{g} / \mathrm{g}-\mathrm{hr}$ & 22.5 & 25 & 50 & 100 & --- & --- \\
\hline $\mathrm{k}_{1 \mathrm{~b}}, 1 / \mathrm{hr}$ & 0.003 & 0.007 & 0.0012 & 0.16 & --- & --- \\
\hline $\mathrm{k}_{2 \mathrm{f}}, \mathrm{g} / \mathrm{g}-\mathrm{hr}$ & 600 & 800 & 700 & 1000 & --- & --- \\
\hline $\mathrm{k}_{2 \mathrm{~b}}, 1 / \mathrm{hr}$ & 0 & 0 & 0 & 0 & --- & --- \\
\hline $\mathrm{S}_{1}^{0}, \mathrm{~g} / \mathrm{g}$ & 0.01 & 0.01 & 0.01 & 0.0048 & --- & --- \\
\hline $\mathrm{S}_{2}^{0}, \mathrm{~g} / \mathrm{g}$ & 0.0018 & 0.003 & 0.00096 & 0.0036 & --- & --- \\
\hline
\end{tabular}


Table 8.2. Sorption/desorption model parameters associated with the Path 1 model fits shown in Figures 8.1 through 8.4, 8.6 and 8.7.

\begin{tabular}{|c|c|c|c|c|c|}
\multicolumn{1}{c}{} & \multicolumn{1}{c}{ Cheto } & PST-1 & Otay (1) & MP-1040 & Otay (2) \\
\hline $\mathrm{k}_{1 \mathrm{f}}, \mathrm{g} / \mathrm{g}-\mathrm{hr}$ & 550 & 900 & 500 & 750 & 20 \\
\hline $\mathrm{k}_{1 \mathrm{~b}}, 1 / \mathrm{hr}$ & 0.2 & 0.26 & 0.3 & 0.12 & 0.0027 \\
\hline $\mathrm{k}_{2 \mathrm{f}}, 1 / \mathrm{hr}$ & 0.009 & 0.02 & 0.04 & 0.0025 & 0.004 \\
\hline $\mathrm{k}_{2 \mathrm{~b}}, 1 / \mathrm{hr}$ & 0.0015 & 0.004 & $0.002 / 0.0015^{1}$ & 0.0005 & 0.0008 \\
\hline $\mathrm{S}_{1}^{0}, \mathrm{~g} / \mathrm{g}^{2}$ & 0.12 & 0.12 & 0.12 & 0.12 & 0.12 \\
\hline $\mathrm{S}_{2}^{0}, \mathrm{~g} / \mathrm{g}^{2}$ & 0.12 & 0.12 & 0.12 & 0.12 & 0.12 \\
\hline
\end{tabular}

${ }^{1} 0.0015$ offered a slightly better fit to the second set of batch 1 Otay colloid experiments.

${ }^{2} \mathrm{~A}$ value of $0.12 \mathrm{~g} / \mathrm{g}$ corresponds to an essentially unlimited number of sites.

\subsection{Procedure for Interpretation of Fracture Transport Experiments}

The interpretation of the fracture transport experiments proceeded in three steps:

(1) The tritium responses in each of the two experiments with the same colloids at different flow rates in a given fracture were simultaneously fitted using a semi-analytical dual-porosity model. These fits were conducted to obtain estimates of mean residence times, Peclet numbers (column length divided by dispersivity, $\mathrm{L} / \alpha$ ), and matrix diffusion mass transfer coefficients $\left(\frac{\phi}{\mathrm{b}} \sqrt{\mathrm{D}_{\mathrm{m}}}\right.$, where $\phi$ is the matrix porosity, $\mathrm{b}$ is the fracture half aperture, $\mathrm{cm}$, and $\mathrm{D}_{\mathrm{m}}$ is the tritium diffusion coefficient in the matrix, $\mathrm{cm}^{2} / \mathrm{s}$ ) in the fractures. The ratio of the mean residence times was constrained to be equal to the ratio of the inverse of the flow rates in the two experiments, and the Peclet numbers and matrix diffusion mass transfer coefficients were constrained to be identical at the two flow rates. The procedure of simultaneously analyzing the tritium responses at different flow rates greatly reduced the nonuniqueness of the transport parameter estimates compared to analyzing the tracer responses individually (Callahan et al., 2000).

(2) Once the mean residence times and dispersivities were established from step 1, these parameters were assumed to apply to the colloids. The colloid responses in each experiment were then fitted by adjusting only a colloid filtration rate constant and a detachment rate constant.

(3) Once the colloid responses were fitted reasonably well, a numerical model was used to obtain fits to the plutonium data by adjusting adsorption and desorption rate constants for $\mathrm{Pu}$ sorption onto the colloids and fracture surfaces. The "two-site" and "Path 1" sorption models used to interpret the batch sorption/desorption experiments were also used in the numerical transport model.

The fits to the tritium and colloid data in steps 1 and 2 were obtained using the RELAP computer model (Reimus and Haga 1999, Appendix A). Briefly, RELAP simulates both sorbing and nonsorbing tracer transport through a "dual-porosity" system in which flow is assumed to occur in parallel-plate fractures that are separated by a matrix containing stagnant water in its pores. One-dimensional advective-dispersive transport is assumed to occur within the fractures, with 
one-dimensional diffusive mass transfer occurring perpendicular to the fracture orientation in the matrix. RELAP is a semi-analytical model that is limited to simulating steady-state flow conditions. The differential equations used in RELAP to simulate solute transport in dualporosity systems are:

Fracture: $\quad \mathrm{R}_{\mathrm{f}} \frac{\partial \mathrm{C}_{\mathrm{f}}}{\partial \mathrm{t}}+\mathrm{v}_{\mathrm{f}} \frac{\partial \mathrm{C}_{\mathrm{f}}}{\partial \mathrm{x}}-\mathrm{D}_{\mathrm{f}} \frac{\partial^{2} \mathrm{C}_{\mathrm{f}}}{\partial \mathrm{x}^{2}}-\left.\frac{\phi \mathrm{D}_{\mathrm{m}}}{\mathrm{b} \eta} \frac{\partial \mathrm{C}_{\mathrm{m}}}{\partial \mathrm{y}}\right|_{\mathrm{y}=\mathrm{b}}=0$

Matrix:

$$
\mathrm{R}_{\mathrm{m}} \frac{\partial \mathrm{C}_{\mathrm{m}}}{\partial \mathrm{t}}-\mathrm{D}_{\mathrm{m}} \frac{\partial^{2} \mathrm{C}_{\mathrm{m}}}{\partial \mathrm{y}^{2}}=0
$$

subject to the following initial and boundary conditions

$$
\begin{aligned}
& \mathrm{C}_{\mathrm{f}}(\mathrm{x}, 0)=0 \\
& \mathrm{C}_{\mathrm{f}}(0, \mathrm{t})=X(\mathrm{t}) \\
& \mathrm{C}_{\mathrm{f}}(\infty, \mathrm{t})=0 \\
& \mathrm{C}_{\mathrm{m}}(\mathrm{y}, \mathrm{x}, 0)=0 \\
& \mathrm{C}_{\mathrm{m}}(\mathrm{b}, \mathrm{x}, \mathrm{t})=\mathrm{C}_{\mathrm{f}}(\mathrm{x}, \mathrm{t}) \\
& \mathrm{C}_{\mathrm{m}}(\infty, \mathrm{x}, \mathrm{t})=0
\end{aligned}
$$

where, $\mathrm{C}_{\mathrm{f}}=$ tracer concentration in solution in fractures, $\mathrm{g} / \mathrm{cm}^{3}$

$\mathrm{C}_{\mathrm{m}}=$ tracer concentration in solution in matrix, $=\mathrm{g} / \mathrm{cm}^{3}$

$X(\mathrm{t})=$ arbitrary injection pulse function, $\mathrm{g} / \mathrm{cm}^{3}$

$\mathrm{v}_{\mathrm{f}}=$ fluid velocity in fractures, $\mathrm{cm} / \mathrm{s}$

$\mathrm{D}_{\mathrm{f}}=$ dispersion coefficient in fractures, $\mathrm{cm}^{2} / \mathrm{s}$

$\mathrm{D}_{\mathrm{m}}=$ molecular diffusion coefficient in matrix, $\mathrm{cm}^{2} / \mathrm{s}$

$\mathrm{R}_{\mathrm{f}}=$ retardation coefficient in fractures (1.0 for tritium)

$\mathrm{R}_{\mathrm{m}}=$ retardation coefficient in matrix (1.0 for tritium)

$\eta=$ porosity within fractures,

$\phi=$ matrix porosity, and

$\mathrm{b}=$ fracture half aperture, $\mathrm{cm}$.

Equations (8.7) and (8.8) assume equilibrium sorption behavior (very fast sorption/desorption kinetics). First-order linear colloid filtration can be accounted for in RELAP using the ratelimited sorption features of the model. Forward and reverse sorption rate constants, which are mathematically equivalent to filtration and detachment rate constants, were adjusted to fit the colloid data. The colloids were assumed to have a matrix diffusion coefficient of zero because of their large size and small diffusivity relative to the tritium. However, some fits were also conducted in which the colloids were assumed to have very small matrix diffusion coefficients (two orders of magnitude smaller than tritium). Ignoring matrix diffusion, the equations used to describe colloid transport through the fractures were: 


$$
\begin{gathered}
\frac{\partial \mathrm{C}_{\mathrm{col}}}{\partial \mathrm{t}}+\mathrm{v}_{\mathrm{f}} \frac{\partial \mathrm{C}_{\mathrm{col}}}{\partial \mathrm{x}}-\mathrm{D}_{\mathrm{f}} \frac{\partial^{2} \mathrm{C}_{\mathrm{col}}}{\partial \mathrm{x}^{2}}+\mathrm{k}_{\mathrm{fcol}} \mathrm{C}_{\mathrm{col}}-\frac{\mathrm{k}_{\mathrm{rcol}} \mathrm{S}_{\mathrm{col}}}{\mathrm{b}}=0 \\
\frac{\partial \mathrm{S}_{\mathrm{col}}}{\partial \mathrm{t}}-\mathrm{b} \mathrm{k}_{\mathrm{fcol}} \mathrm{C}_{\mathrm{col}}+\mathrm{k}_{\mathrm{rcol}} \mathrm{S}_{\mathrm{col}}=0
\end{gathered}
$$

where, $\mathrm{C}_{\mathrm{col}}=$ colloid concentration in solution, $\mathrm{g} / \mathrm{cm}^{3}$

$\mathrm{S}_{\mathrm{col}}=$ colloid concentration on fracture surfaces, $\mathrm{g} / \mathrm{cm}^{2}$

$\mathrm{k}_{\mathrm{fcol}}=$ filtration rate constant $(1 / \mathrm{s})=\lambda \mathrm{v}_{\mathrm{f}}$, where $\lambda=$ filtration coefficient $(1 / \mathrm{cm})$

$\mathrm{k}_{\mathrm{rcol}}=$ reverse filtration (detachment) rate constant, $1 / \mathrm{s}$.

Equations (8.9) and (8.10) were also used to simulate colloid transport through the fractures when plutonium was present in the system. However, a numerical model had to be used to account for $\mathrm{Pu}$ and colloids in the system at the same time (the semi-analytical RELAP model cannot account for species that interact with each other). In this case, $\mathrm{Pu}$ in solution and $\mathrm{Pu}$ sorbed to sites 1 and 2 on colloids were each treated as separate but interacting species. The governing equations for all $\mathrm{Pu}$ species in the case of the two-site model were:

$$
\begin{aligned}
& \frac{\partial \mathrm{C}}{\partial \mathrm{t}}+\mathrm{v}_{\mathrm{f}} \frac{\partial \mathrm{C}}{\partial \mathrm{x}}-\mathrm{D}_{\mathrm{f}} \frac{\partial^{2} \mathrm{C}}{\partial \mathrm{x}^{2}}+\mathrm{k}_{1 \mathrm{f}} \mathrm{CC}_{\mathrm{col}}\left(1-\frac{\mathrm{C}_{1}}{\mathrm{C}_{\mathrm{col}} \mathrm{S}_{1}^{0}}\right)+\mathrm{k}_{2 \mathrm{f}} \mathrm{CC}_{\mathrm{col}}\left(1-\frac{\mathrm{C}_{2}}{\mathrm{C}_{\mathrm{col}} \mathrm{S}_{2}^{0}}\right)+\mathrm{k}_{\mathrm{f}} \mathrm{C}+ \\
& \mathrm{k}_{1 \mathrm{f}} \mathrm{CS}_{\mathrm{col}}\left(1-\frac{\mathrm{C}_{\mathrm{filt}, 1}}{\mathrm{~S}_{\mathrm{col}} \mathrm{S}_{1}^{0}}\right)+\mathrm{k}_{2 \mathrm{f}} \mathrm{CS}_{\mathrm{col}}\left(1-\frac{\mathrm{C}_{\mathrm{filt}, 2}}{\mathrm{~S}_{\mathrm{col}} \mathrm{S}_{2}^{0}}\right)-\mathrm{k}_{1 \mathrm{~b}} \mathrm{C}_{1}-\mathrm{k}_{2 \mathrm{~b}} \mathrm{C}_{2}-\mathrm{k}_{1 \mathrm{~b}} \mathrm{C}_{\mathrm{filt}, 1}-\mathrm{k}_{\mathrm{r}} \mathrm{S}- \\
& \mathrm{k}_{2 \mathrm{~b}} \mathrm{C}_{\text {filt }, 2}-\left.\frac{\phi \mathrm{D}_{\mathrm{m}}}{\mathrm{b} \eta} \frac{\partial \mathrm{C}_{\mathrm{m}}}{\partial \mathrm{y}}\right|_{\mathrm{y}=\mathrm{b}}=0
\end{aligned}
$$

$$
\frac{\partial C_{m}}{\partial t}-D_{m} \frac{\partial^{2} C_{m}}{\partial y^{2}}+k_{f m} C_{m}-k_{r m} S_{m}=0
$$




$$
\begin{gathered}
\frac{\tilde{\mathrm{n}}_{\mathrm{Bf}}}{\eta} \frac{\partial \mathrm{S}}{\partial \mathrm{t}}-\mathrm{k}_{\mathrm{f}} \mathrm{C}+\mathrm{k}_{\mathrm{r}} \mathrm{S}=0 \\
\frac{\tilde{\mathrm{n}}_{\mathrm{B}}}{\phi} \frac{\partial \mathrm{S}_{\mathrm{m}}}{\partial \mathrm{t}}-\mathrm{k}_{\mathrm{fm}} \mathrm{C}_{\mathrm{m}}+\mathrm{k}_{\mathrm{rm}} \mathrm{S}_{\mathrm{m}}=0 \\
\frac{\partial \mathrm{C}_{\text {filt }, 1}}{\partial \mathrm{t}}-\mathrm{k}_{1 \mathrm{f}} \mathrm{CS}_{\mathrm{col}}\left(1-\frac{\mathrm{C}_{\text {filt, } 1}}{\mathrm{~S}_{\mathrm{col}} \mathrm{S}_{1}^{0}}\right)-\mathrm{k}_{\mathrm{fcol}} \mathrm{C}_{1}+\mathrm{k}_{\mathrm{rcol}} \mathrm{C}_{\text {filt }, 1}+\mathrm{k}_{1 \mathrm{~b}} \mathrm{C}_{\text {filt, } 1}=0 \\
\frac{\partial \mathrm{C}_{\text {filt }, 2}}{\partial \mathrm{t}}-\mathrm{k}_{2 \mathrm{f}} \mathrm{CS}_{\mathrm{col}}\left(1-\frac{\mathrm{C}_{\text {filt }, 2}}{\mathrm{~S}_{\mathrm{col}} \mathrm{S}_{2}^{0}}\right)-\mathrm{k}_{\mathrm{fcol}} \mathrm{C}_{2}+\mathrm{k}_{\mathrm{rcol}} \mathrm{C}_{\text {filt }, 2}+\mathrm{k}_{2 \mathrm{~b}} \mathrm{C}_{\text {filt }, 2}=0
\end{gathered}
$$

where, $\mathrm{C}=$ solution concentration of $\mathrm{Pu}$ in fractures, $\mathrm{g} / \mathrm{cm}^{3}$

$\mathrm{C}_{\mathrm{m}}=$ solution concentration of $\mathrm{Pu}$ in matrix, $\mathrm{g} / \mathrm{cm}^{3}$

$\mathrm{S}=$ sorbed concentration of $\mathrm{Pu}$ on fracture surfaces, $\mathrm{g} / \mathrm{g}$

$\mathrm{C}_{1}=$ concentration of $\mathrm{Pu}$ sorbed to site type 1 on mobile colloids, $\mathrm{g} / \mathrm{cm}^{3}$

$\mathrm{C}_{2}=$ concentration of $\mathrm{Pu}$ sorbed to site type 2 on mobile colloids, $\mathrm{g} / \mathrm{cm}^{3}$

$\mathrm{C}_{\text {filt }, 1}=$ concentration of $\mathrm{Pu}$ sorbed to site type 1 on immobile colloids, $\mathrm{g} / \mathrm{cm}^{3}$

$\mathrm{C}_{\text {filt }, 2}=$ concentration of $\mathrm{Pu}$ on sorbed to site type 2 on immobile colloids, $\mathrm{g} / \mathrm{cm}^{3}$

$\mathrm{k}_{\mathrm{f}}=$ rate constant for sorption of $\mathrm{Pu}$ onto fracture surfaces, $1 / \mathrm{s}$

$\mathrm{k}_{\mathrm{r}}=$ rate constant for desorption of $\mathrm{Pu}$ from fracture surfaces, $\mathrm{g} / \mathrm{cm}^{3}-\mathrm{s}$

$\mathrm{k}_{\mathrm{fm}}=$ rate constant for sorption of $\mathrm{Pu}$ onto matrix surfaces, $1 / \mathrm{s}$

$\mathrm{k}_{\mathrm{rm}}=$ rate constant for desorption of $\mathrm{Pu}$ from matrix surfaces, $\mathrm{g} / \mathrm{cm}^{3}-\mathrm{s}$

$\rho_{\mathrm{Bf}}=$ effective bulk density within fractures, $\mathrm{g} / \mathrm{cm}^{3}$

$\rho_{\mathrm{B}}=$ bulk density in matrix, $\mathrm{g} / \mathrm{cm}^{3}$.

For the Path 1 model, equations (8.11), (8.12), (8.13), (8.17), and (8.18) become:

$$
\begin{gathered}
\frac{\partial \mathrm{C}}{\partial \mathrm{t}}+\mathrm{v}_{\mathrm{f}} \frac{\partial \mathrm{C}}{\partial \mathrm{x}}-\mathrm{D}_{\mathrm{f}} \frac{\partial^{2} \mathrm{C}}{\partial \mathrm{x}^{2}}+\mathrm{k}_{1 \mathrm{f}} \mathrm{CC}_{\mathrm{col}}\left(1-\frac{\mathrm{C}_{1}}{\mathrm{C}_{\mathrm{col}}\left(\mathrm{S}_{1}^{0}+\mathrm{S}_{2}^{0}\right)}\right)+\mathrm{k}_{\mathrm{f}} \mathrm{C}+ \\
\mathrm{k}_{1 \mathrm{f}} \mathrm{CS}_{\mathrm{col}}\left(1-\frac{\mathrm{C}_{\text {filt }, 1}}{\mathrm{~S}_{\mathrm{col}}\left(\mathrm{S}_{1}^{0}+\mathrm{S}_{2}^{0}\right)}\right)-\mathrm{k}_{1 \mathrm{~b}} \mathrm{C}_{1}-\mathrm{k}_{1 \mathrm{~b}} \mathrm{C}_{\mathrm{filt}, 1}-\mathrm{k}_{\mathrm{r}} \mathrm{S}-\left.\frac{\phi \mathrm{D}_{\mathrm{m}}}{\mathrm{b} \eta} \frac{\partial \mathrm{C}_{\mathrm{m}}}{\partial \mathrm{y}}\right|_{\mathrm{y}=\mathrm{b}}=0 \\
\frac{\partial \mathrm{C}_{1}}{\partial \mathrm{t}}+\mathrm{v}_{\mathrm{f}} \frac{\partial \mathrm{C}_{1}}{\partial \mathrm{x}}-\mathrm{D}_{\mathrm{f}} \frac{\partial^{2} \mathrm{C}_{1}}{\partial \mathrm{x}^{2}}-\mathrm{k}_{1 \mathrm{f}} \mathrm{CC}_{\mathrm{col}}\left(1-\frac{\mathrm{C}_{1}}{\mathrm{C}_{\mathrm{col}}\left(\mathrm{S}_{1}^{0}+\mathrm{S}_{2}^{0}\right)}\right)-\mathrm{k}_{\mathrm{rcol}} \mathrm{C}_{\text {filt }, 1}+ \\
\mathrm{k}_{1 \mathrm{~b}} \mathrm{C}_{1}+\mathrm{k}_{\mathrm{fcol}} \mathrm{C}_{1}+\mathrm{k}_{2 \mathrm{f}} \mathrm{C}_{1}\left(1-\frac{\mathrm{C}_{2}}{\mathrm{~S}_{2}^{0}}\right)-\mathrm{k}_{2 \mathrm{~b}} \mathrm{C}_{2}=0
\end{gathered}
$$




$$
\begin{gathered}
\frac{\partial \mathrm{C}_{2}}{\partial \mathrm{t}}+\mathrm{v}_{\mathrm{f}} \frac{\partial \mathrm{C}_{2}}{\partial \mathrm{x}}-\mathrm{D}_{\mathrm{f}} \frac{\partial^{2} \mathrm{C}_{2}}{\partial \mathrm{x}^{2}}-\mathrm{k}_{2 \mathrm{f}} \mathrm{C}_{1}\left(1-\frac{\mathrm{C}_{2}}{\mathrm{~S}_{2}^{0}}\right)+\mathrm{k}_{2 \mathrm{~b}} \mathrm{C}_{2}-\mathrm{k}_{\mathrm{rcol}} \mathrm{C}_{\text {filt }, 2}+\mathrm{k}_{\mathrm{fcol}} \mathrm{C}_{2}=0 \\
\frac{\partial \mathrm{C}_{\text {filt }, 1}}{\partial \mathrm{t}}-\mathrm{k}_{1 \mathrm{f}} \mathrm{CS}_{\mathrm{col}}\left(1-\frac{\mathrm{C}_{\text {filt, },}}{\mathrm{S}_{\mathrm{col}}\left(\mathrm{S}_{1}^{0}+\mathrm{S}_{2}^{0}\right)}\right)-\mathrm{k}_{\mathrm{fcol}} \mathrm{C}_{1}+\mathrm{k}_{\mathrm{rcol}} \mathrm{C}_{\text {filt }, 1}+\mathrm{k}_{1 \mathrm{~b}} \mathrm{C}_{\text {filt }, 1}+ \\
\mathrm{k}_{2 \mathrm{f}} \mathrm{C}_{\text {filt }, 1}\left(1-\frac{\mathrm{C}_{\text {filt }, 2}}{\mathrm{~S}_{2}^{0}}\right)-\mathrm{k}_{2 \mathrm{~b}} \mathrm{C}_{\text {filt }, 2}=0
\end{gathered}
$$

$$
\frac{\partial \mathrm{C}_{\text {filt }, 2}}{\partial \mathrm{t}}-\mathrm{k}_{2 \mathrm{f}} \mathrm{C}_{\text {filt }, 1}\left(1-\frac{\mathrm{C}_{\text {filt }, 2}}{\mathrm{~S}_{2}^{0}}\right)-\mathrm{k}_{\mathrm{fcol}} \mathrm{C}_{2}+\mathrm{k}_{\mathrm{rcol}} \mathrm{C}_{\mathrm{filt}, 2}+\mathrm{k}_{2 \mathrm{~b}} \mathrm{C}_{\text {filt }, 2}=0
$$

The numerical model was first run using the Pu-colloid sorption parameters determined from the interpretations of the batch sorption/desorption experiments. The sorption parameters were then adjusted as necessary to obtain better matches to the plutonium transport data. Initial and boundary conditions in the model simulations included

(1) no colloids or plutonium in the fracture initially,

(2) colloid and plutonium injection pulses had the same concentrations and durations as in the fracture experiments, and

(3) the same fraction of plutonium sorbed onto the injected colloids as determined from the interpretations of the batch sorption experiments at the time of injection.

Matrix diffusion was initially included in the simulations, but the results were quite insensitive to this process, so all of the model results reported in the next section were obtained without matrix diffusion in the model (i.e., the model was reduced to a 1-D system). The value of $\mathrm{k}_{\mathrm{f}}$ (rate constant for Pu sorption onto fracture surfaces) was assumed to be very large relative to the rate constants for sorption onto the colloids. Also, the value of $\mathrm{k}_{\mathrm{b}}(\mathrm{Pu}$ desorption rate constant from fracture surfaces) was assumed to be zero. These assumptions had the effect of causing the $\mathrm{Pu}$ to irreversibly adsorb to the fracture surfaces almost immediately after desorbing from colloids (or immediately after entering the fracture if in the solution phase). Although these assumptions are conservative from the standpoint of minimizing the amount of $\mathrm{Pu}$ predicted to transport through the fractures, they are consistent with the observations of (1) no $\mathrm{Pu}$ ever being measured in the solution phase in the effluent water from the fractures and (2) most of the $\mathrm{Pu}$ on the fracture surfaces after the flow systems were dismantled being located near the fracture entrances.

\subsection{Fracture Transport Experiment Interpretations}

To avoid an excessive number of figures in this chapter, all plots showing model simulations of the fracture transport data are provided in Appendices D, E, and F. Appendix D contains plots of simultaneous RELAP model fits to the tritium data in experiments conducted at different flow rates in the same fractures (with the same colloids). Appendix E contains plots of RELAP model 
fits to the colloid data using the mean residence times and Peclet numbers obtained from the fits to the tritium data. Fits assuming a small amount of matrix diffusion and no matrix diffusion of the colloids are shown for each colloid data set. Appendix F contains plots of model simulations of the plutonium data, including the responses predicted from the batch sorption/desorption experiment interpretations as well as "fits" to the data obtained by adjusting the Pu-colloid sorption parameters. To further reduce the volume of this report, only the simulation results using the two-site model are shown in Appendix F. The two-site model always yielded slightly better predictions than the Path 1 model of $\mathrm{Pu}$ transport in the fractures using sorption parameters derived from the batch sorption experiments, and it also required less adjustment of the sorption parameters to obtain reasonable matches to the fracture data.

An example of a simultaneous RELAP fit to the tritium data from two experiments conducted at different flow rates in the same fracture (with the same colloids) is shown in Figure 8.8. Figure 8.9 shows an example of RELAP fits to colloid responses at two different flow rates in the same fracture (both with and without matrix diffusion). Figures 8.10 and 8.11 show the results of numerical simulations of $\mathrm{Pu}$ responses in an Otay montmorillonite experiment and in a clinoptilolite experiment, respectively. These plot in Figures 8.10 and 8.11 were chosen for illustrative purposes because the first is typical of the results for all the montmorillonite and silica colloid experiments (i.e., a significant overprediction of the Pu responses using the sorption parameters derived from interpretion of the batch sorption/desorption experiments), and the second is typical of all the clinoptilolite experiments (i.e., a slight underprediction of the $\mathrm{Pu}$ responses using the sorption parameters derived from batch sorption/desorption experiments).

The best-fitting model parameters associated with the tritium and colloid model fits are provided in Tables 8.3 through 8.13. Table 8.14 lists the Pu-colloid sorption parameters associated with the adjusted best fits to the Pu responses in each fracture experiment. In each case, only $\mathrm{k}_{1 \mathrm{~b}}, \mathrm{k}_{2 \mathrm{f}}$, or the fraction of $\mathrm{Pu}$ assumed to be sorbed onto irreversible sorption sites at the time of injection into the fractures was adjusted to achieve a reasonable fit to the data. Parameter values from Table 8.1 are included in Table 8.14 so that it is easy to see how much the model parameters had to be adjusted from the values derived in the batch experiments to achieve reasonable fits to the $\mathrm{Pu}$ transport data.

The following observations and conclusions can be drawn from the model fits and simulations of the fracture transport data:

- The simultaneous RELAP fits to the tritium responses yielded solute transport parameter estimates that were reasonably consistent in different sets of experiments in the same fractured cores. For instance, the deduced values of fracture apertures (estimated from $2 \mathrm{~b}=\frac{\mathrm{Q} \hat{\mathrm{O}}}{\mathrm{LW}}$, where $\mathrm{Q}$ is the flow rate through the fractures, $\tau$ is the mean tracer residence time, $\mathrm{L}$ is the fracture length, and $\mathrm{W}$ is the fracture width) from the Cheto, silica, and batch 1 Otay experiments in the UE20c 2851 fracture were $0.034,0.060$, and $0.064 \mathrm{~cm}$, respectively. Likewise, the matrix diffusion mass transfer coefficients $\left(\frac{\phi}{b} \sqrt{D_{m}}\right)$ for these same three sets of experiments were $9.4 \times 10^{-3}, 1.3 \times 10^{-2}$, and $1.25 \times 10^{-2} \mathrm{~s}^{-1 / 2}$, respectively. The Peclet 
numbers from these experiments were 38, 26, and 16, indicating an increase of over a factor of two in the apparent longitudinal dispersivity in the cores over time. Similar results were

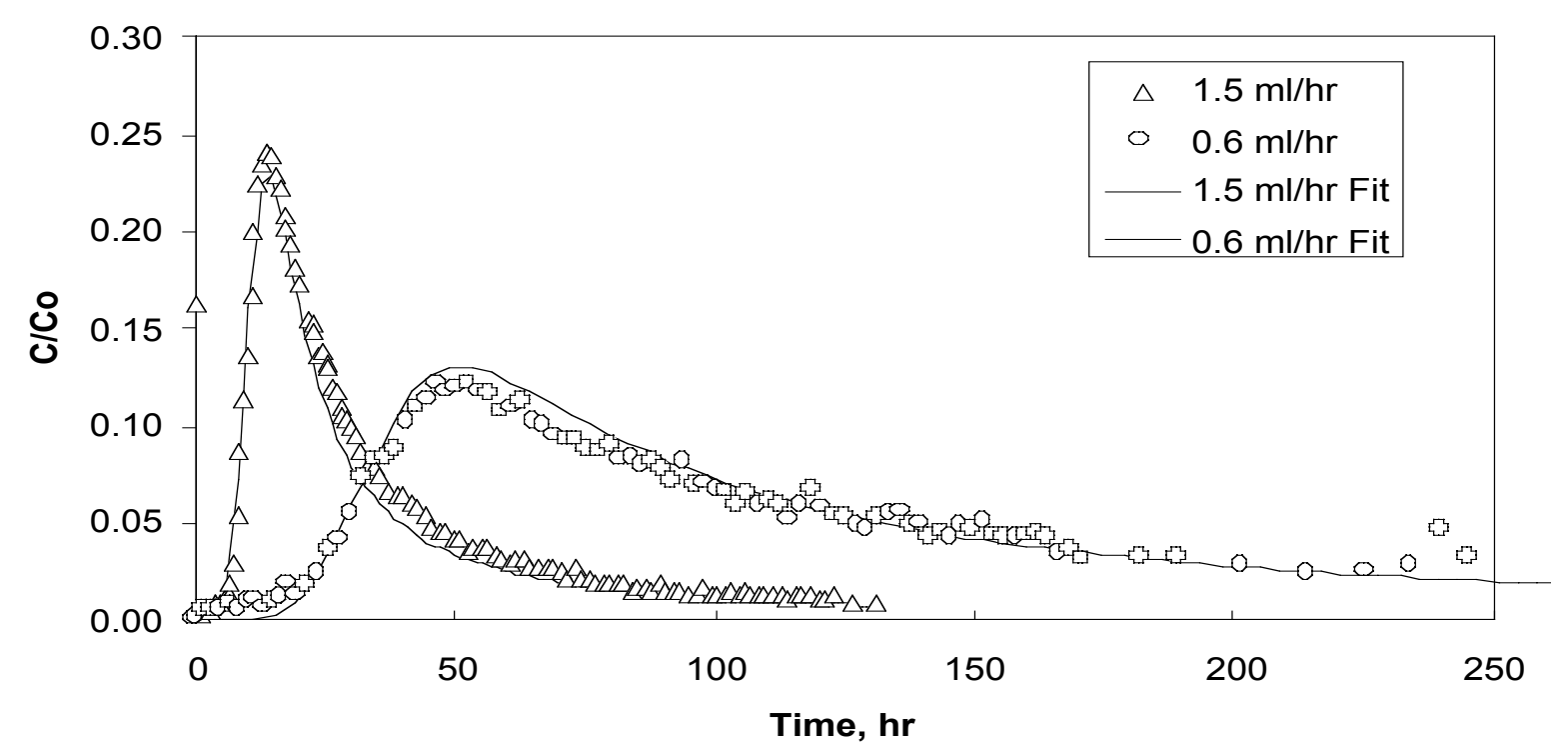

Figure 8.8. Simultaneous RELAP fits to the ${ }^{3} \mathrm{HHO}$ normalized concentrations in the UE20c2858 fracture for the experiments with Cheto montmorillonite colloids ( $1 \mathrm{~b}$ and $2 \mathrm{~b}$ ).

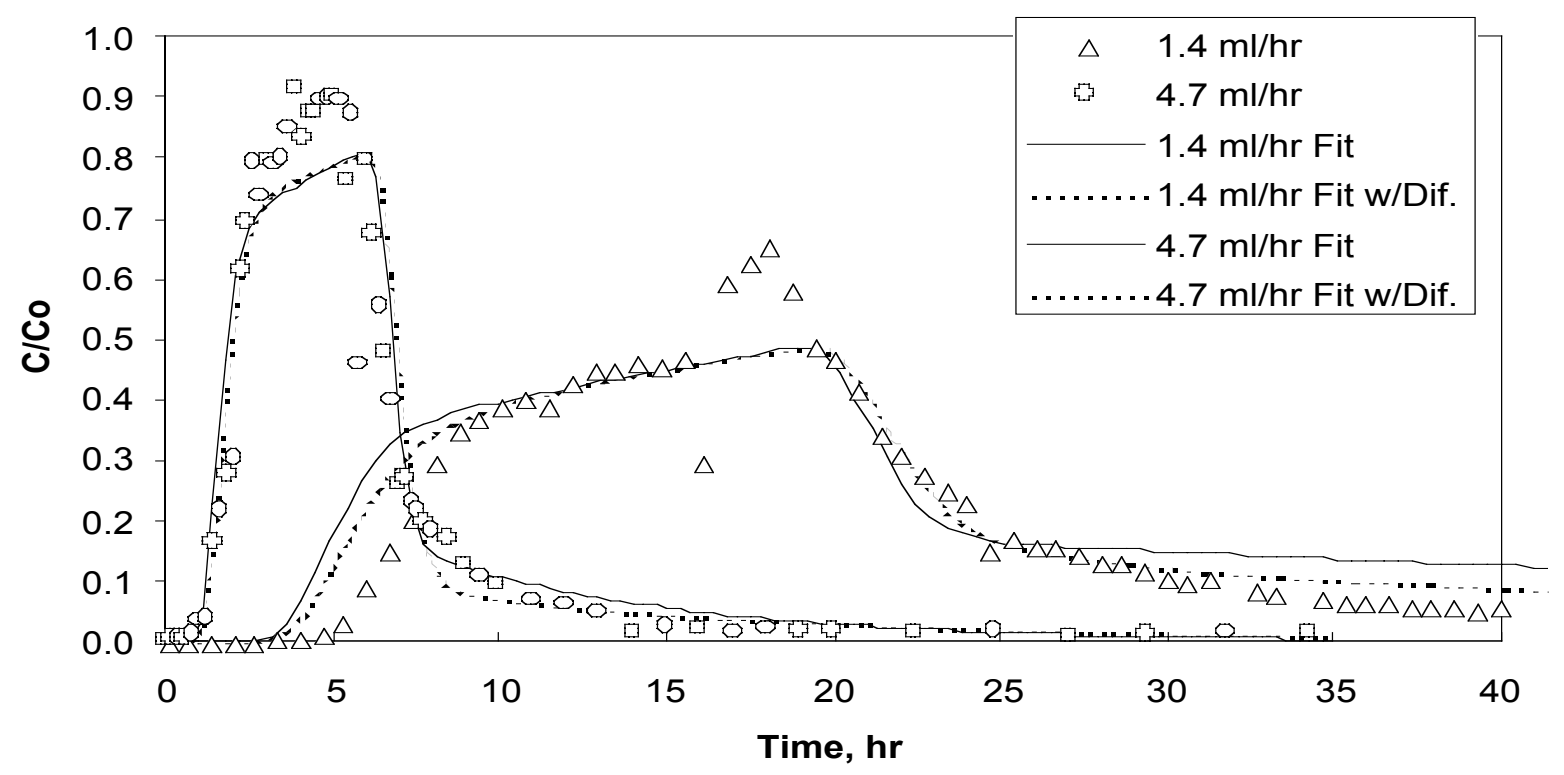

Figure 8.9. RELAP fits to the silica colloid normalized concentrations in the UE20c-2858 fracture for experiments $3 \mathrm{~b}$ and $4 \mathrm{~b}$. 


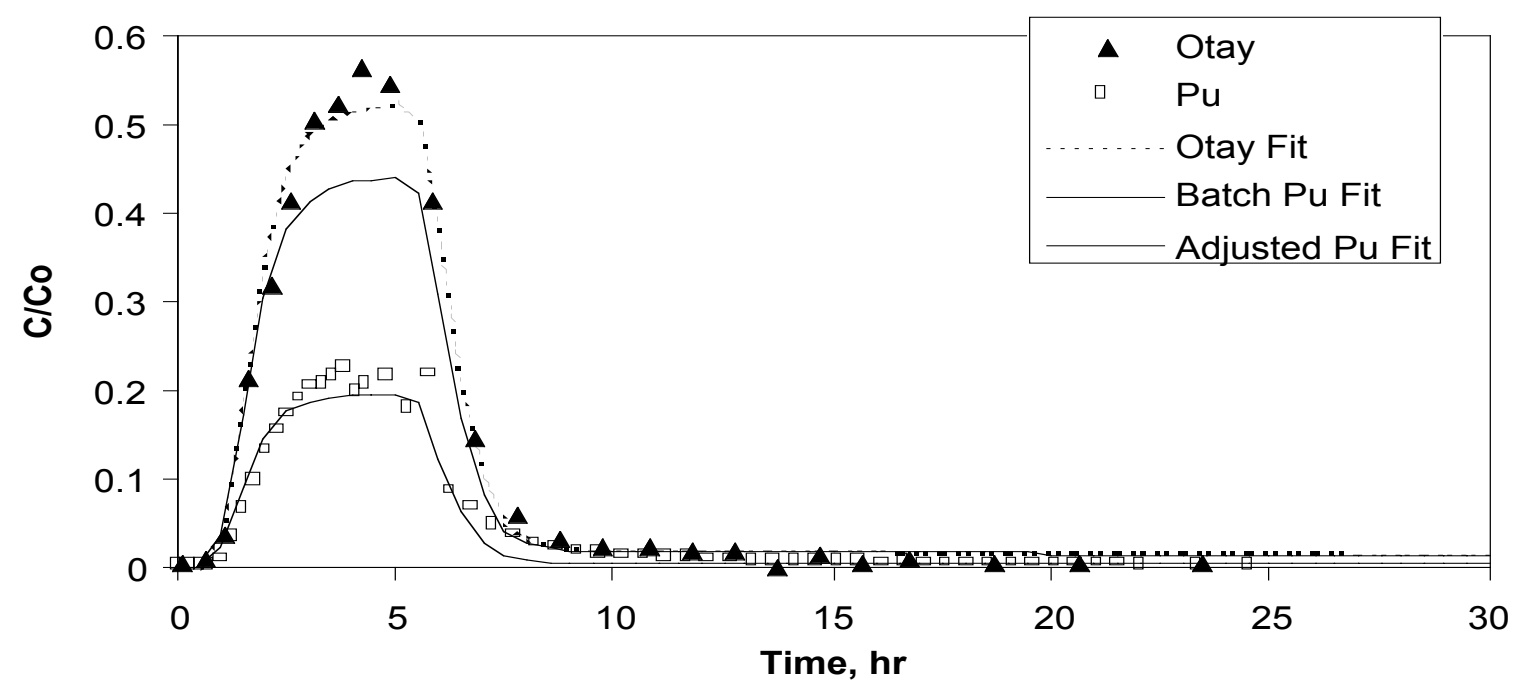

Figure 8.10. Numerical model simulations of batch 1 Otay montmorillonite and Pu responses in the UE20c-2851 fracture as a function of time at $\sim 4.8 \mathrm{ml} / \mathrm{hr}$ in Experiment $6 \mathrm{a}$.

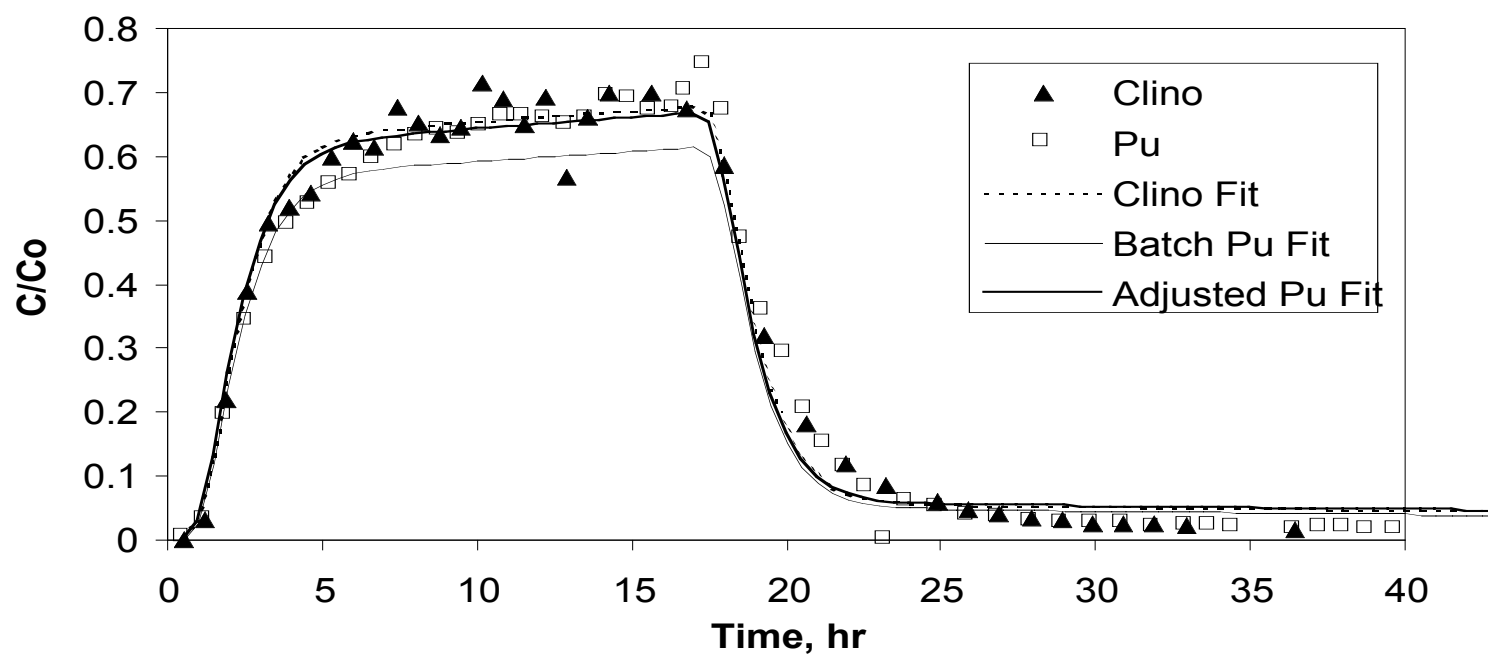

Figure 8.11. Numerical model simulations of clinoptilolite and Pu responses in the PM-2, 4177 fracture as a function of time at $\sim 1.4 \mathrm{ml} / \mathrm{hr}$ in Experiment $7 \mathrm{a}$. 
Table 8.3. Model parameters resulting from fits to the tritium and Cheto montmorillonite colloid data in the fracture from UE20c $2851 \mathrm{ft}$.

\begin{tabular}{lcc}
\hline \hline Model Parameter & $\mathbf{1 . 5 3} \mathbf{~ m l} / \mathbf{h r}$ & $\mathbf{0 . 5 9} \mathbf{~ m l} / \mathbf{h r}$ \\
\hline Mean Fluid Residence Time (hr) & 6.0 & 16.1 \\
\hline Peclet Number & 38 & 38 \\
\hline Dispersivity (cm) & 0.43 & 0.43 \\
\hline Average Fracture Aperture (cm) & 0.064 & 0.064 \\
\hline$\frac{\phi}{\mathrm{b}} \sqrt{\mathrm{D}_{\mathrm{m}}}, \mathrm{hr}^{-1 / 2}$ & 0.56 & 0.56 \\
\hline Colloid filtration rate constant (1/hr) & 0.27 & 0.17 \\
\hline $\begin{array}{l}\text { Fracture half-aperture times colloid } \\
\text { detachment rate constant }(1 / \mathrm{hr})\end{array}$ & 0.014 & 0.0044 \\
\hline \hline
\end{tabular}

Table 8.4. Model parameters resulting from fits to the tritium and Cheto montmorillonite colloid data in the fracture from UE20c $2858 \mathrm{ft}$.

\begin{tabular}{lcc}
\hline \hline Model Parameter & $\mathbf{1 . 5 1} \mathbf{~ m l} / \mathbf{h r}$ & $\mathbf{0 . 5 7} \mathbf{~ m l} / \mathbf{h r}$ \\
\hline Mean Fluid Residence Time (hr) & 5.5 & 14.1 \\
\hline Peclet Number & 42 & 42 \\
\hline Dispersivity (cm) & 0.52 & 0.52 \\
\hline Average Fracture Aperture (cm) & 0.044 & 0.044 \\
\hline$\frac{\phi}{\mathrm{b}} \sqrt{\mathrm{D}_{\mathrm{m}}}, \mathrm{hr}^{-1 / 2}$ & 0.87 & 0.87 \\
\hline Colloid filtration rate constant (1/hr) & 0.34 & 0.25 \\
\hline $\begin{array}{l}\text { Fracture half-aperture times colloid } \\
\text { detachment rate constant (1/hr) }\end{array}$ & 0.018 & 0.0042 \\
\hline \hline
\end{tabular}

Table 8.5. Model parameters resulting from fits to the tritium and silica colloid data in the fracture from UE20c $2851 \mathrm{ft}$.

\begin{tabular}{lcc}
\hline \hline Model Parameter & $\mathbf{1 . 4 3 ~} \mathbf{~ l l} / \mathbf{h r}$ & $\mathbf{4 . 7 0 ~} \mathbf{~ m l} / \mathbf{h r}$ \\
\hline Mean Fluid Residence Time (hr) & 6.0 & 1.83 \\
\hline Peclet Number & 24 & 24 \\
\hline Dispersivity $(\mathrm{cm})$ & 0.70 & 0.70 \\
\hline Average Fracture Aperture (cm) & 0.060 & 0.060 \\
\hline$\frac{\phi}{\mathrm{b}} \sqrt{\mathrm{D}_{\mathrm{m}}}, \mathrm{hr}^{-1 / 2}$ & 0.78 & 0.78 \\
\hline Colloid filtration rate constant (1/hr) & 0.4 & 0.37 \\
\hline $\begin{array}{l}\text { Fracture half-aperture times colloid } \\
\text { detachment rate constant (1/hr) }\end{array}$ & 0.031 & 0.164 \\
\hline \hline
\end{tabular}


Table 8.6. Model parameters resulting from fits to the tritium and silica colloid data in the fracture from UE20c $2858 \mathrm{ft}$.

\begin{tabular}{lcc}
\hline \hline Model Parameter & $\mathbf{1 . 4 3 ~} \mathbf{~ m l} / \mathbf{h r}$ & $\mathbf{4 . 7 5} \mathbf{~ m l} / \mathbf{h r}$ \\
\hline Mean Fluid Residence Time (hr) & 5.5 & 1.66 \\
\hline Peclet Number & 32 & 32 \\
\hline Dispersivity (cm) & 0.68 & 0.68 \\
\hline Average Fracture Aperture (cm) & 0.042 & 0.042 \\
\hline$\frac{\phi}{\mathrm{b}} \sqrt{\mathrm{D}_{\mathrm{m}}}, \mathrm{hr}^{-1 / 2}$ & 0.91 & 0.91 \\
\hline Colloid filtration rate constant (1/hr) & 0.20 & 0.24 \\
\hline $\begin{array}{l}\text { Fracture half-aperture times colloid } \\
\text { detachment rate constant }(1 / \mathrm{hr})\end{array}$ & 0.033 & 0.16 \\
\hline \hline
\end{tabular}

Table 8.7. Model parameters resulting from fits to the tritium and batch 1 Otay montmorillonite colloid data in the fracture from UE20c $2851 \mathrm{ft}$.

\begin{tabular}{lcc}
\hline \hline Model Parameter & $\mathbf{1 . 4 2 ~} \mathbf{~ m l} / \mathbf{h r}$ & $\mathbf{4 . 8 4} \mathbf{~ m l} / \mathbf{h r}$ \\
\hline Mean Fluid Residence Time (hr) & 6.5 & 1.91 \\
\hline Peclet Number & 16 & 16 \\
\hline Dispersivity (cm) & 1.03 & 1.03 \\
\hline Average Fracture Aperture (cm) & 0.064 & 0.064 \\
\hline$\frac{\phi}{\mathrm{b}} \sqrt{\mathrm{D}_{\mathrm{m}}}, \mathrm{hr}^{-1 / 2}$ & 0.76 & 0.76 \\
\hline Colloid filtration rate constant (1/hr) & 0.52 & 0.37 \\
\hline $\begin{array}{l}\text { Fracture half-aperture times colloid } \\
\text { detachment rate constant }(1 / \mathrm{hr})\end{array}$ & 0.012 & 0.016 \\
\hline \hline
\end{tabular}

Table 8.8. Model parameters resulting from fits to the tritium and batch 1 Otay montmorillonite colloid data in the fracture from UE20c $2858 \mathrm{ft}$.

\begin{tabular}{lcc}
\hline \hline Model Parameter & $\mathbf{1 . 4 3} \mathbf{~ m l} / \mathbf{h r}$ & $\mathbf{4 . 7 9} \mathbf{~ m l} / \mathbf{h r}$ \\
\hline Mean Fluid Residence Time (hr) & 5.5 & 1.64 \\
\hline Peclet Number & 16 & 16 \\
\hline Dispersivity (cm) & 1.37 & 1.37 \\
\hline Average Fracture Aperture (cm) & 0.042 & 0.042 \\
\hline$\frac{\phi}{\mathrm{b}} \sqrt{\mathrm{D}_{\mathrm{m}}}, \mathrm{hr}^{-1 / 2}$ & 0.91 & 0.91 \\
\hline Colloid filtration rate constant (1/hr) & 0.46 & 0.47 \\
\hline $\begin{array}{l}\text { Fracture half-aperture times colloid } \\
\text { detachment rate constant }(1 / \mathrm{hr})\end{array}$ & 0.0089 & 0.0096 \\
\hline \hline
\end{tabular}


Table 8.9. Model parameters resulting from fits to the tritium and clinoptilolite colloid data in the fracture from PM-2 $4177 \mathrm{ft}$.

\begin{tabular}{lcc}
\hline \hline Model Parameter & $\mathbf{1 . 4 1 ~} \mathbf{~ m l} / \mathbf{h r}$ & $\mathbf{0 . 5 3 ~} \mathbf{~ l} / \mathbf{h r}$ \\
\hline Mean Fluid Residence Time (hr) & 2.75 & 7.3 \\
\hline Peclet Number & 8 & 8 \\
\hline Dispersivity $(\mathrm{cm})$ & 1.58 & 1.58 \\
\hline Average Fracture Aperture (cm) & 0.036 & 0.036 \\
\hline$\frac{\phi}{\mathrm{b}} \sqrt{\mathrm{D}_{\mathrm{m}}}, \mathrm{hr}^{-1 / 2}$ & 0.54 & 0.54 \\
\hline Colloid filtration rate constant (1/hr) & 0.18 & 0.14 \\
\hline $\begin{array}{l}\text { Fracture half-aperture times colloid } \\
\text { detachment rate constant }(1 / \mathrm{hr})\end{array}$ & 0.014 & 0.0024 \\
\hline \hline
\end{tabular}

Table 8.10. Model parameters resulting from fits to the tritium and clinoptilolite colloid data in the fracture from PM-1 $4823 \mathrm{ft}$.

\begin{tabular}{lcc}
\hline \hline Model Parameter & $\mathbf{1 . 4 1} \mathbf{~ m l} / \mathbf{h r}$ & $\mathbf{0 . 5 4} \mathbf{~ m l} / \mathbf{h r}$ \\
\hline Mean Fluid Residence Time (hr) & 3.5 & 9.1 \\
\hline Peclet Number & 5.5 & 5.5 \\
\hline Dispersivity (cm) & 2.96 & 2.96 \\
\hline Average Fracture Aperture (cm) & 0.034 & 0.034 \\
\hline$\frac{\phi}{\mathrm{b}} \sqrt{\mathrm{D}_{\mathrm{m}}}, \mathrm{hr}^{-1 / 2}$ & 1.22 & 1.22 \\
\hline Colloid filtration rate constant (1/hr) & 0.15 & 0.12 \\
\hline $\begin{array}{l}\text { Fracture half-aperture times colloid } \\
\text { detachment rate constant }(1 / \mathrm{hr})\end{array}$ & 0.017 & 0.0031 \\
\hline \hline
\end{tabular}

Table 8.11. Model parameters resulting from fits to the tritium and batch 2 Otay montmorillonite colloid data in the fracture from PM-2 $4177 \mathrm{ft}$.

\begin{tabular}{lcc}
\hline Model Parameter & $\mathbf{1 . 4 0 ~} \mathbf{~ m l} / \mathbf{h r}$ & $\mathbf{0 . 4 7} \mathbf{~ m l} / \mathbf{h r}$ \\
\hline Mean Fluid Residence Time (hr) & 2.5 & 7.1 \\
\hline Peclet Number & 16 & 16 \\
\hline Dispersivity (cm) & 0.79 & 0.79 \\
\hline Average Fracture Aperture (cm) & 0.032 & 0.032 \\
\hline$\frac{\phi}{\mathrm{b}} \sqrt{\mathrm{D}_{\mathrm{m}}}, \mathrm{hr}^{-1 / 2}$ & 0.54 & 0.54 \\
\hline Colloid filtration rate constant (1/hr) & 0.55 & 0.26 \\
\hline $\begin{array}{l}\text { Fracture half-aperture times colloid } \\
\text { detachment rate constant }(1 / \mathrm{hr})\end{array}$ & 0.018 & 0.00064 \\
\hline \hline
\end{tabular}


Table 8.12. Model parameters resulting from fits to the tritium and and batch 2 Otay montmorillonite colloid data in the fracture from PM-1 $4823 \mathrm{ft}$.

\begin{tabular}{lcc}
\hline \hline Model Parameter & $\mathbf{1 . 6 3 ~} \mathbf{~ m l} / \mathbf{h r}$ & $\mathbf{0 . 4 9} \mathbf{~ m l} / \mathbf{h r}$ \\
\hline Mean Fluid Residence Time (hr) & 2.5 & 8.7 \\
\hline Peclet Number & 2.5 & 2.5 \\
\hline Dispersivity (cm) & 6.5 & 6.5 \\
\hline Average Fracture Aperture (cm) & 0.038 & 0.038 \\
\hline$\frac{\phi}{\mathrm{b}} \sqrt{\mathrm{D}_{\mathrm{m}}}, \mathrm{hr}^{-1 / 2}$ & 0.91 & 0.91 \\
\hline Colloid filtration rate constant (1/hr) & 0.42 & 0.46 \\
\hline $\begin{array}{l}\text { Fracture half-aperture times colloid } \\
\text { detachment rate constant }(1 / \mathrm{hr})\end{array}$ & 0.021 & 0.00077 \\
\hline \hline
\end{tabular}

Table 8.13. Model parameters resulting from fits to the tritium and batch 1 Otay montmorillonite colloid data in the fracture from PM-2 $4177 \mathrm{ft}$.

\begin{tabular}{lc}
\hline \hline Model Parameter & $\mathbf{1 . 5 5} \mathbf{~ m l} / \mathbf{h r}$ \\
\hline Mean Fluid Residence Time (hr) & 2.3 \\
\hline Peclet Number & 16 \\
\hline Dispersivity $(\mathrm{cm})$ & 0.79 \\
\hline Average Fracture Aperture (cm) & 0.032 \\
\hline$\frac{\phi}{\mathrm{b}} \sqrt{\mathrm{D}_{\mathrm{m}}}, \mathrm{hr}^{-1 / 2}$ & 0.54 \\
\hline Colloid filtration rate constant $(1 / \mathrm{hr})$ & 0.145 \\
\hline $\begin{array}{l}\text { Fracture half-aperture times colloid } \\
\text { detachment rate constant }(1 / \mathrm{hr})\end{array}$ & 0.058 \\
\hline \hline
\end{tabular}

obtained for the other cores. Note that the tritium responses from the last three experiments in the PM-2 core were simultaneously fitted (instead of only two data sets) because of the odd number of experiments in that core. Note that mean residence times deduced for tritium indicated linear flow velocities ranging from $\sim 1 \mathrm{~cm} / \mathrm{hr}$ to $\sim 10 \mathrm{~cm} / \mathrm{hr}$ in the fractures. These velocities are probably a little high compared to field velocities, but the lower end of this range was about as low as could be achieved in the lab.

- The quality of the fits to the colloid responses in each core varied considerably. As the figures in Appendix E show, the inclusion of a small amount of matrix diffusion significantly improved the fits to the tails of the colloid responses in most cases. Although true diffusion into the matrix was likely quite minimal for the colloids, it was considered plausible that the colloids could diffuse into stagnant free water in the fractures (either along the rough fracture walls or out of fast-flowing channels into near-stagnant zones in the fractures). This process is mathematically equivalent to matrix diffusion. In the simulations, the colloid matrix diffusion coefficient was set equal to $4 \times 10^{-8} \mathrm{~cm}^{2} / \mathrm{s}$ (the free diffusion coefficient of a 100- 
Table 8.14. Adjusted Pu sorption parameters and/or fractions of Pu assumed to be sorbed onto irreversible sorption sites when injected into fracture; these values were used to obtain the "adjusted" fits to the Pu data in the fracture transport experiments shown in Figures 8.10 and 8.11 and in Appendix F. In all cases, adjustments were made to the two-site sorption model with a fast reversible reaction.

\begin{tabular}{cccc}
\hline Experiment & $\mathbf{k}_{\mathbf{1 b}}, \mathbf{1} / \mathbf{h r} \mathbf{r}^{*}$ & $\mathbf{k}_{\mathbf{2 f}}, \mathbf{g} / \mathbf{g}-\mathbf{h r} \mathbf{r}^{*}$ & $\begin{array}{c}\text { Frac. Pu } \\
\text { Sorbed to Irrev. } \\
\text { Sites** }\end{array}$ \\
\hline $2851,1.5 \mathrm{ml} / \mathrm{hr}$ Cheto-mont. & $1.3 / 0.15$ & $35 / 35$ & $0.6 / 0.6$ \\
2851, 0.6 ml/hr Cheto-mont. & $2.0 / 0.15$ & $35 / 35$ & $0.2 / 0.62$ \\
$2858,1.5 \mathrm{ml} / \mathrm{hr}$ Cheto-mont. & $1.0 / 0.15$ & $35 / 35$ & $0.6 / 0.6$ \\
$2858,0.6 \mathrm{ml} / \mathrm{hr} \mathrm{Cheto-mont.}$ & $2.0 / 0.15$ & $35 / 35$ & $0.42 / 0.62$ \\
$2851,5 \mathrm{ml} / \mathrm{hr}$ Si. & $1.0 / 0.15$ & $0 / 55$ & $0 / 0.42$ \\
$2851,1.5 \mathrm{ml} / \mathrm{hr}$ Si. & $0.9 / 0.15$ & $0 / 55$ & $0 / 0.42$ \\
$2858,5 \mathrm{ml} / \mathrm{hr}$ Si. & $0.75 / 0.15$ & $0 / 55$ & $0 / 0.42$ \\
$2858,1.5 \mathrm{ml} / \mathrm{hr}$ Si. & $0.6 / 0.15$ & $0 / 55$ & $0 / 0.42$ \\
$2851,5 \mathrm{ml} / \mathrm{hr}$ Otay-M. & $1.0 / 0.01$ & $75 / 75$ & $0.31 / 0.79$ \\
$2851,1.5 \mathrm{ml} / \mathrm{hr}$ Otay-M. & $1.0 / 0.01$ & $75 / 75$ & $0.11 / 0.79$ \\
$2858,5 \mathrm{ml} / \mathrm{hr}$ Otay-M. & $1.0 / 0.01$ & $75 / 75$ & $0.07 / 0.79$ \\
$2858,1.5 \mathrm{ml} / \mathrm{hr}$ Otay-M. & $1.0 / 0.01$ & $75 / 75$ & $0.11 / 0.79$ \\
PM-2, 1.4 ml/hr, Clinop. & $0.024 / 0.024$ & --- & $1.0 / 0.91$ \\
PM-2, 0.5 ml/hr, Clinop. & $0.024 / 0.024$ & --- & $1.0 / 0.91$ \\
PM-1, 1.4 ml/hr, Clinop. & $0.024 / 0.024$ & --- & $1.0 / 0.91$ \\
PM-1, 0.5 ml/hr, Clinop. & $0.024 / 0.024$ & --- & $1.0 / 0.91$ \\
PM-2, 1.4 ml/hr, Batch 2 Otay & $1.0 / 0.006$ & $20 / 20$ & $0.32 / 0.62$ \\
PM-2, 0.4 ml/hr, Batch 2 Otay & $1.0 / 0.006$ & $20 / 20$ & $0.05 / 0.67$ \\
PM-1, 1.6 ml/hr, Batch 2 Otay. & $1.0 / 0.006$ & $20 / 20$ & $0.027 / 0.62$ \\
PM-1, 0.4 ml/hr, Batch 2 Otay & $1.0 / 0.006$ & $20 / 20$ & $0.024 / 0.67$ \\
PM-2, 1.5 ml/hr, Batch 1 Otay & $1.0 / 0.01$ & $75 / 75$ & $0.046 / 0.81$ \\
\hline
\end{tabular}

*The first value is the adjusted parameter and the second value is the parameter obtained from fitting the batch sorption/desorption data.

**The fraction of Pu sorbed to irreversible sites is the fraction of the total Pu sorbed (which includes both reversible and irreversible sites). It is not the fraction of the total $\mathrm{Pu}$ in the injection pulse.

nm diameter spherical colloid). Although not shown in Appendix E, fits to the colloid data sets were also obtained assuming irreversible filtration (no detachment). In all cases, the fits were significantly improved by assuming reversible filtration, so only the reversible filtration fits are shown in Appendix E. Also, the parameters from the reversible filtration fits were used in the numerical simulations of $\mathrm{Pu}$ transport in the fractures. 
- For all of the fracture transport experiments not involving clinoptilolite colloids, the $\mathrm{Pu}$ responses predicted using the sorption/desorption parameters from the batch experiments were considerably higher (greater concentrations and mass recoveries) than the observed $\mathrm{Pu}$ responses. The closest matches to the $\mathrm{Pu}$ data in all cases were obtained when using sorption/desorption parameters obtained using the two-site model with one site having fast, reversible sorption kinetics. However, reasonable matches to the $\mathrm{Pu}$ data could be obtained only by (1) significantly increasing the site 1 (fast) desorption rate constants (by nearly 2 orders of magnitude in some cases) and (2) arbitrarily shifting some of the sorbed $\mathrm{Pu}$ mass from sites 2 to sites 1 (the fast, reversible sites). The latter adjustment was made only if the former was incapable of providing a reasonable match to the data. This situation occurred when too much Pu mass was initially sorbed to irreversible sites such that desorption of all of the reversibly-sorbed $\mathrm{Pu}$ could still not get the $\mathrm{Pu}$ concentrations low enough to match the fracture data. Likewise, when the Path 1 model was used, the desorption rate of $\mathrm{Pu}(\mathrm{V})$ had to be increased significantly and either the fraction of $\mathrm{Pu}(\mathrm{V})$ sorbed to the surfaces had to be arbitrarily increased (with $\mathrm{Pu}(\mathrm{IV})$ correspondingly decreased) or the rate of oxidation of $\mathrm{Pu}(\mathrm{IV})$ to $\mathrm{Pu}(\mathrm{V})$ on the surface had to be greatly increased. Similarly, a one-site reversible sorption model provided a reasonable fit to the fracture data only if the desorption rate was increased significantly above that deduced from the batch experiments.

- For the clinoptilolite colloid experiments, the transport model consistently underpredicted the observed $\mathrm{Pu}$ responses. The most likely explanation for this result is that the fraction of $\mathrm{Pu}$ initially sorbed to the colloids must have been larger than that measured in the batch experiments (due to some of the smaller colloids remaining suspended after centrifuging, and the $\mathrm{Pu}$ sorbed to these colloids being counted as soluble $\mathrm{Pu}$ ). Otherwise, the predicted $\mathrm{Pu}$ responses were in good agreement with the observed responses.

\subsection{Discussion}

The results and interpretations of the fracture transport experiments suggest that, for the montmorillonite and silica colloids, the plutonium desorbed from the colloids much faster in the fractures than in the batch experiments. This result would seem to be consistent with the presence of minerals on the fracture surfaces that can effectively compete with the colloids for $\mathrm{Pu}$. Such mineral surfaces were not present in the batch experiments. However, to make the case that competitive sorption is responsible for the experimental observations, one must ignore the relatively slow desorption kinetics from the colloids that was observed in the batch experiments. As the model interpretations suggest, the batch desorption kinetics were too slow to account for the large attenuation of $\mathrm{Pu}$ in the fractures (relative to the colloid recoveries), even though it was assumed that sorption onto the fracture surfaces was very fast and irreversible.

We hypothesize that desorption rates in the fractures were enhanced because of collisions between the colloids and the fracture walls, which offered the opportunity for direct transfer of $\mathrm{Pu}$ from the colloids to fracture surfaces without the intermediate steps of desorption from the colloids, transport to the fracture walls, and adsorption to the fracture walls. Such transfers could occur if the fracture minerals had a higher inherent affinity for Pu than the colloid surfaces. The situation would be analogous in some respects to the ability of EDTA to desorb $\mathrm{Pu}(\mathrm{IV})$ from colloids in the experiments of Chapter 5 (conducted at LLNL). Without the EDTA, the Pu(IV) would presumably have remained strongly sorbed to the colloid surfaces. The desorption 
occurred only because $\mathrm{Pu}(\mathrm{IV})$ complexation with EDTA is energetically more favorable than sorption to the colloids. In the same way, a fracture surface that offers a more energetically favorable sorption site for Pu could effectively "strip" $\mathrm{Pu}$ off the colloids provided the fracture surface comes in contact with the sorbed $\mathrm{Pu}$.

Under laminar flow conditions, collisions would not necessarily be discrete events, but rather the colloids would tend to remain in close proximity to the walls for some time with multiple opportunities to collide with the surfaces during each event and even the possibility of "rolling" or "bouncing" along the surfaces. To explore this hypothesis further, a 2-D particle-tracking model (Reimus, 1995) was used to simulate particles moving through a parallel-plate fracture with a parabolic velocity profile and diffusion of the particles across this profile in the direction perpendicular to flow. The objectives of the simulations were to determine (1) the expected frequency distribution of particle-wall collisions when residence times and fracture apertures were similar to those in the fracture experiments, and (2) how many particles would be expected to pass through the fractures without any wall collisions under these conditions. Although the number of particle-wall collisions in such simulations is dependent on the time and spatial step sizes, it was found that the relative number of particle-wall collisions as a function of residence time or fracture aperture was independent of these time and space discretizations. Also, the fraction of particles passing through the fractures without wall collisions did not change with time and space discretizations. These simulations were expected to provide only a rough indication of the trends that could be expected in the experiments, as the cores certainly did not have parallel fracture surfaces or ideal parabolic velocity profiles.

Figure 8.12 shows typical results from the particle-tracking simulations for cases corresponding to colloids moving through a fracture at two different flow rates differing by about a factor of 2.7. The residence times and fracture aperture corresponded roughly to those deduced from the two Cheto montmorillonite experiments in the UE20c 2851 core. It is apparent that a much higher percentage of the colloids was predicted to move through the core without contacting the fracture walls in the faster flow case than in the slower flow case $(13.6 \%$ vs. $0.5 \%)$. The fractions of particles predicted to move through the fractures without contacting the walls in each fracture experiment (assuming ideal parallel-plate fractures with residence times and apertures deduced from interpretations of the tritium responses) are listed in Table 8.15. Simulation results indicated that the ratio of the total number of particle-wall collisions was almost exactly equal to the ratio of the residence times for experiments conducted in the same cores, which is not surprising. However, the frequency distributions at the two different residence times looked different (e.g., Figure 8.12).

With the exception of the $\mathrm{Pu}$-clinoptilolite experiments, the ratio of $\mathrm{Pu}$ recoveries in paired fracture experiments conducted with the same colloids in the same fractures at different flow rates seems to be correlated reasonably well with the ratio of the number of colloids predicted to pass through the fractures without wall collisions (Figure 8.13). It must be cautioned that the fraction of colloids with no wall collisions in Figure 8.13 should be taken as only a relative measure of colloid-wall interactions, not an absolute one. Also, all collisions would not necessarily be expected to result in $\mathrm{Pu}$ transfer from colloids to fracture surfaces. If we assume that it takes an average of 5 wall collisions to transfer Pu from colloids to fracture surfaces, then we should draw a vertical line at 5 on the $\mathrm{x}$ axis of Figure 8.12 and take the ratio of the values 


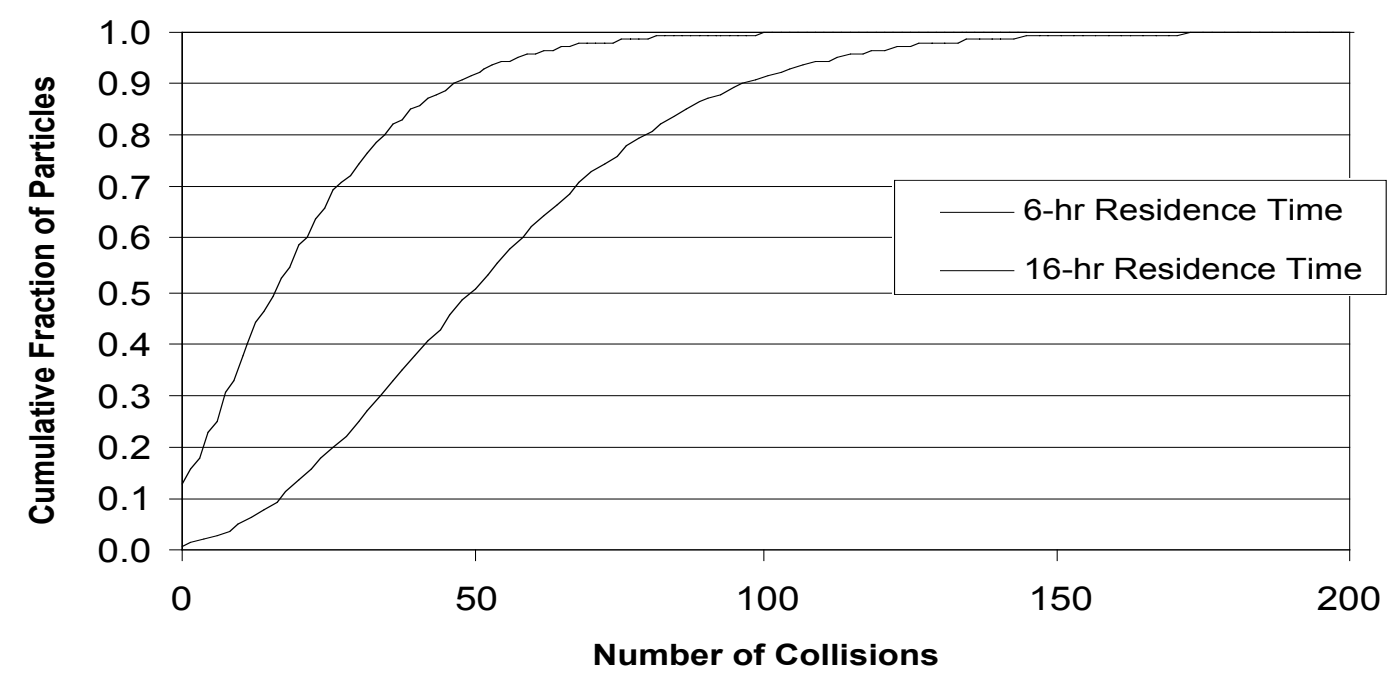

Figure 8.12. Fractions of particles experiencing a given number of collisions for residence times of 16 and 6 hours in a parallel-plate fracture of $0.064 \mathrm{~cm}$ aperture (corresponding approximately to the UE20c, 2851 fracture).

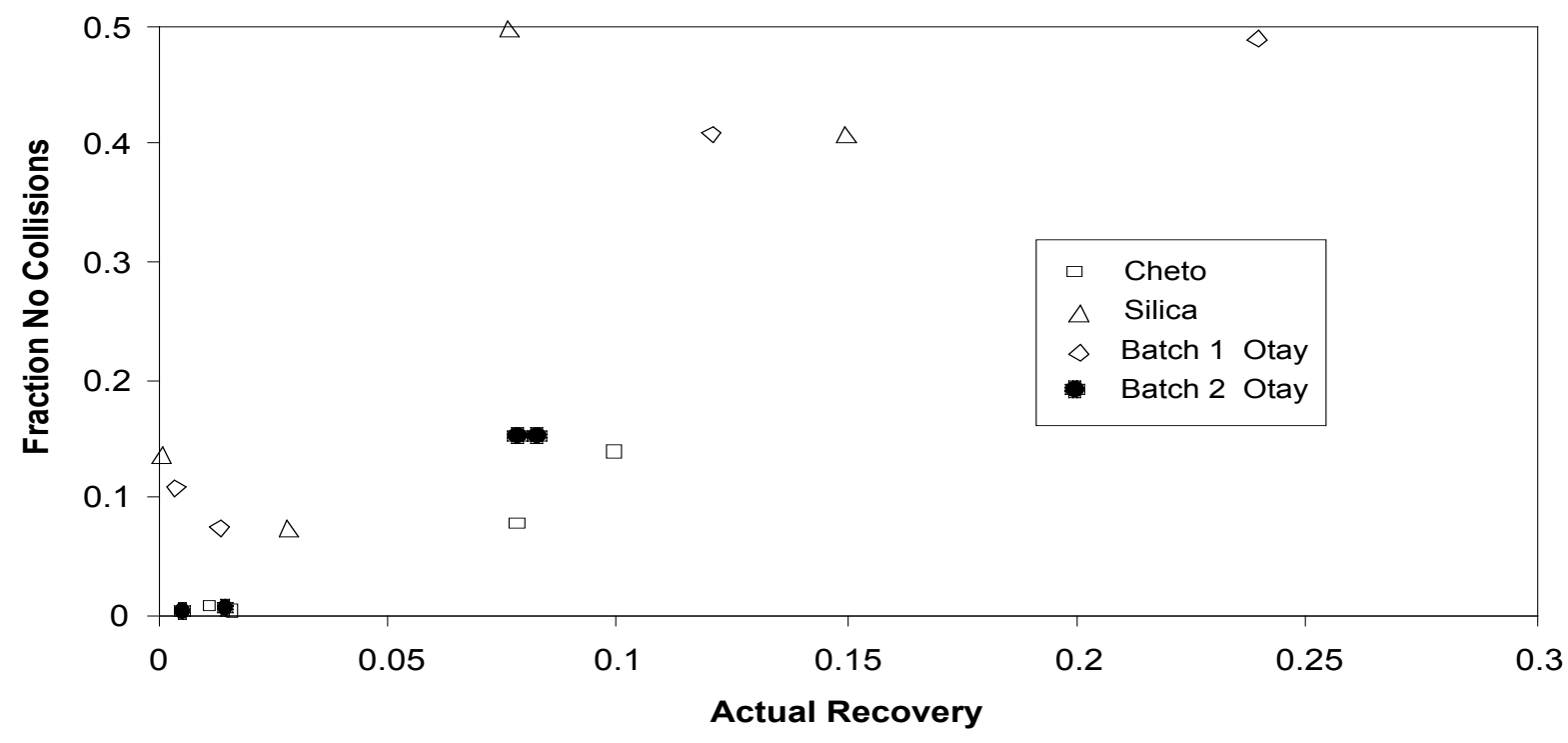

Figure 8.13. Fractions of particles predicted to not collide with fractures walls vs. actual colloid recoveries in the fracture transport tests involving montmorillonite and silica colloids. 
Table 8.15. Fractions of colloids predicted to not collide with fracture walls in the fracture transport experiments (based on particle-tracking simulations).

\begin{tabular}{|c|c|}
\hline Experiment & $\begin{array}{c}\text { Predicted } \\
\text { Fraction of } \\
\text { Colloids not } \\
\text { Colliding with } \\
\text { Walls } \\
\end{array}$ \\
\hline $2851,1.5 \mathrm{ml} / \mathrm{hr}$ Cheto-mont. & 0.136 \\
\hline $2851,0.6 \mathrm{ml} / \mathrm{hr}$ Cheto-mont. & 0.005 \\
\hline $2858,1.5 \mathrm{ml} / \mathrm{hr}$ Cheto-mont. & 0.076 \\
\hline $2858,0.6 \mathrm{ml} / \mathrm{hr}$ Cheto-mont. & 0.002 \\
\hline 2851, $5 \mathrm{ml} / \mathrm{hr} \mathrm{Si}$. & 0.5 \\
\hline $2851,1.5 \mathrm{ml} / \mathrm{hr} \mathrm{Si}$ & 0.136 \\
\hline $2858,5 \mathrm{ml} / \mathrm{hr} \mathrm{Si}$ & 0.41 \\
\hline $2858,1.5 \mathrm{ml} / \mathrm{hr} \mathrm{Si}$ & 0.076 \\
\hline 2851, $5 \mathrm{ml} / \mathrm{hr}$ Otay-M. & 0.49 \\
\hline $2851,1.5 \mathrm{ml} / \mathrm{hr}$ Otay-M. & 0.11 \\
\hline 2858, $5 \mathrm{ml} / \mathrm{hr}$ Otay-M. & 0.41 \\
\hline $2858,1.5 \mathrm{ml} / \mathrm{hr}$ Otay-M. & 0.076 \\
\hline PM-2, 1.4 ml/hr, Clinop. & 0.12 \\
\hline PM-2, $0.5 \mathrm{ml} / \mathrm{hr}$, Clinop. & 0.005 \\
\hline PM-1, 1.4 ml/hr, Clinop. & 0.073 \\
\hline PM-1, $0.5 \mathrm{ml} / \mathrm{hr}$, Clinop. & 0.0006 \\
\hline PM-2, $1.4 \mathrm{ml} / \mathrm{hr}$, Batch 2 Otay & 0.15 \\
\hline PM-2, $0.4 \mathrm{ml} / \mathrm{hr}$, Batch 2 Otay & 0.004 \\
\hline PM-1, $1.6 \mathrm{ml} / \mathrm{hr}$, Batch 2 Otay. & 0.15 \\
\hline PM-1, $0.4 \mathrm{ml} / \mathrm{hr}$, Batch 2 Otay & 0.002 \\
\hline PM-2, $1.5 \mathrm{ml} / \mathrm{hr}$, Batch 1 Otay & 0.18 \\
\hline
\end{tabular}

where the two curves intersect this vertical line as the predicted ratio of $\mathrm{Pu}$ recoveries at the two residence times.

The silica data points on Figure 8.13 may be somewhat higher than the general trend in the figure because a significant fraction of $\mathrm{Pu}$ was predicted to desorb even without collisions with the walls for these colloids (desorption rates were higher from the silica than from the montmorillonite colloids). Figures 8.12 and 8.13 don't explain all of the experimental observations, but they do support the hypothesis that $\mathrm{Pu}$ desorption from colloids may be enhanced by collisions between colloids and fracture walls for some types of colloids. The fact that the $\mathrm{Pu}$ recoveries in the clinoptilolite experiments were essentially identical to the colloid 
recoveries suggests that, if the wall collision hypothesis is correct, the clinoptilolite colloids must have been capable of "hanging on" to all the Pu sorbed onto them during collision events.

The wall collision hypothesis implies that batch sorption experiments (using test tube materials such as Teflon, which have a low affinity for radionuclides) should provide conservative (either low or reasonable) estimates of radionuclide desorption rates that will occur under field conditions (where colloid collisions with sorbing surfaces will inevitably occur). It also implies that to obtain realistic predictions of colloid-facilitated transport in the field it is necessary to conduct experiments in which colloids with sorbed radionuclides come into intimate contact with mineral surfaces expected in the field. 


\subsection{References}

Callahan, T. J., P. W. Reimus, R. S. Bowman, and M. J. Haga (2000). Using Multiple Experimental Methods to Determine Fracture/Matrix Interactions and Dispersion of Nonreactive Solutes in Saturated volcanic Tuff, Water Resour. Res., 36(12), 3547-3558.

Langmuir, D. (1997). Aqueous Environmental Geochemistry. Prentice-Hall, Upper Saddle River, NJ.

Lu, Ningping, J. Conca, G. R. Parker, P. A. Leonard, B. Moore, E. A. Strietelmeier, and I. R. Triay (2000). Adsorption of Actinides onto Colloids as a Function of Time, Temperature, Ionic Strength and Colloid Concentration. LA-UR-00-5121, Los Alamos National Laboratory Unrestricted Report, Los Alamos, NM.

Reimus, P. W. (1995). The Use of Synthetic Colloids in Tracer Transport Experiments in Saturated Rock Fractures, LA-13004-T, Los Alamos National Laboratory Thesis Report, Los Alamos, NM.

Reimus, P. W. and M. J. Haga (1999). Analysis of Tracer Responses in the BULLION ForcedGradient Experiment, LA-13615-MS, Los Alamos National Laboratory Manuscript Series Report, Los Alamos, NM. 


\title{
-Chapter 9- \\ Characterization of Fracture Cores Post Colloid Flow-Through Experiments
}

\author{
Bradley K. Esser and Annie B. Kersting
}

\subsection{Introduction}

This document is a report on surface characterization of natural fracture surfaces in drill core returned from Pahute Mesa wells after laboratory Pu-colloid transport experiments discussed in Chapter 7. In FY 2000 and 2001, the UGTA program sponsored several laboratory experiments conducted at LANL in which $\mathrm{Pu}(\mathrm{V})$ was sorbed onto either montmorillonite or silica colloids, and then the resulting solutions were injected into saturated, fractured cores from Pahute Mesa (along with a conservative tracer, tritiated water). Experiments were also conducted in which $\mathrm{Pu}(\mathrm{IV})$ was sorbed onto clinoptilolite colloids (at LLNL) and then solutions were sent to LANL and injected into fractured cores. After the experiments, the cores were cut open and sent to LLNL for characterization.

One approach to answering or constraining questions regarding the spatial distribution of $\mathrm{Pu}$ on natural fractures and natural fracture coatings in laboratory based Pu-colloid transport experiments is to characterize the surface after the experiment. The goals of this task were to:

- use autoradiography to qualitatively determine the distribution of radionuclides on the surface of natural fractures after Pu-colloid transport experiments,

- use autoradiography to constrain the importance of flow channeling as opposed to sorption in controlling the distribution of colloidal $\mathrm{Pu}$ on natural fracture surfaces in laboratory settings,

- use autoradiography to constrain transport distances for strongly sorbed radionuclides which do not elute during the course of the experiment, and

- use microbeam analysis (SIMS and SEM) to identify specific fracture mineral phases or compositions associated with $\mathrm{Pu}$ sorption or retardation on natural fractures.

The primary rationale for autoradiography is the difficulty of other techniques in characterizing the spatial distribution of trace radionuclides over large surface areas (i.e. many square inches). The primary rationale for secondary ion mass spectrometry (SIMS) is that its combination of spatial resolution (1 micron) and sensitivity (sub-ppm to ppm detection limits) would allow the detection and association of $\mathrm{Pu}$ with specific mineral phases on complex microgranular surfaces. 


\subsection{Characterization of Fracture Cores post Colloid Flow-Through Experiments Samples Examined}

Core 1 (UE20c 2858)

Core 1 is a naturally fractured core section recovered from 2858 feet in drill hole UE20c on Pahute Mesa, NTS. The sample is a devitrified moderately welded tuff from the Pahute Mesa lobe of the Topapah Spring Tuff of the Paintbrush Group (Broxton et al., 1986), and forms a welded tuff aquifer within the Topapah Spring Aquifer.

A previous optical petrography and secondary electron microscopy study characterized the general nature of the fracture coating minerals (Benedict, 2000). An open fracture from sample 2858 had a rough surface with well-developed crystalline coatings and considerable surface porosity. Surface relief was $0.1 \mathrm{~mm}$ with local depressions as deep as $0.3 \mathrm{~mm}$. This texture was described for an adjacent section (UE20c 2851) as a microgranular coated surface of low relief with local sinuous voids providing local depressions. Most of the surface $(60-70 \%)$ on the 2858 open fracture was coated with chalcedony (80$85 \%$ ) and manganese oxides (15-20\%). Manganese oxides occurred on the surface as interlocking fine-grained prismatic crystals; chalcedony occurred as blocky, elongate or fine-grained aggregates.

The core is approximately 8.5 inches long and 3.5 inches in diameter (Figure 1). Core splits received at LLNL after transport experiments and characterization by beta radiography at LANL. The core was subsequently shipped back to LANL, and the concave split (Side A) was sawn into approximate 1.25-1.5 inch sections numbered A7 through A2. The sectioned and intact splits were shipped back to LLNL in February, 2001. In this report, the core is referred to as Core 1, and the intact convex split is referred to as Side B or $1 \mathrm{~B}$. 

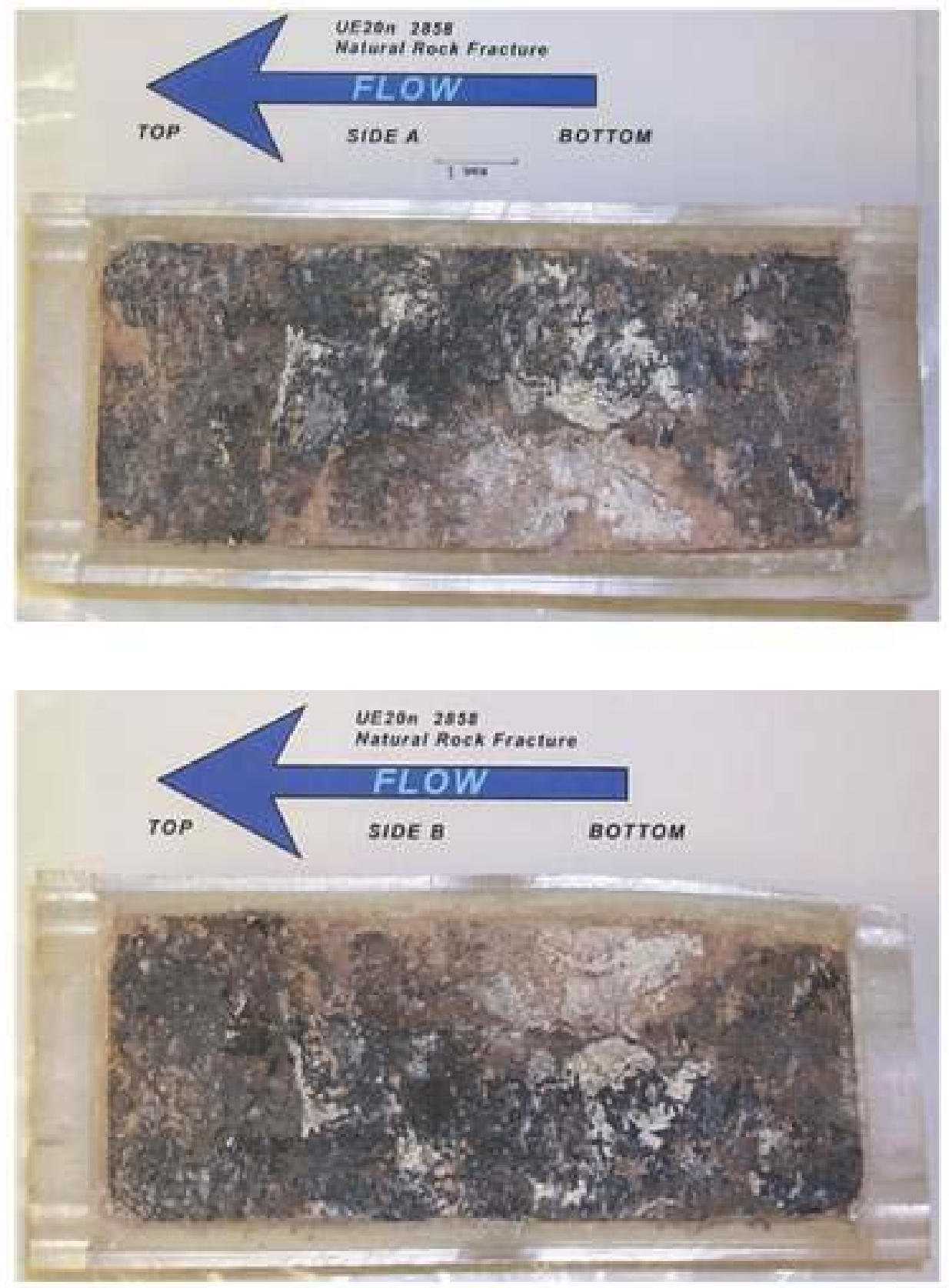

Figure 1. Sides A and B from the UE20c 58528 core sent from LANL. Arrow shows the direction of flow in the LANL transport experiments. Side A was later sent to LANL for sawing into seven sections. 
This sample is a naturally fractured core section recovered from 4823 feet in drill hole PM-1 on Pahute Mesa, NTS. This sample is a quartzofeldspathic altered non-welded tuff from the mafic-poor Bullfrog Tuff of the Crater Flat group (Broxton et al., 1986), and forms a hydrologic confining unit within the Bullfrog Confining Unit.

Secondary electron microscopy (Benedict, 2000) of an open fracture revealed a moderately rough, granular fracture surface. Surface pores were abundant, small (up to $0.02 \mathrm{~mm})$, and angular. Surface relief was low $(0.02 \mathrm{~mm})$. A large proportion of the surface $(90 \%)$ was coated with small crystals and granular aggregates. The fracture coating was comprised of chalcedony (25\%), smectite (65\%), and trace amounts of mixed-metal aggregates. The mixed-metal aggregates contained $\mathrm{Cu}, \mathrm{Fe}, \mathrm{Sn}$, and $\mathrm{S}$. The tentative identification of gypsum and sylvite on the fracture surface suggested that the mixed metal aggregates were likely to be oxides and sulfates.

This core is approximately 7.25 inches long and 3 inches in diameter (Figure 2). The core was received at LLNL in June, 2001, after transport experiments at LANL. In this report, we refer to it as Core 2 with the concave split being referred to as Side A or $2 \mathrm{~A}$ and the convex split being referred to as Side B or $2 \mathrm{~B}$. 

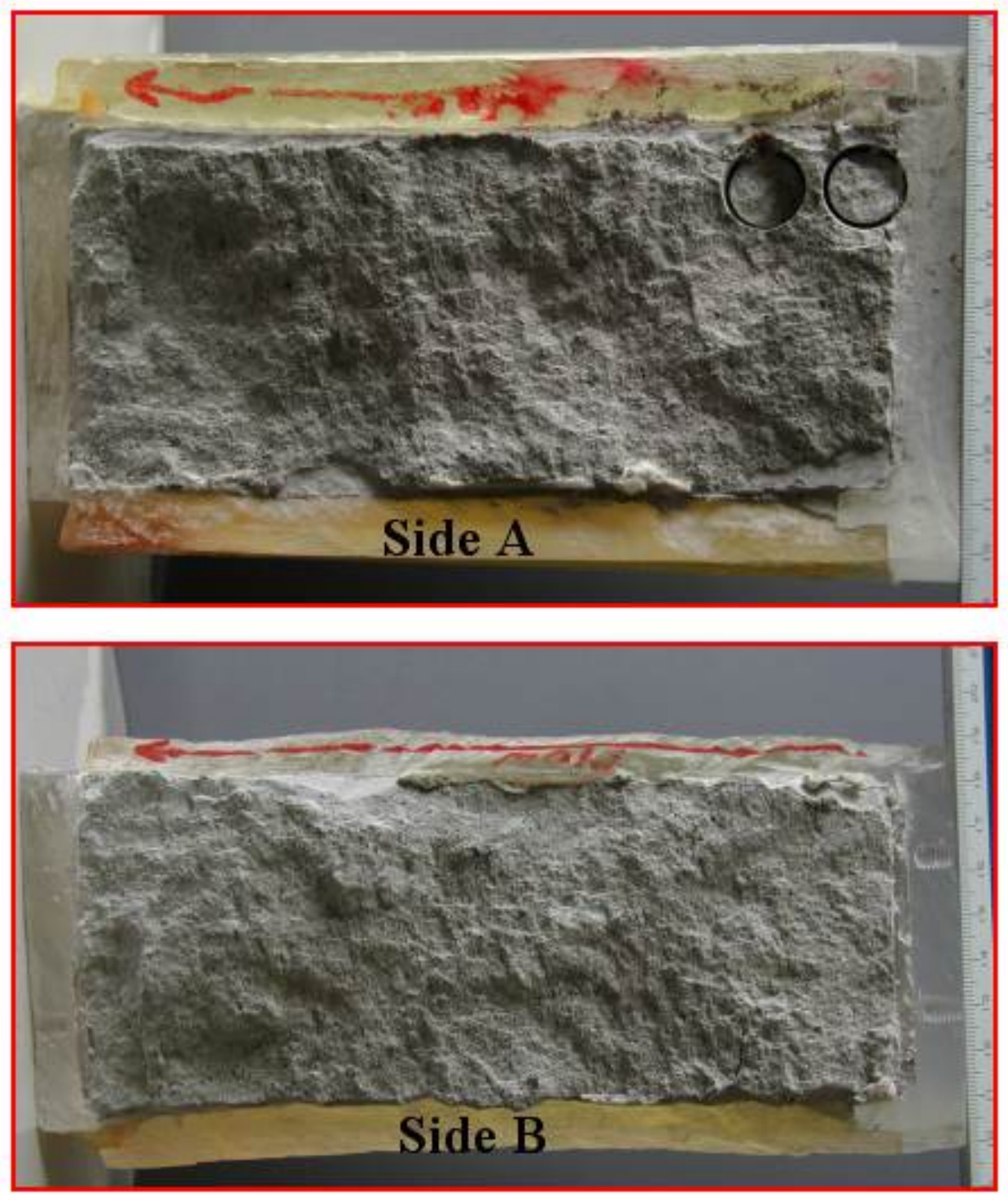

Figure 2. Sides A and B from PM-1 4823. Red arrows show flow direction in LANL flow experiments. Centimeter scale is on stratigraphic bottom of core. Sampling positions for microbeam analysis shown on Side A

\section{Plutonium phases}

A number of transport experiments, using different forms of $\mathrm{Pu}$ (both valence state and substrate), different flow rates and different waters, were conducted at LANL on the 
cores before characterization by LLNL. These experiments are summarized below and in more detail in Reimus (2000)

Table 9.1 Fracture flow experiments conducted at LANL (Chapter 7)

\begin{tabular}{llll}
\hline Core & Pu-phase & Water & Flow Rate \\
\hline & & & \\
UE20c 2858 & Ca-montmorillonite & Syn WW-20 & $1.5 \mathrm{~mL} / \mathrm{h}$ \\
UE20c 2858 & Ca-montmorillonite & Syn WW-20 & $0.6 \mathrm{~mL} / \mathrm{h}$ \\
UE20c 2858 & MP1040 Silica & WW-20 & $1.5 \mathrm{~mL} / \mathrm{h}$ \\
UE20c 2858 & MP1040 Silica & WW-20 & $5.0 \mathrm{~mL} / \mathrm{h}$ \\
UE20c 2858 & Otay Ca-montmorillonite & WW-20 & $1.5 \mathrm{~mL} / \mathrm{h}$ \\
UE20c 2858 & Otay Ca-montmorillonite & WW-20 & $5.0 \mathrm{~mL} / \mathrm{h}$ \\
& & & \\
PM-1 4823 & Colloidal clinoptolite & Syn WW-20 & $1.5 \mathrm{~mL} / \mathrm{h}$ \\
PM-1 4823 & Colloidal Clinoptolite & Syn WW-20 & $0.5 \mathrm{~mL} / \mathrm{h}$ \\
PM-1 4823 & Montmorillonite & WW-20 & $1.5 \mathrm{~mL} / \mathrm{h}$ \\
PM-1 4823 & Montmorillonite & WW-20 & $0.5 \mathrm{~mL} / \mathrm{h}$ \\
& & &
\end{tabular}

UE20c 2858: experiments through October, 2000 (Reimus et al., 2000)

PM-1 4823: experiments through April, 2000 (Paul Reimus, pers. comm., 4/20/01)

\section{Experimental Techniques}

Alpha and Beta Radiography

Alpha autoradiography was performed using TASTRAK CR-39, a polycarbonate plastic alpha-track detector produced by Track Analysis Systems, Ltd. in Bristol, U.K. Small squares of thin plastic $(100 \mathrm{~mm} \times 100 \mathrm{~mm} \times 1 \mathrm{~mm})$ were exposed to fracture surfaces for periods ranging from less than one day to one week. The detectors were placed in as close physical contact as possible with the samples. Sample position was registered on the detector by measurement or by scoring the plastic. After exposure, tracks were etched by heating the plastic in a $6 \mathrm{~N} \mathrm{NaOH}$ solution for 6 hours at $75^{\circ} \mathrm{C}$.

Beta radiography was performed using Kodak Biomax MS film and a Kodak Biomax LE Transcreen. Both 5"x7" and 8"x10" film sheets were used. Biomax MS is a traditional double-sided silver halide emulsion commonly used in film-based macroautoradiography. The Biomax LE Transcreen is a gadolinium oxysulfide: terbium phosphor with a peak output closely matched to the spectral sensitivity of Biomax film; both peak at $540 \mathrm{~nm}$ (Attwood et al., 1999; Vizard et al., 1996). In the Transcreen system, the film is placed between the screen and a reflector, and the sample is placed on or adjacent to the screen. To image fracture surfaces, we most commonly placed the film screen-up in a film tray, attached foam weather-stripping to the Plexiglas border of the core splits or section, and then laid the core face down on the upward-facing film/screen system. A second film tray was taped to first tray, and the whole assembly was placed in a double-layer light-tight change bag. All operations were performed in a darkroom illuminated by a 15-watt GBX- 
filtered incandescent light. For heavier or convex samples, the procedure was modified so that the film was supported by the core. The goal in both cases was to place the screen in close proximity with the fracture surface without actual contact, which we found produced spotting artifacts on the film.

Biomax film was exposed at $-25^{\circ} \mathrm{C}$ for 3-26 days, and subsequently developed using normal Kodak GBX development: GBX Developer (5 minutes with no agitation), water (one minute of vigorous rinsing), GBX Fixer (5 minutes with moderate agitation), and water (one minute of vigorous rinsing). Developed film was allowed to air dry.

\section{Digital Imaging}

Alpha and beta radiographs were digitized using a high-resolution flatbed scanner (Epson Perfection 1640U) with an optical resolution of $1600 \mathrm{dpi}$, and a mechanical resolution of 3200 dpi. The option of using a transparency unit on this scanner allows scanning of either transmitted light (transparency mode) or reflected light (flatbed mode). Beta radiographs (Biomax MS negatives) were scanned with transmitted light; alpha radiographs (etched CR-39 plastic) were scanned in either reflected or transmitted light with image quality being the primary criterion. For complete radiographs, scanning was done with a grayscale depth of 8 bits at $300 \mathrm{dpi}$. Select features on beta radiographs were scanned at high resolution (3200 dpi) to reveal detail.

Digital images were also acquired using digital photography. A 3.3 megapixel Olympus Camedia C-3040 was used for macroscopic imaging, primarily of the rock cores. Pictures were acquired at a resolution of $2048 \times 1536$ pixels as low-compression (1:2.7) JPEG files in 24-bit color. At close focus, the object resolution is approximately 230 pixels/inch. Low-magnification micrographs of the rock cores and of the alpha radiographs were acquired using a Wild binocular microscope and a Polaroid Digital Microscope Camera. Images were acquired as 1600 x 1200 pixel, 8-bit grayscale or 24bit color TIFF files. Object resolution ranged from 4300 to 27,000 pixels/inch.

Digital imagery was processed with Adobe PhotoShop. Overlays were produced by resizing individual images to a common object resolution and using registration marks or recognizable features.

\section{Microbeam Analysis}

After radiography, samples were taken for scanning electron microscopy (SEM) and secondary ion mass spectrometry (SIMS). Samples for microbeam analysis consisted of circular plugs with a diameter of $1.5 \mathrm{~cm}$ and a depth of $0.5 \mathrm{~cm}$. Samples were taken by Ron Pletcher (Energy \& Environment, LLNL) using a low-speed drill cooled with clean proponal. Before each sample, the drill press and bit were cleaned. Low-speed and a nonaqueous coolant were used to preserve the fracture surface and prevent mobilization of $\mathrm{Pu}$. The sample sites were chosen to include fracture surface where radiography indicated that a gradient in $\mathrm{Pu}$ activity existed. Three plugs were taken from the concave side of the UE20c fracture (two from section A7, one from section A6). Two plugs were also taken from the concave side of the PM-1 fracture (from the A split). After sampling, alpha radiographs were acquired for each plug to guide microbeam analysis. 
Secondary ion mass spectrometry was attempted on one sample using a modified CAMECA IMS-3f at LLNL. Analyses were performed by Doug Phinney (C\&MS, LLNL). Fracture surface samples were carbon and gold coated to prevent charging, and were placed in the sample holder with a $0.5 \mathrm{~cm}$ mask to improve secondary ion yield. The instrument was operated in depth profiling mode. NIST SRM 610 (Trace Elements in a Glass Matrix, $3 \mathrm{~mm}$ Wafer) was run immediately prior to running the sample. Surface roughness on the fracture surface samples necessitated optimizing the secondary ion beam at each sampling spot, and prevented setting up an automated transect. At each spot, 10 analyses were performed to allow reconstruction of the depth profile. Isotopes monitored included ${ }^{16} 0,{ }^{23} \mathrm{Na},{ }^{24,25,26} \mathrm{Mg},{ }^{27} \mathrm{Al},{ }^{30} \mathrm{Si},{ }^{39} \mathrm{~K},{ }^{40,42} \mathrm{Ca},{ }^{47,48} \mathrm{Ti},{ }^{54,56} \mathrm{Fe},{ }^{55} \mathrm{Mn}$, ${ }^{138} \mathrm{Ba},{ }^{232} \mathrm{Th},{ }^{238} \mathrm{U}$, and ${ }^{239} \mathrm{Pu}$.

SEM micrographs and qualitative EDS spectra were obtained from the SIMS depth profile spots.

\section{Radiography Standards}

Three sets of sealed-source plutonium standards for radiography were produced by electrochemically depositing $\mathrm{Pu}$ onto 1 -inch steel planchets (Figure 3). The plating was performed by Everett Guthrie (LLNL Chemistry and Material Science). The standards represent the Environmental Monitoring Radiological Laboratory (C\&MS, LLNL) Pu standard, the Pu used at LLNL to prepare Pu-enriched zeolite colloids, and the second batch of $\mathrm{Pu}$ used at LANL to prepare Pu-enriched montmorillonite clays and colloidal silica. These standards will be referred to as EMRL, LLNL, and LANL Pu, respectively. The LANL Pu was prepared in two batches which should be identical. Since the Pu standard solutions were plated without chemical separation of $\mathrm{Pu}$ from other actinides, the plated samples may contain $\mathrm{U}, \mathrm{Np}$ and $\mathrm{Am}$ in addition to $\mathrm{Pu}$. Nominal $\mathrm{Pu}$ activities of the standards were as follows: EMRL 0.2, $0.4 \mathrm{nCi}$; LLNL 0.2, 2.0, $20.0 \mathrm{nCi}$; LANL 0.1, $1.0,10.0 \mathrm{nCi}$. 


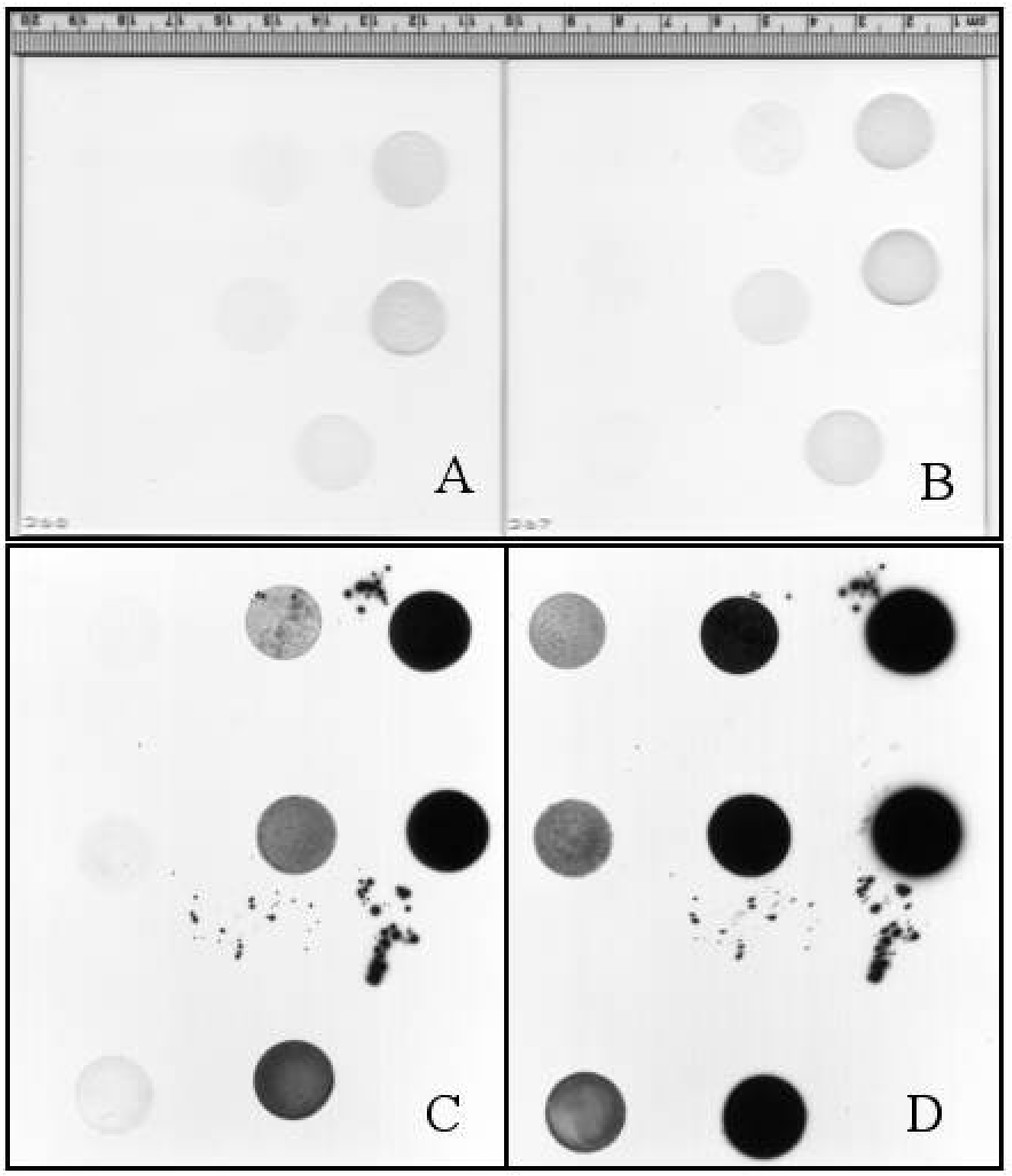

Figure 3. Plutorium radiography standards. A: alpha, $5 \mathrm{~h} ; \mathrm{B}:$ alpha, $13 \mathrm{~h}$; C: beta, $3 \mathrm{~h}$; D: beta, $48 \mathrm{~h}$. Alpha = Tastrak CR-39. Beta = Biomax MS + LE Transcreen. In each image, top row $=\mathrm{LANL}$ at $0.1,1.0$, and $10 \mathrm{nCi}$; middle row $=\mathrm{LLNL}$ at $0.2,2$ and 20 $\mathrm{nCi}$; bottom row $=\mathrm{EMRL}$ at $0.2,4.0 \mathrm{nCi} \mathrm{Pu}$ 
The isotopic composition of the standard $\mathrm{Pu}$ solutions was determined by high-resolution multi-collector inductively coupled plasma mass spectrometry on a MicroMass Isoprobe by Ross Williams (LLNL Chemistry and Material Science). Isotopic compositions were determined both before and after anion exchange separation chemistry. Anion exchange chemistry was performed to separate $\mathrm{Pu}$ from $\mathrm{U}, \mathrm{Np}$ and $\mathrm{Am}$.

For beta radiography, $\mathrm{Pu}$ standards are placed directly on the Transcreen, and the film/screen are placed in a light-tight Kodak cassette which is placed in a change bag. All other protocols for beta and alpha radiography were followed as described.

\section{Results and Discussion}

\section{Plutonium Standards: Isotopic Composition and Radiography}

The isotopic composition for the $\mathrm{Pu}$ standards is presented as atom ratios normalized to ${ }^{239} \mathrm{Pu}$ (Table 9.2). We have not yet completed the determination of ${ }^{238} \mathrm{Pu}$ in the sample, and so cannot calculate atom abundances for the individual $\mathrm{Pu}$ isotopes. Both the LANL and LLNL Pu standards used to prepare $\mathrm{Pu}$-enriched colloid phases contain significantly less than $1 \%{ }^{241} \mathrm{Pu}$, the only $\mathrm{Pu}$ isotope which is a beta emitter. The most surprising result is the high ${ }^{242} \mathrm{Pu}$ content in the LANL $\mathrm{Pu}$. The reported LANL ${ }^{242} \mathrm{Pu} /{ }^{239} \mathrm{Pu}$ data represent three analyses of spiked, unspiked and separated $\mathrm{Pu}$.

The isotopic composition of $\mathrm{Pu}$ is important to understanding the mechanism by which $\mathrm{Pu}$ exposes beta radiography film, which is currently unknown. $\mathrm{Pu} 241$ is a beta emitter with an average beta energy $(5.2 \mathrm{keV})$ approximately equal to that of tritium. We have conducted experiments comparing the sensitivity of Biomax MS film to LLNL and LANL Pu standard versus tritium-implanted silica and silicon (Rose et al., 2000a). Even after correction for self-absorption of the implanted tritium, $\mathrm{Pu}$ standards expose the film at rates orders of magnitude higher than would be expected by ${ }^{241} \mathrm{Pu}$ beta decay alone. We have also considered ${ }^{242} \mathrm{Pu}$ which emits atomic electrons of considerably higher energy (up to $50 \mathrm{keV}$ ). Again however, even after taking into account the spectral sensitivity of the Biomax film/screen combination, ${ }^{242} \mathrm{Pu}$ alone fails to account for the sensitivity of the film to $\mathrm{Pu}$. The qualitative similarity in response to $\mathrm{Pu}$ standards with distinctly different ${ }^{242} \mathrm{Pu}$ contents (Figure 3 and Table 9.2) also argues against ${ }^{242} \mathrm{Pu}$.

Table 9.2. Isotopic composition of plutonium standards

\begin{tabular}{llllllll}
\hline Pu Std & Pu240/ & Pu239 & Pu241/ & Pu239 & Pu242/ & Pu239 & 241Am/ 239Pu \\
& Atom ratio & \pm 2 sigma & Atom ratio & \pm 2 sigma & Atom ratio & \pm 2 sigma & Atom ratio \\
\hline & & & & & & & \\
LANL & 0.06276 & \pm 0.00004 & 0.001086 & \pm 0.000001 & 0.33225 & \pm 0.00013 & 0.00011 \\
LLNL & 0.07165 & \pm 0.00047 & 0.004702 & \pm 0.000030 & 0.00692 & \pm 0.00005 & 0.00251 \\
EMRL & 0.02125 & \pm 0.00030 & $<$ & 0.000042 & $<$ & 0.00013 & 0.00267
\end{tabular}

Isotopic compositions determined by HR-MC-ICPMS at LLNL. 
Alpha and beta radiographs of unseparated $\mathrm{Pu}$ standards (which contain some fraction of $U$ and $\mathrm{Am}$ ) has been acquired (Figure 3), but has not been quantitatively analyzed. Preliminary qualitative analysis indicates no difference in the sensitivity of Biomax MS film (used in conjunction with a Biomax LE Transcreen) to $\mathrm{Pu}$ of different isotopic compositions. This suggests that calibration of alpha and beta $\mathrm{Pu}$ radiography will not be hampered by large isotopic effects.

Core 1 (UE20c 2858) and Core 2 (PM-1 4823) Radiography

Alpha and beta radiography were performed on each split from both cores in an attempt to localize residual $\mathrm{Pu}$ along the fracture plane. A number of general observations apply. Both alpha and beta radiographs give the same patterns on both large and small scales (see discussion below). On all splits (Figures 4, 8, 9 and 10), activity decreases rapidly along the direction of flow in the transport experiments with most activity being concentrated in the first inch of fracture surface along the flow path. Plutonium distribution with depth is not uniform - even at top of the flow path, activity is concentrated either in the center of the core (UE20c, Figure 4) or to one side of the core (PM-1, Figures 9 and 10). In both cores, Pu penetrates to depth along narrow stringers (Figures 8 and 10) which appear to correspond to major relief features in the fracture surface. All of these observations point to flow being a dominant control on the macroscopic distribution of residual $\mathrm{Pu}$ on the fracture surface. 


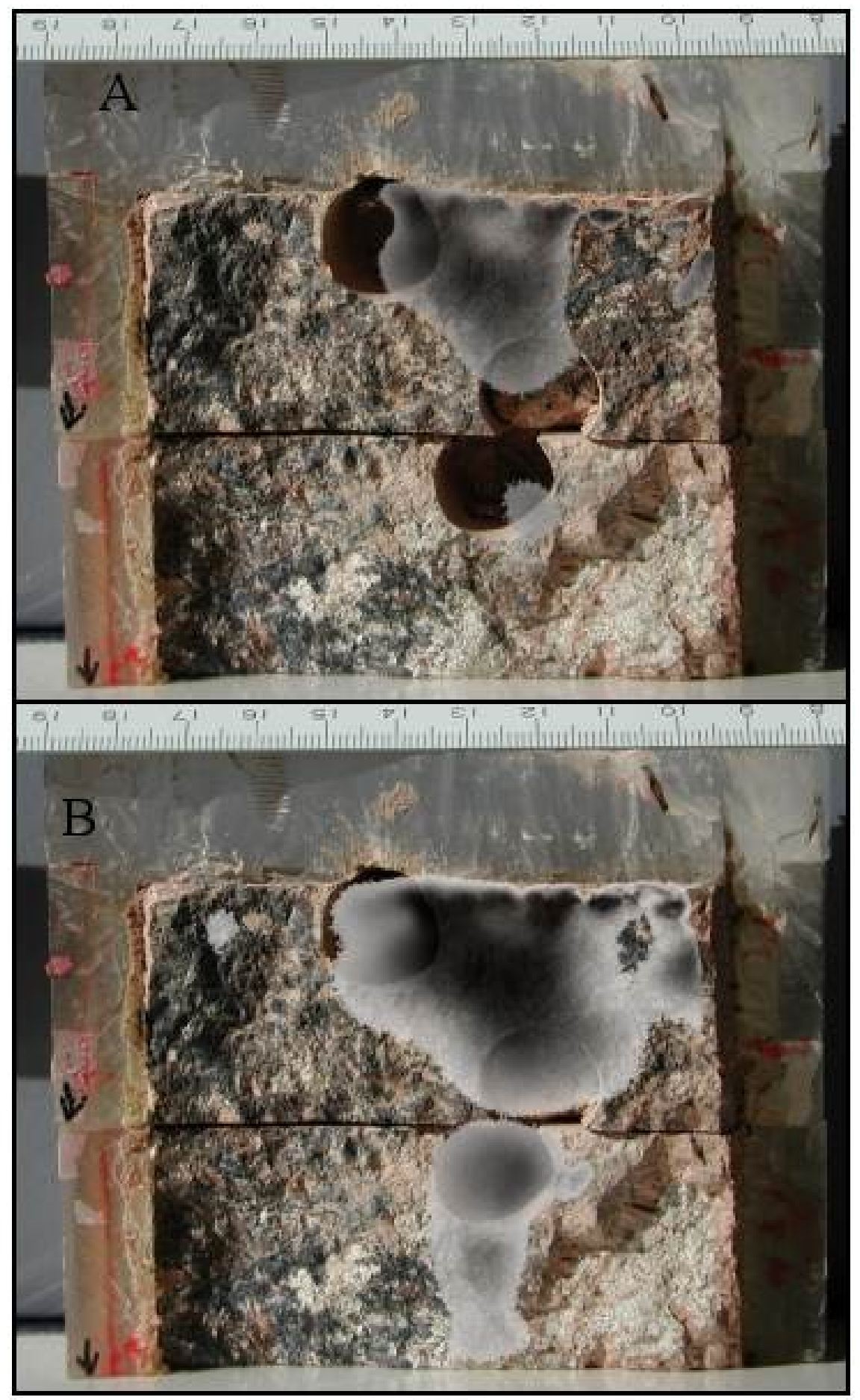

Figure 4. Beta radiographs overlain on the sections $A 7$ and $A 6$ of core UE20c 2858. A: 3 day exposure; $B: 7.4$ day exposure. The lower sample site is A6-1. The radiographs were taken before sampling. 
The radiography gives useful information on a smaller scale as well. Two features can be visually identified in the upper right-hand quadrant of the surface of core split section A7 from core UE20c (Figure 5). A circular feature of approximately $1.5 \mathrm{~mm} \times 2.0 \mathrm{~mm}$ (Figure 6) is clearly visible in both alpha and beta radiography. Resolution is on the order of a few hundred microns for both alpha and beta, with alpha radiography showing better resolution. Discernable features in the radiography appear to correspond to the distribution of a dark crystalline mineral which may be a manganese oxide on a pink aluminosilicate surface which appears to be uncoated tuff. Likewise, a small zigzag of a dark mineral against a pink feldspar and quartz background give rise to similar patterns in both alpha and beta imagery (Figure 7). In this instance, the alpha image clearly has better resolution as would be expected with the shorter range of alpha particles relative to beta particles. In these examples, the distribution of plutonium appears to be correlated with mineralogical differences on the surface. Flow cannot be completely ruled out however, since the dark minerals produced raised surfaces, and both features lie at the edge of significant changes in surface topography. Nevertheless, the correspondence of activity to observable mineralogy on the sub-millimeter scale is consistent with the surface composition and mineralogy playing a role in the distribution of residual $\mathrm{Pu}$ in the cores.
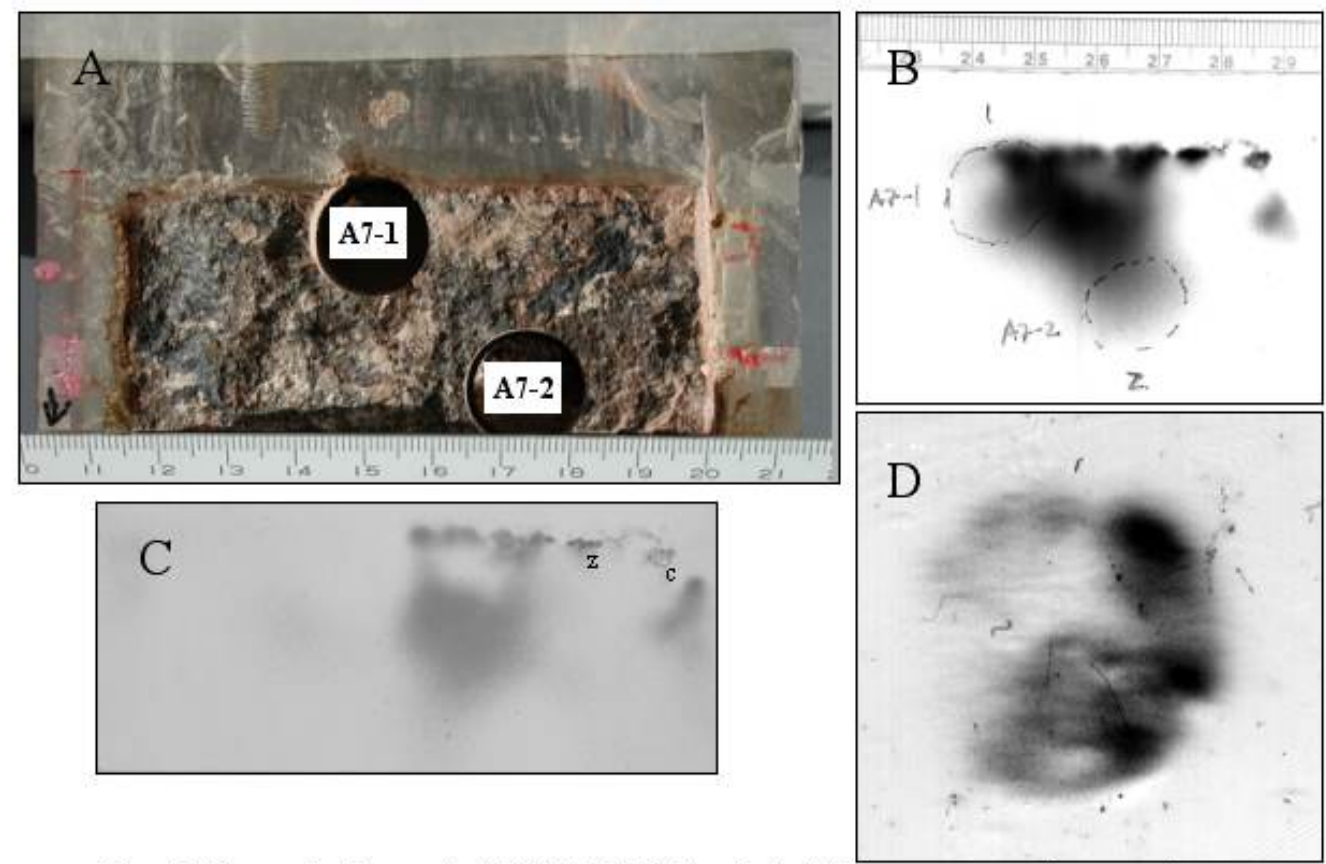

Figure 5. Plug samples from section A7 (UE20c 5828). Flow during LANL transport experiments was down. A: Photograph (with cm scale) showing plug locations. B: Beta radiograph before sampling showing sampling template. C: Alpha radiograph after sampling showing locations of plug samples and small-scale zig-zag (z) and circular (c) features. D:Alpha radiograph of plug A7-1.

The alpha and beta radiographs were used to select sites for taking plugs of the fracture surface for microbeam analysis (Figure 5). Samples were chosen where centimeter scale 
gradients existed in the activity of Pu. This technique worked well. Compare the alpha radiograph of plug A7-1 (Figure 5D) to the beta radiograph template used to guide the drill (Figure 5B). Plugs of $1.5 \mathrm{~cm}$ in diameter were taken using a low-speed drill cooled by clean propanol with the aim of avoiding redistribution of $\mathrm{Pu}$ on the surface by either heat or water.

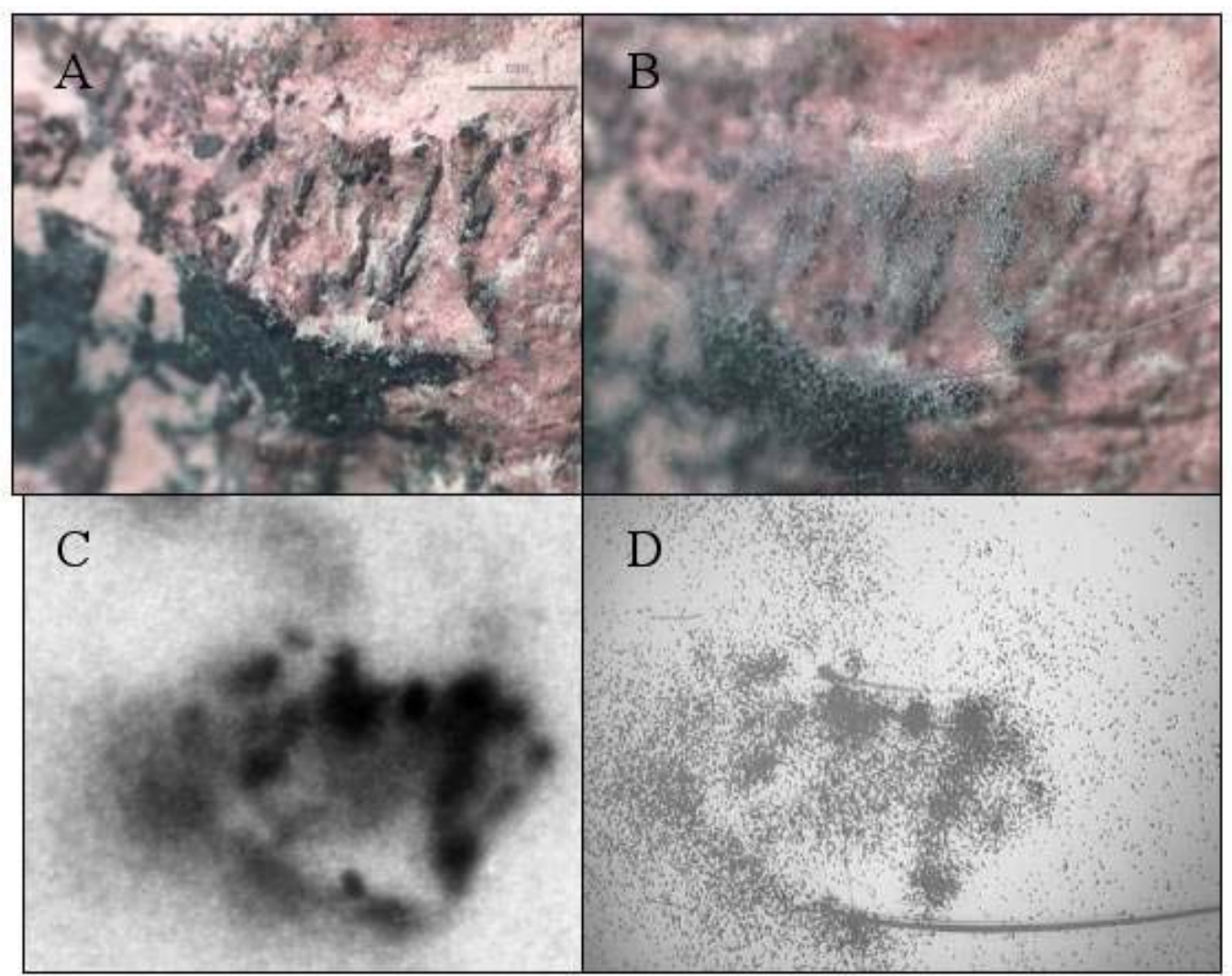

Figure 6. Circular feature in upper right hand comer of sample A7 (UE20c 5828). A: optical microscopy (note 1 rum scale bar). B. overlay of alpha plate on rock sample. C: bet a radiography; D: alpha radiography 


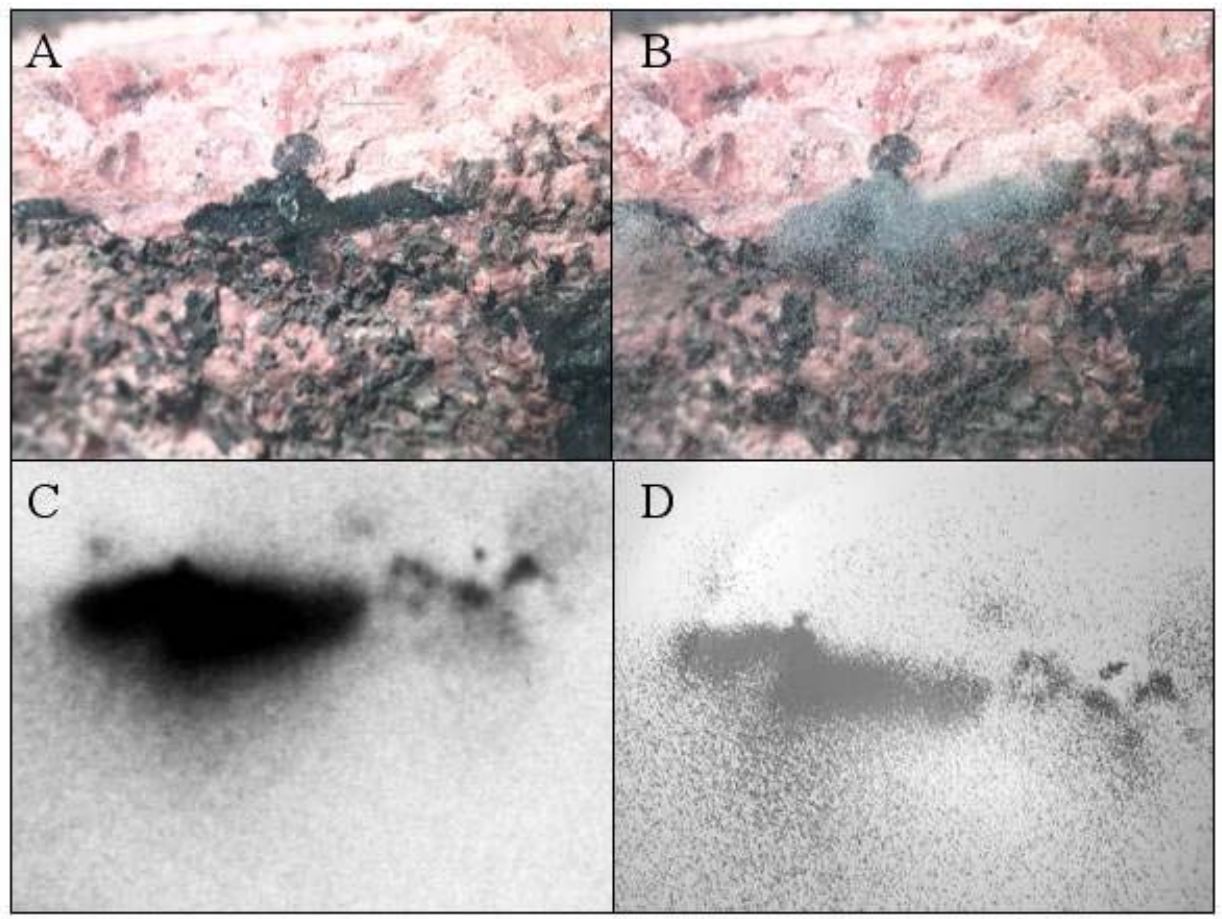

Figure 7. Zig-zag feature in upper right hand comer of sample A7 (UE20c 5828). A: optical microscopy (note $1 \mathrm{~mm}$ scale bar. B. overlay of alpha plate on rock sample. C: beta radiography; D: alpha radiography

\section{Core 1 (UE20c 2858) Microbeam Analysis}

The limited time available for SIMS analysis was used to perform a transect of eight spots across the upper right quadrant of plug A7-1 (UE20c 2858, split A, section A7). Direction in this case is described when the plug is oriented with the flow direction down. Depth profiles were acquired from each of the eight spots. A number of problems were encountered during analysis, all of which are related to the roughness of the fracture surface preserved in the plug samples (Figure 15). Geologic samples are generally presented for SIMS analysis as polished thin sections (Rose et al., 2000b). Because the fracture surface has extremely high surface relief, the surface of the sample cannot be seen in the sample chamber using optical microscopy. The lack of optical location of beam position precludes analysis of visually identified mineral phases or features. The uneven surface also requires that the secondary ion beam be manually refocused and optimized each time the sample is moved. This requirement precludes setting up automated transect or acquiring rastered data. Finally, the uneven surface produces variable and poor secondary ion yield, making quantitation of the data against matrixmatched standards difficult and degrading sensitivity to minor and trace components. 

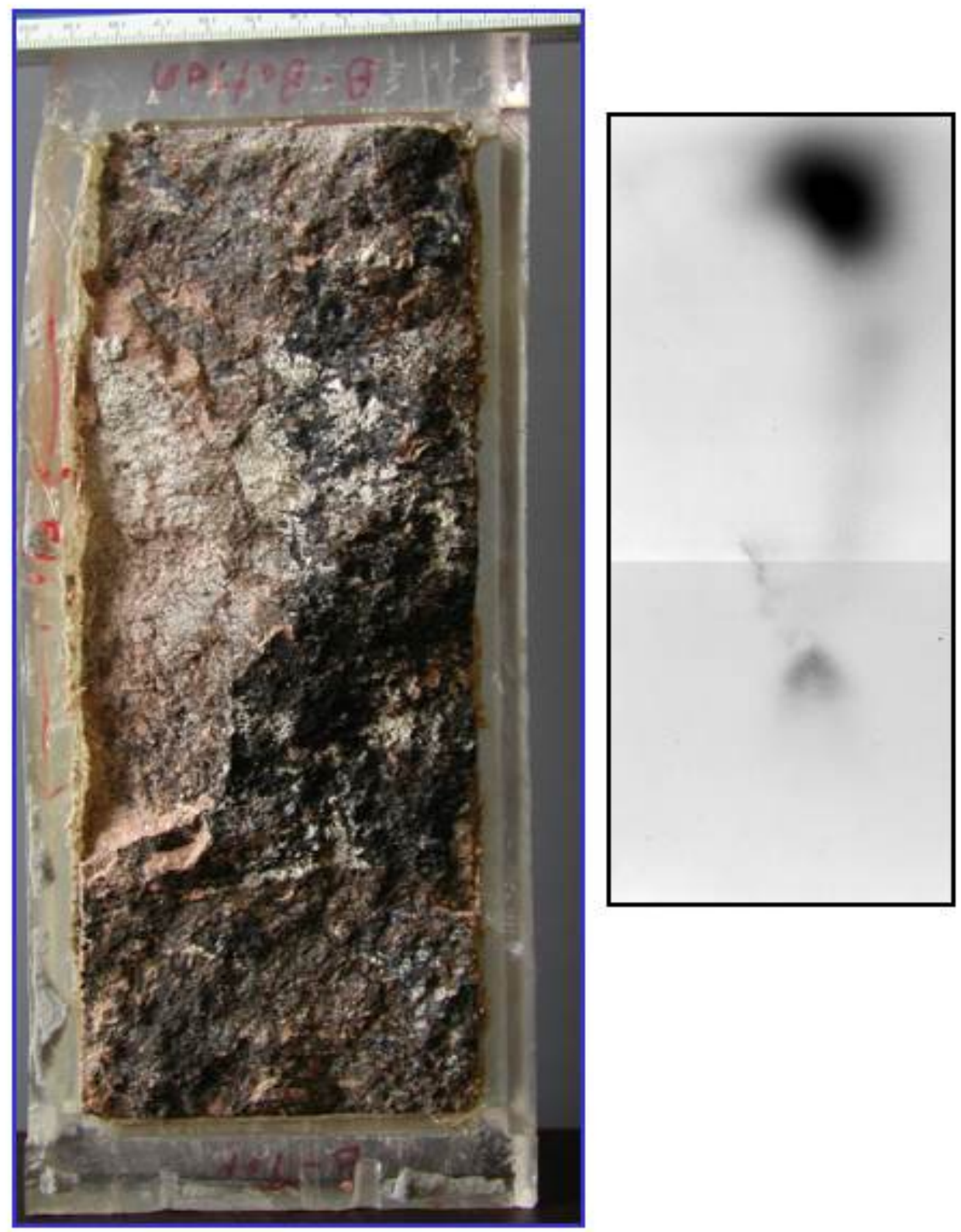

Figure 8. Side $\mathrm{B}$ of core UE20c 2858 and a beta radiograph (4.6 days) shown at the same scale. Note centimeter rule in rock photo. The large spot of activity in at the top of the radiograph is approximately centered on the core.

The SIMS analysis of plug A7-1 indicated an aluminosilicate composition with variable amounts of Mn. In general, quantitation of the data was not possible, and absolute concentrations could not be determined. Normalized count ratio data, however, still yields useful information. During depth profiling, systematic changes were seen in Si- 
normalized $\mathrm{Na}, \mathrm{Al}, \mathrm{K}, \mathrm{Ca}$, and $\mathrm{Mn}$ count ratios (Figure 14). In the initial spots at the beginning of the transect, $\mathrm{Mn} / \mathrm{Si}$ ratios increased with depth, while $\mathrm{Na}, \mathrm{Al}, \mathrm{K}$ and $\mathrm{Ca}$ decreased with depth. This is consistent with a silica and Mn oxide fracture coating overlain by microgranular aluminosilicate debris. Later in the transect, initial $\mathrm{Mn} / \mathrm{Si}$ ratios were lower, and compositional changes with depth were less noticeable, consistent with the profile moving from an area with a fracture coating to an area without a fracture coating . Although the data were suggestive that $\mathrm{Pu}$ was detected in the initial spots where radiography indicated the highest activity (see below for how the spots were localized) and $\mathrm{Mn} / \mathrm{Si}$ count ratios were highest, the count ratio of 239 to 238 was sufficiently low and variable that the 239 signal could not be unambiguously resolved from uranium hydride $\left({ }^{238} \mathrm{U}^{1} \mathrm{H}\right)$. 

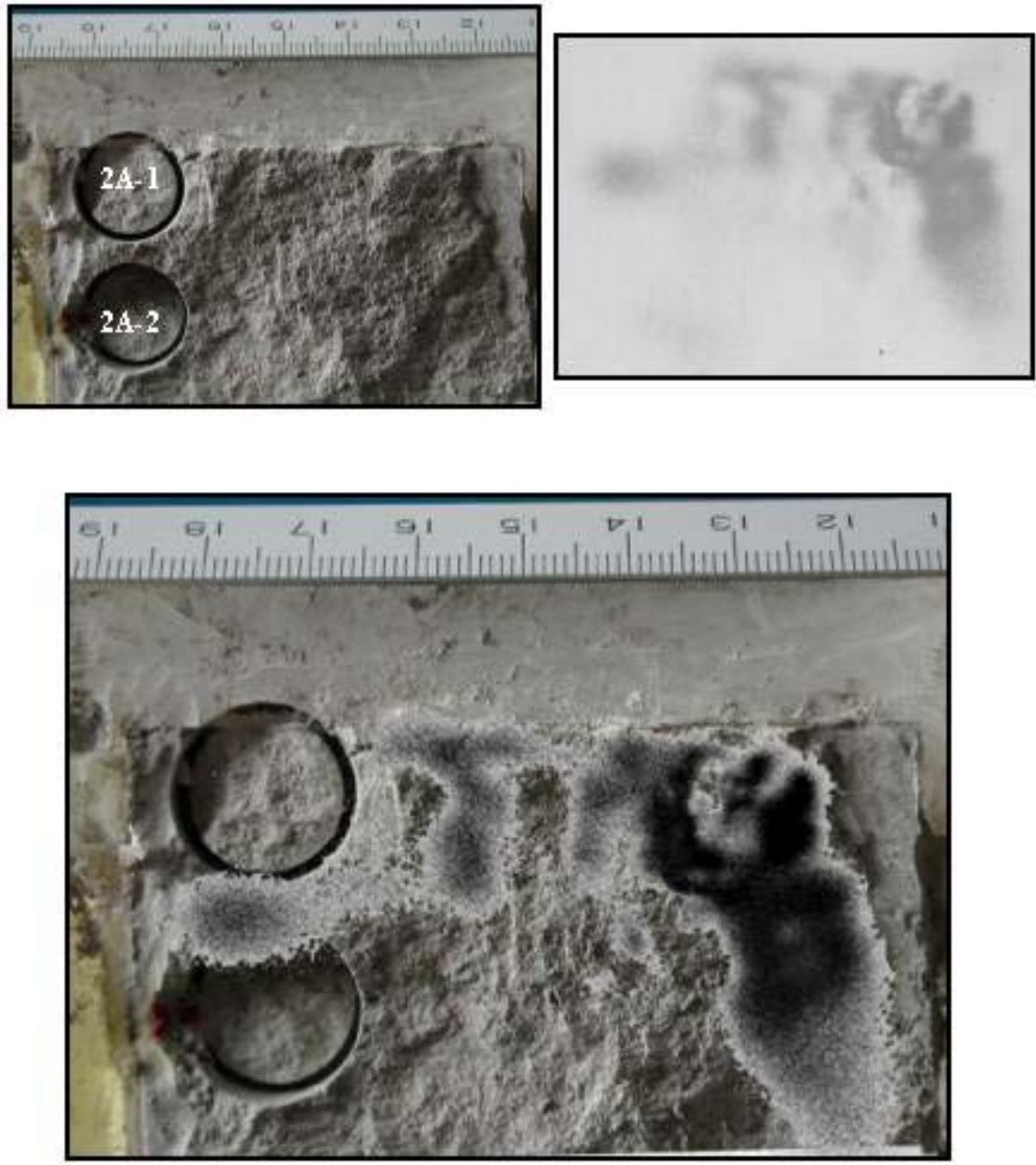

Figure 9. Side B of core PM-1 4823, an alpha radiograph (6.8d), and an overlain image. The radiograph was taker after sampling $2 \mathrm{~A}-1$ and $2 \mathrm{~A}-2$.

The same area on plug A7-1 was imaged by SEM, and we succeeded in finding the SIMS depth profiling spots. In SEM imagery, the SIMS spots appear as circular depressions tens of microns in diameter which at the bottom have a glassy appearance suggestive of heating and partial melting (Figure 16). This texture was not observed outside the hole bottoms. We observed a linear array of six such holes. We were able to relate the location 
of the holes to a flat on the perimeter of the plug by composite imaging (Figure 12). The fracture surface in the vicinity of the SIMS analyses was microgranular, and qualitative EDS spectra indicated a predominantly aluminosilicate composition with variable amounts of Mn (Figures 13 and 16). In Mn rich areas, Ce was detected and Fe was enriched. The Mn-rich regions did not correspond to individual grains or phases on the surface, indicating that $\mathrm{Mn}$ may be a component of the smooth mat-like subsurface underlying the surface grains, and consistent with the sampling volume of the $15.0 \mathrm{kV}$ primary electron beam in relation to the volume of the submicron to 5 micron surface grains. 

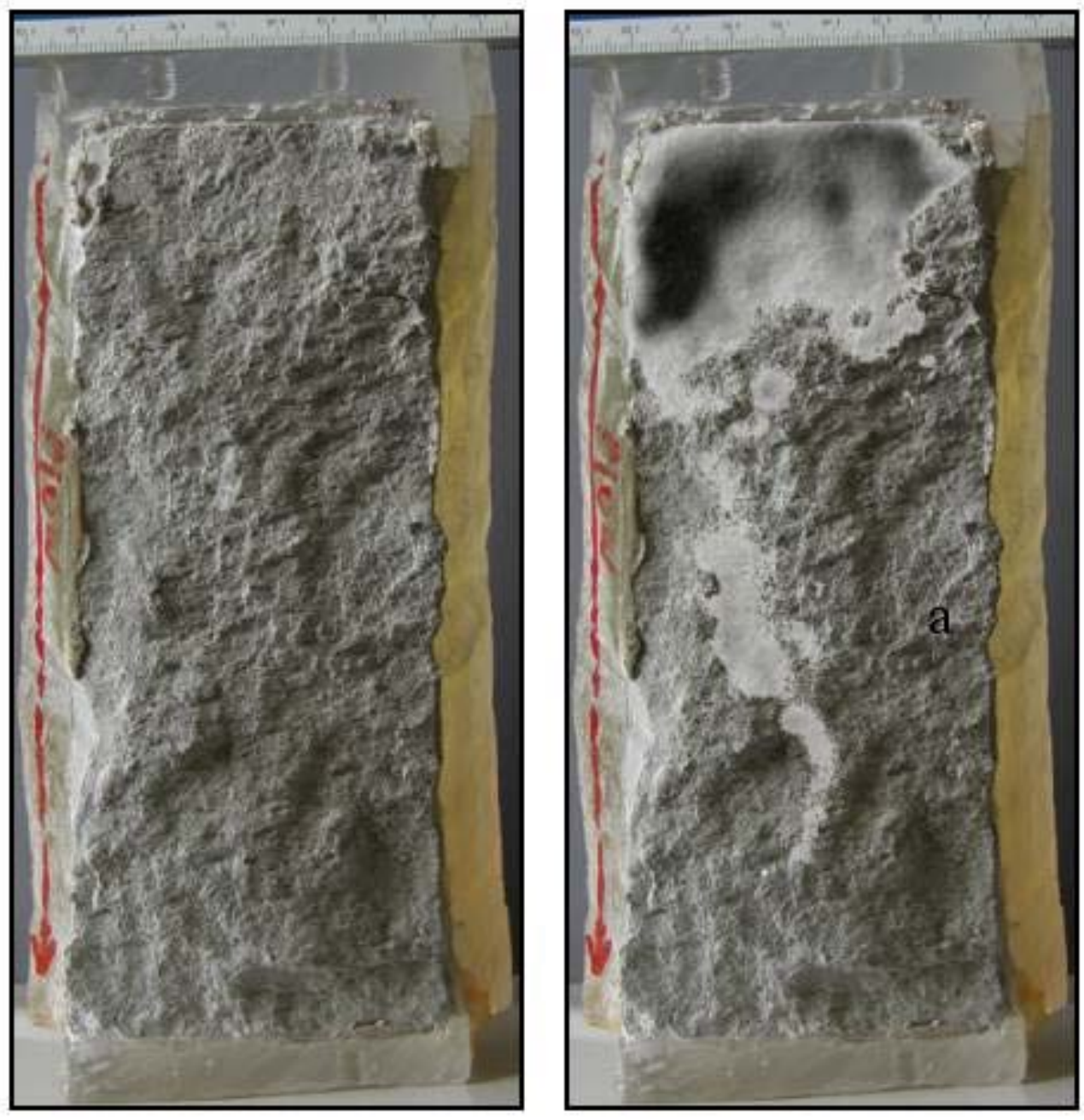

Figure 10. Side B of core PM-1 4823 and an overlain beta radiograph (26 d). The region marked "a" in the overlain image contains no dat a from the radiograph due to artifacts on the film. 


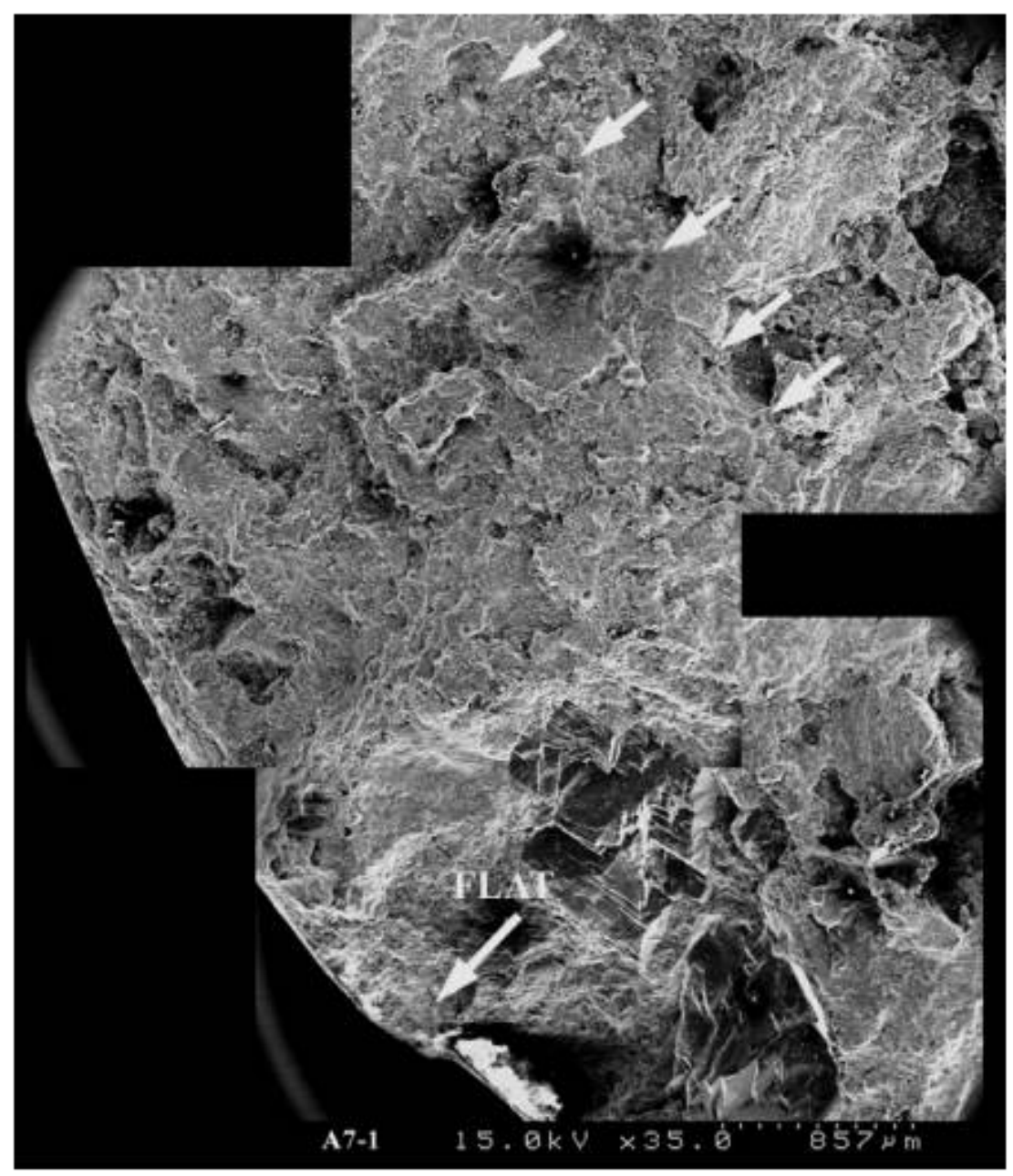

Figure 12. SEM micrograph of SIMS depth profile spots (white arrows) on sample A7-1 (UE20c 5828). The flat on the perimeter is easily identified on the sample plug. 

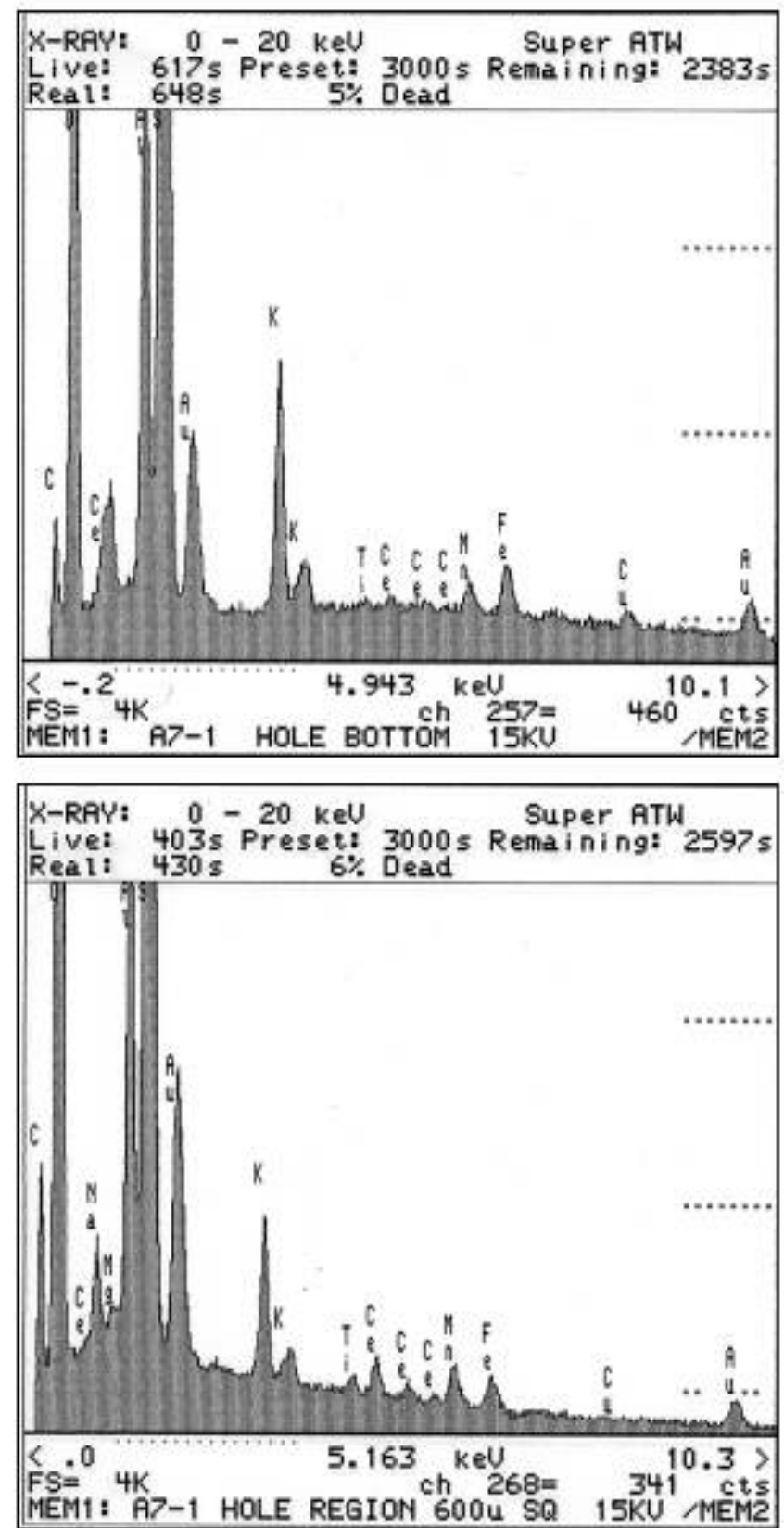

Figure 13. EDS spectra from sample A 7-1 (UE20c 5858). The upper spectrum is from a SIMS crater hole; the lower spectrum is from a flat area outside of a SIMS crater. 

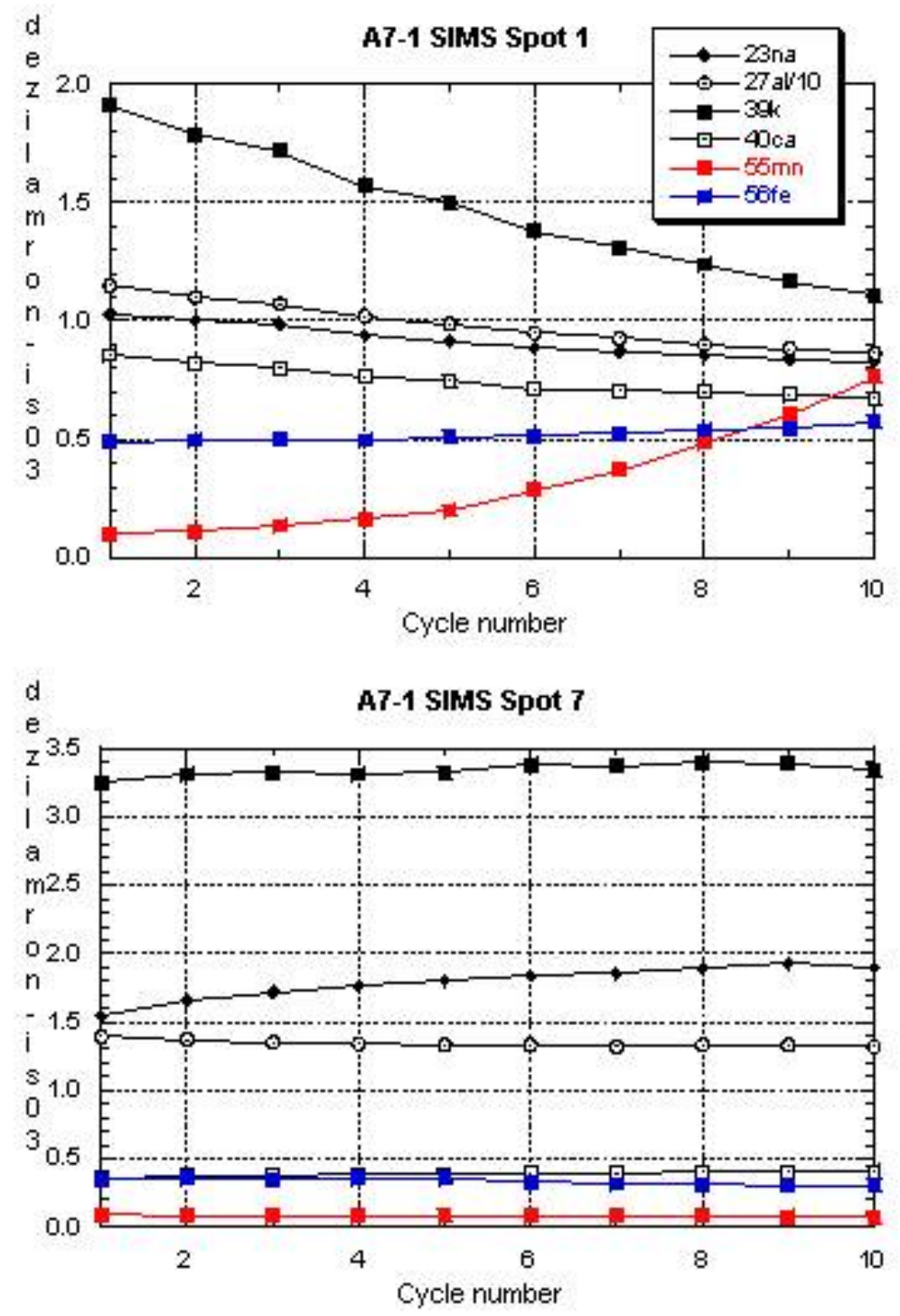

Figure 14. Normalized count rate data from SIMS analysis of plug A 7-1 (UE20c 5828). Depth increases with cycle number. 

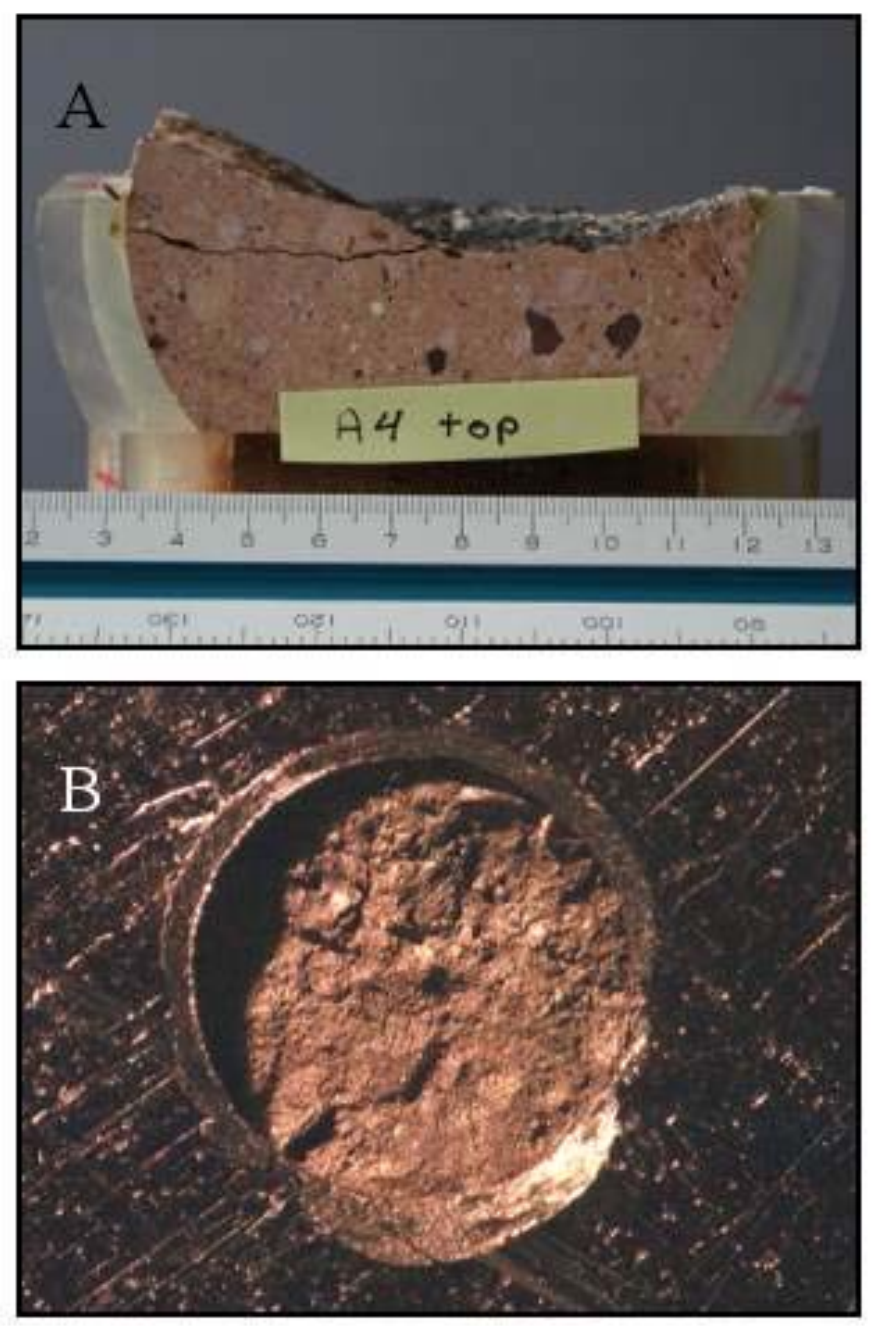

Figure 15. Surface relief on the fracture plane. A: Cross section from core split 1A (UE20c 2858) showing curvature of fracture plane. B: Plug sample in mask for SIMS analysis. 

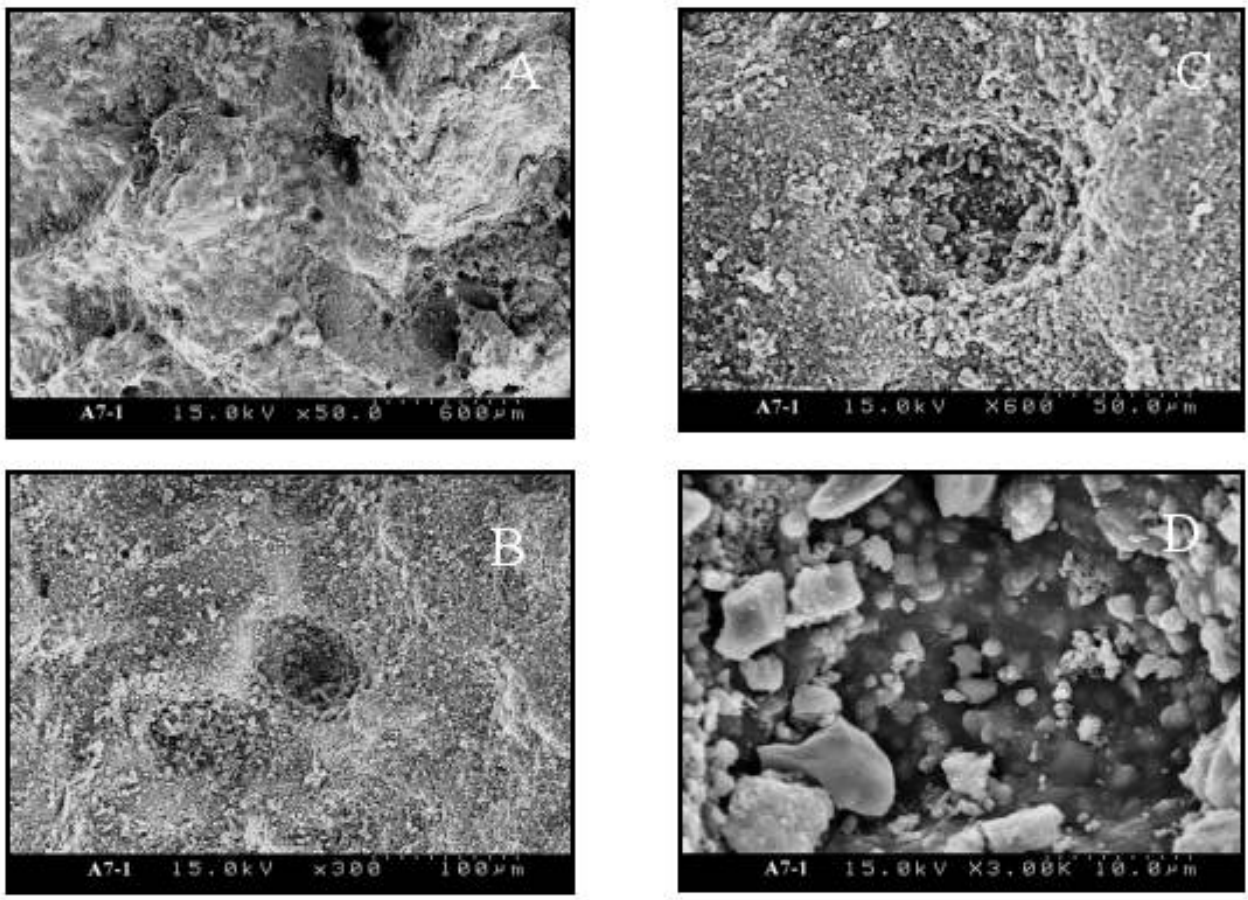

Figure 16. SEM micrographs of a SIMS depth profile spot on sample A7-1 (UE20c 5828). Note the glassy texture at the bottomof the spot hole in image $D$.

\subsection{Summary}

This study has demonstrated the value of radiography in radionuclide transport studies in a number of ways:

- beta radiography film is sensitive to $\mathrm{Pu}$,

- residual $\mathrm{Pu}$ from transport experiments can be located on large rough surfaces using both alpha and beta radiography, and

- $\mathrm{Pu}$ can be localized at sub-millimeter scales using both alpha and beta radiography.

The ability to use both alpha and beta radiography will be extremely useful in designing transport experiments involving both actinides (primarily alpha emitters) and fission products (beta emitters).

Microbeam analysis of rough surfaces is difficult but important to continue to develop.

- This work showed that SIMS is a difficult technique to use with rough surfaces when working on attempting to associate trace nuclide concentrations with specific compositions or surface features, and

- This work just began to show the potential of coupled autoradiography-SEM studies in combining mineral identification with radionuclide localization to 
examine correlation between specific mineral phases and radionuclide retardation on surfaces.

Our findings for the Pahute Mesa cores include the following observations,

- The large (centimeter and greater) scale distribution of residual Pu on the Pahute Mesa core fracture surfaces is consistent with flow channeling being an important mechanism in transport experiments, and

- The small (millimeter and less) scale distribution of residual Pu on the Pahute Mesa core fracture surfaces is consistent with mineralogical control, with manganese oxides being a potential candidate.

- At this time we cannot distinguish whether the alpha activity on the cores is a result of the Pu sorbing to the fracture lining minerals or whether the Pu-colloids were trapped in the fracture surfaces. 


\subsection{References}

Attwood J., Vizard D., Goodkowsky S., and Degregorio J. (1999) Using Intensifying Screens in Autoradiography to Improve Results. American Technology Laboratory (October, 1999).

Benedict C. (2000) Draft Summary of Micrographic Analyses Conducted During FY99 of Selected Core Samples in Support of Diffusion Experiments. HSI Geotrans,

Broxton D. E., Warren R. G., Hagen R. C., and Luedermann G. (1986) Chemistry of Diagenetically Altered Tuffs at a Potential Nuclear Waste Repository, Yucca Mountain, Nye County, Nevada. Los Alamos National Laboratory, LA-10802-MS

Reimus P., Ware S. D., Lu N., Abdel-Fattah A., Kersting A., Hakem N., Zhao P., and Eaton G. (2000) Progress Report on Colloid-Facilitated Plutonium Transport in Fractured Rocks from Pahute Mesa, Nevada Test Site. U.S. Department of Energy,

Rose T. P., Phinney D. L., Hutcheon I. D., Hudson G. B., and Smith D. K. (2000a) Development and Testing of Tritium Reference Standards for the Secondary Ion Mass Spectrometer. Lawrence Livermore National Laboratory, 13 pp.

Rose T. P., Smith D. K., and Phinney D. L. (2000b) Secondary Ion Mass Spectrometry Measurements of Volcanic Tuffs Containing Radionuclides From Underground Nuclear Tests. Radiochim. Acta 88(8), 465-473.

Vizard D., Attwood J., and Mclaughlin W. (1996) A Universal Intensifying Screen System for Enhanced Detection of Low- and High-Energy Isotopes. American Biotechnology Laboratory( October, 1996). 


\title{
-Chapter 10- \\ Colloid Transport Parameters Derived from Field-Scale Tracer Tests and Laboratory Studies
}

\author{
Paul W. Reimus, Amr Abdel-Fattah, and Ioana Anghel
}

\subsection{Introduction}

In this chapter, colloid transport parameter estimates (filtration and detachment rate constants) derived from field and laboratory tests conducted for UGTA and other projects are discussed. First, filtration rate constants obtained from field tracer tests involving polystyrene microspheres are presented. Second, laboratory experiments comparing of the transport of polystyrene microspheres and silica colloids are discussed in the context of assessing whether polystyrene microspheres can be considered conservative surrogates for natural colloids. Finally, the results of microscopic colloid visualization experiments conducted to study and parameterize colloid attachment and detachment processes onto idealized fracture surfaces under flowing conditions are summarized.

\subsection{Field-Scale Tracer Tests in Fractured Media using Polystyrene Microspheres as Colloid Tracers}

Several field tracer tests in saturated fractured media have been conducted in which carboxylatemodified-latex (CML) polystyrene microspheres were used as colloid tracers along with solute tracers. One of these tests, the BULLION forced-gradient experiment in the ER-20-6 wells (\#1, \#2 and \#3) on Pahute Mesa, was conducted for the UGTA project (Reimus and Haga, 1999). Two other tests were conducted in fractured volcanic tuffs at the $\mathrm{C}$ wells complex located about $2 \mathrm{~km}$ southeast of the potential repository footprint at Yucca Mountain. Results from microsphere tests conducted in fractured granite systems at Raymond Quarry, CA and Mirror Lake, $\mathrm{NH}$ are also referred to in this chapter.

Our purpose here is not to describe in detail the manner in which the various tracer tests were conducted, but rather to summarize the pertinent results from the tests for the UGTA project. The microsphere responses in the tracer tests indicated the following:

- Some fraction of the microspheres always moved through the flow systems without being filtered. The fraction transporting without filtration varied significantly from test to test and depended on the travel time and the properties of the flow system.

- The unfiltered fraction of microspheres always arrived at the same time or slightly earlier than nonsorbing solute tracers used in the tests.

- The microsphere responses exhibited long, relatively flat tails consistent with slow, steady detachment of filtered microspheres from fracture surfaces.

- Flow transients, such as pumps stopping and restarting, typically produced short pulses of microspheres at the production well, suggesting that the flow transients were effective in detaching filtered microspheres from fracture surfaces.

In all of the tests, the microsphere responses were interpreted in the same manner as the colloid responses in the fractured core experiments in Chapter 8. It is of interest to plot the filtration rate constants deduced from the field microsphere responses as a function of the mean solute 
residence time in the tracer tests (Figure 10.1). The filtration rate constants reflect the fraction of microspheres that were not filtered during the tests (i.e., the rate constant is

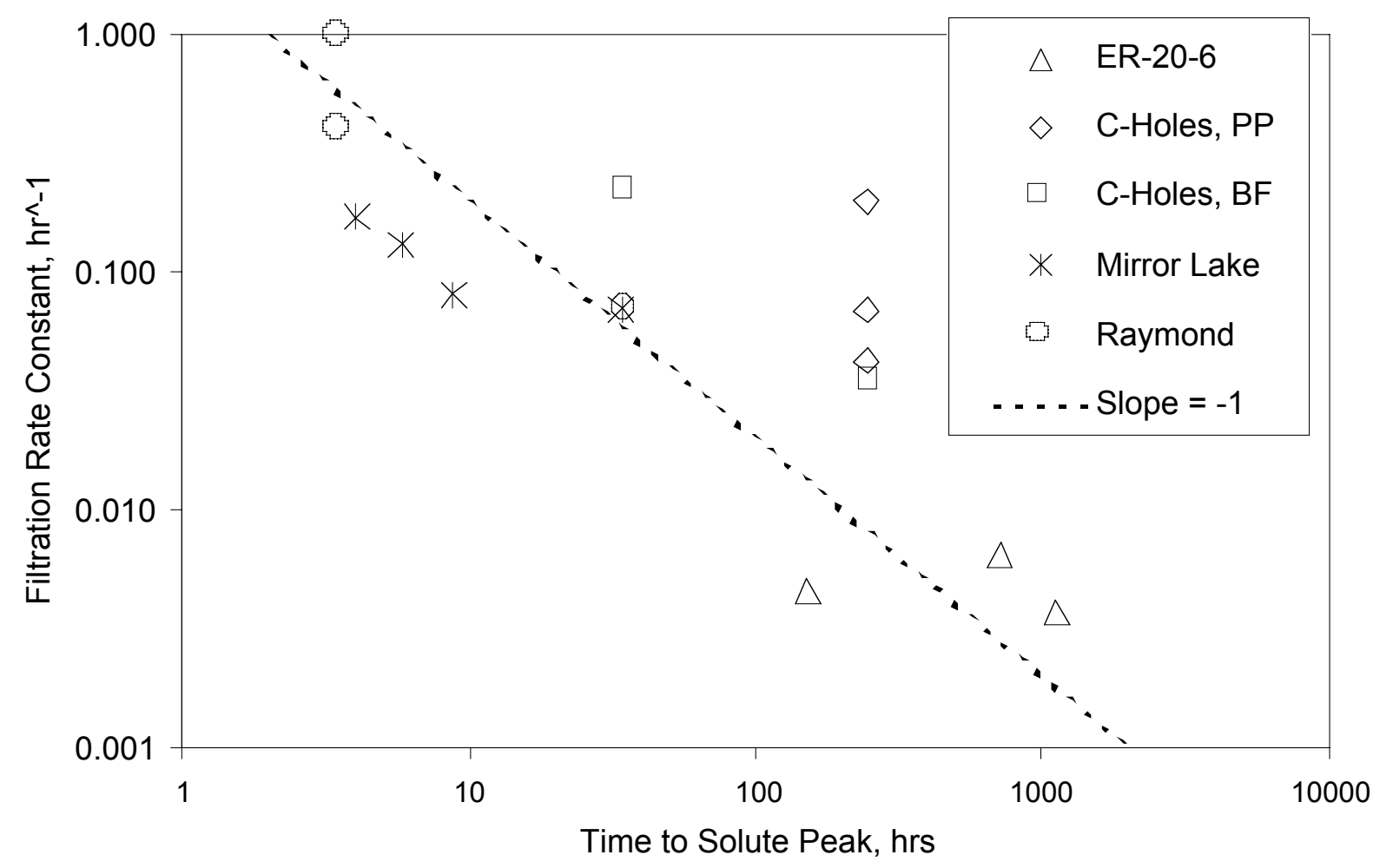

Figure 10.1. Polystyrene microsphere filtration rate constants as a function of time to solute peak concentration in several field experiments in saturated, fractured media. "ER-20-6" refers to the BULLION forced-gradient experiment (Reimus and Haga 1999). "C-holes BF" and "Choles PP" refer to tests in the Bullfrog Tuff and Prow Pass Tuff (both members of the Crater Flat Group), respectively, at the UE25c wells at NTS (Reimus et al. 1999). "Mirror Lake" and "Raymond" refer to tests conducted in fractured granites at Mirror Lake, NH (Reimus and Becker 1999) and Raymond, CA (Becker 1996), respectively. It is important to emphasize that different microspheres were used in the different tests, and also the groundwater chemistry varied from site to site or test to test.

constrained primarily by the magnitude of the early arrival of microspheres). Figure 10.1 shows that, even though different sizes of microspheres were used in the different tests, there is an apparent trend of decreasing filtration rate constant with residence time. The filtration rate constants in the fractured core experiments deduced from the RELAP fits (not shown) follow this trend quite well, although they are clustered at the low residence time end of the plot. The line drawn through the data (not a fit) has a slope of -1 , which implies an inverse time dependence of the filtration rate constant. Schijven et al. (1999) observed a similar decrease in filtration rate constants as a function of distance (proportional to time in their system) for bacteriophage transport in a sandy alluvial aquifer. 
The trend shown in Figure 10.1 suggests that some fraction of microspheres may always transport through a fracture flow system regardless of the time or length scale of the observations. It is tempting to draw the conclusion that filtration rate constants will continually decrease with increasing time scales. However, it may be more appropriate to consider the possibility that, while the majority of microspheres might be filtered quite quickly, there is a small fraction of spheres that are resistant to filtration and therefore capable of traveling large distances over long time periods. This statement implies that there may be a distribution of microsphere filtration rate constants rather than a fixed rate constant that applies to all microspheres. The appearance of a small fraction of microspheres at about the same time as nonsorbing solutes in tracer tests, regardless of the overall time scale of the test, forces filtration rate constants to decrease with time when single rate constants are assumed to apply to all microspheres.

If the concept of a statistical distribution of filtration rates and the possibility of a small fraction of microspheres having very small filtration rates is extended to natural colloids, the implication is that a small fraction of colloids could possibly travel over significant distances in fractured ground water systems. Even if one stays with the interpretation that there is a strong scale dependence of filtration rate constants, the implication is the same. Thus, the microsphere field test results are consistent with, and in fact, could be considered to support, the observations of small amounts of Pu moving significant distances in the subsurface at NTS while very strongly sorbed onto colloids.

\subsection{The Case for Polystyrene Microspheres as Conservative Colloid Tracers in Fractured Media}

To address the suitability of using CML microspheres as surrogates for natural inorganic colloids, a limited number of laboratory experiments were conducted in which the transport behavior of CML microspheres was compared with that of silica colloids in saturated volcanic rock fractures. All tests were conducted using the same CML microspheres (330-nm diameter spheres from IDC dyed with a fluorescent yellow-green dye) that were used in the BULLION forced-gradient experiment. The silica colloids were 100-nm diameter spheres from Nissan Chemical. Table 10.1 provides further information on the two colloid tracers. Silica spheres were used for the comparison studies because the fractured core tests described in Chapter 7 indicated that silica colloids transported with less attenuation than montmorillonite colloids and with about the same attenuation as clinoptilolite colloids. Thus, if CML microspheres were shown to transport conservatively (with minimal attenuation) relative to silica spheres, they would also be expected to transport conservatively relative to clinoptilolite colloids and even more conservatively relative to montmorillonite or smectite colloids.

The 330-nm CML microspheres were selected to be representative of microspheres with diameters ranging from about 250 to $500 \mathrm{~nm}$, which represents a practical size range that can be used in field tests (detection limited at the small end, and cost limited at the large end). Microspheres at the upper end of this size range will settle about twice as fast and diffuse about one-third slower than 330-nm diameter spheres, and microspheres at the lower end of this range will settle about half as fast and diffuse about one-fourth faster than 330-nm diameter spheres. However, the entire CML microsphere size range from 250 to $500 \mathrm{~nm}$ (diameter) will both settle slower and diffuse slower than 100-nm silica colloids. These characteristics (slower settling and 
Table 10.1. Properties of CML Microspheres and Silica Spheres Used in Experiments

\begin{tabular}{|c|c|c|}
\hline Property & $\begin{array}{c}\text { CML } \\
\text { Microspheres }\end{array}$ & Silica Spheres \\
\hline Particle Diameter (nm) & $330 \pm 11$ & 100 \\
\hline$\%$ Solids $(\mathrm{g} / 100 \mathrm{~g})^{(1)}$ & $2 \pm 0.1$ & 40.7 \\
\hline Stock Conc. (number/mL) ${ }^{(1)}$ & $1 \times 10^{12}$ & $3.8 \times 10^{14}$ \\
\hline Density $\left(\mathrm{g} / \mathrm{cm}^{3}\right)$ & 1.055 & 2.65 \\
\hline Dye Excitation/Emission Wavelengths (nm) & $505 / 515$ & No Dye \\
\hline Diffusion Coefficient $\left(\mathrm{cm}^{2} / \mathrm{s}\right)^{(2)}$ & $1.34 \times 10^{-8}$ & $4.43 \times 10^{-8}$ \\
\hline Specific Surface Area $\left(\mathrm{cm}^{2} / \mathrm{g}\right)$ & $1.7 \times 10^{5}$ & $2.3 \times 10^{5}$ \\
\hline Surface Charge (meq/g) & 0.08 & $\mathrm{NM}^{(3)}$ \\
\hline Zeta Potential in U-20WW Water $(\mathrm{mV})$ & $-42.7 \pm 9.1$ & $-41.2 \pm 4.1$ \\
\hline $\begin{array}{l}\text { Zeta Potential in NC-EWDP-19D1 Water } \\
\qquad(\mathrm{mV})\end{array}$ & NM & $-45.15 \pm 2.9$ \\
\hline \multicolumn{3}{|c|}{$\begin{array}{l}\text { (1) Manufacturer's stock solution in deionized water; solutions used in experiments were } \\
\text { diluted in ground water to several orders of magnitude below these concentrations. } \\
\text { (2) } \text { Calculated using the Stokes-Einstein equation, } \mathrm{D}=k \mathrm{~T} /(6 \pi \mu \mathrm{R}) \text {, where } k=\text { Boltzmann's } \\
\text { constant }\left(1.38 \mathrm{X} 10^{-16} \text { ergs } / \mathrm{K}\right), \mathrm{T}=\text { temperature }\left({ }^{\circ} \mathrm{K}\right), \mu=\text { fluid viscosity }(\mathrm{g} / \mathrm{cm}-\mathrm{s}) \text {, and } \\
\mathrm{R}=\text { colloid radius }(\mathrm{cm}) \text {. } \\
\text { (3) } \mathrm{NM}=\text { not measured. }\end{array}$} \\
\hline
\end{tabular}

diffusion) are desirable from the standpoint of reducing the number of colloid collisions with aquifer surfaces. Thus, if electrochemical interactions between colloids and aquifer surfaces are similar for both colloids, the microspheres would be expected transport conservatively relative to the silica.

Testing in fractured volcanic rock was conducted in two different fractured cores from Pahute Mesa at the NTS, with the majority of the testing being done in a fractured lava core. Tables 10.2 through 10.4 provide information on the fractured cores. The two cores differed significantly in their matrix porosities, with the lava core having a very low porosity and the tuff core having a relatively high one. Testing in the lava core was conducted at several different flow rates/residence times. Anghel (2001) provides details of the test procedures and test results associated with the experiments, so they are only summarized here. Only one experiment was conducted in the higher porosity core. Test details for each experiment are provided in Table 10.5. Natural WW-20 water was used in all the experiments (Table 7.1).

Besides their differences in surface characteristics, the silica colloids should have had greater diffusivity than the CML microspheres by a factor of about 3.3 because of their smaller size, and they also should have settled about 2.75 times faster than the CML microspheres because of their greater density. In addition to the colloid tracers, each experiment also included iodide ion as a conservative (nonsorbing) solute tracer. The responses of the colloid tracers could thus be compared to that of a conservative solute to allow better quantification of any colloid retardation 
Table 10.2. Properties of the Fractured Cores Used in the Colloid Transport Experiments: Physical Characteristics

\begin{tabular}{cccc}
\hline Fracture & $\begin{array}{c}\text { Fracture Length } \\
(\mathbf{c m})\end{array}$ & $\begin{array}{c}\text { Fracture Width } \\
(\mathbf{c m})\end{array}$ & Matrix Porosity \\
\hline PM-2, 7032 ft BLS* & 14 & 9 & 0.014 \\
\hline ER-20-6\#1, 2406 ft BLS* & 23.8 & 12.7 & 0.369 \\
\hline
\end{tabular}

* BLS $=$ below land surface.

Table 10.3. Properties of the Fractured Cores Used in the Colloid Transport Experiments: X-ray Diffraction Results (wt \%)

\begin{tabular}{|c|c|c|c|c|c|c|c|c|c|c|c|c|}
\hline Fracture & $\begin{array}{l}\text { Smec- } \\
\text { tite }\end{array}$ & $\begin{array}{l}\text { Zeo- } \\
\text { lite }\end{array}$ & Opal & Quartz & $\begin{array}{c}\text { K- } \\
\text { Spar }\end{array}$ & $\begin{array}{l}\text { Plagio- } \\
\text { clase }\end{array}$ & Hematite & Biotite & Calcite & Chlorite & $\begin{array}{c}\text { Epidote/ } \\
\text { Clinozoisite }\end{array}$ & Total \\
\hline $\begin{array}{l}\text { PM-2 } \\
7032 \mathrm{ft}\end{array}$ & - & - & - & 22.1 & 18.8 & 38.3 & - & 2.0 & 1.2 & 6.7 & 9.6 & 98.7 \\
\hline $\begin{array}{c}\text { ER-20-6\#1 } \\
2406 \mathrm{ft}\end{array}$ & 1.7 & 83.4 & 27.3 & 0.9 & 4.8 & 3.3 & - & 1.5 & - & - & - & 102.7 \\
\hline
\end{tabular}

NOTE: $\quad-=$ not detected

Table 10.4. Properties of the Fractured Cores Used in the Colloid Transport Experiments: Lithologic Descriptions

\begin{tabular}{cl}
\hline Fracture & \multicolumn{1}{c}{ Description } \\
\hline PM-27032 ft & $\begin{array}{l}\text { Devitrified intermediate composition lava from the } \\
\text { andesite of Mt. Helen of the Volcanics of Quartz } \\
\text { Mountain. }\end{array}$ \\
\hline ER-20-6\#1 & $\begin{array}{l}\text { Highly zeolitic, poor to moderately-welded vitric, lithic } \\
\text { tuff from the Calico Hills Formation. }\end{array}$ \\
\hline
\end{tabular}

or early arrival. All tracers were injected simultaneously so that they would have exactly the same injection input function. The iodide was analyzed by ion-selective electrode, the silica spheres by high-sensitivity liquid in-situ particle spectrometery (HSLIS-S50, Particle Measuring Systems, Inc.), and the fluorescent microspheres by fluorometry.

Figures 10.2 through 10.4 show the responses of each of the tracers in the three experiments conducted at the lower flow rates in the fractured lava core. It is clear that at the highest of the flow rates, the CML microspheres and the silica spheres transported almost identically, with some minor attenuation of each colloid. Both colloid tracers became more attenuated at the lower flow rate, with the CML microspheres being consistently less attenuated than the silica spheres. 
Table 10.5. Summary of Experimental Conditions in the Fractured Core Tests

\section{ER-20-6\#1, 2406 ft BLS}

\begin{tabular}{ll}
\hline Flow Rate $(\mathrm{mL} / \mathrm{hr})$ & 4.96 \\
\hline Fracture Orientation & Vertical \\
\hline Injection Duration $(\mathrm{hr})$ & 10.0 \\
\hline & $127 \mathrm{mg} / \mathrm{L}\left(\mathrm{I}^{-}\right)$ \\
& $4.22 \times 10^{7} \mathrm{particles} / \mathrm{mL}(0.33-\mu \mathrm{m}$ yellow-green microspheres) \\
Injection Concentration $^{(1)}$ & $5.2 \times 10^{10} \mathrm{particles} / \mathrm{ml}(0.1-\mu \mathrm{m}$ silica $)$ \\
\hline & $0.85\left(\mathrm{I}^{\mathrm{I}}\right)$ \\
& 0.63 (yellow-green microspheres) \\
& 0.72 (silica) \\
\hline
\end{tabular}

PM-2, 7032 ft BLS

\begin{tabular}{|c|c|c|}
\hline Experiment Number & 1 & 2 \\
\hline Flow Rate $(\mathrm{mL} / \mathrm{hr})$ & 10.02 & 2.5 \\
\hline Fracture Orientation & Vertical & Vertical \\
\hline Injection Duration (hr) & 3.3 & 13.2 \\
\hline Injection Concentration $^{(1)}$ & $\begin{array}{l}127 \mathrm{mg} / \mathrm{L}\left(\mathrm{I}^{-}\right) \\
1.6 \times 10^{7} \text { particles } / \mathrm{mL} \\
(0.33-\mu \mathrm{m} \text { yellow-green microspheres }) \\
6.9 \times 10^{10} \text { particles } / \mathrm{mL}(0.1-\mu \mathrm{m} \text { silica })\end{array}$ & $\begin{array}{l}127 \mathrm{mg} / \mathrm{L}\left(\mathrm{I}^{-}\right) \\
1.9 \times 10^{7} \text { particles } / \mathrm{mL} \\
(0.33-\mu \mathrm{m} \text { yellow-green microspheres }) \\
7.9 \times 10^{10} \text { particles } / \mathrm{mL}(0.1-\mu \mathrm{m} \text { silica })\end{array}$ \\
\hline Tracer Recoveries (Fractional) & $\begin{array}{l}1.09\left(\mathrm{I}^{-}\right) \\
1.09 \text { (yellow-green microspheres) } \\
1.06 \text { (silica) }\end{array}$ & $\begin{array}{l}1.06\left(\mathrm{I}^{-}\right) \\
0.89 \text { (yellow-green microspheres) } \\
0.88 \text { (silica) }\end{array}$ \\
\hline Experiment Number & 3 & 4 \\
\hline Flow Rate (mL/hr) & 0.58 & 0.57 \\
\hline Fracture Orientation & Vertical & Horizontal \\
\hline Injection Duration (hr) & 56 & 56 \\
\hline Injection Concentration $^{(1)}$ & $\begin{array}{l}127 \mathrm{mg} / \mathrm{L}\left(\mathrm{I}^{-}\right) \\
1.9 \times 10^{7} \mathrm{particles} / \mathrm{mL} \\
(0.33-\mu \mathrm{m} \text { yellow-green microspheres }) \\
7.4 \times 10^{10} \text { particles } / \mathrm{L}(0.1-\mu \mathrm{m} \text { silica })\end{array}$ & $\begin{array}{l}127 \mathrm{mg} / 1\left(\mathrm{I}^{-}\right) \\
1.7 \times 10^{7} \mathrm{particles} / \mathrm{mL} \\
(0.33-\mu \mathrm{m} \text { yellow-green microspheres }) \\
7.5 \times 10^{10} \text { particles } / \mathrm{mL}(0.1-\mu \mathrm{m} \text { silica })\end{array}$ \\
\hline Tracer Recoveries (Fractional) & $\begin{array}{l}0.96\left(\mathrm{I}^{-}\right) \\
0.77 \text { (yellow-green microspheres) } \\
0.60 \text { (Silica) }\end{array}$ & $\begin{array}{l}0.93\left(\mathrm{I}^{-}\right) \\
0.77 \text { (yellow-green microspheres) } \\
0.24 \text { (Silica) }\end{array}$ \\
\hline
\end{tabular}

(1) Calculated concentrations for microspheres are as much as $20-30 \%$ of error. However, the normalized concentrations should be accurate to within 5\%. 


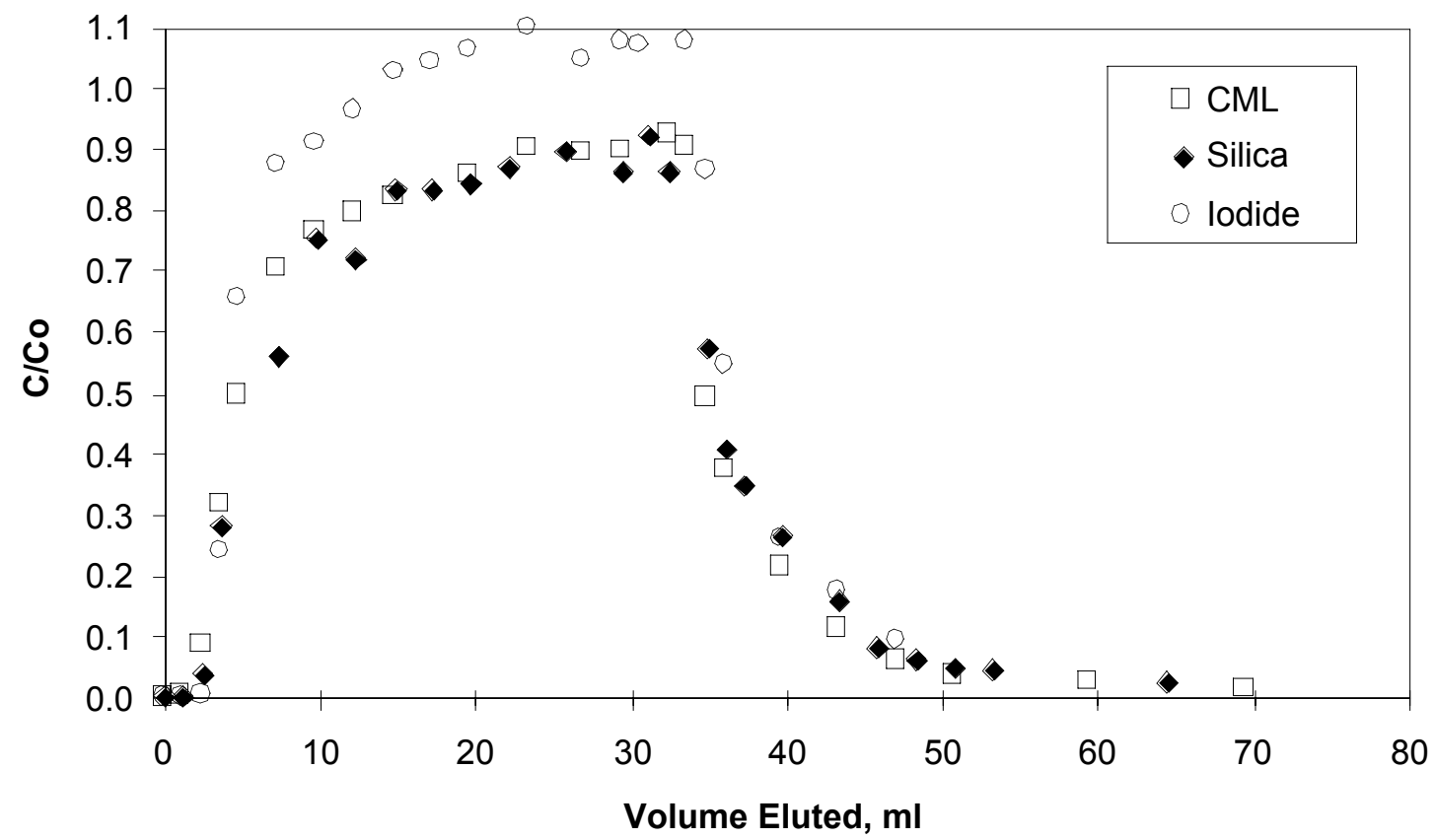

Figure 10.2. Normalized Concentrations of 330-nm Diameter CML Microspheres, 100-nm Diameter Silica Spheres, and Iodide in Fractured Core from PM-2, $7032 \mathrm{ft}$ at an injection flow rate of $2.5 \mathrm{~mL} / \mathrm{hr}$.

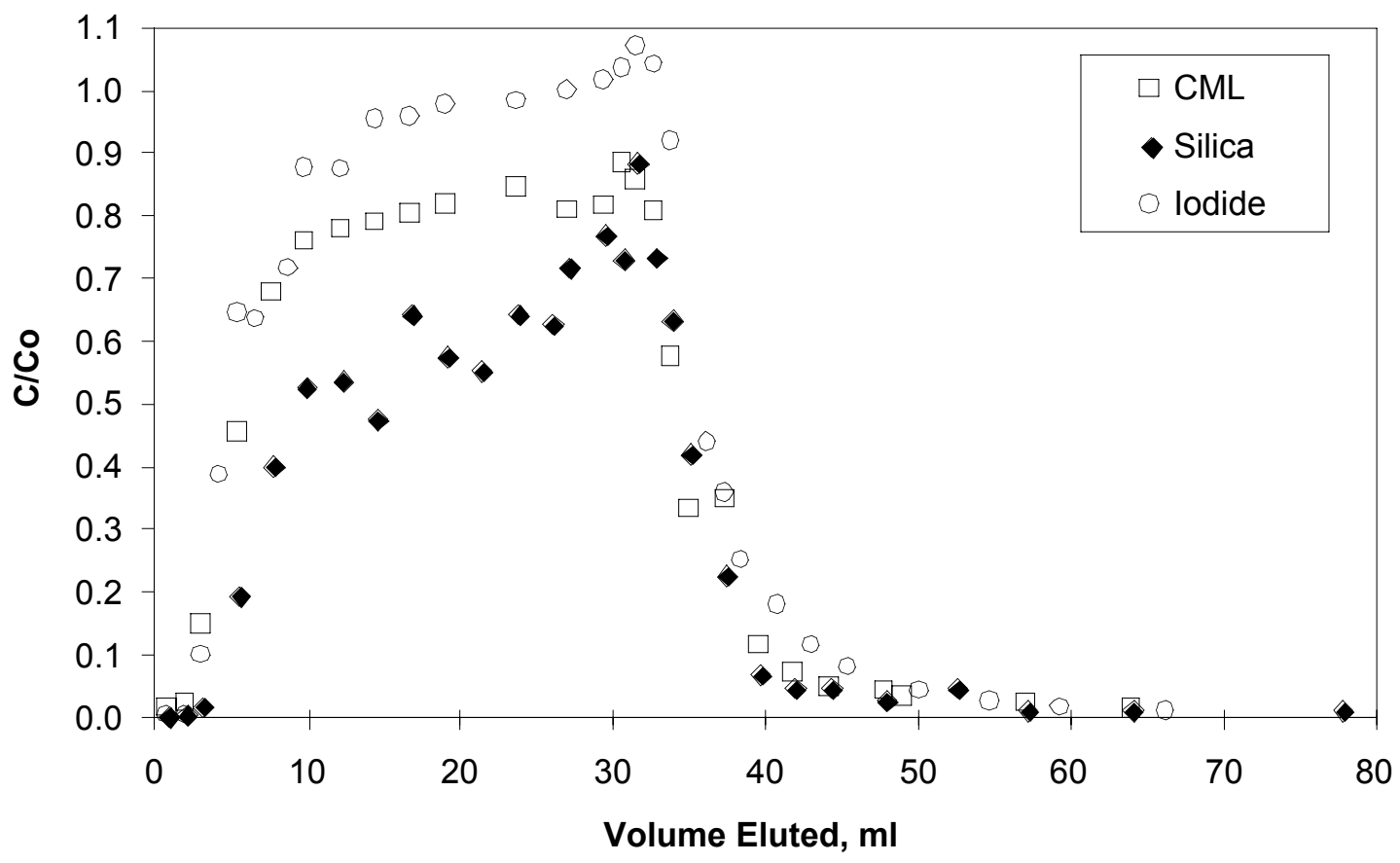

Figure 10.3. Normalized Concentrations of 330-nm Diameter CML Microspheres, 100-nm Diameter Silica Spheres, and Iodide in Fractured Core from PM-2, $7032 \mathrm{ft}$ in Vertical Core Orientation at an injection flow rate of $0.58 \mathrm{~mL} / \mathrm{hr}$. 


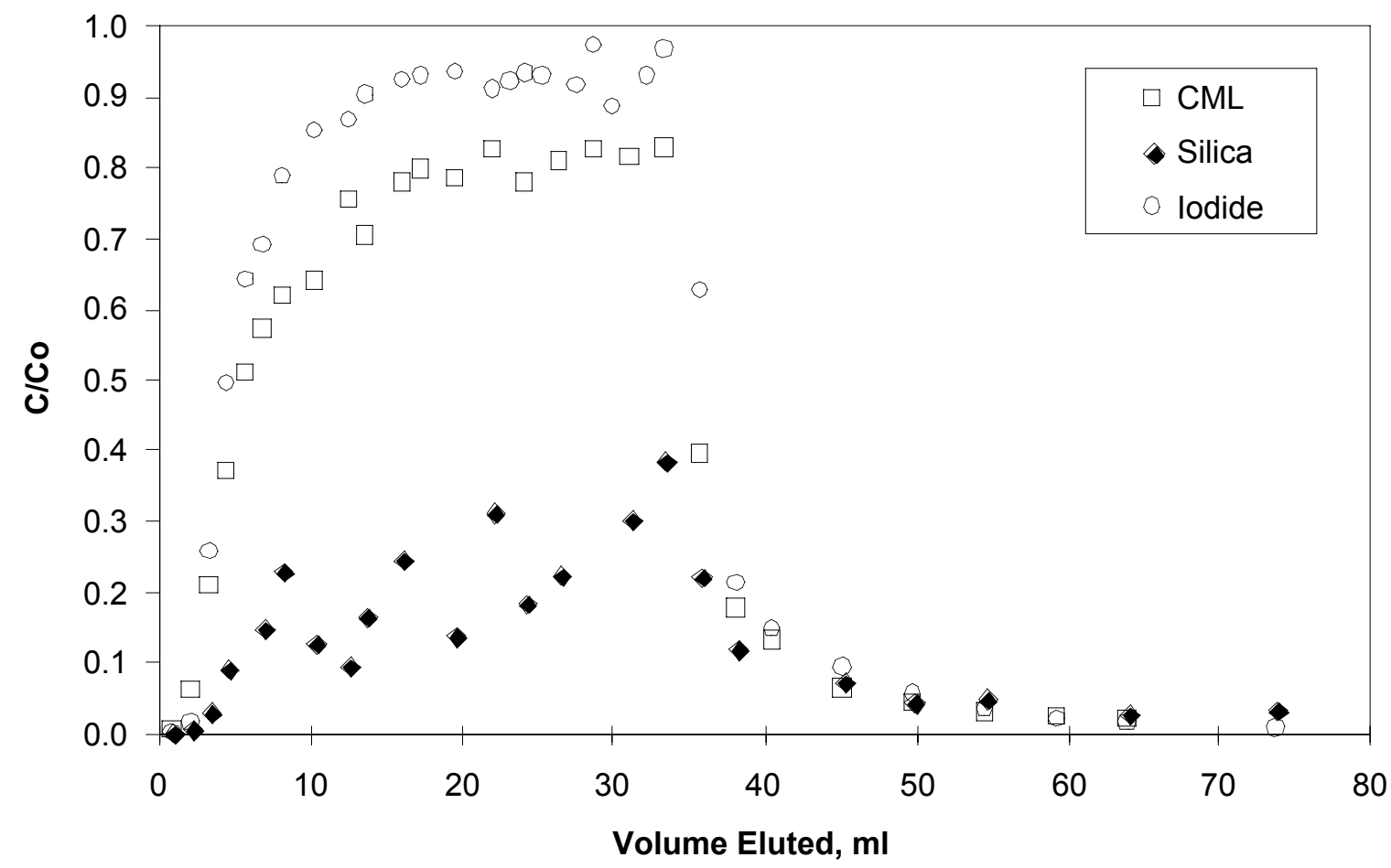

Figure 10.4. Normalized Concentrations of 330-nm Diameter CML Microspheres, 100-nm Diameter Silica Spheres, and Iodide in Fractured Core from PM-2, $7032 \mathrm{ft}$ in Horizontal Core Orientation at an injection flow rate of $0.57 \mathrm{~mL} / \mathrm{hr}$.

Figures 10.3 and 10.4 also show that the silica spheres were more attenuated in the horizontal orientation of the lava fracture than in the vertical orientation at the same flow rate. In this case, a horizontal orientation means that the fractured core was oriented such that the fracture surfaces were parallel to the lab benchtop, whereas a vertical orientation means that the core was stood up on one of its ends with flow from bottom to top.

Quantitative interpretation of the tests in the lava fracture were carried out by Anghel (2001) using the RELAP computer model. The interpretive method was essentially the same as described in Chapter 8 for the colloid portion of the $\mathrm{Pu}$-colloid fractured core experiments. The resulting fitted parameter values are listed in Table 10.6. The single test in the higher-porosity fracture was only qualitatively interpreted because a single test at a constant flow rate does not allow unambiguous estimation of solute or colloid transport parameters. However, it was clear that there was no significant difference in the transport of the two colloid tracers in this fracture. Also, the iodide appeared to experience a significant amount of matrix diffusion in this fracture based on its attenuated peak normalized concentration relative to the colloids and its extended tailing behavior. Colloid recoveries in this fracture suggested a colloid-filtration-rate constant of $\sim 0.2 \mathrm{hr}^{-1}$.

Before drawing conclusions from the results of the fracture transport experiments, it is useful to define two characteristic length quantities: 
Table 10.6. Model Parameters from RELAP Fits of Tracer Breakththrough Curves in Experiments in the Fractured Core from Borehole PM-2

\begin{tabular}{|c|c|c|c|c|}
\hline \multirow{2}{*}{ Model Parameter } & \multicolumn{4}{|c|}{ Flow Rate } \\
\hline & $10 \mathrm{~mL} / \mathrm{hr}$ & $2.5 \mathrm{~mL} / \mathrm{hr}$ & $0.6 \mathrm{~mL} / \mathrm{hV}$ & $0.6 \mathrm{~mL} / \mathrm{hH}$ \\
\hline Mean Fluid Residence Time (hr) & 0.60 & 2.43 & 10.43 & 10.27 \\
\hline Half Aperture (cm) & 0.024 & 0.024 & 0.024 & 0.024 \\
\hline Iodide Peclet Number & 14 & 9.5 & 9.5 & 9.5 \\
\hline Iodide Dispersivity (cm) & 1.0 & 1.5 & 1.5 & 1.5 \\
\hline Colloid Peclet Number & 2.88 & 2.88 & 2.88 & 2.88 \\
\hline Colloid Dispersivity (cm) & 4.86 & 4.86 & 4.86 & 4.86 \\
\hline Iodide Matrix Diffusion Coef. $\left(\mathrm{cm}^{2} / \mathrm{s} \mathrm{x} 10^{7}\right)$ & 5.9 & 14.4 & 14.4 & 14.4 \\
\hline Microsphere Filtration Rate Constant $\left(\mathrm{hr}^{-1}\right)$ & 0.080 & 0.065 & 0.021 & 0.026 \\
\hline Silica Filtration Rate Constant $\left(\mathrm{hr}^{-1}\right)$ & 0.090 & 0.085 & 0.051 & 0.201 \\
\hline
\end{tabular}

NOTE: $\mathrm{V}=$ vertical orientation; $\mathrm{H}=$ horizontal orientation.

$$
\begin{gathered}
L_{\mathrm{D}}=\sqrt{2 D \tau} \\
L_{\mathrm{S}}=\left(\frac{1}{18 \mu}\right)\left(\rho_{\mathrm{P}}-\rho_{\mathrm{f}}\right) g d_{\mathrm{p}}^{2} \tau=V_{\mathrm{S}} \tau
\end{gathered}
$$

where, $L_{\mathrm{D}}=$ characteristic diffusion distance during time, $\mathrm{cm}$

$L_{\mathrm{S}}=$ characteristic gravitational settling distance during time _, $\mathrm{cm}$

$D=$ colloid diffusion coefficient, $\mathrm{cm}^{2} / \mathrm{s}$

_ $=$ residence time in fracture, $\mathrm{s}$

$V_{\mathrm{S}}=$ settling velocity, $\mathrm{cm} / \mathrm{s}$

$\mu=$ fluid viscosity, $\mathrm{g} / \mathrm{cm}-\mathrm{sec}$

$\_\mathrm{p}=$ particle density, $\mathrm{g} / \mathrm{cm}^{3}$

$\__{\mathrm{f}}=$ fluid density, $\mathrm{g} / \mathrm{cm}^{3}$

$g=$ gravitational acceleration, $\mathrm{cm} / \mathrm{s}^{2}$

$d_{\mathrm{p}}=$ particle diameter, $\mathrm{cm}$.

The characteristic lengths $L_{\mathrm{D}}$ and $L_{\mathrm{S}}$ differed for the two colloid tracers in each of the fracture transport tests in the lava fracture (Table 10.7). 
Table 10.7. Characteristic Diffusion and Settling Lengths $\left(L_{\mathrm{D}}\right.$ and $\left.L_{\mathrm{S}}\right)$ for the CML Microspheres and Silica Spheres in the Four Experiments in the Fractured Core from Borehole PM-2.

\begin{tabular}{|c|c|c|c|c|}
\hline \multirow{2}{*}{ Characteristic Length } & \multicolumn{4}{|c|}{ Flow Rate } \\
\hline & $10 \mathrm{~mL} / \mathrm{h}$ & $2.5 \mathrm{~mL} / \mathrm{h}$ & $0.6 \mathrm{~mL} / \mathrm{hV}$ & $0.6 \mathrm{~mL} / \mathrm{hH}$ \\
\hline CML Diffusion Length, $L_{\mathrm{D}}(\mathrm{cm})$ & 0.008 & 0.015 & 0.032 & 0.032 \\
\hline Silica Diffusion Length, $L_{\mathrm{D}}(\mathrm{cm})$ & 0.014 & 0.028 & 0.058 & 0.057 \\
\hline CML Settling Length, $L_{\mathrm{S}}(\mathrm{cm})$ & 0.0007 & 0.0028 & 0.012 & 0.012 \\
\hline Silica Settling Length, $L_{\mathrm{S}}(\mathrm{cm})$ & 0.0019 & 0.0078 & 0.034 & 0.033 \\
\hline
\end{tabular}

NOTE: The average fracture aperture $(2 b)$ was estimated to be $0.048 \mathrm{~cm}$. $\mathrm{V}=$ vertical orientation; $\mathrm{H}=$ horizontal orientation.

With these quantities defined, the following conclusions from the experiments can be stated.

- When the fractures were in the vertical orientation, similar colloid responses were obtained at the same flow rate/residence time provided that $L_{\mathrm{D}} / 2 b<1$ for both colloids, where $2 b=$ average fracture aperture $\left(\mathrm{b}=\right.$ half aperture). At flow rates where $L_{\mathrm{D}} / 2 b>1$ for the silica and $L_{\mathrm{D}} / 2 b<1$ for the CML microspheres, the latter transported with much less attenuation than the former.

- The fracture orientation influenced only the silica colloid transport, which is consistent with the greater silica settling velocity and the fact that $L_{\mathrm{D}} / 2 b \approx 1$ for the silica spheres and $L_{\mathrm{D}} / 2 b$ $<1$ for the CML microspheres. A significant fraction of the silica spheres were apparently able to settle to the lower surface of the horizontally-oriented fracture during their residence time in the flow system, whereas most of the CML microspheres did not settle during this time.

- The "sticking efficiencies" (fractions of colloid-wall collisions that resulted in attachment) of the two colloids appeared to be quite similar. This conclusion is based on comparing the experimental results to colloid deposition theory in a parallel-plate channel in the absence of gravitational effects, which predicts that the filtration rate constant, $k_{\mathrm{f}}$, should be proportional to $\left(\tau d_{\mathrm{p}}^{2}\right)^{-1 / 3}$ if all colloid-wall collisions result in irreversible attachment (Van de Ven 1989). The quantity $d_{\mathrm{p}}^{2}$ for the CML microspheres was 10.9 times greater than that for the silica spheres, so the two colloids would be predicted to have the same $k_{\mathrm{f}}$ values when the residence time _ of the CML spheres is 10.9 times less than that for the silica. In fact, when the residence time was approximately 9 times less for the CML spheres, the filtration rates constants were 0.06 and $0.051 \mathrm{hr}^{-1}$ for the CML and silica spheres, respectively. These rate constants are in relatively good agreement with each other (within experimental error), which suggests that the "sticking efficiencies" of the two colloids were similar. Note that the CML rate constant of $0.06 \mathrm{hr}^{-1}$ was obtained in an experiment conducted at a flow rate of approximately $5 \mathrm{ml} / \mathrm{hr}$ in which only CML microspheres and iodide were used as tracers. This experiment is not presented or discussed here because it did not involve a direct comparison of CML microspheres and silica (all other experiments involved simultaneous injection of both colloid tracers). 
- The above three conclusions suggest that CML microspheres on the order of 250 to $500 \mathrm{~nm}$ in diameter should transport either very similarly to or conservatively relative to natural inorganic groundwater colloids in saturated fractured systems.

\subsection{Summary of Microscopic Attachment and Detachment Studies of Colloids}

Microscopic colloid attachment and detachment visualization experiments were conducted in parallel-plate flow channels for the UGTA program in 1998 and 1999 to study and parameterize colloid attachment and detachment processes onto "smooth" glass surfaces under flowing conditions. The experiments were conducted using the same 330-nm diameter CML polystyrene microspheres that were used in the BULLION forced-gradient experiment. 310-nm diameter silica spheres were also used in the experiments. Colloid attachment and detachment was studied at three different ionic strengths: $0.003 \mathrm{M}$ (synthetic WW20 water), $0.025 \mathrm{M}$, and 0.05 $\mathrm{M}$ (synthetic WW20 water spiked with $\mathrm{NaCl}$ ). For each ionic strength, experiments were conducted at flow rates of $0,0.04,0.06,0.08$, and $0.16 \mathrm{ml} / \mathrm{hr}$ through the flow channels, which corresponded to average flow velocities of $0,0.00069,0.001,0.0014$, and $0.0028 \mathrm{~cm} / \mathrm{sec}$. In each experiment, colloid attachment and detachment events occurring within a small rectangular area on the upper or lower surface of the flow channel were recorded using a microscope system equipped with dark-field illumination that was interfaced to a computer for data acquisition and image capture/processing. The cumulative number of attachment and detachment events and the residence times of individual attached colloids at the flow channel surfaces were among the reduced data that the image processing software was programmed to obtain from the raw images collected. Experiments were conducted for as long as four days.

Details of the experimental methods, the results, and interpretations of the experiments are contained in a final report prepared for the UGTA program in April 2000 (Abdel-Fattah and Reimus, 2000). Additional details and refinements of test interpretations are provided by AbdelFattah (2001). Because the majority of this work has been previously reported to the UGTA program, we summarize only the main conclusions here:

1. Due to flow channel surface heterogeneity and possibly the non-uniformity of colloid surface properties, different sites existed at the surface to which colloids attached with varying degrees of reversibility. Many colloids appeared to be irreversibly attached for the duration of the experiments.

2. The experimental results suggested that there was initially a finite number of sites available for irreversible attachment of colloids on the surfaces. The number of these sites depended on both the ionic strength and flow rate. However, over time, it appeared that sites for irreversible attachment were continuously generated at the surfaces at some relatively low rate that depended on the ionic strength and flow rate. It is possible that such sites could be continuously generated over time in natural systems due to weathering and other geochemical processes.

3. Gravitational effects played a dominant role in the attachment and detachment kinetics of silica colloids, with a net decrease in colloids attached to the upper surface of the flow channel over time but a steady accumulation of colloids at the lower surface of the flow channel. 
4. Both the polystyrene and silica colloids exhibited attachment residence time distributions at the flow channel surface that implied a non-linear rate expression to describe colloid detachment kinetics. These results are in agreement with the results of Luthi and Ricka (1998). The following equation was found to quite accurately describe the attachment residence time distribution of the polystyrene microspheres at all flow rates and ionic strengths (although there was negligible attachment of microspheres at the lowest ionic strength).

$$
\mathrm{P}(\text { res.time } \geq \Delta \tau)=\exp \left(-\left(\frac{\Delta \tau}{\tau_{\text {Det }}}\right)^{1 / 3}\right)
$$

Where, $\mathrm{P}($ res.time $\geq \Delta \tau)=$ probability that a microsphere will remain immobilized at the surface for greater than $\Delta \tau$ time units after it becomes attached. $\tau_{\text {Det }}=$ characteristic time of colloid attachment at the channel surface.

Equation (10.3) implies that while a vast majority of colloids that become attached (reversibly) to surfaces will detach in a relatively short time, a small fraction of them will remain attached for very long times before becoming detached. This result is consistent with the long microsphere tails observed in field tracer tests (mentioned in Section 10.1), as microspheres that remain attached for long times before detaching will arrive late in the tails of tracer response curves.

5. Polystyrene microsphere attachment and detachment rates increased approximately linearly with flow rate at a given ionic strength. This result suggest that flow imparts energy to the colloids allowing them to get over energy barriers more easily both while attaching and detaching from surfaces. The average residence time of the polystyrene microspheres at the surface increased with increasing ionic strength.

6. The polystyrene microsphere attachment rates and the number of sites available for attachment at the surfaces increased significantly as the ionic strength increased, which is in agreement with colloid stability theory.

These conclusions suggest that both colloids and colloid attachment sites on surfaces have distributions of properties (rather than discrete properties), which may result in non-linear macroscopic transport behavior that is not readily described by standard first-order rate expressions in transport models. The fact that these conclusions were reached while studying attachment and detachment of relatively homogeneous polystyrene and silica microspheres onto relatively homogeneous (both chemically and physically) glass surfaces suggests that natural systems could have much wider distributions of properties of both colloids and potential colloid attachment sites. 


\subsection{References}

Abdel-Fattah, A. I. and P. W. Reimus (2000). Colloid Attachment and Detachment Kinetics from Direct Visualization Experiments, UGTA Report, Los Alamos National Laboratory, Los Alamos, NM.

Abdel-Fattah, A. I. (2001). Colloid Deposition onto a Solid Surface Under Low-Flow Conditions in Parallel-Plate Geometry, Ph.D. Dissertation, University of New Mexico.

Anghel, I. (2001). Comparison of Polystyrene and Silica Colloids Transport in Saturated Rock Fractures, M.S. Thesis, University of New Mexico.

Becker, M. W. (1996). Tracer Tests in a Fractured Rock and Their First Passage Time Mathematical Models, Ph.D. Dissertation, University of Texas at Austin.

Luthi, Y. and J. Ricka (1998). Colloidal Particles at the Water-Glass Interface: Analyzing Videomicroscopic Data, J. Colloid Interface Sci., 206, 302-309.

Reimus, P. W. and M. W. Becker (1999). Transport and Attenuation of Carboxylate-Modified Latex Microspheres in Fractured Rock Field Tracer Tests, Proc. Of $127^{\text {th }}$ American Chemical Society Meeting, Anaheim, CA, March 21-25, 1999.

Reimus, P. W. and M. J. Haga (1999). Analysis of Tracer Responses in the BULLION ForcedGradient Experiment, $L A-13615-M S$, Los Alamos National Laboratory Manuscript Series Report, Los Alamos, NM.

Reimus, P. W., A. Adams, M. J. Haga, A. Humphrey, T. Callahan, I. Anghel, and D. Counce (with contributions from USGS staff). 1999. "Results and Interpretation of Hydraulic and Tracer Testing in the Prow Pass Tuff at the C-Holes," Yucca Mountain Site Characterization Project Milestone Report SP32E7M4, Los Alamos National Laboratory, Los Alamos, NM.

Schijven, J. F., W. Hoogenboezem, S. M. Hassanizadeh, and J. H. Peters (1999). Modeling Removal of Bacteriophages MS2 and PRD1 by Dune Recharge at Castricum, Netherlands, Water Resour. Res., 35(4), 1101-1111.

Van de Ven, T. G. M. (1989). Colloidal Hydrodynamics, Academic Press, New York, NY. 


\title{
-Chapter 11- \\ Implications of Experimental Results and Interpretations and Previous Studies for CAU-Scale Colloid-Facilitated Radionuclide Transport
}

\author{
Paul W. Reimus
}

The colloid-facilitated transport model described in Chapter 8 can be used as a tool for assessing the implications of our current understanding of colloid-facilitated transport to the larger time and length scales that are applicable to the CAU modeling strategy of the UGTA program. However, before this is attempted, the material presented in Section 10.3 suggests that a slight modification should be made to the colloid transport portion of the model (equations 8.6 and 8.7). The microscopic attachment-detachment observations clearly indicated that some of the colloid attachments to fracture surfaces could be irreversible. In this case, equation (8.6) should be modified to include an irreversible filtration reaction, and a new equation must be added to the model to account for the accumulation of irreversibly-attached colloids to the fracture surfaces:

$$
\begin{gathered}
\frac{\partial \mathrm{C}_{\mathrm{col}}}{\partial \mathrm{t}}+\mathrm{v}_{\mathrm{f}} \frac{\partial \mathrm{C}_{\mathrm{col}}}{\partial \mathrm{x}}-\mathrm{D}_{\mathrm{f}} \frac{\partial^{2} \mathrm{C}_{\mathrm{col}}}{\partial \mathrm{x}^{2}}+\mathrm{k}_{\mathrm{fcol}} \mathrm{C}_{\mathrm{col}}-\frac{\mathrm{k}_{\mathrm{rcol}} \mathrm{S}_{\mathrm{col}}}{\mathrm{b}}+\mathrm{k}_{\mathrm{fcirr}} \mathrm{C}_{\mathrm{col}}=0 \\
\frac{\partial \mathrm{S}_{\mathrm{col}}}{\partial \mathrm{t}}-\mathrm{b} \mathrm{k}_{\mathrm{fcol}} \mathrm{C}_{\mathrm{col}}+\mathrm{k}_{\mathrm{rcol}} \mathrm{S}_{\mathrm{col}}=0 \\
\frac{\partial \mathrm{S}_{\mathrm{col}, \mathrm{irr}}}{\partial \mathrm{t}}-\mathrm{b} \mathrm{k}_{\mathrm{fcirr}} \mathrm{C}_{\mathrm{col}}=0
\end{gathered}
$$

where, $\mathrm{k}_{\mathrm{fcirr}}=$ irreversible colloid filtration rate constant, $1 / \mathrm{s}$

$\mathrm{S}_{\mathrm{col}, \text { irr }}=$ irrevesibly sorbed colloid concentration on fracture surfaces, $\mathrm{g} / \mathrm{cm}^{2}$

If we assume that most deep natural environmental systems are at a quasi-steady state and that natural colloid concentrations are relatively invariant in time and space, the derivatives of equations (11.1), (8.7), and (11.2) can be set to zero. Setting these derivatives to zero and substituting (8.7) into (11.1), leaves us with the following equation:

$$
\mathrm{k}_{\text {fcirr }} \mathrm{C}_{\text {col }}=0
$$

Equation (11.3) implies that if $\mathrm{k}_{\text {fcirr }}$ is not equal to zero, then $\mathrm{C}_{\mathrm{col}}$ must be zero; that is, there can be no colloids present in the system at steady state because any colloids initially present will eventually become irreversibly filtered. Alternatively, if $\mathrm{k}_{\text {fcirr }}$ is zero, then all colloids present in the system must be the same colloids that were present in the system at time zero. Even if we assume that colloid concentrations can vary in space but not in time (i.e., only the time derivatives in equations (11.1), (8.7), and (11.2) are set to zero), it can be shown that unless $\mathrm{k}_{\text {fcirr }}$ is zero, colloid concentrations will decay to zero at some finite distance from $x=0$. However, we know that colloids are observed in all natural groundwaters, and it also seems very unlikely that $\mathrm{k}_{\mathrm{fcirr}}$ is zero in many natural systems (colloidal material is known to clog fractures). Thus, 
one must either conclude that natural systems are never at true steady state, or if they are, an additional term must be added to equation (11.1) to account for colloid generation:

$$
\frac{\partial \mathrm{C}_{\mathrm{col}}}{\partial \mathrm{t}}+\mathrm{v}_{\mathrm{f}} \frac{\partial \mathrm{C}_{\mathrm{col}}}{\partial \mathrm{x}}-\mathrm{D}_{\mathrm{f}} \frac{\partial^{2} \mathrm{C}_{\mathrm{col}}}{\partial \mathrm{x}^{2}}+\mathrm{k}_{\mathrm{fcol}} \mathrm{C}_{\mathrm{col}}-\frac{\mathrm{k}_{\mathrm{rcol}} \mathrm{S}_{\mathrm{col}}}{\mathrm{b}}+\mathrm{k}_{\mathrm{fcirr}} \mathrm{C}_{\mathrm{col}}-\mathrm{P}=0
$$

where, $\mathrm{P}=$ colloid production rate, $\mathrm{g} / \mathrm{cm}^{3}-\mathrm{s}$

If we again assume steady-state conditions, equation (11.4) yields

$$
\mathrm{k}_{\text {fcirr }} \mathrm{C}_{\text {col }}=\mathrm{P}
$$

which, on rearrangement, yields

$$
\mathrm{C}_{\mathrm{col}}=\frac{\mathrm{P}}{\mathrm{k}_{\text {fcirr }}}
$$

Equation (11.6) implies that measured natural colloid concentrations are a result of the ratio of the colloid production rate to the irreversible filtration rate constant in the system (which may be a local value that depends on flow rates, mineralogy, water chemistry, etc...). We must now add some new terms to the two-site sorption model represented by equations (8.11) through (8.18) to account for mobilization of radionuclides associated with colloid production (including two sorption sites on both the fracture surfaces and the produced colloids), as well as some new equations and terms to account for radionuclide sorption onto irreversibly-attached colloids:

$$
\begin{aligned}
& \frac{\partial \mathrm{C}}{\partial \mathrm{t}}+\mathrm{v}_{\mathrm{f}} \frac{\partial \mathrm{C}}{\partial \mathrm{x}}-\mathrm{D}_{\mathrm{f}} \frac{\partial^{2} \mathrm{C}}{\partial \mathrm{x}^{2}}+\mathrm{k}_{1 \mathrm{f}} \mathrm{CC}_{\mathrm{col}}\left(1-\frac{\mathrm{C}_{1}}{\mathrm{C}_{\mathrm{col}} \mathrm{S}_{1}^{0}}\right)+\mathrm{k}_{2 \mathrm{f}} \mathrm{CC}_{\mathrm{col}}\left(1-\frac{\mathrm{C}_{2}}{\mathrm{C}_{\mathrm{col}} \mathrm{S}_{2}^{0}}\right)+\mathrm{k}_{\mathrm{f}, 1} \mathrm{C}+ \\
& \mathrm{k}_{\mathrm{f}, 2} \mathrm{C}+\mathrm{k}_{1 \mathrm{f}} \mathrm{CS}_{\mathrm{col}}\left(1-\frac{\mathrm{C}_{\text {filt }, 1}}{\mathrm{~S}_{\mathrm{col}} \mathrm{S}_{1}^{0}}\right)+\mathrm{k}_{2 \mathrm{f}} \mathrm{CS}_{\mathrm{col}}\left(1-\frac{\mathrm{C}_{\text {filt }, 2}}{\mathrm{~S}_{\mathrm{col}} \mathrm{S}_{2}^{0}}\right)-\mathrm{k}_{1 \mathrm{~b}} \mathrm{C}_{1}-\mathrm{k}_{2 \mathrm{~b}} \mathrm{C}_{2}-\mathrm{k}_{1 \mathrm{~b}} \mathrm{C}_{\text {filt }, 1}- \\
& \mathrm{k}_{\mathrm{r}} \mathrm{S}-\mathrm{k}_{2 \mathrm{~b}} \mathrm{C}_{\text {filt }, 2}-\left.\frac{\phi \mathrm{D}_{\mathrm{m}}}{\mathrm{b} \eta} \frac{\partial \mathrm{C}_{\mathrm{m}}}{\partial \mathrm{y}}\right|_{\mathrm{y}=\mathrm{b}}=0 \\
& \frac{\partial \mathrm{C}_{1}}{\partial \mathrm{t}}+\mathrm{v}_{\mathrm{f}} \frac{\partial \mathrm{C}_{1}}{\partial \mathrm{x}}-\mathrm{D}_{\mathrm{f}} \frac{\partial^{2} \mathrm{C}_{1}}{\partial \mathrm{x}^{2}}-\mathrm{k}_{1 \mathrm{f}} \mathrm{CC}_{\mathrm{col}}\left(1-\frac{\mathrm{C}_{1}}{\mathrm{C}_{\mathrm{col}} \mathrm{S}_{1}^{0}}\right)-\mathrm{k}_{\mathrm{rcol}} \mathrm{C}_{\text {filt }, 1}+ \\
& \mathrm{k}_{1 \mathrm{~b}} \mathrm{C}_{1}+\mathrm{k}_{\mathrm{fcol}} \mathrm{C}_{1}-\mathrm{PS}_{1}=0 \\
& \frac{\partial \mathrm{C}_{2}}{\partial \mathrm{t}}+\mathrm{v}_{\mathrm{f}} \frac{\partial \mathrm{C}_{2}}{\partial \mathrm{x}}-\mathrm{D}_{\mathrm{f}} \frac{\partial^{2} \mathrm{C}_{2}}{\partial \mathrm{x}^{2}}-\mathrm{k}_{2 \mathrm{f}} \mathrm{CC}_{\mathrm{col}}\left(1-\frac{\mathrm{C}_{2}}{\mathrm{C}_{\mathrm{col}} \mathrm{S}_{2}^{0}}\right)-\mathrm{k}_{\mathrm{rcol}} \mathrm{C}_{\text {filt }, 2}+ \\
& \mathrm{k}_{2 \mathrm{~b}} \mathrm{C}_{2}+\mathrm{k}_{\text {fcol }} \mathrm{C}_{2}-\mathrm{PS}_{2}=0
\end{aligned}
$$




$$
\begin{aligned}
& \frac{\partial C_{m}}{\partial t}-D_{m} \frac{\partial^{2} C_{m}}{\partial y^{2}}+k_{f m} C_{m}-k_{r m} S_{m}=0 \\
& \frac{\tilde{\mathrm{n}}_{\mathrm{Bf}}}{\eta} \frac{\partial \mathrm{S}_{1}}{\partial \mathrm{t}}-\mathrm{k}_{\mathrm{f}, 1} \mathrm{C}+\mathrm{k}_{\mathrm{r}, 1} \mathrm{~S}_{1}+\mathrm{PS}_{1}=0 \\
& \frac{\tilde{\mathrm{n}}_{\mathrm{Bf}}}{\eta} \frac{\partial \mathrm{S}_{2}}{\partial \mathrm{t}}-\mathrm{k}_{\mathrm{f}, 2} \mathrm{C}+\mathrm{k}_{\mathrm{r}, 2} \mathrm{~S}_{2}+\mathrm{PS}_{2}=0 \\
& \frac{\tilde{\mathrm{n}}_{\mathrm{B}}}{\phi} \frac{\partial \mathrm{S}_{\mathrm{m}}}{\partial \mathrm{t}}-\mathrm{k}_{\mathrm{fm}} \mathrm{C}_{\mathrm{m}}+\mathrm{k}_{\mathrm{rm}} \mathrm{S}_{\mathrm{m}}=0 \\
& \frac{\partial \mathrm{C}_{\text {filt }, 1}}{\partial \mathrm{t}}-\mathrm{k}_{1 \mathrm{f}} \mathrm{CS}_{\mathrm{col}}\left(1-\frac{\mathrm{C}_{\text {filt }, 1}}{\mathrm{~S}_{\mathrm{col}} \mathrm{S}_{1}^{0}}\right)-\mathrm{k}_{\text {fcol }} \mathrm{C}_{1}+\mathrm{k}_{\mathrm{rcol}} \mathrm{C}_{\text {filt }, 1}+\mathrm{k}_{\mathrm{lb}} \mathrm{C}_{\text {filt }, 1}=0 \\
& \frac{\partial \mathrm{C}_{\text {filt }, 2}}{\partial \mathrm{t}}-\mathrm{k}_{2 \mathrm{f}} \mathrm{CS}_{\mathrm{col}}\left(1-\frac{\mathrm{C}_{\text {filt }, 2}}{\mathrm{~S}_{\mathrm{col}} \mathrm{S}_{2}^{0}}\right)-\mathrm{k}_{\mathrm{fcol}} \mathrm{C}_{2}+\mathrm{k}_{\mathrm{rcol}} \mathrm{C}_{\text {filt }, 2}+\mathrm{k}_{2 \mathrm{~b}} \mathrm{C}_{\text {filt }, 2}=0 \\
& \frac{\partial \mathrm{C}_{\text {filt,irr,1 }}}{\partial \mathrm{t}}-\mathrm{k}_{1 \mathrm{f}} \mathrm{CS}_{\mathrm{col}, \mathrm{irr}}\left(1-\frac{\mathrm{C}_{\text {filt,irr,1 }}}{\mathrm{S}_{\mathrm{col}, \text { irr }} \mathrm{S}_{1}^{0}}\right)-\mathrm{k}_{\text {firr }} \mathrm{C}_{1}+\mathrm{k}_{1 \mathrm{~b}} \mathrm{C}_{\text {filt,irr,1 }}=0 \\
& \frac{\partial \mathrm{C}_{\text {filt,irr,2 }}}{\partial \mathrm{t}}-\mathrm{k}_{2 \mathrm{f}} \mathrm{CS}_{\mathrm{col}, \mathrm{irr}}\left(1-\frac{\mathrm{C}_{\text {filt,irr,2}}}{\mathrm{S}_{\mathrm{col}, \mathrm{irr}} \mathrm{S}_{2}^{0}}\right)-\mathrm{k}_{\text {firr }} \mathrm{C}_{2}+\mathrm{k}_{2 \mathrm{~b}} \mathrm{C}_{\text {filt,irr,2 }}=0
\end{aligned}
$$

where, $\mathrm{S}_{1}=$ sorbed concentration of $\mathrm{Pu}$ on sites 1 on fracture surfaces, $\mathrm{g} / \mathrm{g}$

$\mathrm{S}_{2}=$ sorbed concentration of $\mathrm{Pu}$ on sites 2 on fracture surfaces, $\mathrm{g} / \mathrm{g}$.

$\mathrm{C}_{\text {filt,irr, } 1}=$ concentration of $\mathrm{Pu}$ sorbed to site type 1 on irreversibly-attached colloids, $\mathrm{g} / \mathrm{cm}^{3}$

$\mathrm{C}_{\text {filt,irr, } 2}=$ concentration of $\mathrm{Pu}$ sorbed to site type 2 on irreversibly-attached colloids, $\mathrm{g} / \mathrm{cm}^{3}$

Note that the above equations assume that colloids are produced from material coating the fracture surfaces, and that radionuclides sorbed to fracture surfaces are added to the inventory of radionuclide mass sorbed to mobile colloids when this material is converted to colloids. For simplicity, it is also assumed that there is only a single reversible sorption site for radionuclides in the matrix. Similar modifications could be made to the equations associated with the Path 1 sorption model of Chapter 8 , but that is not pursued here.

Equations (11.2), (11.4), and (11.6) through (11.13), plus equations (8.10), (8.14) and (8.16) through (8.18) constitute a full model with two sorption sites on both colloids and fracture surfaces to describe colloid-facilitated transport through a fracture system. Although daunting in appearance, this model can be readily implemented in a numerical model to help understand the 
implications of the work conducted to date, both by UGTA and other projects, in predicting colloid-facilitated radionuclide transport at field scales.

First, it should be pointed out that when the best-fitting sorption and colloid transport parameters derived from the fits to the fracture transport data in this report (Chapter 8) are used in the above model to predict the colloid-facilitated transport of $\mathrm{Pu}$ over larger scales, $\mathrm{Pu}$ transport distances of only tens of meters are predicted in all cases except for when $\mathrm{Pu}(\mathrm{IV})$ is sorbed to clinoptilolite colloids. In the case of the clinoptilolite colloids, predicted transport distances are much further because there was essentially no desorption of $\mathrm{Pu}$ from the colloids observed in the fracture experiments, and the colloids themselves were modeled as being reversibly filtered. However, the detachment rate constant obtained for the clinoptilolite colloids must be considered an upper bound estimate because the experiments were not conducted long enough and the colloid measurement method wasn't sensitive enough to determine if all of the clinoptilolite colloids would have eventually exited the fractures. Even if only a small fraction of colloids were irreversibly filtered in a distance of less than $30 \mathrm{~cm}$, the vast majority of such colloids would be predicted to be irreversibly filtered over distances of several $\mathrm{km}$. Likewise, even if the $\mathrm{Pu}(\mathrm{IV})$ desorption rate from the colloids was too small to be observed in the fracture experiments, but was nevertheless nonzero, the vast majority of the $\mathrm{Pu}$ would be predicted to be desorbed from the colloids over the time scales associated with transport of several $\mathrm{km}$ in the field. Using this logic, it would seem unlikely that clinoptilolite colloids could facilitate the transport of Pu over distances of several $\mathrm{km}$.

However, while the inability to measure low-probability events in the clinoptilolite experiments can be used to argue that clinoptilolite colloids will not necessarily facilitate the transport of $\mathrm{Pu}$ over large distances, the same experimental shortcomings make it impossible to conclude that silica or montmorillonite colloids will not facilitate the transport of a small amount of Pu over significant distances. If only a small fraction of the $\mathrm{Pu}$ was irreversibly sorbed to these colloids, and likewise a small fraction of these colloids were resistant to filtration, it is possible that a small fraction of $\mathrm{Pu}$ could be transported over very large distances (even though the vast majority of the $\mathrm{Pu}$ might be desorbed or filtered over very short distances). The fracture experiments were not designed to determine whether a small fraction of colloids were resistant to filtration or whether a fraction of Pu was irreversibly sorbed to the colloids.

Because of these experimental shortcomings, sensitivity analyses were conducted using the above model to determine which model parameters and which processes colloid-facilitated radionuclide transport appears to be most sensitive to and ultimately to determine what conditions, if any, are required to have colloid-facilitated transport over significant distances and time scales. The results of these analyses are presented as a series of plots in which the sensitivity analysis simulations are shown relative to a fixed "base case". The results are also presented in terms of dimensionless parameters so that they can be readily translated from one set of conditions to another. All rate constants were made dimensionless by multiplying them by the mean fluid residence time in the fracture system, $\tau$.

To simplify the simulations, only a single reversible reaction was assumed for radionuclide sorption onto the colloids and another single reversible reaction was assumed for sorption onto the fracture surfaces (i.e., $\mathrm{k}_{2 \mathrm{f}}=0$ and $\mathrm{k}_{\mathrm{f}, 2}=0$ ). The important conclusions from the sensitivity 
analyses can all be obtained using just a single set of reversible reactions (although the model in its entirety may be necessary to evaluate site-specific cases). All simulations assumed

1. A fixed concentration of colloids throughout the system at all times, determined from equation (11.6),

2. A continuous injection of a fixed concentration of a radionuclide (e.g., $\mathrm{Pu}$ ) at the inlet to the system (i.e., a constant step function input). This fixed concentration was assumed be entirely in the solution phase, although it very rapidly partitioned to the colloids and fracture surfaces in all cases.

3. There was no matrix diffusion of either colloids or solute radionuclides - the inclusion of matrix diffusion would decrease predicted radionuclide concentrations but it otherwise does not significantly affect the conclusions from the sensitivity studies.

The base case for the sensitivity studies was chosen to have parameters that result in a minor amount of colloid-facilitated transport. These parameters (dimensionless) are listed in Table 11.1. A minor amount of colloid facilitated transport occurs in the base case because both the irreversible colloid filtration rate constant and the radionuclide desorption rate constant from colloids have dimensionless values of $1.0\left(\mathrm{k}_{\mathrm{fcirr}} \tau=1\right.$, and $\left.\mathrm{k}_{1 \mathrm{~b}} \tau=1\right)$. The effect of having these dimensionless values equal to 1.0 is that the fraction of colloids becoming irreversibly filtered as well as the fraction of radionuclide mass desorbing from colloids during the residence time in the fracture system is $\mathrm{e}^{-1}$ or $\sim 0.37$.

Figure 11.1 shows the effect of colloid concentrations on the simulated results (base case is $5 \mathrm{e}-8$ $\mathrm{g} / \mathrm{ml}$ ). Radionuclide concentrations sorbed to colloids are shown as bold lines and solute concentrations are shown as regular lines. A case with no colloids at all in the system is also shown for comparison. The radionuclides break through at significant concentrations at a dimensionless time of $\sim 1000 \tau$ in this case because the effective retardation factor for solute transport in the fractures is 1000 . The colloid concentrations were increased and decreased by one order of magnitude by increasing or decreasing the colloid production rate, $\mathrm{P}$, by one order of magnitude while holding the irreversible filtration rate constant, $\mathrm{k}_{\text {fcirr }}$, constant. The effect of colloid concentration on radionuclide responses in Figure 11.1 is clear: the concentration of radionuclides associated with colloids that arrive at early times $(\sim \tau)$ is directly proportional to the mobile colloid concentration. Note also that the colloids tend to "pull along" a small concentration of radionuclide in the solution phase, which occurs because of the slow desorption of the radionuclide from the colloids.

The effects of changing the irreversible filtration rate constant, $\mathrm{k}_{\mathrm{fcirr}}$, while keeping all other parameters constant, including colloid concentrations (which were kept constant by increasing or decreasing the colloid production rate, $\mathrm{P}$, by the same amount as $\mathrm{k}_{\text {fcirr }}$ ), are shown in Figure 11.2 (base case is " 1 "). Curves are not shown for $\mathrm{k}_{\mathrm{fcirr}} \tau<0.1$ because they are essentially indistinguishable from the curve for $\mathrm{k}_{\text {fcirr }} \tau=0.1$. This occurs because $\mathrm{k}_{\text {fcirr }} \tau=0.1$ corresponds to a situation where $100\left(\mathrm{e}^{-0.1}\right)$ or $90 \%$ of the colloids are not irreversibly filtered (so decreasing $\mathrm{k}_{\mathrm{fcirr}} \tau$ further has negligible effect). Increasing $\mathrm{k}_{\text {fcirr }} \tau$ by a factor of 10 results in a significant Table 11.1. Model parameters (mostly dimensionless) used in the base case simulation of colloid-facilitated transport. 


\begin{tabular}{cc}
\hline \hline Parameter & Value \\
\hline Peclet Number & 20 \\
\hline Matrix diffusion coefficient, $\mathrm{cm}^{2} / \mathrm{s}$ & 0 \\
\hline $\mathrm{k}_{\text {fcol }} \tau$ & 5 \\
\hline $\mathrm{k}_{\mathrm{rcol}} \tau$ & 1 \\
\hline $\mathrm{k}_{\mathrm{fcirr}} \tau$ & 1 \\
\hline $\mathrm{P} \tau, \mathrm{g} / \mathrm{ml}\left(\right.$ also equal to $\left.\mathrm{C}_{\mathrm{col}}\right)$ & $5 \times 10^{-8}$ \\
\hline $\mathrm{k}_{\mathrm{1f}} \tau, \mathrm{g} / \mathrm{g}$ & 1000 \\
\hline $\mathrm{k}_{\mathrm{b}} \tau$ & 1 \\
\hline $\mathrm{k}_{\mathrm{f}, \mathrm{l}} \tau$ & 1000 \\
\hline $\mathrm{k}_{\mathrm{r}, \mathrm{l}} \tau, \mathrm{g} / \mathrm{ml}$ & 1 \\
\hline $\mathrm{S}_{1}^{0}, \mathrm{~g} / \mathrm{g}$ & $60($ infinite sites $)$ \\
\hline $\mathrm{S}_{2}^{0}, \mathrm{~g} / \mathrm{g}$ & 60 ( infinite sites) \\
\hline
\end{tabular}

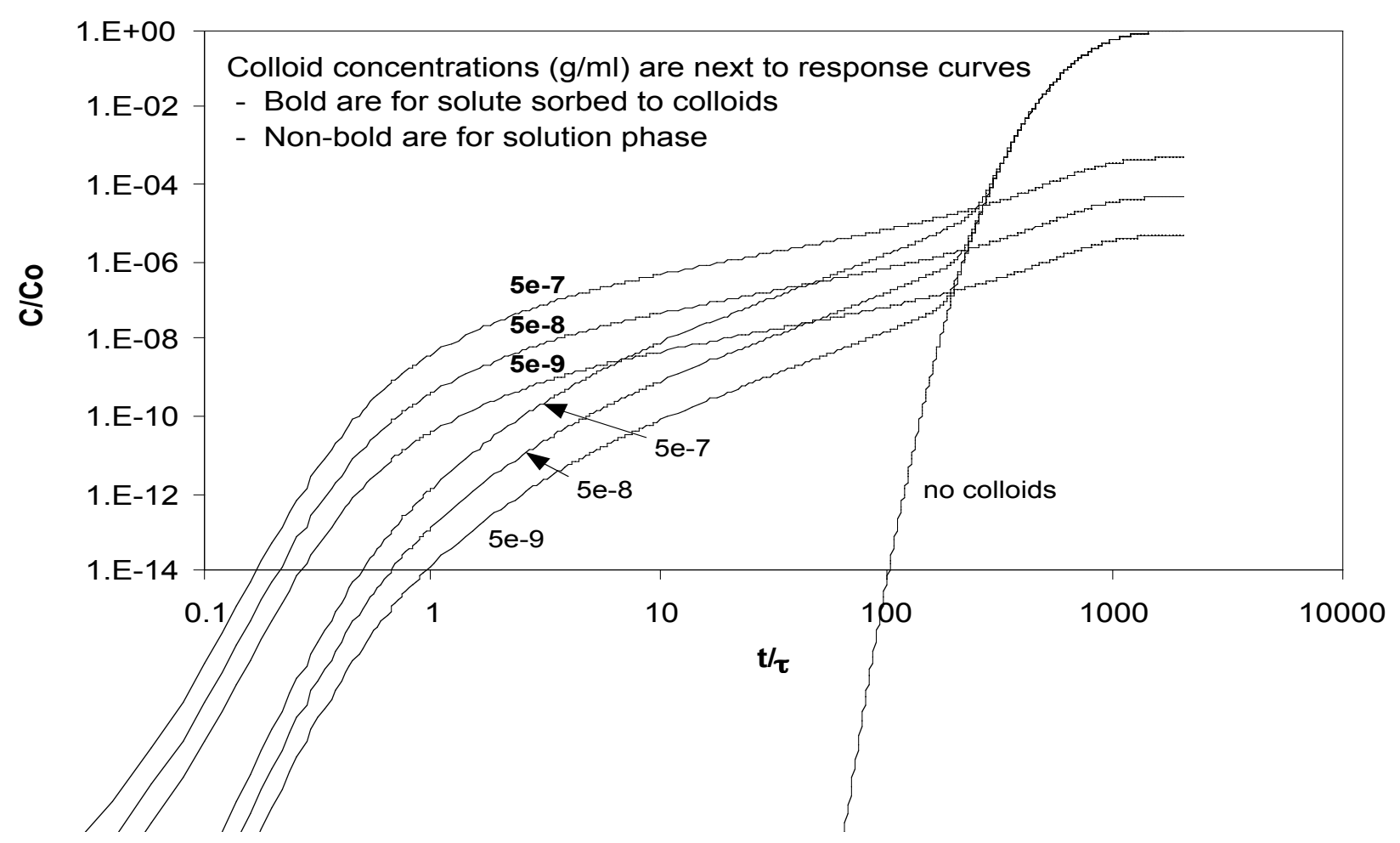

Figure 11.1. Effect of steady-state colloid concentrations on colloid-facilitated transport. 


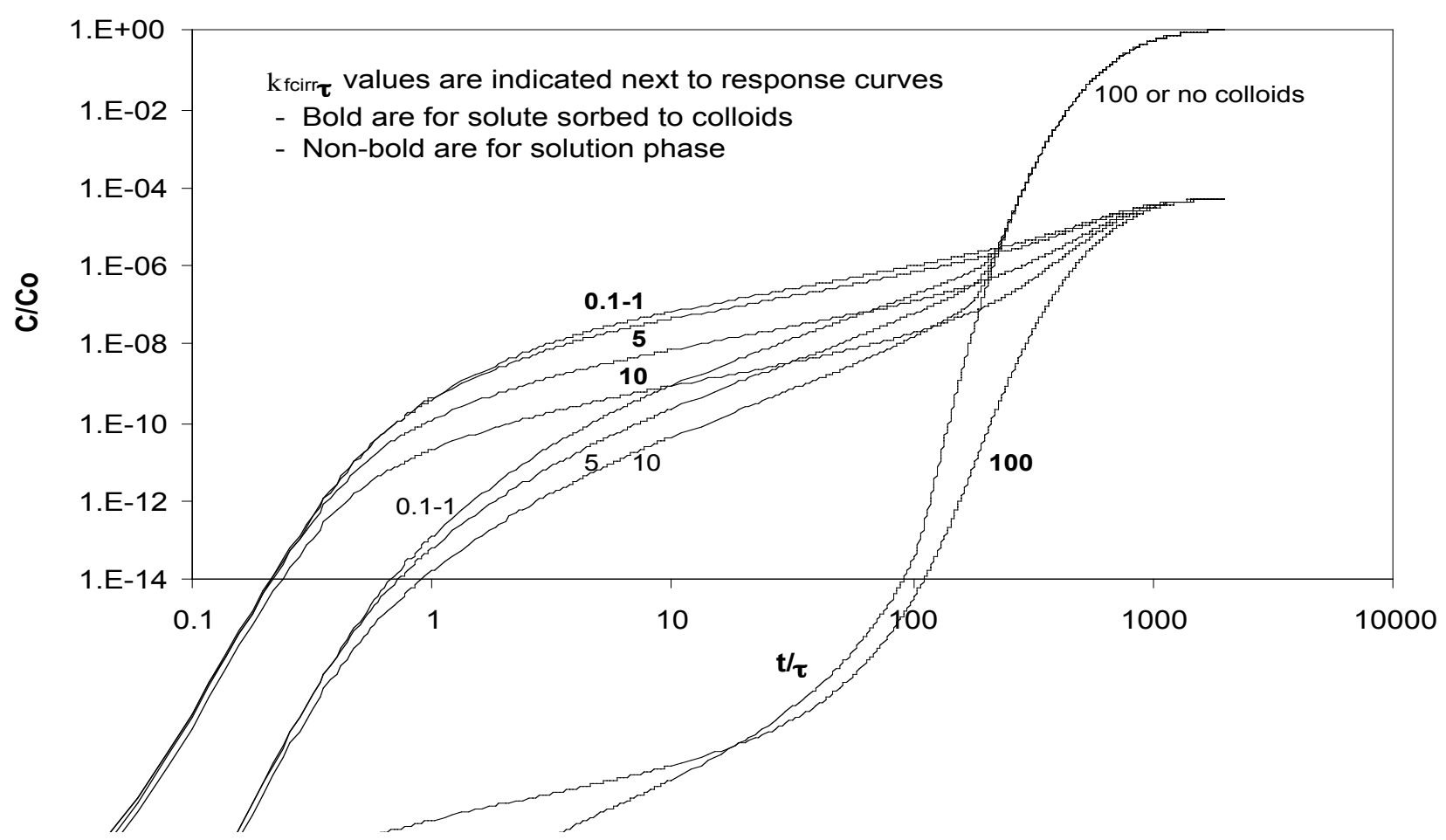

Figure 11.2. Effect of irreversible colloid filtration rate constant on colloid-facilitated transport.

decrease in the concentration of radionuclides associated with the early arrival sorbed onto colloids. As $\mathrm{k}_{\text {fcirr }} \tau$ increases to 100 , colloid-facilitated transport goes away entirely because all of the radionuclide mass associated with colloids becomes irreversibly attached (with host colloids) to fracture surfaces before any can make it through the flow system. Even though the radionuclides can desorb from the colloids, they quickly become sorbed to other colloids or to fracture surfaces.

The effects of changing the reversible attachment and detachment rate constants, $\mathrm{k}_{\text {fcol }}$ and $\mathrm{k}_{\text {rcol }}$, while keeping all other parameters constant, are shown in Figure 11.3 (base case is "5, 1"). Here it is apparent that the rates of reversible attachment and detachment have much less influence on colloid-facilitated transport than the irreversible filtration rate constant (Figure 11.2).

Figures 11.4 shows the effects of changing the forward and reverse rate constants for radionuclide sorption onto the colloids, $\mathrm{k}_{1 \mathrm{f}}$ and $\mathrm{k}_{1 \mathrm{~b}}$, while keeping all other parameters constant (base case is "1000, 1"). It is apparent that increasing the forward sorption rate constant by a factor of 10 without changing the reverse rate constant results in about a factor of 10 increase in radionuclide concentrations associated with colloids at early times. Increasing the ratio of the forward and reverse rates by a factor of 10 has approximately the same effect, provided the reverse rate constant stays less than some critical value. When the value of $\mathrm{k}_{1 \mathrm{~b}} \tau$ increases above 10 , the amount of colloid-facilitated transport drops dramatically, and once it is 100 , there is effectively no colloid-facilitated transport at all. Although not shown, the same results are obtained if $\mathrm{k}_{1 \mathrm{f}} \tau$ drops much below 0.1 , regardless of what the value of $\mathrm{k}_{1 \mathrm{~b}} \tau$ is (an insignificant mass of radionuclide can sorb to the colloids under these conditions). 


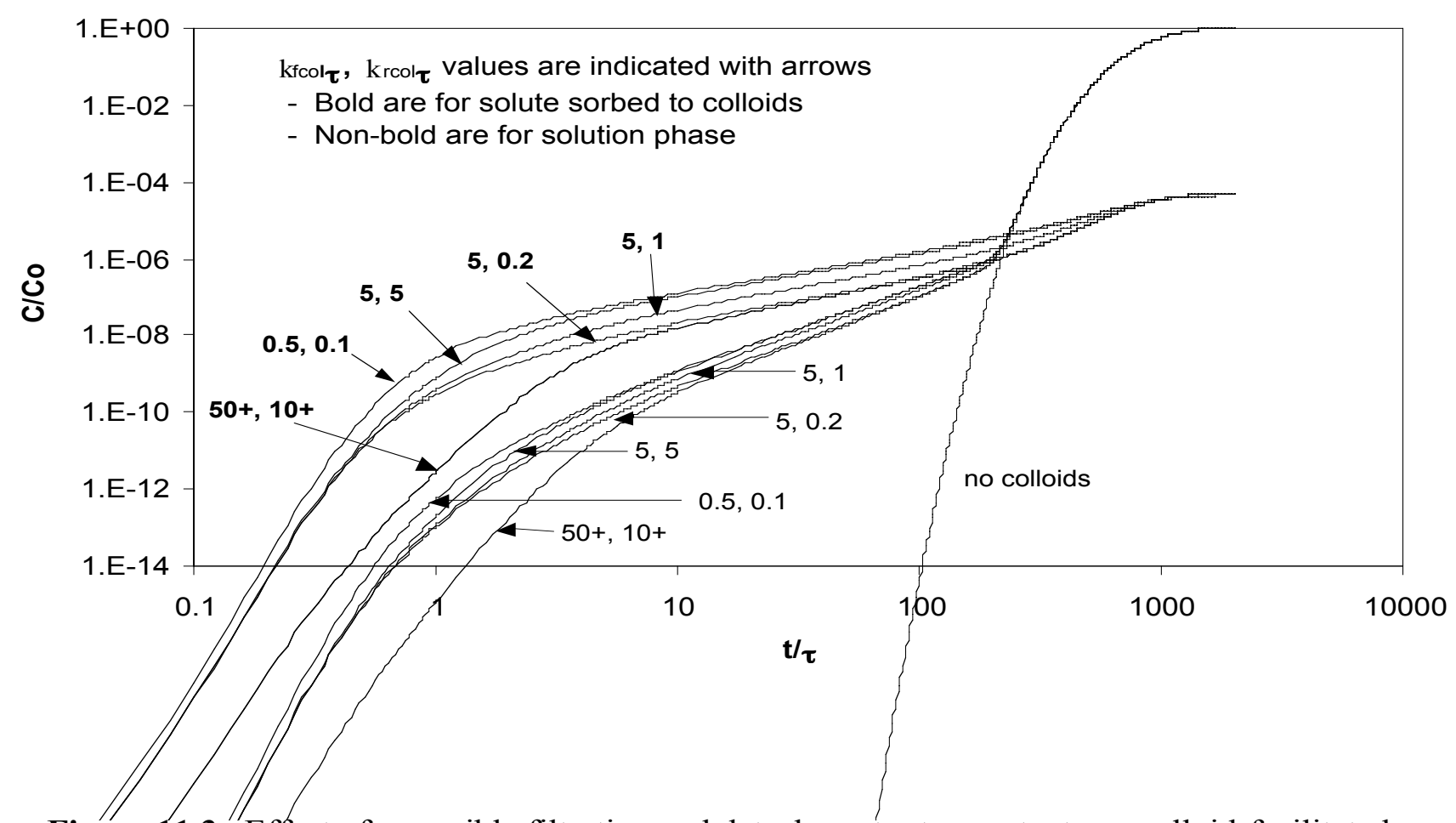

Figure 11.3. Effect of reversible filtration and detachment rate constants on colloid-facilitated transport.

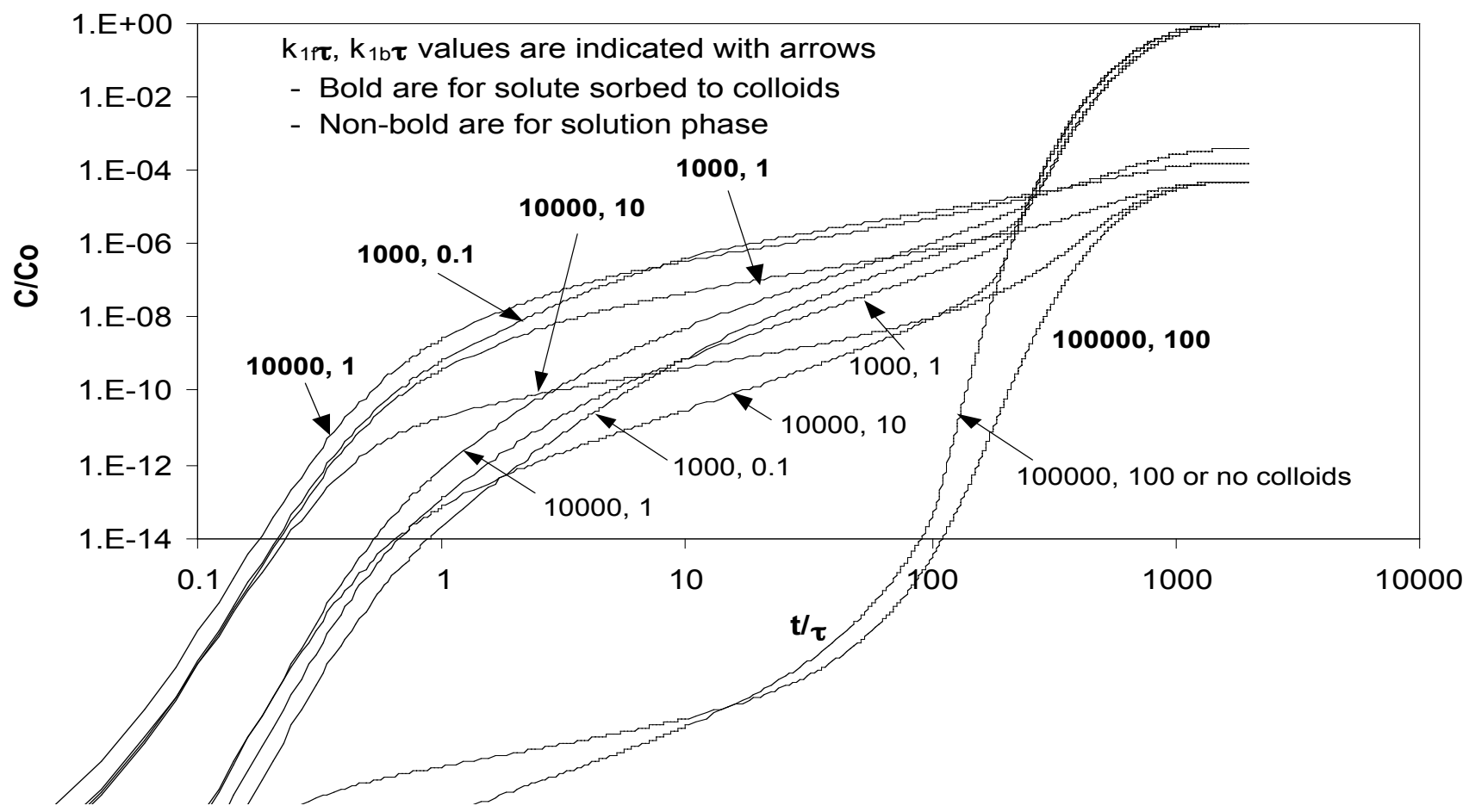

Figure 11.4. Effect of forward and reverse rate constants for radionuclide sorption onto the colloids on colloid-facilitated transport. 
Figure 11.5 shows the effect of increasing the dimensionless quantity $\mathrm{k}_{1 \mathrm{f}} \mathrm{C}_{\mathrm{co}} / \mathrm{k}_{1 \mathrm{~b}}$ while keeping $\mathrm{k}_{1 \mathrm{~b}} \tau=100$ (corresponding to the case in Figure 11.4 where no colloid-facilitated transport occurred; the " 100000,100 " case in Figure 11.4 would be equivalent to $\mathrm{k}_{1 \mathrm{f}} \mathrm{C}_{\mathrm{col}} / \mathrm{k}_{1 \mathrm{~b}}=0.00005$ in Figure 11.5). As $\mathrm{k}_{1 \mathrm{f}} \mathrm{C}_{\mathrm{col}} / \mathrm{k}_{1 \mathrm{~b}}$ increases beyond about 0.5 , the first arrival time of radionuclides associated with colloids effectively starts to decrease. A large ratio of $\mathrm{k}_{1 \mathrm{f}} \mathrm{C}_{\mathrm{col}} / \mathrm{k}_{1 \mathrm{~b}}$ can be achieved with either a high colloid concentration, very large values of $\mathrm{k}_{1 \mathrm{f}}$, or very small values of $\mathrm{k}_{1 \mathrm{~b}}$. With very large values of $\mathrm{k}_{1 \mathrm{f}}$ or $\mathrm{C}_{\mathrm{col}}$, sensitivity simulations indicated that colloid-facilitated transport can be significant even with large values of $\mathrm{k}_{\mathrm{fcirr}} \tau$ (i.e., even when a large fraction of colloids are irreversibly filtered). However, if $\mathrm{k}_{1 \mathrm{f}} \mathrm{C}_{\mathrm{col}} / \mathrm{k}_{1 \mathrm{~b}}$ is large simply because $\mathrm{k}_{1 \mathrm{~b}}$ is very small, values of of $\mathrm{k}_{\text {fcirr }} \tau$ must also be relatively small $(<10)$ for significant colloid-facilitated transport to occur.

Figure 11.6 shows the effect of changing the forward and reverse rate constants for radionuclide sorption onto the fracture surfaces, $\mathrm{k}_{\mathrm{f}, 1}$ and $\mathrm{k}_{\mathrm{r}, 1}$, while keeping all other parameters constant (base case is " 1000,1 "). Here it is apparent that increasing the ratio of the forward to reverse rate without significantly decreasing the forward rate has the effect of (1) decreasing the concentration of radionuclides arriving early associated with colloids and (2) increasing the arrival time of the main "slug" of solute radionuclides. On the other hand, decreasing the ratio of the forward to reverse rate without significantly decreasing the forward rate has the opposite effects. If the forward rate constant is reduced enough, sorption onto the fracture surfaces will become slow enough that a significant fraction of radionuclide mass will move through the system even without colloids being present.

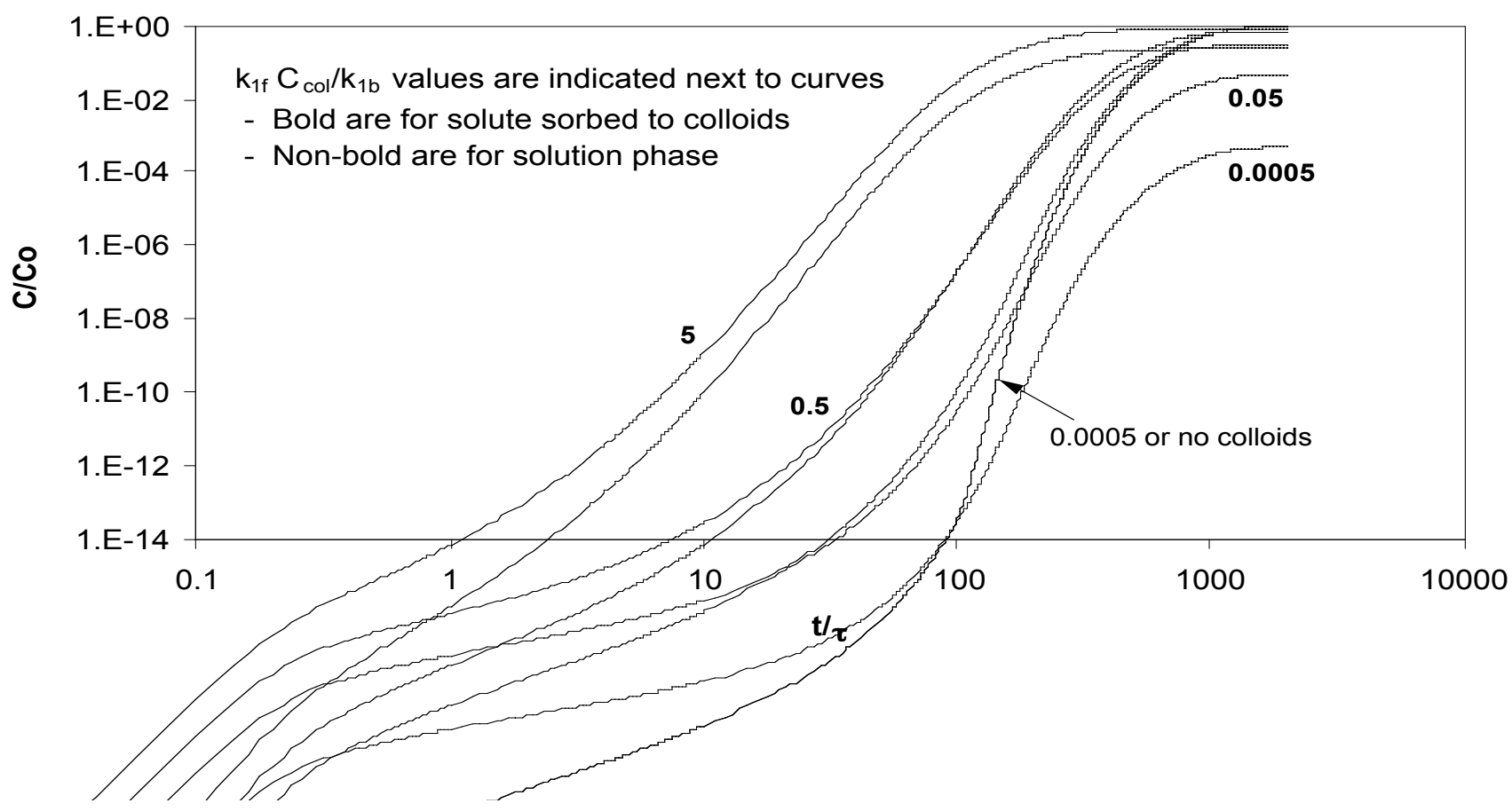

Figure 11.5. Effect of dimensionless quantity $\mathrm{k}_{1 \mathrm{f}} \mathrm{C}_{\mathrm{col}} / \mathrm{k}_{1 \mathrm{~b}}$ (while keeping $\mathrm{k}_{1 \mathrm{~b}} \tau=100$ ) on colloidfacilitated transport. 


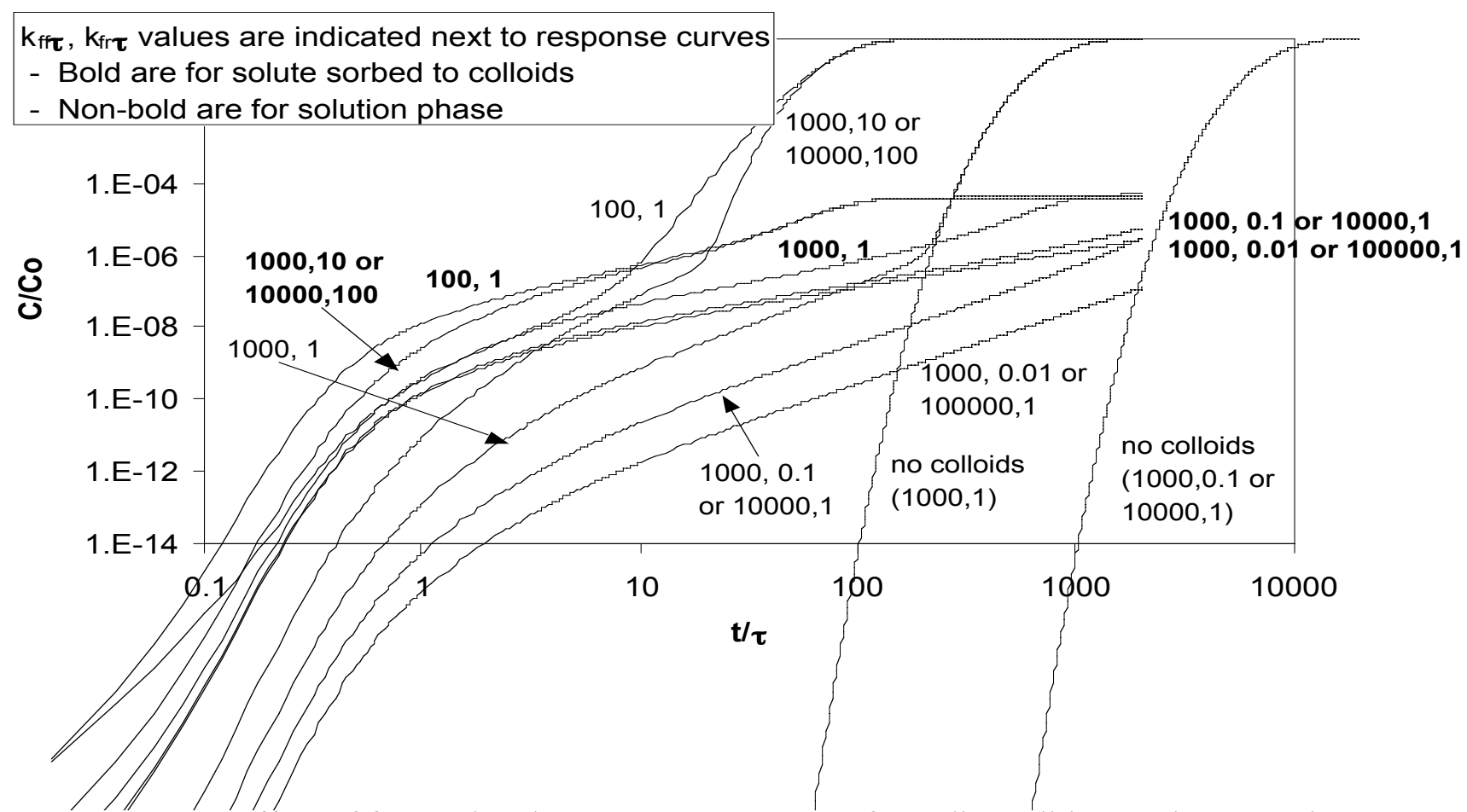

Figure 11.6. Effect of forward and reverse rate constants for radionuclide sorption onto the fracture surfaces on colloid-facilitated transport.

Figure 11.7 shows the effect of a brief but significant increase in the colloid production rate at early times. The production rate is assumed to be 100 times higher than the base case for a period of $0.1 \tau$ starting at $0.2 \tau$. Although the overall effect is minor, this figure illustrates how sudden increases in colloid production rates due to seismic events, flow transients, water chemistry pulses, or other disturbances, could briefly increase concentrations of radionuclides observed at down-gradient locations.

Although Figures 11.1 through 11.7 do not necessarily capture all of the sensitivities of predicted colloid-facilitated transport to values of the model parameters, we believe that the trends shown in these figures capture the most important sensitivities. The sensitivity analyses indicate the following:

- It is not unreasonable to expect a small fraction of radionuclide mass from a discrete source to move essentially at the rate of water flow through fractures over significant distances while sorbed onto colloids. The key requirements for such transport are

(1) the radionuclide desorption rate from the colloids must be slow enough that a fraction of the mass sorbed to the colloids at the source remains sorbed to the colloids during the time it takes to move the distance in question, and

(2) the irreversible filtration rate of the colloids must be slow enough that a fraction of the colloids at the source are not irreversibly filtered during the time it takes to move the distance in question. 


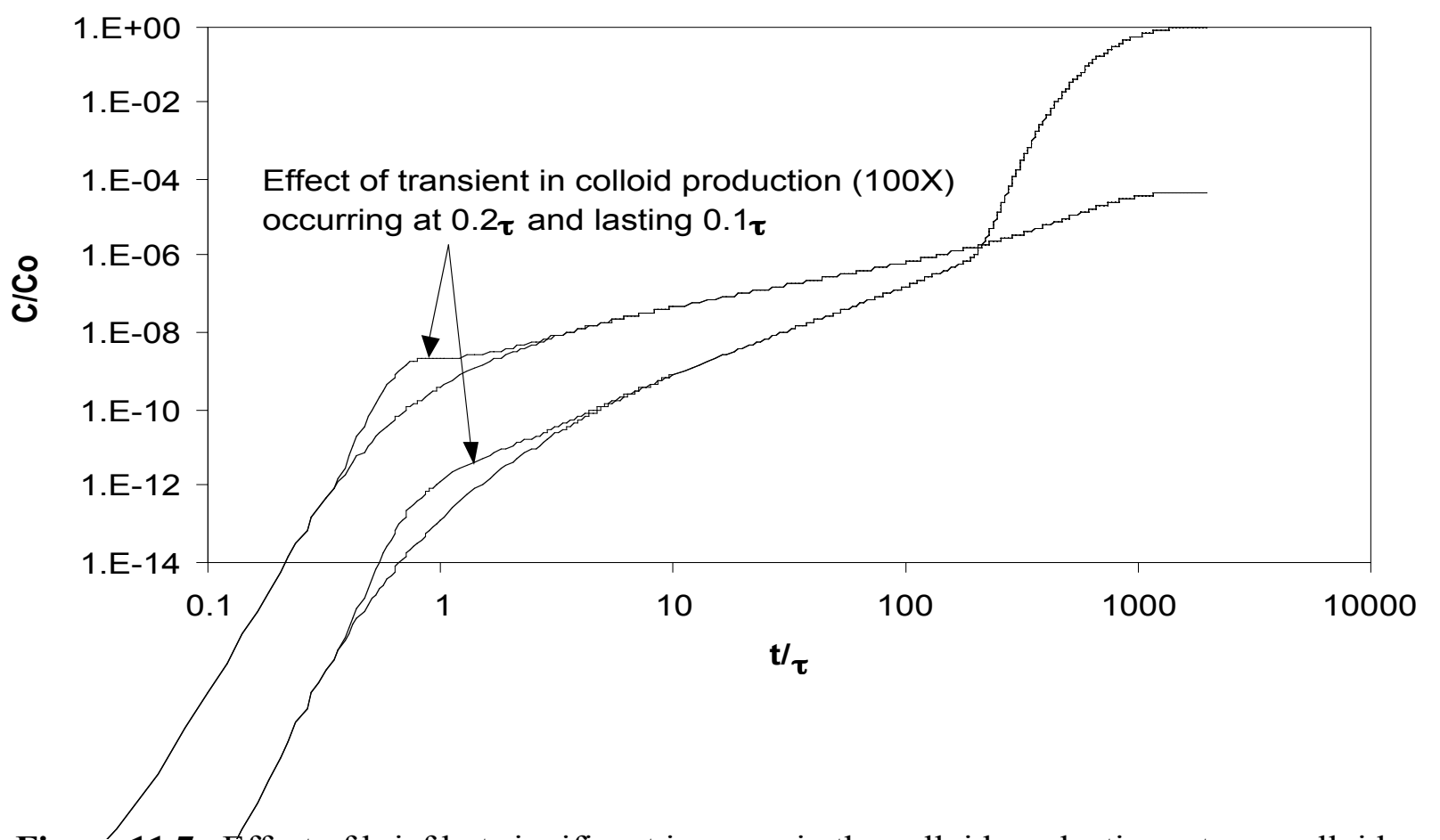

Figure 11.7. Effect of brief but significant increase in the colloid production rate on colloidfacilitated transport.

- The colloid concentrations in the system, the rates of reversible attachment and detachment of colloids onto fracture surfaces, and the rates of radionuclide sorption and desorption onto fracture surfaces all affect the fraction of radionuclide mass from the source that will effectively move at the rate of water flow through the flow system while sorbed to colloids. However, the two conditions listed above must be met before any radionuclide mass will move unretarded through the system.

- The only time colloids can facilitate the transport of radionuclides under conditions of fast radionuclide sorption and desorption rates (e.g., equilibrium conditions) is if the dimensionless quantity $\mathrm{k}_{1 \mathrm{f}} \mathrm{C}_{\mathrm{col}} / \mathrm{k}_{1 \mathrm{~b}}$ exceeds $\sim 0.5$; that is, if there are very high colloid concentrations or very large radionuclide-colloid partition coefficients $\left(\mathrm{k}_{1 \mathrm{f}} / \mathrm{k}_{1 \mathrm{~b}}\right)$. Under these conditions (and assuming $\mathrm{k}_{1 \mathrm{~b}}$ is not very small), colloid-facilitated transport can occur without the conditions in the first bullet being met. Colloid-facilitated transport in this case will be manifested as a decrease in the effective retardation factor of a relatively large fraction of the radionuclide mass in the source (compared to the retardation factor that would be observed if there were no colloids present). The Yucca Mountain Project has derived an effective etardation factor for colloid-facilitated radionuclide transport under these conditions (Arnold and Kuzio, 2001):

$$
\mathrm{R}_{\mathrm{eff}}=\frac{\mathrm{R}_{\mathrm{f}}+\mathrm{k}_{1 \mathrm{f}} \mathrm{C}_{\mathrm{col}} / \mathrm{k}_{1 \mathrm{~b}}}{1+\mathrm{k}_{1 \mathrm{f}} \mathrm{C}_{\mathrm{col}} / \mathrm{k}_{1 \mathrm{~b}}}
$$


where, $\mathrm{R}_{\mathrm{eff}}=$ effective retardation factor for the colloid-facilitated radionuclide transport, and $\mathrm{R}_{\mathrm{f}}=$ retardation factor for radionuclides in the absence of colloids.

The approximate fraction of radionuclide mass that will be transported with the colloids at the reduced retardation factor is

$$
\text { Fraction } \approx \frac{\mathrm{k}_{1 \mathrm{f}} \mathrm{C}_{\mathrm{col}} / \mathrm{k}_{1 \mathrm{~b}}}{1+\mathrm{k}_{1 \mathrm{f}} \mathrm{C}_{\mathrm{col}} / \mathrm{k}_{1 \mathrm{~b}}}
$$

Assessments of colloid-facilitated transport under the conditions of the first bullet (a small amount of radionuclide mass moving unretarded) and the the third bullet (a relatively large amount of radionuclide mass moving with some retardation, but less retardation than a pure solute) may require significantly different types of experiments and measurements. To appreciate this, it must be recognized that the two conditions listed under the first bullet above should not be thought of in terms of discrete radionuclide desorption rates from colloids or discrete irreversible colloid filtration rates. Rather, it is likely that relatively wide distributions of both desorption rates and irreversible filtration rates will occur in natural systems (because there are undoubtedly wide distributions of binding energies of sorption sites on colloids as well as wide distributions of colloid properties). It may be more appropriate to state the two conditions for colloid-facilitated transport listed under the first bullet as:

(1) a fraction of the radionuclide mass must be irreversibly sorbed onto colloids or have very low desorption rates from colloids, and

(2) a fraction of the colloids with irreversibly-sorbed radionuclides must have very small irreversible filtration rates.

By stating the conditions in this way, it is apparent that a small amount of colloid-facilitated transport could occur even if the majority of radionuclide sorption sites on colloids have relatively high desorption rates and the majority of colloids are filtered irreversibly (quite rapidly). Thus, the measurement of bulk radionuclide adsorption/desorption properties and bulk colloid filtration parameters may not be adequate to properly assess colloid-facilitated transport because a small amount of colloid-facilitated transport may occur as a result of outliers in the distributions of both desorption rates and irreversible colloid filtration rates. Figure 11.8 graphically illustrates hypothetical subsets of distributions of both colloid filtration rates and $\mathrm{Pu}$ desorption rates that could result in colloid-facilitated transport over significant distances. Also shown in Figure 11.8 at the right is the hypothetical intersection of the two distributions, which represents the actual fraction of $\mathrm{Pu}$ facilitated by colloids over the time and distance scales of interest. Note that the intersection shown in Figure 11.8 implies a positive correlation between slow $\mathrm{Pu}$ desorption rates and slow irreversible filtration rates. Whether such a correlation actually exists and whether it may be a positive or negative correlation is unknown.

Most laboratory and field tests/measurements, including the ones presented in this report, are not designed to study outliers in parameter distributions. However, field observations of colloidfacilitated radionuclide transport under the condtions of the first bullet are possible because of the extreme sensitivity of radionuclide analytical techniques and the ability to fingerprint sources 
of radionuclides (as for Pu at ER-20-5 \#1 and \#3). Laboratory tests are much better designed to assess colloid-facilitated radionuclide transport that might occur under the conditions of the third bullet listed above because these conditions require large bulk concentrations of colloids and

Figure 11.8. Illustration of hypothetical subsets of distributions of irreversible filtration rates and $\mathrm{Pu}$ desorption rates that could result in colloid-facilitated $\mathrm{Pu}$ transport over significant distances. Shown at the right is the intersection of the two subsets of the distributions, which represents the actual fraction of $\mathrm{Pu}$ facilitated by colloids over the time and distance of interest.

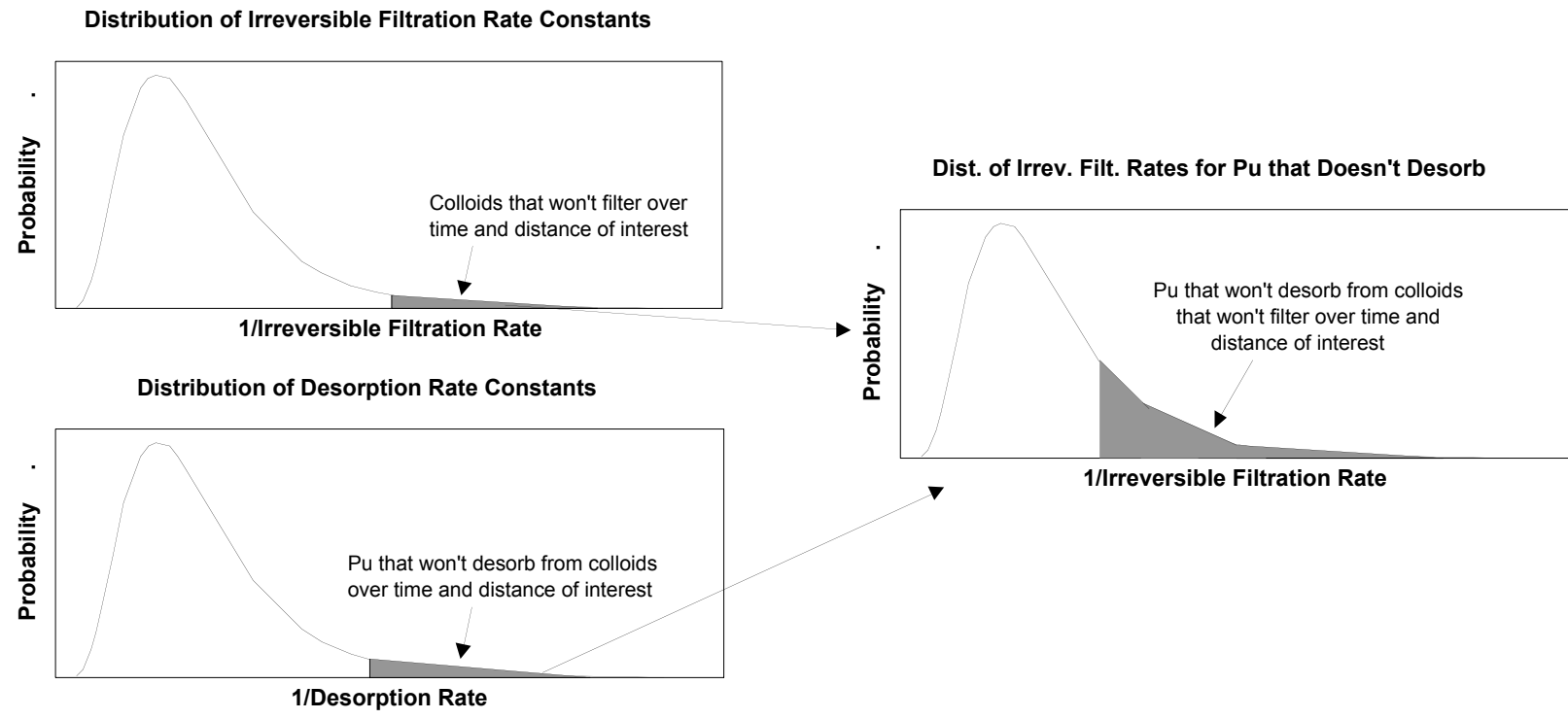

large partition coefficients for radionuclide sorption onto colloids $\left(\mathrm{k}_{1 \mathrm{f}} / \mathrm{k}_{1 \mathrm{~b}}\right.$ values), both of which are parameters that are relatively easily (and commonly) measured in the laboratory.

We believe that observations of Pu in the ER-20-5 wells originating from the BENHAM test are more likely the result of the conditions of the first bullet above being met than of the third bullet being met (assuming they are not entirely the result of "prompt injection"). Pu concentrations have been very low in all ER-20-5 samples analyzed, and they do not appear to be trending upward or downward significantly. These observations are consistent with predicted responses associated with the first bullet. The very low desorption rates of $\mathrm{Pu}$ from the clinoptilolite colloids in the laboratory batch sorption/desorption experiments, as well as the effectiveness of these colloids in facilitating Pu transport through the naturally-fractured cores, suggest that even if a only a small fraction of the natural colloids in Pahute Mesa ground waters are zeolitic, they might be able to facilitate the transport of enough Pu to account for the ER-20-5 observations. Alternatively, it is possible that the observed $\mathrm{Pu}$ could have been incorporated into the matrix of refractory colloids formed at the time of the BENHAM test, which would have resulted in the $\mathrm{Pu}$ being effectively irreversibly adsorbed to (or "engulfed within") these colloids. Even if only a small fraction of such refractory colloids could move as far as the ER-20-5 wells without being irreversibly filtered, they might account for the observations. It is even possible that a small amount of $\mathrm{Pu}$ could have transported while sorbed onto silica or clay colloids provided that there 
was a small fraction of sorption sites with very low desorption rates and a small fraction of colloids were not irreversibly filtered between the source and the ER-20-5 wells. The suggestion of slow desorption rates of $\mathrm{Pu}$ from silica and clay colloids in the multiple-step desorption experiments conducted by the Yucca Mountain Project (Figure 6.1), and the fact that silica colloids transported effectively through the naturally-fractured cores in the experiments described in Chapter 7 lends credence to this possibility.

Perhaps the most important point here is that the observation of Pu from the BENHAM test in the ER-20-5 wells at relatively low concentrations should not be taken as a surprise or even necessarily as a cause for concern. The results of the model sensitivity analyses described above suggest that it is entirely conceivable, and perhaps it should even be expected, that a small fraction of strongly sorbing radionuclides will move over significant distances essentially unretarded because of sorption to either naturally- or artificially-occurring colloids (even without prompt injection). Experiments and measurements to help assess the potential magnitude of colloid-facilitated transport under these conditions are discussed in the recommendations section in Chapter 12. These experiments and measurements would support establishing bounds on the radionuclide mass that could be transported effectively unretarded in association with colloids. 


\section{References}

Arnold, B. W. and S. P. Kuzio (2001). Implementation of Colloid-Facilitated Transport in the Saturated Zone Model for Site-Scale Performance Assessment at Yucca Mountain, presentation at YMP Colloids Integration Meeting, Nov. 13-14, 2001, Las Vegas, NV. 


\title{
-Chapter 12- Recommendations
}

\author{
Annie B. Kersting and Paul W. Reimus
}

\subsection{Recommendations}

The following recommendations are offered for future work that would help reduce uncertainties associated with the prediction of colloid-facilitated radionuclide transport at the NTS.

1. As colloid-facilitated transport is the dominant mechanism controlling transport, efforts to develop a more predictive colloid-transport model must be developed. Our primary recommendation of the report is to analyze colloid samples from the hot wells and analyzed for the radionuclide constituents and concentrations. Based on this analysis a determination may be able to be made as to whether or not colloid transport of radionuclides is a major concern to the UGTA project.

Several unknowns still hamper efforts to model Pu transport. We still do not know the nature of $\mathrm{Pu}$ in groundwater. Is it an intrinisic or pseudocolloid? Does $\mathrm{Pu}$ sorbed to a specific mineral or all minerals present. What is the maximum concentration of $\mathrm{Pu}$ that colloids could transport under conditions at the NTS? The answer to these questions would simplify modeling uncertainties. We proposed the following.

I. Measure the Pu concentration in all available hot wells at the NTS. Correlate the concentrations with groundwater chemistry, colloid mass, rock type, Pu source term information to determine if we can develop a conceptual model for the maximum concentration of $\mathrm{Pu}$ in contact with the source term. Experiments determining the maximum concentration of $\mathrm{Pu}$ for a given mass of colloids could be compared to hot well data. This data would help determine the maximum concentration of $\mathrm{Pu}$ available for transport.

II. Use state-of-the-art nanotechnological techniques to image the $\mathrm{Pu}$ in natural samples. This would include TEM, nanoSIMS and possibly Time-of-Flight Mass Spectrometry to determine if the $\mathrm{Pu}$ is a pseudocolloid or intrinsic colloid. If we could determine the nature of the $\mathrm{Pu}$ in the groundwater, we could simplify tremendously the geochemical modeling currently needed to predict colloidfacilitated transport of $\mathrm{Pu}$. Much of the sorption/desorption data may not be needed.

2. To assess the potential for a relatively large amount of radionuclide mass to move with less retardation than solutes while sorbed to colloids at NTS, sorption measurements of radionuclides such as $\mathrm{Pu}$ onto natural colloids collected from NTS wells should be conducted. Measurements of colloid concentrations and size distributions in NTS ground waters have been and should continue to be conducted, but the collection of sufficient quantities of natural colloids to conduct radionuclide sorption measurements has not been done. Sorption measurements on natural colloids are needed to assess effective radionuclide retardation factors according to equation (11.14) and mass fractions that may move with colloids according to equation (11.15). The use of pure minerals that are known to comprise a fraction of the natural colloid mass, while very informative, is no substitute for conducting 
measurements using actual colloids collected from the flow systems. Collecting enough colloid mass to conduct sorption experiments will require pumping wells for at least a few hours (in most cases) to ensure that non-natural colloids from well casing corrosion or well completion materials are effectively purged from the system before colloid collection begins.

3. Sorption experiments using natural colloids should be accompanied by long-term, multi-step batch or continuous-flow desorption experiments in which the radionuclide-bearing colloids are repeatedly or continuously contacted with radionuclide-free water (similar to the tests described in Section 6.3). These experiments will provide much more accurate and relevant measurements of radionuclide desorption rates than one-step batch desorption experiments. Multi-step or continuous flow desorption tests will allow at least partial quantification of the distribution of radionuclide desorption rates, and, more importantly, they will allow an assessment of what fraction of radionuclide mass has low enough desorption rates to potentially move unretarded over large distances in the NTS subsurface. The radionuclidebearing colloids that remain after several repeat desorption steps or after a long period of continuous contact with radionuclide-free water could then be injected into columns or cores to see if colloid contact with mineral surfaces increases the apparent desorption rate (as it may have for $\mathrm{Pu}$ associated with silica and montmorillonite colloids in Chapter 7). Alternatively, or perhaps additionally, microautoradiography experiments could be conducted with the remaining radionuclide-bearing colloids to determine what natural mineral phases in the colloids are responsible for the lowest desorption rates.

4. Radionuclide-bearing colloids should also be collected, if possible, from nuclear test cavities that are periodically sampled at NTS. If radionuclide concentrations associated with colloids are high enough, multi-step batch or continuous-flow desorption experiments should be conducted to determine the desorption rates of radionuclides from the cavity colloids (and the distribution of these rates). Consideration should also be given to injecting a pulse of radionuclide-bearing cavity colloids through columns or cores to determine whether some of the radionuclides will desorb in the columns/cores faster than they would desorb in batch experiments. Radionuclide concentrations in regularly-sampled high-activity cavities such as ALMENDRO, may be high enough that such tests could be conducted. Of course, these experiments will be more difficult to conduct and interpret than experiments involving introduced radionuclides because the colloids will likely have many different radionuclides associated with them, all of which could have different desorption characteristics.

5. Colloid transport tests capable of quantifying the low-end tail of the distribution of filtration rates of natural colloids should be conducted, as colloids with small irreversible filtration rates are predicted to contribute by far the most to unretarded colloid-facilitated radionuclide transport. One possibility for conducting such tests would be to continuously reinject pulses of colloids that have moved through packed columns or fractured cores and measure the colloid responses at the end of the columns/cores for each repeat pulse. If it is true that some colloids are more resistant to filtration than others (i.e., have lower inherent filtration rates), then these colloids should become concentrated relative to other colloids in each successive pulse, and the corresponding fractional recovery of colloids for each successive pulse will increase. Such results could be interpreted in terms of a distribution of colloid filtration 
rates, with the low-end tail being quantified increasingly better as more pulses are passed through the columns/cores.

6. Equations (11.5) and (11.6) suggest that irreversible colloid filtration rate constants could be indirectly estimated by measuring natural colloid concentrations $\left(\mathrm{C}_{\mathrm{col}}\right)$ in ground waters as well as colloid production rates $(\mathrm{P})$, and then dividing $\mathrm{P}$ by $\mathrm{C}_{\mathrm{col}}$ to obtain an estimate of the irreversible colloid filtration rate constant. The UGTA project has already measured $\mathrm{C}_{\mathrm{col}}$ in many NTS wells. Colloid production rates, however, are largely unknown. These rates could be measured in columns or fractured cores in the laboratory by flowing colloid-free water through the systems and measuring the effluent colloid concentrations. The difference between the concentration of colloids in the water exiting and entering a system would be taken as the colloid production rate in the system. Such experiments could be conducted using a variety of water chemistry conditions, flow rates and different rock types to yield colloid production rates, and hence irreversible filtration rates, as a function of all these variables. These experiments would thus help establish bounds for irreversible filtration rate constants in flow systems. These experiments could be combined with the experiments described in item 4 above by concentrating and then injecting the colloids generated in the columns or cores into other columns/cores to determine the distribution of filtration rates of the produced colloids.

7. Serious consideration should be given to assessing colloid-facilitated transport in future UGTA field cross-hole tracer tests. Such assessments could be made by (1) collecting natural colloids from the test wells during hydraulic testing, (2) adsorbing a stable isotope that is not highly abundant in nature, such as a samarium or cerium isotope, onto the natural colloids, (3) injecting the isotope-tagged colloids in the tracer test, and (4) analyzing for the stable isotope in both solution and colloid fractions of samples collected from the production well by inductively-coupled plasma-mass spectrometry (ICP-MS). Excellent analytical sensitivity should be attainable by either measuring absolute isotope concentrations or isotopic ratios. The information obtained from such a test would support testing and parameterization of a colloid-facilitated transport model at the field scale. Furthermore, it could be obtained without significant additional expense when one considers the overall costs of installing wells and conducting a cross-hole tracer test. This type of assessment of colloidfacilitated transport has a precedent in the international community at the Grimsel Underground Test Facility in Switzerland (completed in a fractured granite), where nonradioactive homologues were co-injected with colloids in field dipole tests in 2001 and similar tests involving radionuclides and colloids are planned for 2002 (Mori and Alexander, 2001).

8. As colloid-facilitated transport is the dominant mechanism controlling transport, efforts to develop a more predictive colloid-transport model must be developed. A colloid-filtration and transport model that is validated by our field and experimental results needs to be developed. This colloid filtration and transport model would build on UGTA's previous investments in geochemical and hydrologic transport codes. Figure 12.1 shows a schematic representation of the input parameters and source codes that would be needed to develop this type of transport model. Our experimental data will be used to develop a geochemical mechanistic description of $\mathrm{Pu}$ that determines the dominant mechanisms that control 
transport. The hydrologic parameters in a fracture-flow environment will provide flow field information that together with colloid characteristics measured from field samples will be linked with the geochemistry to develop a colloid filtration and transport model. This model would help in developing a framework for predicting when colloid-facilitated transport of actinides is important.

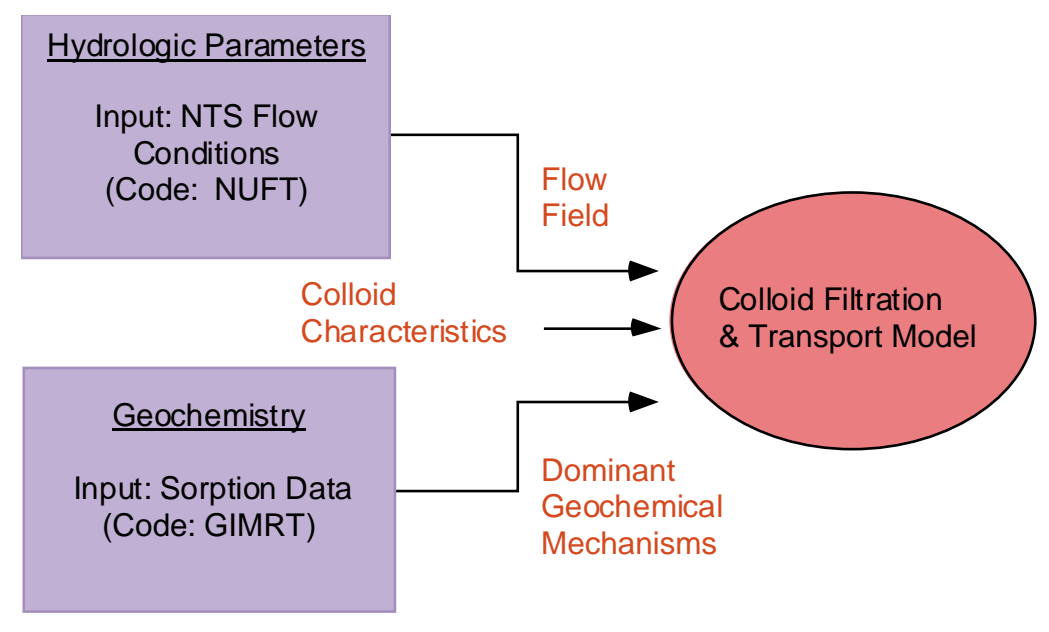

Figure 12.1. Schematic diagram describing the input parameters to developing a colloid filtration and transport model. NUFT= non-isothermal unsaturated flow and transport. GIMRT= Global implicit multi-component reactive transport 


\subsection{References}

Mori, A. and W. R. Alexander (2001). Grimsel Test Site Phase V: CRR - The colloid and radionuclide retardation project. Informal status report of year 2001 activities. Swiss nuclear waste repository program. 


\title{
-Appendix A- \\ Optical Petrography and Scanning Electron Microscope Descriptions of Representative Fracture Surfaces from Cores used in Transport Experiments
}

\author{
F. Chris Benedict and Richard G. Warren
}

\section{Sample UE20C, $2851 \mathrm{ft}$}

\section{General Description}

This material is described as devitrified moderately welded tuff from the Pahute Mesa lobe of the Topopah Spring Tuff of the Paintbrush Group. This material comprises a welded tuff aquifer within the Topopah Spring Aquifer (Drellack and Prothro, 1997).

Multiple fractures are present in the sample. An open fracture is exposed on the fracture surface and intersected at high angle by less prominent fractures which transect the rock matrix. This particular sample has one exposed open fracture face, which is the focus of the SEM characterization.

\section{Optical Petrography}

The polished thin section is a trapezoidal cut of core with a length of $28.5 \mathrm{~mm}$ and widths of 5 and $16 \mathrm{~mm}$, cut close to the plane of foliation. The rock is microspherulitic moderately welded tuff, with brown matrix in plane light. About two-thirds of the polished section is a single spherulitic pumice, brown in plane light, and very crystal-poor, with only 2 large plagioclase phenocrysts. The matrix has common felsic phenocrysts, mostly slightly altered sanidine, much lesser, somewhat altered plagioclase, and rare quartz; scarce to common mafics are mostly blackened biotite, but include some clinopyroxene pseudomorphs. A fracture that borders the edge of the section is filled with fine chalcedony and fine psilomelane that occupies mostly open channels. Other fractures have similar mineralogy but much smaller apertures. Three of these (fractures 1,3 and 4) are subparallel to the exposed fracture surface. One (fracture 2) is at a moderate angle to the exposed fracture surface, and one (fracture 5) is at a high angle to the exposed fracture surface. Fracture 1 exhibits layering of phases and consists primarily of porous chalcedony (5 to $100 \%$ voids) with lesser amounts of porous manganese oxides $(30 \%, 70 \%$ voids) present locally. Fracture 1 exhibits up to $100 \%$ void space indicating that it is, at least locally, accessible to fluids. Fracture 2 contains $20 \%$ chalcedony, $10 \%$ manganese oxides, and $70 \%$ void space. Petrographic data for fractures 3,4 , and 5 are not available.

\section{Scanning Electron Microscopy}

SEM evaluation showed the fracture surface coating to be smooth to finely granular. Surface relief is minimal (on the order of $0.01 \mathrm{~mm}$ or less) with areas of negative relief that are typically smooth in outline and elongate in dimension. The dimensions of these depressions range from up to $0.05 \mathrm{~mm}$ wide to $0.3 \mathrm{~mm}$ long. Depths are on the order of $0.05 \mathrm{~mm}$. One hundred percent of the fracture surface is coated with a very fine granular to "mat-like" covering composed of 80- 
85 percent chalcedony (mixed with small amounts of aluminosilicates and Mn-oxides), 10-12 percent Mn-oxide minerals, 5-8 percent unidentified "clay" minerals, and trace amounts of a Agrich phase. The fracture surface is characterized by a microgranular coated surface of low relief with local sinuous voids providing local depressions. The smooth nature of much of the fracture surface is a result of the "mat-like" accumulation composed of a mixture of manganese oxides and chalcedony. A branching prismatic to dendritic silver-rich phase is present within voids and depressions in the fracture surface.

\section{Sample UE20c, $2858 \mathrm{ft}$}

\section{$\underline{\text { General Description }}$}

This material is described as devitrified moderately welded tuff from the Pahute Mesa lobe of the Topopah Spring Tuff of the Paintbrush Group. This material comprises a welded tuff aquifer within the Topopah Spring Aquifer (Drellack and Prothro, 1997).

An open fracture is exposed on the sample surface. This fracture face is the focus of the SEM characterization.

\section{Optical Petrography}

The polished thin section is a trapezoidal cut of core with lengths of 36 and $38 \mathrm{~mm}$, and widths of 8.2 and $12 \mathrm{~mm}$. It represents microspherulitic moderately welded tuff with common pumice, the largest with granospherulitic interiors and some pumices spherulitic. Pyroclasts are brown in plane light. Common felsic phenocrysts are primarily sanidine, much lesser, slightly altered plagioclase, and rare quartz. Scarce to common mafics are mostly biotite that is generally blackened, and rare clinopyroxene pseudomorphs. One large pumice contains abundant plagioclase and a large clinopyroxene pseudomorph. The section is bounded by an open fracture, which provides a moderately sinuous, irregular surface that has no coatings of secondary minerals or recognizable alteration selvage. The fracture face is poorly preserved in thin section. Therefore a minimal amount of fracture data were generated by the petrographic evaluation.

\section{$\underline{\text { Scanning Electron Microscopy }}$}

SEM evaluation showed the fracture surface coating to be rough with well-developed crystalline coatings exhibiting a variety of morphologies. Surface relief is greater than $0.1 \mathrm{~mm}$ with considerable surface porosity. The dimensions of these local depressions reach $0.2 \mathrm{~mm}$ wide and $0.5 \mathrm{~mm}$ long. Depths are on the order of $0.3 \mathrm{~mm}$. Sixty to 70 percent of the fracture surface is coated with well-developed crystalline coatings of chalcedony (80-85\%) and manganese oxides (15-20\%). The surface coatings have created a significant amount of texture and additional surface area. Chalcedony occurs in 3 different morphologies including blocky crystallites and elongate aggregates. Small isolated fine-grained chalcedony aggregates composed of small numbers of crystallites equivalent to those comprising the elongate prismatic aggregates are locally present. Manganese oxides occur on the surface as interlocking fine-grained prismatic 
crystals.

\section{Sample PM-1, $4823 \mathrm{ft}$}

\section{General Description}

This material is described as quartzofeldspathic altered non-welded tuff from the mafic-poor Bullfrog Tuff of the Crater Flat Group. This material comprises a confining unit within the Bullfrog Confining Unit (Drellack and Prothro, 1997).

An open fracture is exposed on the sample surface.

\section{Optical Petrography}

This polished section is a trapezoidal cut of core with lengths of 35.5 and $37 \mathrm{~mm}$ and widths of 18 and $22 \mathrm{~mm}$. The rock is quartzofeldspathic and minor calcareous and kaolinitic nonwelded tuff with common pumice. Scarce to common felsic phenocrysts are quartz, strongly altered plagioclase, and minor sanidine. Plagioclase is partly replaced by albite and some calcite, and is mantled by adularia. Rare biotite is entirely replaced by chlorite. Lithics are scarce to common. No fractures were observed during the petrographic examination of this sample.

\section{$\underline{\text { Scanning Electron Microscopy }}$}

SEM evaluation identified the fracture surface as a moderately rough, granular surface. Surface pores are abundant, small (up to $0.02 \mathrm{~mm}$ ), and angular. Surface relief is low and on the order of $0.02 \mathrm{~mm} .90$ percent of the fracture surface is coated primarily with small crystals and granular aggregates. The fracture coating is composed of 25 percent chalcedony, 65 percent smectite, and trace amounts of mixed-metal aggregates. The mixed-metal aggregates contain $\mathrm{Cu}, \mathrm{Fe}, \mathrm{Sn}$, and $\mathrm{S}$ in addition to other elements. The tentative identification of gypsum and sylvite on this fracture surface suggests that the mixed metal aggregates are likely to be oxides and sulfates.

\section{Sample PM-2, $4177 \mathrm{ft}$}

\section{$\underline{\text { General Description }}$}

This material is described as devitrified lava from the dacite of Mt. Helen of the Volcanics of Quartz Mountain. This material comprises a lava flow aquifer within the Pre-Belted Range Composite Unit (Drellack and Prothro, 1997).

An open fracture is exposed on the sample surface.

Optical Petrography 
This polished section is a trapezoidal cut of core with lengths of 30 and $40 \mathrm{~mm}$ and widths of 15 and $19 \mathrm{~mm}$. It represents spherulitic, strongly brecciated lava. There is no finely granular matrix typically found in flow breccias, so the fracturing is probably tectonic in origin. Abundant vugs are mostly filled with coarse chalcedony, with some void occasionally remaining, but some vug interiors are filled with coarse carbonate that includes calcite and ankerite, with kaolinite, or cut by carbonate veins. Many chalcedony-filled vugs grade into very sinuous veins that extend for a few $\mathrm{mm}$. Prominent veins are entirely carbonate; the largest are calcite, which is crosscut by ankerite. Several hairline fractures that parallel the largest veins are also filled with carbonate. Plagioclase phenocrysts and biotite are common to abundant. Scarce mafic pseudomorphs include probable hornblende now occupied primarily by chalcedony, and probable clinopyroxene now occupied primarily by illite. Biotite is blackened, but only moderately altered, apatite is unaltered, and plagioclase is mostly, but not entirely altered and replaced by albite and carbonate, either calcite or ankerite. Four fractures are evident in thin section and range in maximum apparent aperture from $0.018 \mathrm{~mm}$ (fracture 4) to $0.64 \mathrm{~mm}$ (fracture 2). Observed relationships indicate a paragenetic sequence of fractures 3 and 4 , followed by fracture 2 , followed by fracture 1. Fractures 3 and 4 contained voids (15 and 70 percent respectively) and chalcedony ( 85 and 30 percent respectively). Fracture 2 contained 100 percent calcite. Fracture 1 contained 100 percent ankerite $\left[\mathrm{Ca}\left(\mathrm{Fe}^{2+}, \mathrm{Mg}, \mathrm{Mn}^{2+}\right)\left(\mathrm{CO}_{3}\right)_{2}\right]$.

\section{$\underline{\text { Scanning Electron Microscopy }}$}

SEM evaluation identified the fracture surface as an irregular smooth to matted surface with blocky offsets. Small surface pores (up to $0.01 \mathrm{~mm}$ ) are smooth and rounded. Surface relief is up to $0.10 \mathrm{~mm}$. One hundred percent of the fracture surface is coated by secondary phases. These include 25 percent calcite as eroded crystals, 25 percent ankerite as blocky crystals and aggregates, 5-10 percent chalcedony as small euhedral crystals, 5-10 percent unidentified clay minerals, 5-10 percent barite, 20-30 percent matted mixture of chalcedony and clay, and a trace amount of amorphous iron-oxide grains. The blocky ankerite fracture filling was partially coated with a smooth, very fine-grained mat-like accumulation of chalcedony and clay (illite?).

\section{References}

Drellack, S. L. Jr., and L. B. Prothro (1997). Descriptive Narrative for the Hydrogeologic Model of Western and Central Pahute Mesa Corrective Action Units. Bechtel Nevada Internal Report. 


\section{-Appendix B- \\ Stability Studies of Ca-Montmorillonite Colloids}

\section{K. Stephen Kung}

Before the Pu-colloid fracture flow-through experiments were conducted as described in Chapter 7, the stability of the montmorillonite colloids were evaluated in different waters so that we could determine the best method of preserving the samples if they could not be analyzed immediately. We studied the stability of montmorillonite in (1) freshly prepared syn. WW20 water, (2) aged synthetic WW20 water, and (3) deionized water. Montmorillonite colloid stock solution was introduced to the desired waters and the total colloid concentrations were monitored for three weeks using the HSLIS-S50.

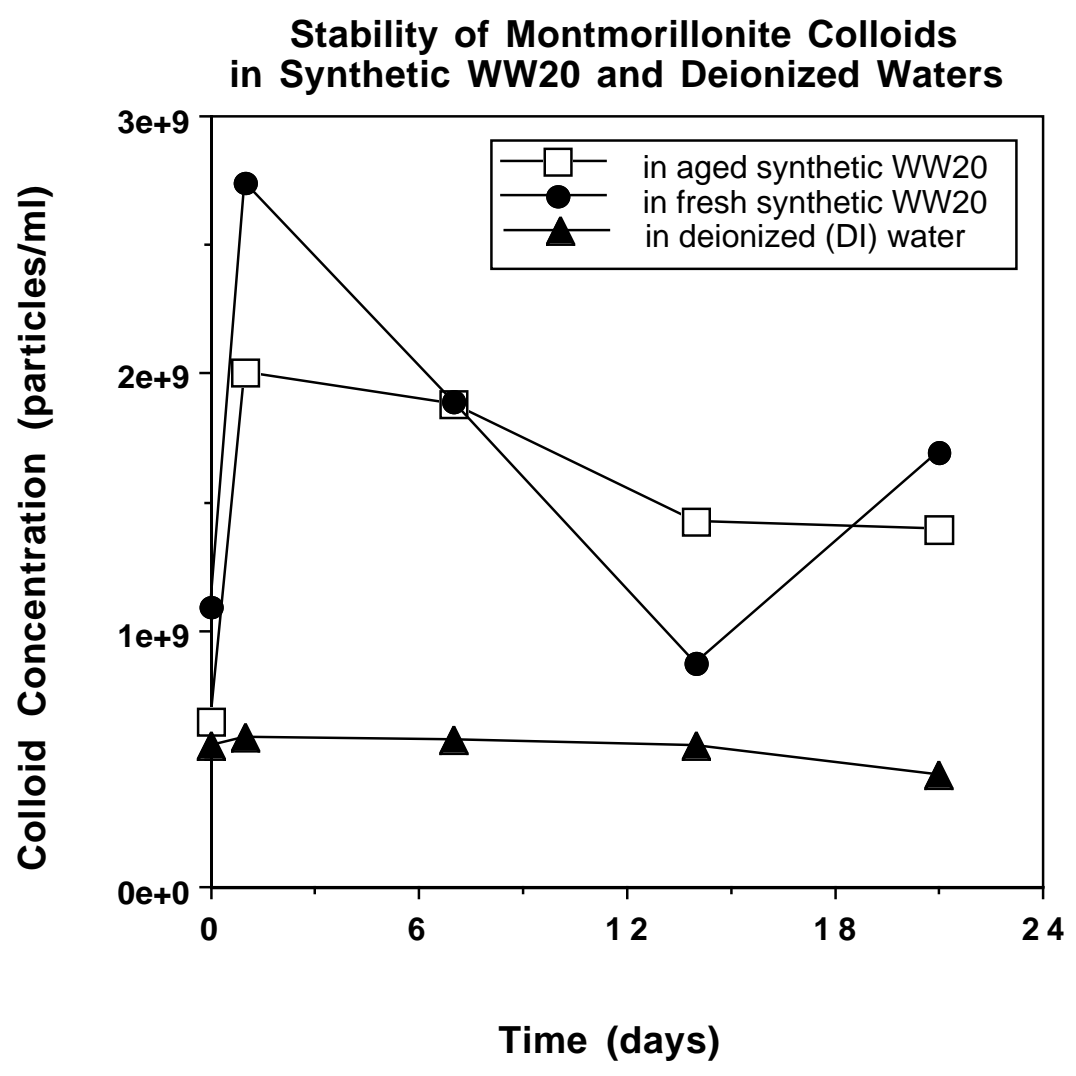

Figure B.1. Stability of montmorillonite colloids in different waters.

The data shown in Figure B.1 indicate that the colloid concentrations increased one day after they were introduced to both freshly prepared and aged syn. WW20 waters. The reason for this 
is unknown. The total concentration of the montmorillonite colloids stored in syn. WW20 water then gradually decreased over the next 20 days. In contrast, colloid concentrations remained stable when diluted in deionized water. For this reason, all fractured column effluent samples were immediately diluted in deionized water to preserve them.

We then diluted all samples that resulted from this study in deionized water to evaluate the effect of DI water dilution on the stability of montmorillonite colloids. The results of this study (show in Figure B.2) confirm that deionized water effectively stabilizes the montmorillonite colloids.

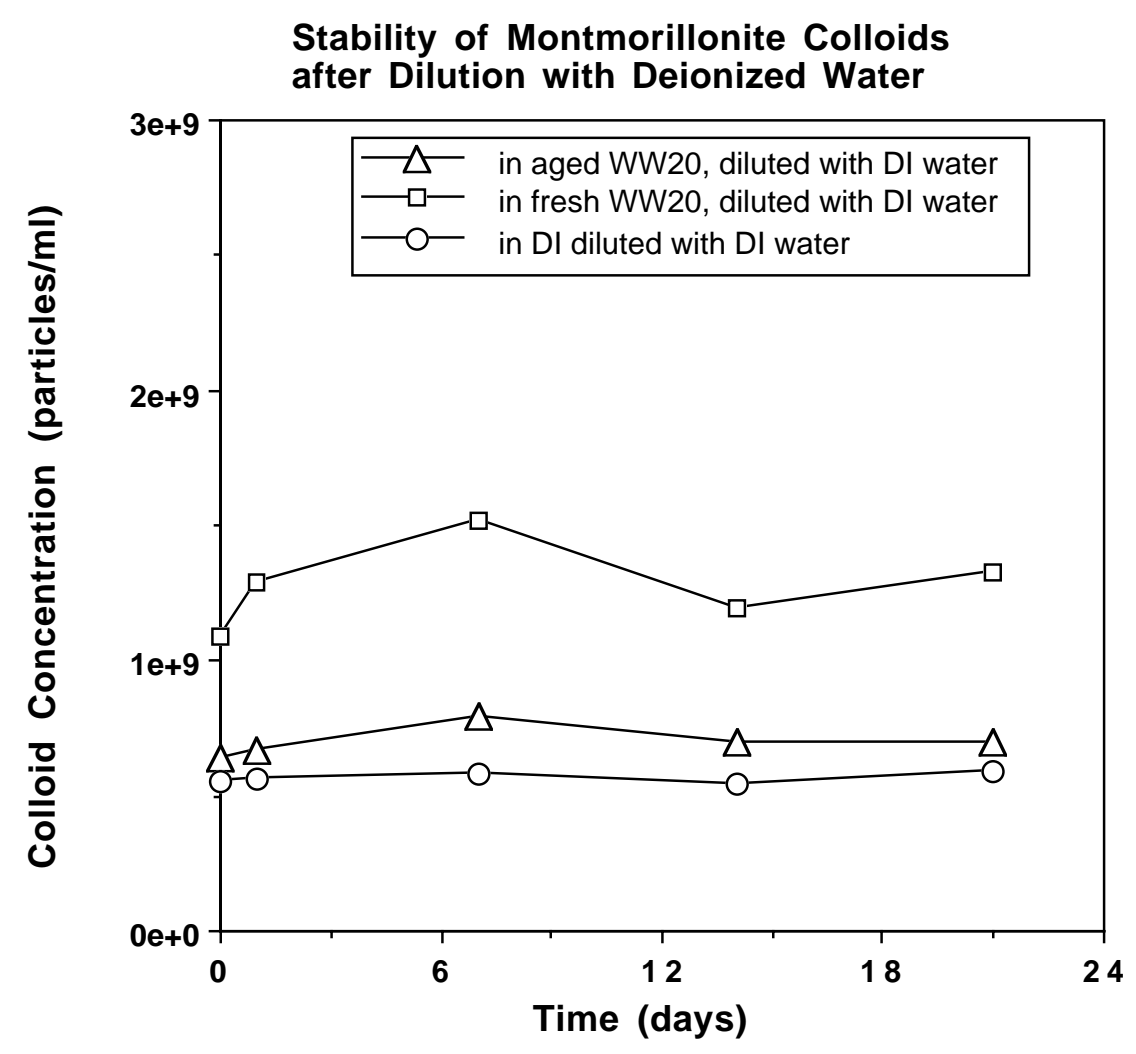

Figure B.2. Stability of montmorillonite colloid after dilution with deionized (DI) water. 


\title{
-Appendix C- \\ Plots of Normalized Concentrations and Recoveries of Tritiated Water, Colloids, and Plutonium in Each of the Fracture Transport Experiments
}

\author{
Paul W. Reimus
}

This appendix contains plots of the normalized concentrations and fractional recoveries of tritiated water, colloids, and plutonium as a function of volume eluted in each of the fracture transport experiments described in Chapter 7.
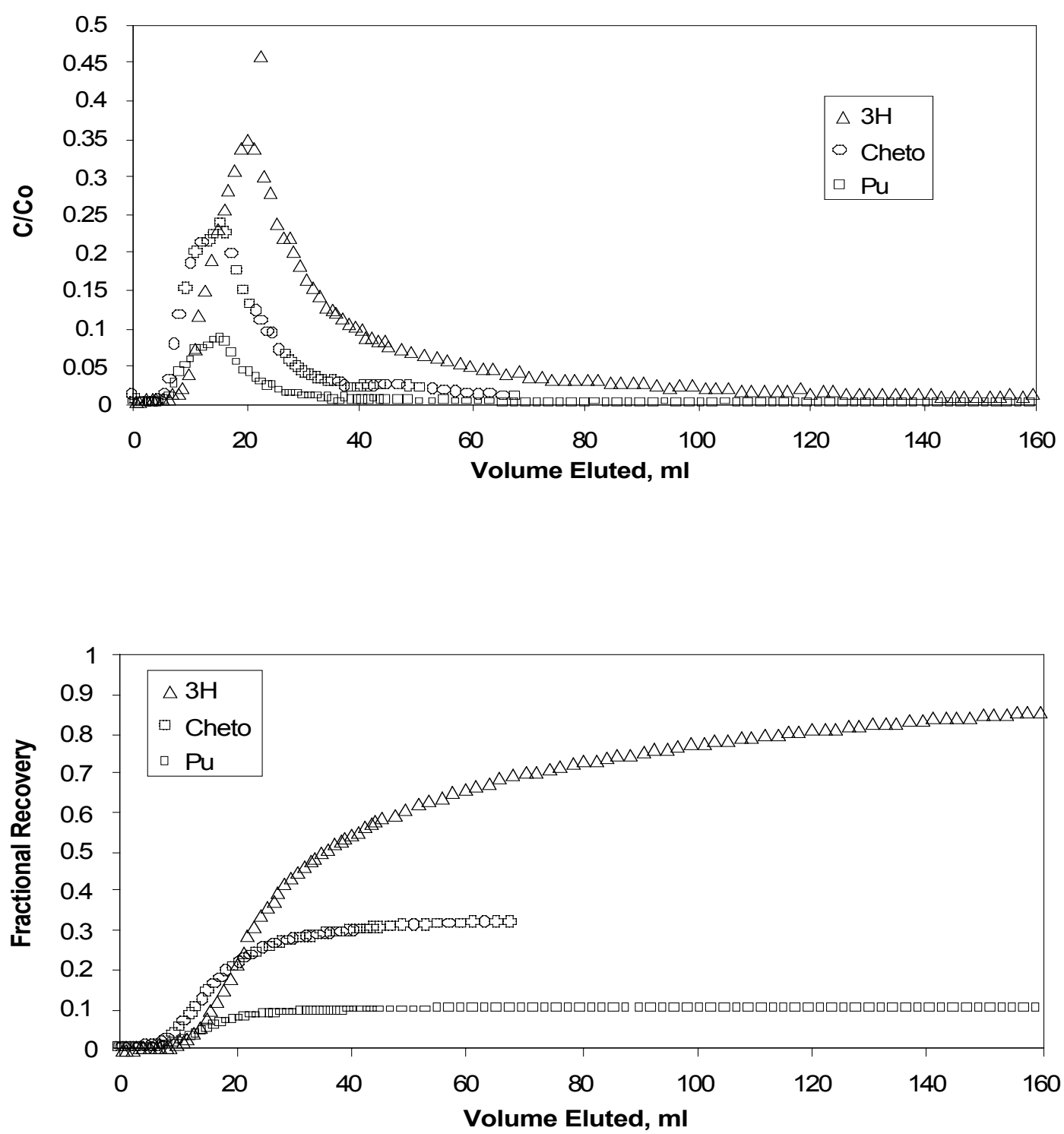

Figure C.1. Normalized concentrations and fractional recoveries in the UE20c 2851 fracture as a function of volume eluted at $\sim 1.5 \mathrm{ml} / \mathrm{hr}$ in Experiment $1 \mathrm{a}$. 

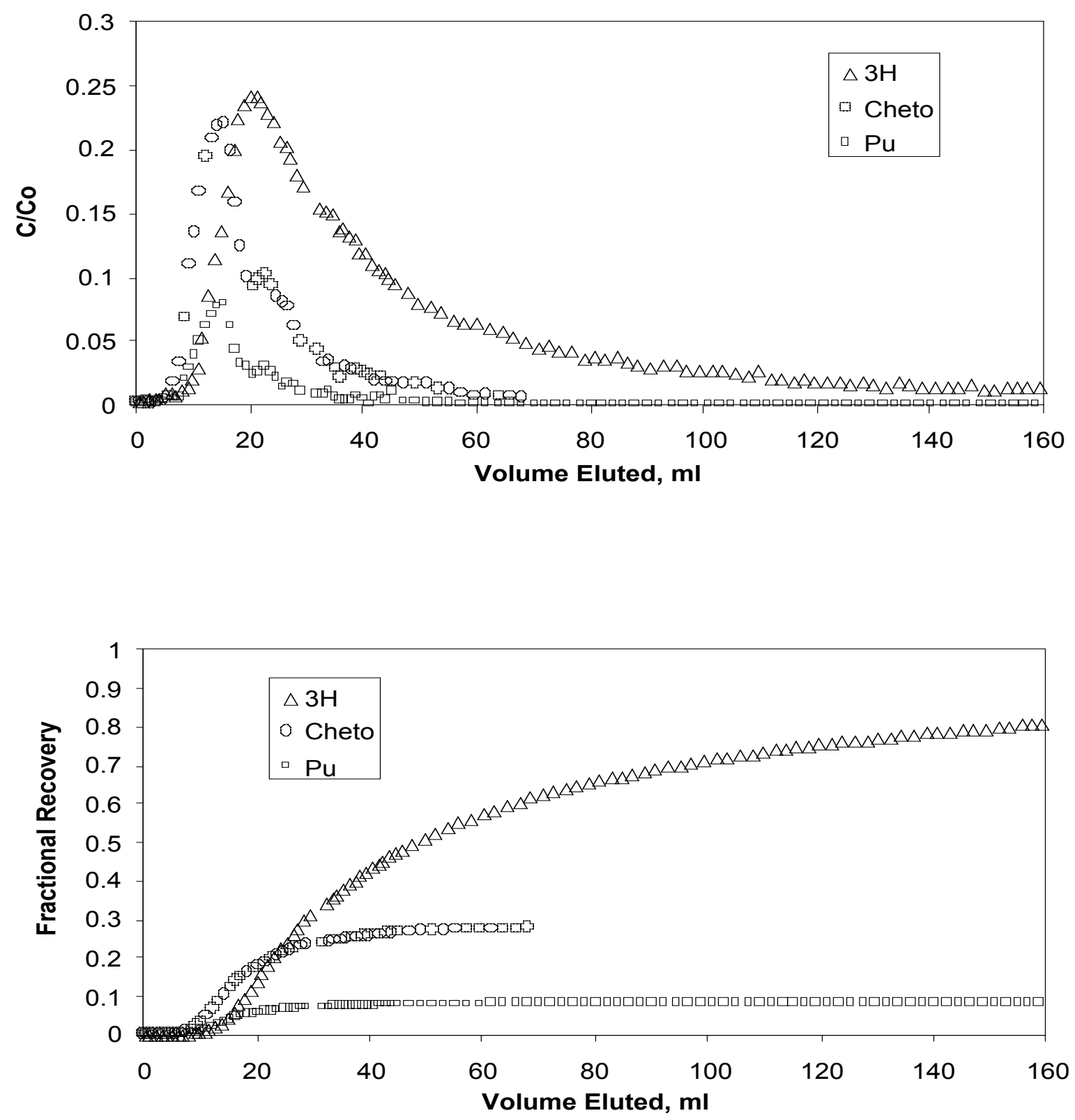

Figure C.2. Normalized concentrations and fractional recoveries in the UE20c 2858 fracture as a function of volume eluted at $\sim 1.5 \mathrm{ml} / \mathrm{hr}$ in Experiment $1 \mathrm{~b}$. 

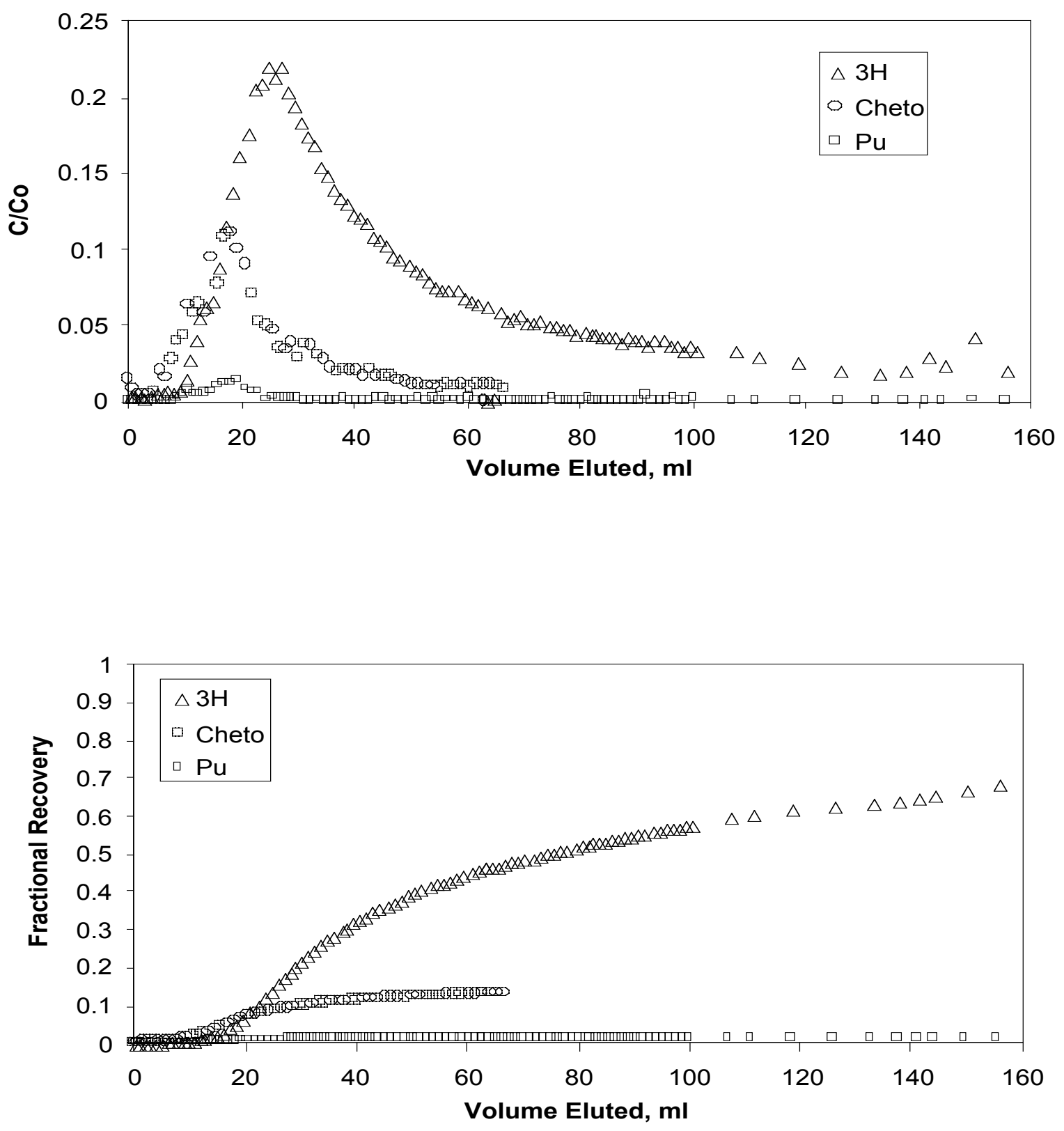

Figure C.3. Normalized concentrations and fractional recoveries in the UE20c 2851 fracture as a function of volume eluted at $\sim 0.6 \mathrm{ml} / \mathrm{hr}$ in Experiment $2 \mathrm{a}$. 

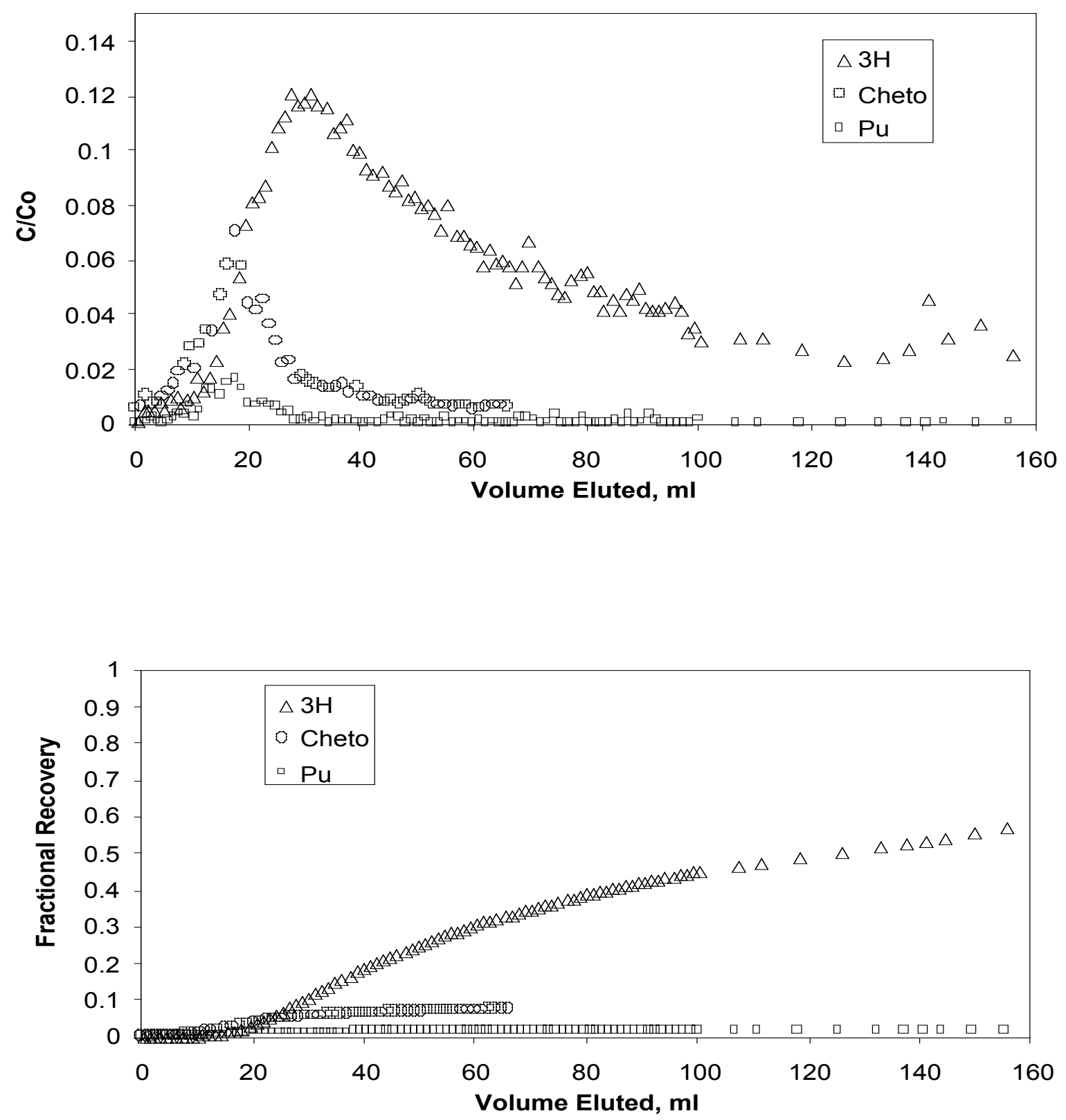

Figure C.4. Normalized concentrations and fractional recoveries in the UE20c 2858 fracture as a function of volume eluted at $\sim 0.6 \mathrm{ml} / \mathrm{hr}$ in Experiment $2 \mathrm{~b}$. 

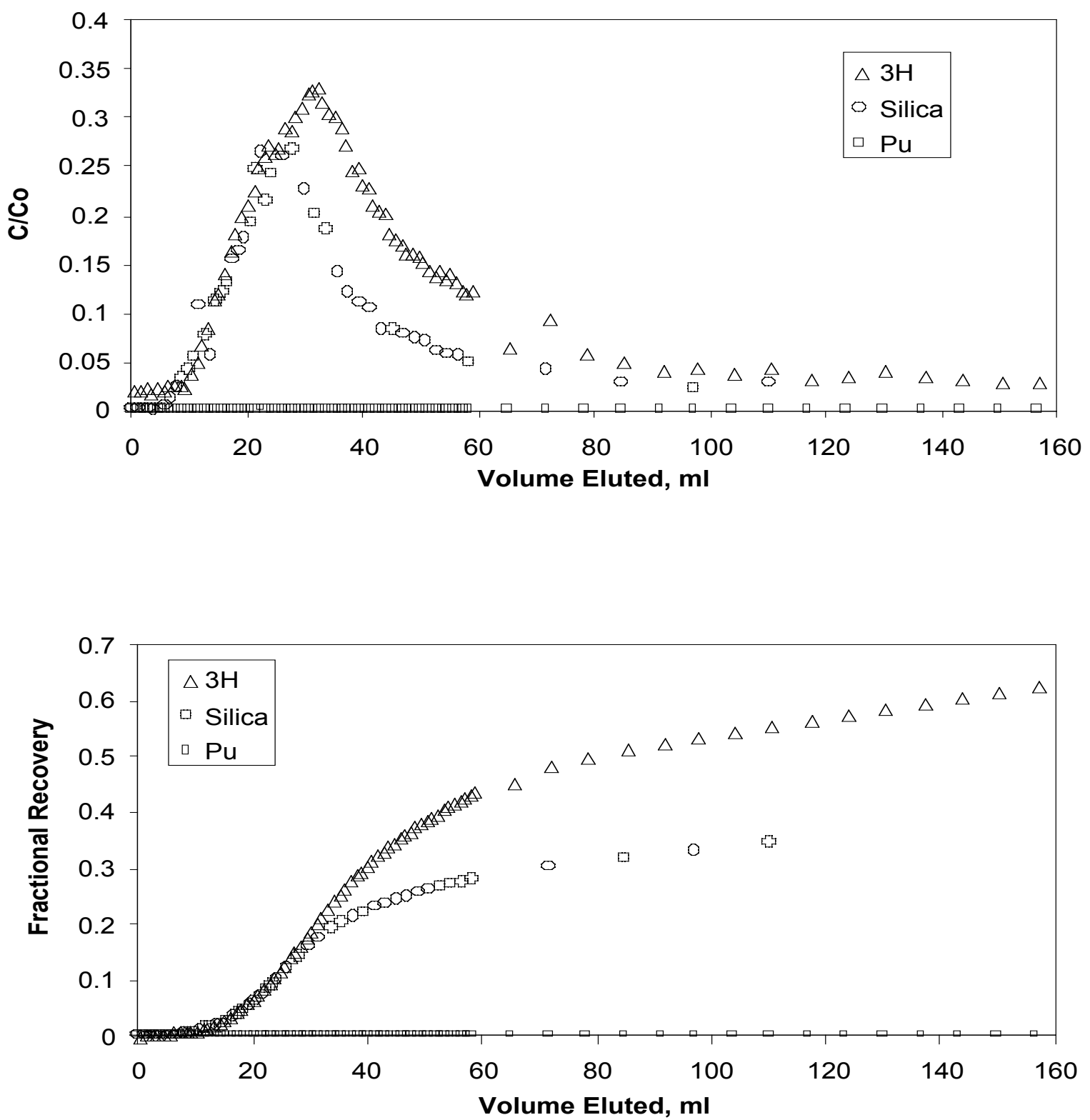

Figure C.5. Normalized concentrations and fractional recoveries in the UE20c 2851 fracture as a function of volume eluted at $\sim 1.5 \mathrm{ml} / \mathrm{hr}$ in Experiment $3 \mathrm{a}$. 

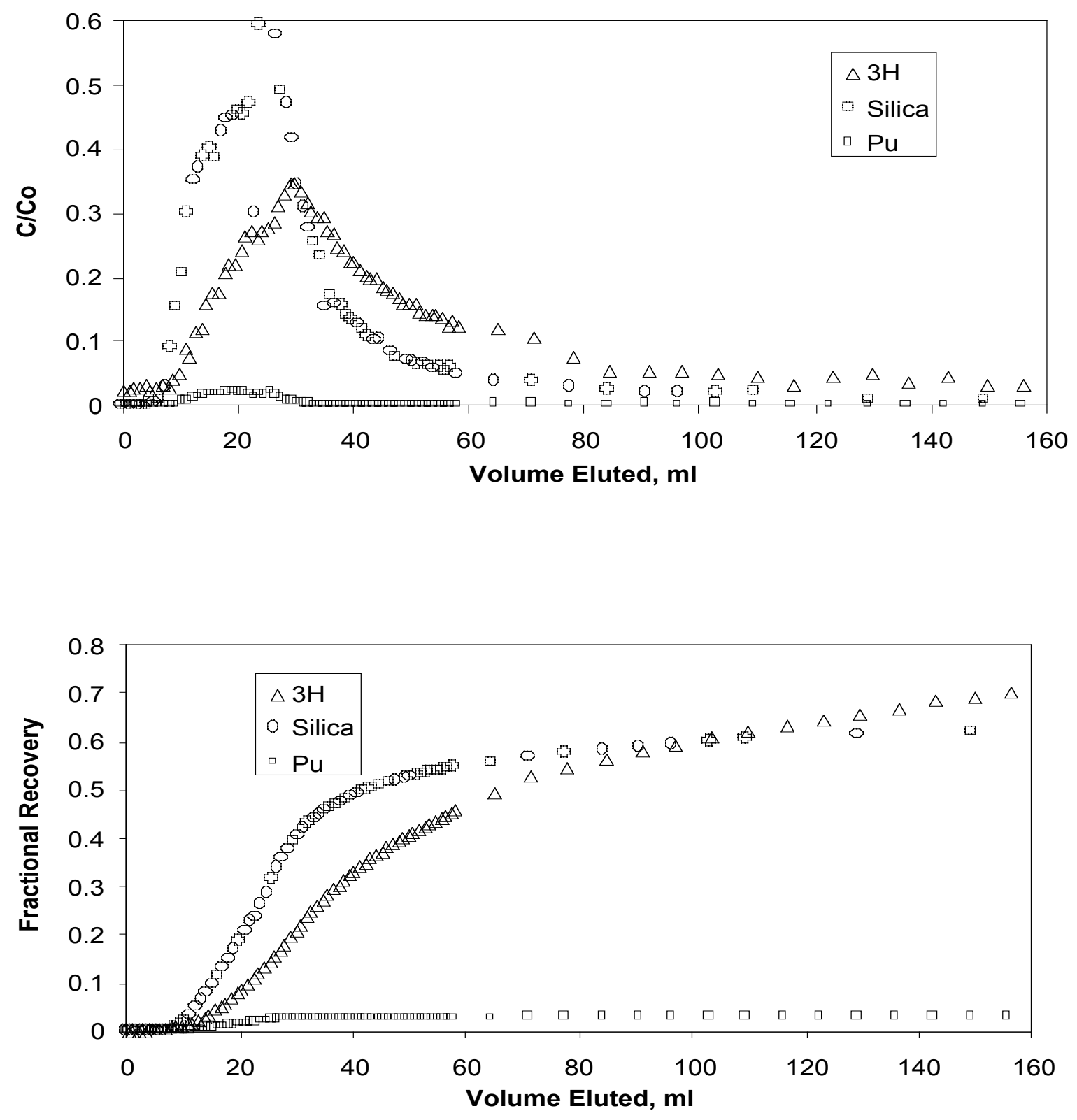

Figure C.6. Normalized concentrations and fractional recoveries in the UE20c 2858 fracture as a function of volume eluted at $\sim 1.5 \mathrm{ml} / \mathrm{hr}$ in Experiment $3 \mathrm{~b}$. 

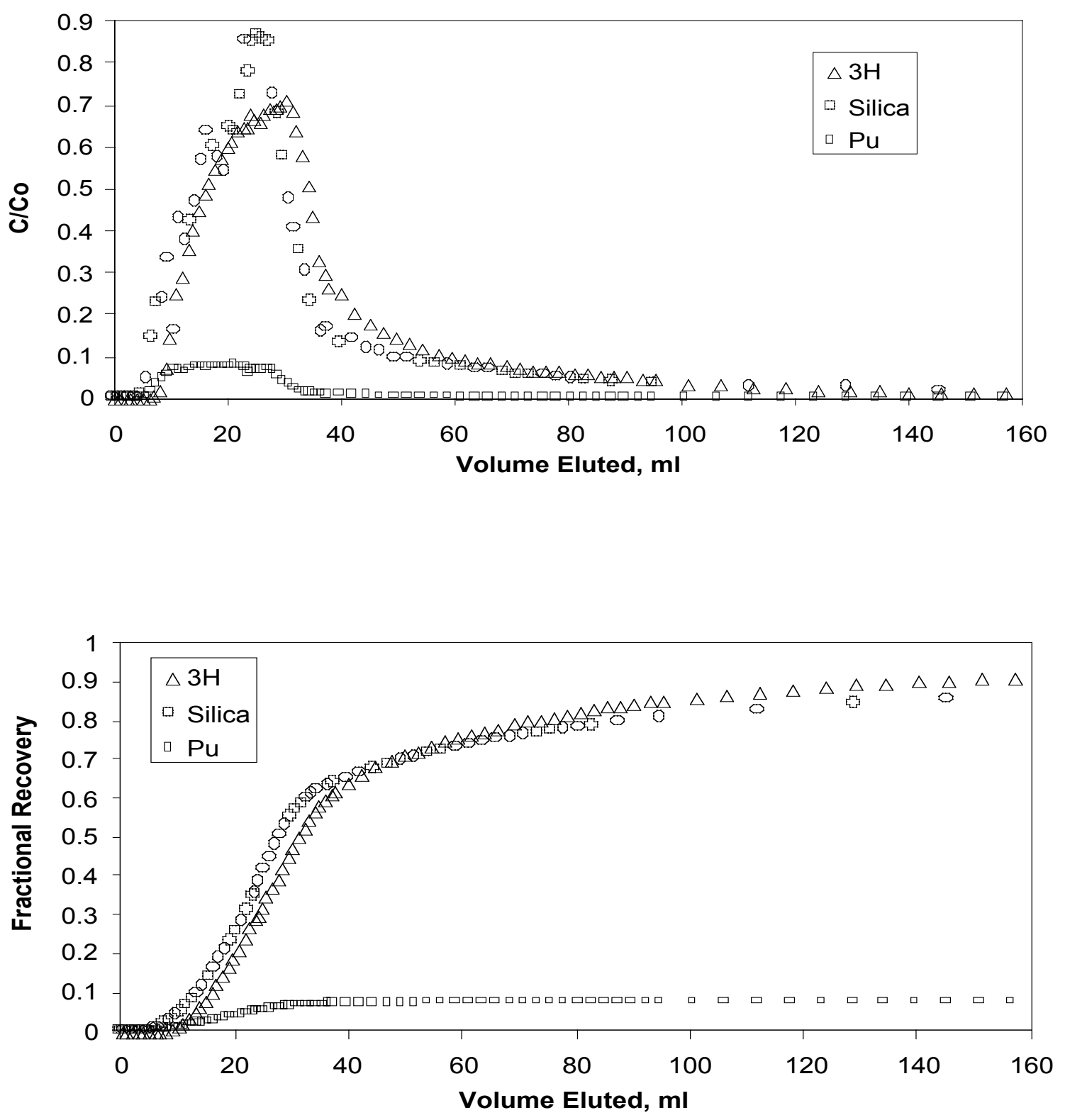

Figure C.7. Normalized concentrations and fractional recoveries in the UE20c 2851 fracture as a function of volume eluted at $\sim 4.7 \mathrm{ml} / \mathrm{hr}$ in Experiment $4 \mathrm{a}$. 

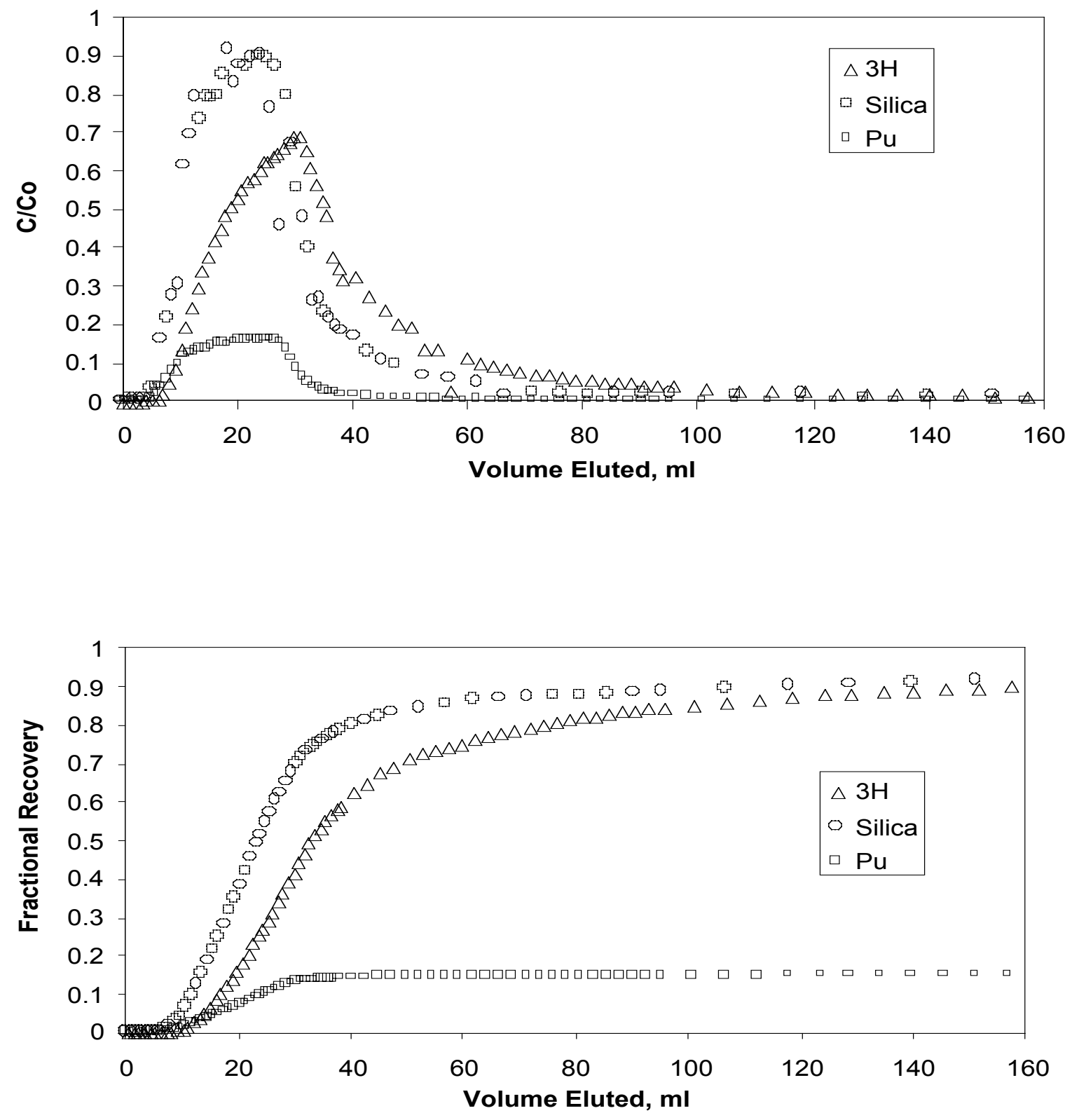

Figure C.8. Normalized concentrations and fractional recoveries in the UE20c 2858 fracture as a function of volume eluted at $\sim 4.7 \mathrm{ml} / \mathrm{hr}$ in Experiment $4 \mathrm{~b}$. 

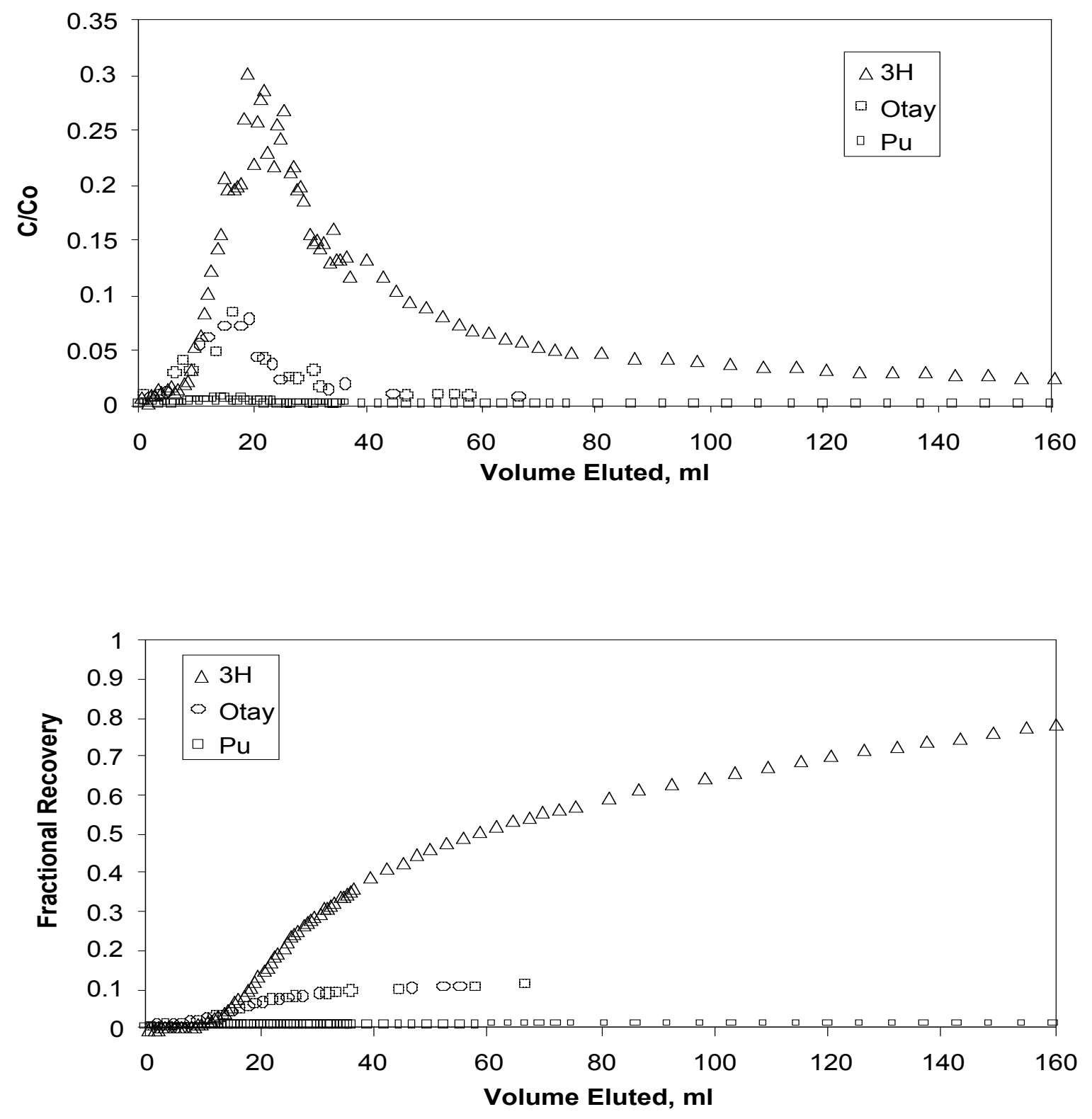

Figure C.9. Normalized concentrations and fractional recoveries in the UE20c 2851 fracture as a function of volume eluted at $\sim 1.5 \mathrm{ml} / \mathrm{hr}$ in Experiment $5 \mathrm{a}$. 

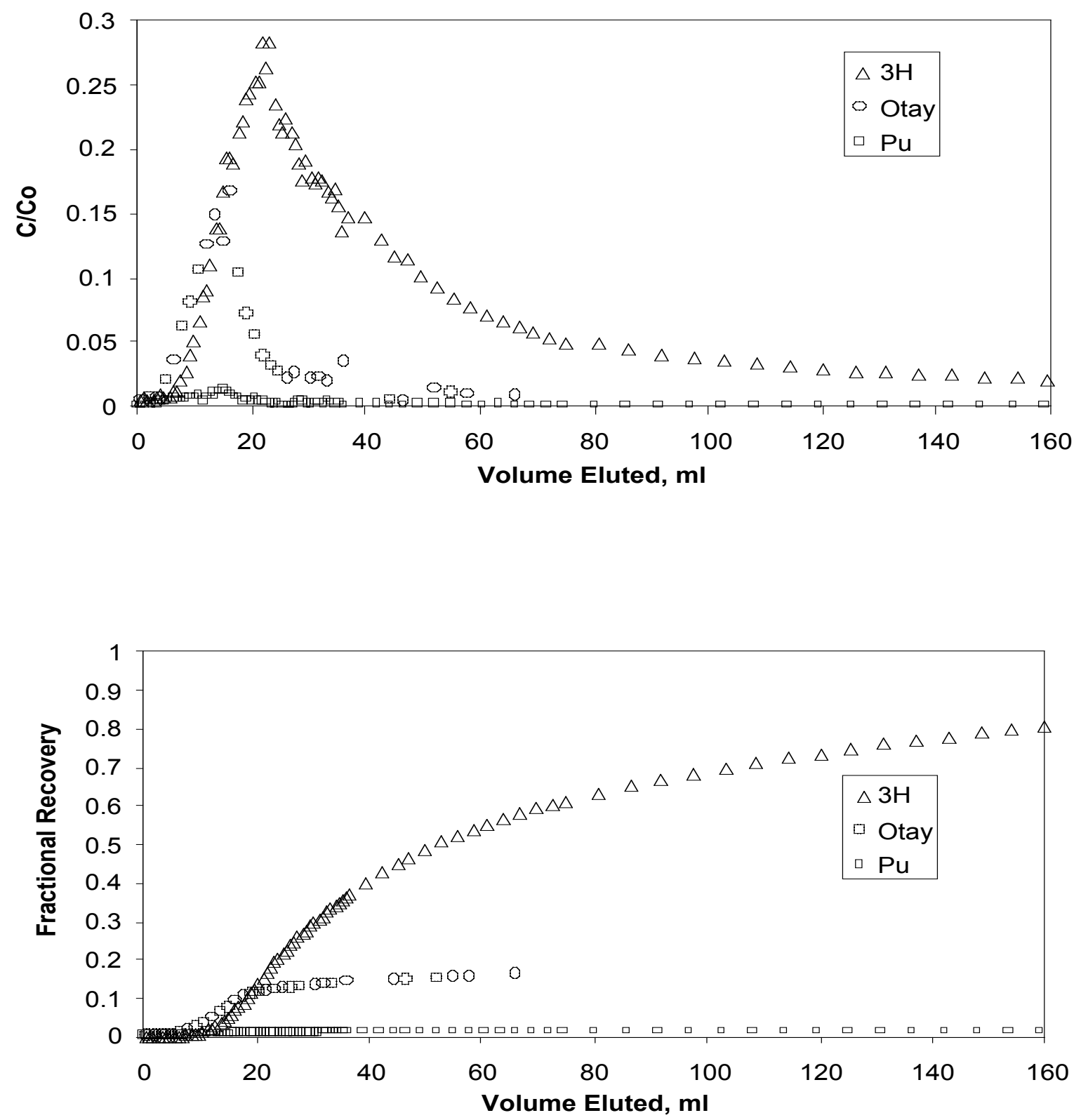

Figure C.10. Normalized concentrations and fractional recoveries in the UE20c 2858 fracture as a function of volume eluted at $\sim 1.5 \mathrm{ml} / \mathrm{hr}$ in Experiment $5 \mathrm{~b}$. 

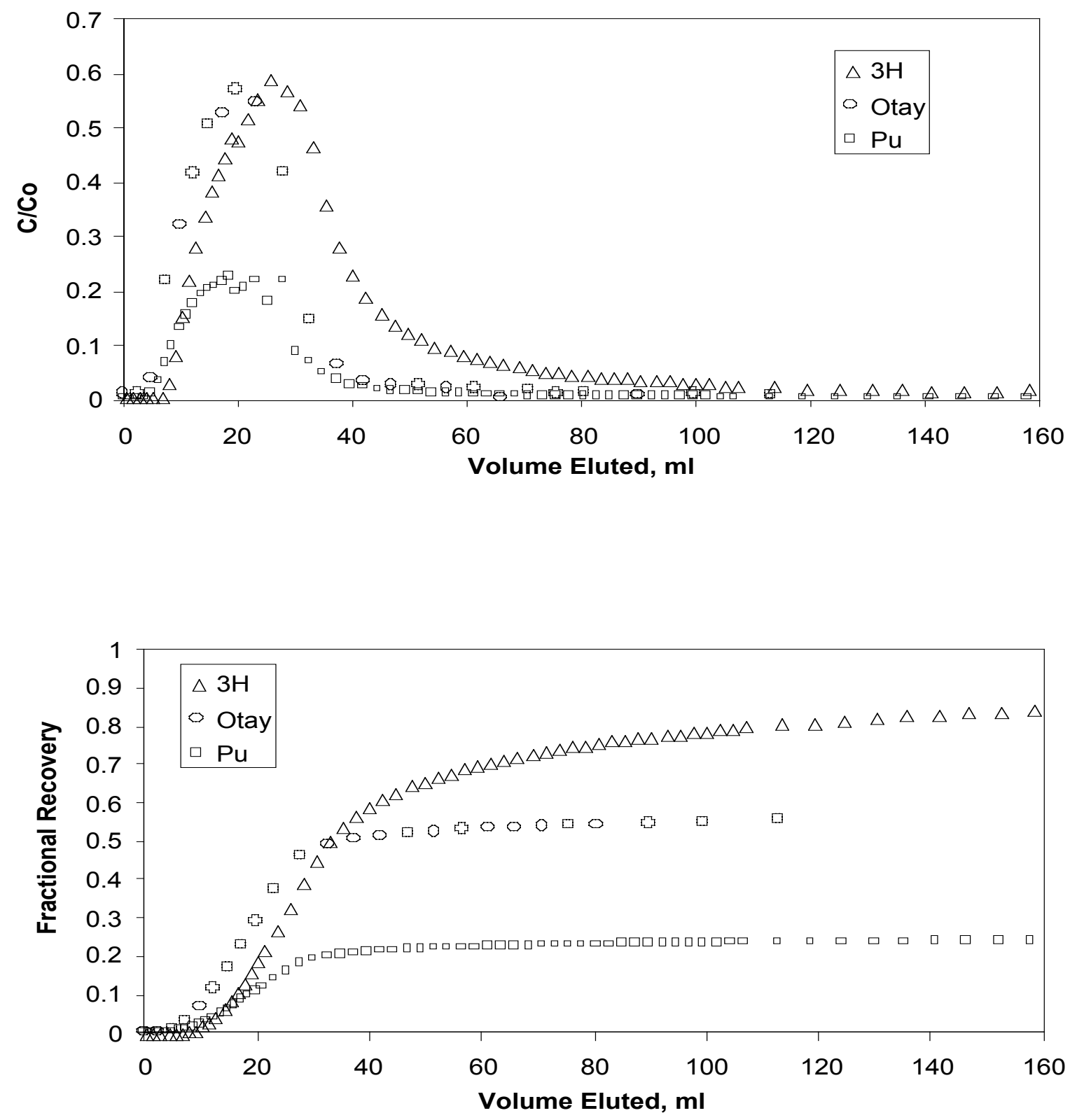

Figure C.11. Normalized concentrations and fractional recoveries in the UE20c 2851 fracture as a function of volume eluted at $\sim 4.8 \mathrm{ml} / \mathrm{hr}$ in Experiment $6 \mathrm{a}$. 

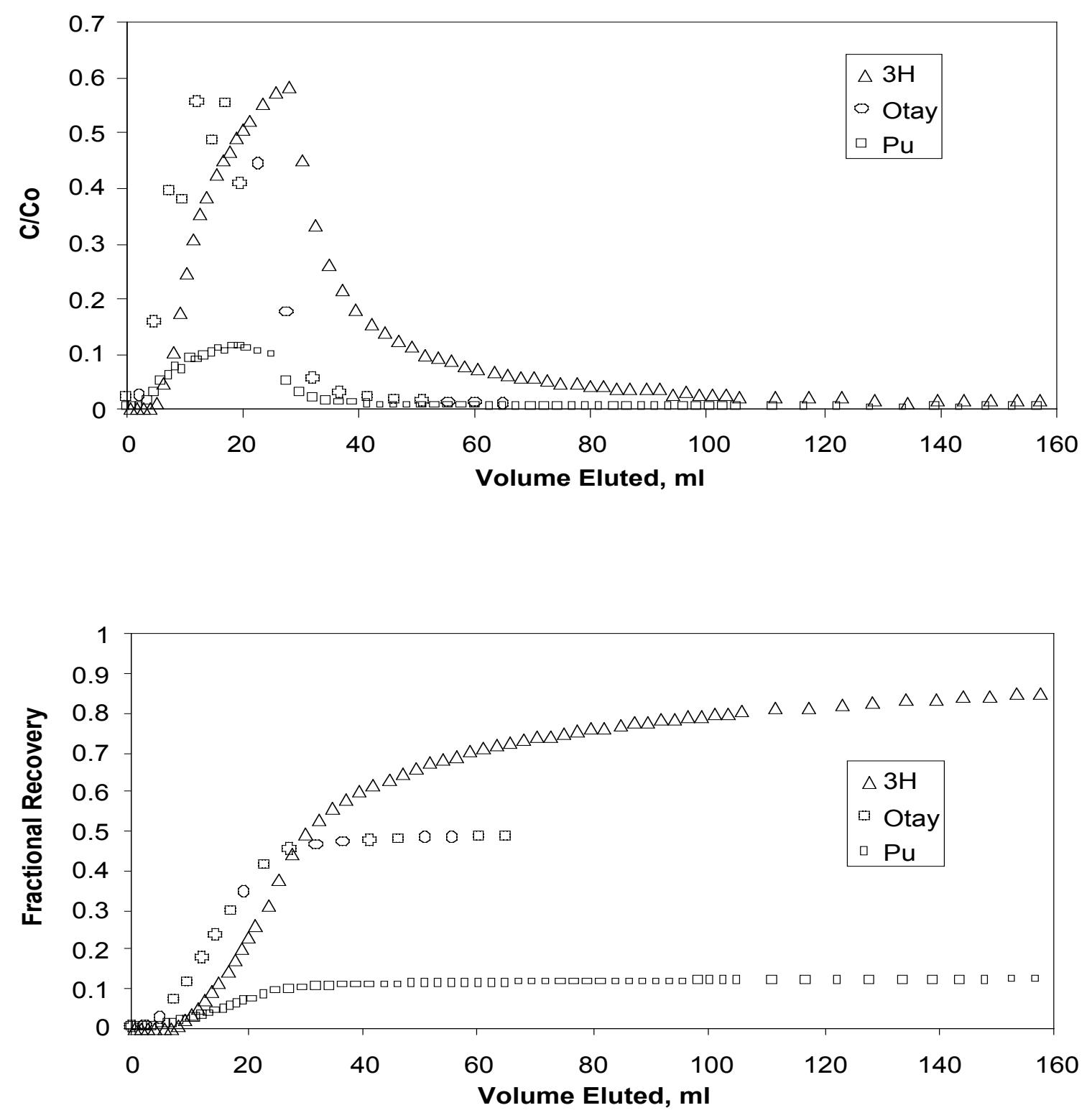

Figure C.12. Normalized concentrations and fractional recoveries in the UE20c 2858 fracture as a function of volume eluted at $\sim 4.8 \mathrm{ml} / \mathrm{hr}$ in Experiment $6 \mathrm{~b}$. 

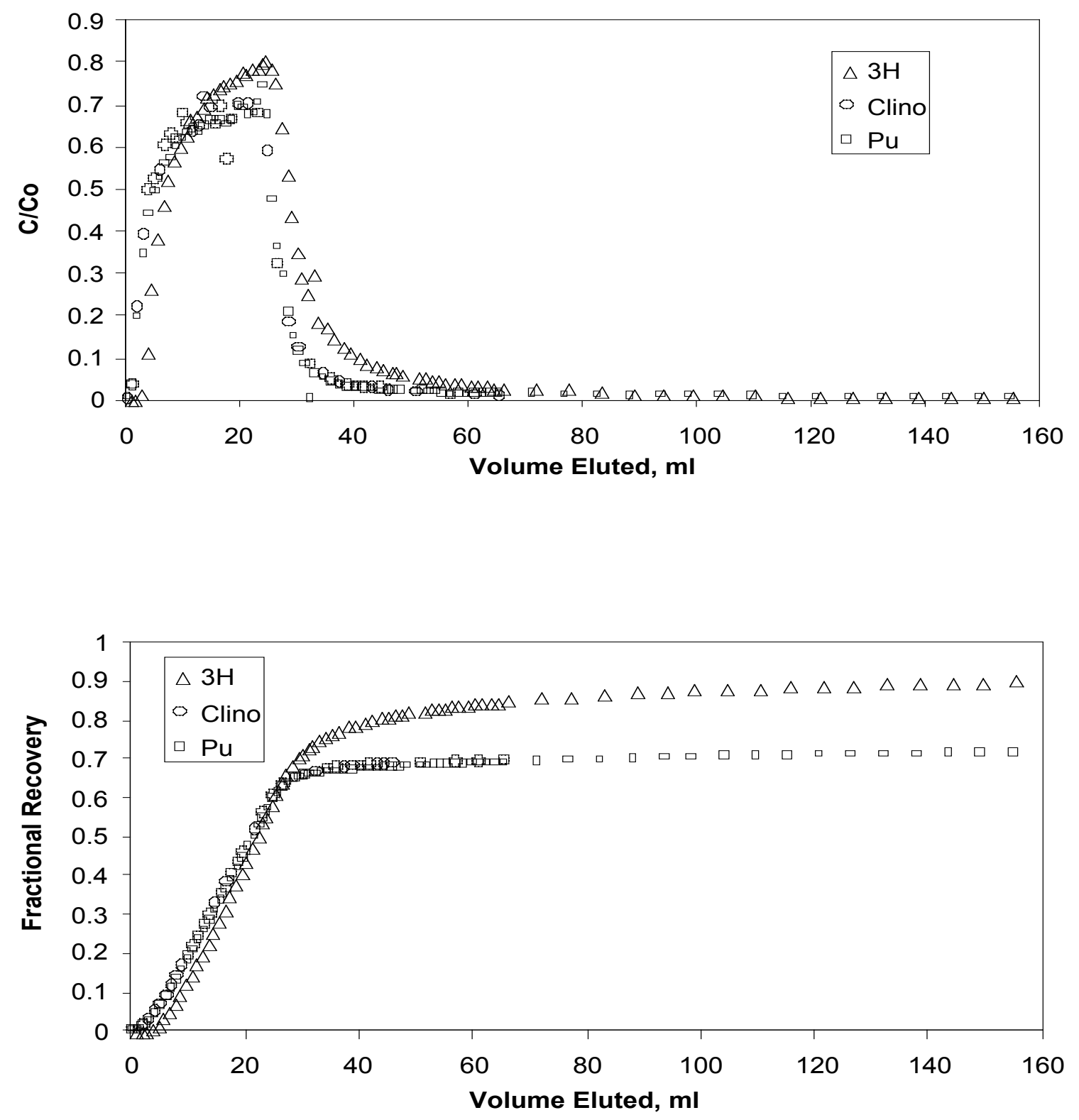

Figure C.13. Normalized concentrations and fractional recoveries in the PM.2, 4177 fracture as a function of volume eluted at $\sim 1.4 \mathrm{ml} / \mathrm{hr}$ in Experiment $7 \mathrm{a}$. 

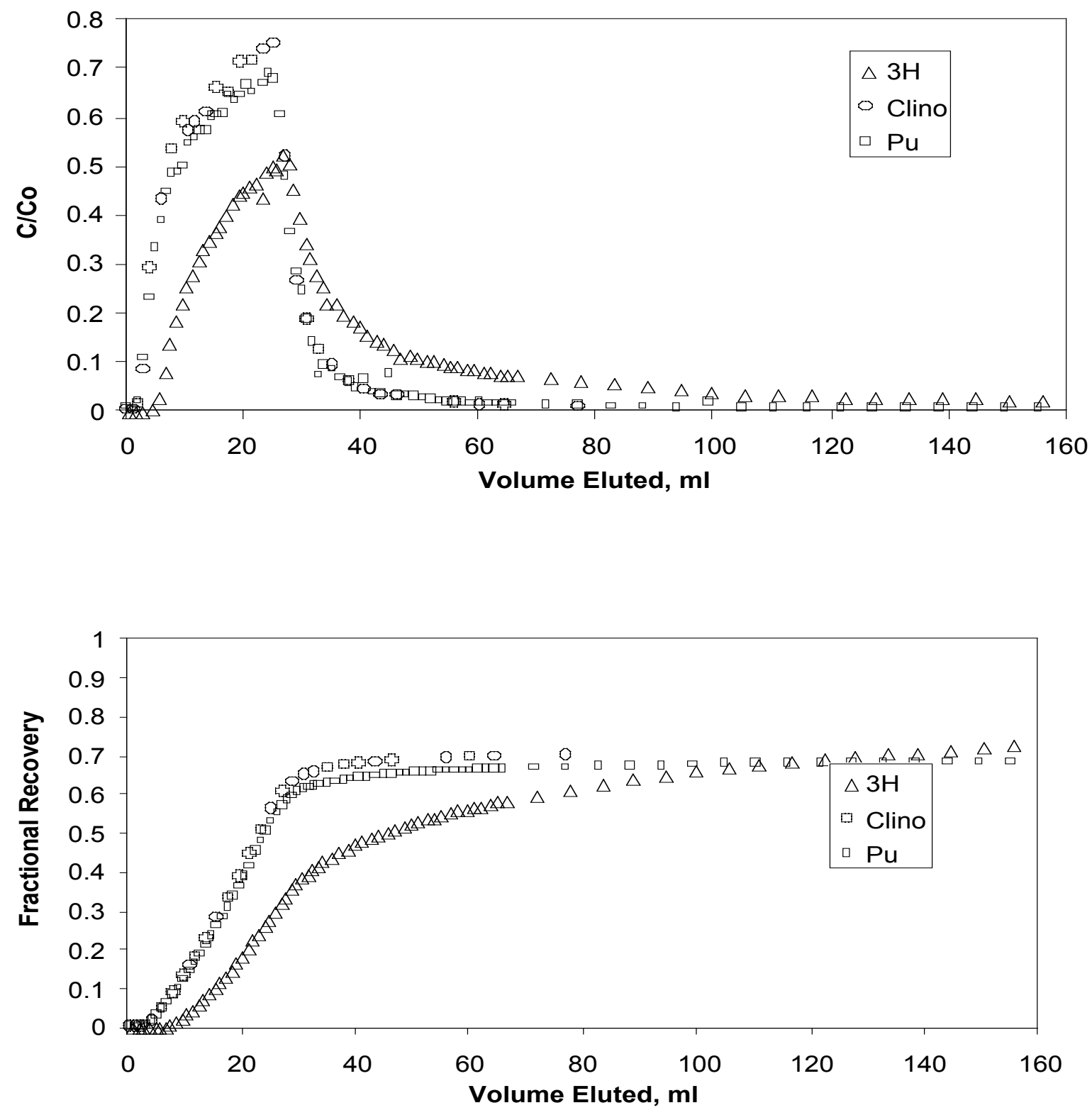

Figure C.14. Normalized concentrations and fractional recoveries in the PM-1, 4823 fracture as a function of volume eluted at $\sim 1.4 \mathrm{ml} / \mathrm{hr}$ in Experiment $7 \mathrm{~b}$. 

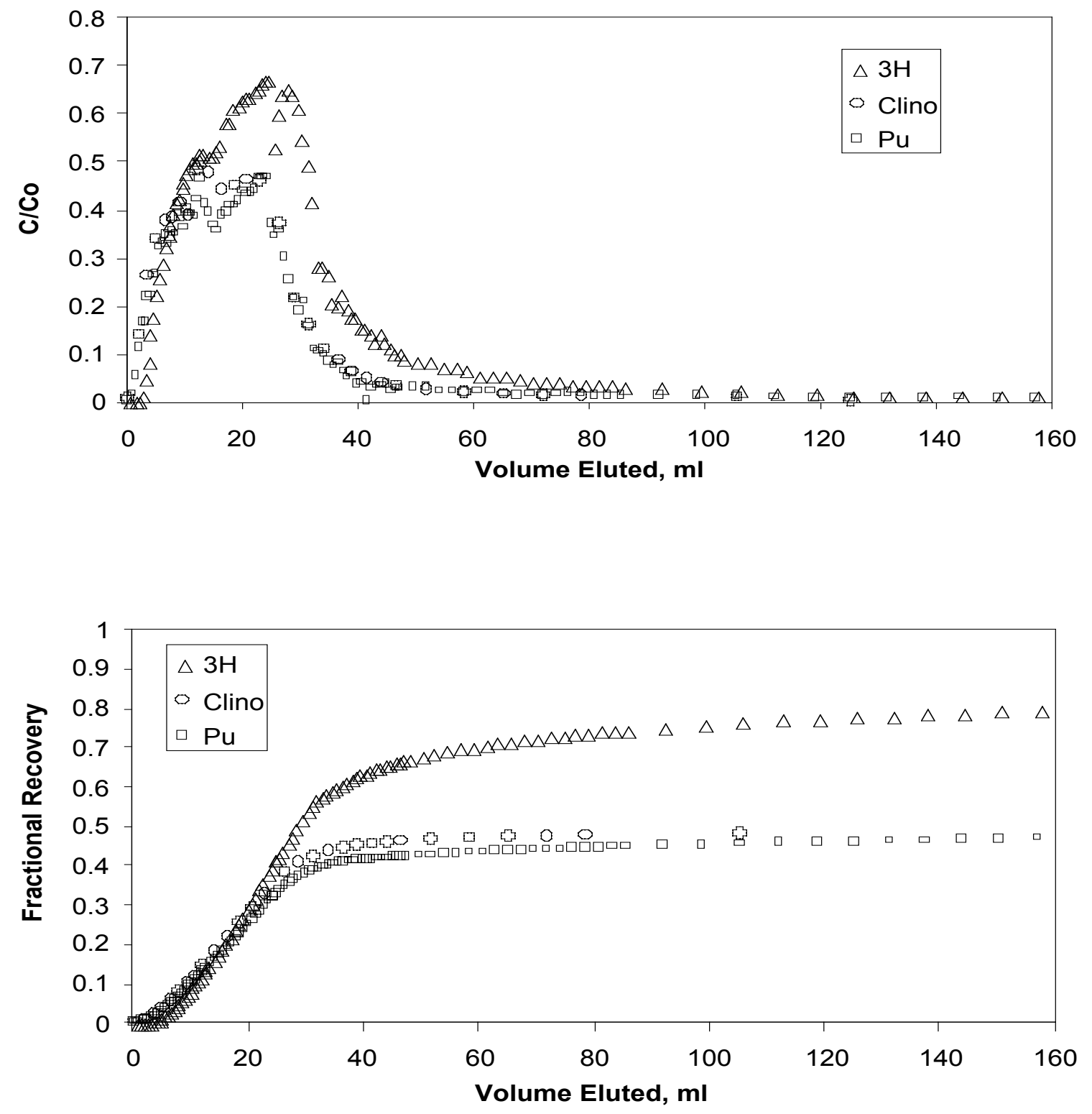

Figure C.15. Normalized concentrations and fractional recoveries in the PM-2, 4177 fracture as a function of volume eluted at $\sim 0.5 \mathrm{ml} / \mathrm{hr}$ in Experiment $8 \mathrm{a}$. 

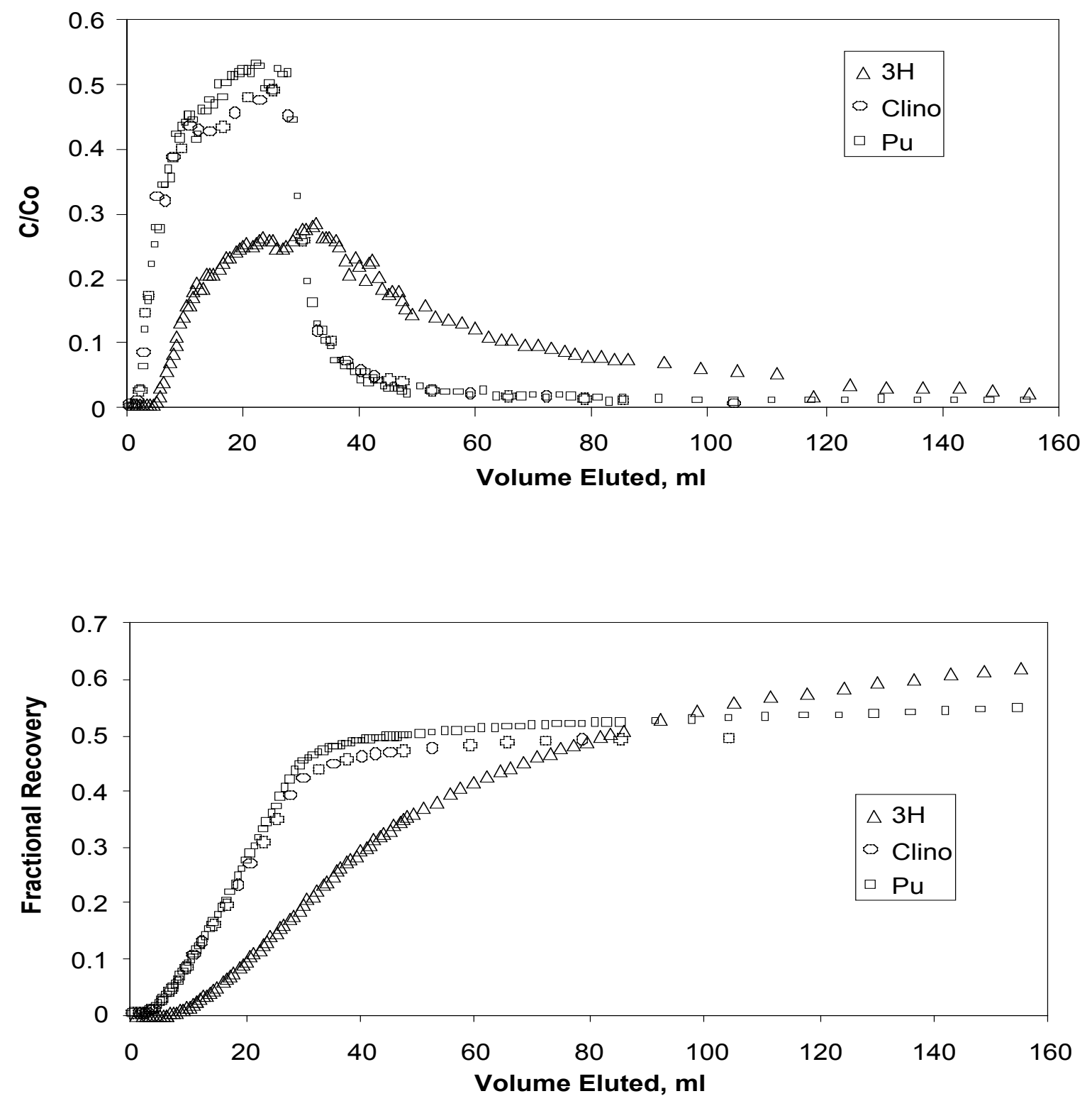

Figure C.16. Normalized concentrations and fractional recoveries in the PM-1, 4823 fracture as a function of volume eluted at $\sim 0.5 \mathrm{ml} / \mathrm{hr}$ in Experiment $8 \mathrm{~b}$. 

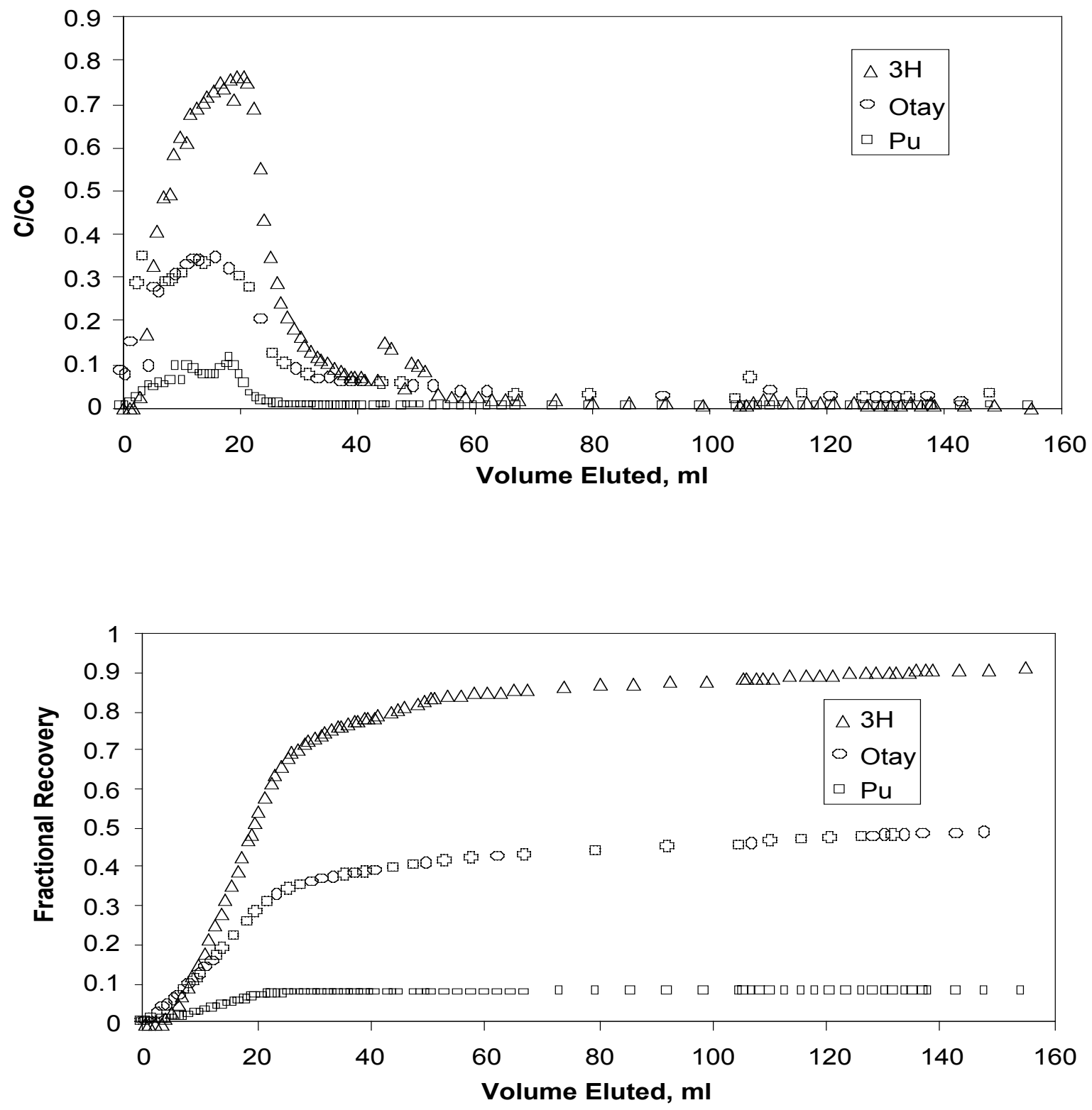

Figure C.17. Normalized concentrations and fractional recoveries in the PM-2, 4177 fracture as a function of volume eluted at $\sim 1.5 \mathrm{ml} / \mathrm{hr}$ in Experiment $9 \mathrm{a}$. 

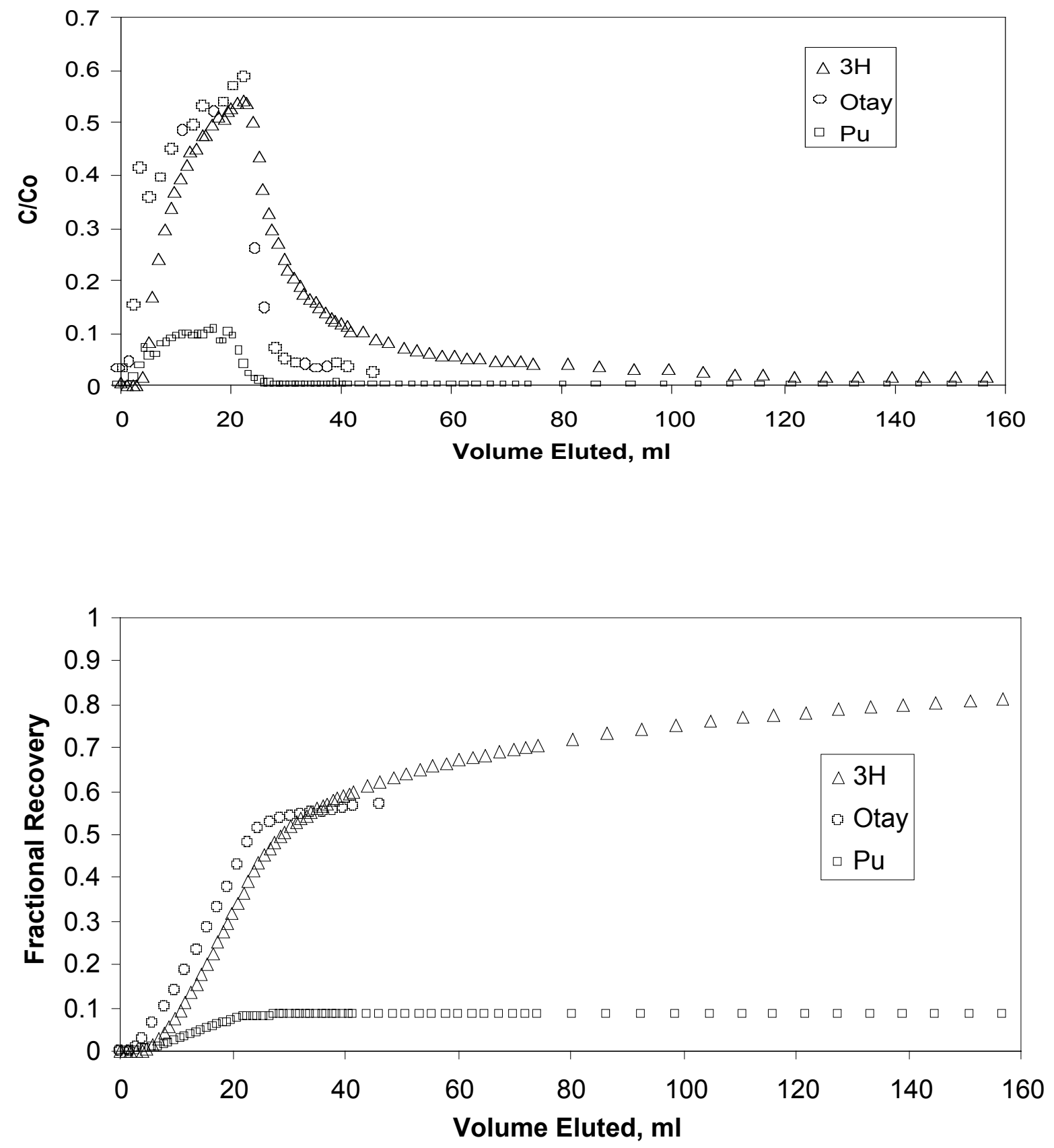

Figure C.18. Normalized concentrations and fractional recoveries in the PM-1, 4823 fracture as a function of volume eluted at $\sim 1.5 \mathrm{ml} / \mathrm{hr}$ in Experiment $9 \mathrm{~b}$. 

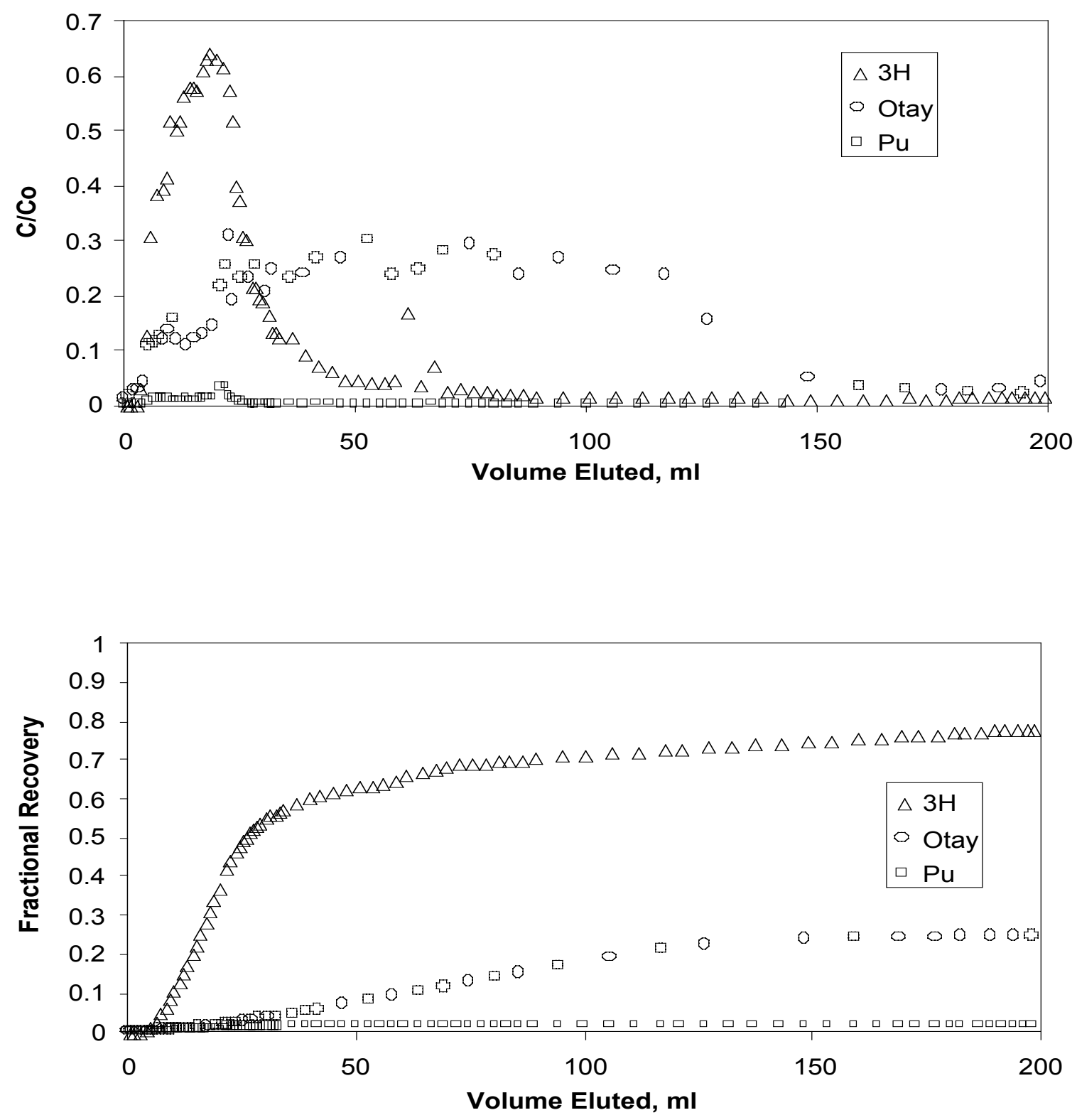

Figure C.19. Normalized concentrations and fractional recoveries in the PM-2, 4177 fracture as a function of volume eluted at $\sim 0.4 \mathrm{ml} / \mathrm{hr}$ in Experiment 10a. 

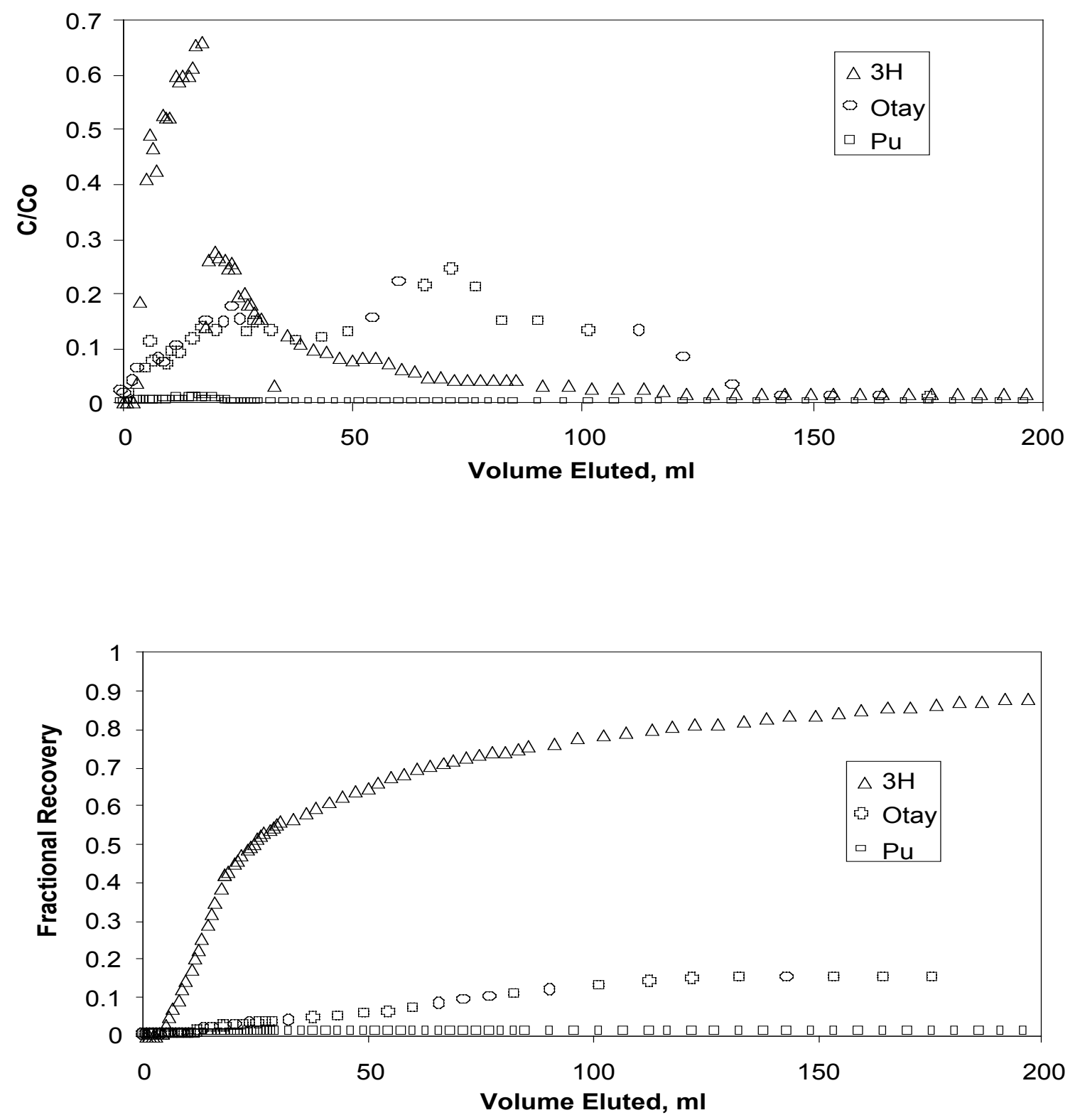

Figure C.20. Normalized concentrations and fractional recoveries in the PM-1, 4823 fracture as a function of volume eluted at $\sim 0.4 \mathrm{ml} / \mathrm{hr}$ in Experiment $10 \mathrm{~b}$. 

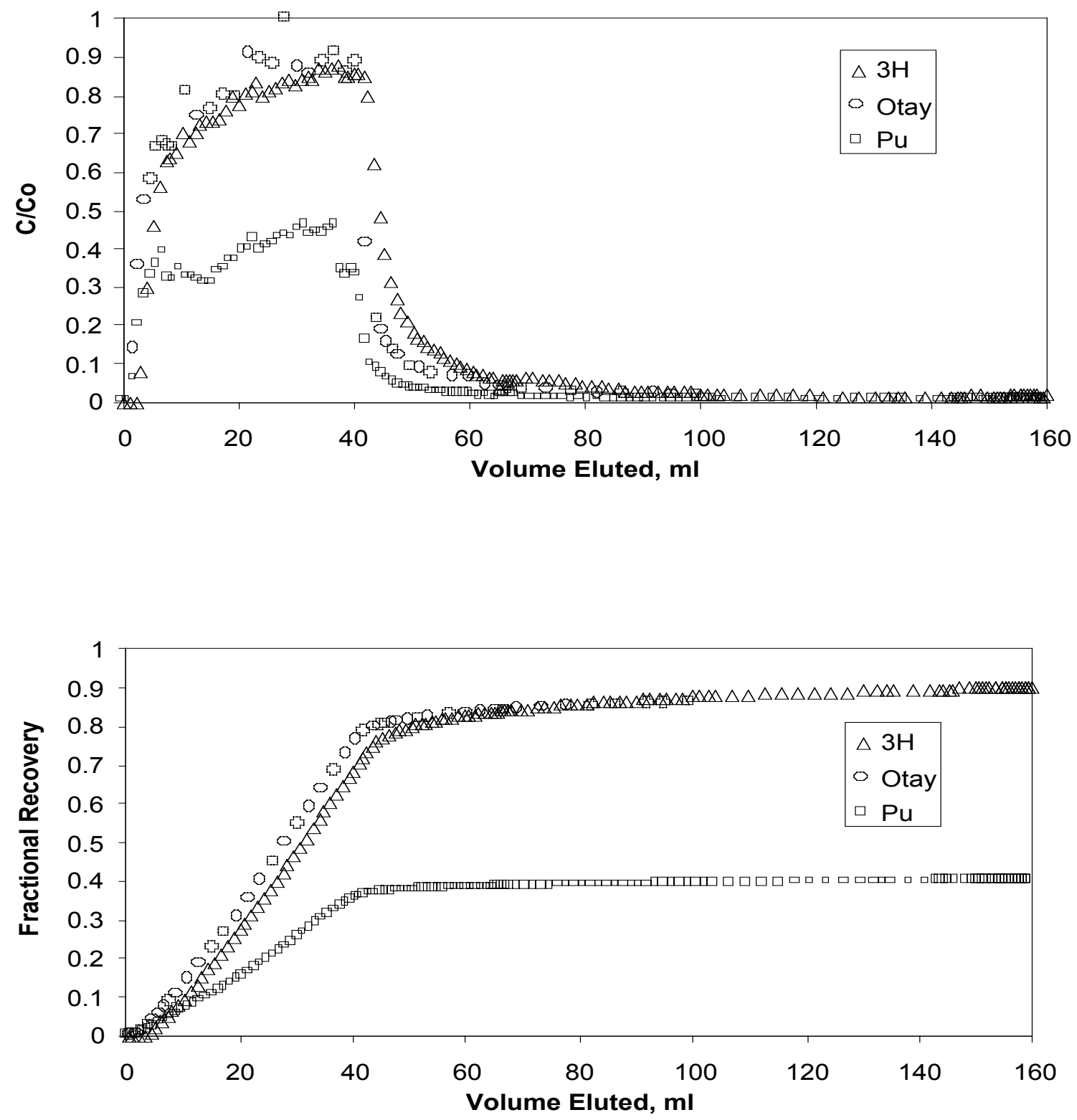

Figure C.21. Normalized concentrations and fractional recoveries in the PM-2, 4177 fracture as a function of volume eluted at $\sim 1.5 \mathrm{ml} / \mathrm{hr}$ in Experiment 11 . 


\title{
-Appendix D- \\ Simultaneous RELAP Fits to Tritiated Water Data in Each Pair of Fracture Experiments for a Given Colloid
}

\author{
Paul W. Reimus
}

This appendix contains plots showing the simultaneous RELAP fits to the normalized concentrations of tritiated water as a function of time in each pair of fracture experiments for a given colloid. The model parameters providing the best fits are listed in tables in Chapter 8.

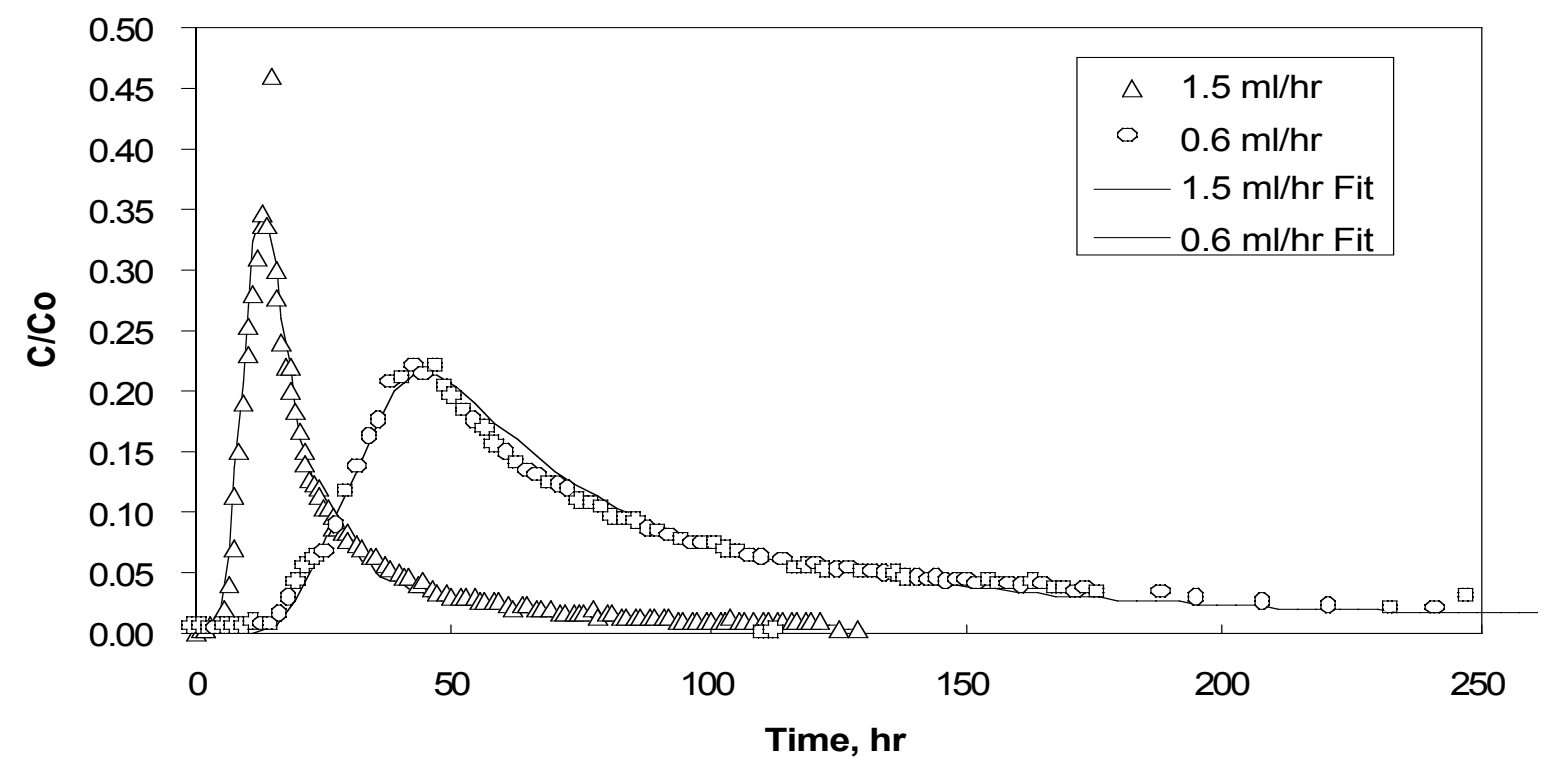

Figure D.1. Simultaneous RELAP fits to the ${ }^{3} \mathrm{HHO}$ normalized concentrations in the UE20c 2851 fracture for the experiments with Cheto montmorillonite colloids (1a and 2a). 


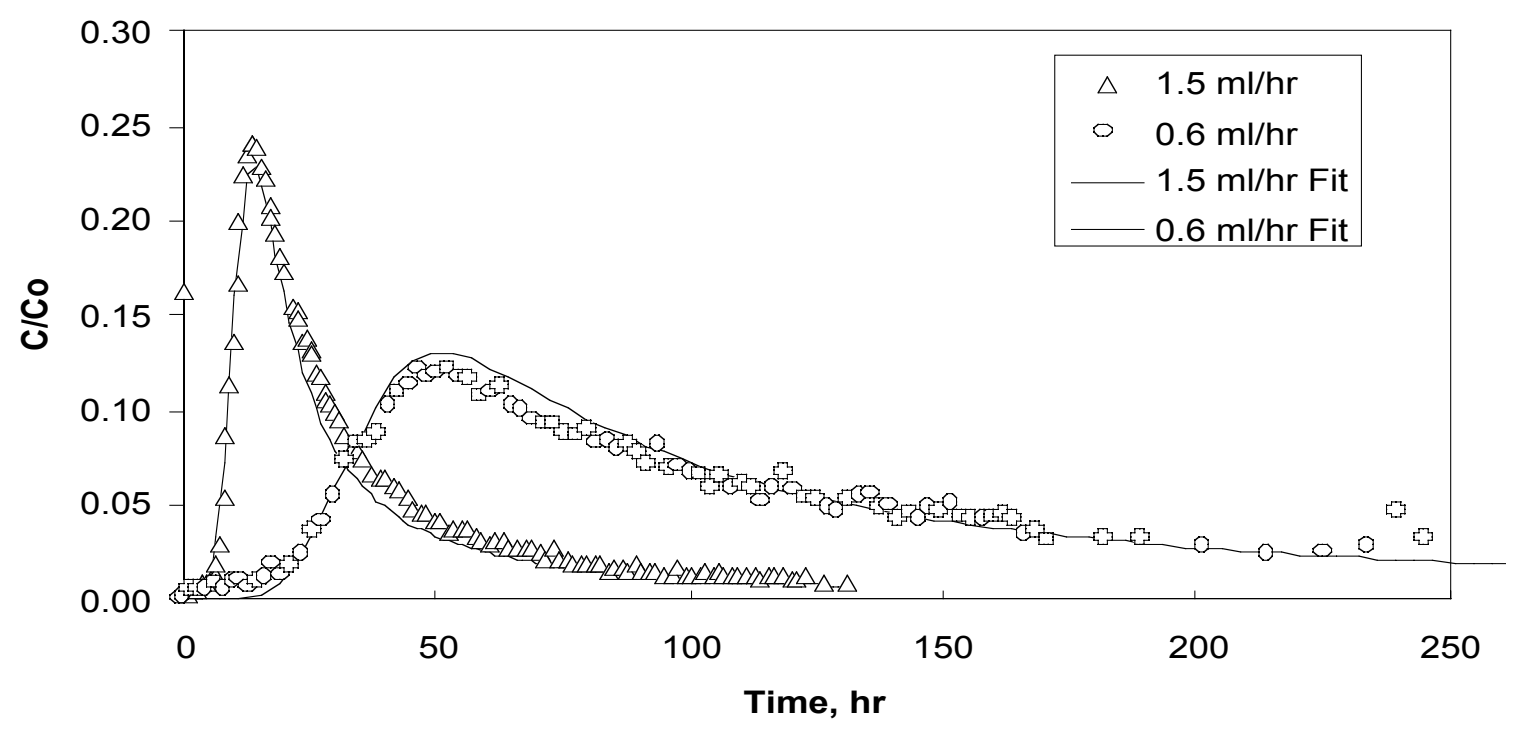

Figure D.2. Simultaneous RELAP fits to the ${ }^{3} \mathrm{HHO}$ normalized concentrations in the UE20c 2858 fracture for the experiments with Cheto montmorillonite colloids ( $1 \mathrm{~b}$ and $2 \mathrm{~b}$ ).

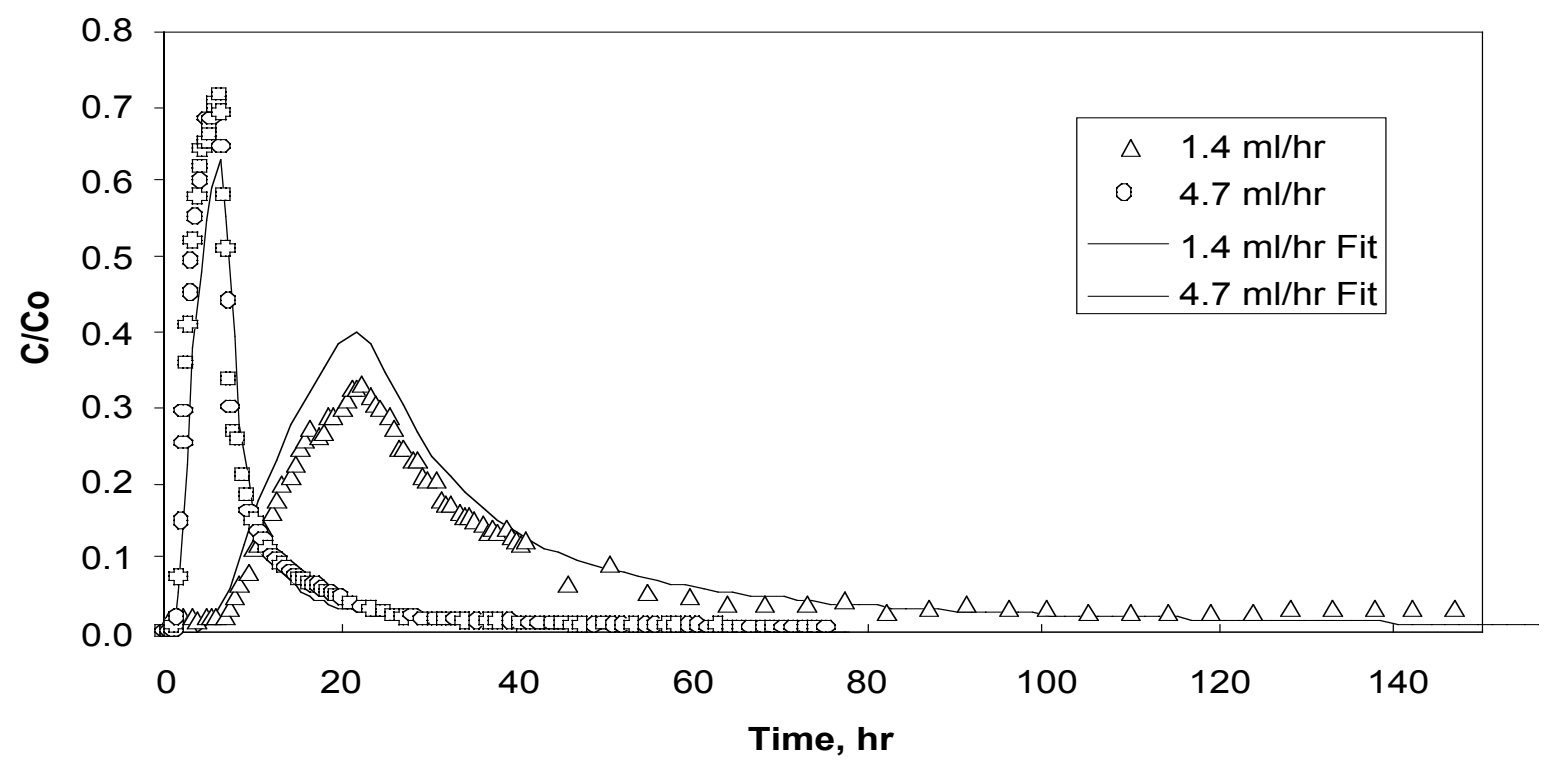

Figure D.3. Simultaneous RELAP fits to the ${ }^{3} \mathrm{HHO}$ normalized concentrations in the UE20c 2851 fracture for the experiments with Silica colloids (3a and 4a). 


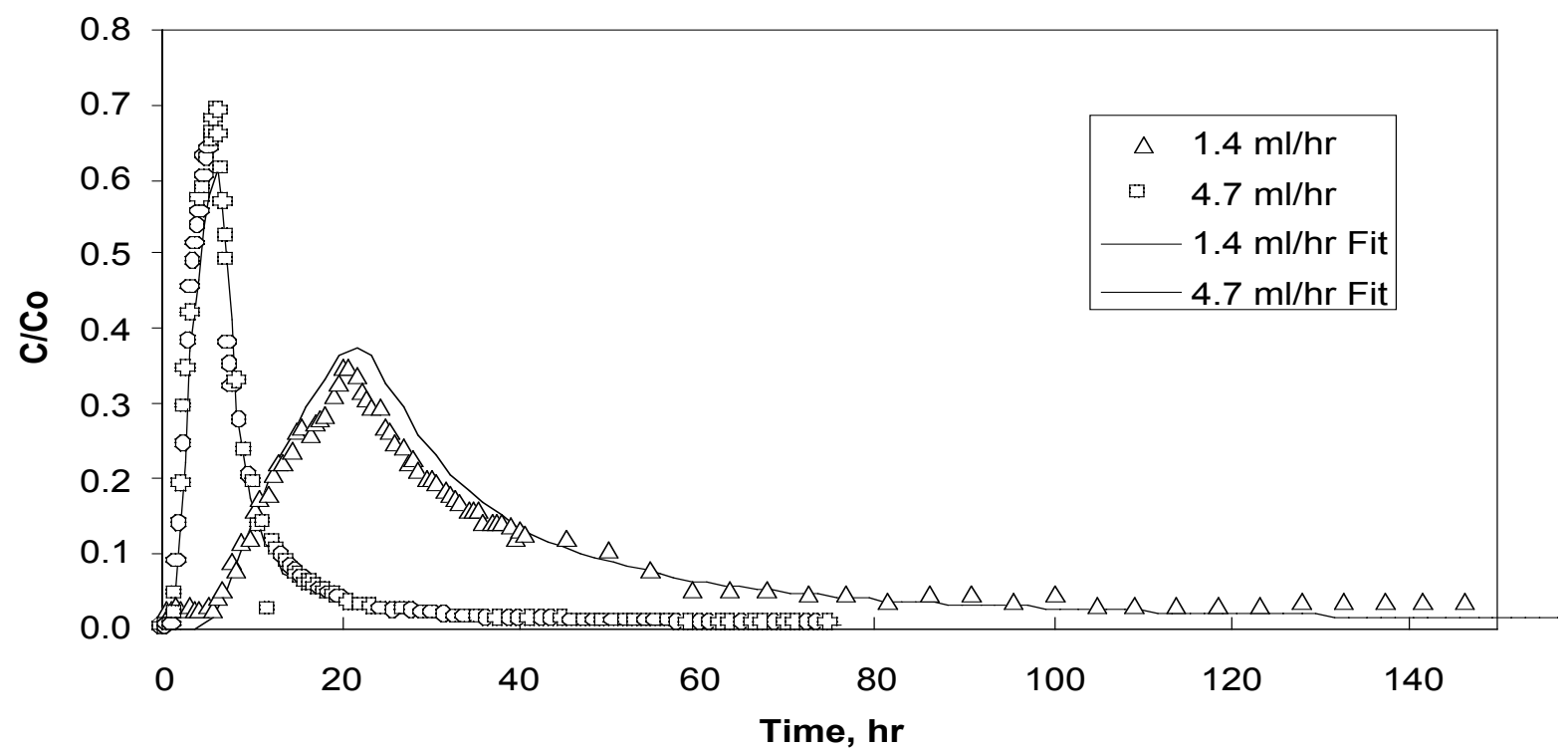

Figure D.4. Simultaneous RELAP fits to the ${ }^{3} \mathrm{HHO}$ normalized concentrations in the UE20c 2858 fracture for the experiments with Silica colloids ( $3 b$ and $4 b)$.

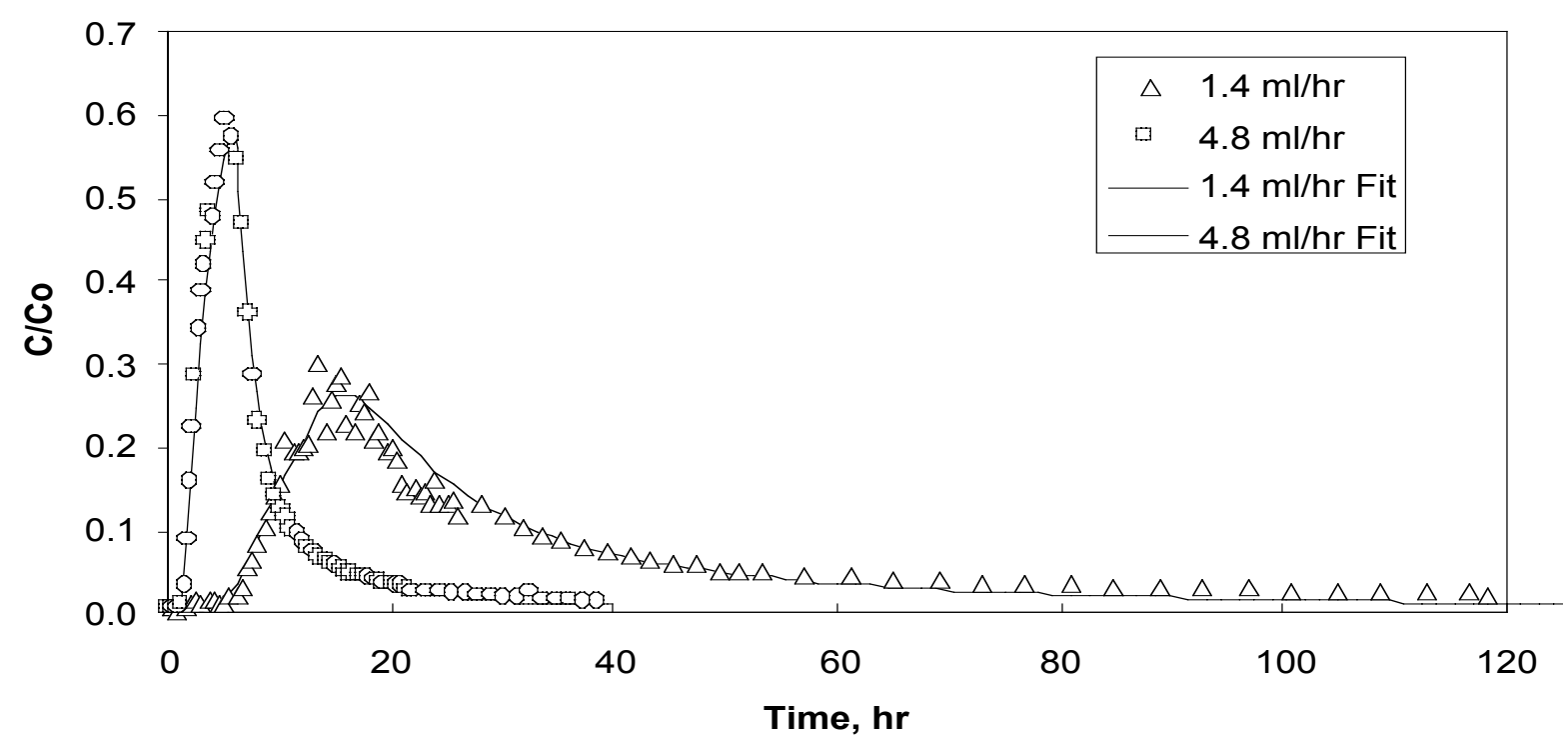

Figure D.5. Simultaneous RELAP fits to the ${ }^{3} \mathrm{HHO}$ normalized concentrations in the UE20c 2851 fracture for the experiments with batch 1 Otay montmorillonite colloids (5a and 6a). 


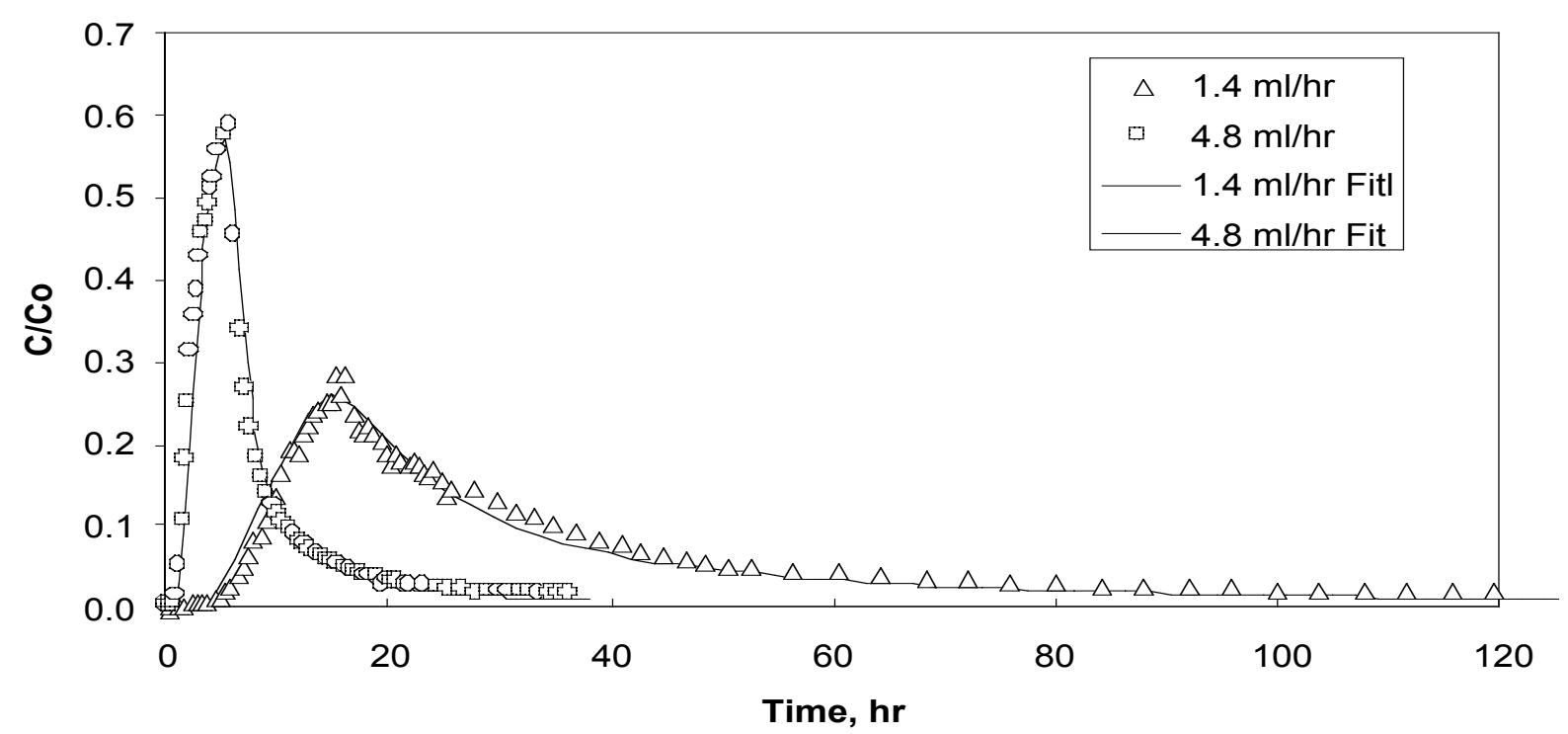

Figure D.6. Simultaneous RELAP fits to the ${ }^{3} \mathrm{HHO}$ normalized concentrations in the UE20c 2858 fracture for the experiments with batch 1 Otay montmorillonite colloids (5b and 6b).

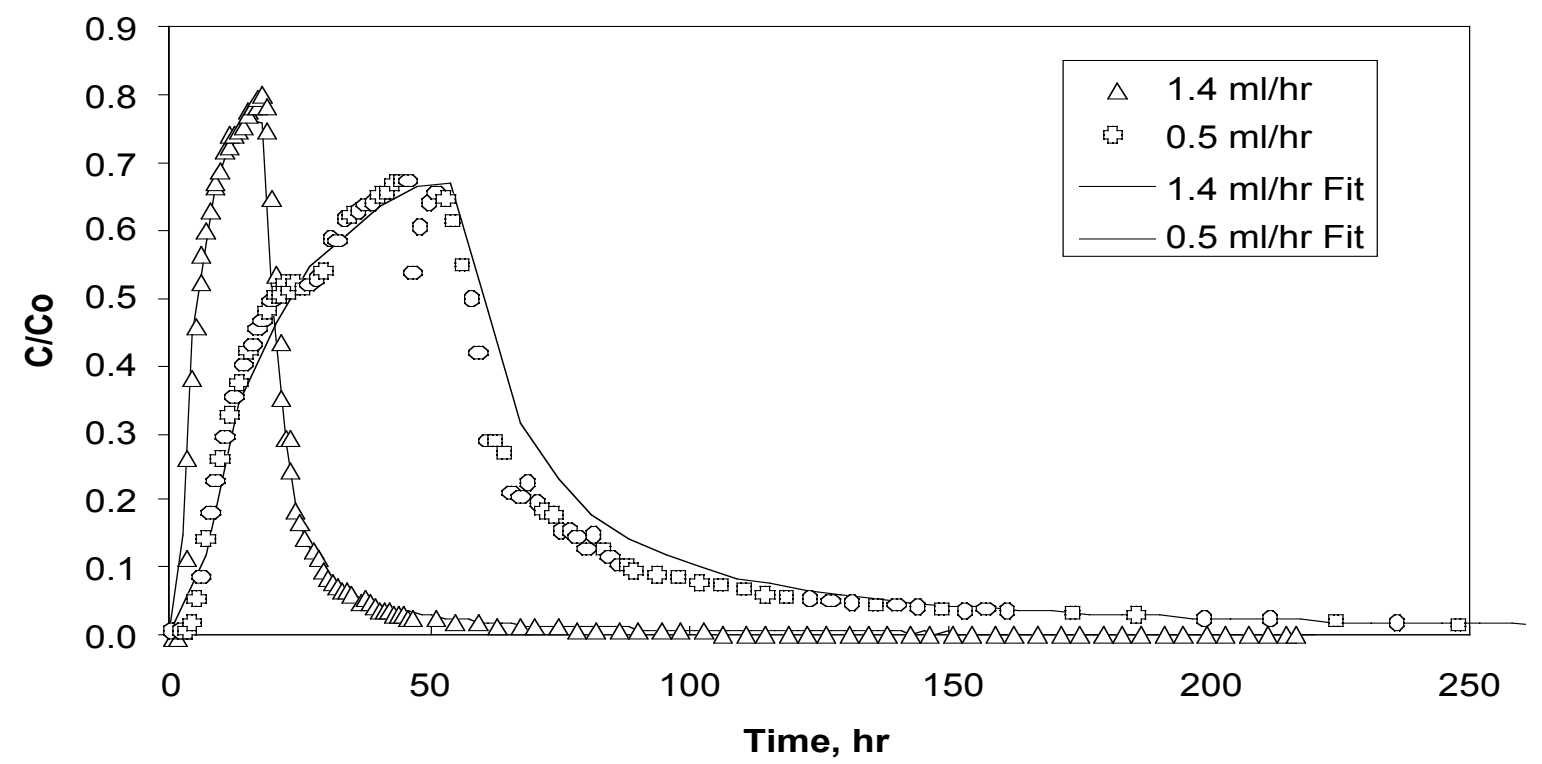

Figure D.7. Simultaneous RELAP fits to the ${ }^{3} \mathrm{HHO}$ normalized concentrations in the PM.-2, 4177 fracture for the experiments with clinoptilolite colloids (7a and 8a). 


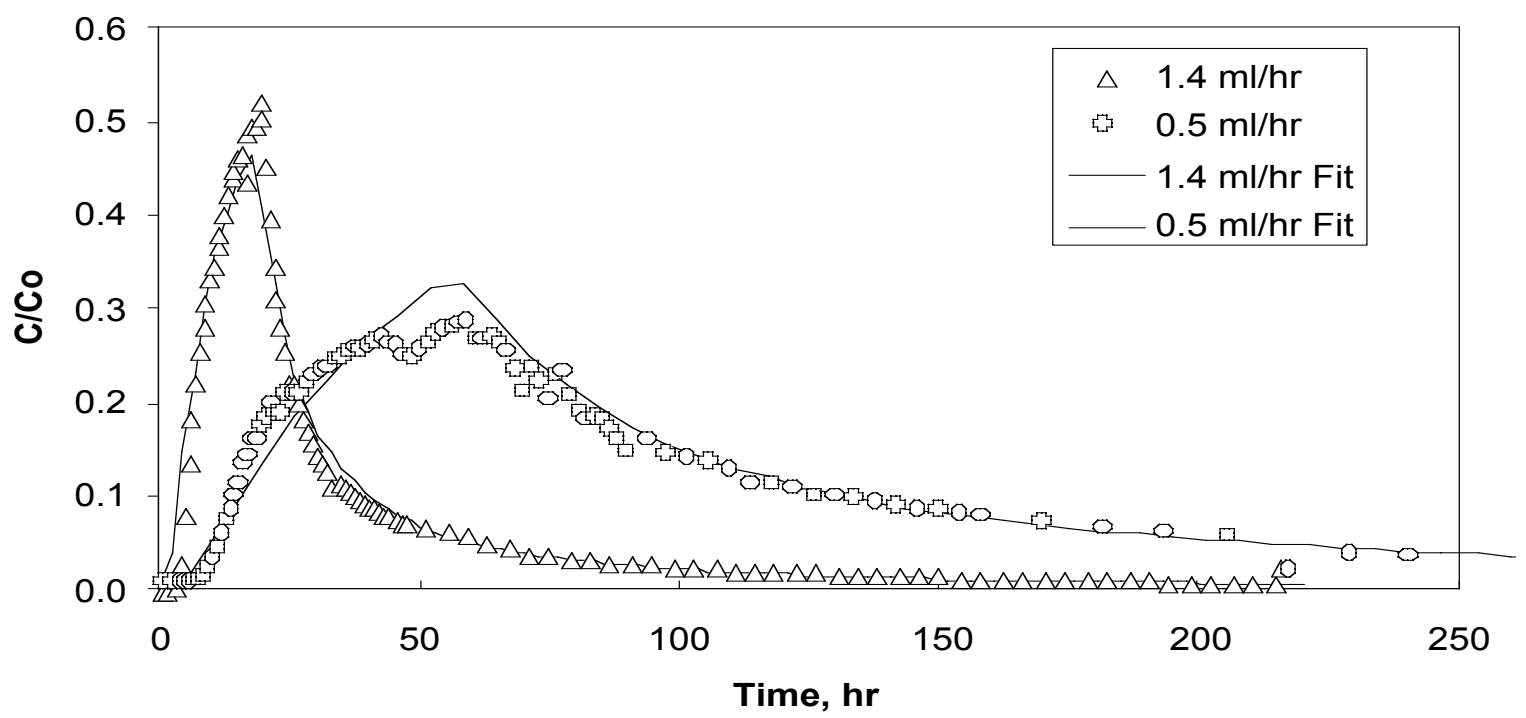

Figure D.8. Simultaneous RELAP fits to the ${ }^{3} \mathrm{HHO}$ normalized concentrations in the PM-1, 4832 fracture for the experiments with clinoptilolite colloids ( $7 \mathrm{~b}$ and $8 \mathrm{~b}$ ).

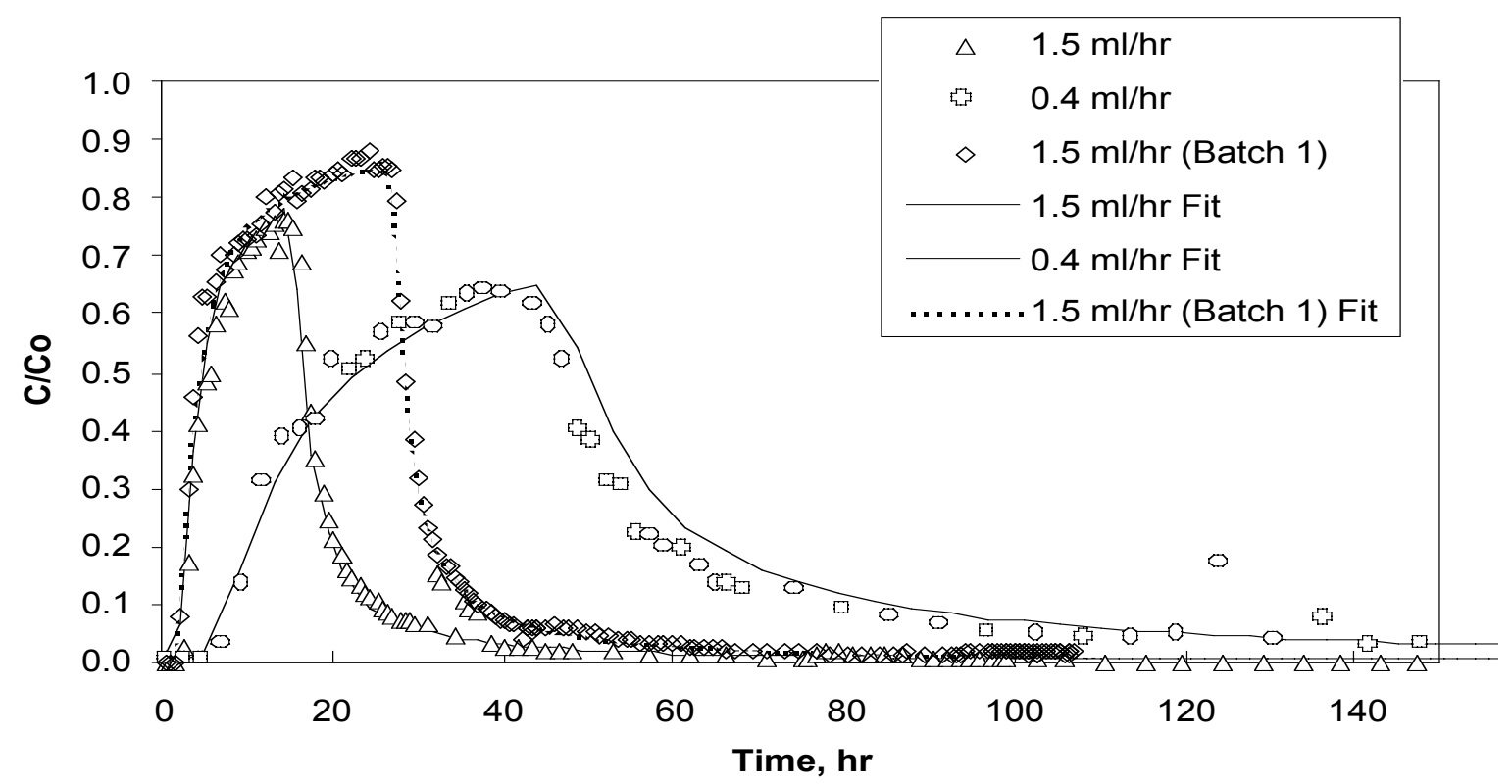

Figure D.9. Simultaneous RELAP fits to the ${ }^{3} \mathrm{HHO}$ normalized concentrations in the PM-2, 4177 fracture for the experiments with batch 2 and batch 1 Otay montmorillonite colloids (9a, $10 \mathrm{a}$ and 11). 


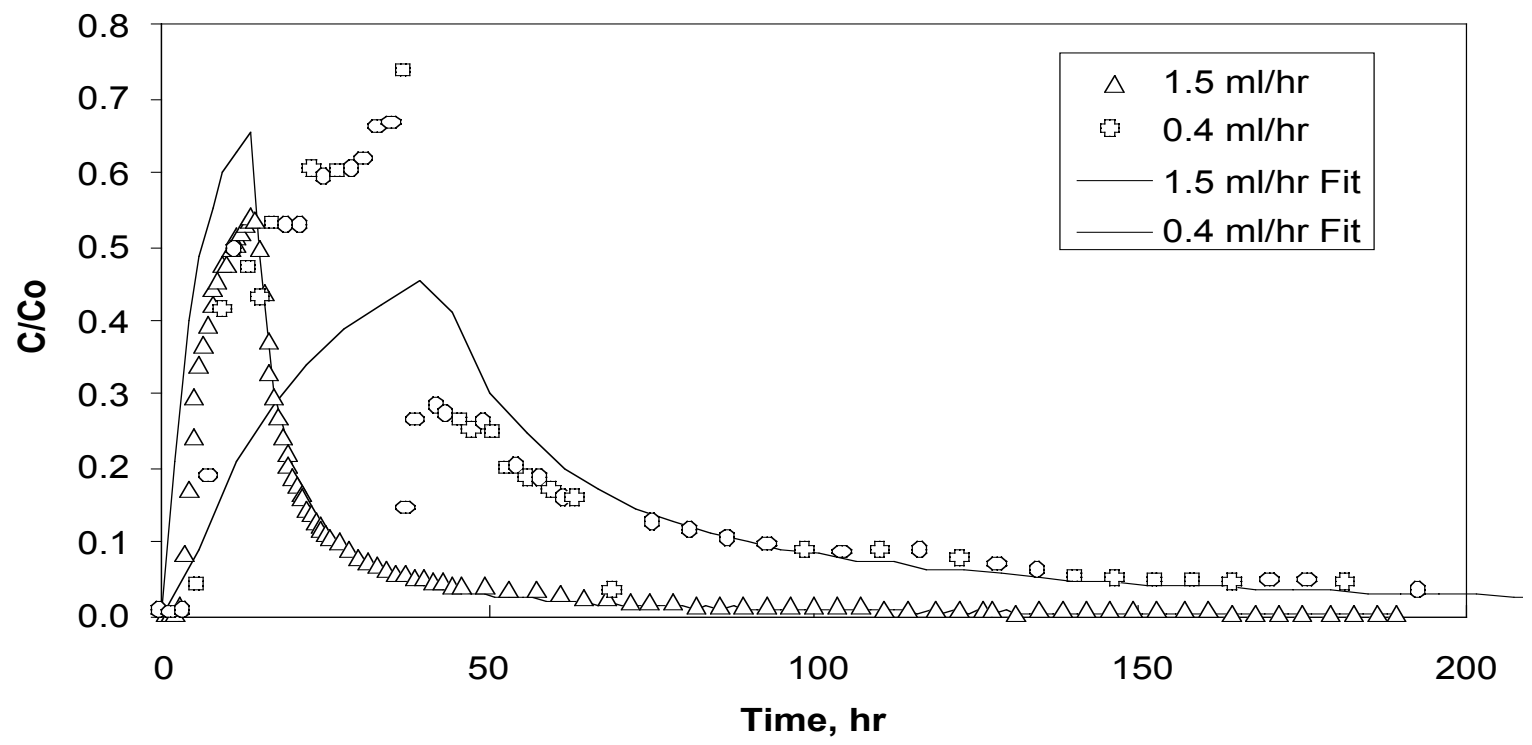

Figure D.10. Simultaneous RELAP fits to the ${ }^{3} \mathrm{HHO}$ normalized concentrations in the PM-1, 4823 fracture for the experiments with batch 2 Otay montmorillonite colloids ( $9 \mathrm{~b}$ and $10 \mathrm{~b}$ ). 


\title{
-Appendix E- \\ RELAP Fits to Colloid Data in Each Pair of Fracture Experiments for a Given Colloid
}

\author{
Paul W. Reimus
}

This appendix contains plots showing the RELAP fits to the normalized concentrations of colloids as a function of time in each pair of fracture experiments for a given colloid. The model parameters providing the best fits are listed in tables in Chapter 8.

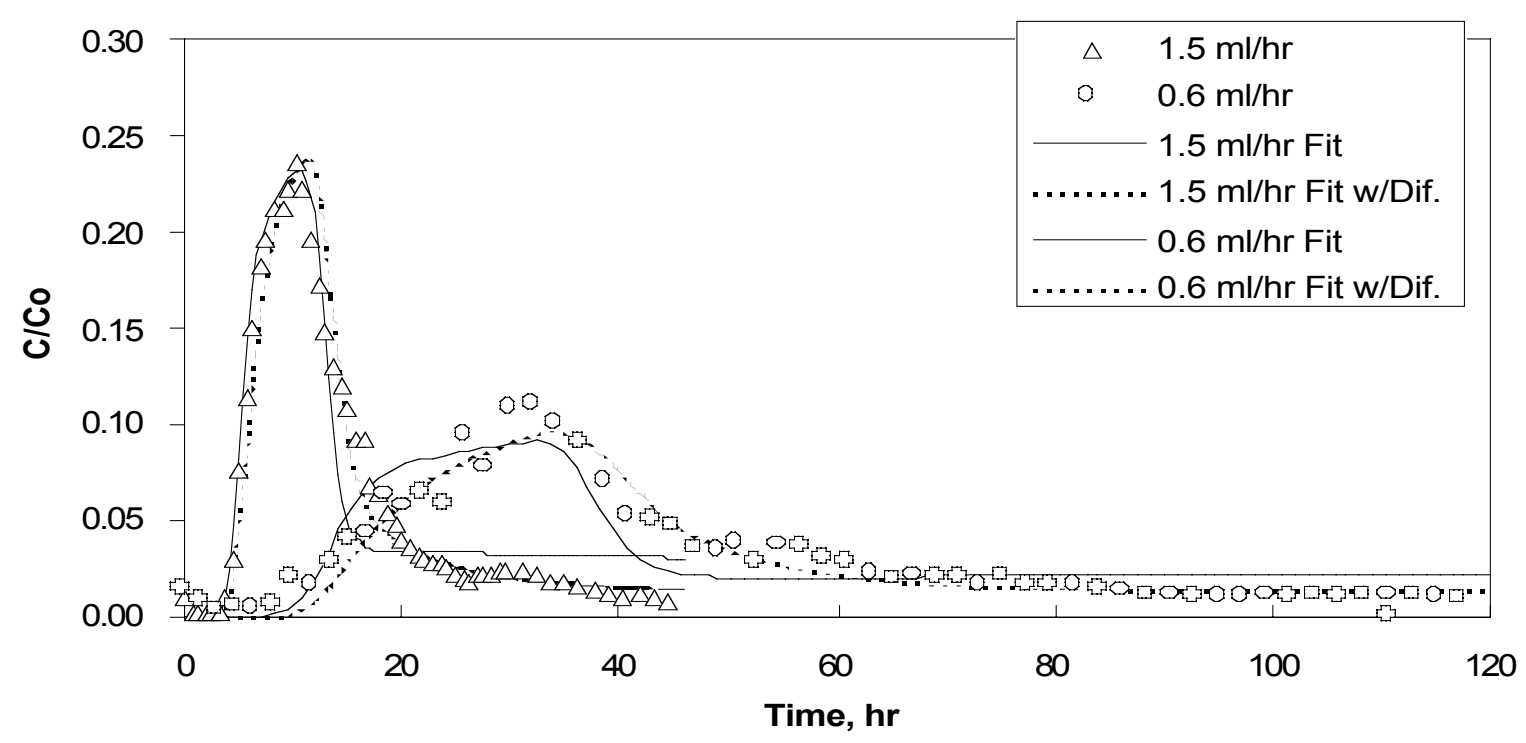

Figure E.1. RELAP fits to the Cheto montmorillonite colloid normalized concentrations in the UE20c 2851 fracture for experiments $1 \mathrm{a}$ and $2 \mathrm{a}$. 


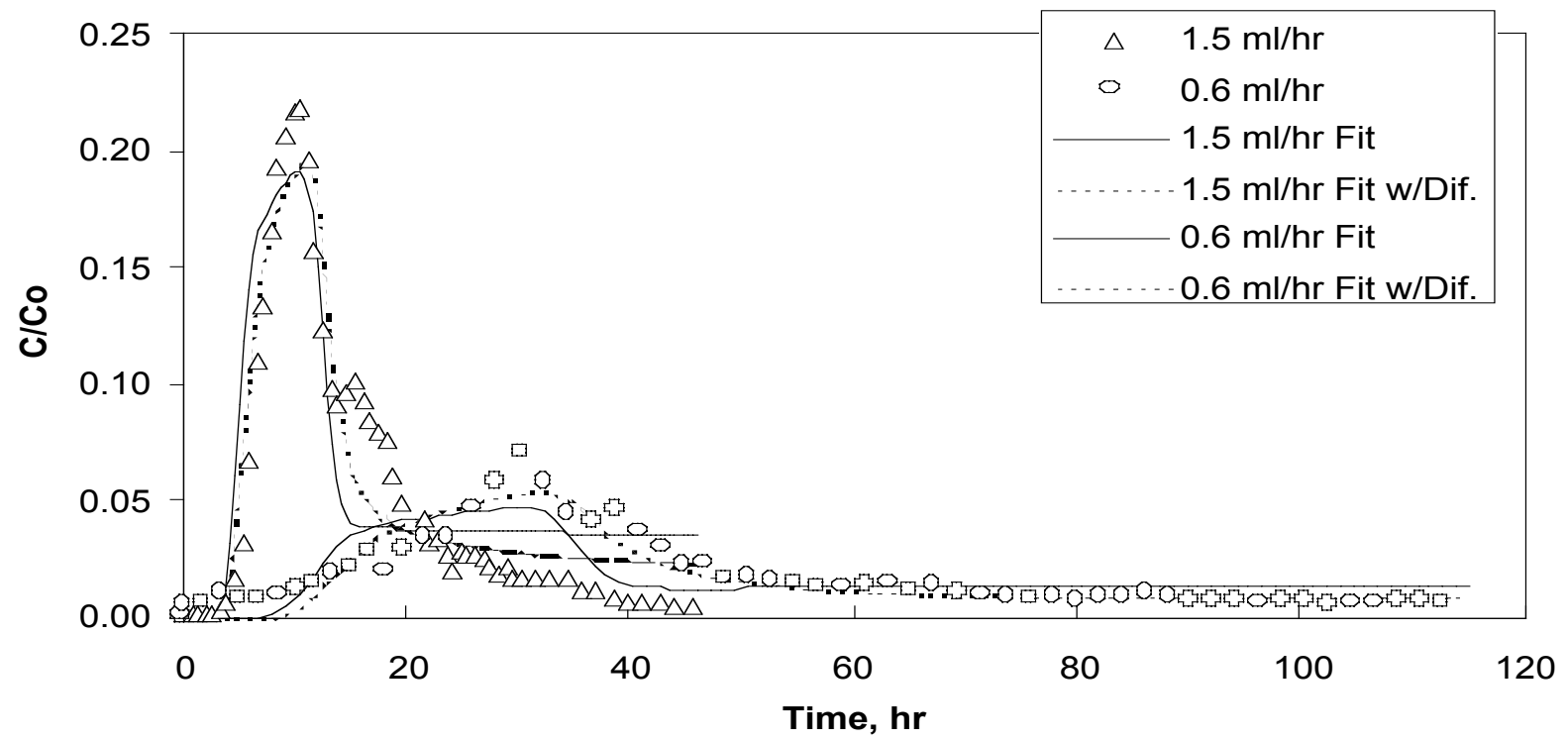

Figure E.2. RELAP fits to the Cheto montmorillonite colloid normalized concentrations in the UE20c 2858 fracture for experiments $1 \mathrm{~b}$ and $2 \mathrm{~b}$.

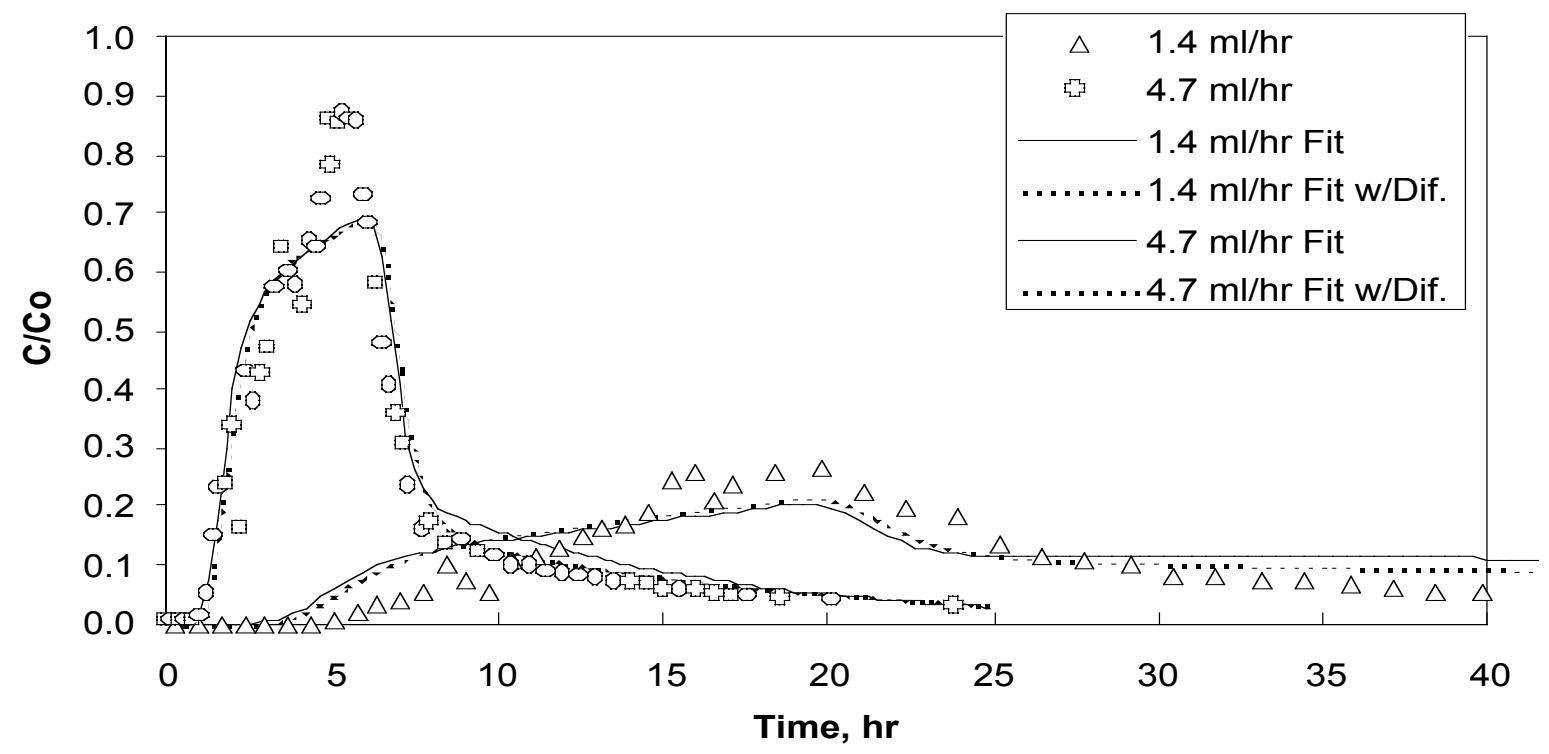

Figure E.3. RELAP fits to the silica colloid normalized concentrations in the UE20c 2851 fracture for experiments $3 \mathrm{a}$ and $4 \mathrm{a}$. 


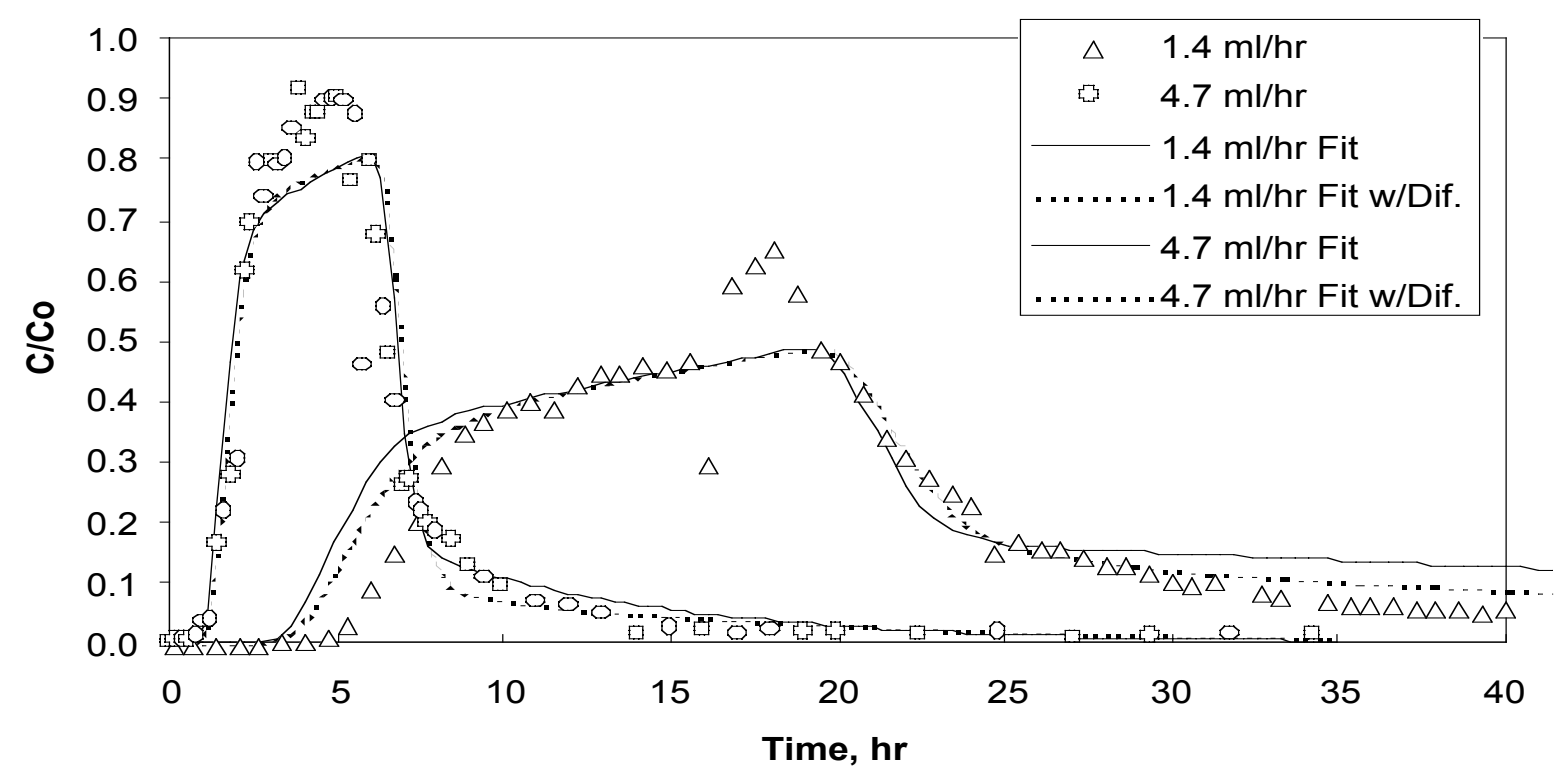

Figure E.4. RELAP fits to the silica colloid normalized concentrations in the UE20c 2858 fracture for experiments $3 \mathrm{~b}$ and $4 \mathrm{~b}$.

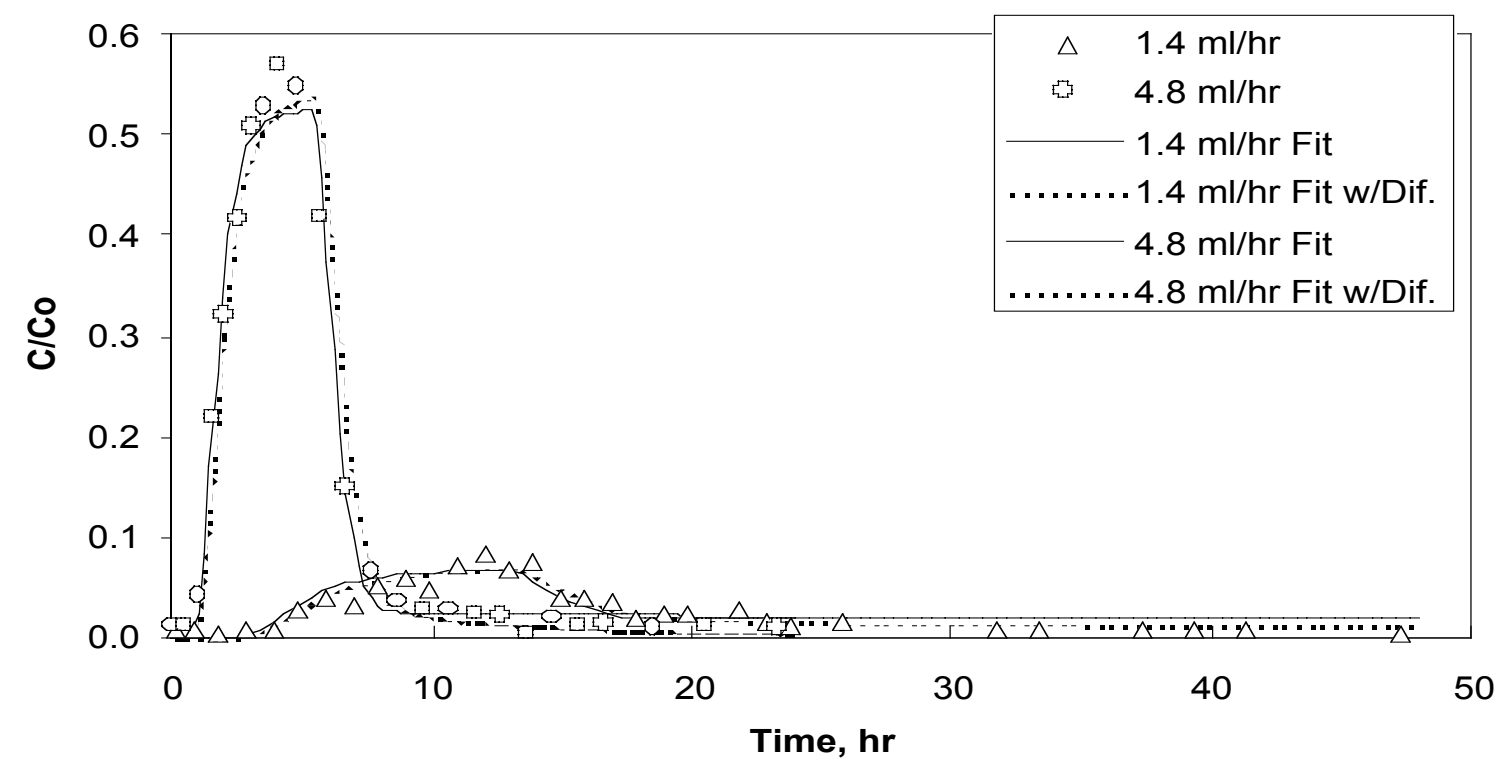

Figure E.5. RELAP fits to the batch 1 Otay montmorillonite colloid normalized concentrations in the UE20c 2851 fracture for experiments 5a and 6a. 


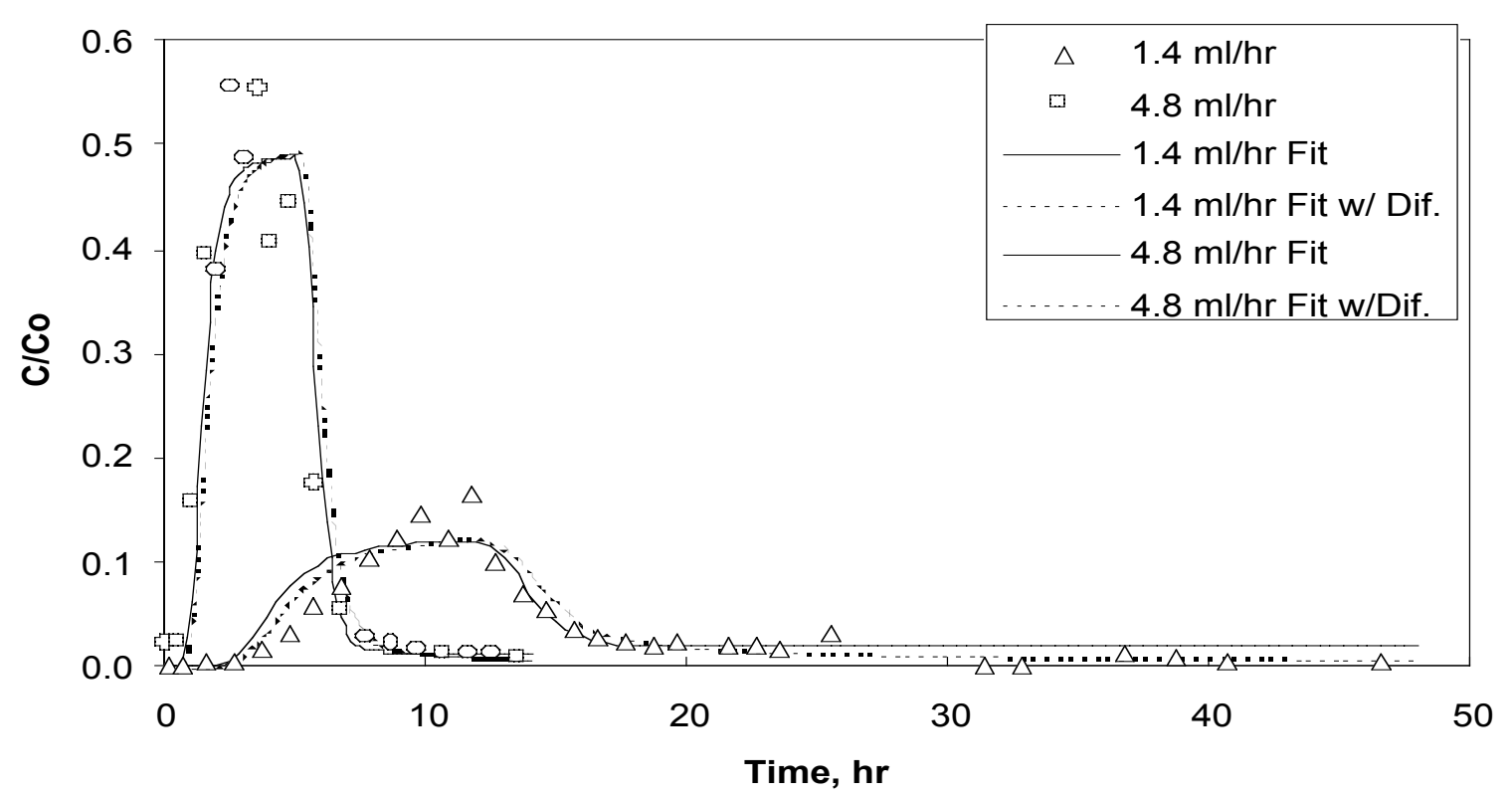

Figure E.6. RELAP fits to the batch 1 Otay montmorillonite colloid normalized concentrations in the UE20c 2858 fracture for experiments $5 \mathrm{~b}$ and $6 \mathrm{~b}$.

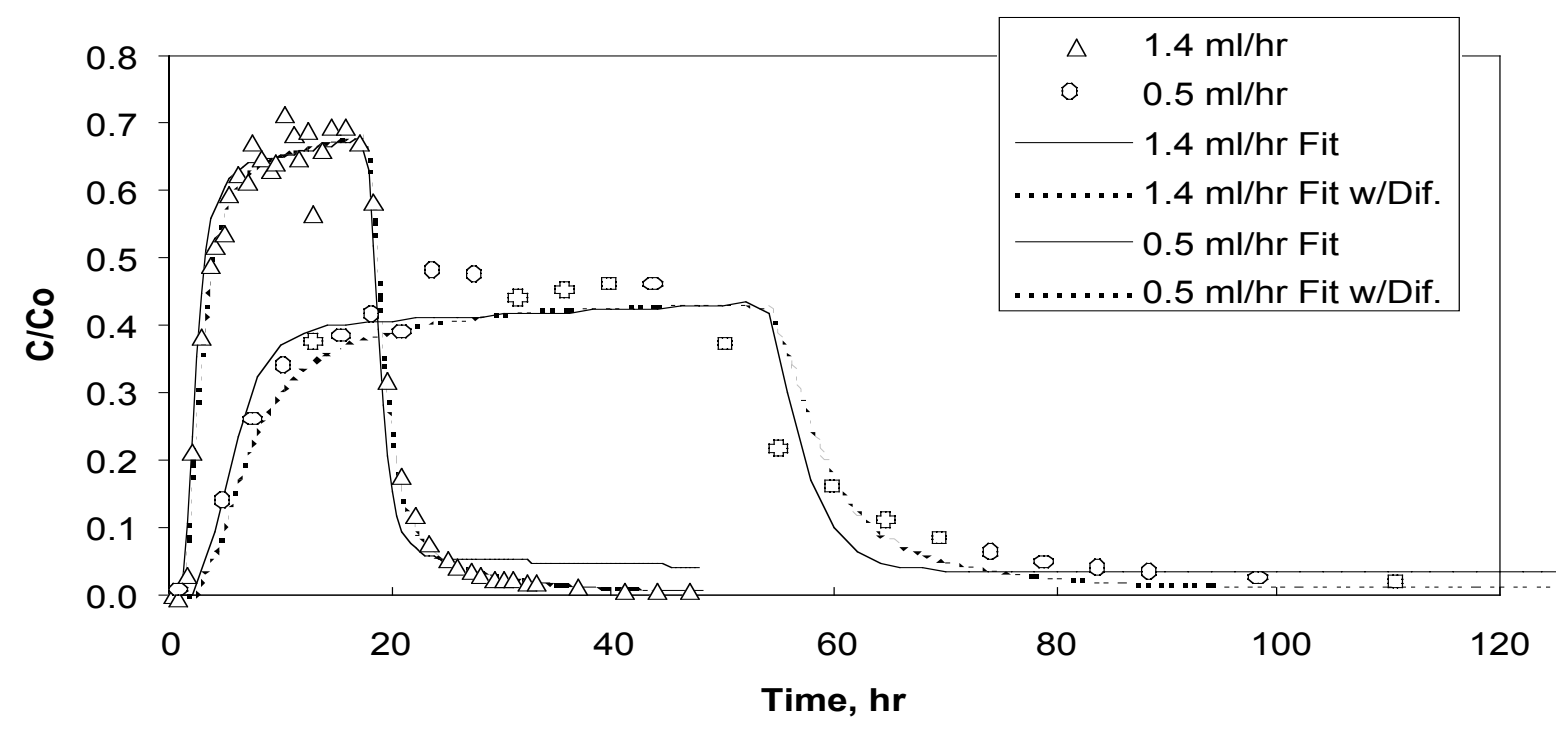

Figure E.7. RELAP fits to the clinoptilolite colloid normalized concentrations in the PM-2, 4177 fracture for experiments $7 \mathrm{a}$ and $8 \mathrm{a}$. 


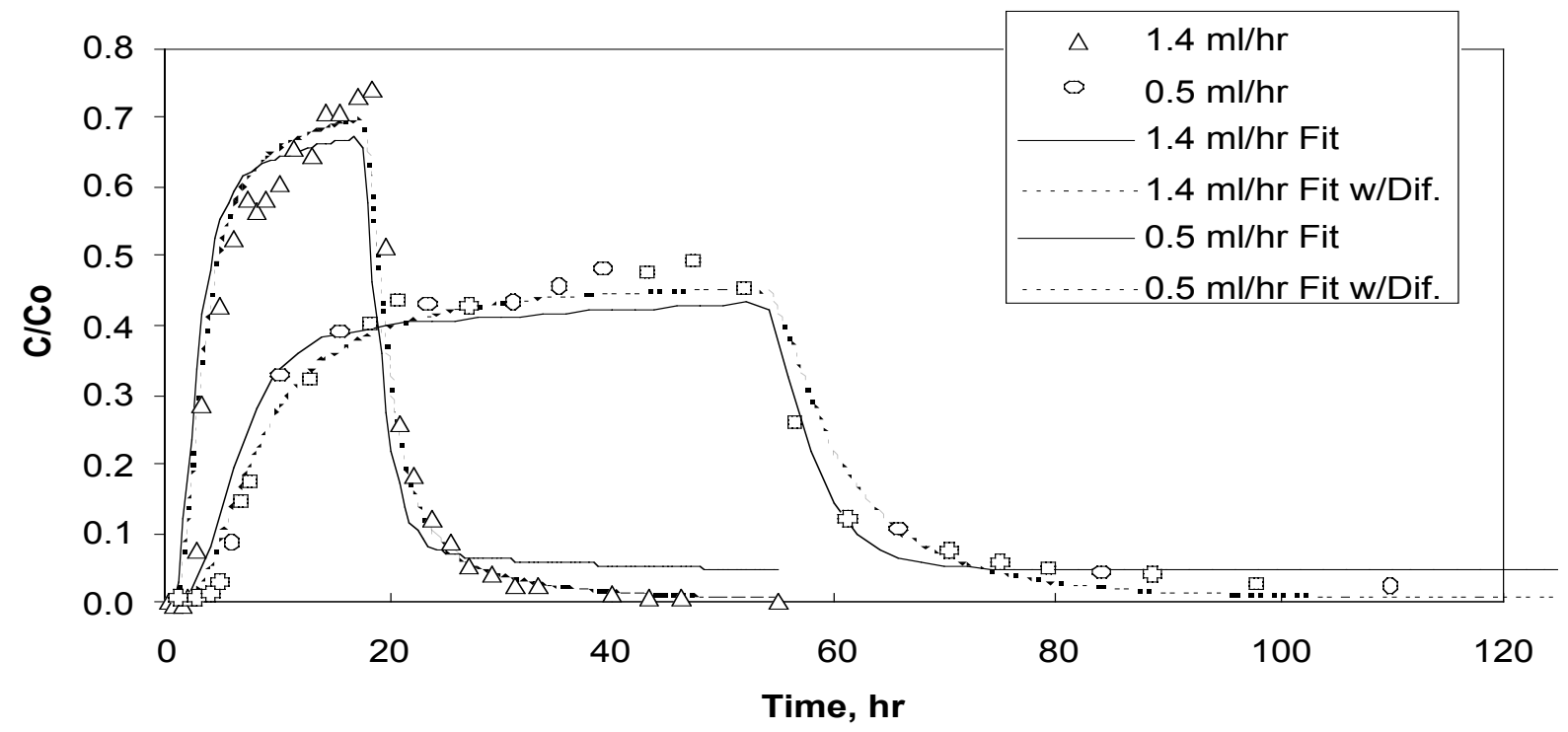

Figure E.8. RELAP fits to the clinoptilolite colloid normalized concentrations in the PM-1, 4823 fracture for experiments $7 \mathrm{a}$ and $8 \mathrm{a}$.

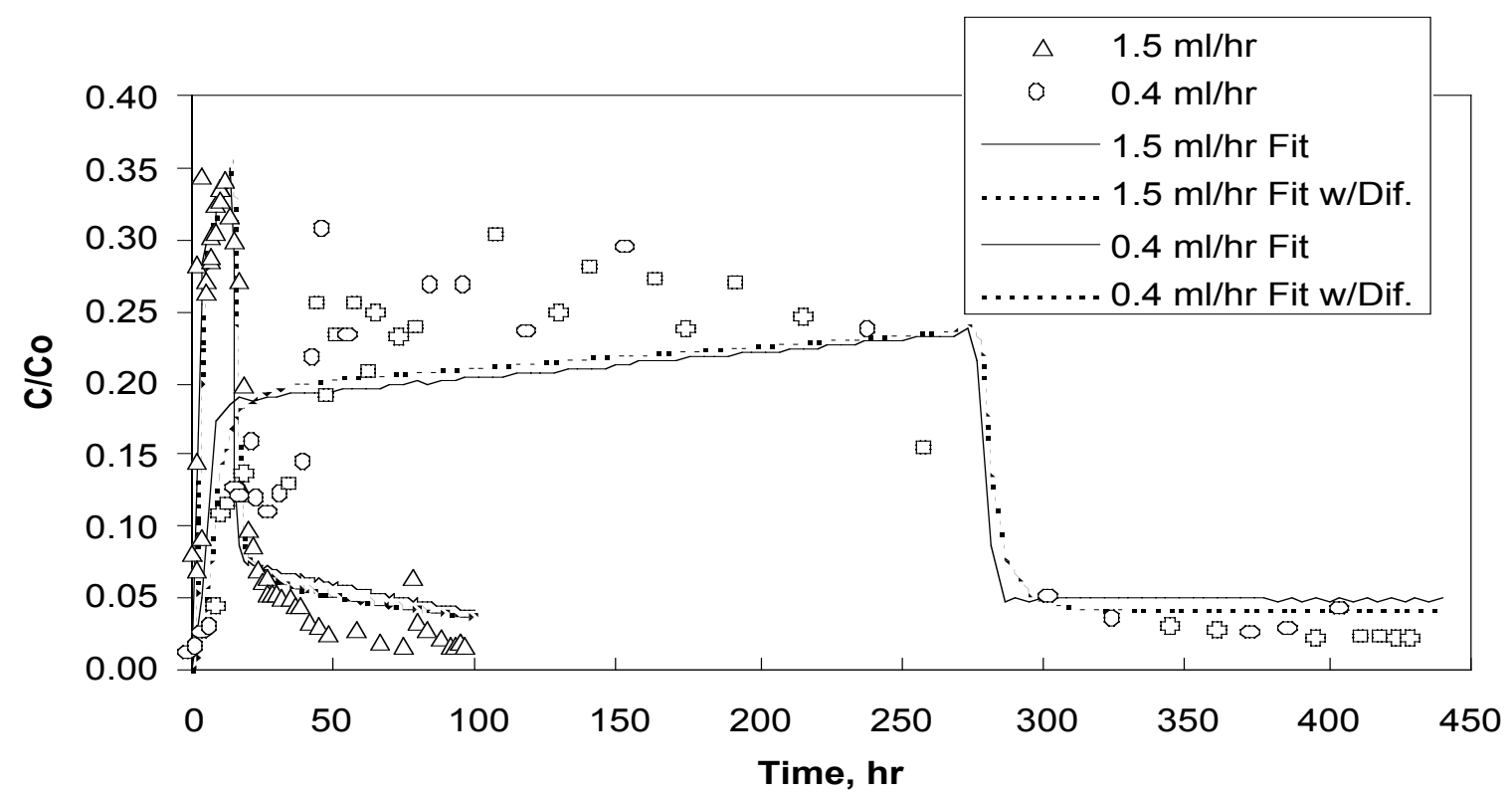

Figure E.9. RELAP fits to the batch 2 Otay montmorillonite colloid normalized concentrations in the PM-2, 4177 fracture for experiments 9a and 10a. 


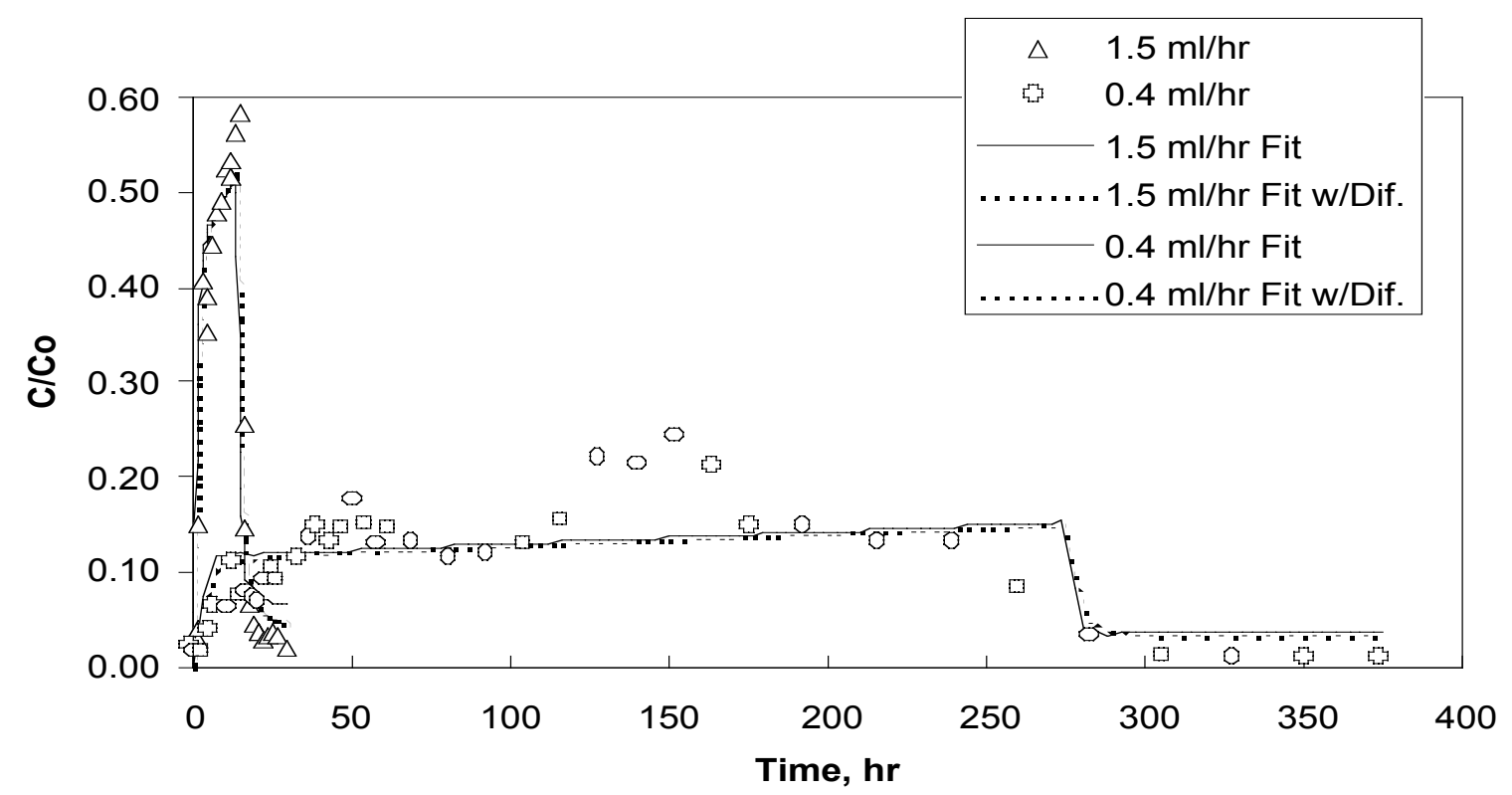

Figure E.10. RELAP fits to the batch 2 Otay montmorillonite colloid normalized concentrations in the PM-1, 4823 fracture for experiments $9 \mathrm{~b}$ and $10 \mathrm{~b}$.

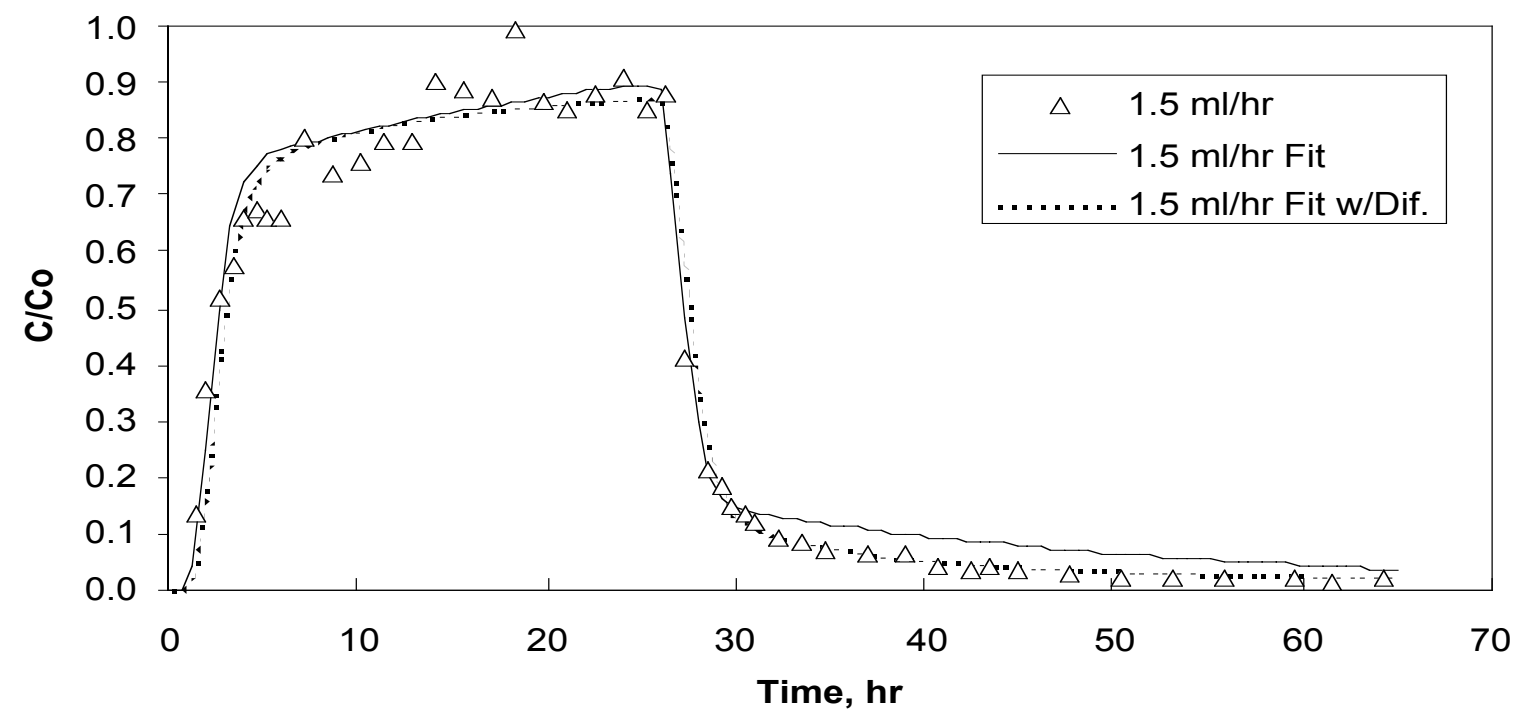

Figure E.11. RELAP fits to the batch 1 Otay montmorillonite colloid normalized concentrations in the PM-2, 4177 fracture for experiment 11. 


\title{
-Appendix F- \\ Model Simulations of Colloid and Pu Responses in all Fracture Transport Experiments Using the Numerical Model Described in Chapter 8
}

\author{
Paul W. Reimus
}

This appendix contains plots of simulated responses of colloid and Plutonium obtained using the numerical model described in Chapter 8. The experimental data are shown in each plot for comparison. Model parameters corresponding to the model simulation curves are listed in tables in Chapter 8.

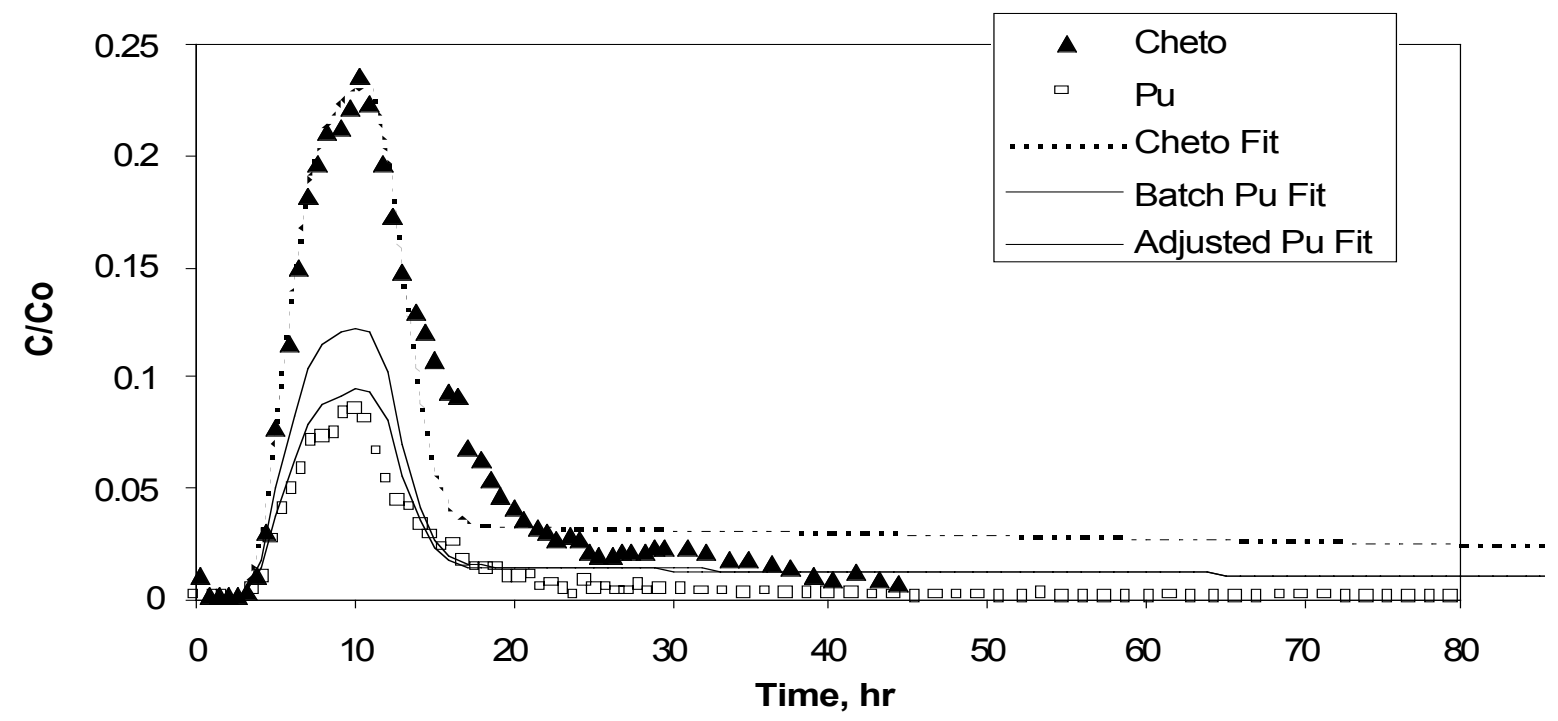

Figure F.1. Model simulations of Cheto montmorillonite and Pu responses in the UE20c 2851 fracture as a function of time at $\sim 1.5 \mathrm{ml} / \mathrm{hr}$ in Experiment 1a. 


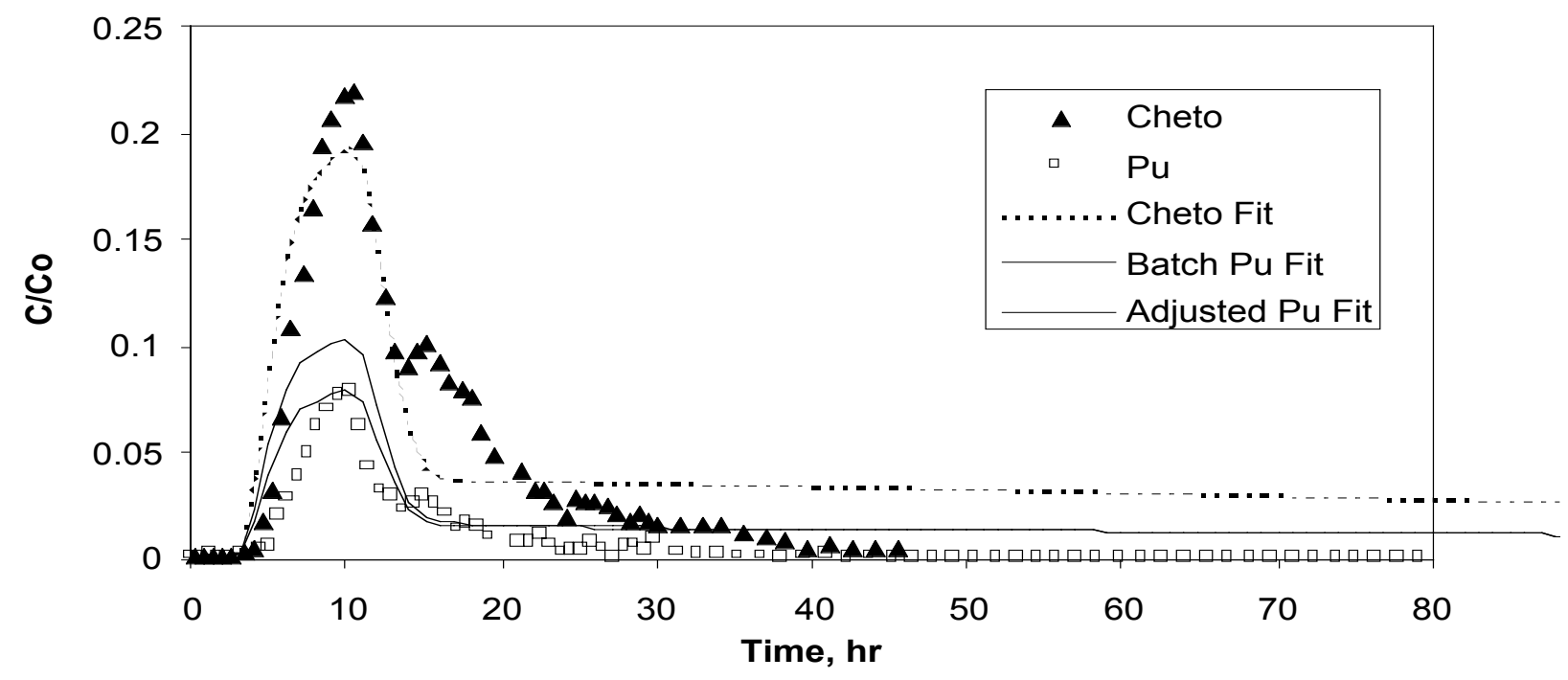

Figure F.2. Model simulations of Cheto montmorillonite and Pu responses in the UE20c 2858 fracture as a function of time at $\sim 1.5 \mathrm{ml} / \mathrm{hr}$ in Experiment $1 \mathrm{~b}$.

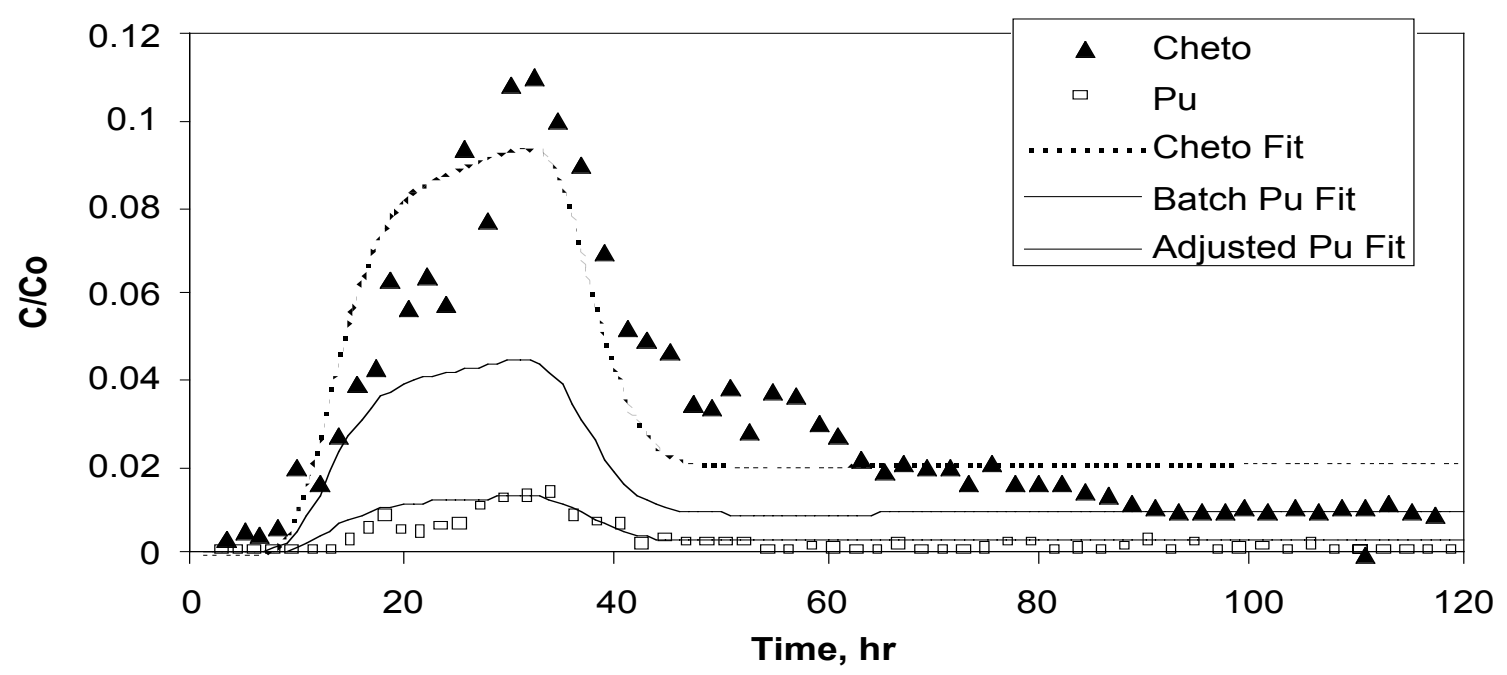

Figure F.3. Model simulations of Cheto montmorillonite and $\mathrm{Pu}$ responses in the UE20c 2851 fracture as a function of time at $\sim 0.6 \mathrm{ml} / \mathrm{hr}$ in Experiment $2 \mathrm{a}$. 


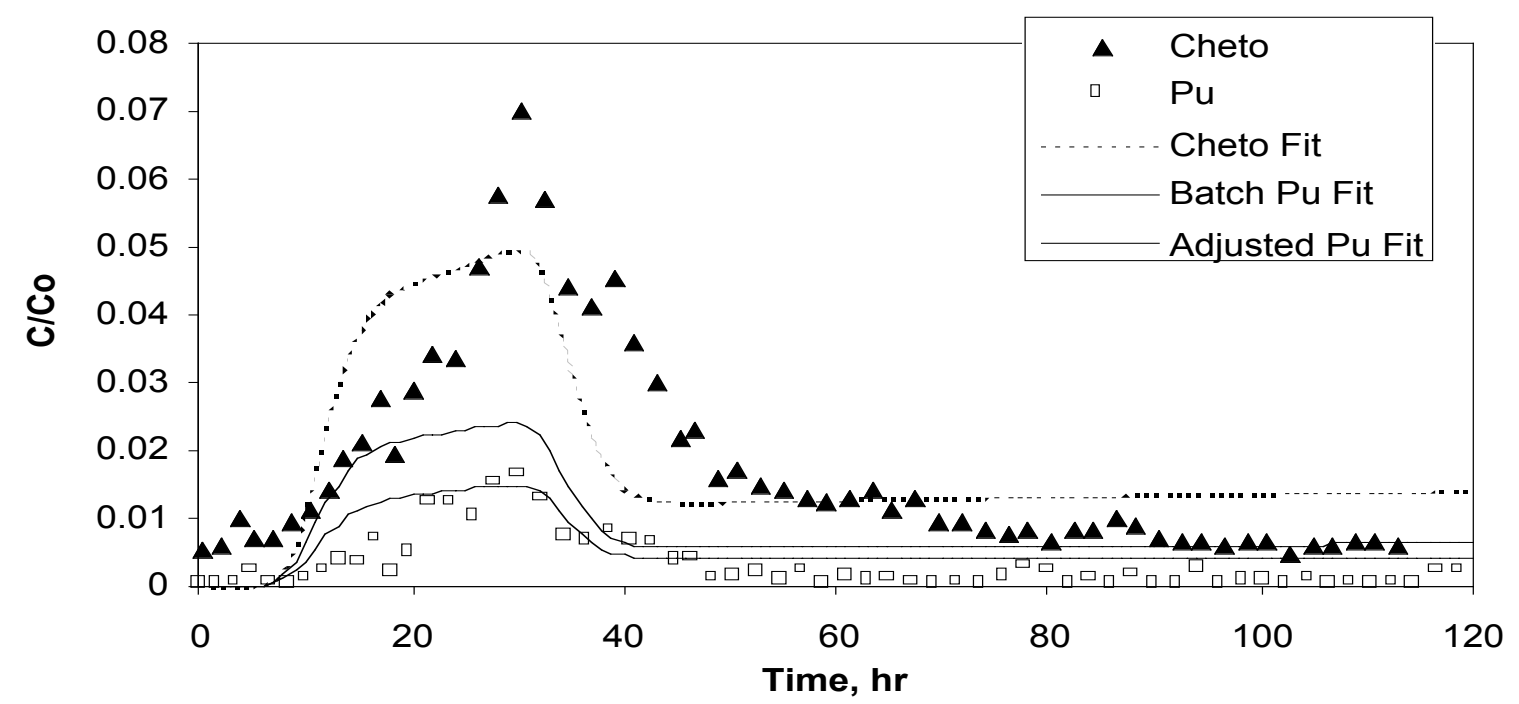

Figure F.4. Model simulations of Cheto montmorillonite and Pu responses in the UE20c 2858 fracture as a function of time at $\sim 0.6 \mathrm{ml} / \mathrm{hr}$ in Experiment $2 \mathrm{~b}$.

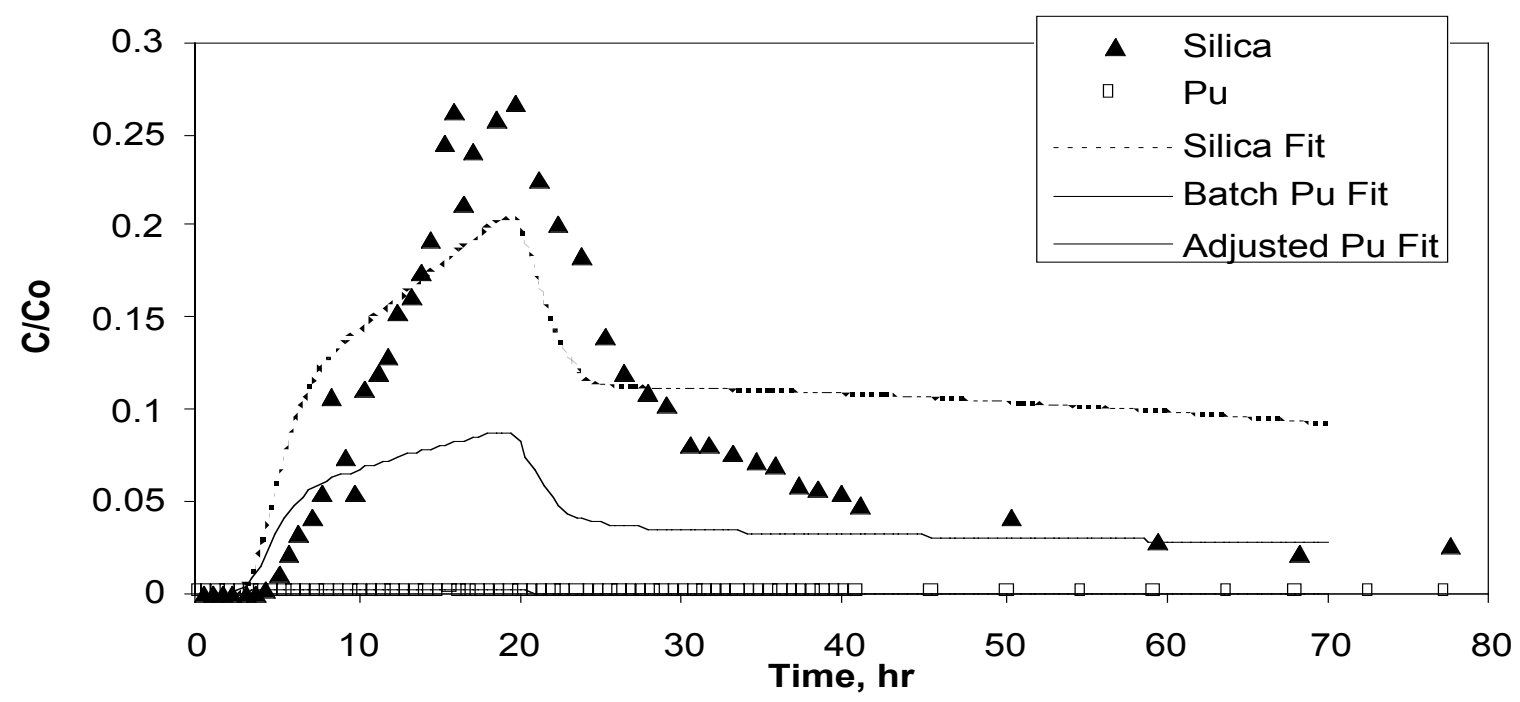

Figure F.5. Model simulations of silica and Pu responses in the UE20c 2851 fracture as a function of time at $\sim 1.5 \mathrm{ml} / \mathrm{hr}$ in Experiment 3a. 


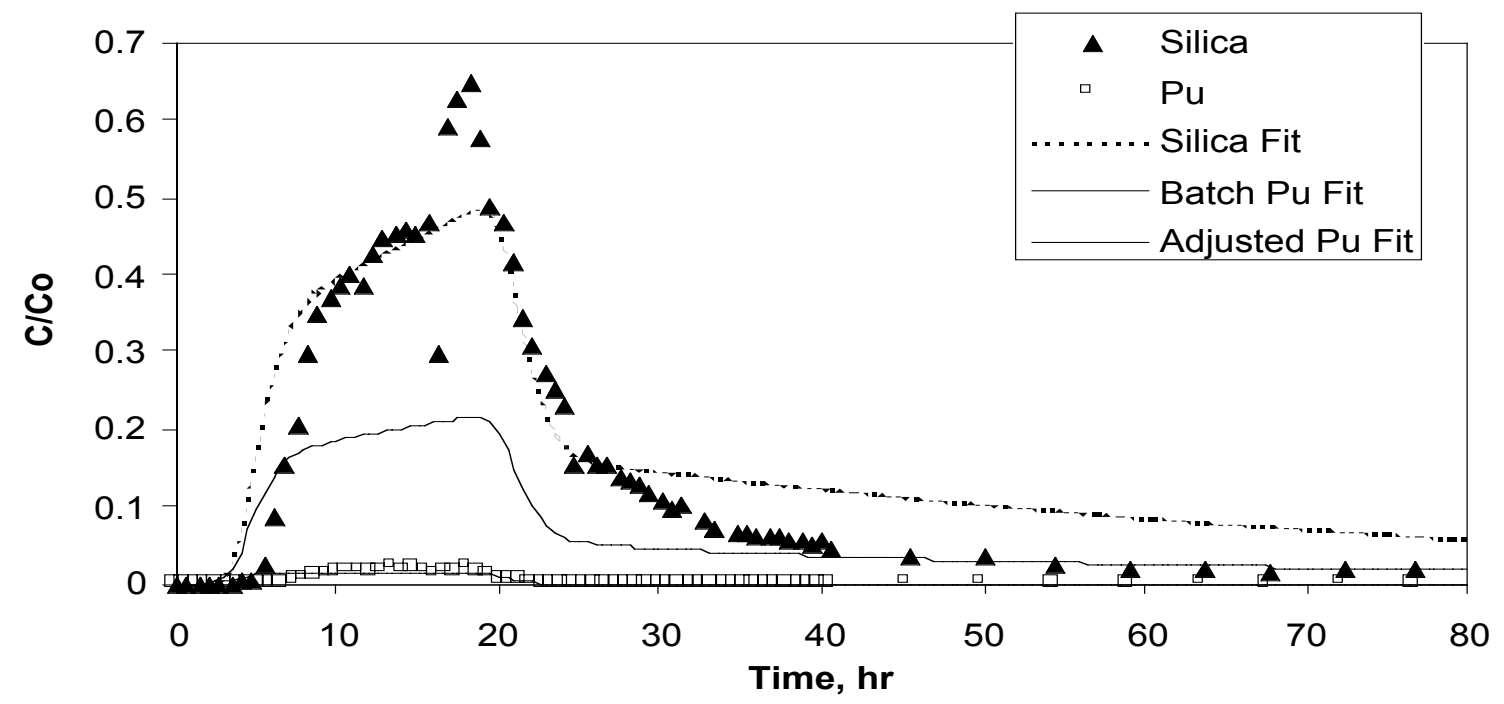

Figure F.6. Model simulations of silica and $\mathrm{Pu}$ responses in the UE20c 2858 fracture as a function of time at $\sim 1.5 \mathrm{ml} / \mathrm{hr}$ in Experiment $3 \mathrm{~b}$.

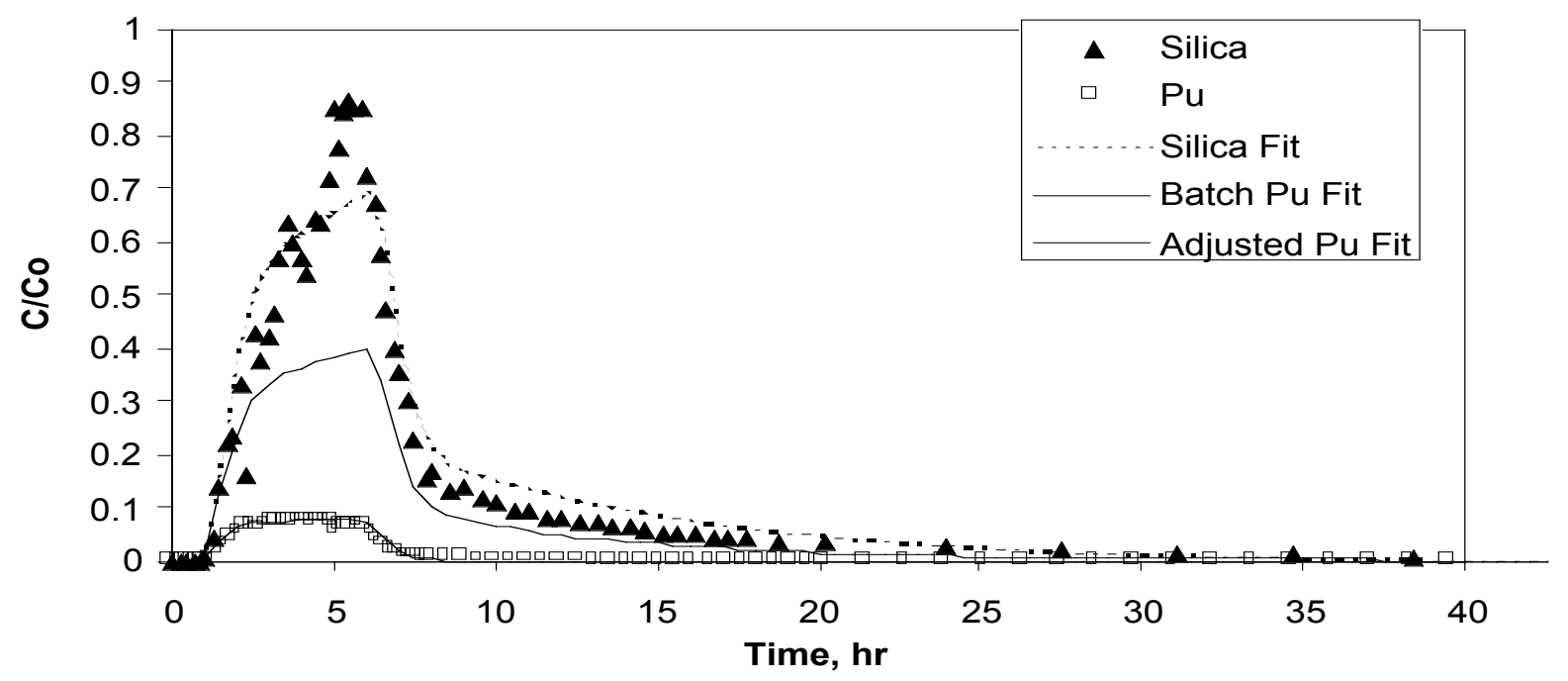

Figure F.7. Model simulations of silica and $\mathrm{Pu}$ responses in the UE20c 2851 fracture as a function of time at $\sim 4.7 \mathrm{ml} / \mathrm{hr}$ in Experiment $4 \mathrm{a}$. 


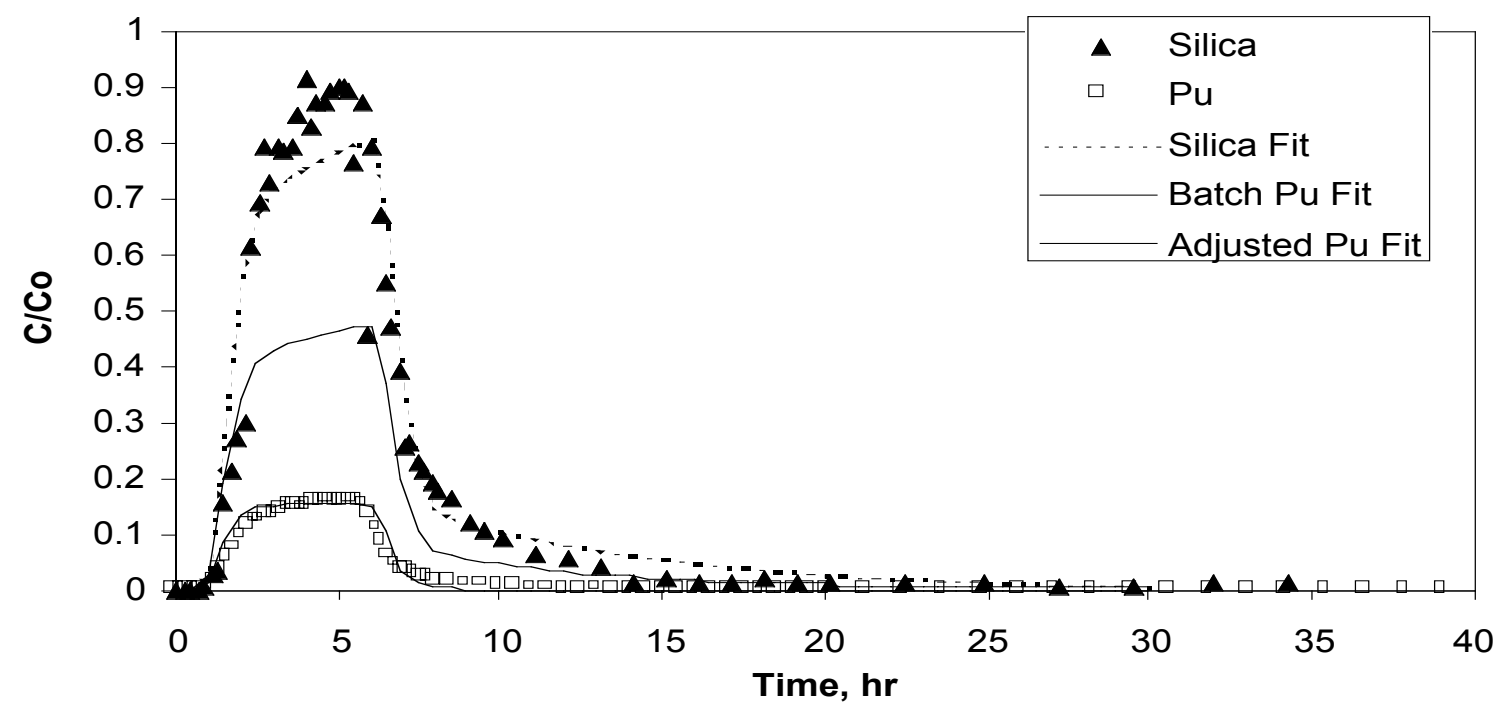

Figure F.8. Model simulations of silica and $\mathrm{Pu}$ responses in the UE20c 2858 fracture as a function of time at $\sim 4.7 \mathrm{ml} / \mathrm{hr}$ in Experiment $4 \mathrm{~b}$.

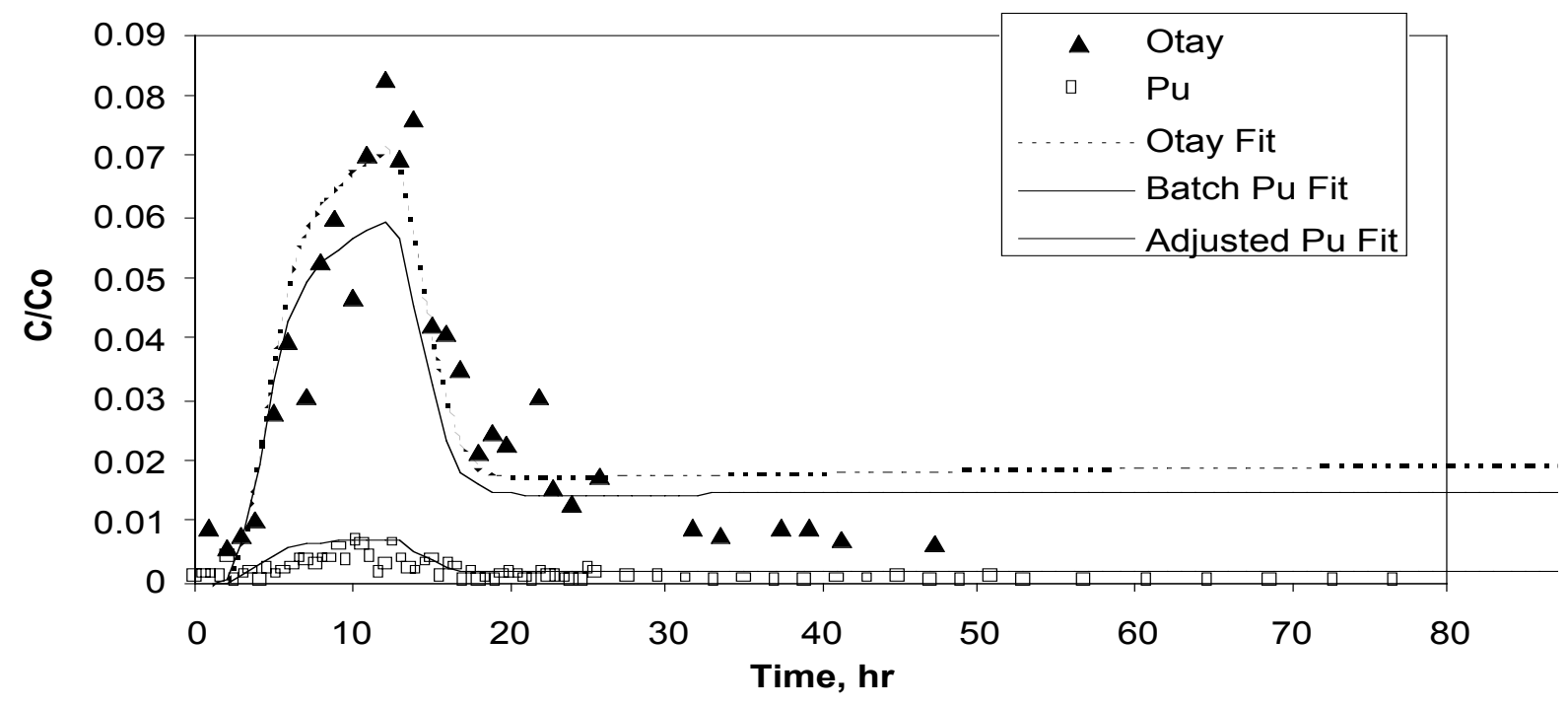

Figure F.9. Model simulations of batch 1 Otay montmorillonite and Pu responses in the UE20c 2851 fracture as a function of time at $\sim 1.5 \mathrm{ml} / \mathrm{hr}$ in Experiment $5 \mathrm{a}$. 


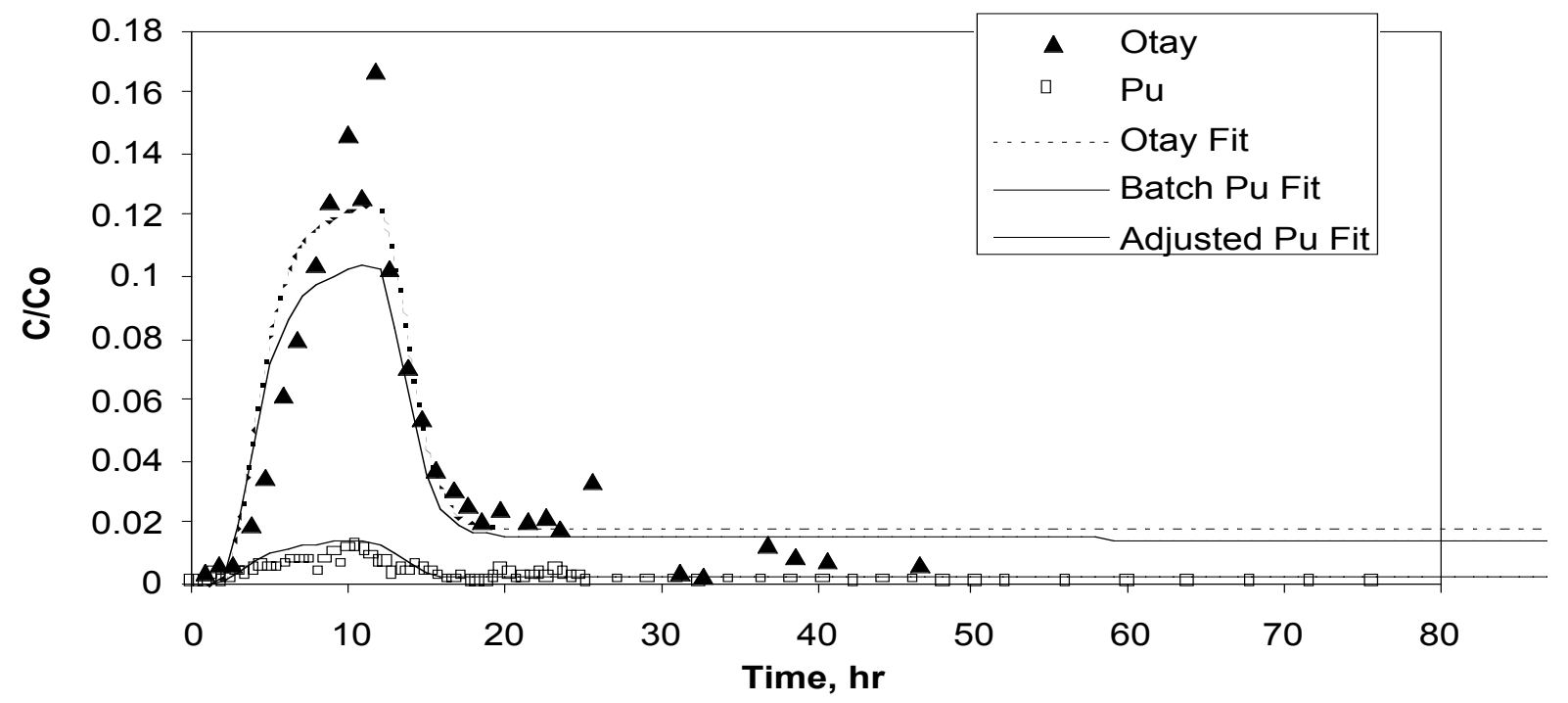

Figure F.10. Model simulations of batch 1 Otay montmorillonite and $\mathrm{Pu}$ responses in the UE20c 2858 fracture as a function of time at $\sim 1.5 \mathrm{ml} / \mathrm{hr}$ in Experiment $5 \mathrm{~b}$.

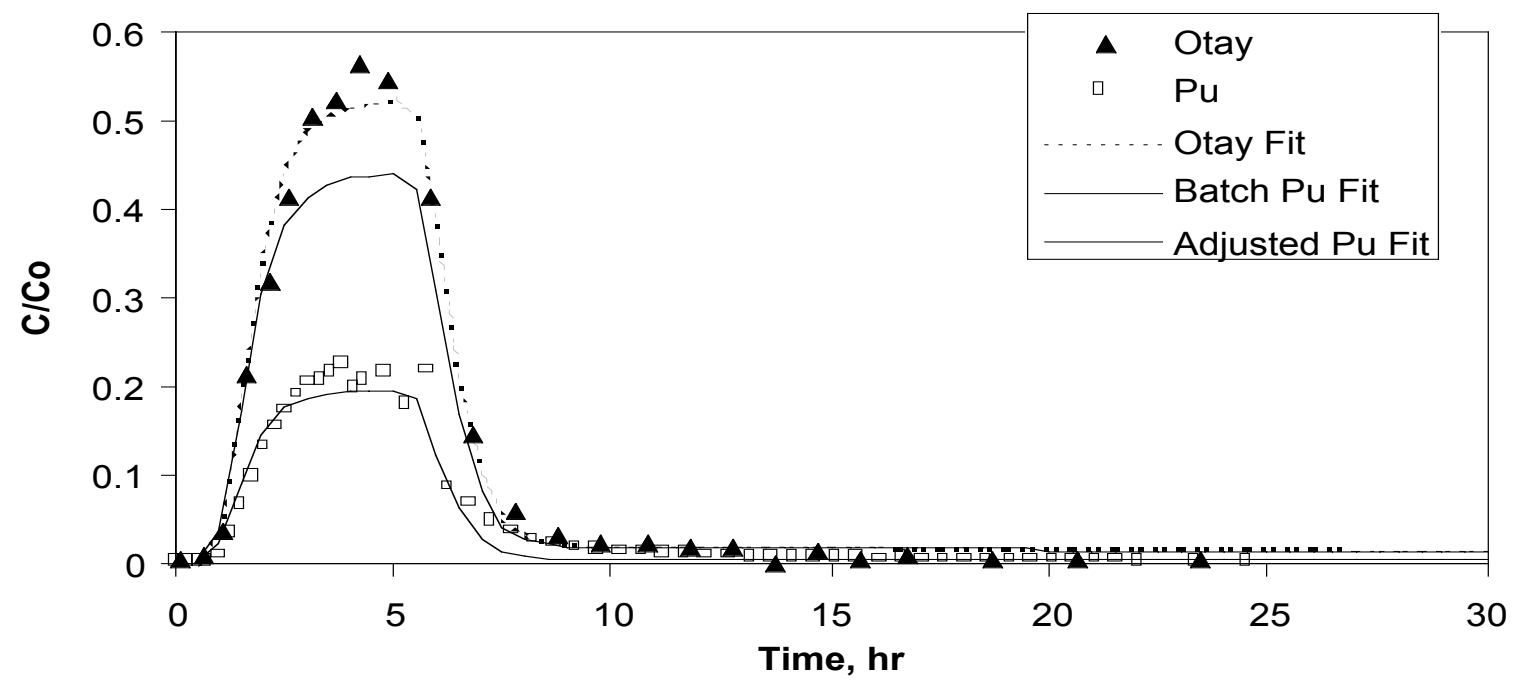

Figure F.11. Model simulations of batch 1 Otay montmorillonite and $\mathrm{Pu}$ responses in the UE20c 2851 fracture as a function of time at $\sim 4.8 \mathrm{ml} / \mathrm{hr}$ in Experiment $6 \mathrm{a}$. 


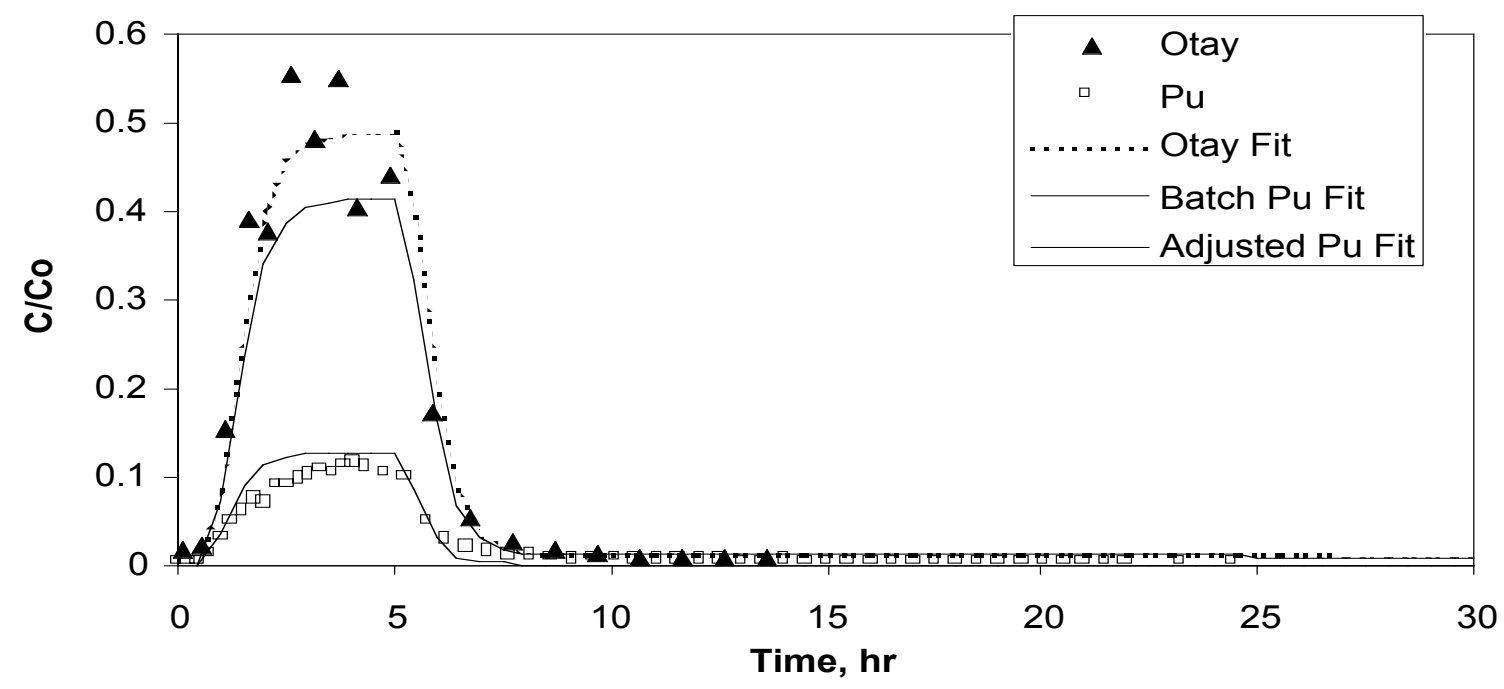

Figure F.12. Model simulations of batch 1 Otay montmorillonite and $\mathrm{Pu}$ responses in the UE20c 2858 fracture as a function of time at $\sim 4.8 \mathrm{ml} / \mathrm{hr}$ in Experiment $6 \mathrm{~b}$.

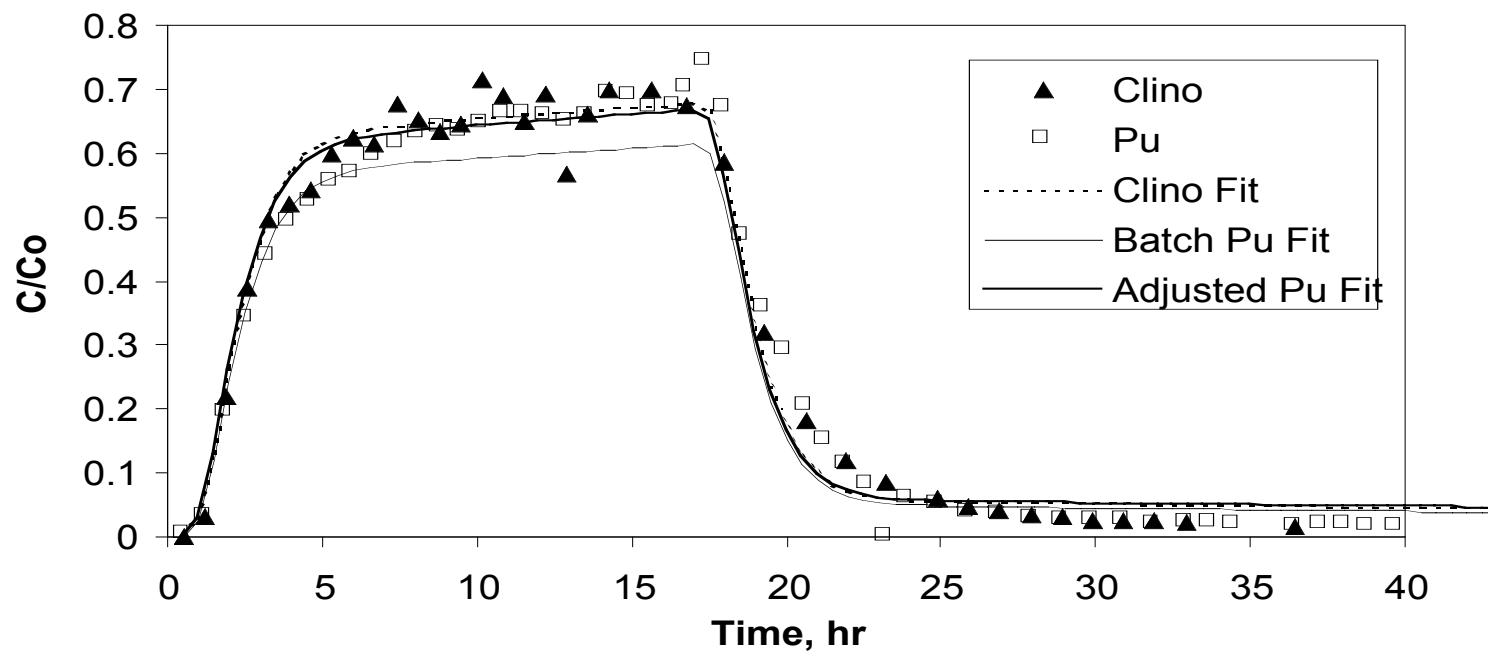

Figure F.13. Model simulations of clinoptilolite and Pu responses in the PM-2, 4177 fracture as a function of time at $\sim 1.4 \mathrm{ml} / \mathrm{hr}$ in Experiment $7 \mathrm{a}$. 


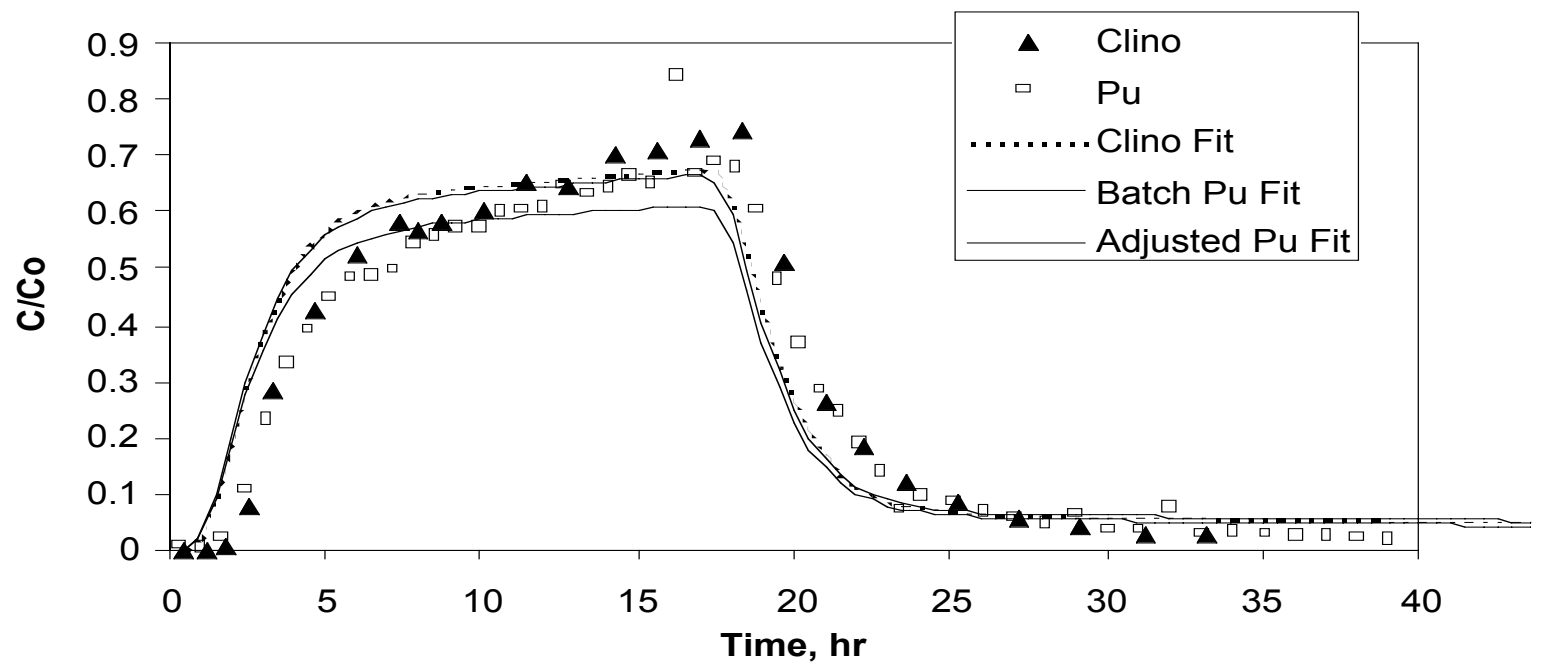

Figure F.14. Model simulations of clinoptilolite and Pu responses in the PM-1, 4823 fracture as a function of time at $\sim 1.4 \mathrm{ml} / \mathrm{hr}$ in Experiment $7 \mathrm{~b}$.

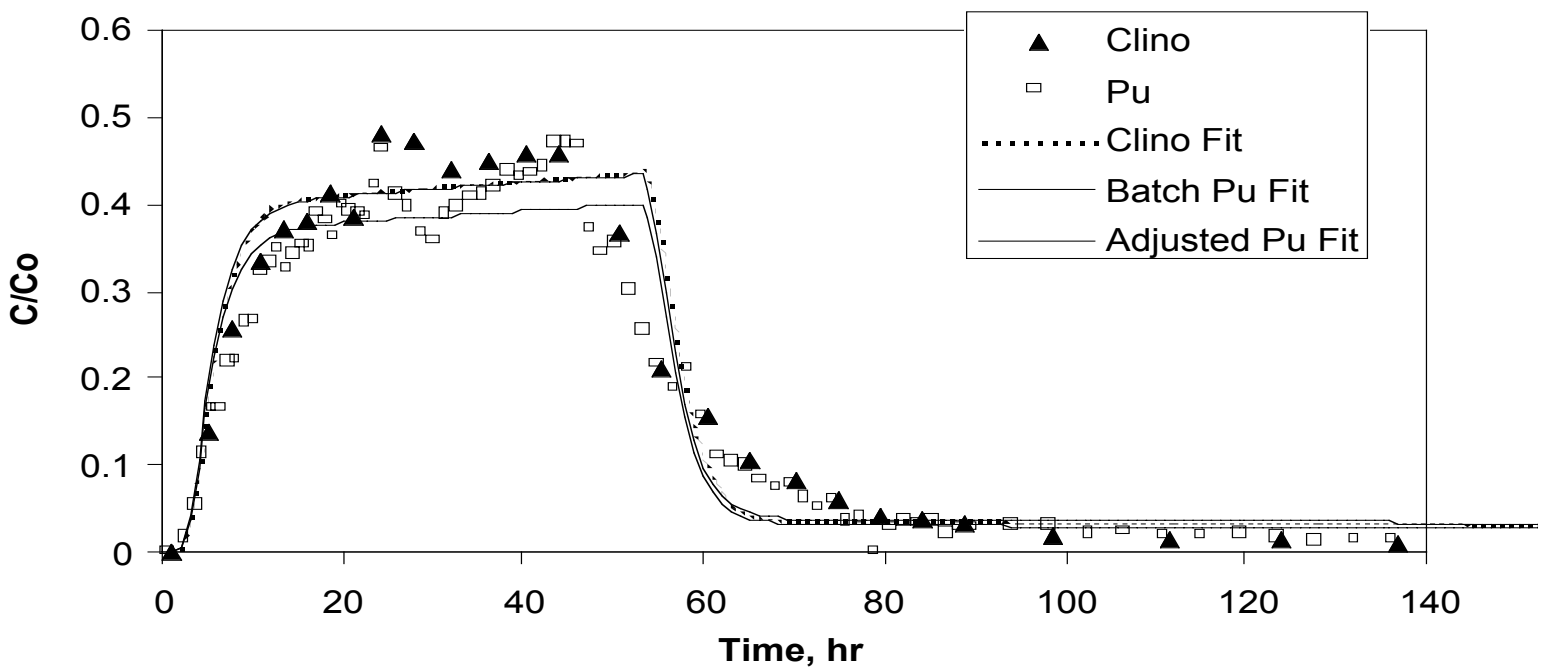

Figure F.15. Model simulations of clinoptilolite and Pu responses in the PM-2, 4177 fracture as a function of time at $\sim 0.5 \mathrm{ml} / \mathrm{hr}$ in Experiment $8 \mathrm{a}$. 


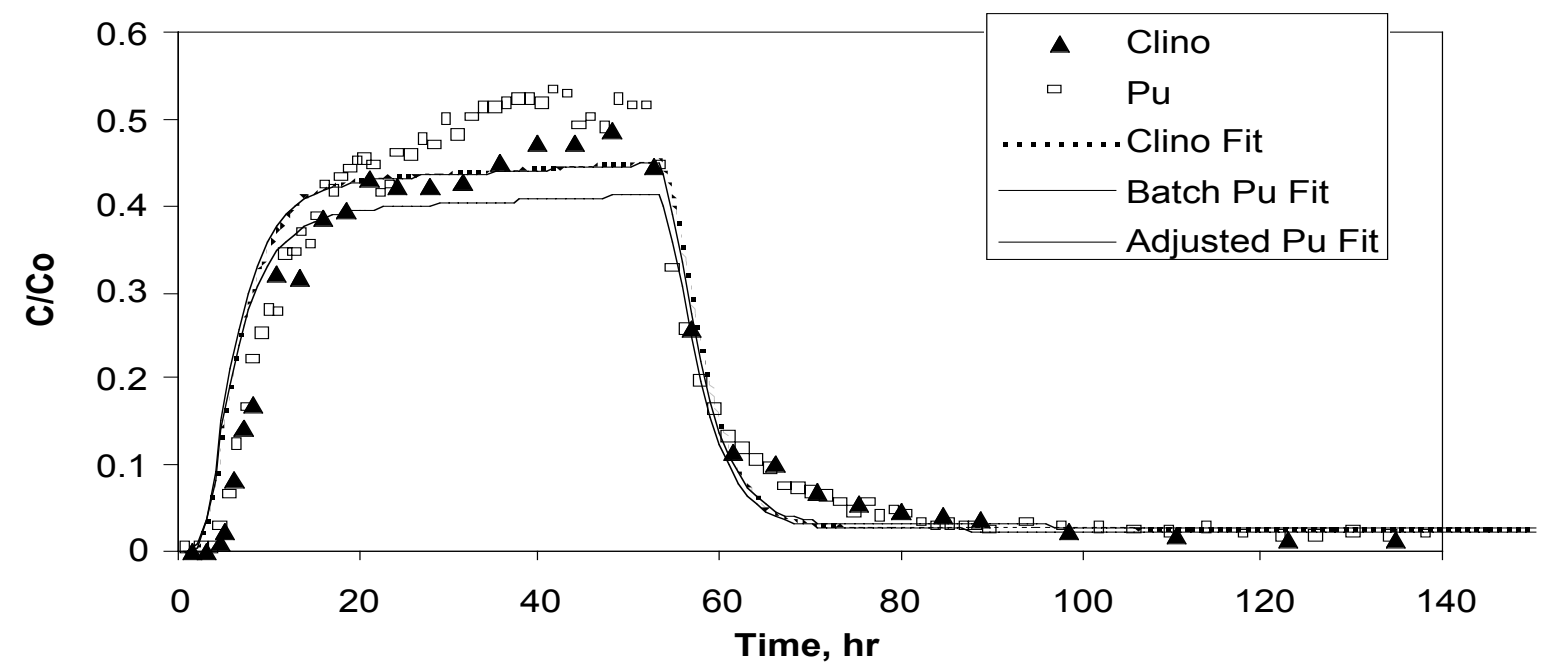

Figure F.16. Model simulations of clinoptilolite and Pu responses in the PM-1, 4823 fracture as a function of time at $\sim 0.5 \mathrm{ml} / \mathrm{hr}$ in Experiment $8 \mathrm{~b}$.

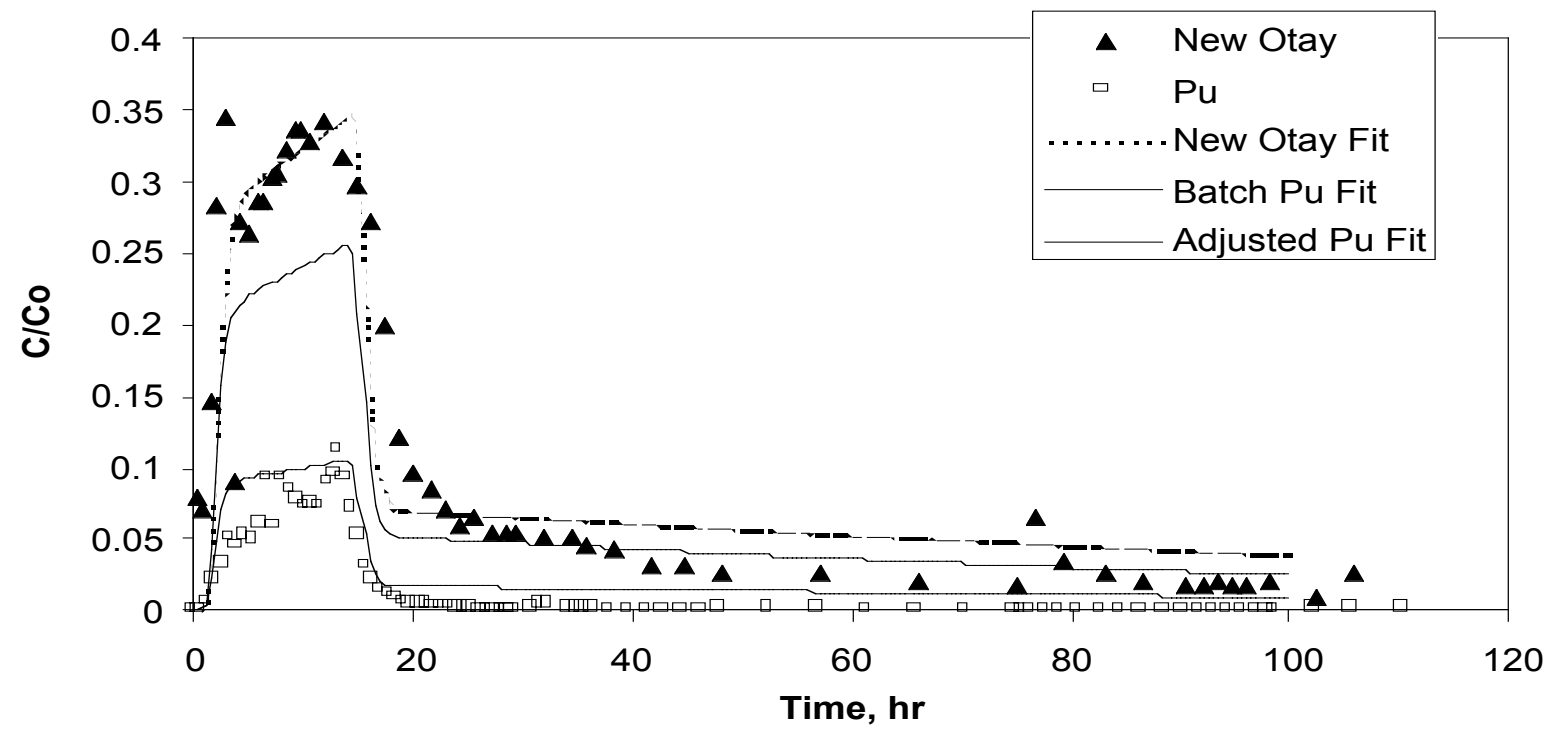

Figure F.17. Model simulations of batch 2 Otay montmorillonite and Pu responses in the PM-2, 4177 fracture as a function of time at $\sim 1.5 \mathrm{ml} / \mathrm{hr}$ in Experiment $9 \mathrm{a}$. 


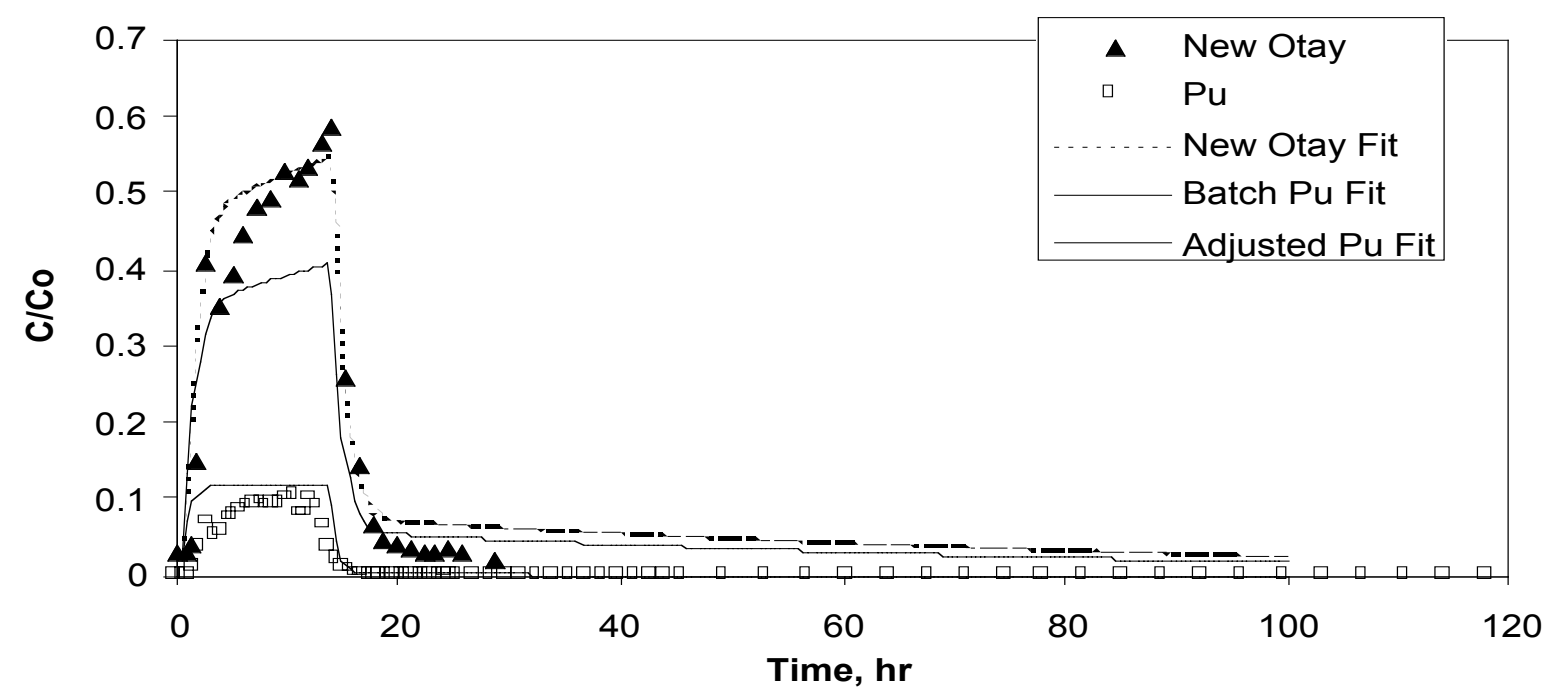

Figure F.18. Model simulations of batch 2 Otay montmorillonite and Pu responses in the PM-1, 4823 fracture as a function of time at $\sim 1.5 \mathrm{ml} / \mathrm{hr}$ in Experiment $9 \mathrm{~b}$.

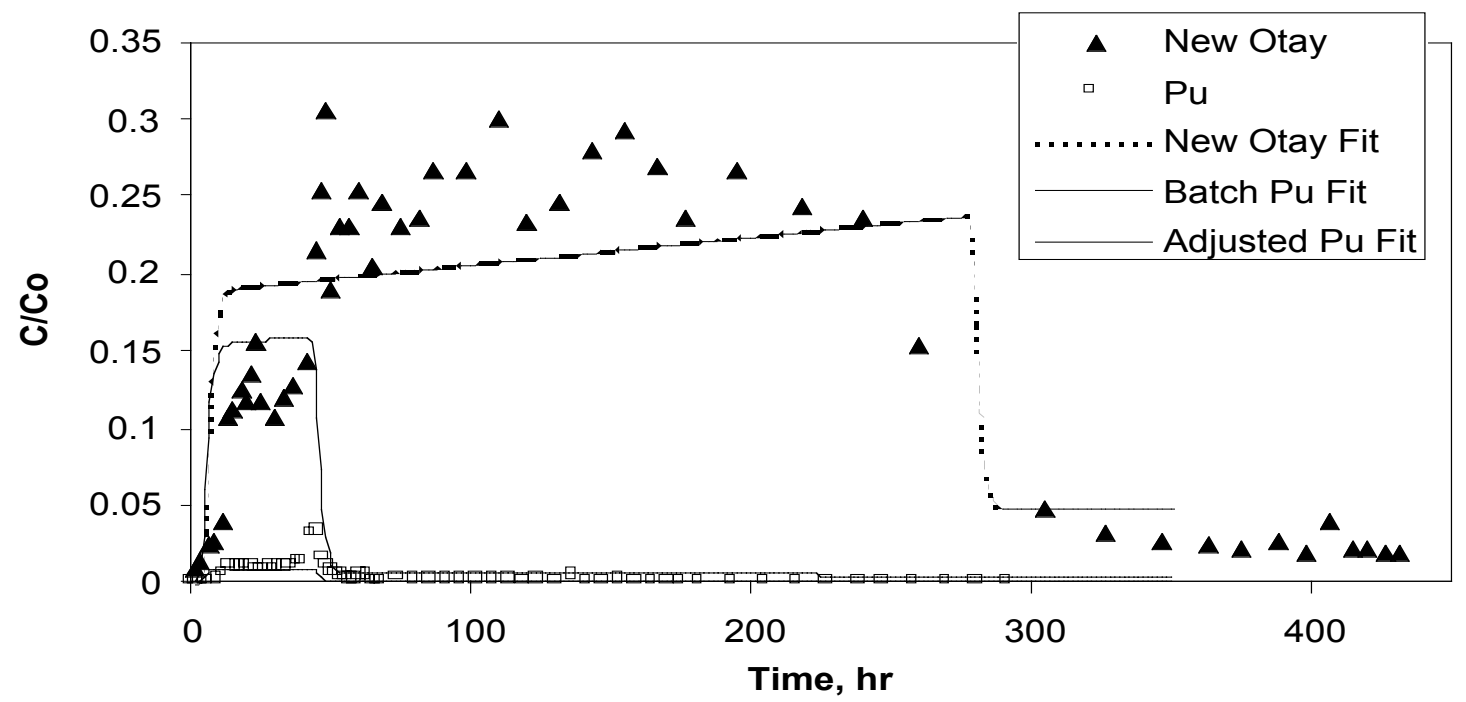

Figure F.19. Model simulations of batch 2 Otay montmorillonite and Pu responses in the PM-2, 4177 fracture as a function of time at $\sim 0.4 \mathrm{ml} / \mathrm{hr}$ in Experiment 10a. 


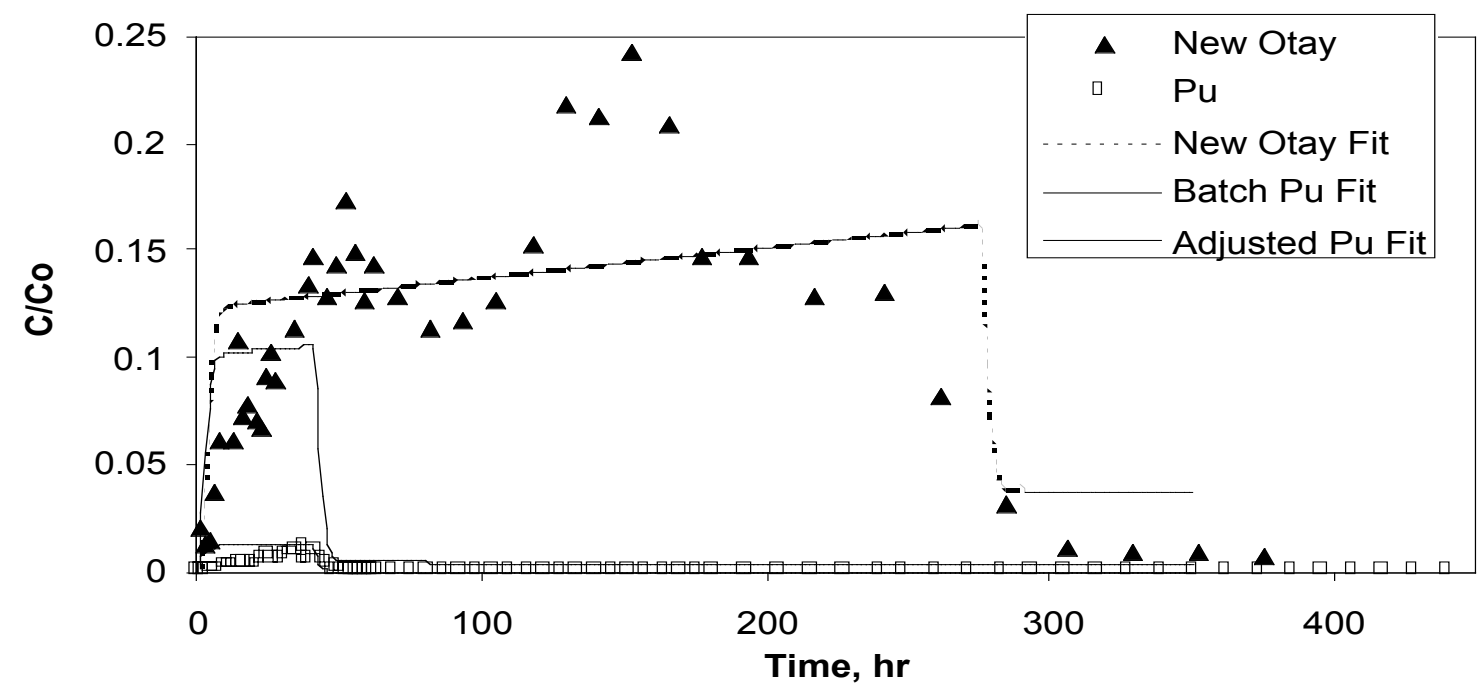

Figure F.20. Model simulations of batch 2 Otay montmorillonite and Pu responses in the PM-1, 4823 fracture as a function of time at $\sim 0.4 \mathrm{ml} / \mathrm{hr}$ in Experiment $10 \mathrm{~b}$.

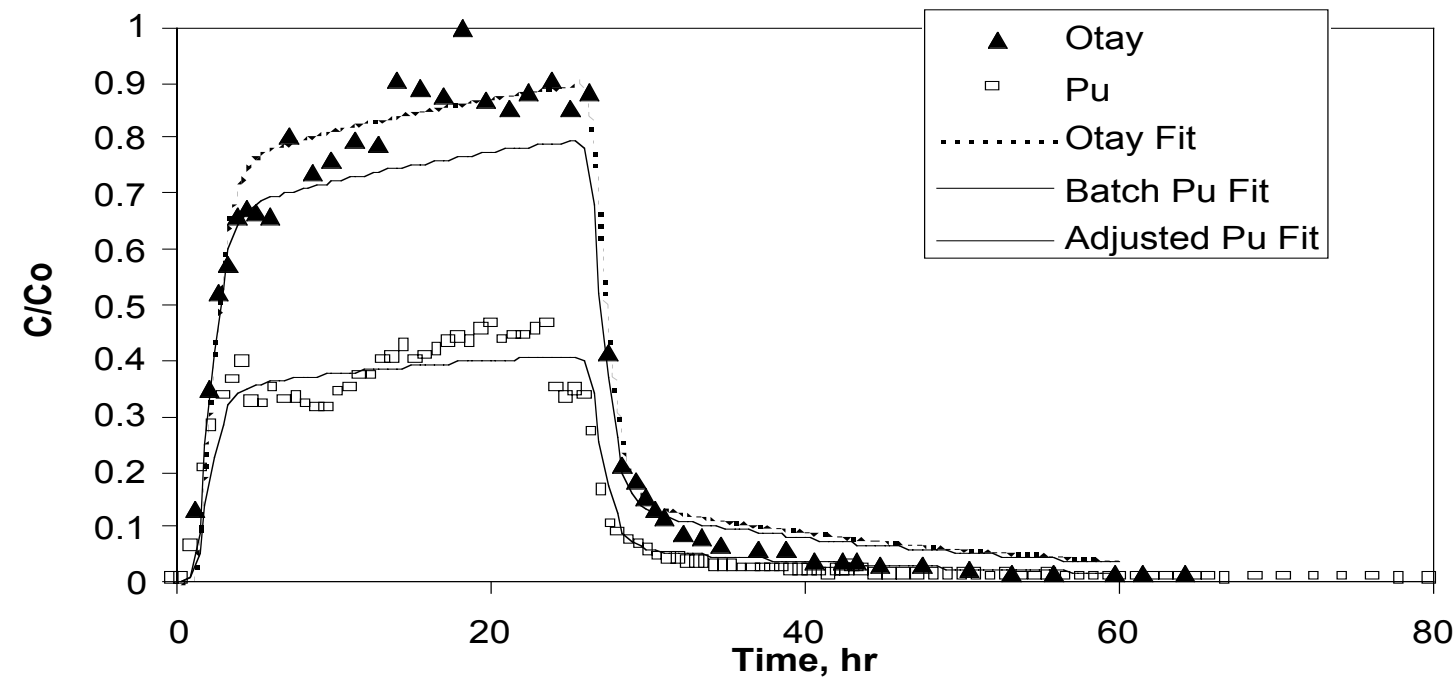

Figure F.21. Model simulations of batch 1 Otay montmorillonite and $\mathrm{Pu}$ responses in the PM-2, 4177 fracture as a function of time at $\sim 1.5 \mathrm{ml} / \mathrm{hr}$ in Experiment 11. 


\title{
-Appendix G - Thermodynamic Data Used to fit Pu Sorption Data
}

\author{
Mavrik Zavarin
}

$\mathrm{Pu}$ speciation information was gathered from a variety of sources for use in modeling the sorption of $\mathrm{Pu}(\mathrm{IV})$ and $\mathrm{Pu}(\mathrm{V})$ discussed in Chapters 4 and 5. References are all cited in Chapter 4. The Pu speciation information is described below. Aqueous speciation of major cations and anions was based on the GEMBOCHS thermodynamic data base version data.com.V8.R6 (Johnson and Lundeen, 1997).

Table G.1 contains the complexation constants used for Pu speciation. The majority of the data was taken from the recent Nuclear Energy Agency (NEA) volume on the chemical thermodynamics of plutonium. However, some additional aqueous species are included in Table G.1 based on fits to amorphous $\mathrm{PuO}_{2}$ solubility data reported in Rai et al. (1999). These data were not included in the NEA volume but the aqueous plutonium carbonate species evaluated based on these data are critical to our evaluation of $\mathrm{Pu}(\mathrm{IV})$ sorption. A short description of these data fits is described below.

Rai et al. (1999) examined the solubility of amorphous $\mathrm{PuO}_{2}$ across a range of $\mathrm{pH}$ and carbonate alkalinity conditions. Between $\mathrm{pH} 8$ and 13, the solubility of amorphous $\mathrm{PuO} 2$ remained constant, with an average aqueous $\mathrm{Pu}(\mathrm{IV})$ concentration of $10^{-10.4} \mathrm{~mol} / \mathrm{L}$. This is in reasonable agreement with the thermodynamic data reported in Table G.1 $\left(10^{-10.87}\right.$ $\mathrm{mol} / \mathrm{L})$. Upon the addition of carbonate ions in solution, the solubility of amorphous $\mathrm{PuO}_{2}$ increases dramatically, suggesting the $\mathrm{Pu}(\mathrm{IV})$-carbonate complexation in solution significantly affects $\mathrm{PuO}_{2}$ solubility. Rai et al. (1999) fitted $\mathrm{Pu}(\mathrm{IV})$ solubility in $\mathrm{KHCO}_{3}$ (with activity correction based on the Pitzer model) using two aqueous $\mathrm{Pu}(\mathrm{IV})$-carbonate species: $\mathrm{Pu}(\mathrm{OH})_{2}\left(\mathrm{CO}_{3}\right)_{2}{ }^{2-}$ and $\mathrm{Pu}\left(\mathrm{CO}_{3}\right)_{5}{ }^{6-}$. We re-fit this data (with activity correction based on the b-dot model) using the amorphous $\mathrm{PuO}_{2}$ solubility product reported in Lemire et al. (1999) which resulted in nearly the same reaction constants (see Figure G.1); these reaction constants are listed in Table G.1.

Rai et al. (1999) also examined amorphous $\mathrm{PuO}_{2}$ solubility in $0.01 \mathrm{~mol} / \mathrm{L} \mathrm{KOH}$ as a function of $\mathrm{K}_{2} \mathrm{CO}_{3}$ concentration. These data were not fitted but suggested that a third $\mathrm{Pu}(\mathrm{IV})$-carbonate species may prevail at high $\mathrm{pH}$. We were able to fit this amorphous $\mathrm{PuO}_{2}$ solubility data using a $\mathrm{Pu}(\mathrm{OH})_{4}\left(\mathrm{CO}_{3}\right)_{2}{ }^{4-}$ aqueous species (Figures G.2 and G.3). Only the $<1 \mathrm{~mol} / \mathrm{L} \mathrm{K} 2 \mathrm{CO} 3$ data were fit since the b-dot activity correction model is unreliable at higher ionic strengths. The 9 day (Figure G.2) and 49 day (Figure G.3) equilibrium solubility data resulted in $\mathrm{Pu}(\mathrm{OH})_{4}\left(\mathrm{CO}_{3}\right)_{2}{ }^{4-}$ reaction $\log \mathrm{K}$ constants of 25.66 and 25.39; the average is reported in Table G.1. Additional evidence for the existence of this species comes from the data of Yamaguchi et al. (1994). Their analysis of amorphous $\mathrm{PuO} 2$ solubility at carbonate/bicarbonate concentrations up to 0.1 molar suggested two dominant $\mathrm{Pu}(\mathrm{IV})$-carbonate species: $\mathrm{Pu}(\mathrm{OH})_{2}\left(\mathrm{CO}_{3}\right)_{2}{ }^{2-}$ and $\mathrm{Pu}(\mathrm{OH})_{4}\left(\mathrm{CO}_{3}\right)_{2}{ }^{4-}$. We refit these data based on the using the amorphous $\mathrm{PuO}_{2}$ solubility product reported in Lemire et al. (1999) which resulted in log K constants of 0.74 and 24.3, respectively (Figures G.4 and G.5). These fitted constants are 2.0 and $1.2 \log \mathrm{K}$ 
lower that those based on the data of Rai et al. (1999). While the difference is significant, it should not be surprising since the speciation is based relative to the solubility product of amorphous $\mathrm{PuO}_{2}$. The variability of amorphous $\mathrm{PuO}_{2}$ solubility imparts significant uncertainty to the aqueous species reaction constants discussed here. Nevertheless, the solubility data of Rai et al. (1999) and Yamaguchi et al. (1994) clearly show that carbonate complexation will increase the solubility of amorphous $\mathrm{PuO}_{2}$ at carbonate concentrations as low as $10^{-3} \mathrm{~mol} / \mathrm{L}$. Since the carbonate alkalinity of J-13 type waters is $\sim 10^{-2.7} \mathrm{~mol} / \mathrm{L}$, the results suggest that $\mathrm{Pu}(\mathrm{IV})$-carbonate complexation will be significant at the NTS.

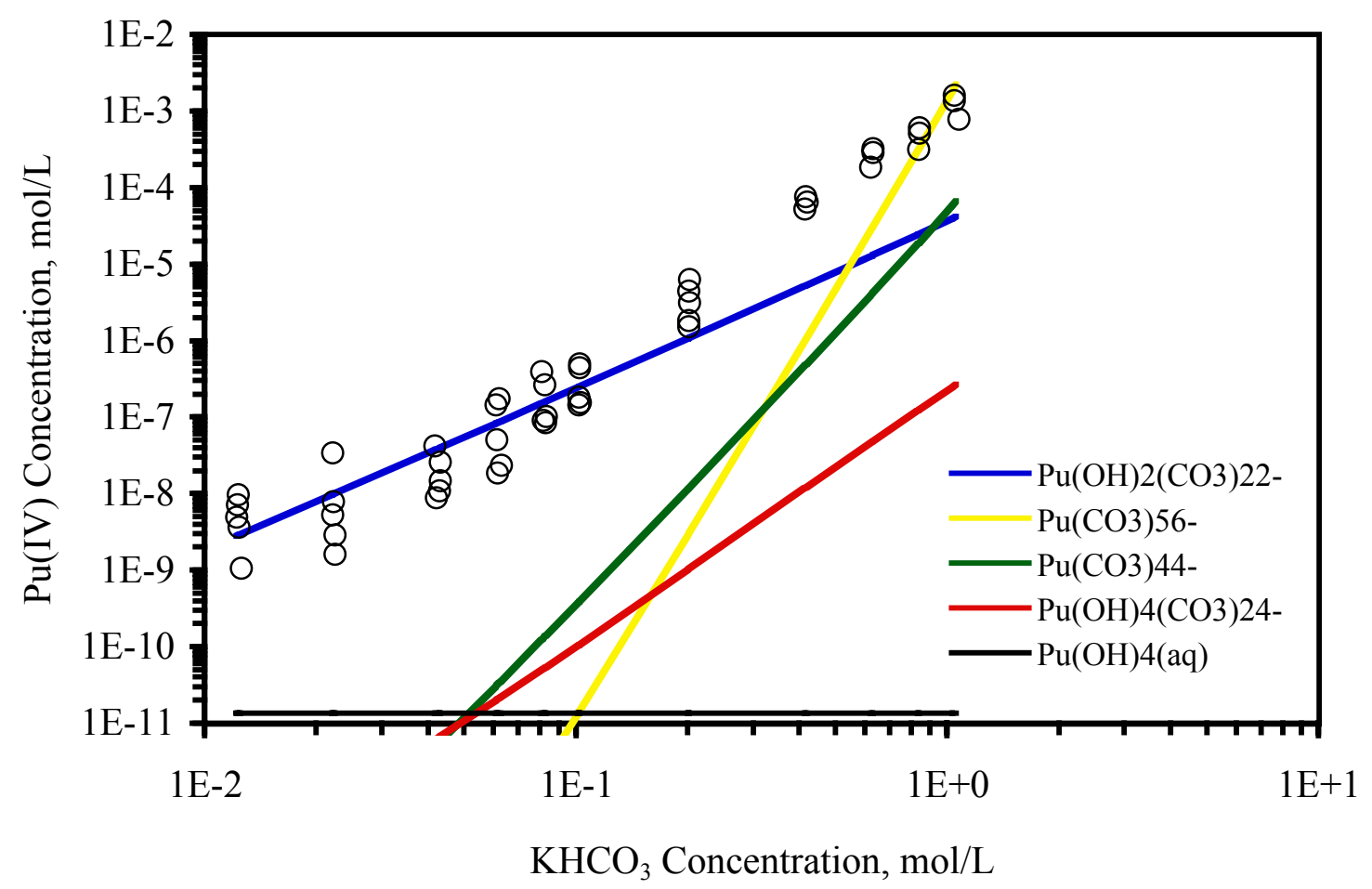

Figure G.1. Fit to amorphous $\mathrm{PuO}_{2}$ solubility as a function of $\mathrm{KHCO}_{3}$ concentration. Solubility experiments performed from oversaturation and undersaturation and 5 to 51 day equilibration time. Data of Rai et al. (1999). 


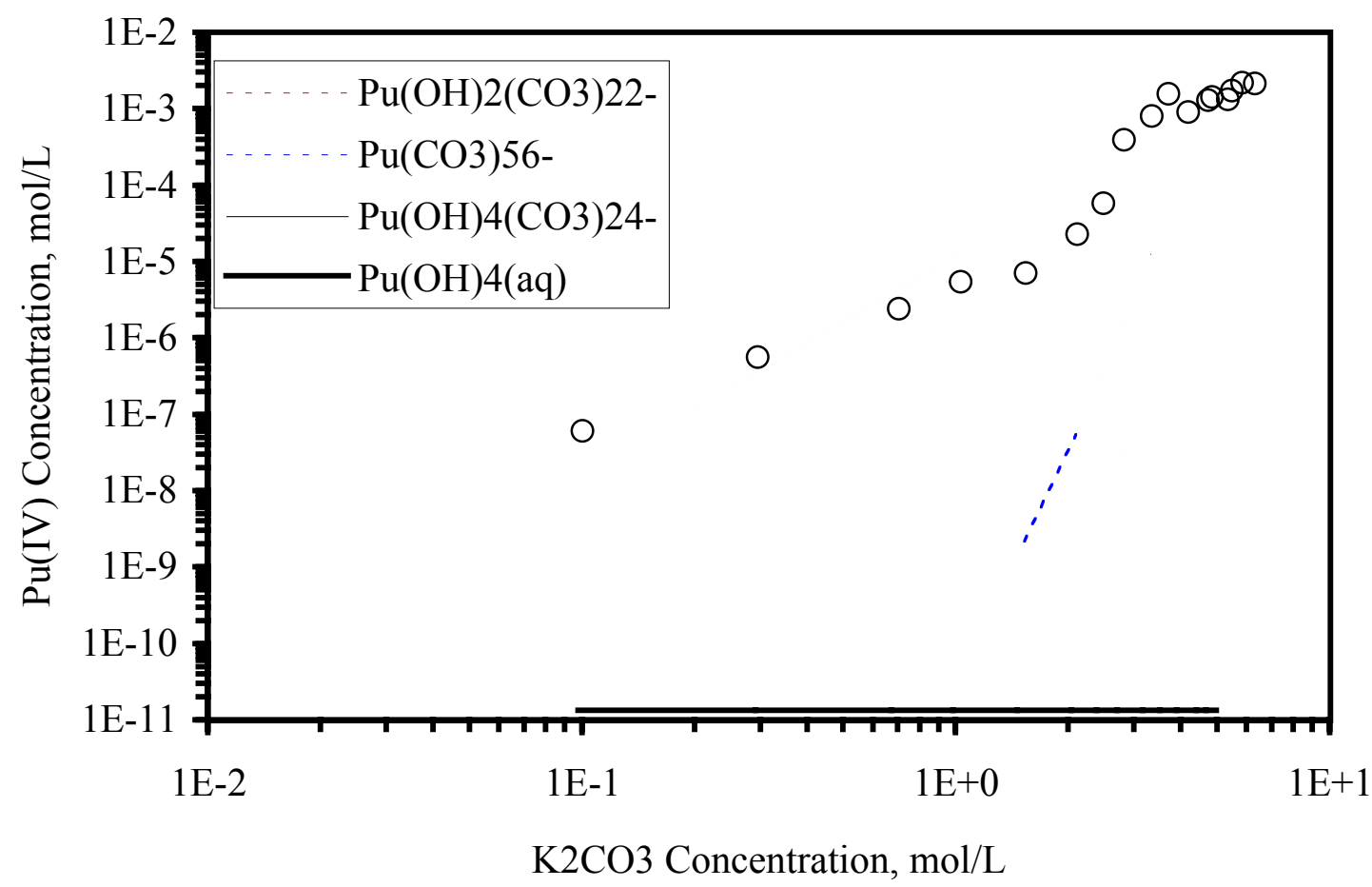

Figure G.2. Fit to amorphous $\mathrm{PuO}_{2}$ solubility as a function of $\mathrm{K}_{2} \mathrm{CO}_{3}$ concentration in $0.01 \mathrm{~mol} / \mathrm{L} \mathrm{KOH}$ solution. Solubility experiments performed from oversaturation and 9 day equilibration time. Data of Rai et al. (1999). Only $<1 \mathrm{~mol} / \mathrm{L} \mathrm{K}_{2} \mathrm{CO}_{3}$ data fitted.

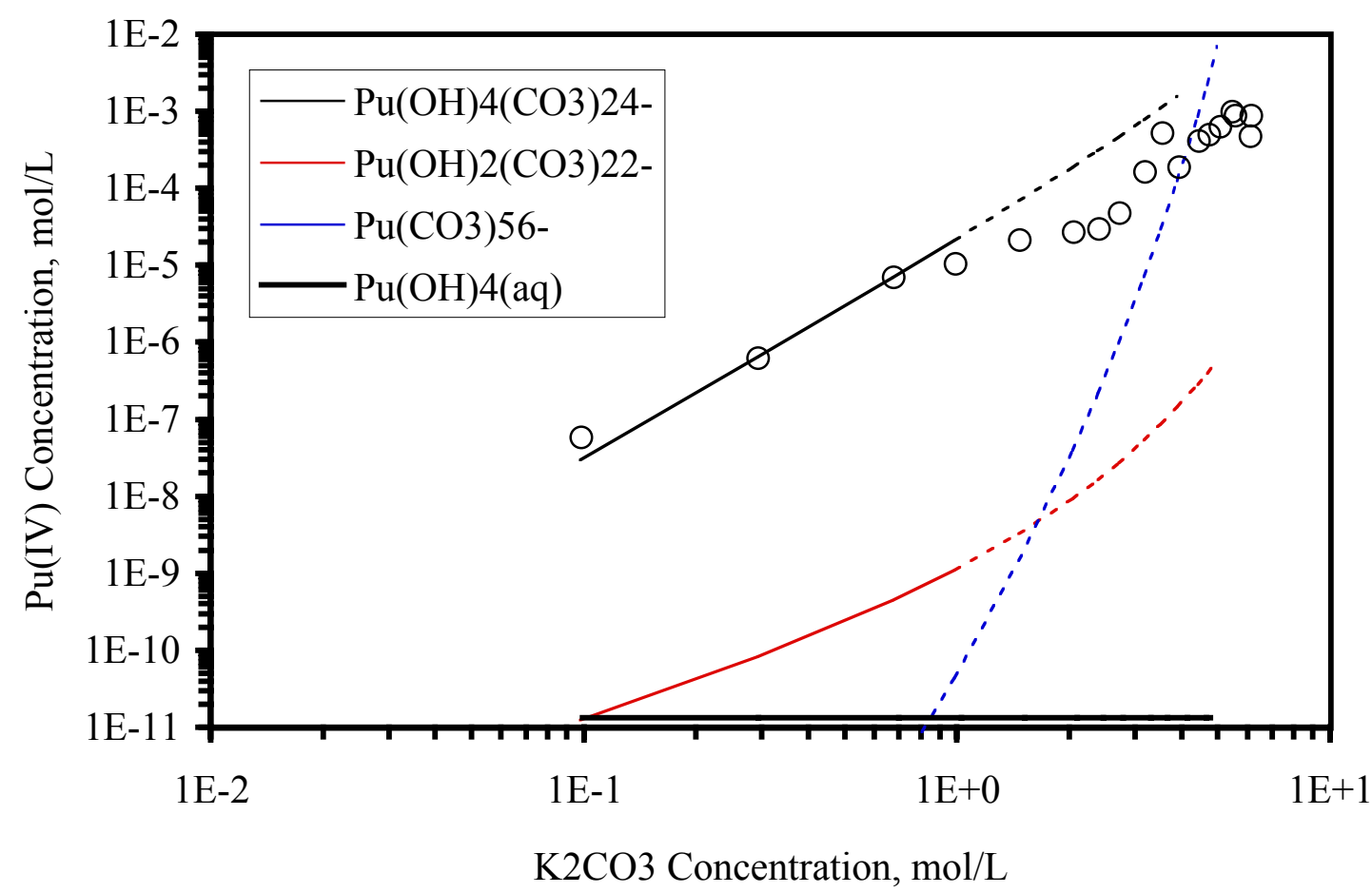

Figure G.3. Fit to amorphous $\mathrm{PuO}_{2}$ solubility as a function of $\mathrm{K}_{2} \mathrm{CO}_{3}$ concentration in $0.01 \mathrm{~mol} / \mathrm{L} \mathrm{KOH}$ solution. Solubility experiments performed from oversaturation and 49 day equilibration time. Data of Rai et al. (1999). Only $<1 \mathrm{~mol} / \mathrm{L} \mathrm{K}_{2} \mathrm{CO}_{3}$ data fitted. 


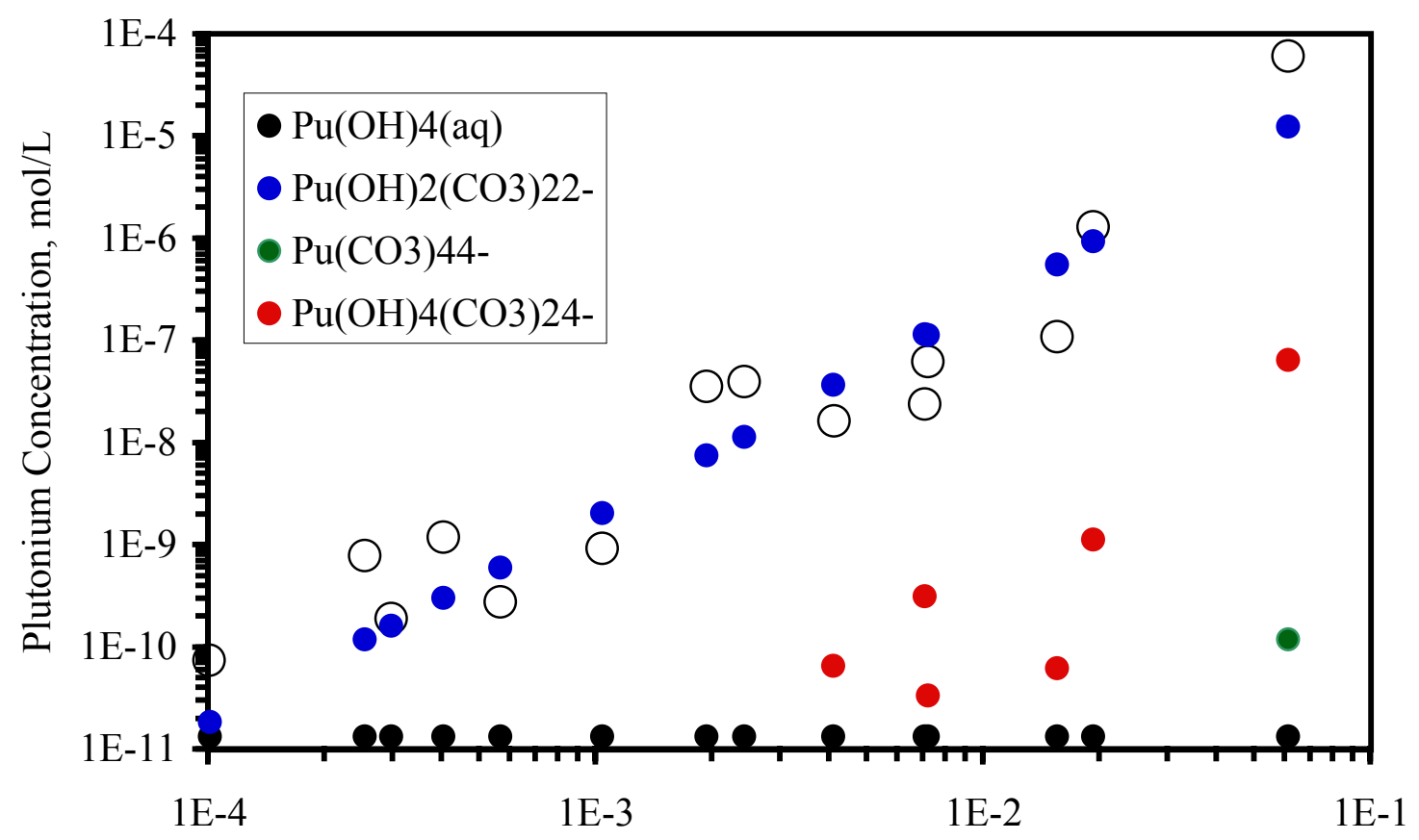

Bicarbonate Concentration, $\mathrm{mol} / \mathrm{L}$

Figure G.4. Thermodynamic data used to fit our experimental data

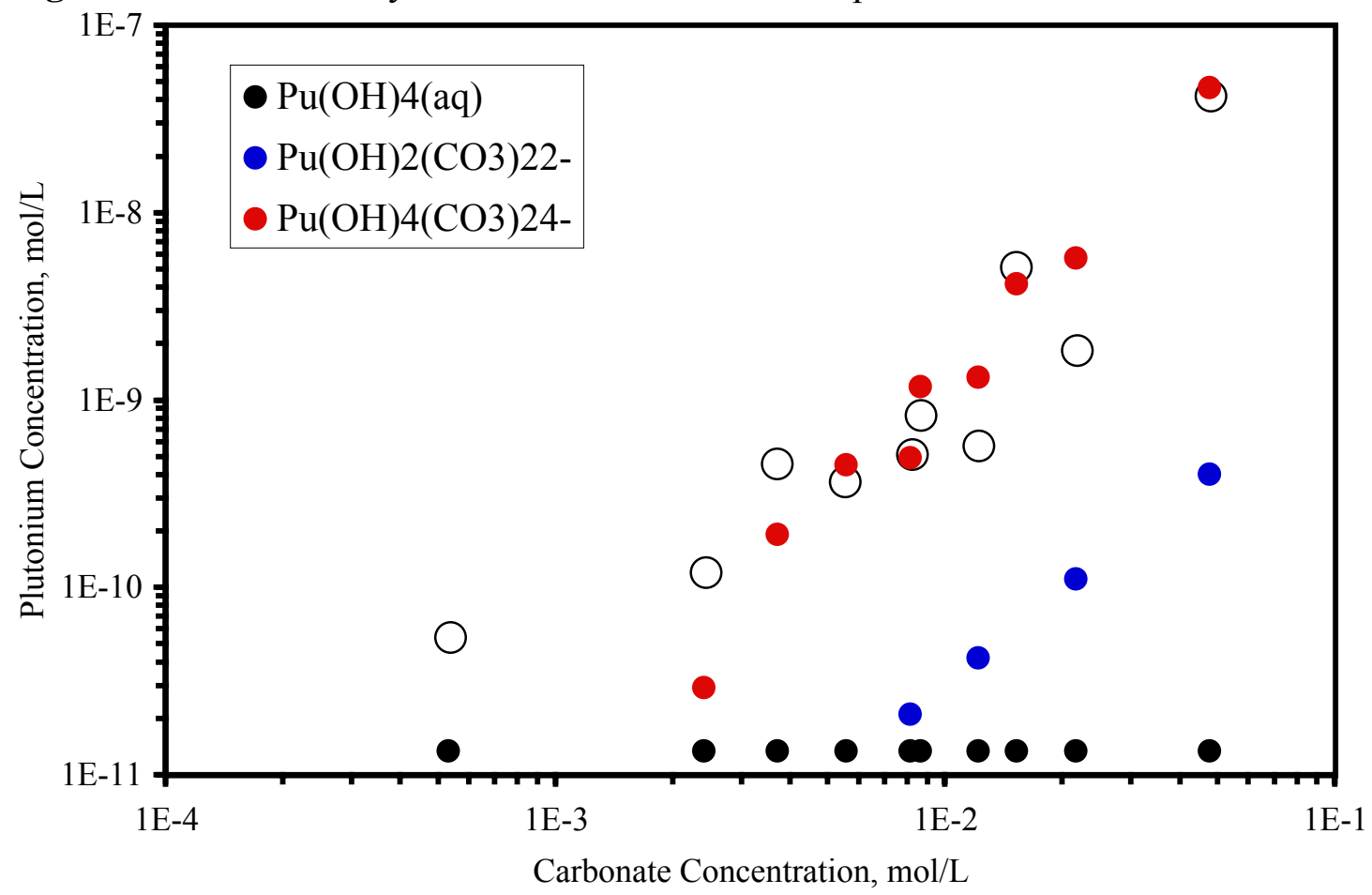

Figure G.5. Fit to amorphous $\mathrm{PuO}_{2}$ solubility as a function of $\mathrm{CO}_{3}^{2-}$ concentration. Open circles $=$ data, filled circles $=$ individual species contributions to fit. The $\mathrm{pH}$ varies from 12 to 13 in these samples. Data of Yamaguchi et al. (1994). Best fit reaction constants reported in text. 
Table G.1. Species used in surface complexation fits.

\begin{tabular}{|c|c|c|c|c|c|c|c|c|c|c|}
\hline Species & $\log \mathrm{K}$ & \# & Component & \# & Component & $\#$ & Component & \# & Component & Reference \\
\hline $\mathrm{Pu}^{3+}$ & 3.74 & -1 & $\mathrm{H}^{+}$ & -0.25 & $\mathrm{O}_{2}(\mathrm{aq})$ & $\begin{array}{c}0 . \\
5\end{array}$ & $\mathrm{H}_{2} \mathrm{O}$ & 1 & $\mathrm{Pu}^{4+}$ & 1 \\
\hline \multicolumn{11}{|l|}{$\begin{array}{l}\mathrm{Pu}^{4+} \\
\text { (primary species) }\end{array}$} \\
\hline $\mathrm{PuO}_{2}^{+}$ & -4.16 & -3 & $\mathrm{H}^{+}$ & 0.25 & $\mathrm{O}_{2}(\mathrm{aq})$ & $\begin{array}{r}1 . \\
5\end{array}$ & $\mathrm{H}_{2} \mathrm{O}$ & 1 & $\mathrm{Pu}^{4+}$ & 1 \\
\hline $\mathrm{PuO}_{2}^{2+}$ & -9.84 & -2 & $\mathrm{H}^{+}$ & 0.5 & $\mathrm{O}_{2}(\mathrm{aq})$ & 1 & $\mathrm{H}_{2} \mathrm{O}$ & 1 & $\mathrm{Pu}^{4+}$ & 1 \\
\hline $\begin{array}{l}\mathrm{PuO}_{2} \\
\text { (amorphous) }\end{array}$ & -2.02 & -4 & $\mathrm{H}^{+}$ & 2 & $\mathrm{H}_{2} \mathrm{O}$ & 1 & $\mathrm{Pu}^{4+}$ & & & 1 \\
\hline $\mathrm{PuOH}^{2+}$ & 6.9 & -1 & $\mathrm{H}^{+}$ & 1 & $\mathrm{H}_{2} \mathrm{O}$ & 1 & $\mathrm{Pu}^{3+}$ & & & 1 \\
\hline $\mathrm{PuOH}^{+3}$ & -0.5 & -1 & $\mathrm{H}^{+}$ & 1 & $\mathrm{H}_{2} \mathrm{O}$ & 1 & $\mathrm{Pu}^{+4}$ & & & 1 \\
\hline $\mathrm{Pu}(\mathrm{OH})_{2}^{+2}$ & 1.66 & -2 & $\mathrm{H}^{+}$ & 1 & $\mathrm{Pu}^{+4}$ & 2 & $\mathrm{H}_{2} \mathrm{O}$ & & & 2 \\
\hline $\mathrm{Pu}(\mathrm{OH})_{3}^{+}$ & 4.62 & -3 & $\mathrm{H}^{+}$ & 1 & $\mathrm{Pu}^{+4}$ & 3 & $\mathrm{H}_{2} \mathrm{O}$ & & & 2 \\
\hline $\mathrm{Pu}(\mathrm{OH})_{4}(\mathrm{aq})$ & 8.85 & -4 & $\mathrm{H}^{+}$ & 1 & $\mathrm{Pu}^{+4}$ & 4 & $\mathrm{H}_{2} \mathrm{O}$ & & & 2 \\
\hline $\mathrm{Pu}(\mathrm{OH})_{2}\left(\mathrm{CO}_{3}\right)_{2}^{-2}$ & 2.75 & -4 & $\mathrm{H}^{+}$ & 2 & $\mathrm{HCO}_{3}^{-}$ & 1 & $\mathrm{Pu}^{+4}$ & 2 & $\mathrm{H}_{2} \mathrm{O}$ & 3 \\
\hline $\mathrm{Pu}(\mathrm{OH})_{4}\left(\mathrm{CO}_{3}\right)_{2}{ }^{4-}$ & 25.53 & -6 & $\mathrm{H}^{+}$ & 4 & $\mathrm{H}_{2} \mathrm{O}$ & 2 & $\mathrm{HCO}_{3}^{-}$ & 1 & $\mathrm{Pu}^{4+}$ & 3 \\
\hline $\mathrm{Pu}\left(\mathrm{CO}_{3}\right)_{5}^{-6}$ & 16.3 & -5 & $\mathrm{H}^{+}$ & 5 & $\mathrm{HCO}_{3}^{-}$ & 1 & $\mathrm{Pu}^{+4}$ & & & 3 \\
\hline $\mathrm{Pu}\left(\mathrm{CO}_{3}\right)_{4}^{-4}$ & 4.62 & -4 & $\mathrm{H}^{+}$ & 4 & $\mathrm{HCO}_{3}^{-}$ & 1 & $\mathrm{Pu}^{+4}$ & & & 4 \\
\hline $\mathrm{PuO}_{2} \mathrm{OH}(\mathrm{aq})$ & 9.73 & -1 & $\mathrm{H}^{+}$ & 1 & $\mathrm{H}_{2} \mathrm{O}$ & 1 & $\mathrm{PuO}_{2}^{+}$ & & & 1 \\
\hline $\mathrm{PuO}_{2} \mathrm{CO}_{3}^{-}$ & 5.21 & -1 & $\mathrm{H}^{+}$ & 1 & $\mathrm{HCO}_{3}^{-}$ & 1 & $\mathrm{PuO}_{2}^{+}$ & & & 1 \\
\hline $\mathrm{PuO}_{2}\left(\mathrm{CO}_{3}\right)_{3}^{5-}$ & 26.0 & -3 & $\mathrm{H}^{+}$ & 3 & $\mathrm{HCO}_{3}^{-}$ & 1 & $\mathrm{PuO}_{2}^{+}$ & & & 1 \\
\hline $\mathrm{PuO}_{2} \mathrm{OH}^{+}$ & 5.5 & -1 & $\mathrm{H}^{+}$ & 1 & $\mathrm{H}_{2} \mathrm{O}$ & 1 & $\mathrm{PuO}_{2}^{2+}$ & & & 1 \\
\hline $\mathrm{PuO}_{2}(\mathrm{OH})_{2}(\mathrm{aq})$ & 13.2 & -2 & $\mathrm{H}^{+}$ & 2 & $\mathrm{H}_{2} \mathrm{O}$ & 1 & $\mathrm{PuO}_{2}{ }^{2+}$ & & & 1 \\
\hline $\mathrm{PuO}_{2} \mathrm{CO}_{3}(\mathrm{aq})$ & -1.27 & -1 & $\mathrm{H}^{+}$ & 1 & $\mathrm{HCO}_{3}^{-}$ & 1 & $\mathrm{PuO}_{2}^{2+}$ & & & 1 \\
\hline $\mathrm{PuO}_{2}\left(\mathrm{CO}_{3}\right)_{2}^{2-}$ & 6.16 & -2 & $\mathrm{H}^{+}$ & 2 & $\mathrm{HCO}_{3}^{-}$ & 1 & $\mathrm{PuO}_{2}^{2+}$ & & & 1 \\
\hline $\mathrm{PuO}_{2}\left(\mathrm{CO}_{3}\right)_{3}{ }^{4-}$ & 13.3 & -3 & $\mathrm{H}^{+}$ & 3 & $\mathrm{HCO}_{3}^{-}$ & 1 & $\mathrm{PuO}_{2}^{2+}$ & & & 1 \\
\hline
\end{tabular}

1: Lemire et al., 2001; 2: Lemire and Tremaine, 1980; 3: Refitted Rai et al., 1999 data; 4:

Lemire et al., 2001 combined with fitted data of Rai et al., 1999. 